RER:IVED

FFR 141997

LA-54B--93-8)

1988 ANNUAL REPORT

Volume VI

$1 A-54 B-.93-81$

PROCESSED FROM BEST AVAILABLE COPY

North Carolina State University
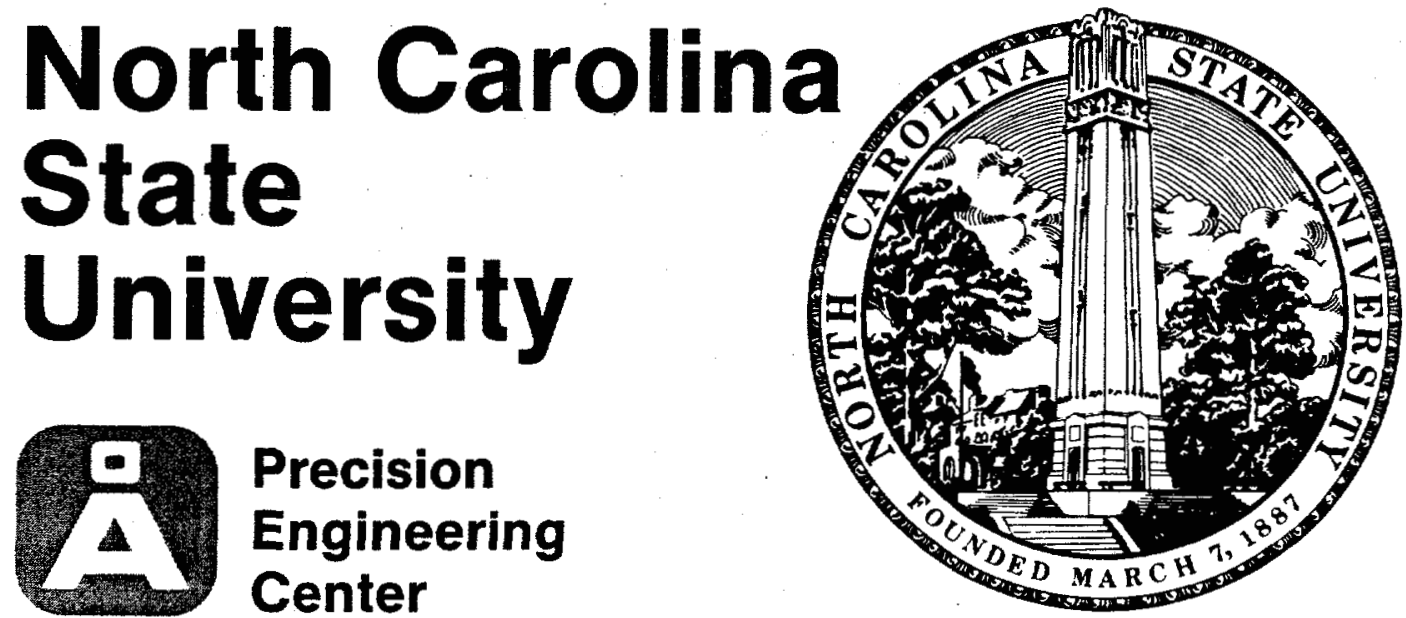

DISTRBBUTON OF THIS DOCUMENT IS UNLRATIED

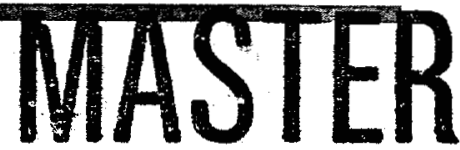




\section{$L A-54 B--93-8$ ) \\ PRECISION ENGINEERING CENTER}

\section{ANNUAL REPORT}

\section{Volume VI}

Office of Naval Research

IBM Corporation

Los Alamos National Laboratory

Moore Special Tool Company

Texas Instruments, Inc.

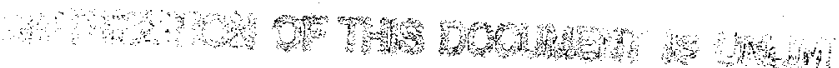

Sponsored by:
Eastman Kodak Company

Lawrence Livermore National Laboratory

Martin Marietta Corporation

Rank Pneumo, Inc.

3M Corporation

\section{Editor}

Thomas A. Dow

Director

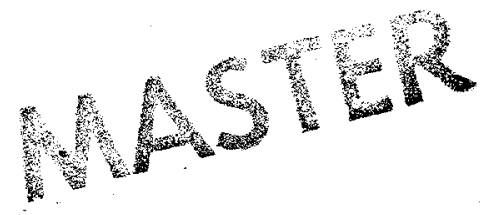

PROCESSED FROM BEST AVAILABLE COPY
Scott Blackley

William Enloe

David Grigg

Michael Loewenthal

Dan Luttrell

John Pellerin

Elizabeth Smith

Ronald Sparks

Authors

PROCESSED FROM BEST AVAILABLE COPY

Graduate Students:

Robert Day

Karl Falter

Gary Hiatt

Kyle Loseke

Hakan Ozisik

Gordon Shedd

Mary Beth Smith

Michael van Haaren
Joseph Drescher

Steve Fawcett

Byron Knight

Michael Luh

Harlow Paul

Denise Skroch

Stan Smith

\section{PRECISION ENGINEERING CENTER STAFF}

Kenneth P. Garrard Martha U. Neeley

Cheryl K. Reynolds Anne Marie Smith

Lauren Taylor 
DISCLAMMER

Portions of this document may be illegible in electronic image products. Images are produced from the best available original document. 


\section{CONTENTS}

SUMMARY 1

MEASUREMENT AND ACTUATION

1 Design and Calibration Of A Scanning 11

Tunneling Microscope for Large

Machined Surfaces

2 Atomic Resolution Images of Graphite $\quad 27$

In Air

3 Atomic Force Microscope $\quad 33$

4 Measuring Depth Profiles of Residual 47

Stress With Raman Spectroscopy

5 Mapping Residual Stress Fields From 61

Vickers Hardness Indents Using

Raman Microprobe Spectroscopy

6 Pressure Induced Phase Transitions

In Ceramic Compounds Containing

Tetragonal Zirconia

7 Structural Response Synthesis

8 Structural Power Flow Measurement $\quad 87$

REAL-TIME CONTROL

9 Diamond Turning Machine Controller 99

Implementation

10 A Heterogeneous Hierarchical Architecture 117

For Real Time Computing

11 Multiprocessor Programming Environment 
12 Fabrication of Brittle Materials

- Current Status

13 Diamond Turning of $\mathrm{Si}$ and Ge Single Crystals

14 Diamond Turning of Thermoplastic

Polymers

15 Diamond Turning of Glass

16 Precision Diamond Grinding of 173 Ceramics and Glass

17 The Effects of Machine Parameters on Residual Stress Determined Using Micro-Raman Spectroscopy

18 Atomic Structure of Machined Semiconducting Chips: An X-Ray Absorption Spectroscopy Study

19 Observation of Diamond Turned OFHC Copper Using Scanning Tunneling Microscopy

20 Analysis of The Influence of Tool 207 Dynamics in Diamond Turning

21 Measurement of Tool Forces 223 in Diamond Turning

22 Simulations of Ductile Flow In Brittle Material Processing

23 Fracture Mechanics Applied to 257 The Machining of Brittle Materials 
25 Development of A Focused Ion

Beam Micromachining System

26 Nanofabrication With The

Scanning Tunneling Microscopy

TECHNOLOGY TRANSFER 


\section{MANAGEMENT SUMMARY}

\section{BACKGROUND}

To reverse the downward trend in the balance of trade, American companies must concentrate on increasing research into new products, boosting productivity, and improving manufacturing processes. The Precision Engineering Center at North Carolina State University is a multi-disciplinary research and graduate education program dedicated to providing the new technology necessary to respond to this challenge. One extremely demanding manufacturing area is the fabrication and assembly of optical systems. These systems are at the heart of such consumer products as cameras, lenses, copy machines, laser bar-code scanners, VCRs, and compact audio discs - products that the Japanese and other East Asian countries are building dominance. A second critical area is the fabrication of VLSI and ULSI circuits. The tolerances required to produce the next generation of components for such systems have created the need for new approaches - approaches that could either make or break America's competitive position.

Precision Engineering involves striving to improve the precision - the accuracy and repeatability - of a mechanism or process. It has been defined as painstaking attention to detail, and thus is not so much a technique or process as it is an attitude. However, such attention to detail often requires a multi-disciplinary approach because errors may be caused by thermal effects, mechanical distortions, misalignment, material variations, or wear.

Progress in precision manufacturing has been largely due to improvements in metrology. The ability to understanding a mechanism or process is predicated on the capability to measure shape, surface finish, temperature, motion, or material properties. Modern sensors take advantage of a variety of physical phenomena and provide the ability to see and comprehend at a level not previously available. For example, the scanning tunneling microscope (STM) allows a surface to be mapped at a scale the size of a molecule, and Raman spectroscopy creates the possibility of non-destructive measurement of surface residual stresses.

If the ability to measure forms the foundation for Precision Engineering, the computer is the superstructure. Current manufacturing processes rely on the mechanical structure of the machine tool to provide accuracy and repeatability in the finished product. However, the intelligence of modern computers, combined with metrology systems designed to measure the status of the product and/or the process, creates the potential for real-time control to realize greater precision in the resulting components.

The challenge for improving the precision of fabricated components is to integrate the measurement function into the manufacturing process. A group of faculty interested in studying the issues involved with this integration have created the Precision Engineering 
The education aspect of the program is also important. Industry has shown a keen interest in the Precision Engineering Center graduates because of their multidisciplinary background. These graduates, along with the technology developed, will strengthen the country's competitive position in the high technology area.

\section{FACILITIES}

The Precision Engineering Center began in the corner of a heat transfer laboratory in 1982, while a $1500 \mathrm{ft}^{2}$ facility was being constructed. This facility consisted of a temperaturecontrolled laboratory, a workroom, a library/conference area, and offices for staff and several graduate students. During the month of February 1988, the Center moved to a new facility on the NCSU Centennial Campus. It now occupies a $11,000 \mathrm{ft}^{2}$ ground floor of the first research building on this new campus. The Precision Engineering Center facility was designed to provide the quality space necessary for optical and metrological research and fabrication. The facility includes four temperature-controlled and vibration-isolated laboratories ( $400 \mathrm{ft}^{2}$ each) as well as workrooms and office space for 24 graduate students, a staff of 5 , and the 7 faculty members involved with the Center.

The Center is equipped with state-of-the-art measurement computational and fabrication facilities including Talysurf 4, Talystep, and Zygo Maxim 3D surface profilometers, scanning tunneling microscopes, vibration isolated tables, digital storage oscilloscopes, a dual-channel FFT spectrum analyzer, PDP-11 and MassComp data acquisition systems, a high-speed digital controllers based on Intel and Texas Instruments microprocessors, linear and phase-measuring interferometry systems, a Zeiss metalographic microscope, a long range microscope with video camera, several types of piezoelectric (PZT) actuators and amplifiers, and a full complement of sensors including capacitance gages, eddy current sensors, linear variable differential transformers, and accelerometers. In addition to this metrology equipment the Center has also purchased or built laboratory-scale and production-scale diamond turning machines, grinding apparatus, elastic emission polisher, and a computer-controlled focused ion beam machining system.

The Center has a cluster of three networked DEC Micro-Vax computers which are also linked to the campus computer network. This network connects the Departmental and University computers and includes links to the major national computer networks through the Triangle University's Computer Center (TUCC). Other mainframe computers available at TUCC are an IBM 3083, an IBM 4341, and an FPS 164.

A variety of facilities are located at North Carolina State University and available to the faculty and students. These include an electron microscope facility (SEM, TEM, X-ray analyzer, ion beam microprobe) and a microelectronics facility for the production of unique semiconductors and gate array chips. Instrumentation at the Microelectronics Center of North Carolina including a state-of-the-art SEM has also been made available for the research program. 


\section{TECHNICAL PROGRAM REVIEW}

\section{Measurement and Actuation}

The concept of real-time, closed-loop feedback control of a manufacturing process depends heavily on sensors for error or motion detection or measurement of surface characteristics (reflectivity or residual stresses). In addition to real-time control information, sensors such as the Scanning Tunneling Microscope (STM) and Raman Spectrometer allow one to view the result of a fabrication process in detail to corroborate theoretical models. Once a desired response has been determined the actuator/structure must change the process in a rapid, controlled fashion. A summary of the progress made over the past year in the areas of measurement sensors and actuation is presented below.

Scanning Tunneling Microscope A major effort has been undertaken to develop the STM as a precision profilometer. The principal advantage of this device is the excellent horizontal resolution-several orders of magnitude better than such profilometers as the Rank Talystep or Wyco Topo-2D. The sensitivity of the STM is related to its operating principal but it can lend to phantom features if the shape of the scanning tip are not well controlled. This is particularly true for the non-atomically smooth surfaces resulting from precision fabrication processes. The principal limitation of the STM for surface profile studies is the small area of measurement. The largest scan currently possible is $3 \mu \mathrm{m}$ and many surface features are larger than this. However, the exceptional horizontal magnification allows the high frequency fractures to be effectively imaged.

- Design and Calibration - To measure machined fractures from turning, grinding, and polishing operations, a STM for large surfaces has been designed, fabricated, and calibrated. The piezoelectric tube used for the 3-dimensional scans has been calibrated by capacitance and other gages as well as corrected for non-linear effects due to hysteresis.

- Atomic Resolution Images - The original STM built in the Center has achieved atomic-scale resolution images in air during the past year. An image of a graphite surface is presented and discussed in light of the theoretical model of the atomic structure.

- Atomic Force Microscope - If the superb resolution of the STM could be extended to non-conducting materials, then many important optical materials could be studied. A preliminary design for such an instrument is presented along with development plans.

Raman Spectroscopy There are several optical techniques which can provide information about the atomic structure of a material. The Raman effect is strong in most 
semiconductor materials and can provide residual stress information from machined surfaces.

- Stress Depth Profiles - While Raman measurements are routinely made with a lateral resolution of $1 \mu \mathrm{m}$, the variation of stress with depth has not received much attention. A novel technique has been developed that allows quantitative measurement of the variation of the residual stress with depth resolution of $10 \mathrm{~nm}$.

- Mapping the Residual Stress Field - The measurements provided by the Raman technique are related to the average stress throughout the measurement volume, but the direction of this stress would be valuable addition to lead the theoretical development of the model. The orientation of the laser light's polarization is changed and the resulting Raman scattered light is being analyzed to study the orientation of the surface stress components. To develop the technique, Micro-Raman spectroscopy is being used to map the well defined residual stress field caused by a Vicker's hardness indenter.

Structural Dynamics Controlling the dynamic response of a machine structure is an important challenge in the goal of real-time control.

- Structural Response Synthesis - Closed loop control techniques depend upon prior knowledge of the structure to design a filter which can avoid the resonances and control the response. Input signal tailoring can also be used in a similar manner leading to the possibility of more rapid response. Differences between this open-loop technique and digital, closed-loop control are discussed.

- Structural Power Flow - The measurement of power flow is a potentially powerful method of machine analysis. Over the past year, techniques for such measurements have been refined and a transfer function correction technique has been employed to compensate for instrumentation errors. Unfortunately, power flow measurements are more applicable to beam-like structures which are seldom used in machine tool design. Therefore, the usefulness of this technique is severely limited.

\section{Real Time Control}

There are two complementary approaches to the elimination of errors in a mechanical systems. The first relies on the reduction of errors by eliminating their sources, and using such techniques, impressive precision is obtainable in machining operations. A second approach involves closed-loop feedback control of the process. The rationale behind closedloop control is that there are some errors which cannot be effectively eliminated at their source or are from unknown sources. A combination of closed-loop control with careful 
elimination of errors will result in a system with better precision than that obtainable by applying either approach independently.

The emphasis during the past year has been divided into two thrusts:

1. Application of commercial computer hardware to the control of the diamond turning machine and the development of software to control this fabrication process and,

2. Design, prototype development, and programming of a new multi-processor computer designed for high-speed real-time control applications.

Diamond Turning Machine Controller A custom high-performance controller has been applied to the DTM. The details of the computer hardware and the software developed for machine control are discussed.

H$^{2}$ ART A new multi-processor design named Heterogeneous Hierarchial Architecture for Real Time has been implemented over the past year. The architecture, operating system, and programming language are discussed as well as the reduced interprocessor communication time resulting from this unique hardware arrangement.

Programming Multiprocessor Systems The focus of this project is to provide new tools and techniques by which programmers with experience on single processor systems can make the transition to multi-processor systems. A discussion of languages and compilers developed for $\mathrm{H}^{2} \mathrm{ART}$ are discussed.

\section{Fabrication Technology}

The goal of the Precision Engineering Center involves in-process control of precision fabrication processes. This is the rationale for research into sensors and control systems. However, these areas represent only part of the problem. A key question left unresolved is what parameter of the process should be changed to produce the desired results. The answer to this question requires a thorough understanding of the process and the influence of the available physical variables on the quality and dimensions of the product. Therefore, a crucial task of the Center is to study fabrication processes and build theoretical models of these processes to provide a basis for control strategies.

Fabrication of Brittle Materials A review of the Center's past programs related to fabrication of brittle materials is presented. This review summarizes the progress made to date and gives an agenda for continuing research. In addition, the details of several material studies are presented. 
- Diamond Turning of Si and Ge - A new process model is presented for diamond turning which is based on the critical depth of cut for plastic flow-to-brittle fracture transition. A procedure for interrupting the cutting process to measure the critical depth parameter is also described.

- Diamond Turning of Polymers - Diamond turning studies of a series of thermoplastic polymers has been completed. These materials had a range of glass transition temperatures and were selected as analogs for glass. Variations in surface morphology and roughness of the polymers were observed as a function of cutting speed, an observation that can be attributed to the material's response to shear hearing from the cutting process.

- Diamond Turning and Grinding of Glass - New research initiatives to study fabrication of brittle, amorphous materials have begun. These studies are designed to provide insight into the ductile-to-brittle transitions and to define operating regimes which control the surface damage. Material properties which relate to the surface damage such as crack toughness will be measured for several model brittle materials.

Surface Features of Machined Surfaces Several important new techniques are unveiled for the characterization of diamond turned surfaces. Raman Spectroscopy measures the change in residual stress that occurs as a function of tool rake angle in machined silicon and germanium. A technique using light of different wavelengths, drawing on the different penetration depths, allowed determination of this stress profile as a function of depth into the sample. $X$-ray Absorption Spectrum has been used to define the atomic structure of a collection of Ge machining chips. By comparing this XAS profile to that of the original Ge crystal signature and oxidized $\mathrm{Ge}$, an estimate of the maximum chip temperature can be made. The $S T M$ has been used to study the features and diamond turned surfaces. Some puzzling observations of these surfaces are presented as well as insight into the potential of the STM for high-resolution surface profilometry.

Dynamic Tool Forces Measurements of static and dynamic tool forces during diamond turning for a variety of materials are presented. The influence of machine resonances are discussed and a dynamic model of the tool/workpiece interface is presented. This model displays a surprising low interface stiffness. Three-dimensional force measurements are also reported for OFHC copper and 6061-T6 aluminum. A model including the effects of work hardening from previous cuts is outlined.

Simulation of the Machining Process Two papers are presented which describe the current status of the modeling effort. The first describes the thermal environment which exists in machining. Different tool and workpiece conductivities are modeled, the resulting temperature distributions are illustrated, and the potential impact on material flow is discussed. The second paper describes preliminary results of a fracture machanics model 
applied to machining of a brittle material. A comparison to the results of machining silicon indicates that the model may be useful in predicting material response.

Elastic Emission Polishing The results of an extensive series of experiments using this technique to polish brittle materials are presented. The quantitative results are similar to those published in the literature but the absolute magnitudes are an order of magnitude less. A plan for continued research is presented.

Micro and Nano Fabrication A focused ion beam machining system has been assembled over the past year including an ultra-high vacuum UHV system, a liquid metal ion gun, and a control and data acquisition computer system. This system is being used to study and predict the features produced by ion beam machining. As a companion project, a UHV Scanning Tunneling Microscope (STM) is being built to study the potential of the STM for the manipulation of nanometer-scale particles produced by the FIB source. The STM will be positioned directly beneath the ion beam and thus can take advantage of the long range imaging capability of the FIB which can, in turn, utilize the high-resolution of the STM for imaging micro machined features. 


\title{
1 DESIGN AND CALIBRATION OF A SCANNING TUNNELING MICROSCOPE FOR LARGE MACHINED SURFACES
}

\author{
David A. Grigg \\ Graduate Student \\ Phillip E. Russell \\ Associate Professor, MTE \\ Thomas A. Dow \\ Professor, MAE
}

During the last year the large sample STM has been designed, built and used for the observation of several different samples. Calibration of the scanners for proper dimensional interpretation of surface features has been a chief concern, as well as corrections for nonlinear effects such as hysteresis during scans. Several procedures used in calibration and correction of piezoelectric scanners used in the laboratory's STMs are described. 



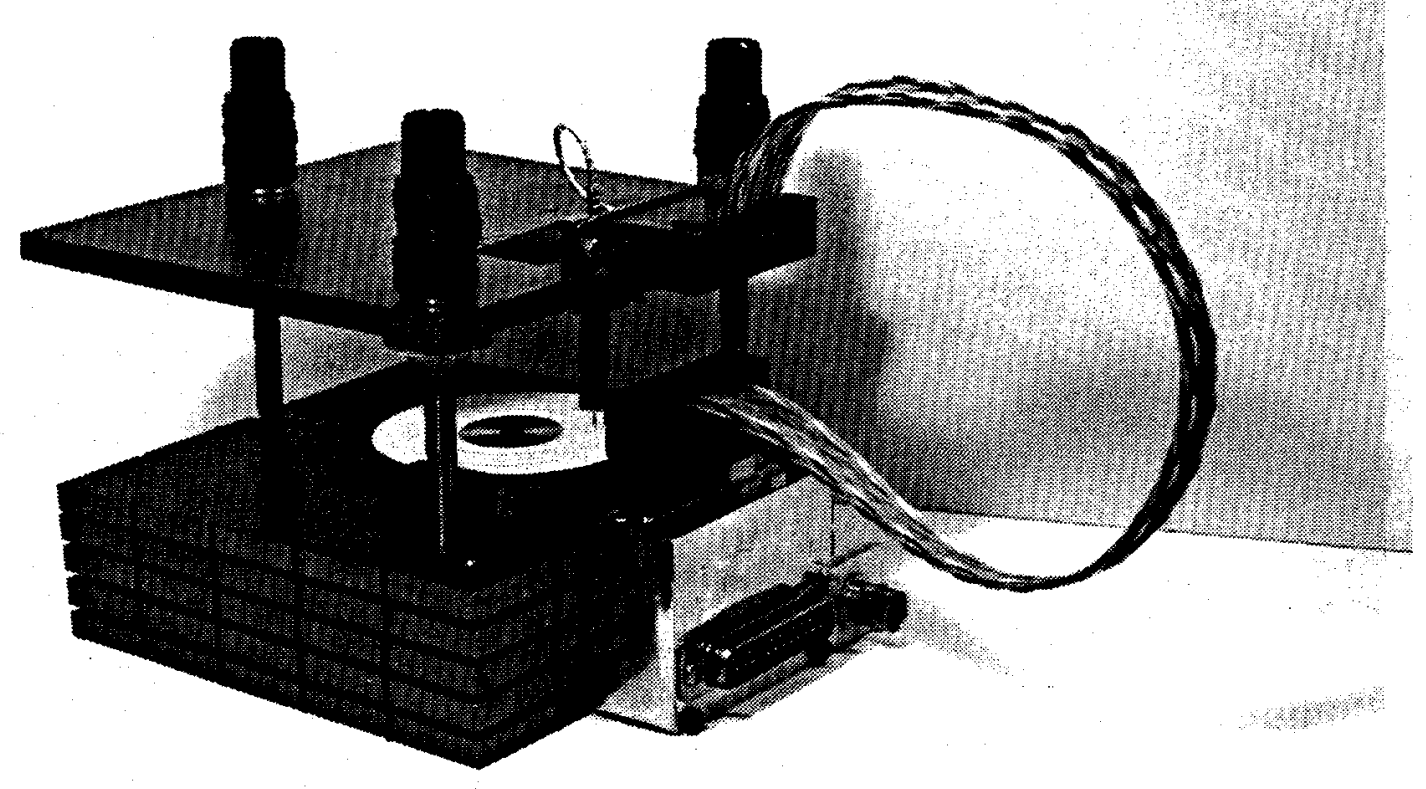

Figure 1: Large Sample STM

\subsubsection{Tube Scanner Design}

The tube scanner was designed and fabricated at the Precision Engineering Center. Features common to other designs include; capillary tube tip mounting, shielded signal wire running down the tube axis and, separation of the outer electrode for mechanical decoupling of the z-axis. Figure 2 shows the tube assembly after construction and mounting onto the head of the large sample STM. The X and $\mathrm{Y}$ axis are electrically contacted using the four spring loaded pogo pins seen in Figure 2. The PZT tube (dimensions) is made of PZT-5H material [9].

One popular method for tip mounting involves a capillary tube into which the tip is inserted. This arrangement provides quick and convenient tip changes. While other designers [6] have mounted the capillary tubes to the inner or outer wall of the tube, this method was not used here because of possible secondary piezoelectric deformation effects which can displace the tip holder not only relative to the sample but also relative to the tube itself. This is not a problem if the effect is coupled with just one axis, $\mathrm{X}$ or $\mathrm{Y}$ because calibrations will minimize these effects. However, if the secondary effect causing relative displacement of the tip is due to a $\mathrm{Z}$ axis control signal, the cross coupling between $\mathrm{X}$, $\mathrm{Y}$, and $\mathrm{Z}$ becomes a greater problem. For this reason, the capillary tube was epoxied to a boron nitride end-cap. In this configuration, the radial expansion of the piezoelectric tube element near the tip has a negligible effect on the tip position due to the cancelation of expansion of opposing sides and the restriction to expansion imposed by the epoxied end-cap. 


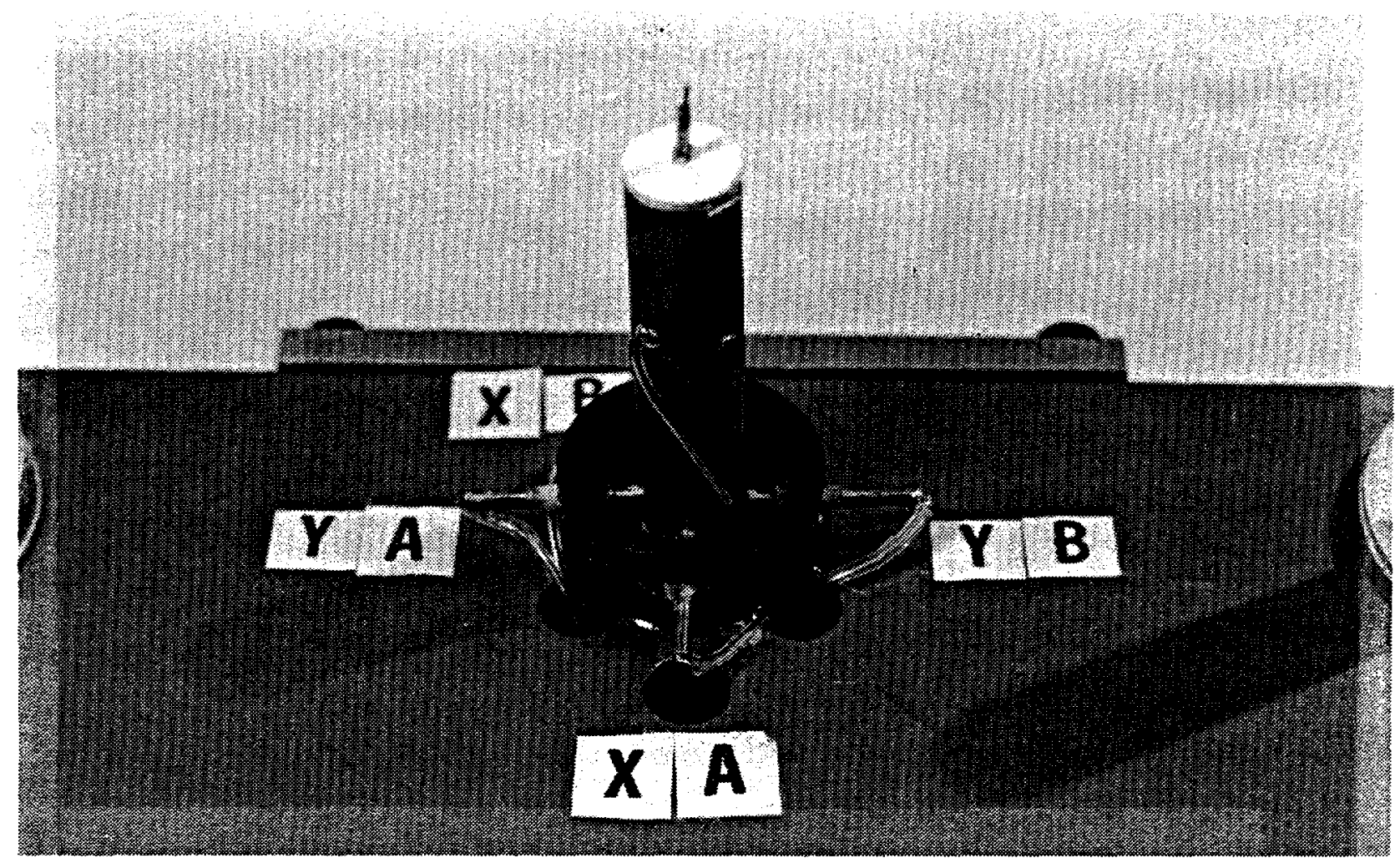

Figure 2: Tube Scanner

The central location of the capillary tube allowed the implementation of a ground shield running down the axis of the tube which contained the tip signal wire. This makes the exchange of tube scanners much easier by reducing the number of exposed wires leading from critical points on the tube.

Figure 3 shows the assembly of the tube scanner. The boron nitride (BN) base-cap was tapped for a stainless steel \#4-40 screw which had been drilled for passage of the tip signal wire. The screw threaded through the $\mathrm{BN}$ base-cap and into a stainless steel tube which lead directly to the base of the capillary tube used to hold the scanning tip. The stainless steel tube maintained clearance between the BN end-cap and the capillary tube such that there remained freedom of flexural and axial motion of the tube scanner. High dielectric constant tubing was then used to insulate the gold wire from the surrounding stainless steel tube and screw. The gold wire was indium soldered to the crimped end of the capillary tube. The stainless steel screw and tube was then electrically connected to the signal ground for proper shielding of the tunneling signal.

This arrangement maximizes the shielding of the signal wire in two respects. First, since the tube has been built such that the $Z$ axis driving signal is on the outer surface of the tube and the $\mathrm{Z}$ axis $\mathrm{DC}$ offset signal is on the inner electrode, the dynamic control signal to the $\mathrm{Z}$ axis is spatially and electrically separated from the inner cavity of the tube scanner. The inner electrode, which is maintained at a DC level acts as a potential plane by which the high voltage field lines from the outer electrode may terminate. Any stray fields which continue towards the central axis are then terminated with the additional 


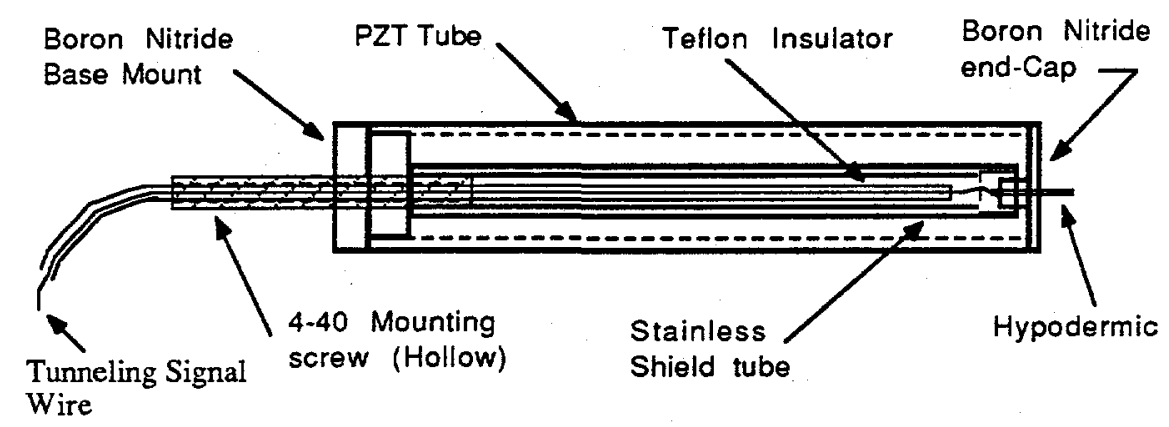

Figure 3: Tube Scanner Assembly

shielding provided by the stainless steel tube.

\subsubsection{Tip-sample Approach}

The tip to sample approach was developed to be very simple in design and operation. Lever demagnification was the most straight forward method. Three 80 pitch, or 80 turns per inch, lead screws were used in the design to achieve a satisfactory amount of control over the tip to sample position. As seen in Figure 1, the tube scanner has been located close to the pivot line of the back two lead screws. This allows coarse adjustment of the tip height with these lead screws and fine positioning control using the front screw.

The axis of the tube scanner has been positioned approximately $0.4 \mathrm{~mm}$ inch from the pivot line of the back two lead screws, and the distance between the front and back lead screws was set at $100 \mathrm{~mm}$. Therefore, the demagnification factor for motion of the front lead screw becomes approximately $\frac{1}{64} \mathrm{X}$. Therefore, for a $10^{\circ}$ turn of the front 80 pitch lead screw the motion realized at the tip becomes approximately $0.13 \mu \mathrm{m}$, assuming no backlash or nonlinearities in the lead screw.

The tube scanner was placed half way between the back two lead screws such that a factor of $\frac{1}{2} \mathrm{X}$ was realized in the motion of the tip for adjustments made to the back lead screws. Therefore, for a $10^{\circ}$ turn of one of the back lead screws, the tip would move approximately $4.34 \mu \mathrm{m}$. These back lead screws were used for coarse positioning of the tip to the sample as well as leveling of the head prior to scanning.

It was necessary to level the head with respect to the sample because of sample slope. Too 
great of a slope would cause the controller to go out of range near the ends of the $\mathrm{X}$ or $\mathrm{Y}$ axis scans. Any additional slope remaining in the scan could be removed by corrections in the scanning voltages or in slope removal routines in the image analysis program. Corrections made to the scanning voltages involved summing a proportional amount of the $\mathrm{X}$ or $\mathrm{Y}$ axis signal to the $\mathrm{Z}$ axis, therefore, correcting the $\mathrm{Z}$ height as $\mathrm{X}$ and $\mathrm{Y}$ axis are scanned. In the image analysis routines, slope is removed from the images by plane subtraction. A plane was fit to a set number of points in the image, 16k points per image on average. Then, the plane was subtracted from the image point by point.

The tip was viewed by a $50 \mathrm{X}$ optical microscope during the coarse approach phase. This allowed the positioning of the tip to within a few turns of the fine adjust lead screw. Then, with the controller turned on, the fine adjust lead screw was slowly turned until tunneling was achieved. Subsequent scans have shown no effects of tip crashes using this technique.

\subsubsection{Vibration Isolation}

The vibration isolation was achieved using two stages of isolation; four stacks of Viton O-ring, as used in the first STM designed at the Precision Engineering Center [10], and air bags supporting a granite slab on which the STM rested.

Four stacks of Viton were used to give additional isolation to the STM. Two accelerometers were used to measure the isolation effectiveness of the Viton stacks. One of the accelerometers was placed on the base of the STM and the other on the sample plate. By exciting the base of the STM and comparing the frequency content from each accelerometer a frequency response function was obtained. Figure 4 shows a plot of the frequency response function of the Viton isolation system using this method. The first fundamental frequency for the system was found to be approximately $28 \mathrm{~Hz}$. Effective isolation occurs at frequencies past the point of the $0 \mathrm{db}$ crossover. The $0 \mathrm{db}$ crossover occurs at approximately $43 \mathrm{~Hz}$ with approximately a $-2 \mathrm{db} /$ decade drop. Therefore, the amplitude of any excitations, greater than $43 \mathrm{~Hz}$, induced into the base of the STM are reduced by 2 decibels for every $10 \mathrm{~Hz}$ above $43 \mathrm{~Hz}$.

However, there still existed a $31 \mathrm{~Hz}$ frequency in the laboratory and in the tunneling signals. Therefore, additional isolation was required to minimize the effects of any lower frequencies prevalent in the laboratory. A granite slab, approximately $65 \mathrm{Kg}$, was placed on top of three air bags to provide additional isolation. The resonant frequency of the granite slab system was found to be approximately $5.6 \mathrm{~Hz}$ with a $-2 \mathrm{db} /$ decade drop. The $0 \mathrm{db}$ crossover point for the granite slab system was found to be approximately $12 \mathrm{~Hz}$. This provided an adequate amount of isolation and has been successfully used in imaging. 


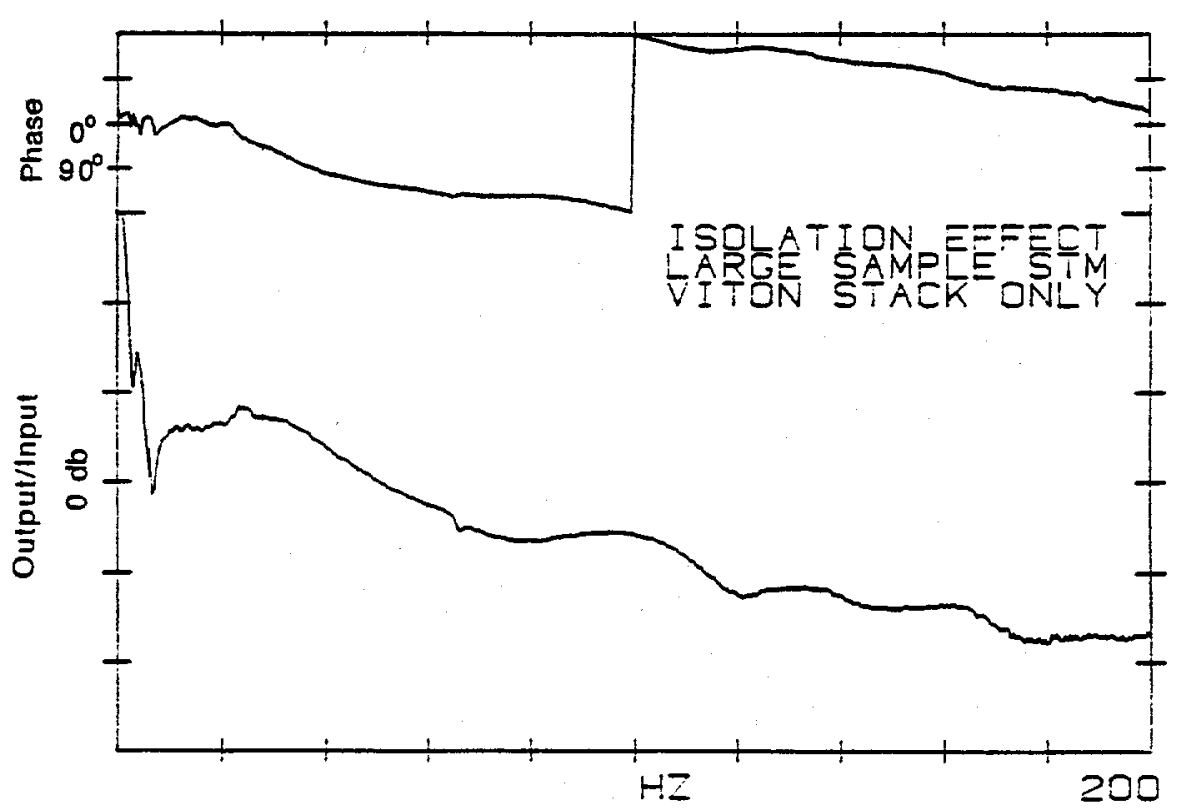

Figure 4: Isolation Effectiveness of the Viton O-ring Stacks

\subsection{CALIBRATION}

Several methods are available which can be used to measure displacements with sufficient accuracy for calibration of piezoelectrics. Two such methods are described which have been used to measure the static and dynamic displacements of piezoelectric tubes. Capacitance gages were used as the first method for static displacements. Then, because of concerns with possible field effects from the piezoelectrics upon the operation of the capacitance gage, an optical probe, OSSEM (Optical Sensor for Structural Energy Measurement) [8], was used to verify the capacitance measurements.

\subsubsection{Capacitance Method}

A capacitance gage is used to measure displacement by a change in gap distance between two surfaces, ie, the probe and the sample, or target. The capacitance between two parallel plates is given by:

$$
C=\frac{K \epsilon A}{X_{\circ}}
$$

where: 


$\begin{array}{ll}C & \text { Capacitance, Farads } \\ K & 8.7 \times 10^{-15} \text { Farads } / m m \\ \epsilon & \text { dielectric constant }(\epsilon=1 \text { for air) } \\ A & \text { Plate area, } \mathrm{mm}^{2} \\ X_{0} & \text { Plate separation, } m m\end{array}$

The sensitivity of a capacitor to changes in the plate separation, or gap distance can be obtained by differentiating equation 1 :

$$
\frac{d C}{d X_{0}}=\frac{-K \epsilon A}{X_{0}^{2}}
$$

Therefore, both the capacitance and the sensitivity increase with a decrease in the gap size. Capacitance gage electronics are then used to convert the very small change in capacitance of the probe into a usable electrical signal. Several methods are used to convert this change in capacitance into a linearized output signal. The Equal Ratio Arm Transformer Bridge (ERATB) is the most common circuit employed. Typical performance values for the ADE Microsence capacitance probes are: [7]

Probe active diameter

Minimum distance from probe center

to target edge for accurate measurements

Nominal standoff range

Linearity

Resolution

Frequency Response

Analog output

\section{$1.7 \mathrm{~mm}$}

$1.7 \mathrm{~mm}$

$75 \mu \mathrm{m}$ to $125 \mu \mathrm{m}$

$100 \mu \mathrm{m}$ of full travel

$25 \mathrm{~nm}$

$40 \mathrm{kHz}$. (3 db down)

$400 \mathrm{mv} / \mu \mathrm{m}$

Experimental Method and Results For X, Y, and Z axis calibration using an ADE Microsence capacitance gage, it was necessary to supply a conducting surface by which the capacitance probe could measure. For the $Z$ axis, a second $B N$ end-cap was used, as shown in Figure 3. This end-cap was placed backwards onto the protruding capillary tube using the hole existing in the center and provided a flat surface facing outward for measurement. A piece of reflective metallic tape (3M Scotch Brand 850 Silver Polyester Film) was cut to fit the flat face of the end-cap. This provided the conducting surface by which capacitance measurements could be made.

For the $\mathrm{X}$ and $\mathrm{Y}$ axis measurements, it was necessary to construct a target to measure lateral displacements of the tube. A tungsten tip was epoxied, on one end, to a small plastic plate approximately $0.05 \mathrm{in}$. thick and cut to cover an area approximately equal to the size of the capacitance gage head. On the opposing side from the epoxied tip, another piece of reflective metallic tape was applied such that capacitance measurements could be made. Therefore, when the epoxied tip was placed into the capillary tube, as a standard 


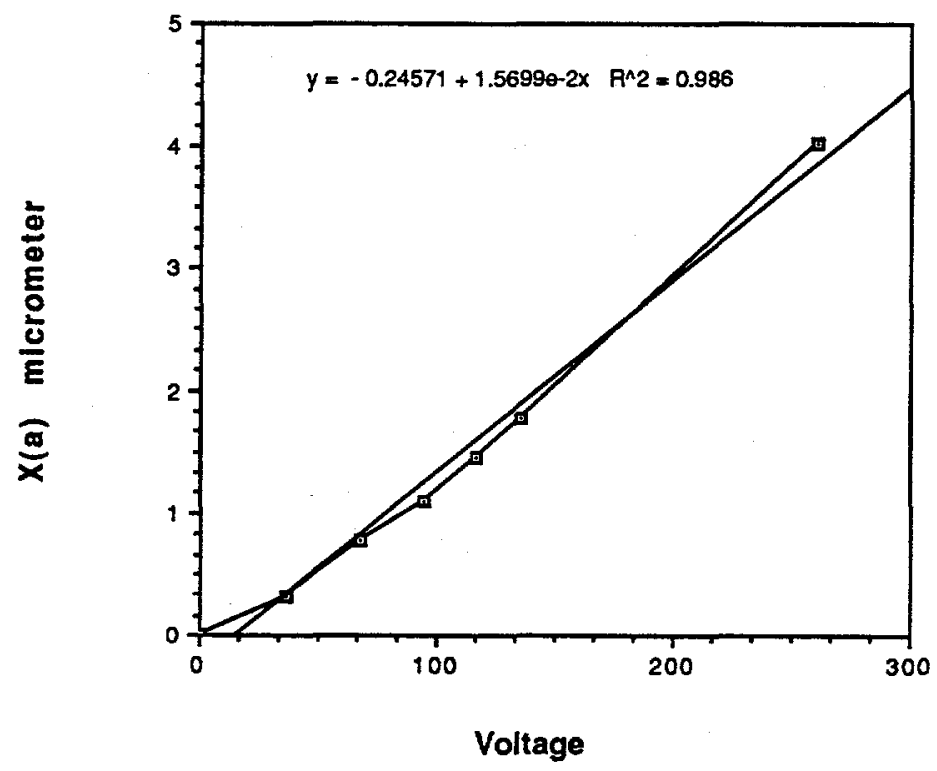

Figure 5: Static Calibration Curve for the X Axis using Capacitance Gage

tip would be during operation, the surface was parallel to the axis of the tube. Then, by simply rotating the target such that it rested parallel to the piezoelectric quadrant of interest, the capacitance gage could be positipned to measure lateral deflections of the tube.

The head of the large sample STM was clamped horizontally onto a 90 degree mounting bracket which was clamped to an isolated optical table. Therefore, the tube was positioned such that the axis of the tube was parallel to the table top. Static deflections of the tube were measured by application of a high voltage to individual sections of the tube using a Burleigh, Model PZ-70, high voltage op-amp. DC output of the capacitance gage was observed using an oscilloscope.

Figure 5 shows a calibration curve obtained for the $\mathrm{x}$ axis. A straight line has been fit through the data in Figure 5 to estimate the slope. From this curve it was determined that the gain for the $\mathrm{X}$ axis, over the operating range of $260 \mathrm{~V}$, was $156 \AA / \mathrm{V}$. It can also be seen that the curve is slightly nonlinear due to material creep and hysteresis.

\subsubsection{Optical Method (OSSEM)}

The Optical Sensor for Structural Energy Measurements (OSSEM) operates on the principle of a change in intensity of reflected light from a surface as the distance from source to target changes. Light from a light emitting diode is coupled into a center fiber (emitter) of a seven fiber bundle, consisting of a single emitter surrounded by six receiving fibers 
(receivers). Because light exits the central fiber in a uniangular cone, upon reflection from a nearby target the light will illuminate a specific area surrounding the fiber [8]. The intensity and the area covered by this reflection are specifically related to the distance of the target from the fiber bundle. The surrounding fibers receive the reflected light and pass this light to a photo-diode detector to measure its intensity. This measured intensity is output as a voltage which is directly proportional to target displacement.

The output signal is not linear over the entire range of operation. In fact, there are two regions which have opposite slopes. From zero standoff to approximately 0.1 in. the output voltage rises to a peak and then drops again with increasing gap distance. The first region is the most sensitive and is highly linear for relatively small displacements. Sensitivities on the order of $560 \mathrm{mV} / \mu \mathrm{m}$ are typical. Performance values for the OSSEM probe are [8]:

Probe active diameter

$1.2 \mathrm{~mm}$

Minimum distance from probe center

to target edge for accurate measurements

Nominal standoff range

$0.6 \mathrm{~mm}$

Linearity

$0.5 \mathrm{~mm}$

Resolution

$0.25 \mathrm{~mm}$ in near field zone

Frequency Response

$10 \mathrm{~nm}$

Analog output

$100 \mathrm{kHz}$.

$560 \mathrm{mv} / \mu \mathrm{m}$

Experimental Method and Results "The experimental setup for the optical sensor probe was identical to that for the capacitance probe. The reflective metallic tape used for the capacitance gage was also used for the optical probe. It was necessary, however, to recalibrate the optical probe each day for the specific type of reflective surface used in any experiment. Therefore, calibration of the optical probe was first performed to obtain the proper sensitivity. Calibration of the OSSEM probe was performed using a micrometer over $2.5 \mathrm{~mm}$ travel to determine the near field linear zone. Figure 6 shows the calibration curve used to obtain the sensitivity for the experiment. A straight line has been fit through the data to determine its slope.

Curves were obtained for $\mathrm{X}, \mathrm{Y}$ and $\mathrm{Z}$ axis. A comparison of the results for optical and capacitance measurements is discussed next.

\subsubsection{Comparison of Methods}

Figure 7 shows how well the capacitance and optical methods matched in the $Z$ axis. For the $\mathrm{Z}$ axis, the two methods compared within $5 \%$. Based on these results it could be assumed that the effects of stray fields upon the operation of the capacitance gage is negligible. Therefore, further investigation upon scanner displacements may be conducted using the capacitance gage. It will also be shown in the next section that images obtained with the 


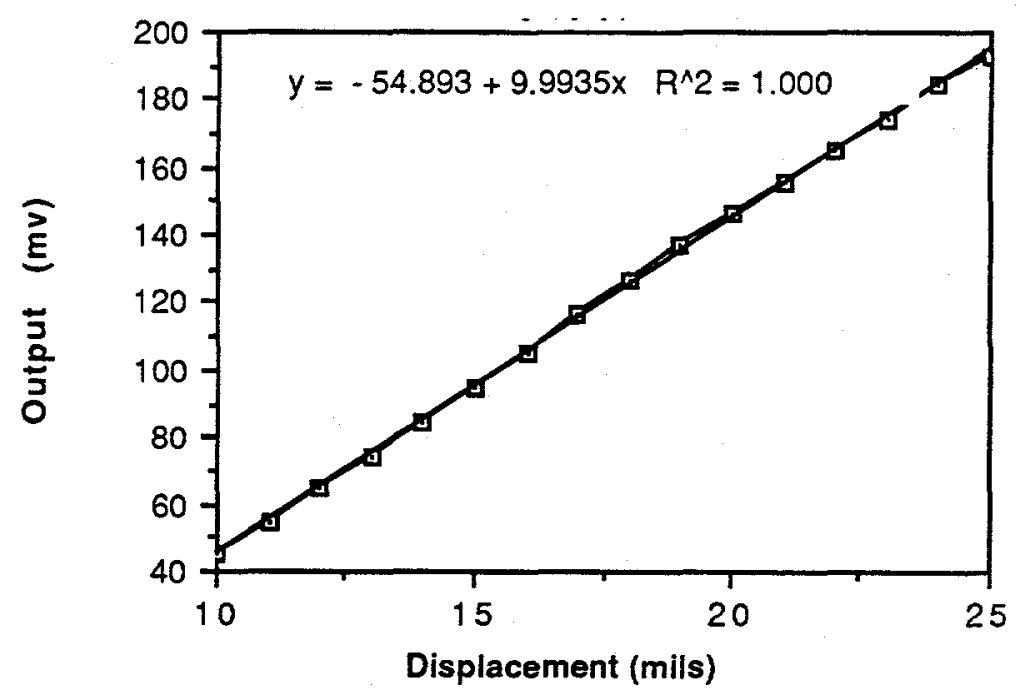

Figure 6: Calibration Curve for the Optical Probe

STM conformed closer to the capacitance results in X and $\mathrm{Y}$ than that obtained with the OSSEM gage. The capacitance method is preferred to the optical method in practice only because of the need to calibrate to each reflective surface for any experiment using the OSSEM probe because of its nonlinear nature. This is not necessary for the capacitance probe. Also, the capacitance probe is linear to $0.4 \%$ over its entire range therefore offsets along its calibrated range will not change its apparent gain. It was necessary to use the OSSEM gage, however, to determine that the stray fields of the PZT's were not effecting the capacitance measurements. The OSSEM probe is very useful, and out performs the capacitance gage, in measurements with large standoff range and high frequency demands. However, OSSEM's use as a static displacement probe are only parallel, at best, with the capacitance gage.

\subsubsection{Calibration Results in Imaging}

Figure 8 shows an S.E.M. image of an optical disk sample coated with $50 \AA$ of Platinum. According to the manufacturer the grooves have a spacing of $0.6 \mu \mathrm{m}$ and widths of 1.0 $\mu \mathrm{m}$. Therefore, for calibrations giving approximately $150 \AA / \mathrm{V}$ in the $\mathrm{X}$ and $\mathrm{Y}$ axis, scans of $215 \mathrm{~V}$ should produce images covering approximately $3.25 \mu \mathrm{m}$. An STM image of 3.25 $\mu \mathrm{m}$ should cover two complete grooves in any position.

Figure 9 shows an STM image of the same optical disk grooves in Figure 8. From this image it can be seen that the $150 \AA / V$ prediction is correct, however, dimensional variations from left to right of the thickness of each groove are due to hysteresis effects in the scanner. 


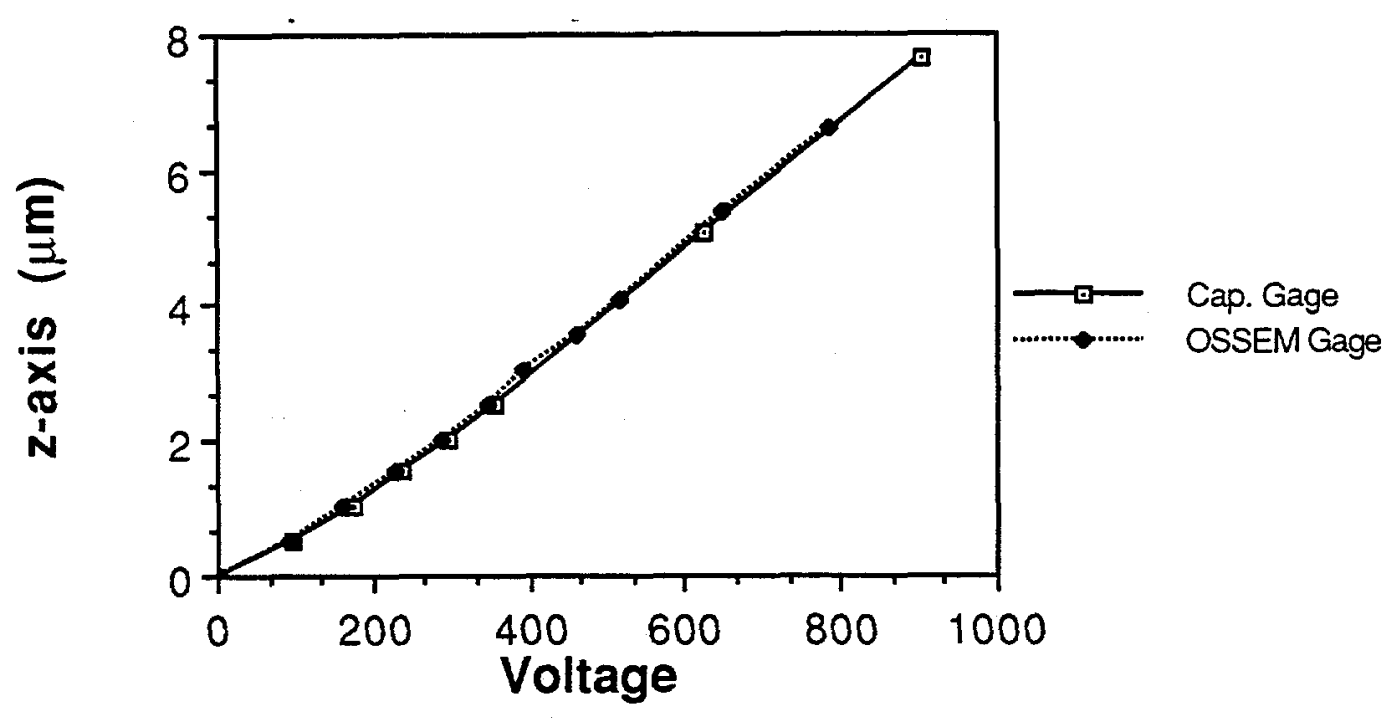

Figure 7: Comparison of Static Calibration Curve for Z Axis

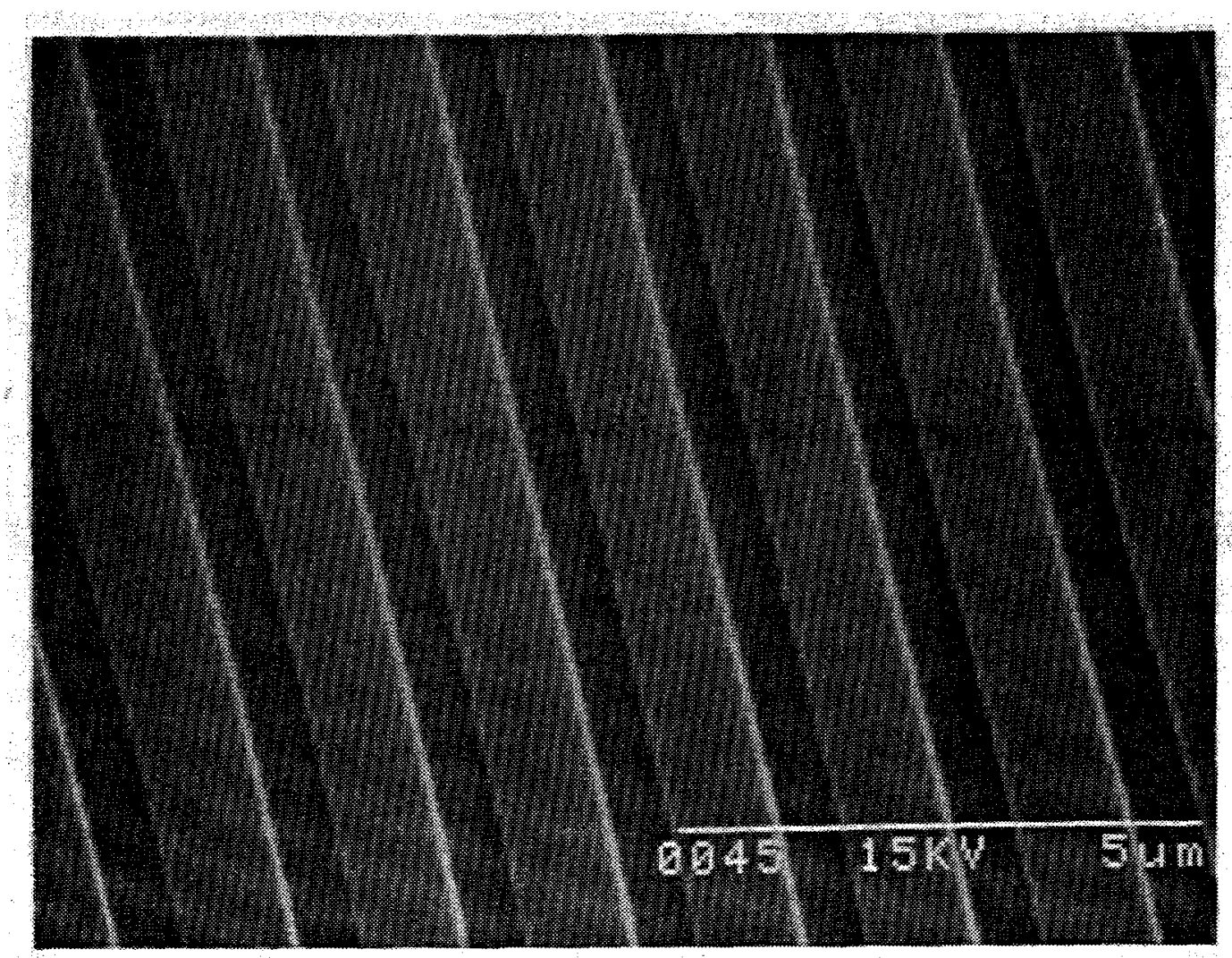

Figure 8: S.E.M. Micrograph of Optical Disk Grooves 


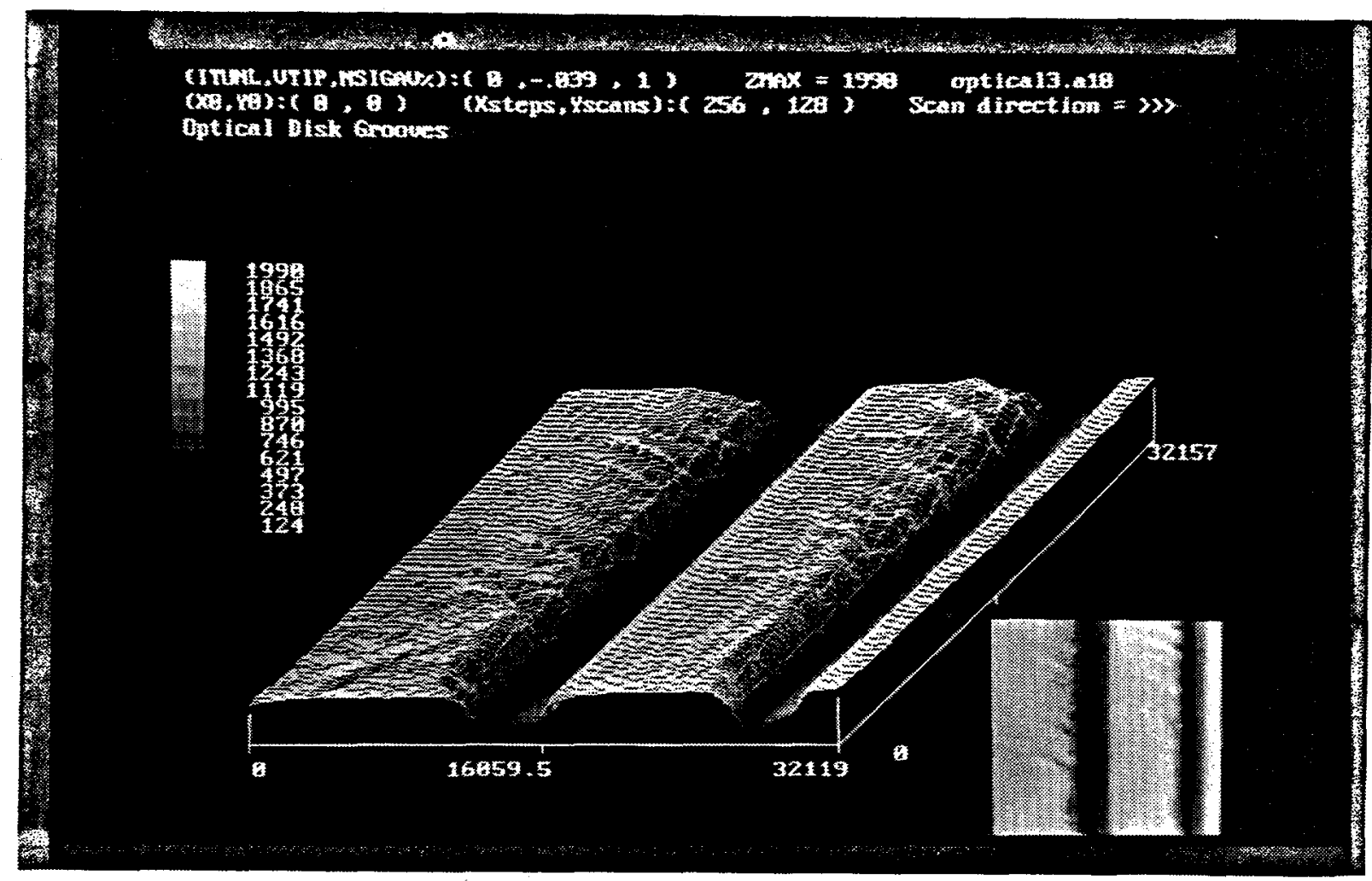

Figure 9: $3.21 \times 3.21 \mu \mathrm{m}$ STM Image of Optical Disk Grooves

The height of the grooves are in conflict between the STM image and the manufacturer's dimensions. This is believed to have been due to the fact that during calibration of the scanners, inadvertent depoling of the piezoelectrics occurred. The Burleigh PZ-70's used for calibration and hysteresis effects were defective such that voltage spikes occurred during power off. These large voltage spikes across the thin walls of the piezoelectric scanners $(0.5 \mathrm{~mm})$ can cause depoling of the scanners and subsequently changes in gain. This did not happen for the $\mathrm{X}$ and $\mathrm{Y}$ axis, however, the $\mathrm{Z}$ axis has been effected. Further work has followed to build a new tube. Calibrations in the $\mathrm{Z}$ axis for the newer tubes is presently under way and subsequent imaging of optical disk grooves will follow.

\subsection{HYSTERISIS CORRECTIONS}

When a cyclic voltage is applied to piezoelectric ceramics a closed hysteresis curve of displacement against voltage is obtained. Figure 10 shows a curve obtained on an $X-Y$ plotter, using the capacitance transducer and a $50 \mathrm{~Hz}$ triangular waveform, output from the STM scan generator. Nishikawa, Tomitori, and Minakuchi [3] have shown similar curves for PZT ceramics and PLZT $\left[P b_{1-x} L a_{x}\left(Z r_{y} T i_{1-y}\right)_{1-x / 4} O_{3}\right.$, where $\left.\mathrm{x}=3 \%, \mathrm{y}=52 \%\right]$ stick scanners. They show that the PLZT material has less hysteresis than PZT materials. Because the PZT materials have a higher mechanical gain than the PLZT materials, the initial choice for scanners was the PZT type. The PZT-5H material [9] has approximately 


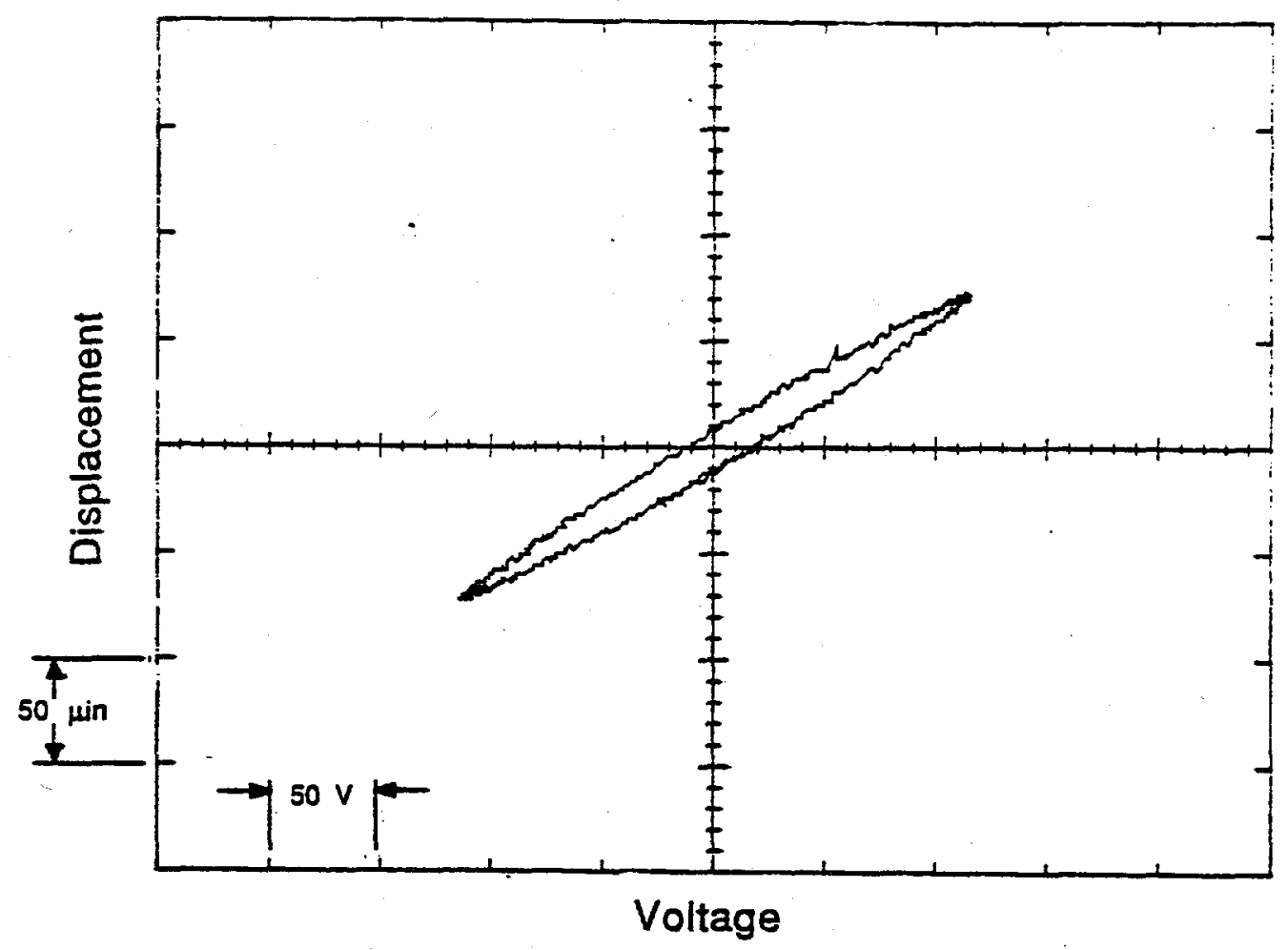

Figure 10: Hysterisis loop of PZT-5H tube, $50 \mathrm{~Hz}$ triangle wave, $250 \mathrm{~V}$

$35 \%$ increase in mechanical gain over the PLZT materials.

Corrections for the effects of hysteresis may still be necessary in the PLZT or electrostrictive ceramics. These corrections are necessary for proper interpretation of the longer range STM images since the effects of hysteresis become more prevalent for larger voltage swings.

\subsubsection{Measurement Methodology and Setup}

The capacitance gage was used for all of the hysteresis measurement experiments. The same setup was used as in the static calibration experiments. However, instead of using the PZ-70 high voltage amplifier to drive the scanner, the STM scan generator was used. Concerns with the effects of target vibration during hysteresis measurements were eliminated since the measurements were made at relatively low frequencies compared to the high natural frequency of the target $(\sim 200 \mathrm{~Hz})$. Most scans acquired using the STM over large scan ranges were limited to speeds less than $50 \mathrm{~Hz}$ in the $\mathrm{X}$ axis direction and $1 \mathrm{~Hz}$ in the $\mathrm{Y}$ axis direction. Therefore, it was not necessary to determine the effects of hysteresis at higher scan frequencies.

The STM scan generator has a $10 \mathrm{~V}$ output channel proportional to the scan voltage for each axis. This was used to monitor the high voltage output of the scan generator. The capacitance gage has $a \pm 10 \mathrm{~V}$ output channel that is proportional to the displacement of the transducer. These two signals, the scan generator output and the capacitance gage output, 
were then input to a MASSCOMP 5400 data acquisition computer. The MASSCOMP was used to acquire the two simultaneous channels of input and store the data into files which were then downloaded to a Digital MicroVAX system for data manipulation. The MicroVAX was used to evaluate the hysteresis curves and obtain polynomial expressions for each leg of the hysteresis loops.

Since images are acquired in both directions of the $\mathrm{X}$ axis scan, polynomial expressions for both legs of the hysteresis loop can be used. Polynomials obtained for the first path on the hysteresis loop were used for corrections in the primary scan direction images, ie. scans obtained in the direction from $-260 \mathrm{~V}$ to $0 \mathrm{~V}$ in the $\mathrm{X}$ axis. Polynomials obtained for the second path on the hysteresis loop were used for corrections in the secondary scan direction images. Second order polynomials were sufficient to accurately reproduce the hysteresis loops. By keeping the polynomial expressions to a minimum order, the speed for image correction in the post-processing routine could be kept high.

\subsubsection{Application of Hysteresis Data to Image Processing}

Figure 11 shows an STM image before and after hysteresis correction. From the first image in Figure 11 features on the right side are thinner than those on the left. After correction, features on the left have been reduced in width and features on the right have been increased in width.

\subsection{CONCLUSIONS AND FUTURE CONSIDERATIONS}

Error mapping of mechanisms used for precise positioning which has been used for many years. Applying an experimentally determined displacement function to the measured position output remains a highly useful method for inherent error correction. The problems of nonlinearities and hysteresis are only small parts of a larger problem to be dealt with in the future of STMs. Since the accuracy of an instrument can be no better than its resolution, the STM has limitations in its accuracy. By departing from a single given linear gain to a nonlinear functional expression for displacement, the accuracy of the STM is increased. This allows the STM to become a more useful tool in spatial measurements on surfaces as when using the instrument for surface science research. It has been shown that functional fits to displacement curves can be used to properly display surface details, thereby increasing the instrument accuracy.

By using components which are more linear, the accuracy of the STM is higher when considering linear gains. However, remaining hysteresis effects make the more linear components still less accurate than corrected elements having less linearity. Therefore, unless scanner elements are linear to within the resolution of the STM, nonlinear corrections can still increase the STM's accuracy. 


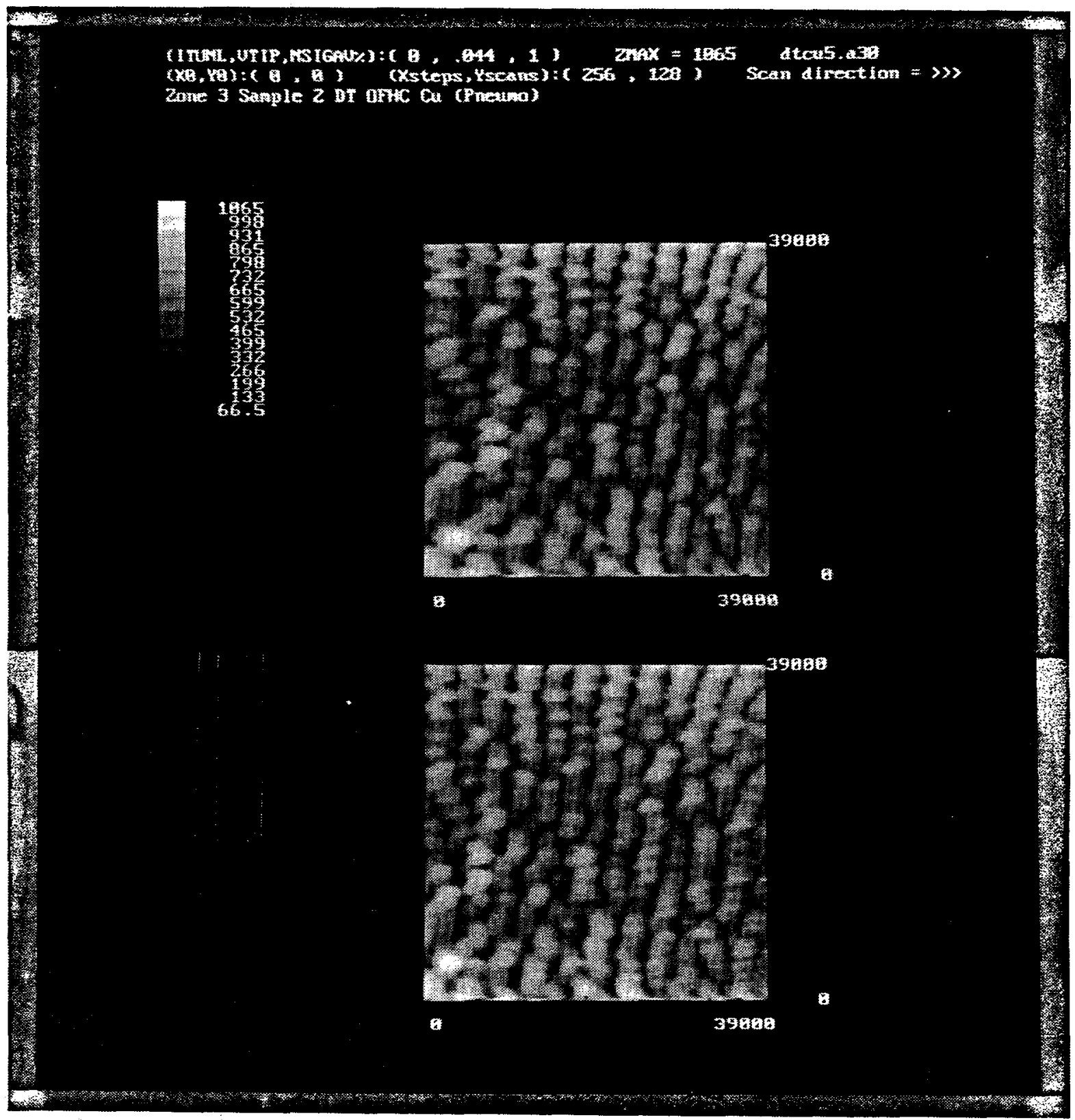

Figure 11: STM image before and after hysteresis correction. 
The remaining question to the problem is in regard to the repeatability of the system. How repeatable is the hysteresis over time? Creep and aging of piezoelectric elements can change their properties and hence decrease the accuracy of the system. Therefore, to maintain the system within acceptable bounds, frequent calibrations are necessary.

In the future, however, for more precise measurements of surface features, it becomes inevitable that direct measurement of displacement of the scanner is necessary, particularly over large ranges. Instruments, such as laser interferometers, can be used which are less prone to aging effects and changes in repeatability, however, precautions and adjustments must be made for such variables as atmospheric conditions. 


\section{References}

[1] G. Binnig, H. Rohrer, Ch. Gerber, and E. Weibel, "Surface Studies by STM", Physical Review Letters, Vol. 49, No. 1, 1982, p. 57.

[2] S. Vieira, "The Behavior and Calibration of Some Piezoelectric Ceramics Used in the STM",IBM Journal of Research and Development, Vol. 30, No. 5, September 1986, pp. 553-556.

[3] Osamu Nishikawa, Masahiko Tomitori and, Akira Minakuchi, "Piezoelectric and Electrostrictive Ceramics for STM".

[4] T.M. Tritt, D.J. Gillespie, G.N. Kamm, and A.C. Ehrlich, "Response of Piezoelectric bimorphs as a Function of Temperature", Review of Scientific Instruments, Vol. 58, No. 5, May 1987, pp. 780-783.

[5] M. Gehrtz, H. Strecker, and H. Grimm, "Scanning Tunneling Microscopy of Machined Surfaces",Journal of Vacuum Science and Technology, Vol. 6, No. 2, March/April 1988, pp. 432-435.

[6] Mechanical design as exhibited by the Nanoscope I instrument manufactured by Digital Instruments, Inc. Goleta, CA.

[7] Thomas G. Bifano, "Fundamentals of Capacitance Gaging for Precise Displacement Measurements",Precision Engineering Center Annual Report, January 1985, pp. 818.

[8] Steven C. Fawcett, "Analysis and Development of a Multi-Channel Fiber Optic Probe for Structural Vibration Measurement", North Carolina State University Master Thesis, 1988.

[9] "Piezoelectric Technology Data for Engineers", Vernitron Piezoelectric Division, Bedford, Ohio.

[10] David A. Grigg, Phillip E. Russell, and Thomas A. Dow, "Design and Characterization of a Scanning Tunneling Microscope", Precision Engineering Center Annual Report, December 1987, pp. 65-84.

[11] David A. Grigg, Phillip E. Russell, and Thomas A. Dow, "Observation of Diamond Turned OFHC Copper Using Scanning Tunneling Microscopy",Precision Engineering Center Annual Report, December 1988. 


\title{
2 ATOMIC RESOLUTION IMAGES OF GRAPHITE IN AIR
}

\author{
David A. Grigg \& Gordon M. Shedd \\ Graduate Students \\ Dieter Griffis \\ Research Associate, ERSD \\ Phillip E. Russell \\ Associate Professor,MTE
}

One sample used for proof of operation for atomic resolution in STM is highly oriented pyrolytic graphite (HOPG). This sample has been imaged with many different STM's obtaining similar results. Atomic resolution images of HOPG have now been obtained using an STM designed and built at the Precision Engineering Center. This paper discusses the theoretical predictions and experimental results obtained in imaging of HOPG.

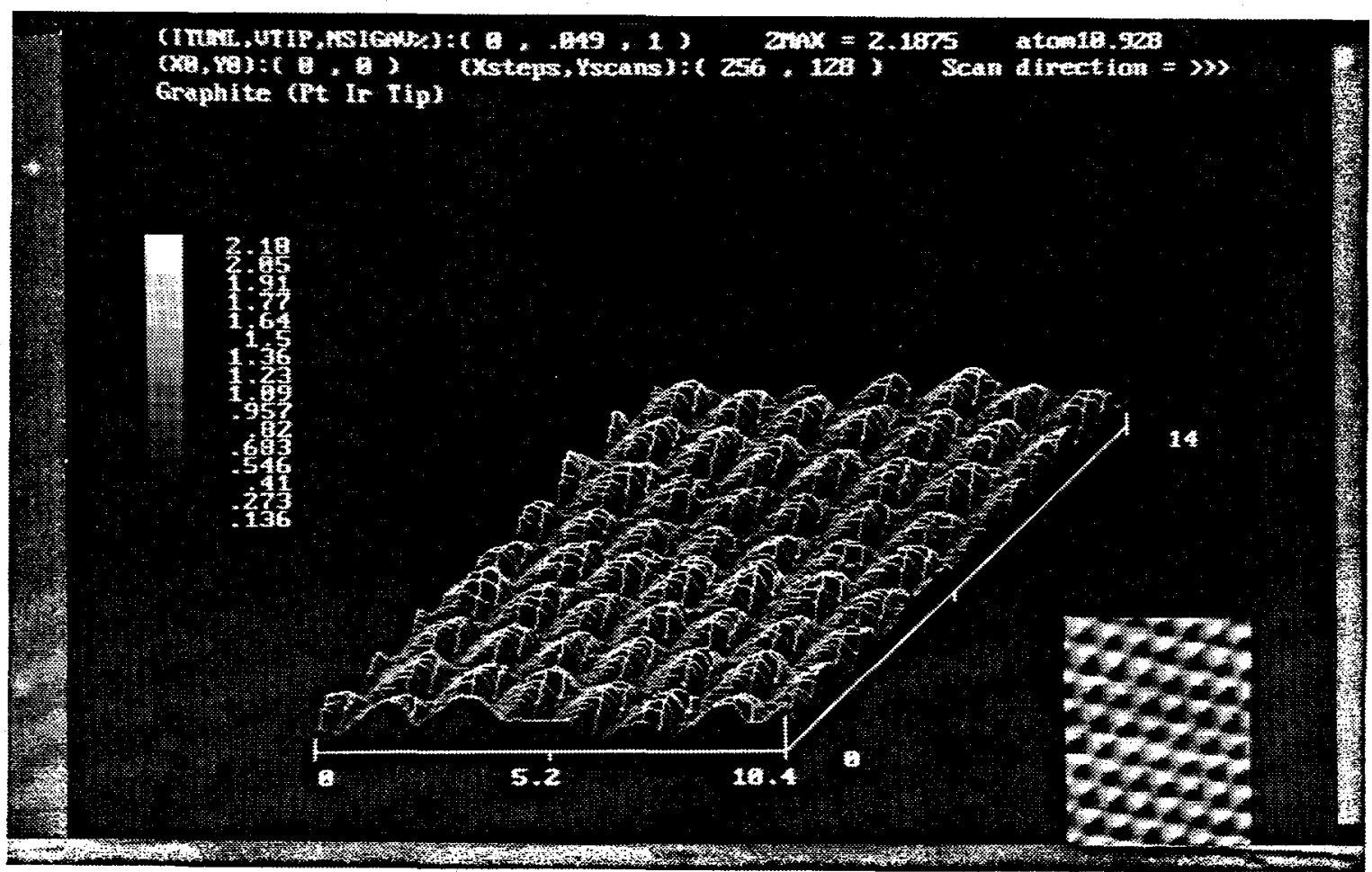




\subsection{INTRODUCTION}

The Scanning Tunneling Microscope (STM) has become an instrument with a variety of surface science applications. Work by Park and Quate in 1986 [1] demonstrated that atomic resolution images of graphite can be obtained in air. This has aided in the development of theoretical modeling of the tunneling phenomena during scanning and the role of tip geometry in surface reproduction [2].

A tomic resolution images of graphite in air at room temperature have been obtained over the past year. Each of these images has similar periodicity, however, details within the waveform of the images differ. Similar findings have been reported and explained as asymmetric tip effects by several groups including J. Schneir, R. Sonnenfeld, P.K. Hansma, and J. Tersoff [2].

A brief explanation to the nature of graphite in its bulk state will be presented as well as several images showing atomic resolution and the effects of tip asymmetries.

\subsection{EXPERIMENTAL METHOD}

The details of operation for the prototype design used in these experiments has been previously discussed [3]. The sample was highly oriented pyrolytic graphite (HOPG) [6]. HOPG remains oxide free in air and fresh surfaces can be easily prepared, once mounted, by cleaving the sample using scotch tape. The tips used were made from $0.25 \mathrm{~mm}$ diameter platinum-iridium (PtIr) wire, which had been sharpened to a point by mechanical shearing. These tips are used because PtIr is relatively oxide free, however, the mechanical shearing can cause tips to be asymmetric. Asymmetry occurs more frequently for mechanically sharpened tips than tips produced by etching techniques [2]. The process for etching PtIr tips is slightly more complex than that for Tungsten (W) tips, currently used in the PEC. Work is underway to process PtIr tips by etching methods which should produce tips of similar aspect ratios to the $\mathrm{W}$ tips, but without the oxide build-up, which plagues the $\mathrm{W}$ tips [5].

All images were taken with a tip current of $2 \mathrm{nA}$ and a tip bias of $+50 \mathrm{mV}$. Scan speeds for each image varied slightly. The time constant of the feedback loop was set as low as possible to increase the speed of the analog controller and the gain set as high as possible before instabilities in the controller caused oscillations of the tip. When the current signal was noisy and could not be maintained within an acceptable limit $(\approx 10 \mathrm{mV})$, the tip was cleaned by increasing the controller gain to cause tip oscillation. However, if the signal did not improve after oscillation of the tip two or three times, the tip was replaced. 


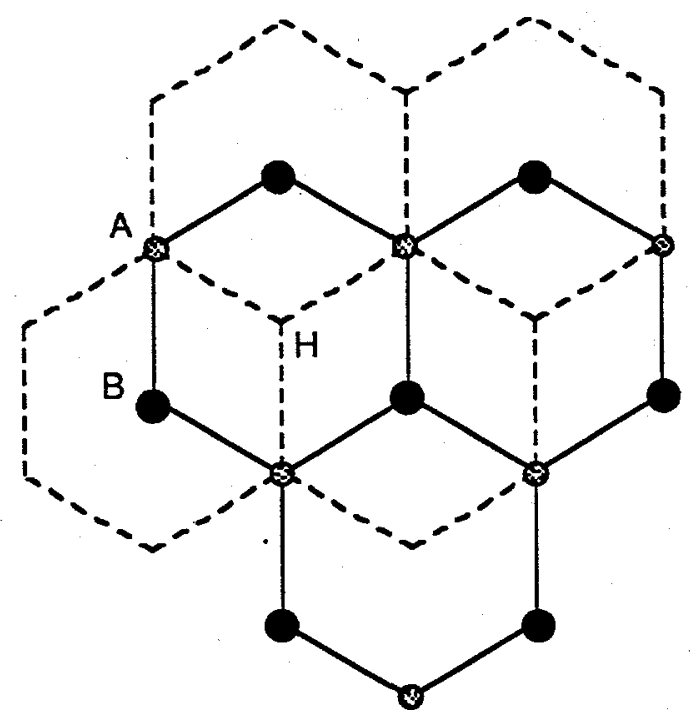

Figure 1: Two staggered layers of the (0001) graphite surface shown by solid and dotted lines. Two different (A and B) atomic sites in the top layer are indicated by small and large dots, respectively. The $\mathrm{H}$ site is the center of the hexagon.

\subsection{THEORY}

Highly Oriented Pyrolytic Graphite (HOPG) has a hexagonal close packed (HCP) lattice structure. Two layers of carbon atoms are stacked in a repetitive pattern (ABAB...) pattern with each atom of one layer fitting between atoms on the other layers forming a staggered hexagonal structure. This is illustrated in Figure 1. The upper B atoms dominate the shape of the local density of states on the surface, therefore, they become the prominent structure in the STM image. The lower A atoms can be recognized when conditions are proper. Because the lower $A$ atoms support the upper $B$ atoms, their effect can be seen as a rise in the local density of states between upper B atoms compared to holes in the $\mathrm{H}$ sites (The $\mathrm{H}$ site is the center of the hexagon). Therefore, in the STM image, which maps the local density of states, the lower A atom sites should map at a higher level relative to the $B$ sites. All three levels should be noticeable in the STM image; the upper $B$ level, lower A level, and the $\mathrm{H}$ hole.

\subsection{EXPERIMENTAL RESULTS}

Figure 2 shows an STM image of graphite in air. From the perspective view only the prominent $B$ level atoms can be seen, however, from the top view (lower right in Figure 2) the three fold symmetric shape expected in graphite can be observed. 


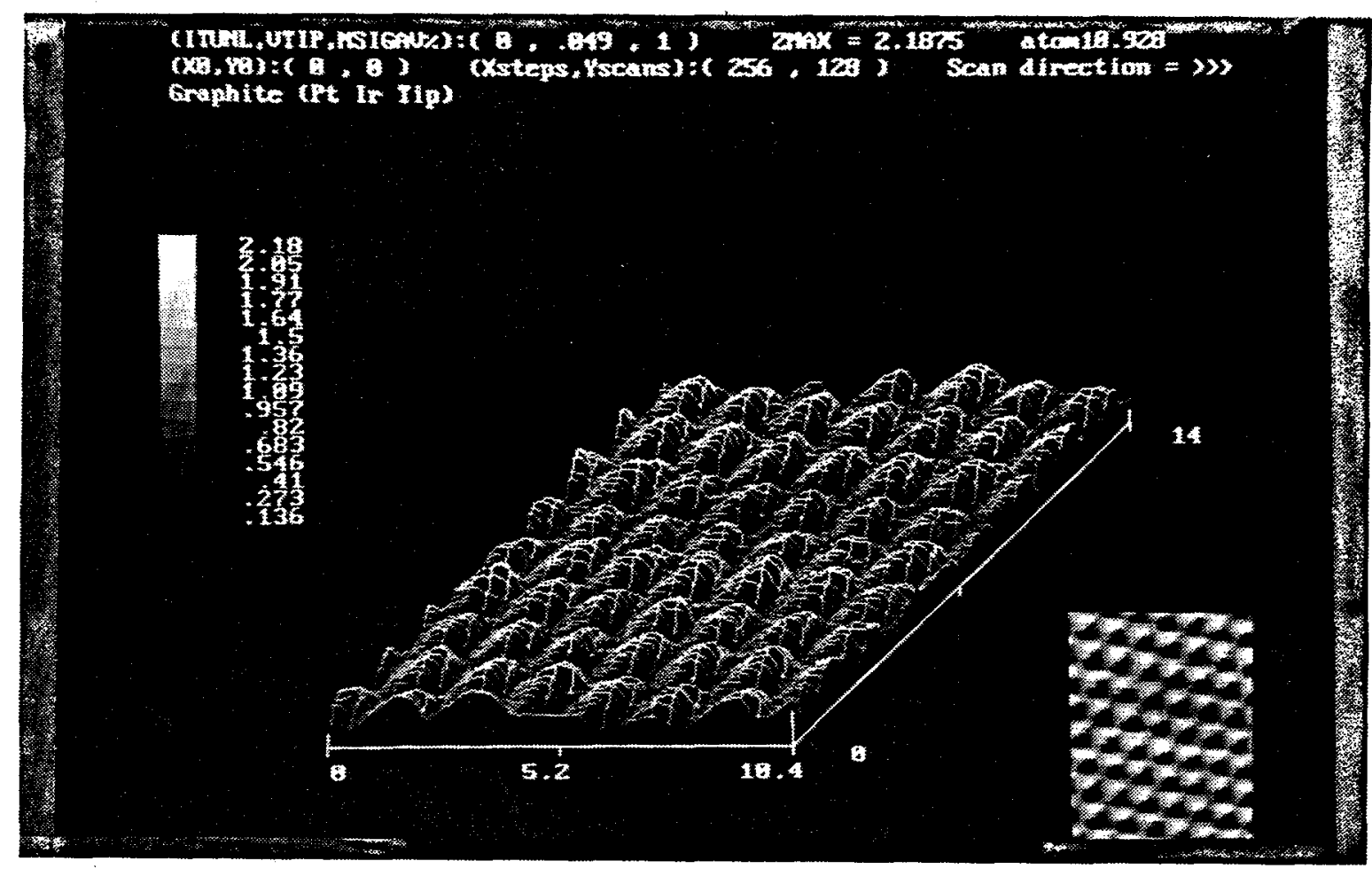

Figure 2: STM image of Graphite

Figure 3 shows an expanded top view of the same image depicting the HCP structure. A hexagonal overlay has been added to the image to aid in depicting the graphite structure. Comparison of this image to Figure 1 shows close agreement to theoretical predictions of the lattice shape. Since the lattice parameter is known to be $2.54 \AA$, the $\mathrm{X}$ and $\mathrm{Y}$ piezoelectrics can be calibrated.

Figure 4 shows an example of an asymmetric image. This effect may be due to a tilted sample or an asymmetric tip, as suggested by Binnig et al [4]. Studies have shown [2] that asymmetric images are more prevalent when using the mechanically sheared PtIr tips as opposed to chemically etched PtIr tips. For this reason, chemical etching techniques for PtIr tips are being studied. 


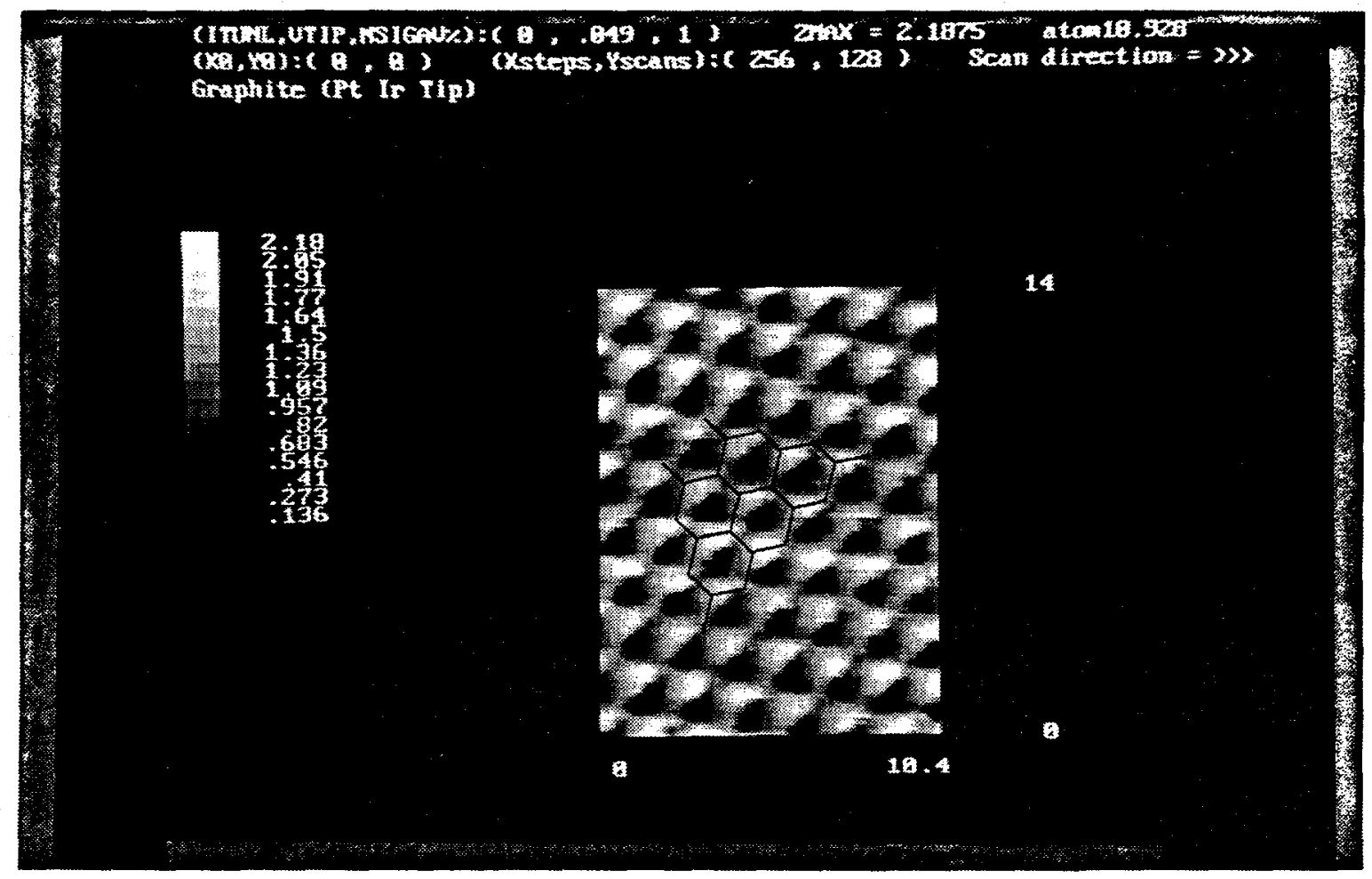

Figure 3: Top view image of Graphite showing HCP structure.

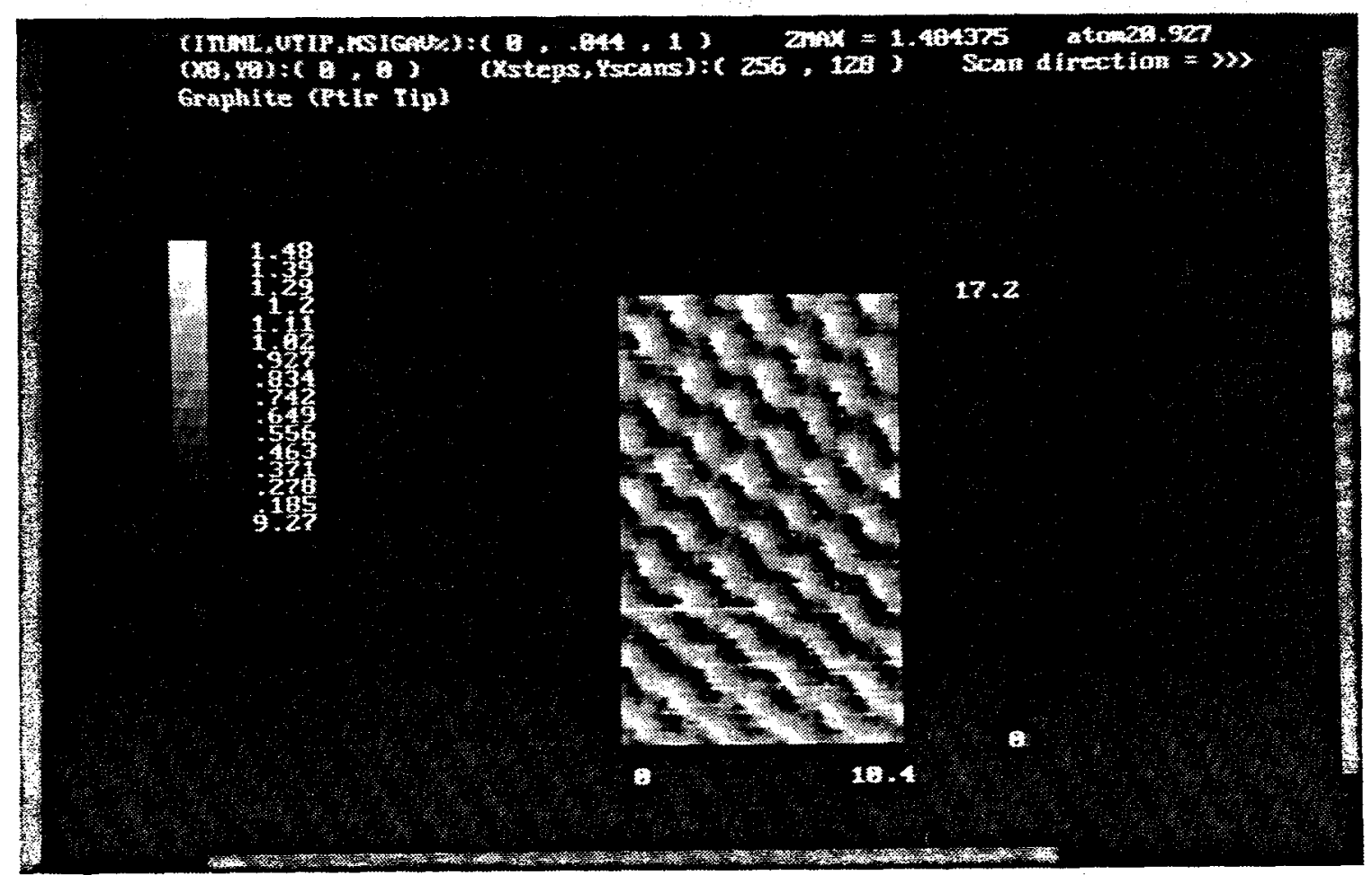

Figure 4: STM image of graphite showing asymmetric effects in imaging 


\section{References}

[1] Sang-Il Park, and C.F. Quate, "Tunneling Microscopy of Graphite in Air", Applied Physics Letters, Vol. 48, No. 2, p. 112, 1986.

[2] J. Schneir, R. Sonnenfeld, P.K. Hansma, and J. Tersoff, "Tunneling Microscopy Study of the Graphite Surface in Air and Water",Physical Review, Vol. B34, No. 8, Part I, p. 4979, October 1986.

[3] Gordon M. Shedd, and Phillip E. Russell, "Designing a Scanning Tunneling Microscope (STM) for the Characterization of Precisely Machined Surfaces", Precision Engineering Annual Report, Vol. 4, p. 8, January 1986.

[4] G. Binnig, H. Fuchs, Ch. Gerber, H. Rohrer, E. Stoll, and E. Tosatti, "EnergyDependent State-Density Corrugation of Graphite Surface as Seen by Scanning Tunneling Microscopy", Europhysics Letters, Vol. 1, No. 1, p. 31, 1986.

[5] I. H. Musselman, P.A. Peterson, B. Day, and P.E. Russell, "Preparation and Surface Analysis of Tungsten Tips for Scanning Tunneling Microscopy", Poster Presentation at Seventh Annual Symposium on Advances in Microscopy Sponsored by the Duke University Medical Center and the North Carolina Society for Electron Microscopy and Microbeam Analysis, September 24, 1988.

[6] "Pyrolytic Graphite Monochromator for X-ray and Neutron Analysis", Union Carbide, Cleveland, Ohio. 


\title{
3 ATOMIC FORCE MICROSCOPE
}

\author{
Robert D. Day \\ Graduate Student \\ Phillip E. Russell \\ Associate Professor, MTE
}

The Atomic Force Microscope (AFM) is a recently developed instrument that has achieved atomic resolution imaging of both conducting and non-conducting surfaces. Because the $A F M$ is in the early stages of development, and because of the difficulty of building the instrument, it is currently in use in fewer than ten laboratories worldwide [11]. It promises to be a valuable tool for obtaining information about engineering surfaces and aiding the study of precision fabrication processes. This paper gives an overview of AFM technology and presents plans to build an instrument designed to look at engineering surfaces.

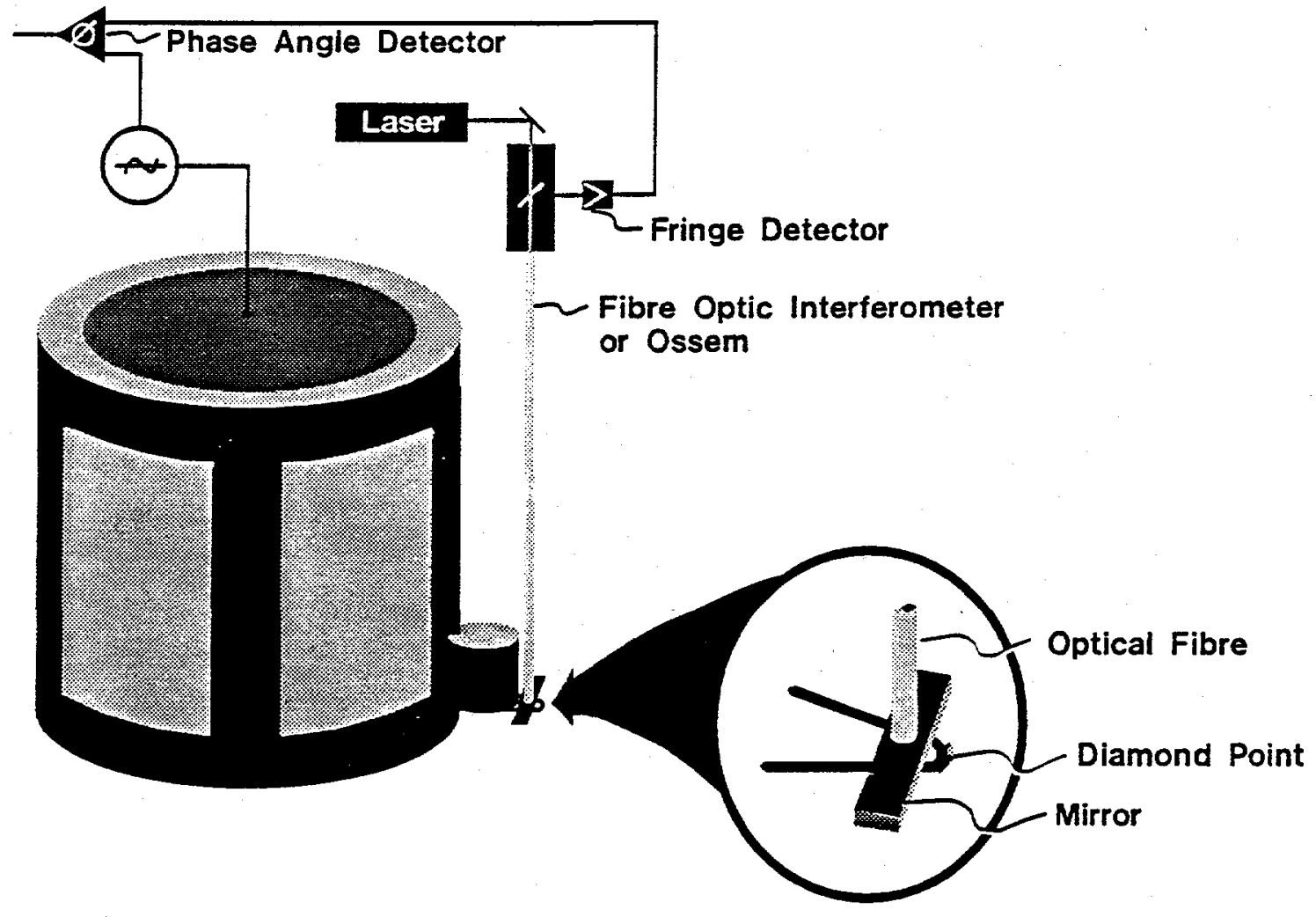




\subsection{BACKGROUND}

The Atomic Force Microscope (AFM) was invented in 1986 by G. Binnig, C. F. Quate, and $\mathrm{Ch}$. Gerber [1]. The concept for it stemmed from earlier experiments on electron tunneling. E. C. Teague commented on the van der Waals forces of attraction when performing vacuum tunneling experiments using two gold spheres [2]. The idea for using atomic forces as a means for producing high resolution images was born out of the Scanning Tunneling Microscope (STM) work.

To obtain a feel for the magnitude and scale of the atomic force interactions, we will consider a thin cantilever spring with a small tip placed on its end that has an atomically sharp point. As this tip is moved within very close proximity to a "flat" surface, van der Waals forces will try to pull the tip and the surface closer together. This will result in a bending of the cantilever spring such that the amount of bend in the spring multiplied by its spring constant will just offset the attractive force. As the separation distance decreases this attractive force increases resulting in a compensating deflection of the cantilever spring. When the tip and surface become very close together the negative charges from the outermost electrons of the closest atoms begin to repel each other exerting a force in the opposite direction to the van der Waals force. The result is that the force on the cantilever spring becomes less so its deflection also becomes less. Upon further decreases in the separation distance, the repulsive force cancels and eventually becomes much greater than the attractive force. This causes the spring deflection to change from a positive direction (defined as being towards the sample) to a negative direction.

A force vs. distance plot [3] for a tungsten tip approaching a graphite surface is shown in Figure 1. This work was done by Mate, et al. [3] and it illustrates what has just been described. In the upper right hand corner of the graph is a schematic drawing of a cantilever spring with three dark circles on its end. These dark circles represent an atomically sharp tip. The atoms on the surface are also represented by dark circles below the tip. The man hanging from the tip is to demonstrate that when the tip is this far from the surface attractive forces bend the end of the cantilever toward the sample. It can be seen from the graph that this effect begins to take place when the tip and the sample are separated by a distance of between 50 and $100 \AA$. At a little less than $50 \AA$ the repulsive forces begin to come into play causing the spring to begin to deflect away from the surface. This is represented by the drawing in upper left hand corner of the graph. The man is now pushing up on the tip (deflecting the spring in the negative direction) to illustrate the effect of the repulsive forces on the spring. The plot shows that the repulsive forces dominate the interaction at very close tip and sample distances. It should be noted that the zero value for the sample position is defined to be the point where the repulsive force and the attractive force cancel each other, which is not necessarily the point where the sample and the tip touch. 


\section{ATOMIC FORCES ACTING AGAINST STYLUS}

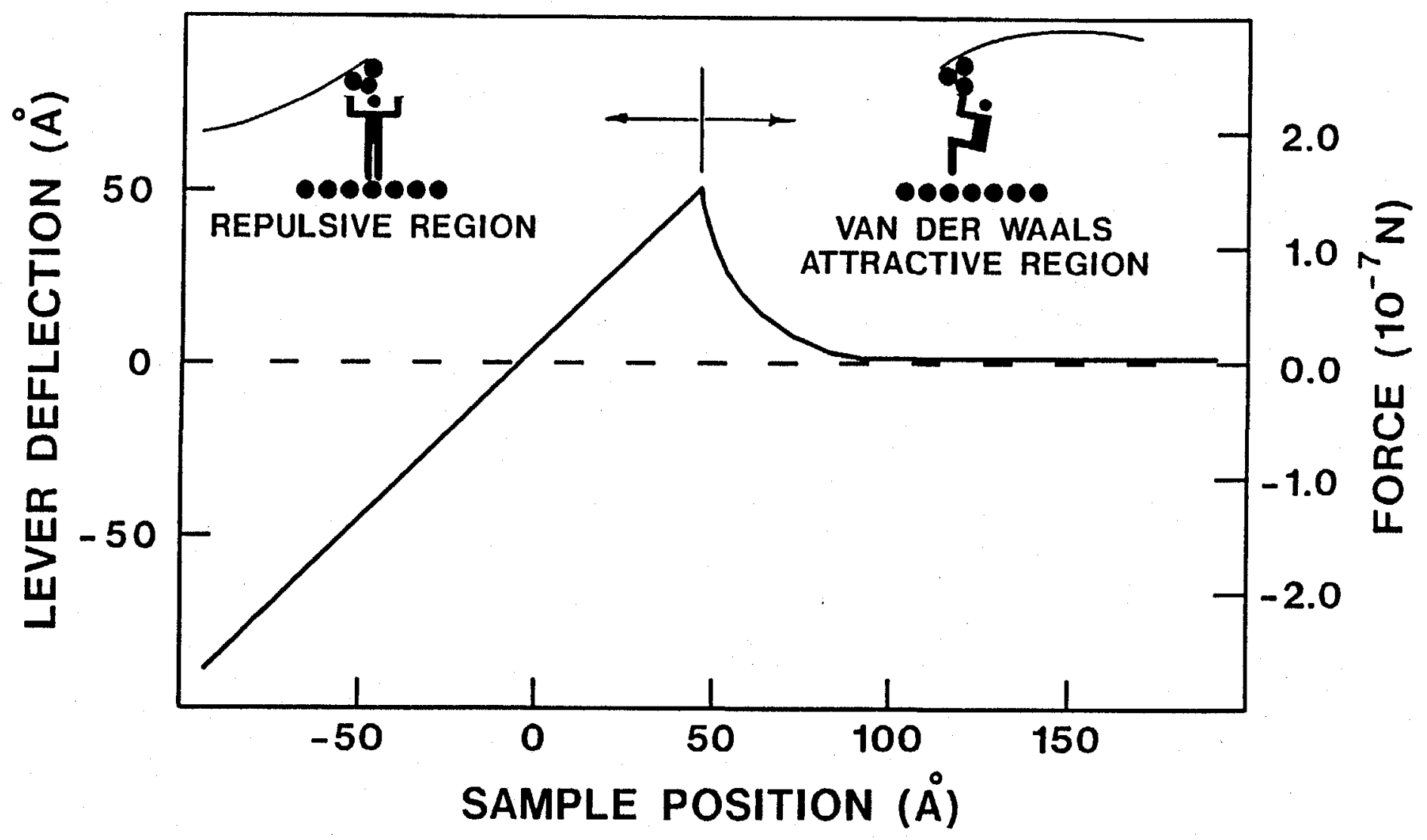

Figure 1: Force vs. distance plot for tungsten against a graphite surface. This illustrates the attractive and repulsive force regimes encountered as an AFM tip approaches a sample's surface. [3] 


\subsection{USING ATOMIC FORCES TO OBTAIN TOPOGRAPHI- CAL IMAGES}

Ore conclusion to be drawn from the above discussion is that by monitoring the amount of deflection of the spring (assuming its spring constant is known) the interaction of the forces fields between individual atoms on the tip and on the sample can be determined. Another way to state this conclusion is that if a constant amount of deflection is maintained as the tip is scanned across the surface then constant force contours can be plotted as a function of the tip's position. Since the magnitude of force is a strong function of tip-to-sample spacing, high resolution topographical images of the samples surface can be acquired.

The two basic image forming processes stated above are illustrated in Figure 2. The upper portion of the illustration depicts the constant force mode. In this mode a known deflection is applied to the spring. This amount of deflection is maintained as the tip is scanned across the surface by the feedback loop connected to the z-portion of the piezoelectric tube scanner shown. If for example, the surface has a peak on it the spring will be forced to deflect upward to rise over the peak. Since the feedback loop is set to maintain a constant spring deflection, a voltage will be applied to z-axis piezoelectric driver when the peak is encountered which will raise the tip holder in concert with the surface profile. If the voltage applied to the $z$-axis is plotted vs. $x$ and $y$ position a line scan image of the surface topography will be generated.

The second mode of image formation is the constant height mode (also called the variable deflection mode) as shown in the lower portion of Figure 2 . In this mode the tip is brought close enough to the sample surface so the atomic forces can interact. As the tip is scanned across the surface the voltage to the z-axis is held constant. This means that the cantilever's base is held at a constant height above the sample surface (disregarding $x$ and $y$ tilts of the sample which can be subtracted from the data). Any roughness in the sample will induce force fluctuations on the tip causing the cantilever to be deflected. This deflection can be plotted directly as a function of position in a manner similar to the constant force mode, or it can be converted into force contour lines and plotted as a top view false color image.

The chief advantage of the AFM over the STM for producing images of surfaces is that the AFM will image both conductors and insulators whereas the STM will only image conductors or semiconductors. This is a very important consideration because many of the surfaces of importance to the precision engineering field are either insulators or have a thick insulating oxide surface layer which makes them effectively insulators by STM standards. 


\section{Basic Image Formation Processes 1. Constant Force Mode}

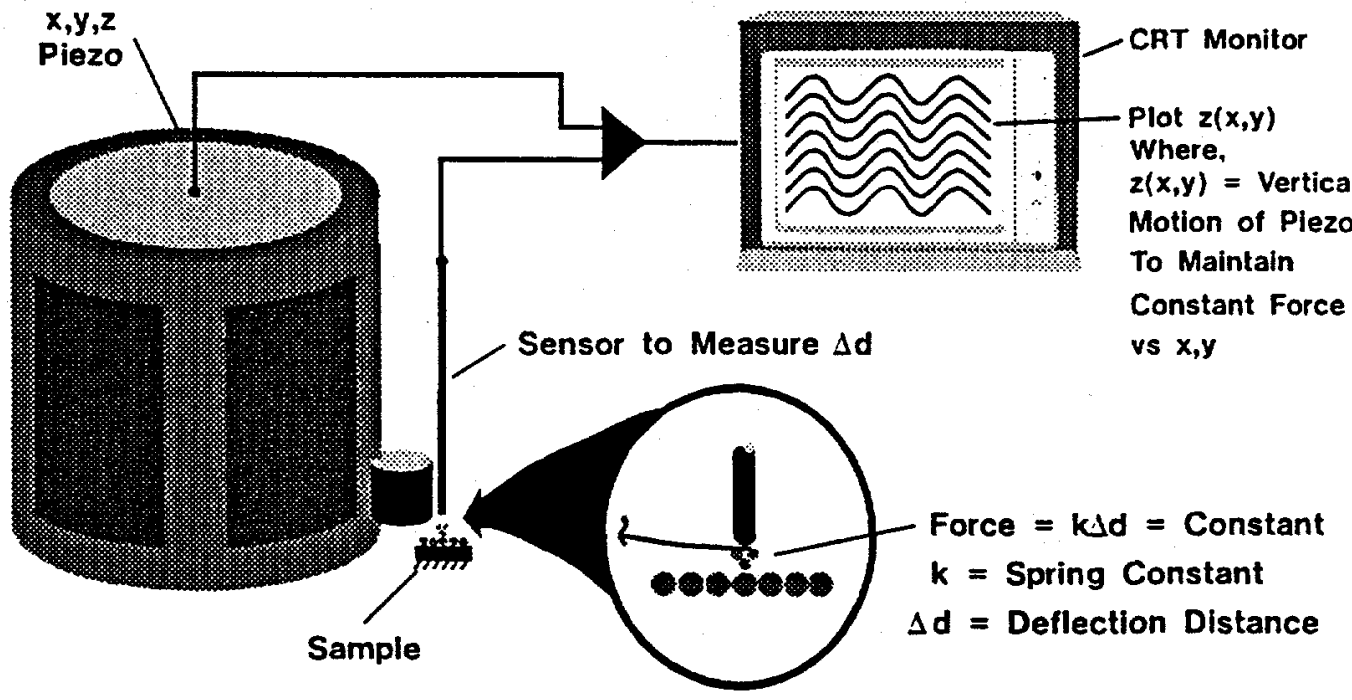

\section{Constant Height Mode}

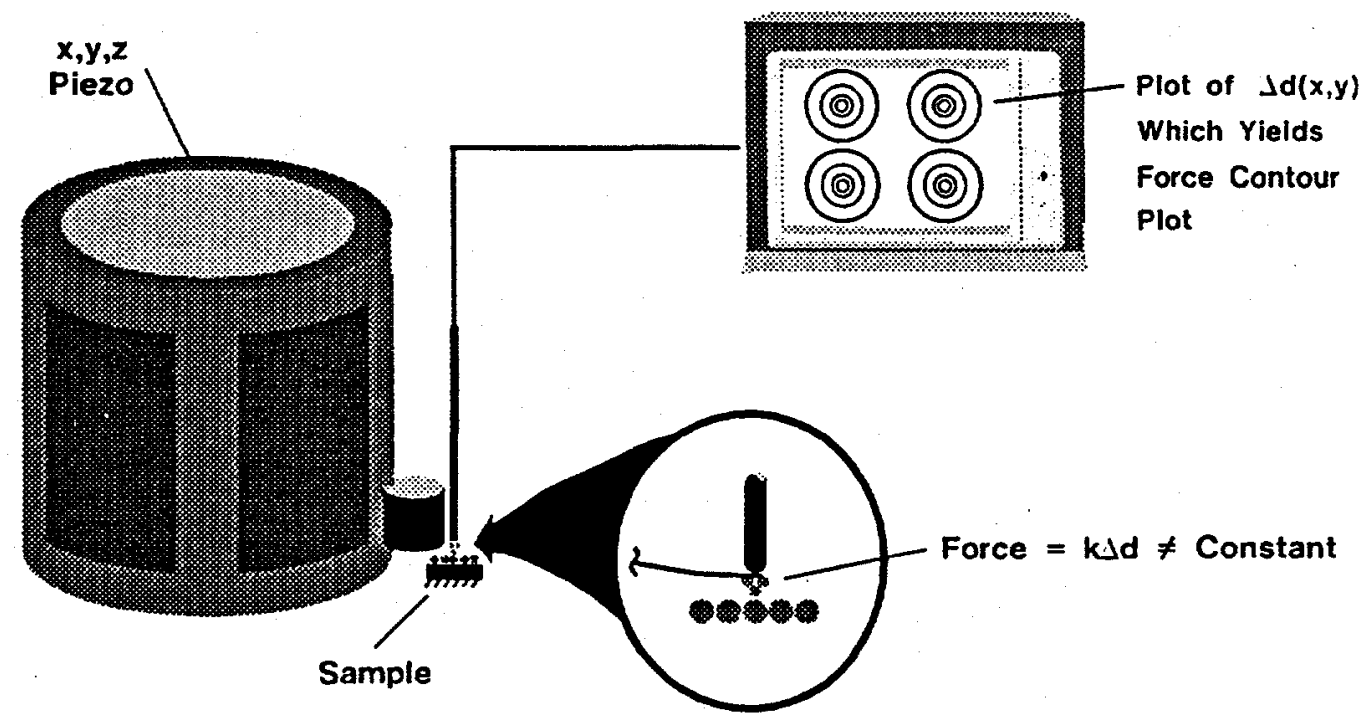

Figure 2: Illustration of the two basic image formation processes. 


\subsection{SOME RECENT USES FOR THE AFM}

The AFM is being used as a diagnostic tool for many types of experiments. The vast majority of these to date have been imaging experiments of one form or another. It has been used to image a variety of nonconducting surfaces [11 and 18] including biological specimens [11 and 15] and polymers [16]. Other uses have been to measure friction coefficients at the atomic level [5], high-resolution imaging of magnetic domain walls [17], studying force interactions between various materials [10], and simultaneously taking STM and AFM images to separate contributions from the geometric and electronic structure factors of a sample [19].

\subsection{AFM DESIGN CONSIDERATIONS}

The objective is to design and fabricate an AFM that can be used to perform high resolution imaging of surfaces of interest to the precision engineering field. These surfaces would include those found on optics, diffraction gratings, optical disks, diamond cutting tools, and precision bearings. We envision it as being an instrument that can help us to understand material removal processes on a very fine scale.

The instruments in use have concentrated on atomic images of surfaces which are quite flat. An AFM for examining engineering surfaces would not need atomic scale resolution (although it would not hurt to have it) but would need to accurately image rough surfaces. The resolution requirements for such and instrument would be $10 \AA$ laterally and less than $1 \stackrel{A}{ }$ in the vertical direction. The range requirements would be at least 3 microns laterally and at least 0.5 micron vertically. It would also be convenient to operate in the STM mode or the AFM mode with the same tip. The fact that most AFM's raster the sample instead of the tip places severe limitations on the sample size, and renders them ineffective for many precision engineering applications. Therefore, an important requirement is that the tip move over the sample.

Since the inspiration for the AFM came from the STM its mechanical design has benefited directly from the generations of improvements made upon its predecessor. The main focus of the current development efforts lie in the areas of producing the cantilever spring and in sensing its deflection. Another area that is beginning to receive some attention is the role of the tip in image formation [16], [19-21].

\subsubsection{Cantilever Design}

The two main design considerations for the cantilever are its spring constant and its natural frequency. When choosing a spring constant the amount of required force resolution must first be determined. This choice depends upon the scale and types of surfaces to be imaged. As an example, Alexander [4] determined that when imaging the native oxide of silicon 
the change in force as the tip passes over an atom is $4 \times 10^{-10} \mathrm{~N}$. Therefore if this is the sample of interest, a force resolution of at least $1 \times 10^{-10} \mathrm{~N}$ would be required (to be safe). If a deflection sensor with $1 \AA$ resolution is used, then a spring constant of $1 \times 10^{-10} \mathrm{~N} / \AA$ (or $1 \mathrm{~N} / \mathrm{m}$ ) is required. To put this into perspective, $1 \mathrm{~N} / \mathrm{m}$ is about the spring constant of a slinky. However the slinky's natural frequency is about $1 \mathrm{~Hz}$, and in order to take data at a reasonable rate, the cantilever should have a natural frequency of around 4,000 $\mathrm{Hz}$. The natural frequency for a cantilever can be calculated by using Equation (1):

$$
\omega_{n}=\sqrt{3 E I /(0.23) l^{3} m}
$$

where,

$\omega_{n}=$ the natural frequency in radians / sec,

$E=$ Young's Modulus for the cantilever material,

$I=$ the moment of inertia,

$l=$ the length of the cantilever,

$m=$ the mass of the cantilever.

The spring constant for a cantilever spring is given by Equation (2)

$$
K=3 E I / l^{3}
$$

where,

$K=$ the spring constant, and the other variables are the same as for Equation (1).

The expression for the moment of inertia of a cantilever with a rectangular cross section is given by Equation (3):

$$
I=b h^{3} / 12
$$

where,

$b=$ the cantilever's width, and

$h=$ the cantilever's thickness.

The mass is given by:

$$
m=\rho V=\rho b h l
$$

where, 
$\rho=$ the spring material's density.

By substituting Equations (3) and (4) into Equations (1) and (2) and rearranging, the expressions for the natural frequency and the spring constant in terms of the geometrical and material parameters are:

$$
\begin{gathered}
\omega_{n}=(1.043)\left(h / l^{2}\right)(\sqrt{E / \rho}) \\
K=(E b / 4)(h / l)^{3}
\end{gathered}
$$

Equations (5) and (6) illustrate that a small spring constant and a high natural frequency can be simultaneously achieved by having a short, and thin spring made out of a low density material. For example, a piece of beryllium $2 \mathrm{~mm}$ long $\times .25 \mathrm{~mm}$ wide $\mathrm{x} .008 \mathrm{~mm}$ thick has a spring constant of $1.2 \mathrm{~N} / \mathrm{m}$ and a natural frequency of $4,200 \mathrm{~Hz}$. A piece of aluminum $2 \mathrm{~mm}$ long $\times .25 \mathrm{~mm}$ wide $\times .012 \mathrm{~mm}$ thick has a spring constant of $0.93 \mathrm{~N} / \mathrm{m}$ with a natural frequency of $2,500 \mathrm{~Hz}$. These two examples illustrate that material and geometry parameters can be varied to achieve an acceptable design. Most of the AFM's currently in service use tungsten wires as the spring material [4-6]. This violates the concept of using a low density material for the cantilever, but tungsten wire is readily available (another important consideration) in a variety of small sizes so the geometry can be altered to achieve a usable design. Alexander [4] used a 20 micron diameter tungsten wire bent at a 90 degree angle (to give it lateral stability) which resulted in a spring constant of $10 \mathrm{~N} / \mathrm{m}$ with a $6,000 \mathrm{~Hz}$ natural frequency.

\subsubsection{Deflection Sensor}

Since the force varies linearly with the cantilever's deflection, the AFM's force resolution and dynamic range depend directly upon the deflection sensor. The first method used to detect the spring deflection was that of tunneling [1], [7]. A tunneling probe was placed directly behind the end of the cantilever so any deflection of it would result in a spacing change between the probe and the cantilever. This would induce a change in the tunneling current. Subangstrom movements of the spring could easily be resolved with this detection method. Its dynamic range is restricted somewhat by the need for the tunneling probe to be within a very few angstroms of the spring before a tunneling current can be detected. Therefore the maximum spring deflection that can be sensed is only a few angstroms. The dynamic range of this sensor can be increased by placing a piezoelectric actuator in the structural loop between the spring and the probe. This allows the undeflected spring to be moved closer to or further away from the tip so that the mean preload force between the spring and the sample can be changed [1]. This scheme of deflection detection has problems; most users complain that these instruments are unreliable. The general conclusion is that contamination between the probe and spring is the major contributor to this unreliability. 
It should be noted, however, that P. Bryant has built a series of atomic force detectors [8] that mount to the Nanoscope I [9] which use this principal and achieve reliable results. Most researchers have abandoned this sensing method, however, and are looking into other means of detection.

Other candidate sensing methods are optical and capacitive [3] with the optical approaches getting the most attention. There are a variety of optical techniques being investigated, including:

1. reflecting a laser beam off of the cantilever into a position sensor to measure its angular deflection as a function of force changes [4], [6]

2. heterodyne interferometry [10]

3. fiber optic interferometry [11], [12]

4. fiber optic displacement sensor [13]

The first technique was used by Alexander [4], and Meyer and Amer [6]. A displacement resolution of less than $0.1 \dot{A}$ at the AFM tip was achieved. For the second technique Martin et al. [10] oscillated the spring at its natural frequency and monitored the amplitude of change of the cantilever as it interacted with the sample. Using this procedure, the heterodyne interferometry method can measurement displacements as small as $1 \times 10^{-4} \AA$. The third method was used by Drake and Leiner [12] to measure the vibration characteristics of a cricket's posterior tympanic membrane. Sensitivities of less than $0.1 \AA$ were reported. In a review article on the STM and the AFM, Hansma, [11] reported that a fiber optic interferometer has recently been employed by scientists at IBM Almaden Research Center as a deflection sensor. An upgraded design of the OSSEM could also be used with a potential dc sensitivity of 0.25 to $0.75 \AA$ /microvolt [14].

The advantages of the optical methods over the tunneling method is an increase in reliability and an increase in dynamic range. One disadvantage is that the hardware is generally larger than that required for the tunneling probe. The fiber optic techniques (interferometer and OSSEM), however, can be implemented in very small spaces in the region near the AFM tip which makes them quite attractive. For instance a sensing area of less than $0.2 \mathrm{~mm}$ in diameter on the back of the cantilever is all that is needed for the fiber optics displacement sensor.

\subsubsection{Role of the Tip in Producing Images}

The shape of the tip directly influences the quality of the image obtained by the AFM. To achieve atomic resolution an atomically sharp tip is necessary. As difficult as this may sound it has been accomplished quite routinely by many investigators $[4,7,8,15,18$, 
20]. The saving grace for atomic imaging is that the sample is very flat over the imaging region of approximately $200 \AA$. Therefore if one atom is closer to the sample than any other atom on the tip it will be the one that produces the image. For rougher surfaces (which is the case for most engineering samples) the image produced is a convolution of the surface topography and the macroscopic (relative to atomic scale) tip shape. The effect of tip shape on image quality for rough surfaces has been very graphically demonstrated by Musselman and Peterson [22] for STM tips. Albrecht et al. [16] also discuss this problem for AFM tips. So an instrument designed for rougher surfaces must have a sharp and stable tip of known geometry. This is an area that needs quite a bit more work.

\subsection{CURRENT AFM DEVELOPMENT PLANS AT THE PRE- CISION ENGINEERING CENTER}

A two pronged approach to AFM development is being implemented the PEC. Initially an AFM probe similar to the one P. Bryant describes in reference [8] will be built and attached to the STM scanning head. This probe should produce AFM images and has the capacity to image in both the STM and AFM modes. Since it will mount to the STM heads in place of the STM tunneling tip, lateral movements from subangstrom to nine micrometers will be possible. Vertical displacements will range from subangstrom to three micrometers. This AFM probe consists of a $0.25 \mathrm{~mm}$ diameter tungsten wire that has a $80 \%$ platinum - 20attached to its end. The various cantilevers can range in thickness from 2 to 10 micrometers. Each cantilever has a triangular shape with a length ranging from $0.1 \mathrm{~mm}$ to $0.25 \mathrm{~mm}$. The apex forms an angle of about 40 degrees. This apex is turned downward to form the contouring tip. The deflection sensor is a tunneling probe that is glued to the tungsten wire. A schematic of this AFM probe is shown in Figure 3. Bryant reported spring constants ranging from 0.1 to $200 \mathrm{~N} / \mathrm{m}$, with natural frequencies ranging from 10 to $100 \mathrm{kHz}$. The forces exerted on samples have varied from $1 x 10^{-7} \mathrm{~N}$ to $1 x 10^{-4}$ N.

This probe's main disadvantage is its lack of flexibility. Because the tunneling tip is glued in position, the distance from the tip to the AFM probe, is set during fabrication and is not adjustable. Thus the only way to bring the tunneling tip into position is to load the AFM cantilever beam against the surface. Depending on the initial relative position, different loads will be required to obtain tunneling for each probe. Therefore the only way to vary the force would be to build many of these probes and calibrate each one - and unappealing option. Another disadvantage of this design is that due to the preload of the AFM cantilever necessary to obtain tunneling, the probe can only operator in the repulsive force regime.

Because of the inflexibility of Bryant's probe and the horror stories about tunneling deflection sensors, an optical deflection sensor will be investigated. Electronics are being produced by Optoacoustic Sensors which will detect signals from either a fiber optic bun- 


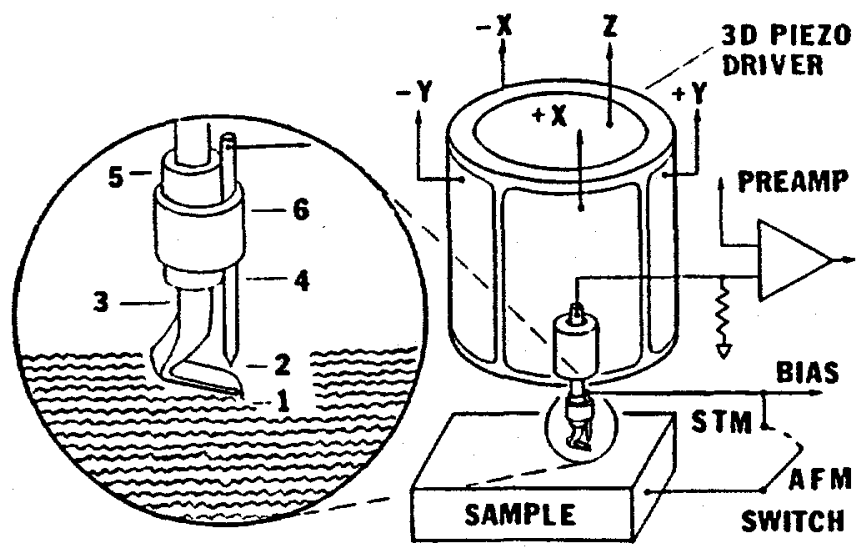

Figure 3: Schematic representation of an AFM probe designed by P. Bryant [8] mounted to a tube scanner. Note that it can be easily switched from the AFM to the STM mode. 
dle (similar to that used in the OSSEM instrument) or a fiber optic interferometer. The fiber optic bundle will use seven $0.125 \mathrm{~mm}$ fibers with a sending/detecting configuration that is optimized for sensitivity. Sensitivities of from 0.25 to $0.75 \AA / \mu v$ are anticipated with this scheme. The actual sensitivity and stability of this sensor will have to measured when the hardware arrives. We plan to purchase the components to build a fiber optic interferometer for deflection sensing. This promises to have better sensitivity than the fiber optic bundle.

Beryllium cantilevers have been ordered with a range of spring constant from $1 \mathrm{~N} / \mathrm{m}$ to 50 $\mathrm{N} / \mathrm{m}$. The current plan is to use a diamond for the AFM tip. This could either be a piece of large lapping grit or a fractured piece from a larger gem quality diamond [4].

This hardware will be small enough to mount to the end of a tube scanner without degrading its vibration characteristics. If this can be done it removes the need to move the sample (as is done with most AFM's ).

\subsection{CONCLUSIONS AND FUTURE WORK}

The AFM should be a useful tool at the PEC and to the precision engineering field in general because of its ability to image conducting or non-conducting surfaces with nanometer or better spatial resolution. The following objectives represent the planned activities for 1989.

- AFM design will have the tip raster across the surface as opposed to rastering the sample. This should make the instrument more valuable for precision engineering studies.

- An AFM probe that will mount in any of the STM tube scanners interchangeably with a STM tip. This probe will use tunneling current feedback as the tip deflection sensor and will be able to operate either in the STM or AFM mode. Its disadvantages are that it can only operate in the repulsive force region and its force sensing dynamic range is extremely limited.

- In addition, an AFM probe using optical position feedback via a fiber optic cable will be investigated. This technique may provide much more force sensing dynamic range, operation in either the attractive or repulsive regimes, enhanced reliability, and simultaneous STM and AFM images (depending on tip material). 


\section{References}

[1] G. Binning, C.F. Quate, and Ch. Gerber, Phys. Rev. Lett. 12, (1986), pages 930-933.

[2] E. C. Teague, Room Temperature Gold-Vacuum-Gold Tunneling Experiments (University Microfilms International. Ann Arbor, Michigan, 1978), Chap. 4, pp. 141-148.

[3] C. Mathew Mate, Ragnar Erlandsson, Gary M. McClelland, and Shirley Chiang, J. Vac. Sci. Technol. A 6 (3), May/Jun 1988, pages 575-576.

[4] S. Alexander, L. Hellemans, O. Marti, J. Schneir, V. Elings, P. K. Hansma, An Atomic Resolution Atomic Force Microscope Implemented Using an Optical Lever, to appear in the Journal of Applied Physics.

[5] C. Mathew Mate, Gary M. McClelland, Ragnar Erlandsson, and Shirley Chiang, Phys. Rev. Lett. Vol. 59, No. 17, 26 Oct. 1987, pages 1942-1945.

[6] G. Meyer and N. M. Amer, Appl. Phys. Lett. 53 (12), 19 Sep. 1988, pages 1045-1047.

[7] O. Marti, B. Drake, S. Gould, P. K. Hansma, Atomic Resolution Atomic Force Microscopy of Graphite and the "Native Oxide" on Silicon, to appear in the J. Vac. Sci. \& Technol.

[8] P. J. Bryant, R. G. Miller, and R. Yang, Appl. Phys. Lett. 52 (26), 27 June 1988, pages 2233-2235.

[9] Digital Instruments, Inc., 135 Nogal Drive, Santa Barbara, CA 93110.

[10] Y. Martin, C. C. Williams, and H. K. Wickramasinghe, J. Appl. Phys. 61 (10), 15 May 1987, pages 4723-4729.

[11] P. K. Hansma, V. B. Elings, O. Marti, C. E. Bracker, Science Vol. 242, 14 Oct. 1988, pages 209-242.

[12] A. D. Drake, D. C. Leiner, Rev. Sce. Instrum. Vol. 55 No. 2 Feb 1984, pages 162-165.

[13] S. Fawcett and R. F. Keltie, Precision Engineering Center Annual Report vol. IV 1986, pages 24-29. Sensor developed at NCSU and Optoacoustic Sensors, 1706 Medfield Rd., Raleigh, NC.

[14] Based on discussions with Reg Cook of Optoacoustic Sensors.

[15] O. Marti, B. Drake, S. Gould, and P. K. Hansma, J. Vac. Sci. Technol. A 6 (3), May/Jun 1988, pages 2089-2092.

[16] T. R. Albrect, M. M. Dovek, C. A. Lang, P. Grutter, C. F. Quate, S. W. J. Kuan, C. W. Frank, R. F. W. Pease, J. Appl. Phys. 64 (3), 1 Aug. 1988, pages 1178-1184.

[17] Y. Martin, D. Rugar, and H. K. Wickramasinghe, Appl. Phys. Lett. 52 (3), 18 Jan 1988, pages 244-246. 
[18] T. R. Albrect and C. F. Quate, J. Appl. Phys. 62 (7), 1 Oct. 1987, pages 2599-2602.

[19] P. J. Bryant, R. G. Miller, R. Deeken, R. Yang, and Y. C. Zheng, to be published.

[20] T. R. Albrect and C. F. Quate, J. Vac. Sci. Technol. A 6, (1988), pages 271-276.

[21] J. B. Pethica, W. C. Oliver, Phys. Scripta T19, (1987), pages 61-66.

[22] I. H. Musselman and P. A. Peterson, Poster presentation at Second Annual Symposium Organized by the North Carolina Section of the American Chemical Society on Chemistry at Surfaces and Interfaces, Duke University, Sep. 9, 1988, worked performed at NCSU Precision Engineering Center. 


\title{
4 MEASURING DEPTH PROFILES OF RESIDUAL STRESS WITH RAMAN SPECTROSCOPY
}

\author{
W.S. Enloe and R.G. Sparks \\ Graduate Students \\ Michael A. Paesler \\ Associate Professor, Physics
}

Knowledge of the variation of residual stress is a very important factor in understanding the properties of machined surfaces. The nature of the residual stress can determine a part's susceptibility to wear, deformation, and cracking. Raman spectroscopy is known to be a very useful technique for measuring residual stress in many materials. These measurements are routinely made with a lateral resolution of $1 \mu \mathrm{m}$ and an accuracy of $0.1 \mathrm{kbar}$. The variation of stress with depth; however, has not received much attention in the past. A novel technique has been developed that allows quantitative measurement of the variation of the residual stress with depth with an accuracy of $10 \mathrm{~nm}$ in the $z$ direction. Qualitative techniques for determining whether the stress is varying with depth are presented. It is also demonstrated that when the stress is changing over the volume sampled, errors can be introduced if the variation of the stress with depth is ignored. Computer aided data analysis is used to determine the depth dependence of the residual stress.

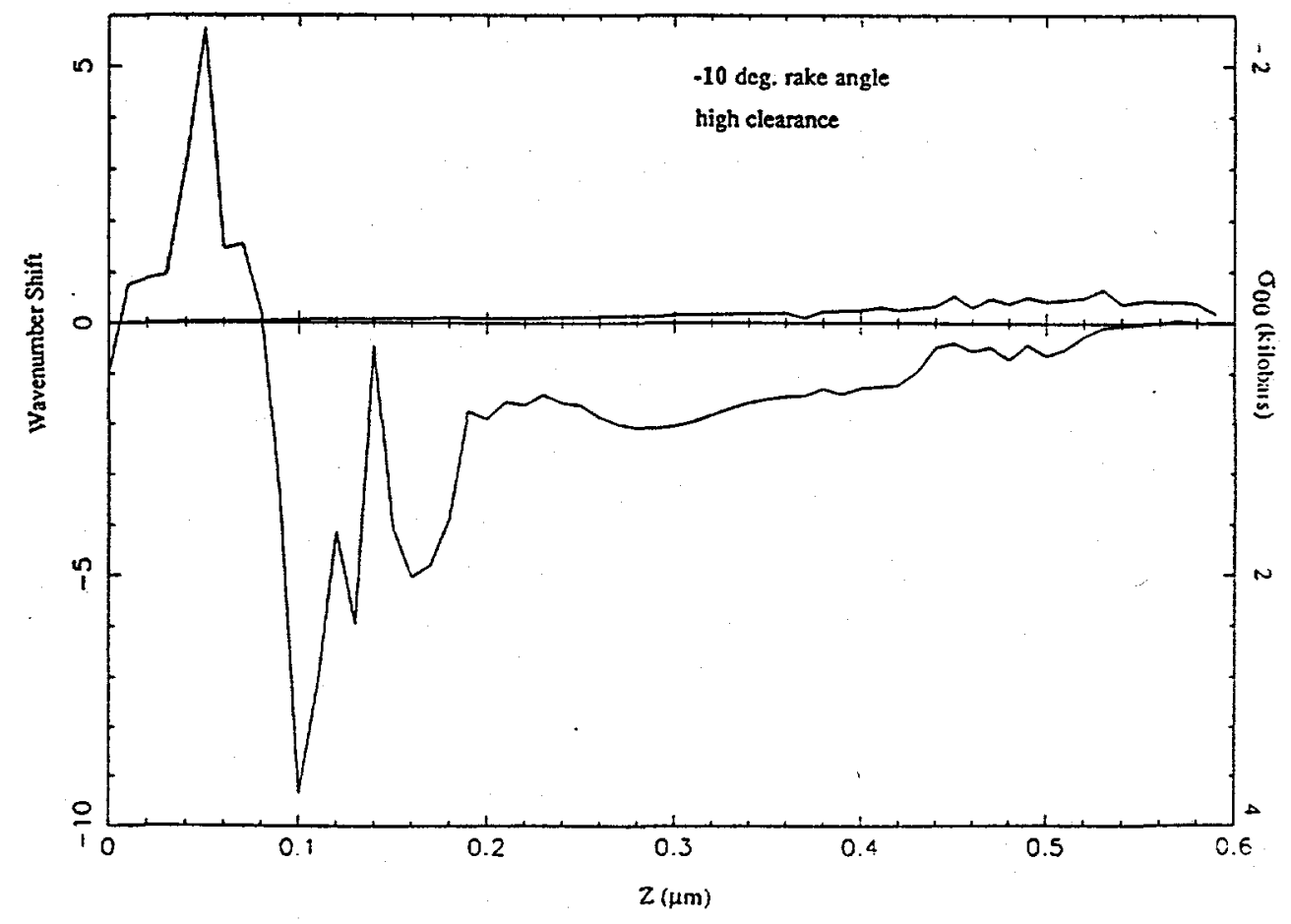




\subsection{INTRODUCTION}

Raman spectroscopy is an important technique for measuring a number of important material parameters [1] such as phase, residual stress, crystalinity, etc. The addition of microscope focusing optics $[2,3,4]$ has allowed Raman spectra to be obtained with a lateral resolution of $1 \mu \mathrm{m}$ [5]. With the possible exception of very small particles, spectra obtained in the micro-Raman configuration can be interpreted in the same manner as those obtained in a standard configuration. Micro-Raman spectroscopy has successfully been used to measure a number of material parameters with high lateral resolution; however, the variation of the Raman signal with depth into the sample has received little attention. This paper will concentrate on the application of micro-Raman spectroscopy to residual stress measurements, and specifically on the possibility of obtaining depth profiles of residual stresses in semi-conducting materials. A technique will be considered to be a successful method of determining depth profiles if an unambiguous determination of the variation of residual stress can be made in either a qualitative or quantitative sense. Particular emphasis will be placed on the influence of a priori assumptions, and on any inherent limitations of the technique.

A previous work by Cheong, Marcus, and Adar [6] describes a technique for measuring the residual stress around a heterogeneous interface. The system that they considered was that of thin Si films deposited on quartz substrates, with the residual stress due to the difference in thermal expansion coefficients of these materials. This is a theoretically and experimentally complex problem due to the fact that the conditions of equilibrium require that there be zero net stress in the material and that the residual stress variations through the sample be complex. The residual stress was assumed to be constant in the film and to decay exponentially in the substrate. The procedure used was to make assumptions about the nature of the residual stress and then calculate the response of the film/substrate system to this stress. Since the substrate is transparent, the residual stress can be measured by focusing on the interface and the surfaces of the film and substrate. It was determined that the stress contained in the substrate penetrates to a depth of 1-2 times the film thickness.

While this technique is useful for determining the depth variation of the residual stress, it suffers from a number of obvious defects. First, the calculation of the expected residual stress is quite cumbersome, even in a relatively simple system such as this one. Secondly, it depends on stringent a priori assumptions about the nature of the residual stress (i.e. constant stress in the film and exponential decay of the stress in the substrate). Finally, the fact that the measured values are actually averaged over the probe thickness was not taken into account. It was reported that the actual stresses might be as much as 5-10 times higher than the measured values, in general agreement with calculational studies reported below.

A technique will now be described that can successfully be used to determine the depth variation of residual stress. The volume average character of the measured values is treated 
explicitly. Furthermore, there is no need for complicated stress calculations, and no a priori assumptions about the nature of the residual stresses are necessary.

\subsection{ABSORPTION COEFFICIENTS}

When light of intensity $I_{o}$ penetrates a solid, the intensity at any depth $z$ will be $I_{0} e^{-\alpha z}$, where $\alpha$ is the absorption coefficient,and $1 / \alpha$ defines a characteristic depth for light in the sample known as the penetration depth. In the case of Raman scattering, the light must penetrate to a depth $z$ before being scattered and then it must return to the surface in order to be collected; therefore, the intensity of the Raman signal from any depth $z$ will be proportional to $e^{-2 \alpha z}$ (the fact that the absorption coefficient for the scattered light is slightly different from that of the incident light will not be considered in this analysis). The value of the absorption coefficient depends on both the material of interest and the energy of the incident light. A plot of the absorption coefficient vs. energy for $\mathrm{Si}, \mathrm{Ge}$ and GaAs is shown in Figure 1. It is the exponential decay of the intensity of the Raman signal with depth into the sample combined with the fact that the value of the absorption coefficient varies with the energy of the light that makes determination of stress profiles from Raman spectra feasible.

Raman scattering is typically done in the visible region with the green $(514.5 \mathrm{~nm})$ and blue (488 $\mathrm{nm}$ etc) lines of an Ar ion laser. This light has an energy of approximately $2.5 \mathrm{ev}$. In this region the absorption coefficient shows drastically different behavior for different materials. For Si, the absorption coefficient, and therefore the penetration depth, is rapidly changing over this region, but it is relatively low. In $\mathrm{Si}$, green light would have a penetration depth of just under one $\mu \mathrm{m}$. Germanium, on the other hand, has a much higher absorption coefficient. Green light has a penetration depth of approximately 0.15 $\mu m$ in Ge. The disadvantage is that the absorption coefficient changes very slowly with energy over this range. The absorption coefficient of GaAs is intermediate between that of $\mathrm{Si}$ and $\mathrm{Ge}$ and it varies either rapidly or slowly depending on the exact energy of the light used.

The absorption coefficient, or alternatively the penetration depth, of the light used determines the effective volume sampled by the Raman signal. Thus, by adjusting the energy of the light used or the material being studied, it is possible to study the variation of stress over different depths in the material. Another important consideration is that when the absorption coefficient is changing rapidly with energy, it may no longer be valid to assume that the absorption coefficient is the same for the incident and the inelastically scattered light. 


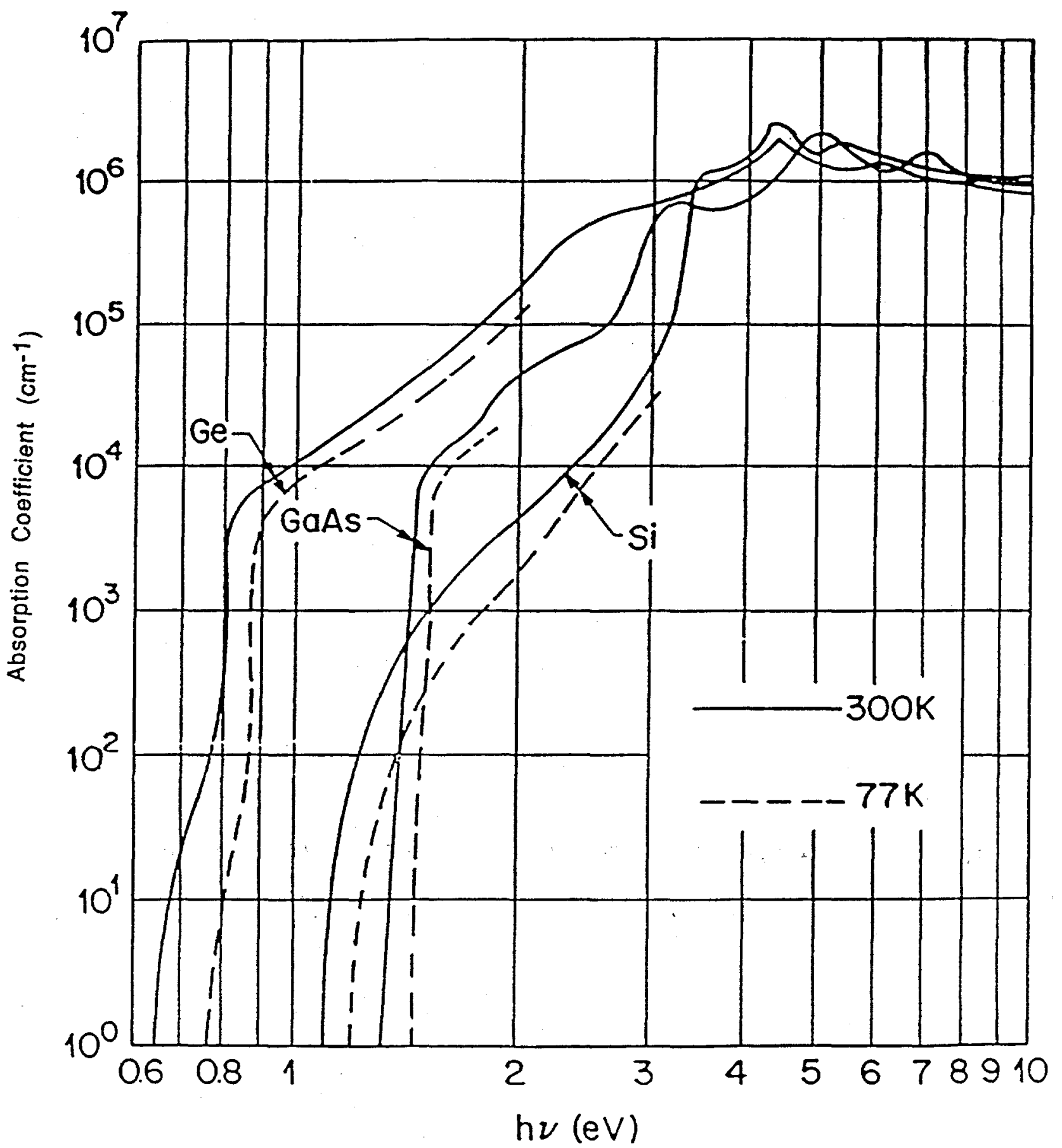

Figure 1: Measured absorption coefficients near and above the fundamental absorption edge for pure $\mathrm{Ge}, \mathrm{Si}$, and $\mathrm{GaAs}$ [7]. 


\subsection{THEORETICAL MODEL}

In this analysis, it will be assumed that the Raman signal has a Gaussian lineshape. Thus, the analytical expression for the Raman signal is:

$$
R(w)=A \int \exp [-2 \alpha z] \exp \left[-(\omega-\omega z) 2 / 2 \sigma^{2}\right] d z+b k
$$

where

$$
\omega_{z}=\omega_{o}+S(z)
$$

This is actually a simple function consisting of three parts. The first is an integral over all depths. The second is an exponentially decaying intensity factor. Finally there is a Gaussian lineshape located at $\omega_{z}$ and having a natural line width of $2 \alpha^{2}$. Amplitude and background factors ( $A$ and $b k$ ) are included so that calculated values will correspond to actual data. The depth $z$ dependence enters this expression at two points. These are the exponentially decaying intensity factor and $\omega_{z}$. The term $\omega_{z}$ is the location of the unshifted Raman signal, $\omega_{0}$, plus the shift, in wavenumbers, due to the stress at each depth $z$. This shift function, $S(z)$, will hereafter be referred to as the stress profile.

The factors which will effect the nature of the Raman signal are the functional form of the stress profile and the value of the absorption coefficient (or alternatively the energy of the light used to excite the Raman signal). Since the Raman function is somewhat complicated, it is instructive to make some assumptions about the functional form of the stress profile and calculate the Raman signal expected for different values of the absorption coefficient. To this end, assume that the stress profile is characterized by some finite surface stress and that the stress goes to zero beyond some depth, $z_{0}$. This stress profile is assumed to have the functional form of a damped cosine, or

$$
S(z)=s_{0} e^{-\left(a z / z_{0}\right)} \cos \left(3 \pi z / 2 z_{0}\right)
$$

where $s_{o}$ is the value of the stress at the surface, $z_{0}$ is the depth at which the stress goes to zero, and $a$ is a constant that determines the total area under the curve. Here $a$ was chosen so that the net stress would be zero.

This stress profile, along with the exponentially decaying intensity of four different colors of light, is plotted in the upper portion of Figure 2. Here $z$ is the normalized depth into the material and the absorption coefficients are expressed in terms of $z_{0}$, the characteristic depth of the stress profile. The lower portion of this figure shows the calculated Raman signal corresponding to each of the four absorption coefficients chosen. The stress profile was divided into four regions (three regions where the stress is changing and the unstressed 

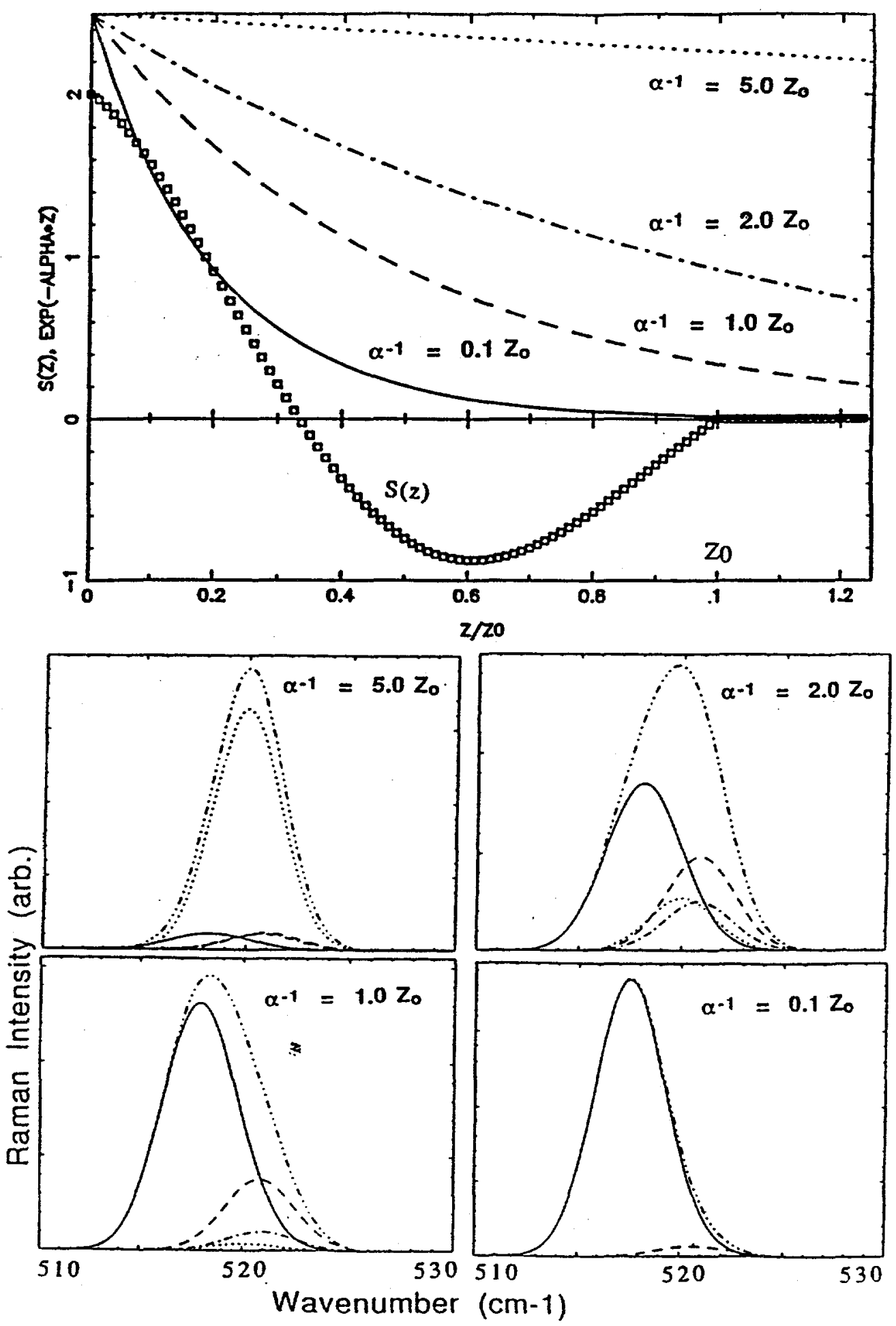

Figure 2: The top of the upper portion of the figure contains a hypothetical stress profile having the functional form of a damped cosine. Also shown are the exponential decay of four different wavelengths of light ranging from red (dotted line) to blue (solid line). The penetration depth, $\alpha^{-1}$, is expressed in terms of the maximum depth of the stress profile, $z_{o}$, for each wavelength. The lower portion of the figure shows calculated Raman spectra from each of the four wavelengths. In each graph, the upper curve is the total Raman signal, and the other curves are contributions from successively deeper regions into the sample. 
bulk material) and the contribution from each region was calculated along with the total Raman signal. Thus, in each graph, the upper curve represents the total Raman signal, the solid curve is the surface contribution, and the dotted line is the contribution from the unstressed bulk material. The surface stress was taken to be -3 wavenumbers and $z_{o}$ was set equal to one.

In the graph in the upper left the penetration depth, $1 / \alpha$, is much greater than the characteristic depth of the stress profile, $z_{0}$. Here the Raman signal is mainly from the unstressed bulk material and is thus unshifted $\left(\omega_{0}\right.$ was taken to be $\left.520 \mathrm{~cm}^{-1}\right)$ and only very slightly broadened. In the other extreme, when the penetration depth is much less than $z_{\mathrm{o}}$ (lower right), the total Raman signal is essentially all from the surface variation of the stress profile. Here the Raman signal is both shifted and, more importantly, asymmetrically broadened. For intermediate cases, when the penetration depth is on the order of $z_{0}$, varying amounts of shift and asymmetry are observed. The important thing to notice here is that the presence of broadening and asymmetries in the Raman signal conclusively indicate that the stress is changing over the volume probed. When this is the case, it is inappropriate to characterize the stress by the peak value of the Raman signal.

\subsubsection{Qualitative Analysis}

It is evident that qualitative studies are possible by carefully varying the penetration depth of the light used. For the stress profile under consideration here, the high penetration depth data can be used to put an upper bound on $z_{o}$. The data for $\alpha^{-1}=5.0$ is unshifted and unbroadened indicating that this depth contains mostly unstressed material. The $\alpha^{-1}=$ 2.0 data is substantially broadened and unshifted. This would indicate that 2.0 would be an appropriate upper bound for $z_{0}$. The data taken with the light having the smallest penetration depth indicates that the surface stress is tensile (negative shift), and has a magnitude of at least 2.5 wavenumbers. The fact that the curves are asymmetrically broadened to the higher wave number side indicates the presence of compressive stress. However, it is not possible to draw any conclusions about the magnitude or location of this compressive stress other than the fact that it probably occurs at a dimensionless depth greater than 0.1. Considering the complexity of the problem, these estimates compare quite favorable with the known values of $s_{o}=-3.0, z_{o}=1.0$ and the zero crossing at 0.33 .

The ability to make qualitative estimates of this sort will be severely limited in practice. In order to successfully determine a stress profile, the stress must be changing over the penetration depth of the light used. Furthermore, it is necessary to use light with penetration depths both greater and substantially less than the maximum depth of the stress profile. The range of absorption coefficients, or penetration depths, is strongly material dependent (for instance the penetration depth of visible light in $\mathrm{Si}$ is on the order of one $\mu m$ ). The ability to vary the penetration depth is limited by the availability of appropriate laser lines, as well as the nature of the absorption coefficient vs. energy curve. It will be seen that these factors limit the ability to make quantitative estimates as well. 


\subsubsection{Quantitative Stress Profles}

At this point it would seem that to determine a stress profile, all that is necessary is to make appropriate assumptions about the functional form of the stress profile and then apply an appropriate least squares data analysis. In some instances [6] this may be sufficient but the results will then be biased by the choice of $S(z)$. For machined brittle materials such as $\mathrm{Si}$ or $\mathrm{Ge}[8,9]$, the appropriate theoretical analog for residual stress calculations $[10,11]$ is the plastic flow model of micro-hardness indentation [10-15]. The expected residual stress profile is characterized by compression within a zone of plastic deformation near the surface and tensile stresses in the bulk of the sample which responds elastically. The residual stress profile is expected to be discontinuous, or nearly so, at the elastic/plastic boundary with maximum tensile and compressive stresses on either side of this boundary. In these studies, the important factors are the extent of the plastic deformation zone and crack initiation. As a result, the form of the compressive stress is typically unspecified at the surface [13].

The dilemma is that the expected functional form of the stress profile extremely complicated and the behavior is unspecified over much of the region of interest. The problem is how does one apply least squares data analysis to a nonlinear function of unspecified form that in all likelihood is also nonanalytical. The inherent complexity of this problem suggests a novel solution. One, in fact, that does not require any a priori assumptions about the form of the stress profile.

The integral in the Raman function is naturally divided into two regions, greater and less than $z_{0}$. Where the stress is zero $\left(z>z_{0}\right)$, the Gaussian lineshape can be taken outside the integral and the remaining expression can easily be solved analytically. For $0<z<z_{o}$, the stress is changing and the integral can only be solved numerically. Numerical integration would involve dividing the interval into $\mathrm{dz}$ elements and then evaluating the function at each $z$. Therefore the stress function, $S(z)$, must be evaluated at each $z$ regardless of its functional form. The stress at each depth can then be made a parameter that is free to vary.

The function to be fitted then becomes

$$
\begin{array}{r}
\left.R(\omega)=\sum A \exp [-2 \alpha z j] \exp \left[-\left(\omega-\left[\omega_{\circ}+S(z j)\right]\right)^{2} / 2 \sigma^{2}\right]\right] d z \\
+A m p(1 / 2 \alpha) \exp \left[-2 \alpha z_{\circ}\right] \exp \left[-\left(\omega-\omega_{\circ}\right)^{2} / 2 \sigma^{2}\right] \\
+b k
\end{array}
$$

The first term is the integral from zero to $z_{0}$. The second term is the integral from $z_{o}$ to infinity and is constant for a given $z_{o}$. And the third term is simply a background correction. The only parameters free to vary are the $S(z j)$ 's, the residual stress at each depth. Thus the stress profile can then assume any form, even if it is discontinuous. 
The procedure is to chose some depth, $z_{o}$, beyond which the stress will be zero by assumption. The interval from zero to $z_{0}$ is divided into an appropriate number of intervals, each having a width, $d z$. The Raman function is approximated by a sum, and the stress at each depth is varied until the calculated Raman signal corresponds to the one obtained experimentally.

Least squares data analysis involves minimization of the sum of the squares of the differences between the experimental and the calculated data. This sum of squares is referred to as $X^{2}$, and

$$
X^{2}=\sum 1 / \sigma^{2}\left(Y_{\exp }-Y_{f i t}\right)^{2},
$$

where $\sigma^{2}$ is the experimental uncertainty associated with each data point. Since the Raman data are collected in a photon counting mode, the data follow Poison statistics [16] and the uncertainty is equal to the square root of the number of counts.

Thus $X^{2}$ is a function of the experimental uncertainties and the parameters $S(z j)$. These parameters span a $j$ dimensional space known as parameter space. There are a number of standard methods [16] for minimizing the function $X^{2}$, and they all involve searching parameter space for the smallest value of $X^{2}$. The simplest method is known as grid search least squares. In this case each parameter, $S(z j)$, is varied independently until $X^{2}$ is minimized, then the next parameter is adjusted, following a grid in parameter space. This is done until the change in $X^{2}, \Delta X^{2}$, is less than some specified value (typically $1 \%$ ). This method has the advantage of being analytically simple, it is not prone to numerical instabilities, and convergence is guaranteed. The disadvantage of this method is that it is very slow. A second difficulty is that each parameter is adjusted independently, holding all the others constant, and implicitly assuming that they are optimal, or near optimal. In the initial stages of the fit (i.e. far from the minimum $X^{2}$ ), this is certainly not the case and this is one source of the slowness of this technique.

One significant problem associated with optical measurements of depth profiles is that the intensity is a maximum at the surface and decays exponentially into the sample. This makes optical measurements subject to cumulative errors traceable to the surface data [17]. Surface measurements made with light of an appreciable penetration depth are thus unreliable. If one tries to address this problem by using light with a shallower penetration depth, the data from deeper within the sample becomes unreliable. Another problem [17] is that the absorption coefficient, (penetration depth) $[7,18]$, is sensitive to the defect density, band structure, and doping level of the sample, particularly near the absorption edge. Once again, the ability to adjust the penetration depth of the light is often severely limited by the absorption coefficient vs. energy curve and the availability of appropriate laser lines.

As previously mentioned, the availability of laser lines and the value of the absorption coefficient are determined by materials properties, so that there is little prospect for im- 
provement here. However, it has been suggested that data from several laser lines can often be used to make qualitative estimates; with the high penetration depth data providing bounds on the stress profile deep in the material, and the low penetration depth data is used to determine the nature of variations near the surface. The problem of cumulative errors in quantitative estimates can only be solved to the extent that data taken with different laser lines can reliably be included in the data analysis.

It has already been decided that goodness of fit would be determined by minimizing $X^{2}$. If data are taken with green and blue laser lines, it would certainly be possible to define a $X_{b l u e}^{2}$ and $X_{\text {green }}^{2}$ to measure the deviations from the blue and green data respectively. The most simple solution to this problem would be to minimize the total $X^{2}$ defined as the sum of $X_{\text {blue }}^{2}$ and $X_{\text {green }}^{2}$, or

$$
X_{\text {tot }}^{2}=X_{\text {blue }}^{2}+X_{\text {green }}^{2}
$$

This technique could easily be extended to three or more laser lines; however, one would want to be careful that other statistical problems do not arise. Typically the green data will converge much more rapidly than the blue data, It has also been observed that any instabilities in the estimates are associated with regions covered only by the green light, thus supporting the conclusions above about accumulated errors.

\subsection{APPLICATIONS}

Figure 3 shows a typical stress profile obtained from machined Ge using the technique described above. The upper graph is the spectra obtained with blue $(488 \mathrm{~nm})$ light and the middle graph is that obtained with green $(514.5 \mathrm{~nm})$ light, while the lower graph is the stress profile. The depth resolution of this technique is determined by the distance between the $S(z j)$ 's. A value of $10 \mathrm{~nm}$ is typically used for this spacing and changes in the residual stress have been observed over this scale, indicating that this value is a good upper-bound for the depth resolution in this system. The maximum depth resolution for this technique is determined by the value of the absorption coefficient for the light used, with higher absorption coefficients giving greater depth resolution.

It has been shown that when the stress is changing over the volume sampled, it is inappropriate to determine the magnitude of the stress from the peak value of the Raman signal. A new technique has been presented which allows residual stress profiles to be determined from Raman Spectra with high depth resolution. This technique is analytically very simple and independent of any a priori assumptions about the nature of the stress profile. 
This data analysis technique has been used to evaluate the residual stress in machined semiconductors and details of the stress fields produced while machining are described in Section 10. The technique developed should also be applicable to other systems such as strained layers, superlattices, and hardness indents. The application to hardness indents is the subject of Section 5 .
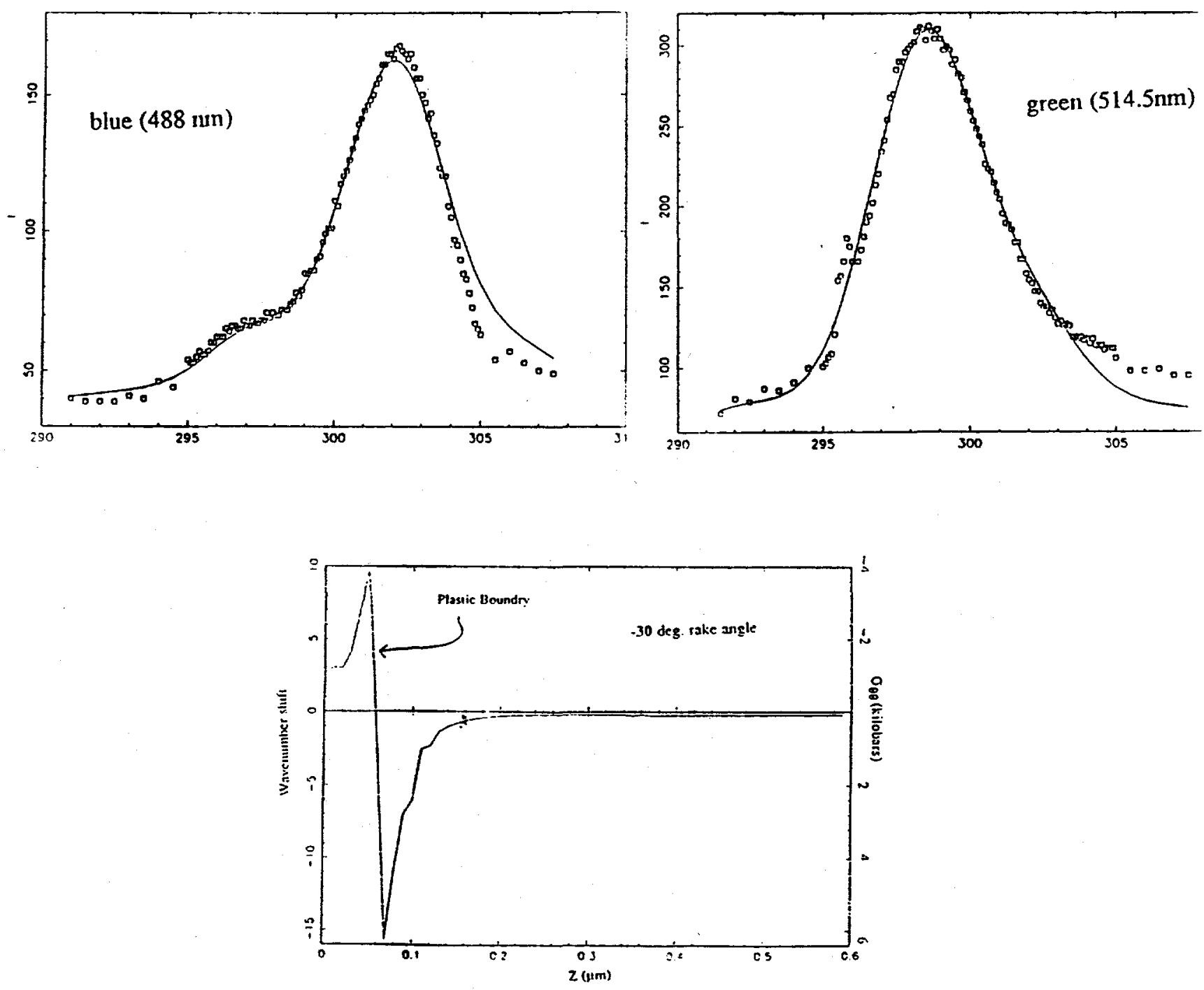

Figure 3: This figure contains fitted Raman data (upper two graphs) and the associated stress profile (lower graph). The spectra taken with the blue light is shifted to higher wavenumbers, indicating that the surface is compressively stressed, but broadened to the side of tension. The green spectra is shifted in the tensile direction, but broadened to higher wavenumbers, again indicating that the surface is compressive. The fitted stress profile shows that the transition from tensile to compressive stress takes place on a scale 
of $10 \mathrm{~nm}$. This abrupt transition defines a elastic/plastic boundary between the plasticly deformed surface and the elastic bulk material. 


\section{References}

[1] Nemanich, R.J., "Raman Spectroscopy for Semiconductor Thin Film Analysis". Mat. Res. Soc. Symp. Proc. Vol. 69, 1986 p.23.

[2] Etz, E.S., "Raman Microprobe Analysis: Principles and Applications". SEM 1979-I, p.67.

[3] Rosasco, Gregory, "Raman Microprobe Spectroscopy". in Advances in Infrared and Raman Spectroscopy, Vol 7. Clark and Hester Ed. Heyden and Son Ltd. 1980, p.223.

[4] Sparks, R.G. and M.A. Paesler. "Micro-Raman Analysis of Stress in Machined Silicon and Germanium". Precision Eng. 10(4), 1988 p.191.

[5] Adar, Fran and D.R. Clarke. "Raman Microprobe Spectroscopy of Ceramics". Microbeam Analysis - 1982, p.307.

[6] Cheong, Y.M., H.L. Marcus and F. Adar. "Raman Microprobe Measurements of Residual Strains at the Interfaces of Si on Quartz". J. Mater. Res. 2(6), 1987 p.902.

[7] Sze, S.M., Physics of Semiconductor Devices, 2nd ed. John Wiley \& Sons, NY 1981.

[8] Johansson,Stefan and Jan-Ake Schweitz. "Contact Damage in Single Crystalline Silicon Investigated By Cross-Sectional Transmission Electron Microscopy". JACS $71(8), 1988$ p.617.

[9] Badrick, A.S.T. et al.. "Scratches on near (111) silicon slices". J. Phys. D 10, 1977 p.195.

[10] Chaing, S.S., D.B. Marshall, and A.G. Evans. "The Response of Solids to Elastic/Plastic Indentation. I Stresses and Residual Stresses". J. Appl. Phys. 53(1), 1982 p.298.

[11] Chaing, S.S., D.B. Marshall, and A.G. Evans. "The Response of Solids to Elastic/Plastic Indentation. II Fracture Initiation". J. Appl. Phys. 53(1), 1982 p.312.

[12] Bradshaw, W., "Stress Profile Determination in Chemically Strengthened Glass Using Scattered Light". J. Mat. Sci. 14, 1979 p. 2981.

[13] Lawn, B.R. and M.V. Swain. "Microfracture Beneath Point Indentations in Brittle solids". J. Mat. Sci. 10, 1975 p.113.

[14] Lawn, Brian and Rodney Wilshaw. "Review: Indentation Fracture: Principles and Applications". J. Mat. Sci. 10, 1975 p.1049.

[15] Marshall, B. and B.R. Lawn. "Residual Stress Effects in Sharp Contact Cracking: Part 1 Indentation Fracture Mechanics". J. Mat. Sci. 14, 1979 p. 2001. 
[16] Bevington, P.R., Data Reduction and Error Analysis for the Physical Sciences. McGraw -Hill Book Co., NY 1969.

[17] Possin, B.E. and C.G. Kirkpatirck. "Electron Beam Depth Profiling in Semiconductors". SEM 1979-I, p.245.

[18] Pankove, J.I., Optical Processes in Semiconductors. Dover Publications, Inc., NY 1971. 


\title{
5 MAPPING RESIDUAL STRESS FIELDS FROM VICKERS HARDNESS INDENTS USING RAMAN MICROPROBE SPECTROSCOPY
}

\author{
R. G. Sparks and W. S. Enloe \\ Graduate Students \\ Michael A. Paesler \\ Associate Professor, Physics
}

Micro-Raman spectroscopy is used to map the residual stress fields in the vicinity of Vicker's hardness indents. Both 514.5 and $488.0 \mathrm{~nm}$ light is used to excite the effect and the resulting shifted and broadened Raman peaks are analyzed using computer deconvolution. Half-wave plates are used to vary the orientation of the incident laser light's polarization state with respect to crystal orientation. The Raman scattered light is then analyzed for polarization dependences which are indicative of the various components of the Raman scattering tensor. Such studies can yield valuable information about the orientation of stress components in a well known stress field. The results can then be applied to the determination of stress components in machined semiconductor materials.

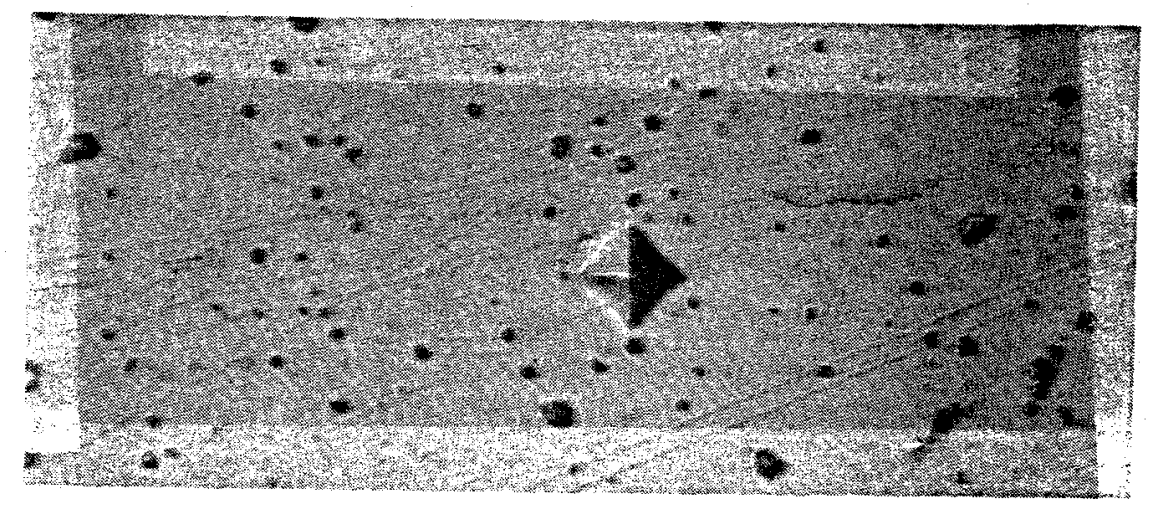




\subsection{INTRODUCTION}

The response of solids to indentation has provided information about phenomena such as erosion, wear, machining damage and surface fracture strength. There exists a considerable amount of experimental data which shows variations of indentation microhardness with load, temperature, and surface contact environment. In brittle materials such as semiconductors, indentation fracture dictates the material degradation process [1]. The magnitude of the elastic/plastic stress field as well as the extent of plasticity are of primary importance to the indentation fracture process. The theory of rigid perfectly elastic solids can be used to predict indentation pressures, using wedge-shaped or conical indenters, which depend only on the geometry of the indenter and the yield stress of the material [2]. Micro-Raman spectroscopy has proved to be a powerful tool for determinations of residual stresses in materials. Raman spectroscopy in conjunction with computer deconvolution techniques have provided depth profiles of the residual stress field in machined materials. Studies have been undertaken to map the residual stress fields using micro-Raman spectroscopy in the vicinity of Vicker's hardness indents. Since such indents produce stress fields which are more fully understood than in the case of single point diamond turning, important insights into the material removal process can be obtained from the comparison of indents with diamond turned samples. Because the various components of the Raman signal are polarization dependent in single crystals, polarization analysis of the Raman spectra with respect to known crystal directions can provide determination of stress components around the region of indentation. These techniques can presumably be applied to the determination of stress components in machined materials.

\subsection{EXPERIMENT}

The experimental apparatus consists of an argon ion laser with strong outputs at 514.5 $\mathrm{nm}$ and $488.0 \mathrm{~nm}$. The light from the laser is plane polarized in a direction normal to the plane formed by the optical table on which the laser is mounted. The plane polarized, monochromatic beam is then steered using plane mirrors through an achromatic half-wave plate (Fresnel rhomb). The effect of the half wave plate is to rotate the vertical polarization state of the incident beam by an known angle with respect to the original polarization direction. Since the Fresnel rhomb produces some ellipticity in the emerging beam a GlanThompson prism polarizer mounted to a calibrated rotation stage is used to provide a pure linear polarization state at a known angle (Fig.1). The light is then steered into the microRaman apparatus where the known polarization state is focused by a microscope objective on the sample with a particular orientation to the sample's crystal direction. The $1 \mu m$ spot can then be moved to various positions around and in Vicker's hardness indents with micro-Raman spectra obtained for both $514.5 \mathrm{~nm}$ and $488.0 \mathrm{~nm}$ wavelengths at each point. Since the different components of the Raman tensor display different polarization states with respect to crystal direction, an analyzer is used in the scattered beam to select one particular Raman polarization state. Because the holographic gratings in the spectrometer 
are more efficient reflectors for polarization states perpendicular to the plane of the grating, a second Fresnel rhomb is used to rotate the Raman scattered light to this position. By varying both the orientation of the exciting polarization state using the first Fresnel rhomb relative to the position of the crystal, analyzer and second Fresnel rhomb determinations of the various stress components can be obtained. Since in the case of Vicker's hardness indents the behavior of such components is well modeled, these studies provide verification of the technique which may then be applied to machined samples.

\subsection{PRELIMINARY RESULTS}

Initial studies of Vicker's indents result in Raman spectra that are shifted and broadened in contrast to the symmetric and unshifted spectrum indicative of unstressed single crystal samples. A systematic study of indents of varying depths is underway and will be reported in the future. Preliminary results point toward a strong verification of the Raman depth profiling technique as an accurate means of determining stress profiles. 


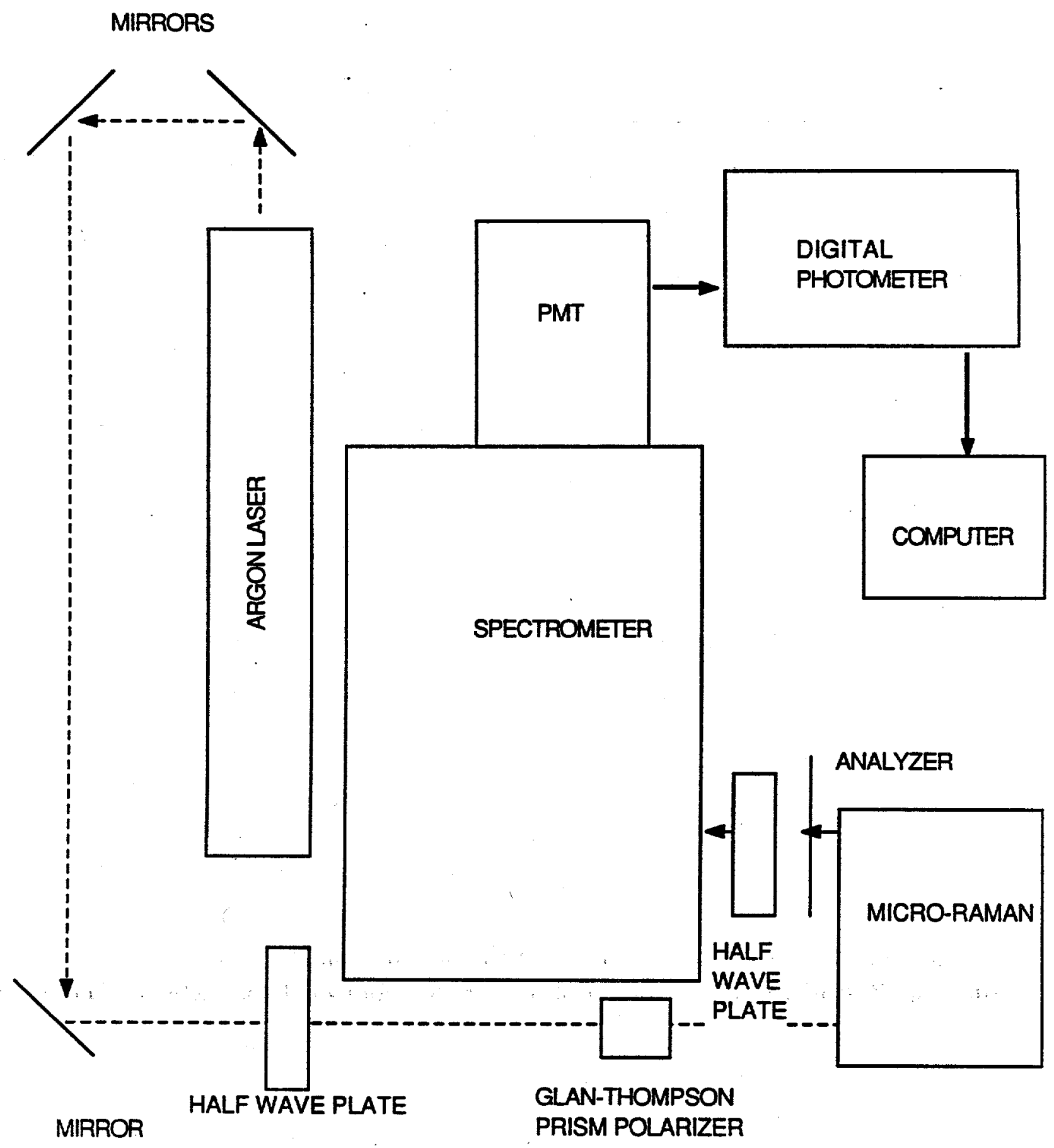

Figure 1: The experimental apparatus. The Raman effect is excited using the argon laser 


\section{References}

[1] Chaing, S. S., Marshall, D. B. and Evans, A. G. J Appl Phys Vol. 53, p. 1,1982.

[2] Johnson, K. L., J Mech Phys Solids Vol. 18,1970. 

 IN CERAMIC COMPOUNDS CONTAINING TETRAGONAL ZIRCONIA
}

\author{
R.G. Sparks and G. Pfeiffer \\ Graduate Students \\ Michael A. Paesler \\ Associate Professor, Physics
}

Stabilized tetragonal zirconia compounds exhibit a transformation toughening process in which stress applied to the material induces a crystallographic phase transition. The phase transition is accompanied by a volume expansion in the stressed region thereby dissipating stress and increasing the fracture strength of the material. The hydrostatic component of the stress required to induce the phase transition can be investigated by the use of a high pressure technique in combination with Micro-Raman spectroscopy. The intensity of Raman lines characteristic for the crystallographic phases can be used to calculate the amount of material that has undergone the transition as a function of pressure. It was found that pressures on the order of $2-5 \mathrm{kBar}$ were sufficient to produce an almost complete transition from the original tetragonal to the less dense monoclinic phase; while a further increase in pressure caused a gradual reversal of the transition back to the original tetragonal structure.

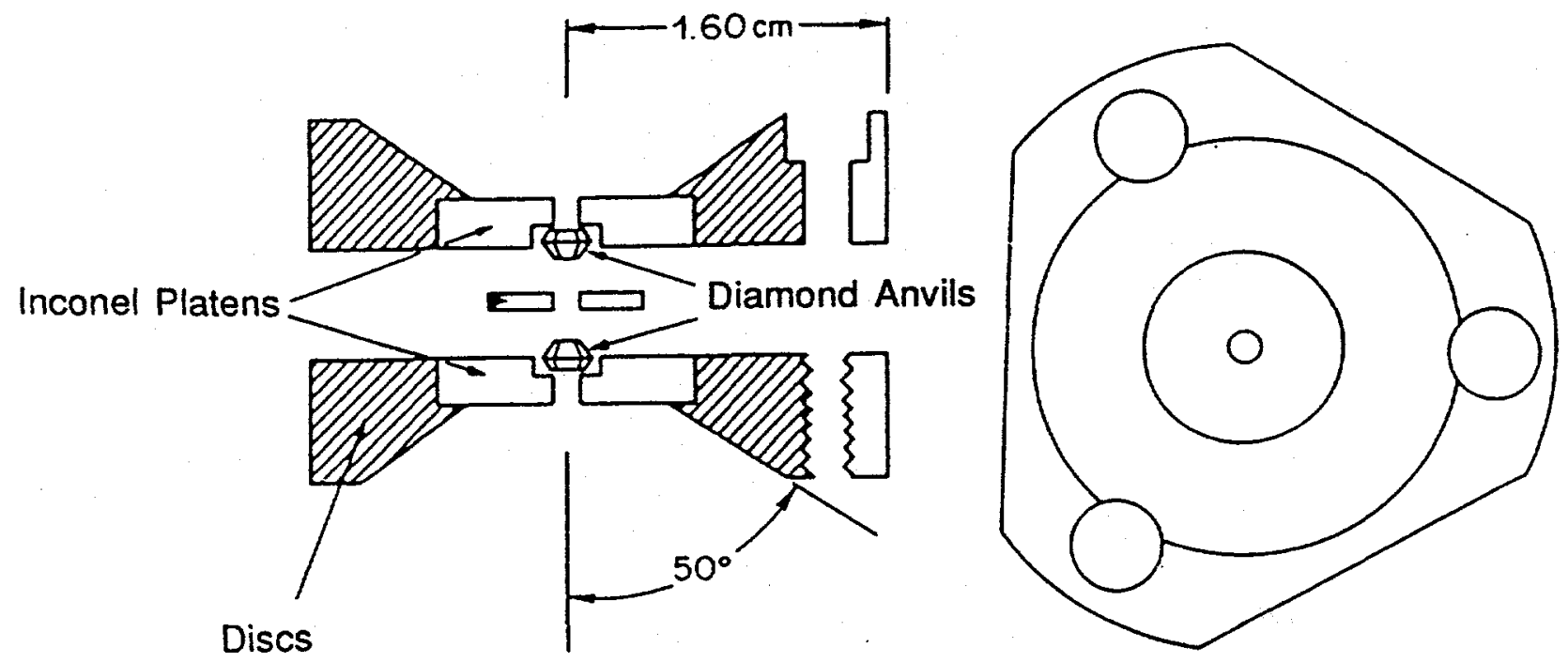




\subsection{INTRODUCTION}

Ceramics containing tetragonal zirconia have received a great deal of attention as high strength ceramic materials because of their fracture toughening properties. If mechanical stress is applied to these materials, they undergo a crystallographic phase transition from tetragonal to monoclinic accompanied by a volume expansion on the order of a few percent. This volume expansion in return causes stress, concentrated for example in the region of a crack, to be dissipated and thereby preventing the specimen from fracturing.

The tetragonal and monoclinic phase are very well characterized by their Raman spectra since each phase displays a number of unique lines. In indentation experiments, the size of the transformation zone has been determined by micro-Raman spectroscopy [1] under the assumption that the transformation is irreversible. However, the magnitude of the shear and hydrostatic components of the residual stress in the crack region is not very well known. A combination of the high pressure diamond anvil cell to produce hydrostatic pressures and Raman spectroscopy to determine the stress field offers a unique tool for examination of the pressure dependence of the tetragonal to monoclinic phase transition in zirconia.

\subsection{EXPERIMENTAL METHODS}

In the past decade, high pressure diamond anvil cells (DAC) have become the standard method for studying phase transitions as a function of hydrostatic pressure. For light scattering experiments such as Raman spectroscopy, the geometry of the Merrill-Bassett DAC allows convenient optical access to the sample through the transparent diamond anvils (Fig. 1). The two diamonds anvils, held by steel backing plates, are pressed against each other while being separated by a thin metal foil. At the center of the culet tip, a $200 \mu \mathrm{m}$ hole drilled through the metal foil serves as the sample chamber. The sample, measuring about $120 \mu \mathrm{m}$, and a small ruby chip are placed in the chamber together with a mixture of methanol and ethanol as a pressurizing fluid. The pressure is increased by tightening the screws that connect the two pressure plates. Pressure calibration is accomplished by spectroscopically determining the wavelength of a fluorescence line from the ruby chip placed next to the sample.

Raman spectra as a function of pressure have been taken with the micro-Raman Spectrometer using a $10 \mathrm{x}$ objective that results in a light spot of $20 \mu \mathrm{m}$ in diameter. Since the spot size is much larger than the average grain size of the $\mathrm{ZrO}_{2}$ particles embedded with random crystallographic orientations in a $\mathrm{MgO}$ matrix, the spectra did not show the strong polarization dependence of $\mathrm{ZrO}_{2}$ single crystals. The intensity of the Raman signal is very low due to reflection losses at the diamond surfaces so that a scan rate of $1 \mathrm{~cm}^{-1} \mathrm{~min}^{-1}$ was required to obtain a sufficiently low noise level at a spectral resolution of $2.5 \mathrm{~cm}^{-1}$. 


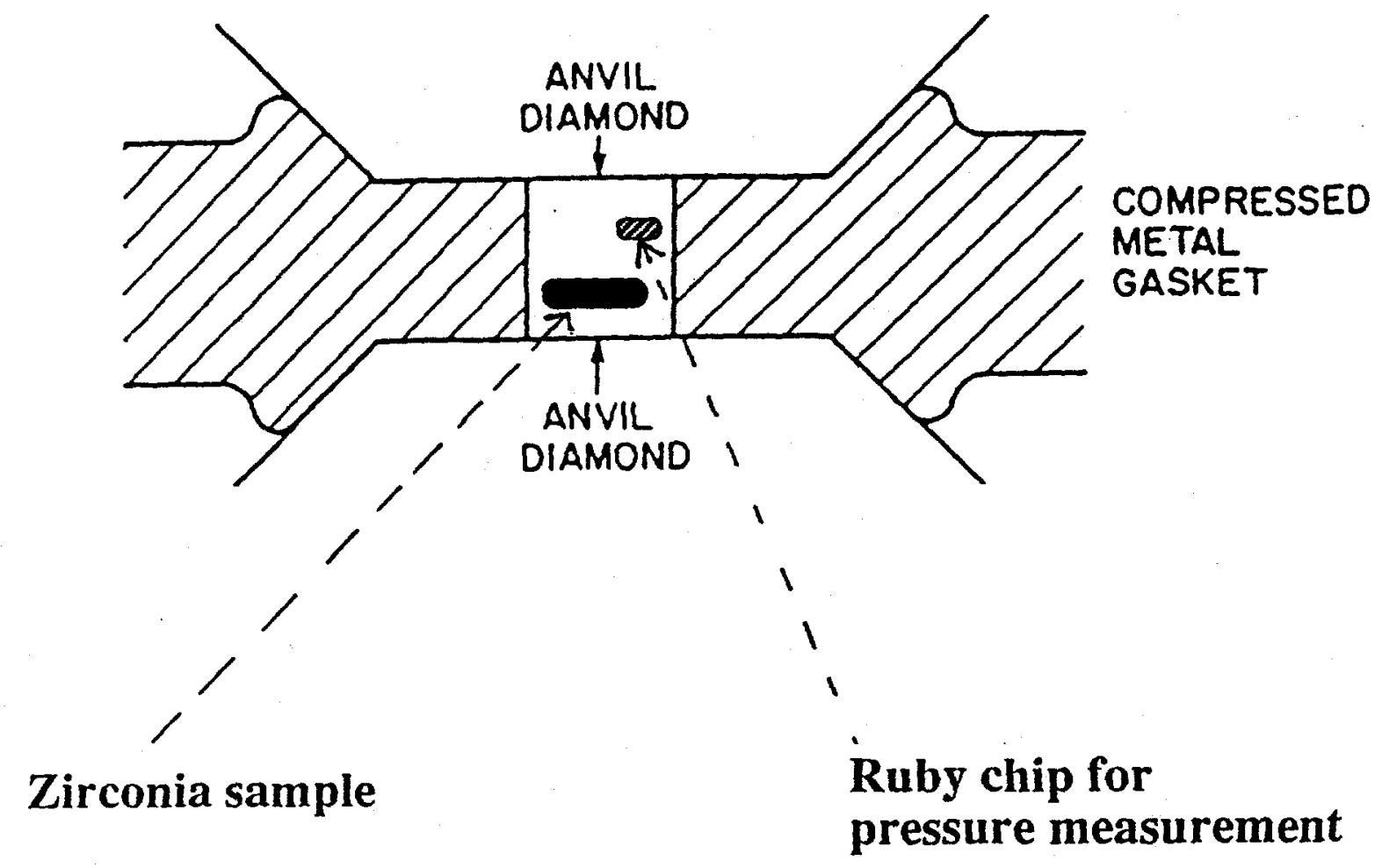

Figure 1: Cross sectional view of the sample chamber in a diamond anvil high pressure cell. The opposed diamond anvils compress the metal gasket and create a high pressure region in the volume containing the sample, a ruby calibration chip and the high pressure medium-a mixture of methanol and ethanol. 


\subsection{EXPERIMENTAL RESULTS}

The tetragonal phase of $\mathrm{ZrO}_{2}$ shows six(6) Raman active bands whereas the monoclinic phase displays sixteen(16) Raman active lines. A clear distinction between the two crystallographic structures is only possible for frequencies less than $300 \mathrm{~cm}^{-1}$ since many of the lines overlap and/or are weak. The characteristic bands are 148 and $264 \mathrm{~cm}^{-1}$ for the tetragonal structure and 181 and $192 \mathrm{~cm}$ for the monoclinic structure. Figure 2 shows the Raman spectra for pressures from 1.2 to $31 \mathrm{kBar}$ and an unpressurized reference sample for a compound of $25 \% \mathrm{ZrO}_{2} / \mathrm{MgO}$. In addition to the six tetragonal lines, the monoclinic doublet at $181 / 192 \mathrm{~cm}^{-1}$ is present as a weaker feature in the spectrum for the reference sample. With increasing pressure, the intensity of the monoclinic doublet first increases to a point where it dominates the spectrum and then returns to a value close to the unpressurized sample. The intensities of the characteristic lines can be used to calculate the amount of monoclinic phase present in the mixed phase sample by using an expression adapted from $\mathrm{x}$-ray scattering experiments $[1,2]$ :

$$
C_{m}=\frac{\left(I_{m}^{181}+I_{m}^{192}\right)}{\left.\mu\left(I_{T}^{148}+I_{T}^{248}\right)+I_{m}^{181}+I_{m}^{192}\right)}
$$

with $\mu=0.97$.

In Figure 3 the monoclinic concentration of the mixed phase computed from the experimentally measured spectra is shown as a function of pressure. Note that the amount of monoclinic phase increases from about $7 \%$ to more than $75 \%$ with the application of about $3 \mathrm{kBar}$ of pressure. A further increase in pressure results in a gradual decrease and return to the original structure which is strongly dominated by the tetragonal phase. A certain amount of monoclinic phase present in the mixed phase sample is associated with two values for the pressure, one on the increasing and the other on the decreasing part of the curve in Figure 3. Therefore the material undergoes a reversible phase transition from tetragonal to monoclinic back to tetragonal. 


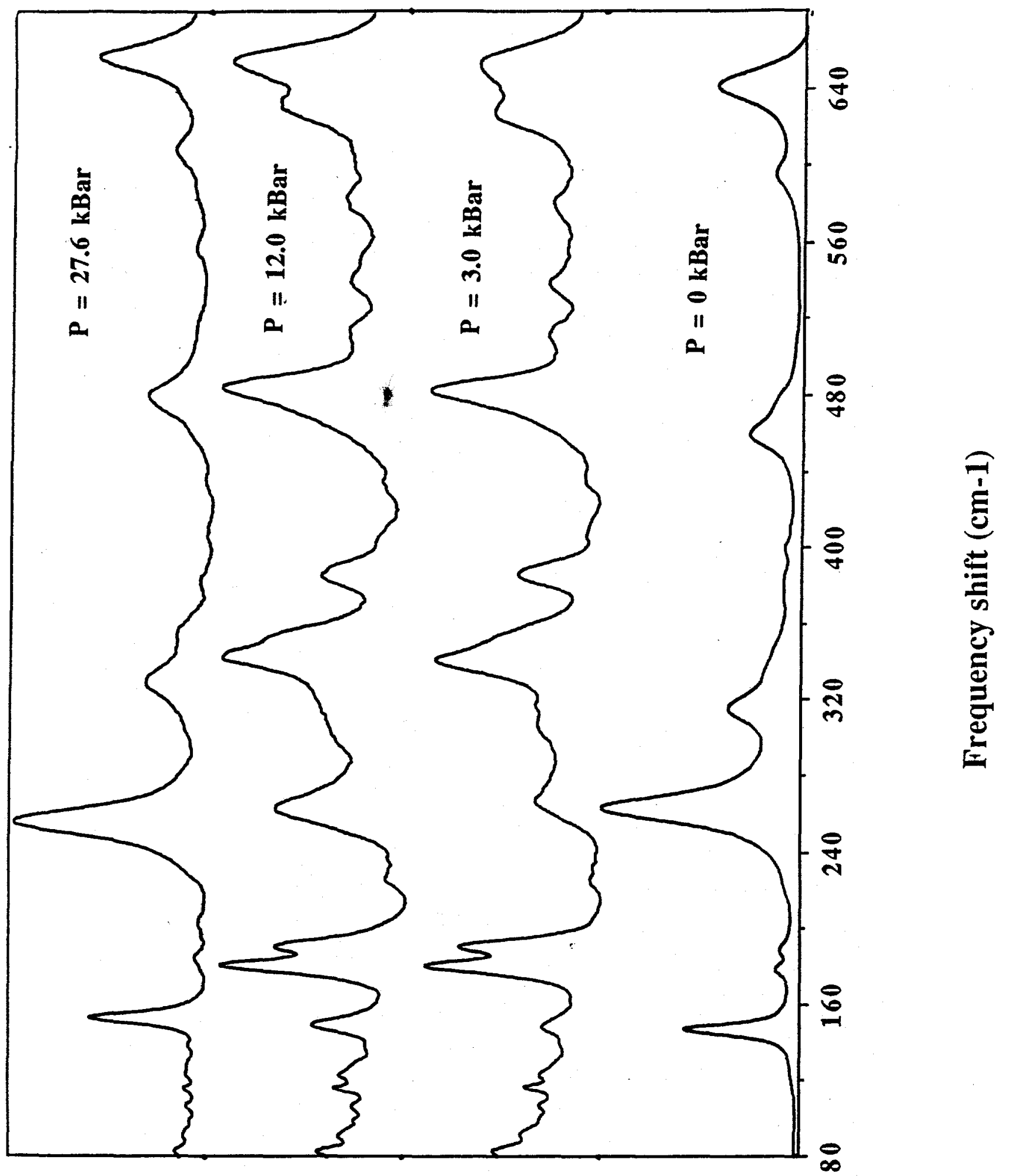

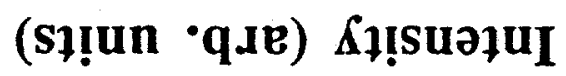

Figure 2: Micro-Raman spectra taken on the sample of zirconia at the pressures indicated. Phase transformations are readily apparent as features in the Raman spectra grow and then shrink with increasing pressure. 


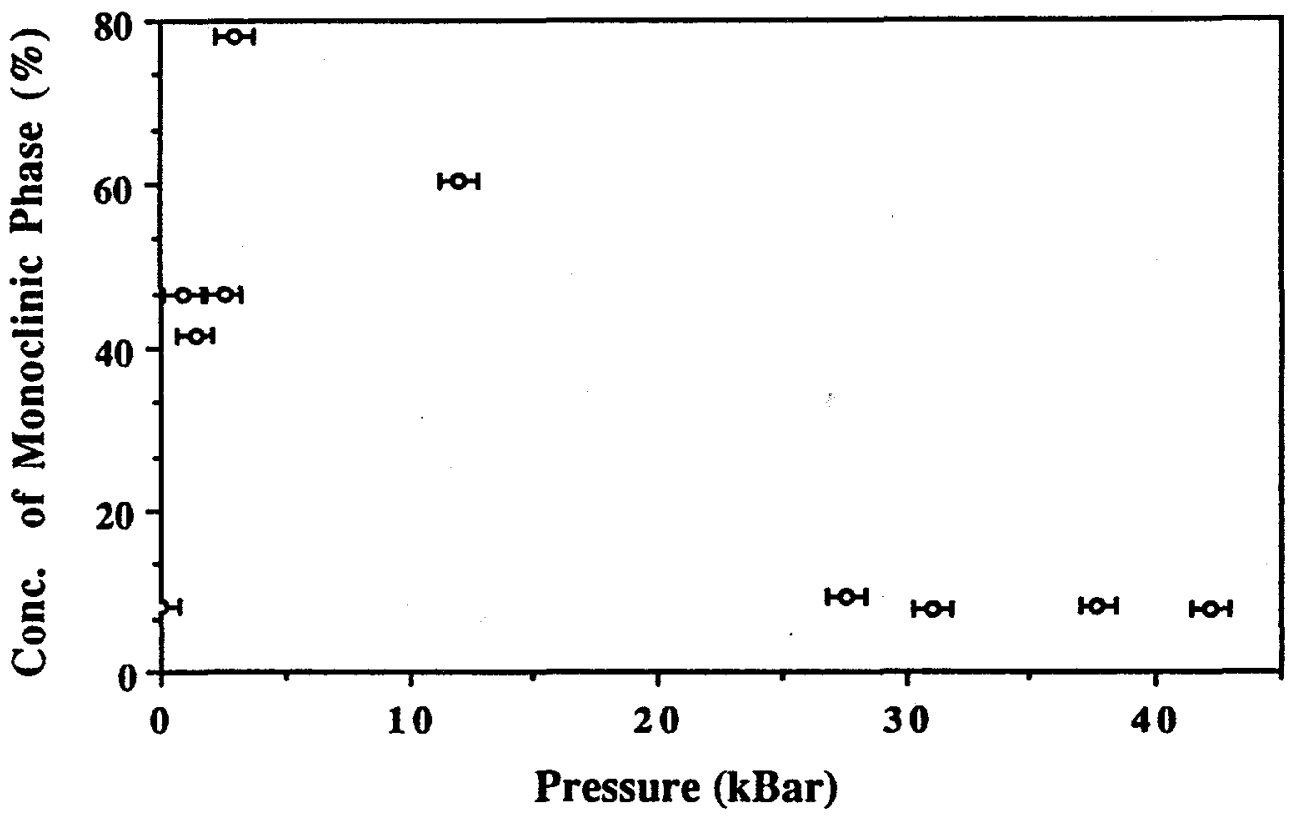

Figure 3: The concentration of the monoclinicphase of zirconia as a function of pressure. Because a curve through the experimental points is not a monotonic function of pressure, one cannot use Raman features indicative of the monoclinic phase as a measure of pressure.

\subsection{CONCLUSIONS}

The high pressure DAC technique in combination with micro-Raman spectroscopy is a very powerful method to characterize phase transformations under hydrostatic pressure. The experiments have yielded interesting new information about the phase transition from tetragonal to monoclinic in the transformation toughening process in ceramics containing tetragonal $\mathrm{ZrO}_{2}$. The data suggest that the initial tetragonal to monoclinic transformation induced by pressures of up to $5 \mathrm{kBar}$ is completely reversible by increasing the pressure to values on the order of 20-30 kBar. It is to be concluded that the concentration of the monoclinic phase, as determined from the intensities of the characteristic lines (Eq. 1) alone, is not a sufficient measure to determine the hydrostatic component of the residual stress in the transformation zone. Since the frequency of the characteristic lines is a monotonic function of pressure, the exact form of this dependence will be established through further experiments the result of which will be improved micro-Raman characterization techniques for this class of materials. 


\section{References}

[1] Clarke, D. R. and Adar, F., J. Am. Ceram. Soc.65, 284 (1982).

[2] Garvie, R.C. and Nicholson, P.S, Am. Ceram. Soc.55, 303 (1972). 



\title{
7 STRUCTURAL RESPONSE SYNTHESIS
}

\author{
Hakan Ozisik \\ Graduate Student \\ Richard F. Keltie \\ Associate Professor, MAE
}

The open loop control technique of predicting a conditioned input signal based on a specified output response for a second order system has been analyzed both analytically and numerically to gain a firm understanding of the method. Differences between this method of control and digital closed loop control using pole cancellation were investigated as a follow up to previous experimental work. Application of the technique to diamond turning using a fast tool servo is also discussed.

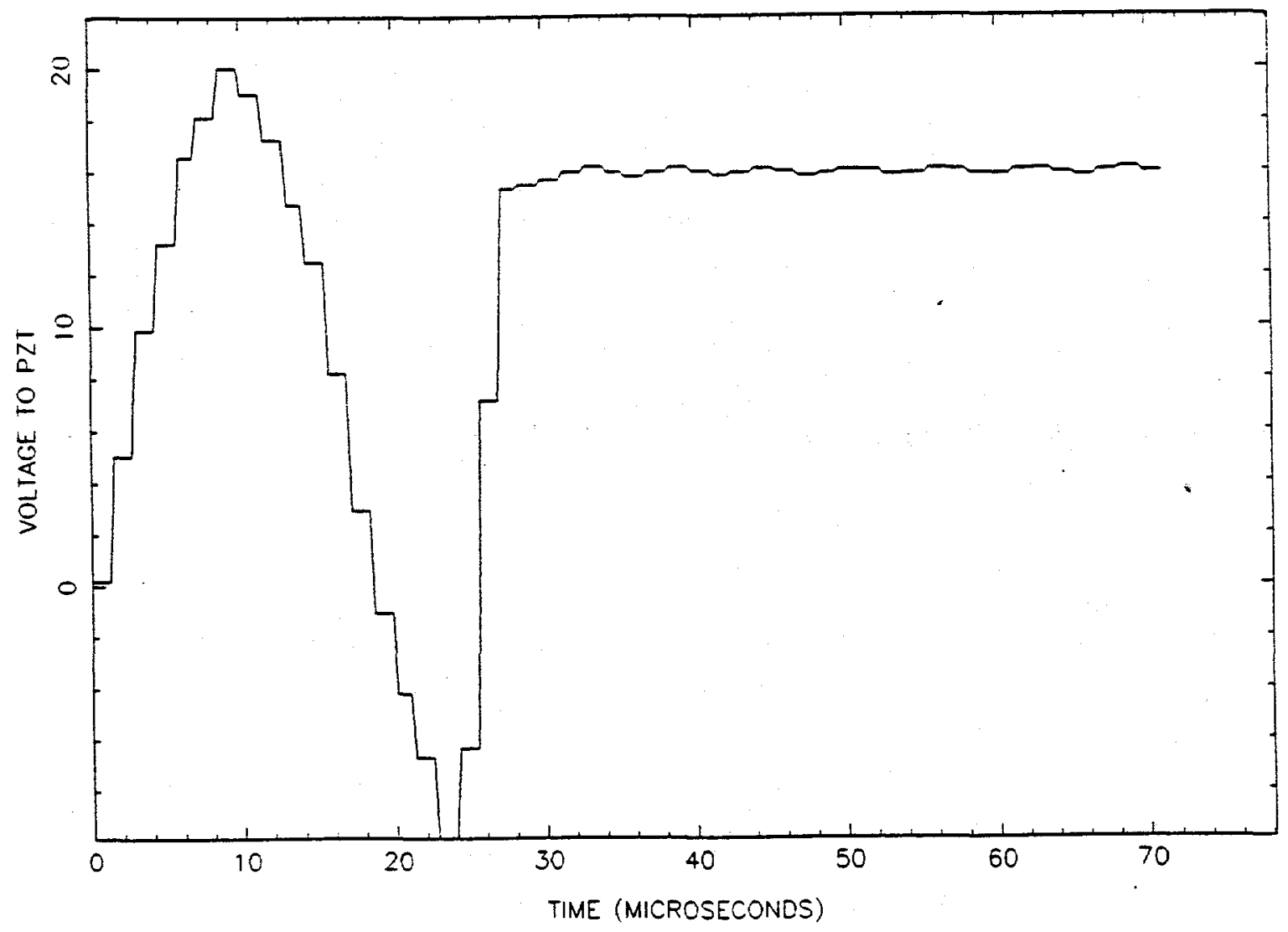




\subsection{INTRODUCTION}

The dynamic control of elastic structures is an integral part of many automated manufacturing processes to assure quality control of the finished product. This is especially true when a precision process is coupled with dynamic response times on the order of the natural period of the structure. Under these circumstances residual vibration of the structure occurs, limiting the achievable accuracy of the process. An appropriate method of system control must therefore be developed and incorporated into the dynamic process.

Because such problems are commonplace, the dynamic control of systems has been an area of much interest and investigation for many years. This topic lends itself to a wide variety of dynamic control applications ranging from large flexible structures used in robotic and spacecraft control [1-2] to reducing residual vibrations in printer carriages [3]. The method of control implemented may be broadly cast in one of the two distinct areas of either closed or open loop controls. Quite often closed loop control of a system is preferred due to its flexibility and robustness with respect to open loop controls. When the process, however, is repetitive and has insignificant parameter variations, an open loop method can provide a simple and effective solution [4].

Controlling the response of a system to a step input is a common application of dynamic controls. A traditional closed loop system may use pole cancellation of the plant along with integration of the error signal to assure steady state accuracy. The system's final output becomes a compromise between reducing the peak overshoot and maintaining a quick rise time. Since the controlled output is a smooth signal with finite rise time, one might consider inputting a more physically acceptable signal to the system in order to reduce the amount of residual vibration [5],[6]. Control of structures by this technique may be viewed as open loop.

Structural response synthesis is a method of open loop control which specifies a smooth output signal based on the criteria of zero acceleration and velocity at the point where residual vibrations usually occur. This specified dynamic response of the structure is then achieved by positioning the structure based on a unique input signal generated from physical parameters and constraints of the system. The input signal is computed numerically via a fast fourier transform (FFT) and inverse FFT, given the system transfer function and desired output time signal.

Technological advances in high speed digital signal processors have made the method applicable to precision positioning [4]. Analysis of the method is discussed in the following sections along with applications to diamond turning using a fast tool servo. 

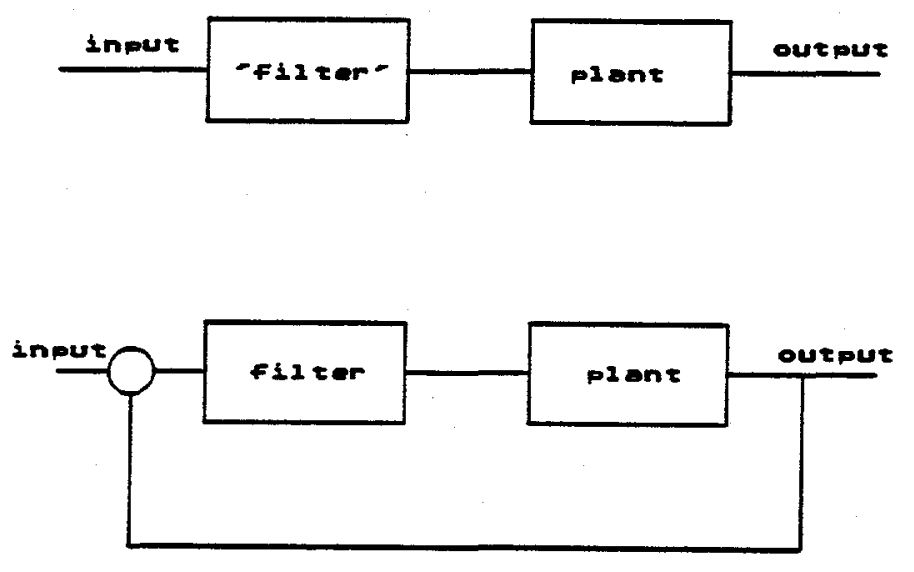

Figure 1: Open loop and closed loop block diagrams

\subsection{METHOD ANALYSIS WITH RESPECT TO CLOSED LOOP CONTROLS}

The method of synthesizing an input signal [4] was experimentally implemented on a physical structure [7] and the results compared with a closed loop experiment on the same structure. The results of this comparison indicated that open loop control of the structure allowed a rise time approximately three times faster than that of the closed loop control. Since this was a significant improvement, an investigation into apparent differences between the two techniques was conducted.

To compare the two methods, both systems were reduced to a single transfer function with the same input, in this case a step in time, and then analyzed. This was no problem for the closed loop system. The open loop method, however, had to be viewed as as a closed loop system consisting of a filter and a plant. Figure 1 shows the two systems in block diagram form, the top diagram representing open loop and the bottom representing closed loop. The input to both systems is a step but the the effective filter for the open loop system is unknown and had to be calculated using the step input and the plant input used in the actual experiment.

The input signal to the plant in the open loop case was obtained numerically from the specified output signal, $y(t)$, which was mathematically represented by

$$
y(t)= \begin{cases}\sin ^{3}\left(\omega_{0} t\right) & 0 \leq t: \leq \pi / 2 \omega_{o} \\ 1 & t>p i / 2 \omega_{0}\end{cases}
$$




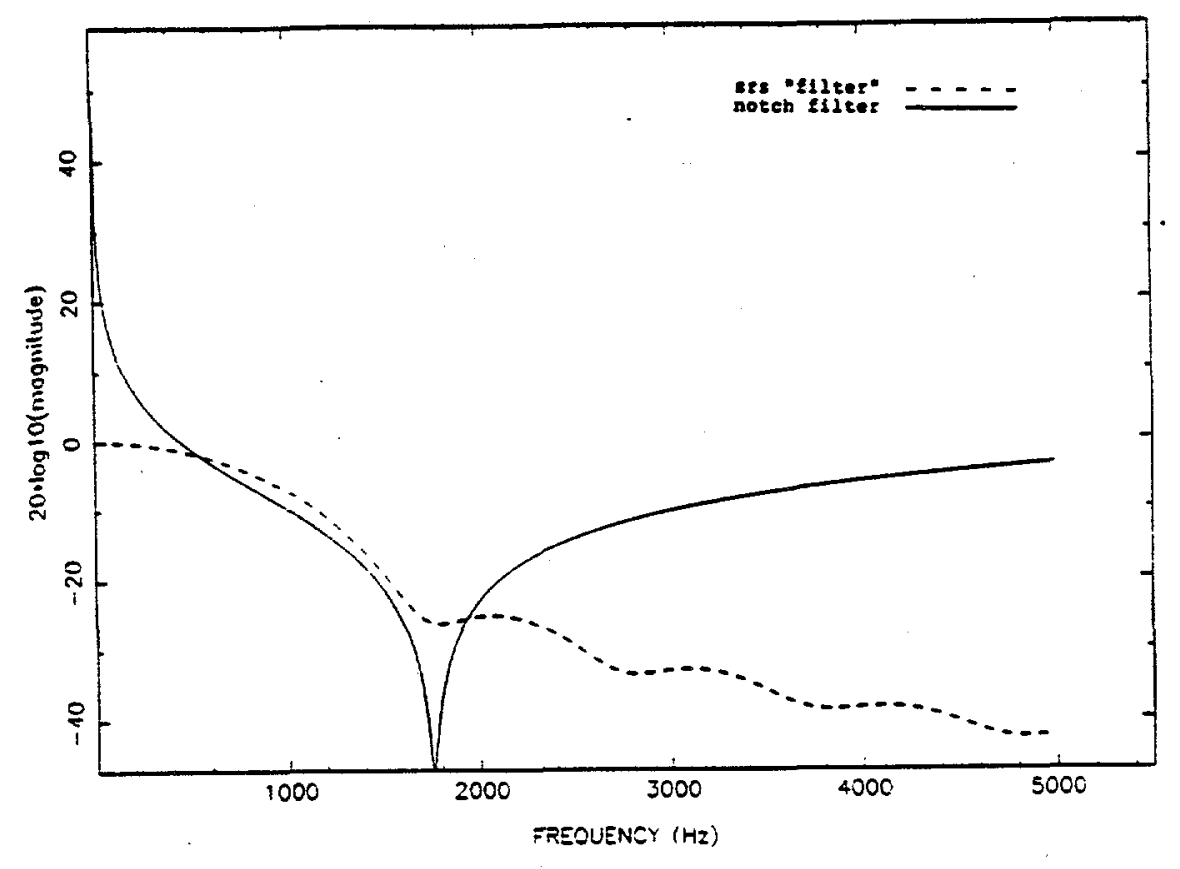

Figure 2: Notch filter and open loop 'filter'

where the parameter $\omega_{o}$ controls the rise time of the specified output signal. For the experiment this parameter had been adjusted so that the rise time ( 90 percent of the steady state value) of the plant was around $1 \mathrm{~ms}$ as compared to $3 \mathrm{~ms}$ in the closed loop experiment. As long as the rise time is larger than the natural period of the structure (plant), the input and output time signals calculated by the response technique will be essentially the same. Faster responses result in signal breakdown which is discussed in Section 1.4. Since the natural period of the plant was around $0.5 \mathrm{~ms}$, Equation (1.1) may also be used to describe the required input signal to the plant. This allowed the transforms of the step function and the input function to the plant to be expressed analytically assuring correct calculation of the 'filter' transfer function in the open loop method. Figure 2 shows transfer functions of the two filters. The closed loop filter produces a notch in the frequency spectrum corresponding to the fundamental frequency of the plant. The open loop filter slowly rolls off and appears to act as a low-pass filter to eliminate many of the higher frequencies not filtered by the notch filter. This is quite helpful when dealing with continuous structures with two or more closely spaced resonance frequencies. Placing more than one notch filter (i.e. pole cancellation with the plant) in the model increases the order of the effective filter. When used digitally in a control algorithm this increases the number of required calculations.

Finally the closed loop transfer functions for both methods were obtained and the frequency responses are plotted in Figure 3. The closed loop experiment transfer function tapers off slowly exhibiting no peaks at the fundamental frequency. The structural response synthesis (SRS) transfer function does show a peak at resonance but a very sharp cutoff around $2000 \mathrm{~Hz}$. This type of behavior indicates that when input and output signals, calculated using the SRS method, are the same, the effect 


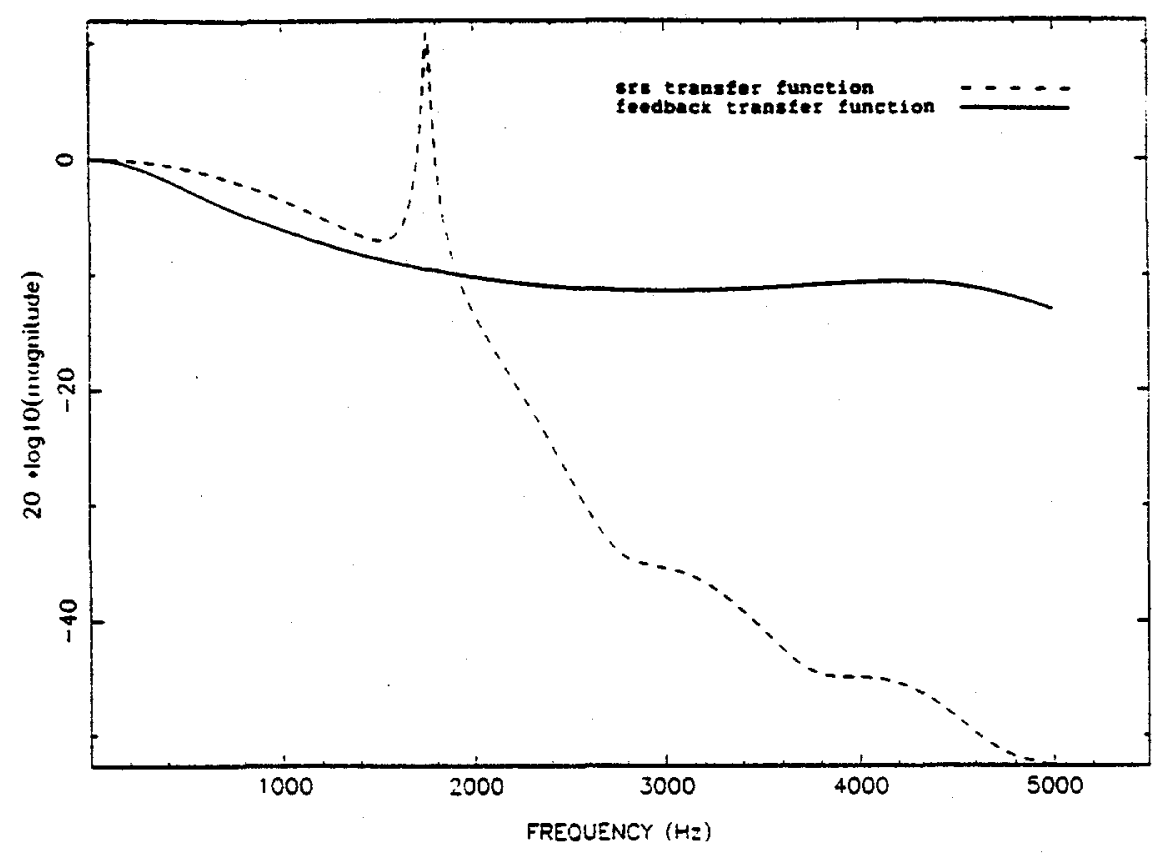

Figure 3: Closed loop frequency responses

is similar to that of a low pass filter. The cutoff frequency is determined by the parametric value of $\omega_{0}$ which is in turn determined by the desired output signal. This is not the case when the ratio of the specified rise time to natural period of the structure is less than unity.

The closed loop system shown in Figure 1 was independently analyzed using the simulation language ACSL and also by the z-transform technique. Both methods accounted for digital sampling using a zero order hold. The results of the simulation showed that the closed loop system should have been able to rise faster than $3 \mathrm{~ms}$ and at least as fast as the SRS method (rise time $1 \mathrm{~ms}$ ). This is unfortunate in that no firm conclusion may be established as to why experimental results are better for SRS while simulation shows an equally fast response time for the closed loop system.

\subsection{RISE TIME LIMITATIONS}

The goal of the present method is the same as any other control method, which is, practically speaking, to rise as quickly as possible (with respect to a step response) while still containing peak overshoot to a minimum. The limitations of this method were investigated for an output of the form given by equation (1.1). The parameter $\omega_{0}$ was varied and the required input signals were calculated. The crucial parameter is the ratio of the rise time (RT) to the natural period (NP) of a structure (modeled as a single degree of freedom system with $\mathrm{NP}=0.5 \mathrm{~ms}$ ). As the ratio $\mathrm{RT} / \mathrm{NP}$ approaches unity signal breakdown occurs between the specified output and the calculated input signals. This means that the input signal begins to deviate from the 


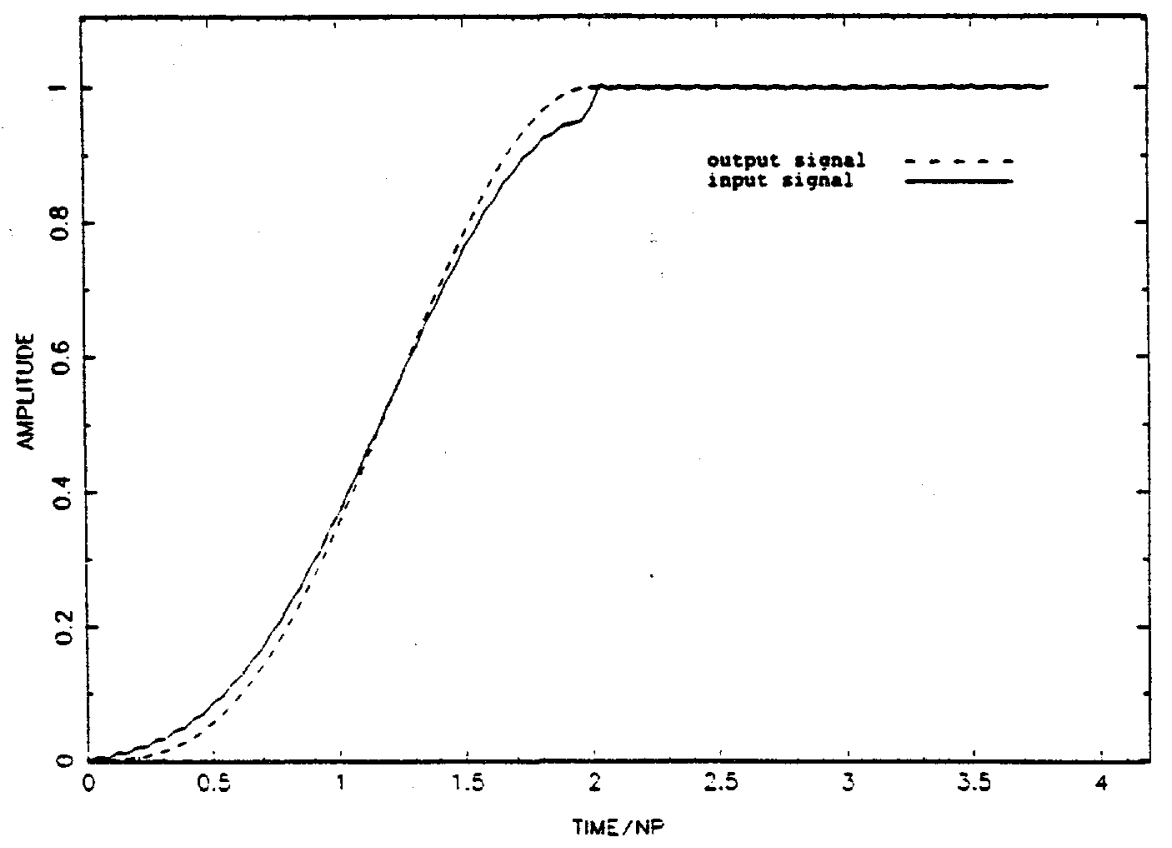

Figure 4: Input and output signals $\mathrm{RT} / \mathrm{NP}=2$

output signal in order to compensate for the decrease in rise time. Mathematically speaking, the point at which the input signal changes direction can be related to inflection points on the output signal ( change of radius of curvature).

Figure 4 shows input and output signals plotted against time for $R T / N P=2$. The two signals are very similar with the most noticeable deviation occurring near the peak as might be expected since the output was specified to allow no overshoot. Compensation for this imposed constraint must therefore occur somewhere in the interval $0 \leq t \leq R T$. Figure 5, and Figure 6 show the same trend, although it becomes more pronounced as the value of RT/NP is decreased to 0.7. At this point the structure is rising faster than its natural period while maintaining the imposed constraint. Positioning as fast or faster than the natural period has also been demonstrated by other investigators [1],[8]. Finally, Figure 7 shows the case $\mathrm{RT} / \mathrm{NP}=0.2$. The attempt to compensate for changes in the acceleration of the specified output can be clearly seen by the radical change of the required input signal. There are two factors which limit the ability to reproduce the required input signal. First of all, components in the experimental apparatus must have a bandwidth capable of handling speeds on the order of the rise time. The other consideration is immediately noted from the amplitudes of the input signal. The amplifier's slew rate must be able to handle the load and the actuator (usually a piezoelectric crystal in micro positioning) must be able to supply the required forces. Even if all these requirements are met,the final limitation is set by the structure itself. For example, in a pinned-free beam structure in bending, commonly used to model flexible robotic arms, a physical limit to achievable response time is set by time required for a wave to travel the length of the member [1]. 


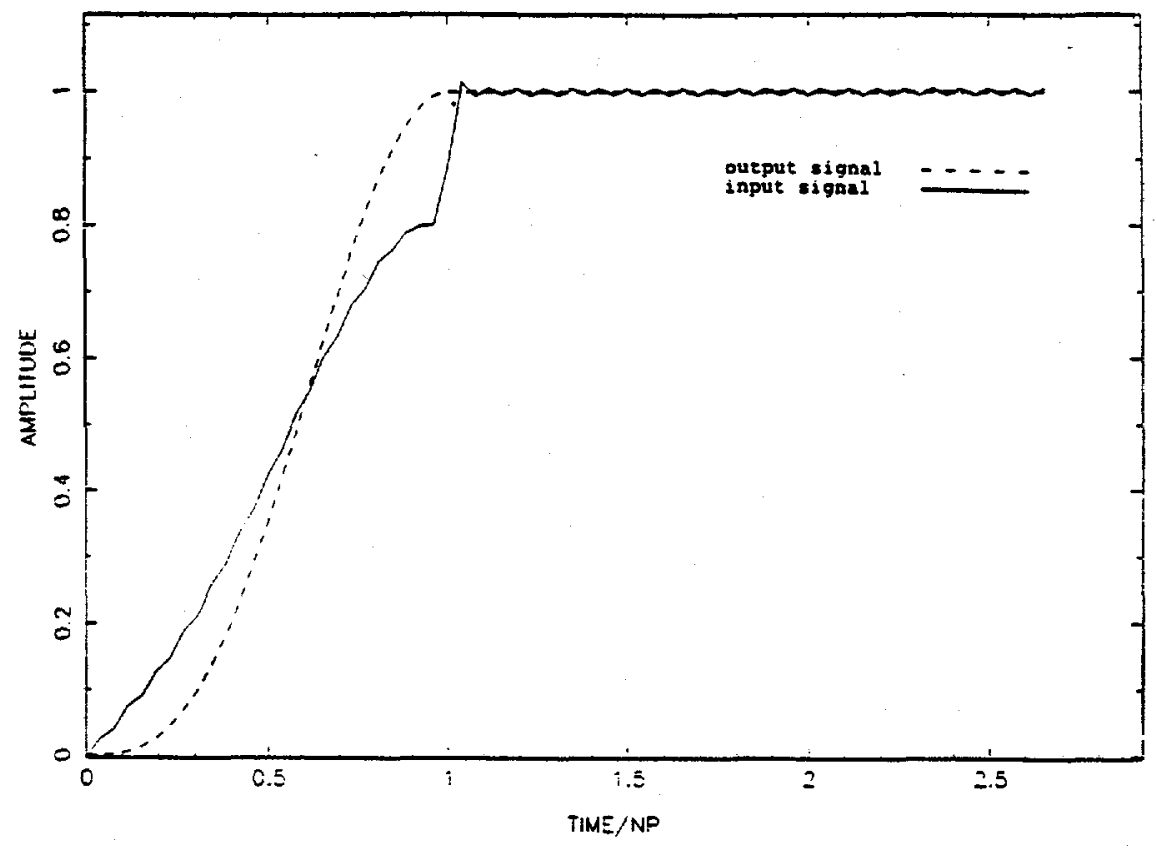

Figure 5: Input and output signals $\mathrm{RT} / \mathrm{NP}=1$

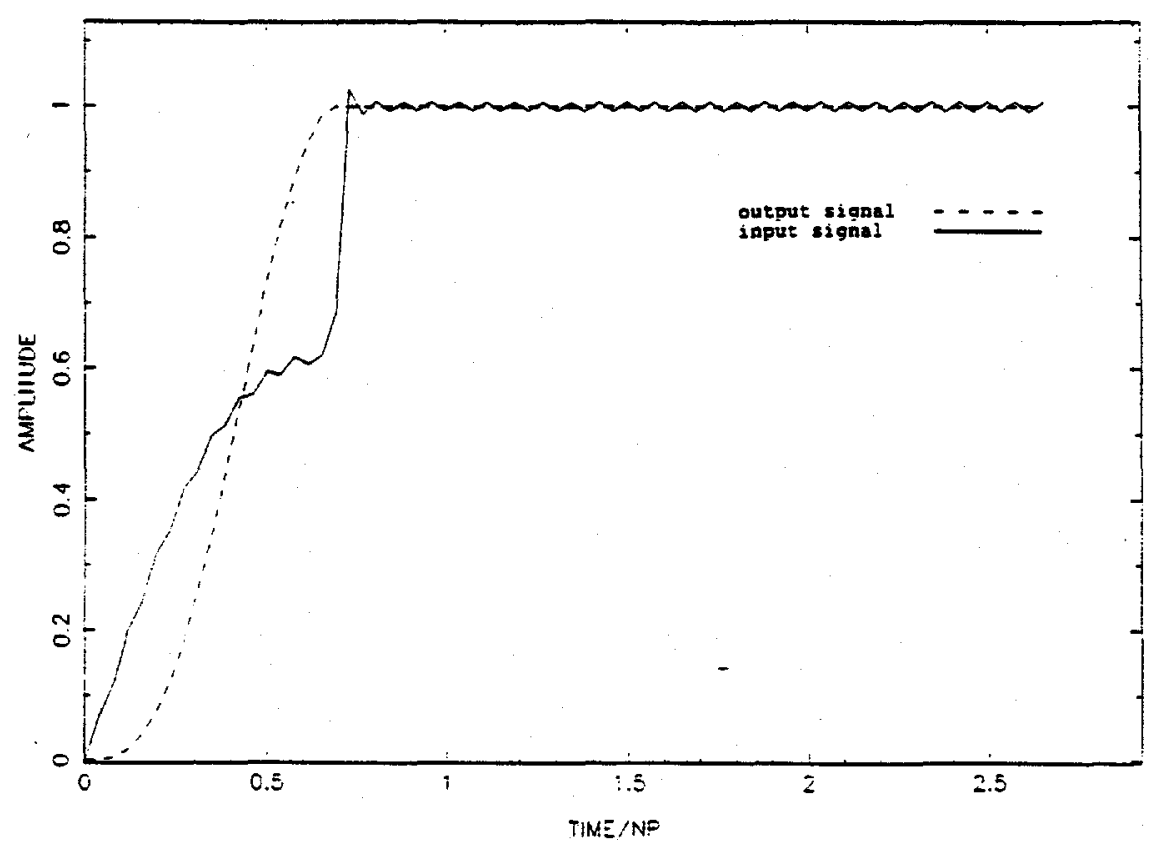

Figure 6: Input and output signals $\mathrm{RT} / \mathrm{NP}=0.7$ 


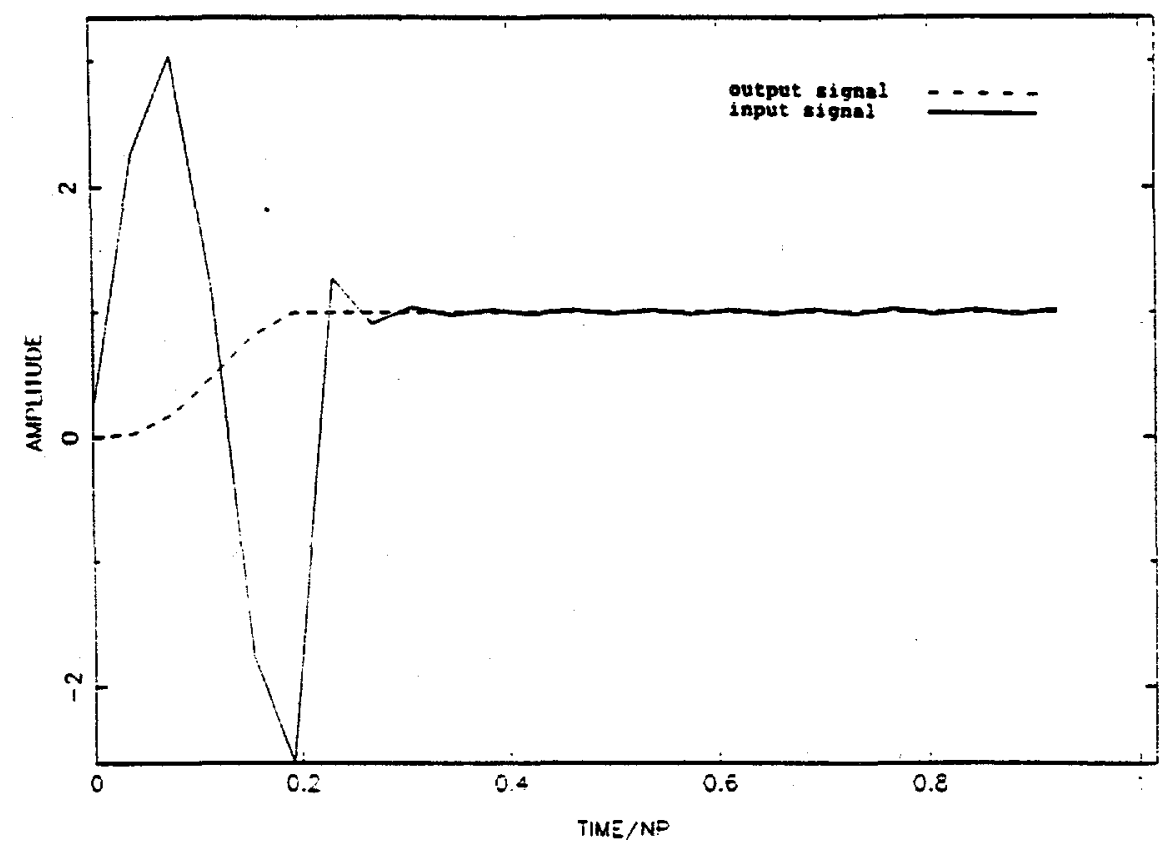

Figure 7: Input and output signals $\mathrm{RT} / \mathrm{NP}=0.2$

\subsection{DIAMOND TURNING APPLICATIONS}

The application of this method of control to diamond turning appears possible but will require a very high speed digital to analog converter (DAC) along with an amplifier capable of supporting the required slew rates to move the tool and current draw during the process. Such an attempt was made earlier this year, using the Masscomp 5400, but output speed $(300 \mathrm{kHz})$ and buffer recycling created problems in outputting data.

Interest in this project was generated by results of diamond turned surfaces using the fast tool servo and a $40 \mathrm{kHz}$ DAC to produce asymmetric cuts in brass at a depth of $0.33 \mu \mathrm{m}$ [9]. The points of tool entry and exit during the cutting process produced residual vibrations of the tool while in contact with the workpiece, resulting in ripples on the surface of the flat during tool retraction and in the cut itself during tool entry. Since the cuts were produced using the TMS32010 high speed digital signal processor (DSP) which has $25 \mu$ second sampling time, application of the conditioned signal must occur during this period of time. This will require a very high speed DAC so that the conditioned signal may be generated during this interval of time.

As discussed in the previous section, signal breakdown occurs when applying this method to the servo. The first fundamental frequency of the servo during the cutting process for brass has been estimated at $13 \mathrm{kHz}$ [9]. Using this information along with the rise time of the desired output signal, the profile of the required input signal can be determined. The desired output shape will be the same as Equation (1.1) but $\omega_{0}$ is altered to yield a rise time of $25 \mu$ seconds. The ratio RT/NP is 


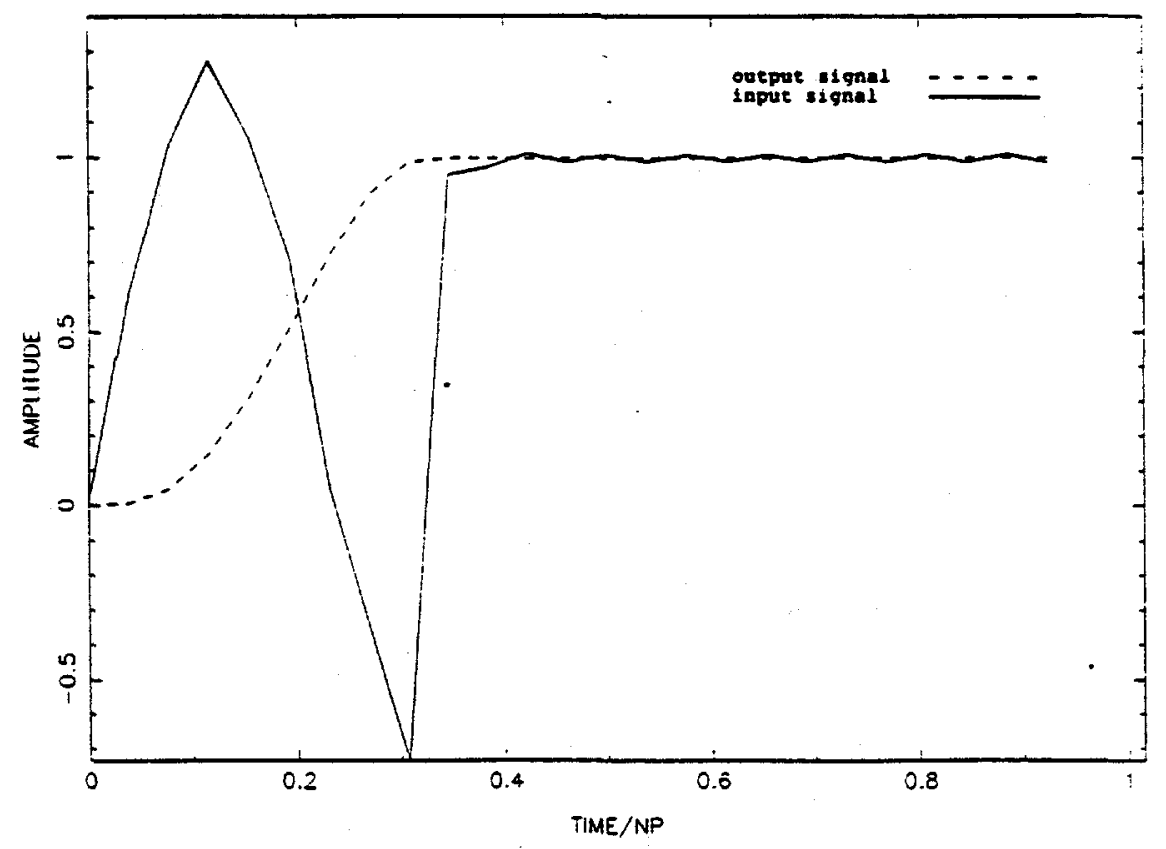

Figure 8: Input and output signals for cutting $\mathrm{RT} / \mathrm{NP}=0.325$

around 0.325 , indicative of signal breakdown. Figure 8 shows desired output and required input signals for the cutting process. Dimensionless time is calculated as the ratio of dimensional time to the servo's natural period $(1 / 13000 \mathrm{sec}$.). The system is assumed to be linear so that the amplitude of 1 may be translated into $0.33 \mu$ inches. The maximum value of the input signal is the estimated to be 0.43 $\mu$ inches. The smoothness of the curve is due to the numerical transform used to obtain the profile. Importance is given mainly to the trend of the curve and not it's exact shape.

The input signal of Figure 8 in now translated into a plot of input voltage required for the piezoelectric actuator (PZT) in the servo versus real time. The scaling of the voltage is based on a (PZT) coefficient of 21 nanometers per volt [9]. The sampling of the signal is around $1.6 \mu$ seconds and is based on a zero order sample and hold.

Consideration must be first give to a DAC capable of these speeds. One candidate is a high speed signal processing board produced by the Markenrich Corporation. Top output speed of this board is 20 megahertz. The data (input signal) is stored in RAM and the buffer may be recycled upon triggering by an external source, which in this case will be the digital encoder on the fast tool servo. Digital resolution based on 0 to 5 volts positive output is 0.04 volts per bit. The low resolution is a trade off for the high DAC speeds required, but appears to be more than adequate based on Figure 9.

Once again looking at Figure 9, an overshoot and undershoot of the steady state value occurs to compensate for momentum of the system. Without this motion ringing would theoretically occur. To estimate a realizable slew rate (volts/second), the over and undershoot portions of the input curve are viewed as symmetrical. This 


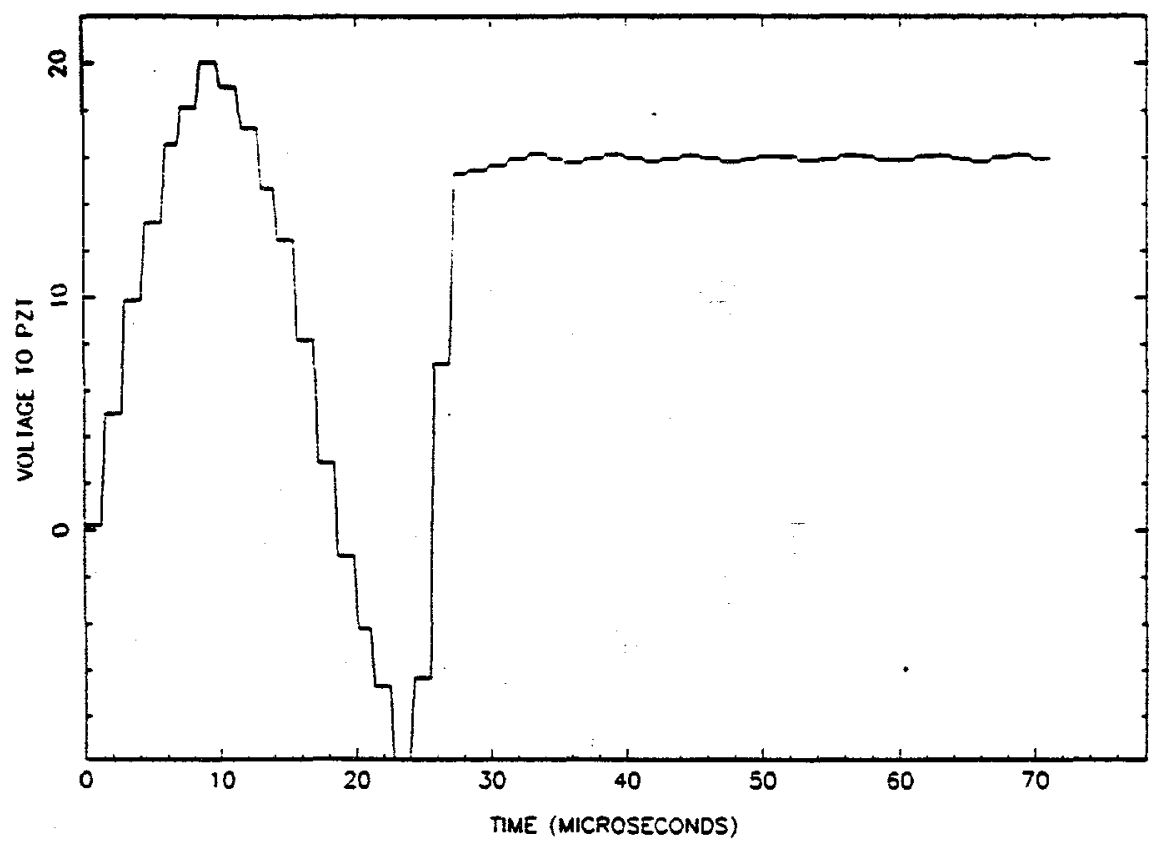

Figure 9: Sampled input signal based on voltage

is justified since concern is with the overall shape of the input signal. Using the required DAC speed and, largest voltage difference (about 5 volts) in the overshoot portion of the curve, a slew rate of about 3 volts per $\mu$ second is obtained. This is not unrealistic since the Burleigh PZ-70 amplifier can handle a slew rate of up to 5 volts per $\mu$ second. The approximate current draw of the PZT stack is estimated at around $1 \mathrm{amp}$ which is very high and may create a serious problem. This calculation is based on the PZT capacitance of $0.3 \mu \mathrm{F}$.

\subsection{CONCLUSION}

The application of this method to the diamond turning process is possible but the restricting factor at the moment is an amplifier which can handle the current requirements of the PZT. Options at present are to reduce the amplitude of the signal thus reducing the required current, but this would defeat the purpose of this specific demonstration. A better option is to use a different desired output curve shape in the same period of time so that the input signal will not require such a high voltage draw. This option will involve some form of an optimization process.

At the present time work is continuing on finding a solution to this problem so that the experiment may be conducted as planned. Specifically, attention is being given to finding a different form for the output signal. Such a solution would allow the use of existing equipment thus making the method practical for very high speed applications. If this is not possible for some reason an alternative plan will be to either decrease the desired output signal amplitude or to increase the rise time. Decreasing the output signal will maintain the same input signal but reduce the 
required voltage. Increasing the rise time, however, will change the input signal, again reducing the required voltage.

Specific tasks include finding a solution to the problem discussed, and then implementing the method in a cutting process, demonstrating a reduction of residual vibration at points of tool entry and exit during the cut. 


\section{References}

[1] R.H. Cannon, Jr and Eric Schmitz,"Initial Experiments on the End-Point Control of a Flexible One-Link Robot", The International Journal of Robotics Research, Vol.3, No.3, Fall 1984, p.62.

[2] C.P. Neuman and P.K. Khosla, "Parameter Identification for Robot Control", Dynamic Systems: Modeling and Control, Vol. 1, Fall 1985, p.213.

[3] I. Yamada and M. Nakagawa, "Reduction of Residual Vibrations in Positioning Control Mechanism", Journal of Vibration, Acoustics, Stress, and Reliability in Design, Vol.107, January 1985, p.47.

[4] H. Ozisik and R.F. Keltie, "Structural Response Synthesis", Precision Engineering Center Annual Report, Vol.5, 1987, p.128.

[5] P.H. Meckl and W.P. Seering, "Minimizing Residual Vibration for Point-toPoint Motion", Journal of Vibration, Acoustics, Stress, and Reliability in Design, Vol.107, October 1985, p.378.

[6] D.M. Aspinwall, "Acceleration Profiles for Minimizing Residual Response", Journal of Dynamic Systems, Measurement, and Control, Vol.102, 1980, p.3.

[7] D.E. Luttrell and T.A. Dow, "Development of a High Speed System to Control Dynamic Behavior of Mechanical Structures", Precision Engineering Center Annual Report, Vol.4, 1986, p.59.

[8] T.G. Bifano and T.A. Dow, "Application of a Novel Two Step Digital Control Algorithm for Precision Actuation", Second Annual Report on Precision Engineering-SRO154, January 1985, p.99.

[9] P.J. Falter and T.A. Dow, "Diamond Turning of Rotationally Asymmetric Specimens", Precision Engineering Center Annual Report, Vol.5, 1987, p.186. 


\title{
8 STRUCTURAL POWER FLOW \\ MEASUREMENT
}

\author{
Karl J. Falter \\ Graduate Student \\ Richard F. Keltie \\ Associate Professor, MAE
}

\begin{abstract}
Previous investigations of structural power flow through beam-like structures resulted in some unexplained anomalies in the calculated data. In order to develop structural power flow measurement as a viable technique for machine tool design, the causes of these anomalies needed to be found. Once found, techniques for eliminating the errors could be developed. Error sources were found in the experimental apparatus itself as well as in the instrumentation. Although flexural waves are the carriers of power in the experimental apparatus, at some frequencies longitudinal waves were excited which were picked up by the accelerometers and altered power measurements. Errors were found in the phase and gain response of the sensors and amplifiers used for measurement. $A$ transfer function correction technique was employed to compensate for these instrumentation errors.
\end{abstract}

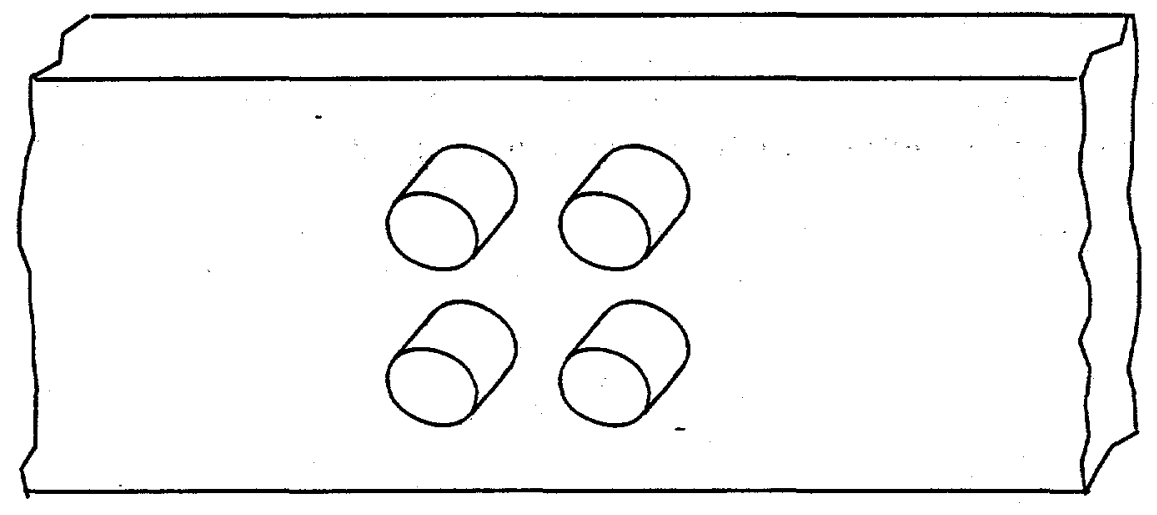




\subsection{INTRODUCTION}

Structural power flow transmitted by simple flexural vibration may be measured using a two accelerometer technique. Power flow may be determined using the imaginary part of the cross spectrum between two accelerations at closely spaced points. For the case of a thin beam, the time averaged power flow is given by:

$$
\text { power }=\frac{2 \sqrt{E I \rho}}{\Delta} \int_{0}^{\infty} \frac{\operatorname{Im}\left\{G\left(a_{1}, a_{2}\right)\right\}}{\omega^{2}}
$$

where $E$ is the modulus of elasticity, $I$ is the cross sectional area moment of inertia, $\rho$ is the mass per length, $\Delta$ is the spacing of the accelerometers, $\omega$ is the circular frequency and $\operatorname{Im}\{G()\}$ indicates the imaginary part of the cross spectrum [1].

Previousiy [2], this technique was used to study the transmission efficiency of various types of bolted joints in a beam using the apparatus shown in Figure 1 . The 20 foot long 0.5 by 2.0 inch steel beam was suspended by four wires with its 2.0 inch dimension vertical. The beam was driven at one end with an electrodynamic shaker while the other end was buried in sand to absorb energy. Two pairs of accelerometers were used. Pair ' $A$ ' was mounted mid-way between the shaker and the joint and pair ' $B$ ' was mounted mid-way between the joint and the sandbox. The beam was driven using pure tone excitation at various frequencies. At each frequency the transmission efficiency, or power measured by pair ' $B$ ' divided by power measured by pair ' $A$ ', was calculated.The transmission efficiency

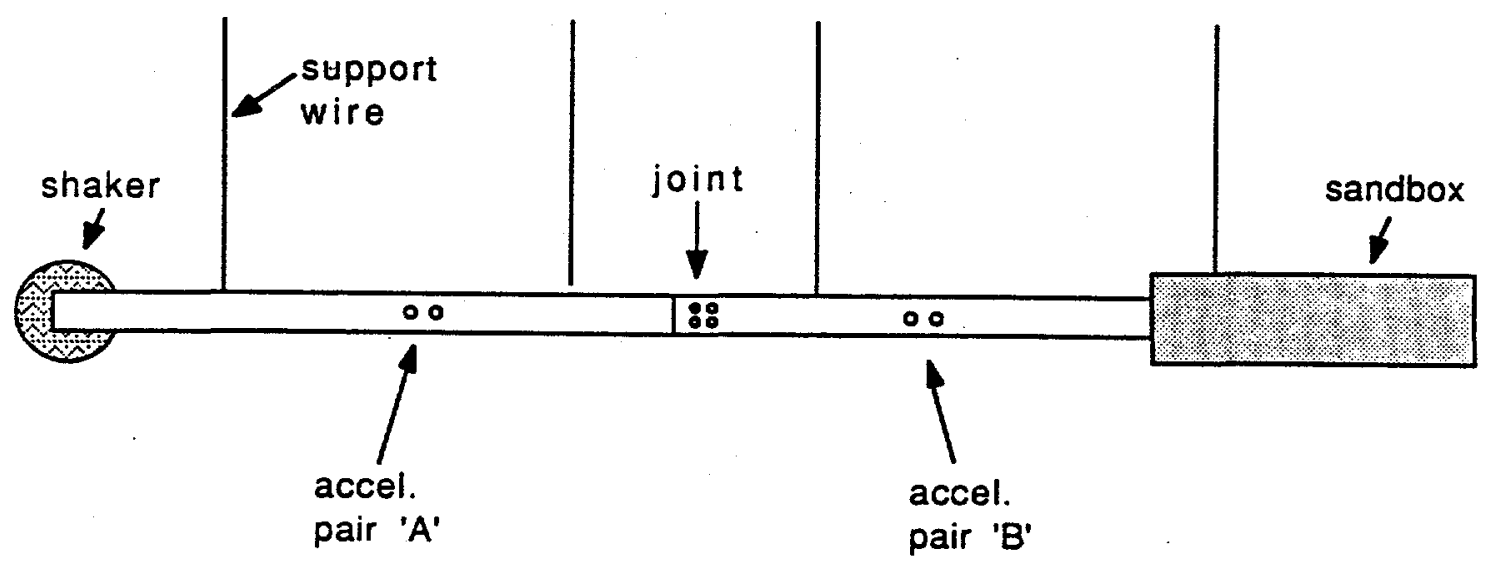

Figure 1: Experimental apparatus used to measure transmission efficiency of bolted joints. 


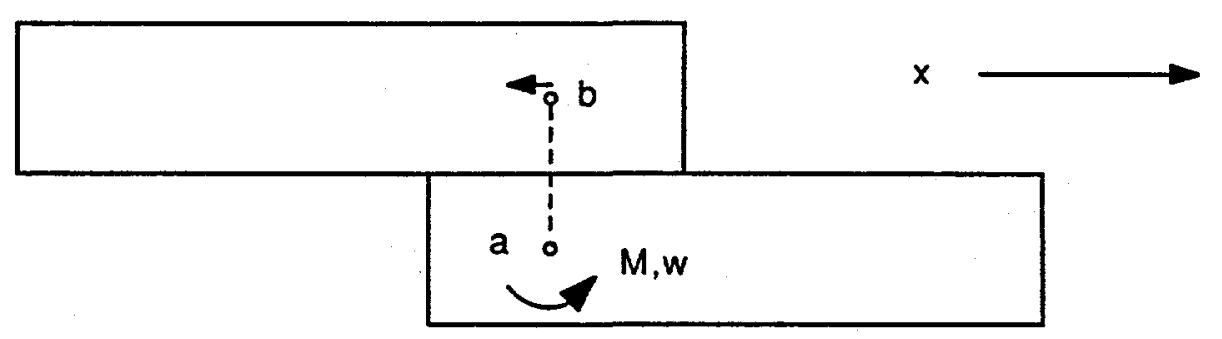

Figure 2: Origin of longitudinal waves in lap joint.

in some cases was found to be greater than 100 percent. Obviously this an erroneous result since no power is added to the system at the joint. To develop structural power flow measurement as a viable technique for machine tool development, the causes of this anomaly and their solutions were required. This effort formed the basis of the present study.

\subsection{INDENTIFICATION OF ERRORS}

Several sources of the erroneous results were investigated. It was thought that modes of vibration other than just flexural vibration were being excited in the experimental beam and that these secondary vibrations were somehow corrupting the measurement of flexural vibration. Sources of error in the instrumentation itself such as phase and gain mismatch between channels were also pursued.

\subsubsection{Longitudinal Waves In Beam}

One possible explanation for the anomalous data observed in the previous power flow studies was the presence of longitudinal waves originating in the lap joint. As shown in Figure 2 , the angular velocity of the joint due to the flexural wave, $w$, causes point $b$ to move in the $\mathbf{x}$ - direction relative to point a. This relative movement would induce a longitudinal wave in the beam [3]. It was thought that these longitudinal waves might cause transverse motion of the beam through Poisson's effect, resulting in the beam bulging as 


\begin{tabular}{||l|l|l||}
\hline \multicolumn{3}{||c||}{ Longitudinal Natural Frequencies } \\
\hline \multicolumn{3}{|c||}{ of Beam (Hz) } \\
\hline mode & calculated & measured \\
\hline \hline 1 & 419 & 425 \\
\hline 2 & 837 & 800 \\
\hline 3 & 1256 & 1275 \\
\hline 4 & 1675 & 1700 \\
\hline
\end{tabular}

Table 1: Longitudinal natural frequencies of the beam.

the longitudinal compression waves travel along its length. This bulging could add constructively or destructively to the flexural wave thus affecting the power flow measurement.

The theoretical longitudinal natural frequencies for the beam used in the experiments were calculated and are shown in Table 1 . These values were calculated assuming free-free end conditions and neglecting the joint in beam. The natural frequencies were also found experimentally by measuring the longitudinal frequency response of the beam. The longitudinal motion of the beam was thought to be excited by flexural waves. Thus the input for the frequency response analysis, the flexural motion, was measured with an accelerometer oriented laterally, mounted midway between the shaker and the joint. The output, longitudinal motion, was measured with an accelerometer oriented longitudinally at the joint(see Figure 3).The beam was driven with white noise and the transfer function up to $5 \mathrm{kHz}$ was measured. The measured response spectrum showed peaks which corresponded well with the calculated natural frequencies.

Calculations were made to determine how much influence Poisson's effect would have on the transverse motion of the beam. It was found that less than 0.5 percent of the longitudinal motion of the beam would be transformed into transverse motion. The small amount of resulting signal contamination was not judged to be important. Although longitudinal waves do not affect the measurement of power flow through Poisson's effect, they do however appear to affect the measurement by means of the accelerometer's transverse sensitivities. These accelerometers have published transverse sensitivities ranging from 2.7 to 3.8 percent. Depending on whether or not the longitudinal wave is in phase with the flexural wave, the measured flexural accelerations may be too high or too low. Thus, the longitudinal wave could affect the flexural power measurements, although not through the Poisson effect. 


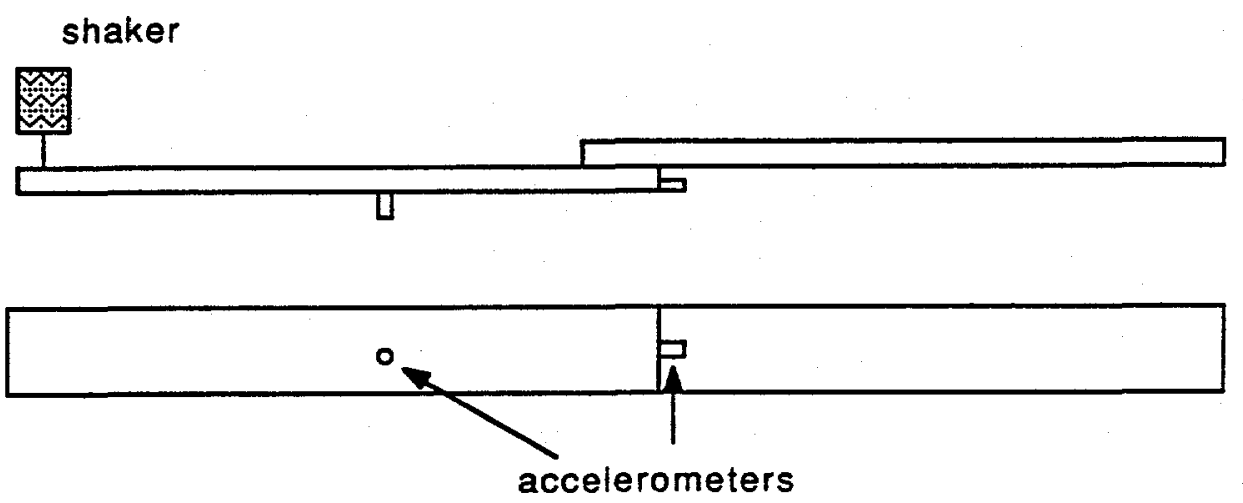

Figure 3: Experimental set up to find longitudinal natural frequencies of the beam.

\subsubsection{Errors In Instrumentation}

The largest sources of instrumentation error in the measurement of power flow were found to be phase response differences between channels and deviation of the response of the transducers and amplifiers from nominal values. A phase mismatch of 0.1 degree between a pair channels results in a error in power of approximately 1.0 percent. Any error in gain causes a similar error in power. For example, a 2 percent error in overall sensitivity causes a 2 percent error in power. The phase and gain mismatch between channels of accelerometers and amplifiers was measured by mounting the accelerometers to a common vibration target, in this case directly on the electrodynamic shaker used to excite the beam. The outputs of two of the accelerometer amplifiers were sent to a Nicolet 660B digital waveform analyzer and the transfer function between the two signals was calculated. The accuracy of the Nicolet $660 \mathrm{~B}$ was also checked by sending identical white noise signals into both of its channels and checking to see if the calculated transfer function indicated any phase or magnitude differences. Slight errors were indeed found. These errors can be compensated for when comparing accelerometer channels in two ways. The first method is to perform the transfer function once then repeat it with the inputs to the Nicolet $660 \mathrm{~B}$ switched. The difference in the two transfer functions then is due to true differences in the inputs to the analyzer and not inherent to the analyzer itself. The other method is to simply divide the transfer function of the accelerometer channels by the calculated transfer function of the same signal fed into both channels of the analyzer. This division is accomplished by dividing the complex values of the transfer function at each bin frequency of interest. The movement of the shaker was also checked to ensure that all points where the accelerom- 


\begin{tabular}{||l|l|l||}
\hline \multicolumn{3}{||c||}{ Complex Transfer Between Two } \\
\hline \multicolumn{2}{||c||}{ Nominally Identical Channels } \\
\hline Freq (Hz) & Real & Imaginary \\
\hline \hline 500 & 0.981 & 0.0031 \\
\hline 800 & 0.982 & 0.00296 \\
\hline 1000 & 0.980 & 0.00351 \\
\hline 1200 & 0.981 & 0.00430 \\
\hline 1500 & 0.984 & 0.00864 \\
\hline 2000 & 0.978 & 0.00713 \\
\hline
\end{tabular}

Table 2: Transfer function between two nominally identical channels of instrumentation.

eters were mounted did indeed move synchronously. This was accomplished using two accelerometers. The first was attached to the shaker at an arbitrary point. The second was moved to various locations. At each location a transfer function was performed. If the shaker moved as a rigid body the transfer function should be independent of the location of the second accelerometer. The shaker did prove to move as a rigid body.

The accelerometer and amplifier sets were also compared two at a time at various pure tone frequencies. Table 2 shows the results for two nominally identical channels. The transfer function is expressed in real and imaginary components. The real part is defined as the magnitude times the cosine of the phase angle, the imaginary part is the magnitude time the sine. These results show the slight frequency dependence of the channels of instrumentation.

\subsection{CORRECTION OF ERRORS}

\subsubsection{Transfer Function Correction Technique}

Based on the results obtained in the previous section, it was decided to apply a transfer function correction technique to the two accelerometer power flow measurement technique [4]. The desired quantity to be measured is the imaginary part of the complex cross spectrum of the accelerations at the two closely spaced points. These quantities depend on the magnitude of each acceleration and the phase difference between the accelerations in the measurement pair. The desired quantities are altered in phase and magnitude at each stage of processing from the beam to the spectrum analyzer. These alterations may be expressed as the multiplication of the acceleration of the beam by an overall complex 
gain for the particular instrumentation channel. Differences in these complex gains between channels may be corrected in the following manner.

As depicted in Figure 4 the four accelerometers are mounted to the shaker driven at a particular frequency. All accelerometers are subjected to the same acceleration. As the accelerations are converted to voltages each acceleration, $S$, is effectively multiplied by the overall complex gain (i.e. $I_{0}$ yielding $S * I_{0}$ ). Performing the transfer function on two signals is essentially the same as dividing the complex signals. For example the transfer function between $S * I_{0}$ and $S * I_{1}$ may be represented as:

$$
\frac{S * I_{1}}{S * I_{0}}=\frac{I_{1}}{I_{0}}
$$

In this way the complex gains of one channel may be compared to that of another. When measuring power flow the two pairs are mounted to the beam and the again the accelerations are multiplied by the complex gain of each channel. The cross spectrum is equivalent to multiplying complex signals. Thus, the cross spectrum of an accelerometer pair is than the acceleration at one point multiplied by a complex instrument gain times the acceleration at the second point multiplied by its instrument gain. For example the cross spectrum of pair ' $\mathrm{A}$ ' is $\left(a * I_{0}\right) *\left(b * I_{1}\right)$. If the cross spectrum of a pair is divided by the transfer function measured when the pair was mounted on the shaker, the result is equivalent to the product of both acceleration in the pair multiplied by the same instrument gain. For example:

$$
\frac{\left(a * I_{0}\right) *\left(b * I_{1}\right)}{\frac{I_{1}}{I_{0}}}=\left(a * I_{0}\right) *\left(b * I_{0}\right)
$$

In this way phase and gain differences between instrument channels in a pair can be eliminated. Since the same instrument was used to compute both the transfer function and the cross spectrum, errors between channels of this instrument are also eliminated when the cross spectrum is divided by the transfer function. To make comparative power flow measurements between two pairs of accelerometers it is necessary only to correct for gain differences between pairs. This may be done by divided the corrected cross spectrum of pair ' $B$ ' by the square of ratio of gain of ' $B$ ' divided by the gain of 'A'.

$$
\frac{\left(c * I_{2}\right) *\left(d * I_{2}\right)}{\left|\frac{I_{2}}{I_{0}}\right|^{2}}=\left(c * I_{0}\right) *\left(d * I_{0}\right)
$$

Thus the transfer function correction technique may be used to correct the response of all the instrument channels relative to the response of one master channel (in this case channel 0 ). This correction is sufficient for comparative power flow measurements. To make absolute measurements it would be necessary calibrate the master. 


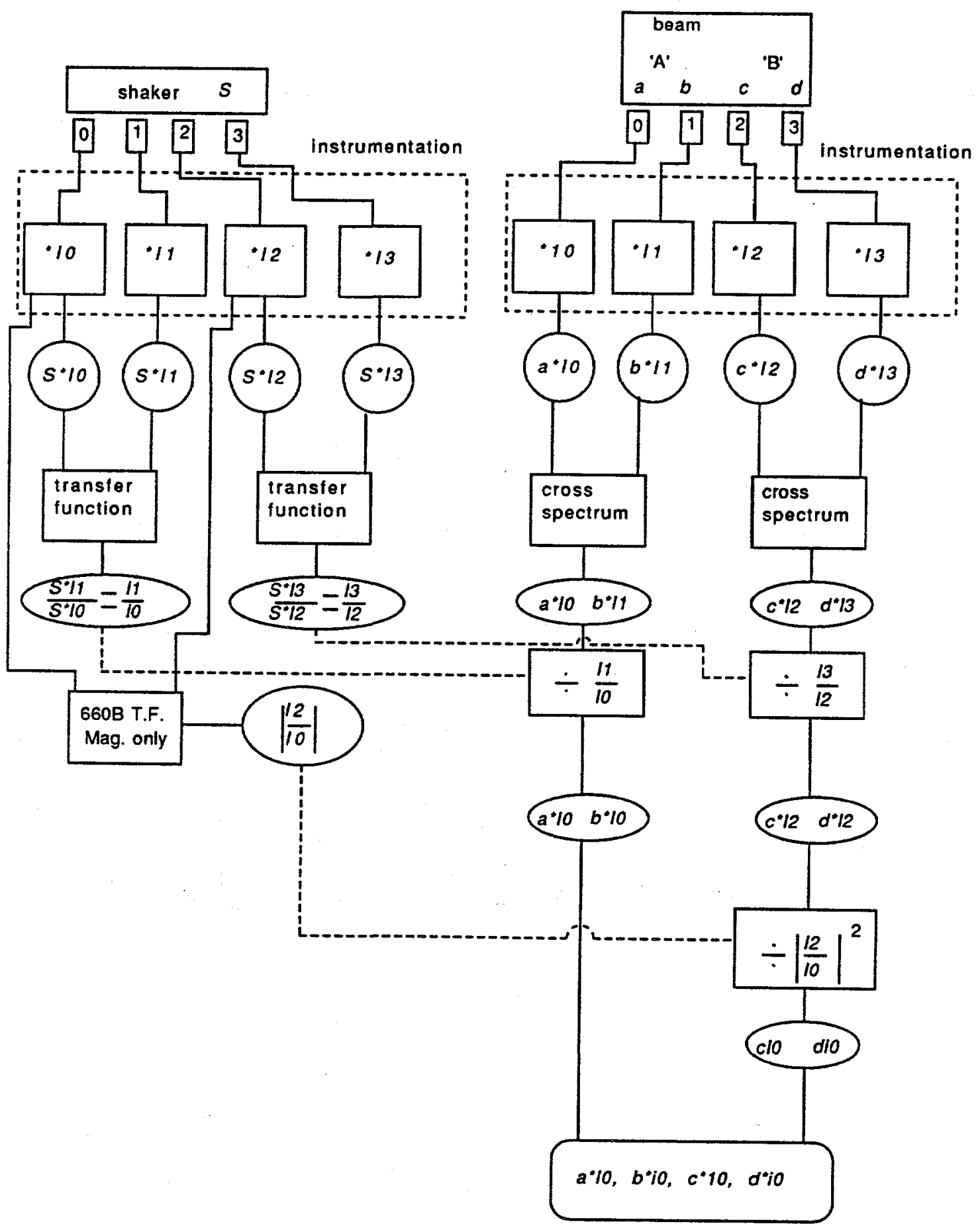

Figure 4: Schematic diagram of transfer function correction technique. 


\begin{tabular}{||l|l|l||}
\hline \multicolumn{3}{||c||}{ Transmisson Efficiencies } \\
\hline & \multicolumn{2}{|c|}{ Pair ' $\mathrm{B}$ ' / Pair ' $\mathrm{A}$ ' } \\
\hline Freq (Hz) & nominal & corrected \\
\hline \hline 500 & 0.819 & 0.862 \\
\hline 800 & 0.964 & 1.005 \\
\hline 1000 & 0.914 & 0.951 \\
\hline 1200 & 0.902 & 0.997 \\
\hline 1500 & 0.979 & 0.999 \\
\hline 2000 & 1.038 & 1.034 \\
\hline
\end{tabular}

Table 3: Nominal and corrected transmission efficiencies.

\subsubsection{Results}

Power flow measurements were performed using two pairs of accelerometers. Transmission efficiencies across the bolted joint were calculated, using both the nominal instrumentation sensitivities and also the transfer function correction technique. In this instance the nominal sensitivities gave transmission efficiencies that appeared to be too low. In earlier experiments with different accelerometers transmission efficiencies that were too high (greater that 100 percent) were calculated. As shown in Table 3 the transmission efficiencies calculated using the transfer function correction technique are closer to 100 percent than the nominal results. At some frequencies the corrected transmission efficiency is greater than 100 percent. In an effort to eliminate the effect of longitudinal waves both pairs of accelerometers were placed at the same location along the beam as shown in Figure 5. By placing both pairs of accelerometers at the same location on the beam, both pairs should be subject to the same accelerations regardless of what type of vibration causes them. As shown in Table 8.3.2 by applying the transfer function correction technique, the differences are quite small. Notable exceptions are at 500 and $1200 \mathrm{~Hz}$. These frequencies are close to a longitudinal natural frequencies. Thus the transverse sensitivities of the accelerometer is important at these frequencies. The accelerometers in pair ' $B$ ' have higher transverse sensitivities and those in pair ' $A$ '. This appears to be the cause of the greater than unity ratio. One important conclusion here is that the relatively large longitudinal acceleration associated with longitudinal resonances, coupled with the accelerometers' transverse sensitivity, can effectively mask the transverse acceleration component necessary for power flow measurement using the two-accelerometer technique. 
shaker

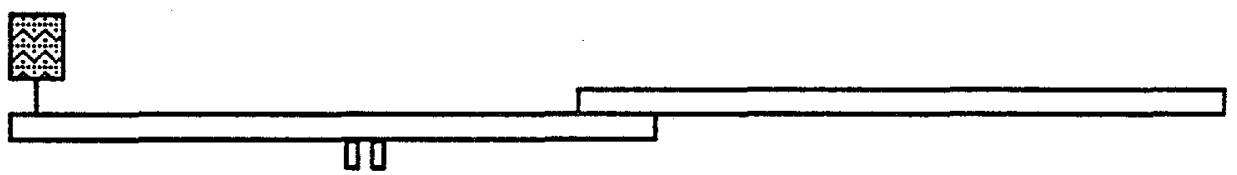

पण

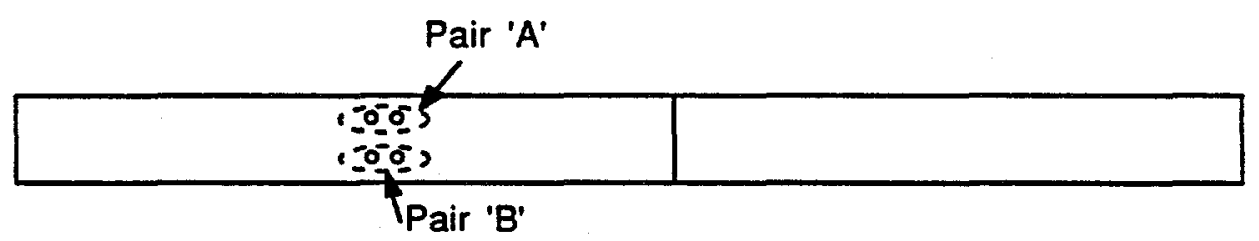

Figure 5: Apparatus set-up for comparative power measurement

\begin{tabular}{||l|l|l||}
\hline \multicolumn{2}{||c||}{ Comparison of Measured Power Flow } \\
\hline & \multicolumn{2}{|c||}{ Pair 'B' / Pair 'A' } \\
\hline Freq (Hz) & nominal & corrected \\
\hline \hline 500 & 0.914 & 1.048 \\
\hline 800 & 0.879 & 0.998 \\
\hline 1000 & 0.922 & 1.017 \\
\hline 1200 & 0.930 & 1.083 \\
\hline 1500 & 0.935 & 0.998 \\
\hline
\end{tabular}

Table 4: Comparison of power flow with two pairs of accelerometers. 


\subsection{CONCLUSION}

The two accelerometer power flow measurement technique appears to be accurate enough to make application to some types real world structures viable. One application that might be pursued is the analysis of vibration transmission paths in structures comprised of simple beam or plate-like elements. This could be done by exciting the structure at some point and 'tracking' the vibrational energy to its dissipation point by measuring the direction and magnitude of power flow at various points. Another application of structural power flow measurement would be noise source identification in machines. Structural power flow measurement has advantages over simple, single accelerometer spectrum analysis in that it can detect direction as well as magnitude of power flow instead of just vibrational amplitude.

When using the two point power flow measurement technique it is recommended that all accelerometer instrumentation channels be checked for phase and overall sensitivity errors. This may be accomplished by placing the accelerometers on a common target and checking the transfer function between channels. Care should also be taken to account any errors in the instrumentation used to measure transfer function are. Such errors may be found by measuring the transfer function between two signals which are known to be identical and then checking if any phase or magnitude differences are erroneously calculated. If phase and overall sensitivity errors are found in the instrumentation, the transfer function correction technique is effective for compensating these errors especially when performing comparative measurements using two pairs of accelerometers. Care should also be taken to ensure that other types of waves in the structure are sufficiently small so as not to effect measurement of the desired wave type through transverse sensitivity of the accelerometers.

Given the current state of development, power flow measurement is best suited to structures with plate and beam-like elements which are not found in machine tools. In addition, the difficultly in measuring vibration in precision machine tools is compounded by the small amplitudes which require high amplification which may lead to greater phase and gain mismatch between channels. These difficulties limit the present potential of structural power flow measurement as a useful tool in precision machine tool analysis. 


\section{References}

[1] Verheij, J.W., "Cross Spectral Density Methods for Measuring Structure Borne Power Flow on Beams and Pipes", Journal of Sound and Vibration, 1980, 70(1), 133-139.

[2] Allred, C. K., "Experimental Investigation of Flexural Power Flow Through Structure Interfaces", M.S. Thesis, North Carolina State University, Raleigh, NC, 1987.

[3] Cremer, L., M. Heckl and E. E. Ungar, "Structure Borne Sound", Springer Verlag, 1973, Chapter 5.

[4] Waser, M. P. and M. J. Crocker, "Introduction to the Two-Microphone CrossSpectral Method of Determining Sound Intensity", Noise Control Engineering Journal, May-June 1984, 76-85. 


\title{
9 DIAMOND TURNING MACHINE CONTROLLER IMPLEMENTATION
}

\author{
Kenneth P. Garrard and Lauren W. Taylor \\ Research Assistants \\ Byron F. Knight \\ Graduate Student \\ Robert J. Fornaro \\ Associate Professor, CSE
}

The standard controller for a Pnuemo ASG 2500 Diamond Turning Machine, an Allen Bradley 8200, has been replaced with a custom high-performance design. This controller consists of four major components. Axis position feedback information is provided by a Zygo Axiom 2/20 laser interferometer with 0.1 micro-inch resolution. Hardware interface logic couples the computer's digital and analog $I / O$ channels to the diamond turning machine's analog motor controllers, the laser interferometer, and other machine status and control information. It also provides front panel switches for operator override of the computer controller and implements the emergency stop sequence. The remaining two components, the control computer hardware and software, are discussed in detail below.

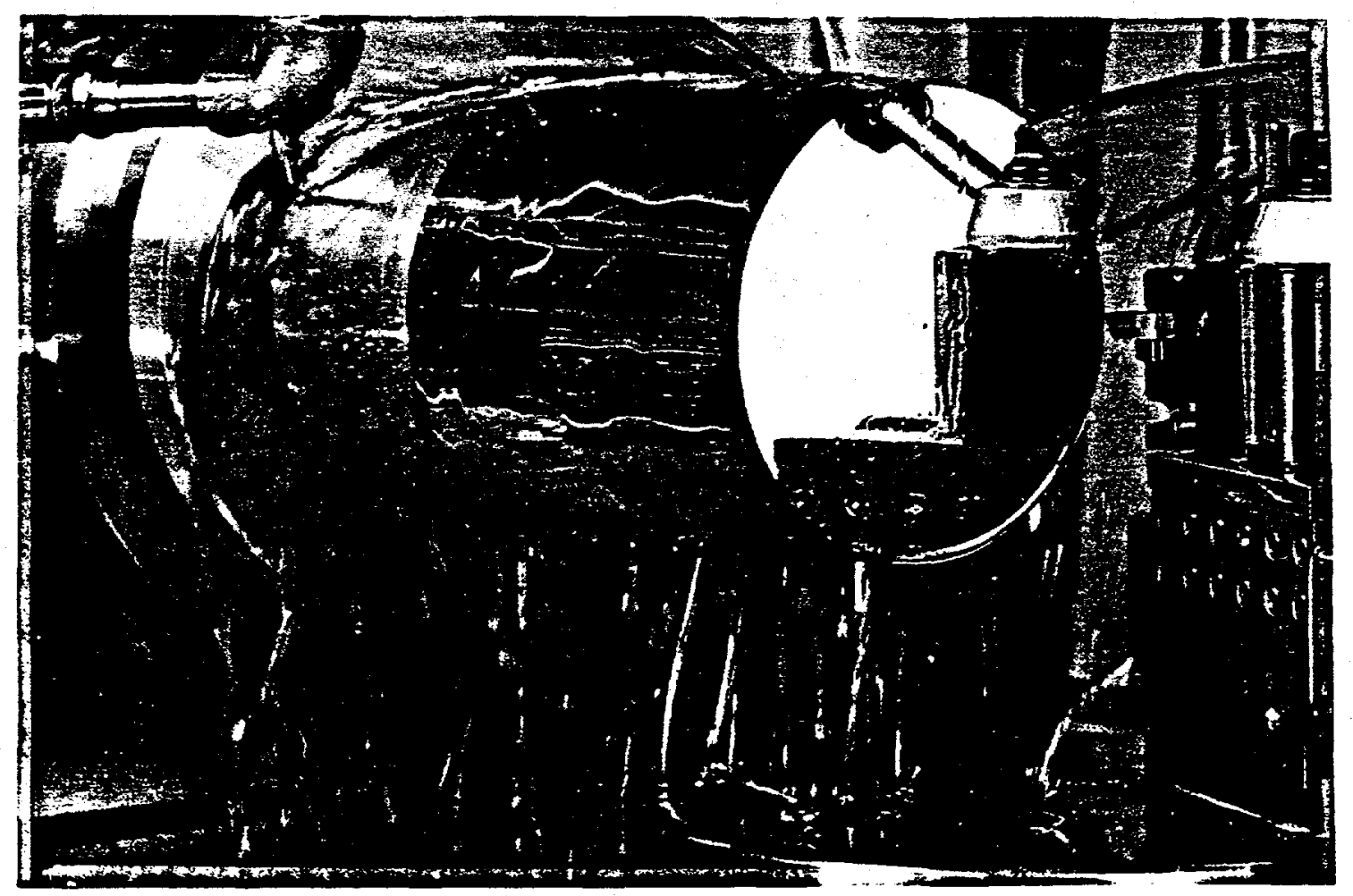




\subsection{INTRODUCTION}

The PC-AT based diamond turning machine controller is operational. The goal of a 1 millisecond loop closure cycle has been achieved. Furthermore, multi-step control sequences do not suffer from missed update cycles (i.e., there is no block update timing anomaly). The control algorithm currently in use is a integral controller with a single adjustable gain for each axis. Position feedback information is filtered via a running average of successive laser interferometer inputs at 1 millisecond intervals. This results in slower, but more stable machine response.

Elementary controller functions are accessible to the user through a keyword set of operator commands. Generic high-level control programs for cutting flats have also been implemented. Only linearly interpolated cuts are available to the user. Circular interpolation has been implemented and is currently being tested. Multi-step cuts, with full axis control for the entire sequence of operations, are provided by storing coordinate, feedrate tuples in a file and specifying the file name as a parameter to a control program. The complete set of user commands and control programs implemented are described in the appendix.

DTM status is continuously displayed in real-time on a color video monitor with separate windows reserved for axes position updates, command scrolling, error messages and part program information. Extensive command line editing, history and recall functions are available. These single line editing functions allow sequences of commands to be easily repeated with a minimum of keystrokes by the user.

The present controller software provides a foundation for the further development of control algorithms. Both open and closed loop performance monitoring of the response of an axis to an arbitrary input (e.g., step, constant velocity, sine wave, etc.) is a permanent feature of the control software. Servo outputs and laser position inputs can be recorded over adjustable sample intervals with or without data averaging. These data sampling and storage features do not interfere with the cycle time of the control loop. The high-level control programs written in $\mathrm{C}$ can be easily modified to accommodate proposed controller designs. The collection and correlation of control outputs and axis position feedback information should simplify the task of evaluating controller performance.

\subsection{SYSTEM CONFIGURATION}

\subsubsection{Computer Hardware}

The current computer system configuration (Figure 1) is based on a Zenith PC-AT microcomputer. The PC-AT processor board has an Intel $80286 \mathrm{cpu}$ with an 80287 numeric co-processor and 512K bytes of RAM. The PC-AT also has one parallel port and one serial port. Mass storage is provided by a $20 \mathrm{M}$ byte hard disk and a $1.2 \mathrm{M}$ byte floppy disk. 
A $512 \mathrm{~K}$ byte memory expansion card provides additional on-line storage capacity. The operator's console is a high-resolution analog RGB color video monitor supported by an EGA card.

The computing power of the PC-AT is enhanced by the addition of a Definicon Systems 68020 co-processor board. This board is equipped with a 68882 floating point accelerator chip and $4 \mathrm{M}$ bytes of 32 bit RAM, of which $64 \mathrm{~K}$ bytes are dual-ported into the PC-AT address space. Both processors operate at a $20 \mathrm{MHz}$ clock rate. The Definicon processor board also has two serial ports, a 16 bit programmable timer, and a socket for the 68851 memory management unit.

Two Burr-Brown PCI-20001C-2 I/O carrier boards provide the interface to the DTM hardware. Each carrier board has 32 channels of digital $1 / O$, and three slots for analog or digital modules. Two of the slots on each carrier board are occupied by a PCI-20003M-2 analog output instrument module and a PCI-20004M-1 digital I/O instrument module. These modules provide 2 analog output channels, and 32 additional digital input/output channels on each carrier for controlling the DTM hardware. One module slot on each carrier remains unused. A total of 50 channels of digital I/O are unused in the present configuration. One analog output channel is also available. The configuration of the DTM hardware and $I / O$ interfaces are described in detail in [1].

Expansion beyond the PC-AT chassis is also possible. A PC-bus to Multibus adapter package allows the PC to access any resource of the Multibus through a re-configurable memory mapped window. Interrupts can be transmitted across the interface. The present version of the DTM controller does not make use of this capability. However, access to the multibus will allow subsequent implementations of the controller to utilize other computing resources such as the 80186/TMS320C25 dual digital signal processor architecture. This DSP architecture, known as $\mathrm{H}^{2}$ ART, was developed specifically for high-speed real-time applications and is described in the next section (Section 10) of this report.

\subsection{COMPUTER SOFTWARE}

The PC-AT operating environment is MS-DOS 3.2. The controller software running on the 80286 is written in C. Program development is supported by Turbo C 2.0. This package includes an integrated $\mathrm{C}$ development system, an-ässembler, linker, librarian and a source code debugger.

Programming on the Definicon 68020 processor board is supported by an assembler, linker, source code debugger, and a $\mathrm{C}$ compiler. These tools all run directly on the 68020 processor. A loader program which executes on the $\mathbf{8 0 2 8 6}$ provides a transparent user interface and allows programs executing on the 68020 to access all hardware facilities of the PC-bus. This user interface is perfectly adequate for supporting the system development tools or running computationally intensive user programs. However, the performance requirements 


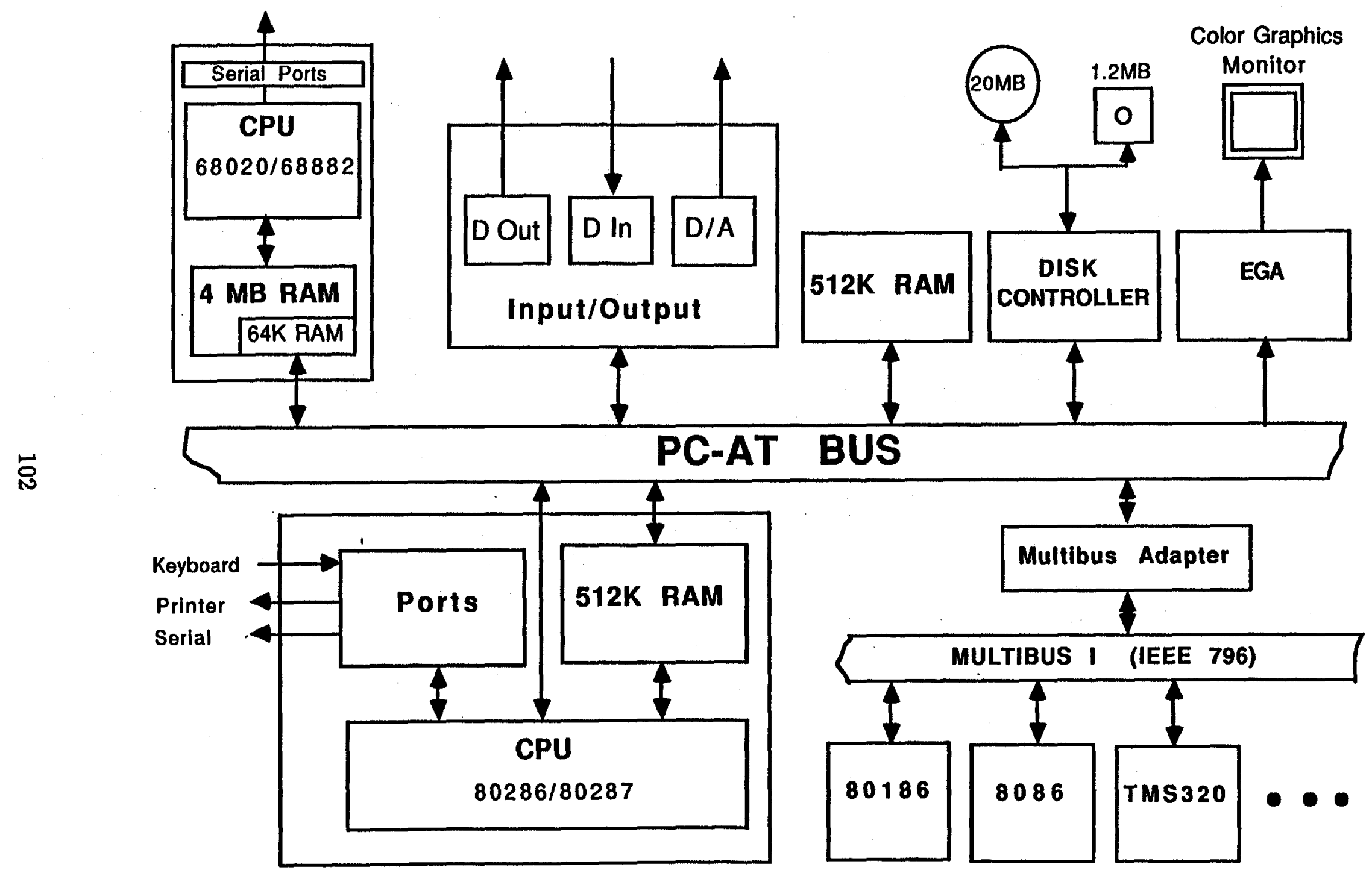

Figure 1: DTM System Configuration 
of the DTM controller far exceed the capabilities of Definicon's loader. A 68020 program simply cannot access PC-bus I/O devices fast enough through Definicon's loader interface. To overcome this difficulty and produce an operational controller, a customized monitor program was written for the $\mathbf{8 0 2 8 6}$ to provide fast access to the hardware facilities of the PC-bus from an executing 68020 control program.

\subsection{6 / 68020 MEMORY MAPPED INTERFACE}

A 68020 control program accesses the PC-bus I/O devices that interface to the DTM through polled loop synchronization. The $64 \mathrm{~K}$ bytes of RAM on the 68020 board which are accessible to the 80286 are used to store critical data structures common to the programs executing on each processor. Access to this shared data is arbitrated via the usual hardware interlock mechanism used to control dual ported memory. The hardware guarantees uninterrupted access by one processor to a single byte of memory for one bus cycle. Synchronization of the processors is achieved by using one byte of the shared memory as a 68020 status and control byte. Codes chosen for this byte indicate the type of service requested by a 68020 program and its current execution status. These requests include laser input, motor controller output, machine status and fault checks, single channel digital switch operations, spindle operations, and disk file operations. Execution status values indicate whether the processor is running, has detected an error condition, or has completed its control program.

After starting a 68020 control program using Definicon's loader, the 80286 executes a function which simply waits for and processes service requests from the 68020 . The 80286 cpu executes a polled synchronization loop until the status and control byte indicates that the 68020 requires service or that an error has occurred. During this time period the 68020 has exclusive access to the shared data. It posts a service request by changing the control byte. The 80286 now has exclusive access to the shared data. The 68020 waits for completion of its request by looping until the control byte value is reset by the 80286 .

Immediately after restarting the 68020 the 80286 executes functions to test the keyboard status and update the real-time display by writing a single character directly to the EGA video memory. During this time interval, the 68020 is busy performing control calculations after obtaining laser feedback inputs or waiting for the update cycle timer interrupt after updating the motor controller analog outputs.

\subsection{BACKUP COMPUTER SYSTEM}

During the past year a backup computer system was purchased. This system is also a Zenith PC-AT with an $80286 \mathrm{cpu}$ and 512K bytes of RAM. It does not have the 80287 numeric co-processor. A Definicon 68020 co-processor board and the accompanying software development tools were also obtained. The 68881 floating point accelerator has been 
upgraded to a 68882 . The software for the original 68020 was upgraded also. The backup system and the operational controller system are identical except for the 80287 and the Burr-Brown I/O boards. Besides the obvious reduction in down-time in the event of computer component failure, the backup system provides two other benefits.

- Further development of the controller software can proceed without limiting access to the DTM. In particular, a substantial amount of the program development involved in implementing our high-level, mnemonic part programming language can be done with very little testing on the DTM control computer. This development activity is not restricted to editing and compiling source code files, extensive testing can be performed as well.

- Controller software can be effectively tested even though the backup computer system does not have an I/O interface to the actual DTM, by providing simulated laser interferometer feedback. Very little of the computer software depends on the actual DTM responses for logical correctness. Thus the simulated laser feedback functions simply assume an ideal machine environment (i.e., no runtime faults) and return the expected axis position based only on the current output to the servo motors for each axis and the update time interval. This produces an ideal response curve for any output to the simulated DTM. Conditional compilation at the lowest level of the source code is used to produce either an operational controller or a simulated response controller. The foremost advantage of this approach is that the execution speed of the controller is not diminished since the simulated controller is produced entirely at compile time. Since only one set of source code files have to be maintained, compatability errors are eliminated. By minimizing the amount of code affected by the two modes of compilation, the ability to test the software off-line is enhanced. Lastly, extensions to include the dynamic rather than "ideal" DTM response can be easily accommodated.

\subsection{CONTROLLER SOFTWARE EXTENSIONS}

Several software extensions to the DTM controller are planned using the current computer system configuration. A high-level, mnemonic part programming language will be defined and implemented. The existing controller keyboard commands described in the Appendix will form the basis for this language. Conditional operations and loop control features as well as a single-step trace capability will also be implemented. In addition, data archive and performance monitoring commands will be included as a permanent feature of the part programming language. A program debugging mode that enables the operator to view a graphical display of the proposed tool path prior to cutting is also planned. As the operator interface commands and the part programming language become more complex, a simple on-line help facility and user documentation become increasingly important. The help facility and the user documentation should be essentially the same product. 
The integration of the circular interpolation and geometric error compensation algorithms into the controller should not present any performance difficulties. Techniques for geometric error compensation have been formulated; however, geometric error data has not been systematically measured. Another source of error is the change in wavelength of the laser beam due to environmental changes.

\subsubsection{Laser Wavelength Correction}

Background The laser interferometer feedback system has an air path and no correction is made for variations in the wave length due to atmospheric changes. With the DTM in a fixed position, variation in the wavelength due to possible atmospheric changes can produce position errors. With a design goal to produce samples with figure accuracy of no more than $25 \mathrm{~nm}$ on a five inch diameter disk, an estimate of the potential error caused by wavelength variations must be made.

The accuracy of the laser interferometer is directly affected by the changes in the wavelength, which is influenced by the changes in the refractive index of the pathway medium. The DTM uses air as its medium. The refractive index of air $(n)$ is related to that of vacuum by

$$
n_{\text {air }} \lambda_{\text {air }}=\lambda_{\text {vac }}
$$

In 1965, B. Edlén published a formula known commonly as the Edlén Equation for calculating the refractive index of air, dependent upon air pressure, air temperature, water vapor pressure, and $\mathrm{CO}_{2}$ content [2]. Edlén estimated the uncertainty of his formula to be $\pm 5 \times 10^{-8}$.

The Edlén Equation is

$$
n-1=\frac{D * 0.104127 * 10^{-4} * P}{1+0.3671 * 10^{-2} * T}-0.42063 * 10^{-9} * F
$$

Where:

$$
\begin{gathered}
D=0.27651756 * 10^{-3} *\left(1+0.54 * 10^{-6} *(C-300)\right) \\
\mathrm{P}=\text { pressure of air in } \mathrm{Pa} \\
\mathrm{T}=\text { air temperature in }{ }^{\circ} \mathrm{C} \\
\mathrm{F}=\text { water vapor pressure in } \mathrm{Pa} \\
\mathrm{C}=\mathrm{CO}_{2} \text { content in ppm. }
\end{gathered}
$$


Since the publication of the Edlén Equation, simple corrections for the effects of changes in the atmosphere on the refractive index have been possible. These corrections can be modeled into the closed-loop control system, resulting in improved positioning and accuracy. Schellekens [3] used this equation to support his design of an interference refractometer. He stated that the uncertainty in the measurement of a length is limited by the accuracy of the index measurement.

$$
\frac{\Delta L}{L} \propto \frac{\Delta n}{n}<10^{-7}
$$

Other studies were performed on the refractive index of Nitrogen [4] and Helium [5]. The value $n-1$ is the difference in the index of refraction for a given gas and the index of a vacuum (1.0).

The Ultraprecision 84 inch Diamond Turning Machine (No. 3) constructed by the Lawrence Livermore Laboratory utilizes a helium pathway medium [6]. The pathway is sealed with an oil seal and a helium regulator is used to maintain an absolute pressure of $0.1 \mathrm{~mm}$. Helium was chosen because for a given change in pressure and temperature, it has only $10 \%$ the effect on the refractive index when compared to air. The pressure change would only result in a change of $0.003 \mathrm{ppm}(\Delta \mathrm{L} / \mathrm{L})$. The overall effect is a worst case error of $10 \mathrm{~nm}$.

Calculations of the Refractive Index Three gases were modeled for this scenerio, air, helium, and nitrogen. The variables pressure and temperature were included in the calculations of all three gases, while humidity and $\mathrm{CO}_{2}$ content were included in the calculations of air only. The variations are chosen to model potential changes in the atmosphere in the DTM laboratory, not actual measured results, and are the same for all three gases modeled. The index of refraction $\Delta$ is the inaccuracy in the measured length $(\Delta n / n)$.

The data in Table 1 illustrate the effects of the four variables on the calculation of the refractive index of air. For each calculation of a variable range, the three other variables were held constant.

The value for the pressure variation was estimated by the National Weather Service for a difference over a 15 minute period. The temperature range was limited to $\pm 0.06^{\circ} \mathrm{C}$, as the room is designed to be controlled to that range. The vapor pressure was chosen for a $45-55 \%$ range in the relative humidity. The $\mathrm{CO}_{2}$ content (300-1200) is a common range used for standard variations in air.

It is generally accepted that helium exhibits about $10 \%$ of the change in index of reflection as does air. Using the formulas presented by Mansfield and Peck [5], the results for this model are presented in Table 2. 


\begin{tabular}{|l|c|c|c|}
\hline Variable & Low & High & $\Delta$ \\
\hline Pressure (Pa) & 100988 & 101015 & 27 \\
Index (n-1) & .000270381 & .000270453 & $.072 \mathrm{ppm}$ \\
\hline Temperature $\left({ }^{\circ} \mathrm{C}\right)$ & 19.94 & 20.06 & 0.12 \\
Index (n-1) & .000270473 & .000270362 & $0.111 \mathrm{ppm}$ \\
\hline $\mathrm{H}_{2} \mathrm{O}$ Pressure (Pa) & 1080 & 1032 & 48 \\
Index (n-1) & .000270468 & .000270367 & $.101 \mathrm{ppm}$ \\
\hline $\mathrm{CO}_{2}$ Content (ppm) & 300 & 1200 & 900 \\
Index (n-1) & .000270417 & .000270549 & $.132 \mathrm{ppm}$ \\
\hline
\end{tabular}

Table 1: Calculated Effects on the Index of Refraction of Air

\begin{tabular}{|l|c|l|c|}
\hline Variable & Low & \multicolumn{1}{|c|}{ High } & $\Delta$ \\
\hline Pressure (atm) & .9998688 & 1.0001312 & .0002624 \\
Index (N-1) & .000032569 & .000032578 & $.009 \mathrm{ppm}$ \\
\hline Temperature $\left({ }^{\circ} \mathrm{C}\right)$ & 19.94 & -20.06 & 0.12 \\
Index (N-1) & .000032567 & .000032554 & $0.013 \mathrm{ppm}$ \\
\hline
\end{tabular}

Table 2: Calculated Effects on the Index of Refraction of Helium 


\begin{tabular}{|l|c|c|c|}
\hline Variable & Low & High & $\Delta$ \\
\hline Pressure (atm) & .9998688 & 1.0001312 & .0002624 \\
Index (N-1) & .000278602 & .000278675 & $.073 \mathrm{ppm}$ \\
\hline Temperature $\left(^{\circ} \mathrm{C}\right)$ & 19.94 & 20.06 & 0.12 \\
Index (N-1) & .000278695 & .000278581 & $0.114 \mathrm{ppm}$ \\
\hline
\end{tabular}

Table 3: Calculated Effects on the Index of Refraction of Nitrogen

The calculations for nitrogen were performed for two reasons. They were used to compare with the air effects from the Edlén Equation, and also to see if a controlled environment of dry nitrogen provided any benefits in laser accuracy. Table 3 presents the results.

Conclusions With the results for air, the four variables sum to a total error of $.416 \mathrm{ppm}$, and this multiplied by the $0.8 \mathrm{~m}$ laser path (.4m each for send/return), a $330 \mathrm{~nm}$ variation results.

The results from the helium model illustrate the benefits from using this gas, with only $.022 \mathrm{ppm}$ variation, or $17 \mathrm{~nm}$ error from the $0.8 \mathrm{~m}$ laser path. While this error is acceptable for the DTM, it could involve extensive modifications.

The nitrogen results support the Edlén Equation calculations, with almost the same variations for pressure and temperature. This follows, as air is $78 \%$ nitrogen. The advantage from this gas medium would be a combined variation of only $.187 \mathrm{ppm}$ or $150 \mathrm{~nm}$ for the $0.8 \mathrm{~m}$ path length. This is still too large for the control restraints, but is greatly improved over the results for air.

One solution to the wavelength correction is to collect the pertinent information in the control computer and update the wavelength calculation at regular intervals. Measurements of the changes in pressure and humidity will be made in the DTM room and predictions of the maximum wavelength error will be made. If necessary, sensors will be integrated into the controller to reduce the effect of environmental changes. If the results of further study show that even this solution is unacceptable, a helium path could be constructed.

\subsubsection{Control Algorithm Development}

The present controller software presents an ideal test bed for the development of higher order control algorithms. Structured programming techniques have isolated into a few small modules the time critical code that would have to be modified to implement another control scheme. Control algorithms for the $X$ and $Z$ axes are of course independent and thus need not be identical. The real-time performance monitoring and data collection 


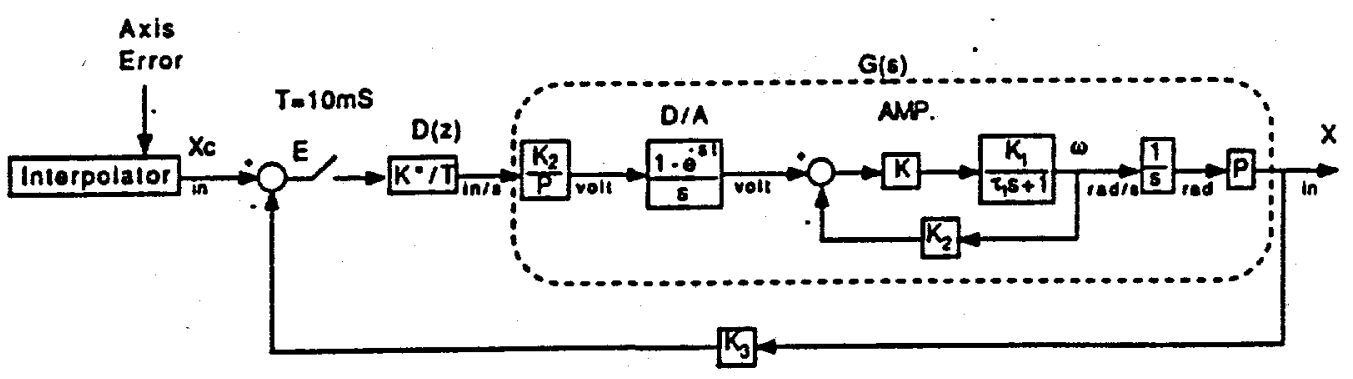

$K=$ Amplifier gain
$K^{*}=$ Compensalor gain $\left(K^{*} / T\right)$
$K_{1}=5.7364$ (Rad/(Sec-Volt))
$K_{2}=$ Velocity feedback gain $(=.40107$ VolvRad/Sec))
$K_{3}=$ Position (eedback gain $(=1.0)$
$\eta_{1}=0.01156$ Rad-Sec
$P=.03135352$ in/rad (pilch)
$=$

Figure 2: Diamond Turning Machine Controller - Block Diagram

capabilities of the control programs provide a good basis for experimentation. A more realistic implementation of the simulated feedback mode of operation will also aid in the coding and testing of future control systems. Providing user access to the simulated single channel digital inputs will allow full testing of the fault detection logic.

System Model The technology used to manufacture diamond turned parts is based on the premise that the path traced out by a numerically controlled tool combined with the shape of the cutting edge corresponds exactly to the surface created in a workpiece. The basis of this technology is the accurate control of the tool path during a cutting process. The control of the $Z$ axis slide on the Pneumo DTM has been analyzed and the system has been modeled to predict the performance characteristics of the slide.

A block diagram is shown in Figure 2 illustrating the slide system with a model of a digital controller, a dc motor, with tach feedback and laser interferometer position feedback. Example time responses of the modeled system to step inputs of $2.5 \mu \mathrm{m}$, and $25 \mu \mathrm{m}$ are given as well as the measured step response of the Z-slide in Figure 3. In comparing the time response of the modeled and the actual system, the percent difference in rise time to these step inputs is less than $10 \%$ verifying the form of the modelled system. For this test, a feedrate of $0.12 \mathrm{~mm} / \mathrm{sec}(0.005 \mathrm{in} / \mathrm{sec})$ was used, and the system was modeled using Advanced Continuous Simulation Language (ACSL). The step inputs were modeled as a series of steps separated by the $10 \mathrm{~ms}$ update time, which is typical of a commercial controller.

The electro-mechanical system model illustrated here is being used to develop an improved 


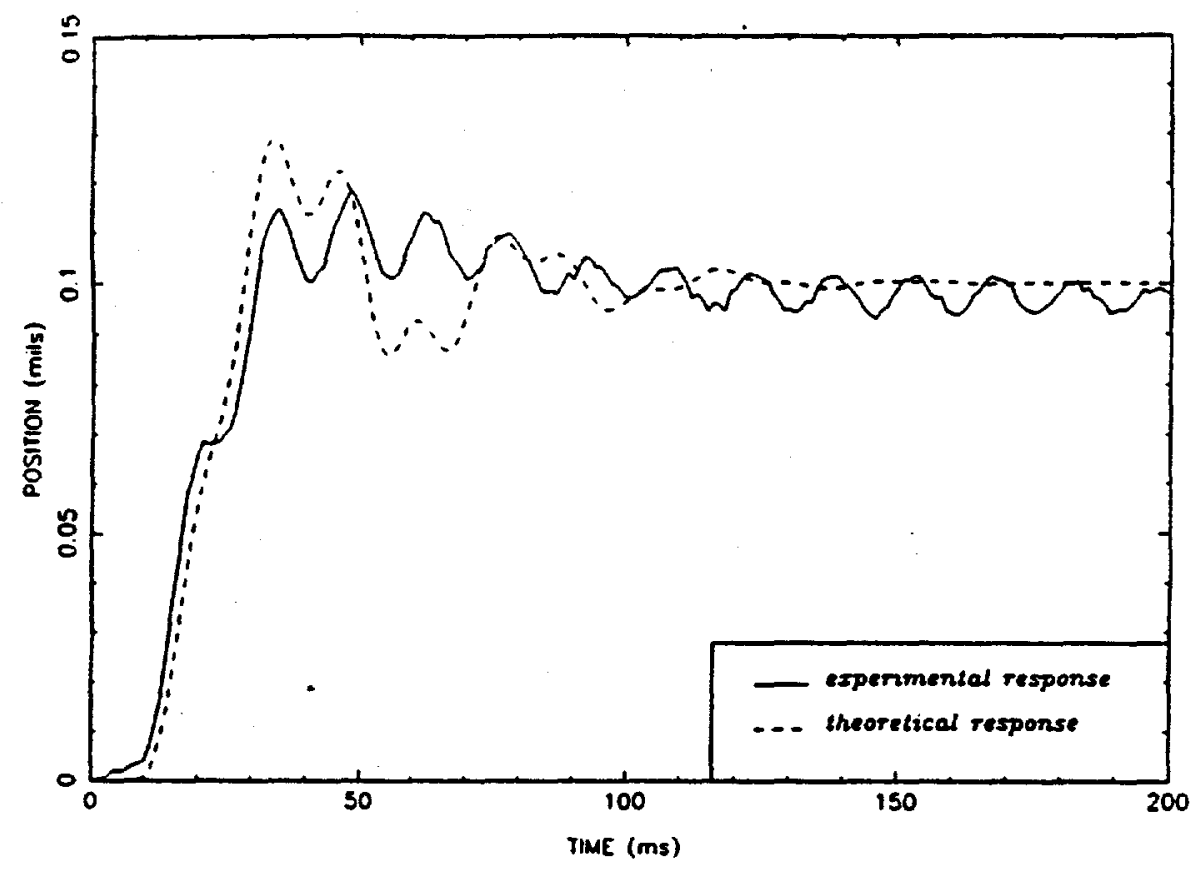

Figure 3(a): DTM Response to $2.5 \mu m(100 \mu$ in $)$ Step At A Feedrate of $0.12 \mathrm{~mm} / \mathrm{sec}$

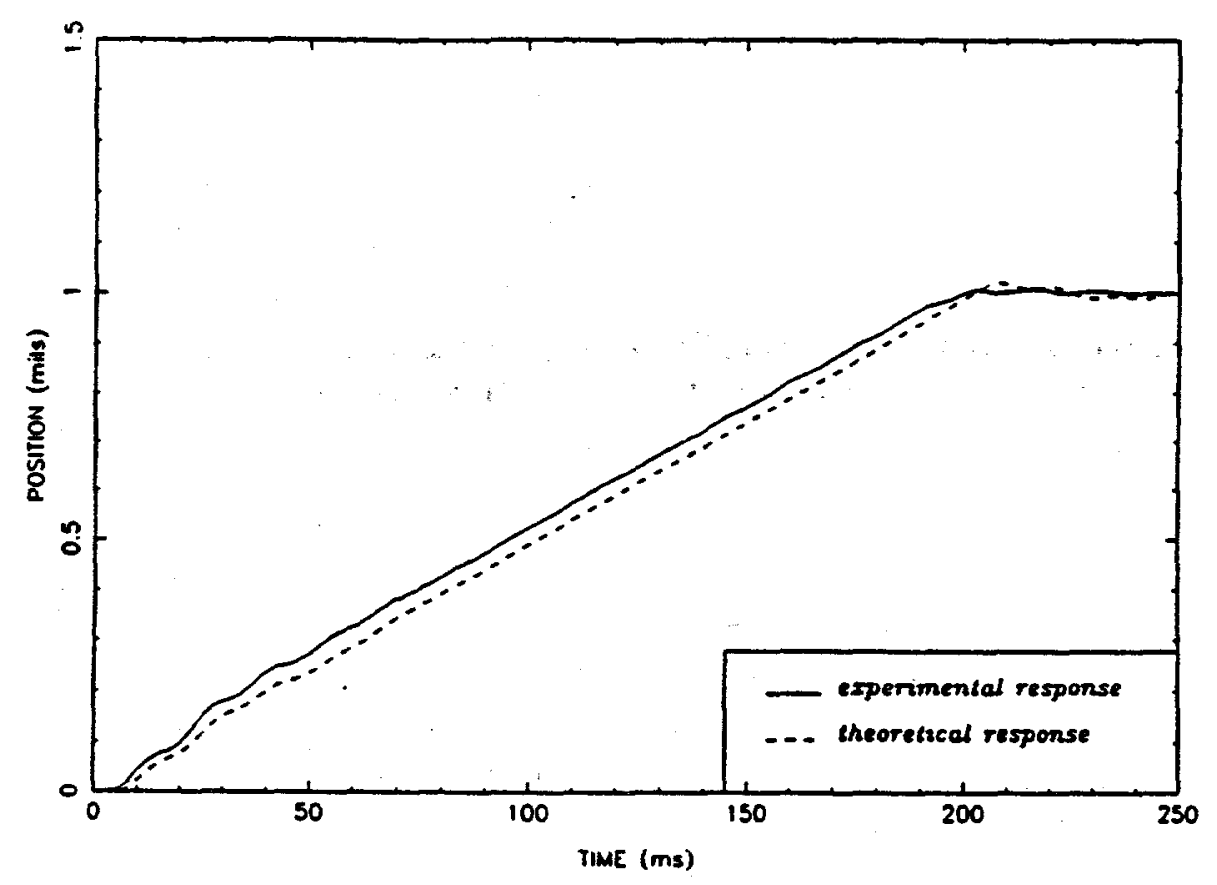

Figure 3(b): DTM Response to $25 \mu m(1000 \mu$ in) Step At A Feedrate of $0.12 \mathrm{~mm} / \mathrm{sec}$ 
digital controller for the DTM with an update time of $1 \mathrm{~ms}$, allowing faster start-up response and better disturbance rejection. The current digital control algorithm is an integral type with a single gain. The software environment described here will allow the development of a digital control algorithm of a first or second order type to improve the time response characteristics of the system.

\subsection{FUTURE WORK}

The present configuration suffers from an I/O bottleneck through the PC-AT bus. Operation speed is limited by the communication interface between the 68020 controller software and the $80286 \mathrm{I} / \mathrm{O}$ interface software. Response time of the controller is thus limited by the response time of the 80286 to I/O requests by the 68020 .

The processing power of the 68020 is considerable, though not unlimited. With the addition of geometric error compensation and more sophisticated control algorithms, the processor will eventually be overloaded. In particular, the Fast Tool Servo cannot be controlled by the 68020 in addition to the $\mathrm{X}$ and $\mathrm{Z}$ axes without increasing the update cycle interval.

The 80186/TMS320C25 dual digital signal processor architecture (i.e., $\mathrm{H}^{2} \mathrm{ART}$ ) is a potential candidate for furthering the development of the DTM controller. The very high speed processing capability and double buffered memory organization provide a means of controlling each axis with a different processor. The PC-bus I/O bottleneck would be eliminated by providing each processor with the necessary interfaces to the DTM. Thus the 80286 would no longer be directly involved in the real-time control of the cutting operations; it would serve only as the user interface processor and program development system. Communication with the control processors would be achieved via memory to memory transfers by the 80186 DSP control processor. This communication would primarily be of a non-time critical, informative nature.

The principle problems associated with porting the control software to the TMS320C25 environment are the absence of hardware floating point operations and the relatively small amount of memory available to each processor. The linear interpolation and basic proportional control algorithms have been ported to the DSP environment. Experiments are currently underway to measure the relative speed of the DSP and 68020 architectures and evaluate the consequences of using fixed point or software floating point calculations.

The DSP architecture has been implemented as a wire-wrapped prototype and a printed circuit design is in progress. The printed circuit version will include expanded TMS320C25 memory. Each processor board is expected to have the full $64 \mathrm{~K}$ words of program memory and $64 \mathrm{~K}$ words of data memory directly addressable by the TMS320C25. The processors will also have their own digital and analog I/O interface boards.

The next generation DSP processor, the TMS320C30, is also being evaluated as a candidate for the implementation of future control systems. It's major features include 40-bit 
floating point operations, concurrent DMA, $16 \mathrm{M}$ word address space, $60 \mathrm{n} \mathrm{sec}$ instruction cycle time, and two independent counter/timers. Processor throughput of 33M flops is claimed if the on-chip multiplier and ALU are operated in parallel. Program development is supported by both a macro.assembler/linker and a C compiler. A software simulator for the entire instruction set is also available. The cycle counting feature of the simulator allows algorithm implementations to be accurately timed prior to their integration into a complete system. Experimental versions of these software tools have been obtained from Texas Instruments.

\section{References}

[1] Taylor, Lauren W. "Hardware Configuration of the Diamond Turning Machine Controller," Precision Engineering Center Technical Report, January 1989.

[2] Edlén, B., "The Refractive Index of Air," Metrologia, Vol. 2, No. 2, 1966, pp. 71-80.

[3] Schellekens, P., "Design and Results of a New Interference Refractometer Based on a Commercially Available Laser interferometer," Annals of the CIRP, Vol. 35, No. 1, 1986, pp. 387-391.

[4] Peck, E.R. and Khanna, B.N., "Dispersion of Nitrogen," Journal of the Optical Society of America, Vol. 56, No. 8, August 1966, pp. 1059-1063.

[5] Mansfield, C.R. and Peck, E.R., "Dispersion of Helium," Journal of the Optical Society of America, Vol. 59, No. 2, February 1969, pp. 199-204.

[6] Bryan, J.B., "Design and Construction of an Ultraprecision 84 inch Diamond Turning Machine," Precision Engineering, Vol.1, No.1, 1979, pp. 13-17

[7] Baumeister, T, Avallone, E.A., and Baumeister, T. III, Mark's Standard Handbook for Mechanical Engineers, $8^{\text {th }}$ edition, McGraw-Hill, 1978. 


\section{APPENDIX - CONTROLLER KEYBOARD COMMANDS}

All controller functions are initiated with the following keyboard commands. The axes position and machine error displays are maintained during command entry. Any command can be abbreviated with its shortest unambiguous leading character sequence. The command line can be edited with a a variety of single keystroke functions. The 16 most recent commands entered by the user can be recalled for editing and re-execution. Both insert and overwrite data entry modes are available.

1.) home $\{$ " $x " \mid$ "z" $\}$

The home command moves both axes to their predefined home positions $(x=1.0, z=5.0)$. The optional arguments specify the desired axes to "home". If no arguments are specified, the $z$ axis is moved to its home position follored by the $x$ axis.

2.) jog "slog" | "s" | "modium" | "m" | "fast" | "f" | <speed>

The jog command enables operation of the hand held manual jogger at the specified axis velocity.

$$
\begin{aligned}
& \text { "slov" | "s" } \Rightarrow .023 \mathrm{in} / \mathrm{min} \\
& \text { "medium" | "m" } \Rightarrow 10.0 \mathrm{in} / \mathrm{min} \\
& \text { "fast" | "f" } \Rightarrow 20.0 \mathrm{in} / \mathrm{min} \\
& \text { 〈speed> } \quad \Rightarrow \text { user defined jog relocity in in/min }
\end{aligned}
$$

Axis velocity can be changed vithout exiting jogger mode with the cursor and control keys as follors. The jogger is disabled if any other keystroke is entered.

$\begin{array}{lll}\text { up_arror } & = \\ \text { dorn_arror } & \text { Increase speed } 1.0 \mathrm{in} / \mathrm{min} \\ \text { page_up } & =>\text { decrease speed } 1.0 \mathrm{in} / \mathrm{min} \\ \text { page_dorn } & =\Rightarrow \text { decrease speed } 5.0 \mathrm{in} / \mathrm{min} \\ \text { right_arror } & =\Rightarrow \text { increase speed } 0.1 \mathrm{in} / \mathrm{min} \\ \text { left_arror } & =\Rightarrow \text { decrease speed } 0.1 \mathrm{in} / \mathrm{min} \\ \text { ctrl-right_arror } & =>\text { increase speed } 0.01 \mathrm{in} / \mathrm{min} \\ \text { ctrl-left_arror } & =>\text { decrease speed } 0.01 \mathrm{in} / \mathrm{min} \\ \text { home } & \Rightarrow \text { return to original } \mathrm{relocity}\end{array}$




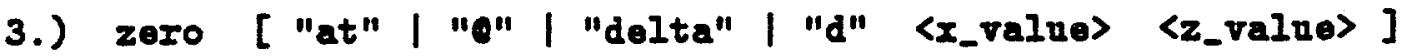
setx [ "at" | "e" | "delta" | "d" <x_value> ] .
setz [ "at" | "a" | "delta" | "d" 〈z_value〉]

Defines the relative coordinate system on both axes (zero), the $x$ axis (setx) or the $z$ axis (setz). If optional arguments are omitted, relative zero is set equal to the current absolute axes position. The relative coordinate system can be defined in either of tro rays.

In terms of absolute axes coordinates, "at" | "Q", $\operatorname{rel}(0,0)=\left(\left\langle x_{\text {_value }}\right\rangle,\left\langle z_{-}\right.\right.$value $\left.\rangle\right)$

In terms of the current relative coordinates, "delta" | "d", $\operatorname{rel}(0,0)=\operatorname{rel}(0,0)+\left(\left\langle x_{-}\right.\right.$value,$\left.\left\langle z_{\text {_ralne }}\right\rangle\right)$

\section{4.) spin 〈rpms〉 [ "cr" | "cct"]}

The spin command starts the spindle at the specified speed in revolutions per minute. The motor is ramped up to the desired relocity. The default direction is clockrise: Counter-clockrise rotation is obtained by specifying "ccr".

5.) Iun <control_program \{ <argument> $\}$

The run command executes a 68020 control program. All coordinates are expressed in the relative coordinate system and all feedrates are in inches per minute. Unless otherwise specified, the maximum feedrate alloved during cutting is 5 in/min and the maximn depth of cut is 1 mil. The run command vill not execute a program unless the machine home has been found, the relative coordinate system defined, and the spindle started. It is assumed that the relative zero position is defined at the center of the spindle and the surface of the vorkpiece. The consistency of the direction of spindle rotation with all $x$ axis coordinates is also 
checked.

The folloring control programs are currently available,

"crtrel" $x \geq f$

Hoves the axes to position $(x, z)$ at feedrate $f$.

"cutrel2" $x 1 \quad z 1 \quad f 1 \quad x 2 \quad z 2 \quad f 2$

Moves the axes to position $(x 1, z 1)$ at feedrate 11 , follored by position $(x 2, z 2)$ at feedrate $f 2$.

"ctrlv" $\quad x \quad 2 \quad f$

Same as cutrel, except that the control algorithm filters the input rith a 10 sample running average of input laser positions.

"ctrlv2" $\quad x 1 \quad 21 \quad f 1 \quad x 2 \quad 22 \quad \pm 2$

Same as cutrel2, except that the control algorithm filters the input rith a 10 sample running average of input laser positions.

"box" xc zC z1 fz $x 1$ fz [ fo ]

Hoves the axes in a rectangular pattern equivalent to, cutrel xc zc fo

cutrel $x \mathrm{z} 1 \mathrm{fz}$

cutrel $x 1$ z1 $f x$

cutrel $x 1$ zc $f 0$

The box program uses the same input filter as ctrlv and ctrlv2. The optional feedrate fo defaults to $4 \mathrm{in} / \mathrm{min}$. Maximum valne of $f z$ is 1 in/min. Haximum value of $f x$ is $5 \mathrm{in} / \mathrm{min}$.

"mstep" part_file

Hoves the axes to the positions as described in the specified input file. Jses the same input filter as ctrlv and ctrlv2. 411 feedrate, spindle and coordinate restrictions are tested prior to starting execution of the first move.

6.) Miscellaneous commands. 


$\begin{array}{ll}\text { nospin } & \text { Stops the spindle. } \\ \text { chip } & \text { Turns the chip extractor on. } \\ \text { nochip } & \text { Turns the chip extractor off. } \\ \text { Inbe } & \text { Turns the coolant sprayer on. } \\ \text { nolube } & \text { Turns the coolant sprayer off. } \\ \text { clear } & \text { Clears the error, axis velocity and user } \\ \text { display rindors. } & \begin{array}{l}\text { Temporary exit to the operating } \\ \text { system. The machine is shntdorn, but } \\ \text { controller parameters (e.g. relative } \\ \text { coordinate system) are retained. }\end{array} \\ \text { quit } & \text { Terminates execution of the DTy controller. }\end{array}$




\title{
10 A HETEROGENEOUS HIERARCHICAL ARCHITECTURE FOR REAL-TIME COMPUTING
}

\author{
Denise A. Skroch \\ Graduate Student \\ Robert J. Fornaro \\ Associate Professor, CSE
}

The need for high-speed data acquisition and control algorithms has prompted continued research in the area of multiprocessor systems and related programming techniques. The result presented here is a unique hardware and software architecture for high-speed real-time computer systems. The implementation of a prototype of this architecture has required the integration of architecture, operating systems and programming languages into a cohesive unit. This report describes a Heterogeneous Hierarchial Architecture for Real-Time $\left(H^{2} A R T\right)$ and system software for program loading and interprocessor communication.

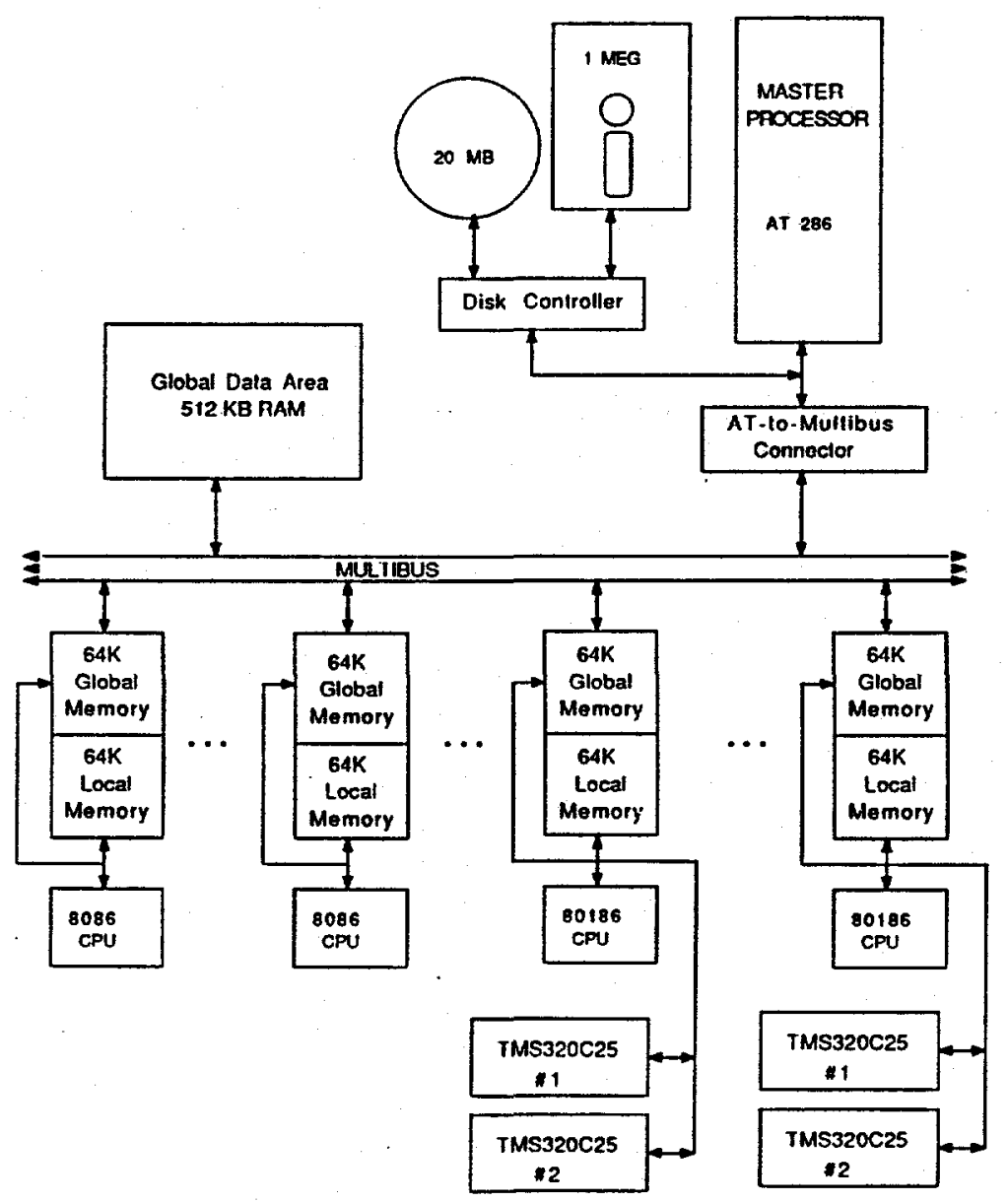




\subsection{INTRODUCTION}

The primary goal of the computer science group within the Center has been to contribute to the solution of computational problems arising from control and signal processing needs of Precision Engineering applications. The interaction necessary to define these problems and iterate on solutions has, in turn, stimulated interest in several topics which have been separately identified by others as legitimately in need of research from a computer science perspective. The work reported in this section, along with results described in Sections 9 and 11, represent a blend of real-time programming and numerical control machining. Recently, Stanbovic [1] has described the state of the art in real-time systems and outlined a wide range of research problems. Grossman [2] has presented the history of numerical control programming and suggests that speed, precision and function in numerical control machining could all be improved by developing and integrating better computer science tools.

The primary concern in real-time programming is to guarantee that results of a computation are available to meet timing requirements. Fast computing is distinguished from real-time computing in that speed alone does not necessarily guarantee timing. One approach is for real-time systems to periodically re-examine deadlines and dynamically adjust scheduling of computations to meet these deadlines. This is an attempt to achieve high utilization for processor along with guaranteed timing response. Algorithms to accomplish this efficiently are very difficult and the subject of much research.

Another approach, as suggested by Wirth [3], is to assign only a single software task to each processor. This is necessary if a subset of sensor monitoring and computational requirements fully utilize a processor. In this case, multiple processors are required to guarantee real-time constraints. However, suitable hardware architectures and software techniques for multiprocessor systems are also active research areas.

Requirements for precision engineering applications have steered our research efforts toward process-per-processor systems. The first objective of this work has been to identify a computing engine with the performance and flexibility required for such applications. A range of commercially available systems and components have been evaluated and none have been adequate. Thus, the decision was made to build a prototype architecture better suited to real-time requirements. A Heterogenous Hierarchical Architecture for Real-Time $\left(\mathrm{H}^{2} \mathrm{ART}\right.$ ) has been developed. This system utilizes multiple processors of different types arranged in a hierarchy of busses sharing address and memory modules. The primary software paradigm for this system is the process-per-processor model.

The details of this architecture and preliminary software to enable its use are presented below. 


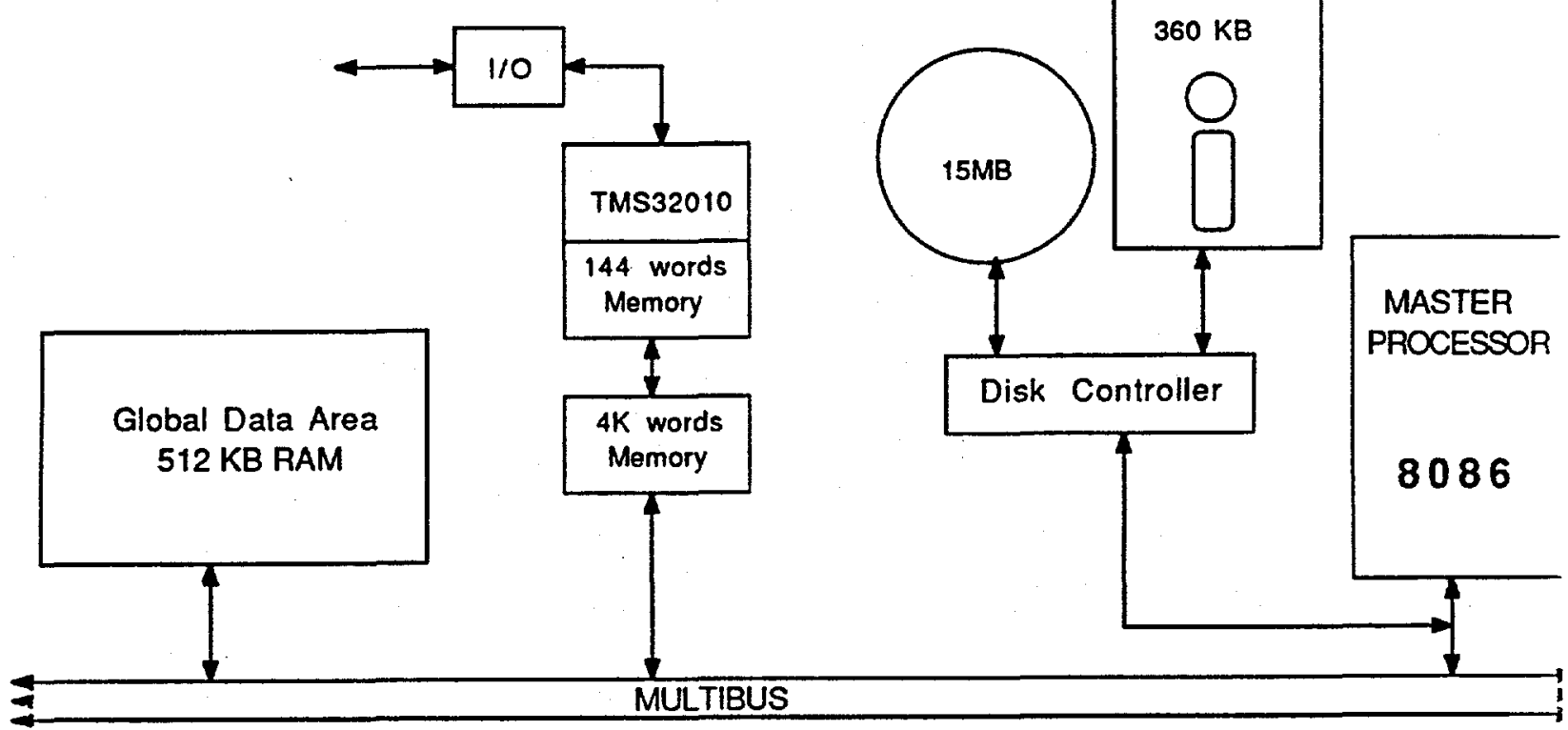

Figure 1: TMS32010 Multiprocessor System

\subsection{HARDWARE ARCHITECTURE}

The original multiprocessor system that was investigated is composed of a single INTEL $8086 / 8087$ processor acting as a master to a TMS32010 slave processor. Though this system had limited multiprocessor capabilities, it offered some insight into the development of later systems. The architectural structure of this system is shown in Figure 1. Details of this architecture and its applications have been previously reported and can be found in [4], [5], and [6]. In order to better examine multiprocessor systems, it was necessary to develop a system with more parallel capability than the TMS32010 system.

Hardware architectural development continued with a Homogenous Architecture for RealTime (HART). This architecture makes use of off-the-shelf hardware and offers an excellent testbed for both system and application software. HART is a multibus based architecture based on a shared memory model. The system is currently configured with one master processor and four slave processors, though the system can be configured to support up to eight slave processors. The HART master processor is an Intel 8086 processor running MSDOS. Each processing node consists of a single Intel 8086 processor and can be configured with or without an Intel 8087 math co-processor. Address mapping is used to configure local and global memory. An individual processing node has $64 \mathrm{~K}$ of global memory visible to the rest of the system and $64 \mathrm{~K}$ of local memory. Program execution and data storage can make use of either memory. The global memory of each processing node along with 


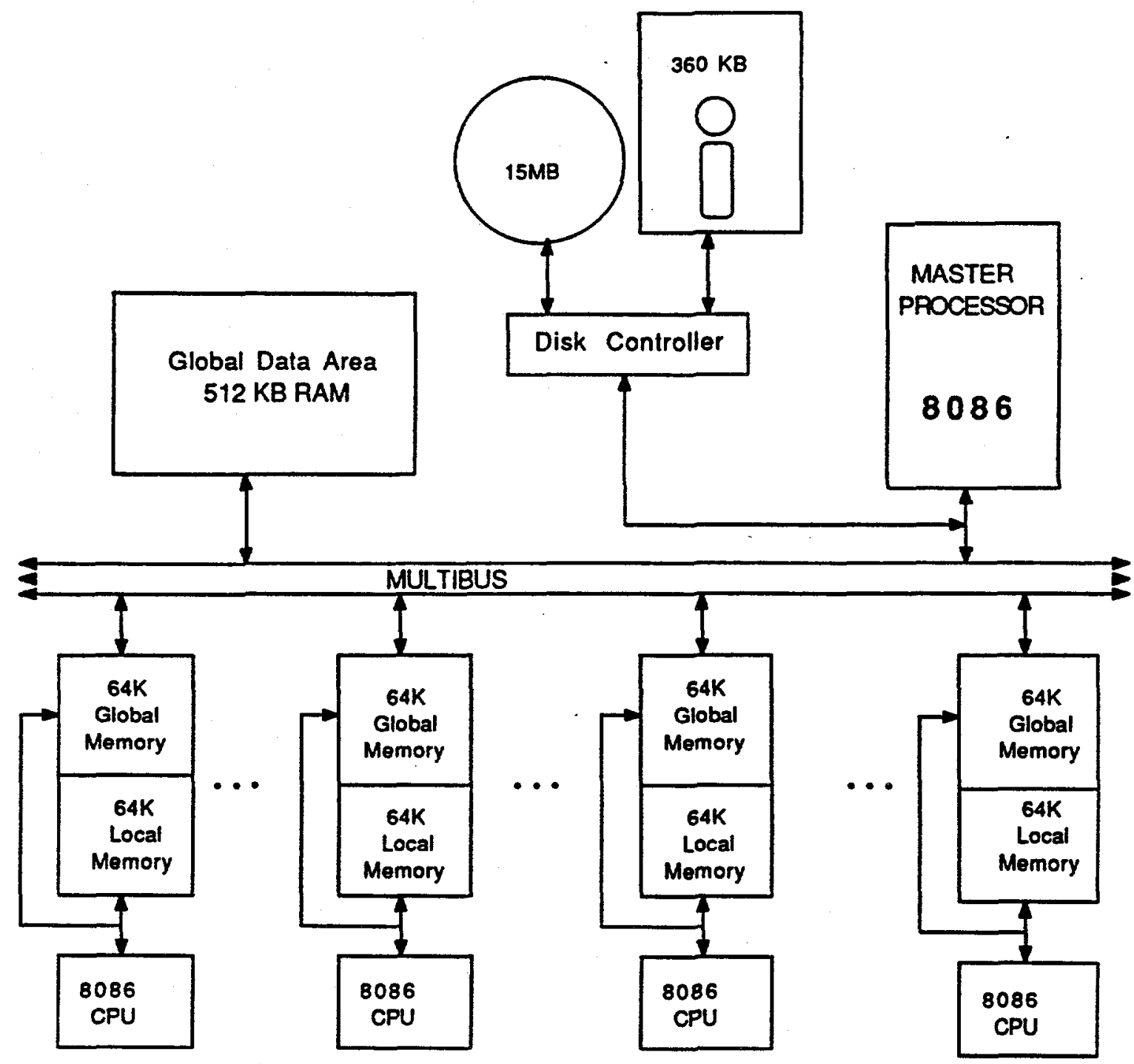

Figure 2: The HART Multiprocessor Architecture

512K global system memory are used for system communication and data transfer. For system configuration details, see Figure 2.

The performance characteristics of several application algorithms were examined on the HART multiprocessor system. Among these applications were fast fourier transforms, matrix manipulations, control filters and recursive least squares. The details of these experiments have been previously reported [7]. One of the main goals of these experiments was to establish the computational power of HART. Unfortunately, very few of the implementations succeeded in producing theoretically expected results. The major contributing factor in this failure was the inter-processor communication overhead introduced in the parallel implementation. However, this failure did not outweigh the advantages of parallel computation. Therefore, the goal of the next development phase was to decrease 
the communication cost within the multiprocessor system. This can be accomplished by overlapping the communication and computation within the system.

To improve communication and speed, HART has been modified to a Hierarchical Heterogenous Architecture for Real-Time $\left(H^{2} \mathrm{ART}\right)$. Like HART, $\mathrm{H}^{2} \mathrm{ART}$ is a bus-based architecture with shared memory, configurable up to eight processing nodes. An architectural overview is presented in Figure 3. The $\mathrm{H}^{2} \mathrm{ART}$ processing node Figure 4, consists of an Intel 80186 control processor and two TMS320C25 digital signal processors (DSPs). The DSPs have dual-ported, double-buffered data memories allowing simultaneous computation and data transfer within each processing node. In the HART system, communication required that all processing on a node cease for the duration of the inter processor communication. On a $\mathrm{H}^{2} \mathrm{ART}$ processing node, the double data memories permit the DSP to continue processing from one memory while data is simultaneously transferred to or from the other memory.

Each DSP's program memory and two data memories are mapped into the address space of the 80186 . The 80186 also has $64 \mathrm{~K}$ of global memory and $64 \mathrm{~K}$ of local memory. The 80186 processor is considered a control node, because it determines whether the DSP has access to its program and data memories. This is accomplished by writing to memory mapped locations on the $\mathbf{8 0 1 8 6}$ which correspond to ports on the DSPs. Communication between DSPs on a processing node, must also go through the control node. Within a processing node, the two DSPs communicate by way of direct memory access (DMA) transfers also controlled by the 80186. This incorporates the fastest method of communication, that of a simple address change. The dual data memory characteristic of the DSP allows for data transfer and computation to occur simultaneously, thus increasing the amount of parallel activity in the system. A DSP has no means of directly accessing the multibus, therefore all communication with the rest of the system must go through its control processor ( the 80186).

Currently, I/O capability (digital and analog) is being added to $\mathrm{H}^{2} \mathrm{ART}$ node and testing is underway to verify real-time performance of the system. The design of this $1 / 0$ allows for data to be collected at the DSP level and buffered up to the control node level and then distributed throughout the system as deemed necessary by the application. Thus, real-time capability is increased by separating the communication and I/O. Computation can take place at the $\mathrm{I} / \mathrm{O}$ site.

\section{$10.3 \quad$ SYSTEM SOFTWARE}

The host environment for the $\mathrm{H}^{2} \mathrm{ART}$ system is an AT/286. Software development, both system software and applications software, is written in a combination of Turbo ' $C$ ' and Assembly language under MSDOS version 3.21.

A multiprocessor control program, MPC, is the user interface to the $\mathrm{H}^{2} \mathrm{ART}$ multiproces- 


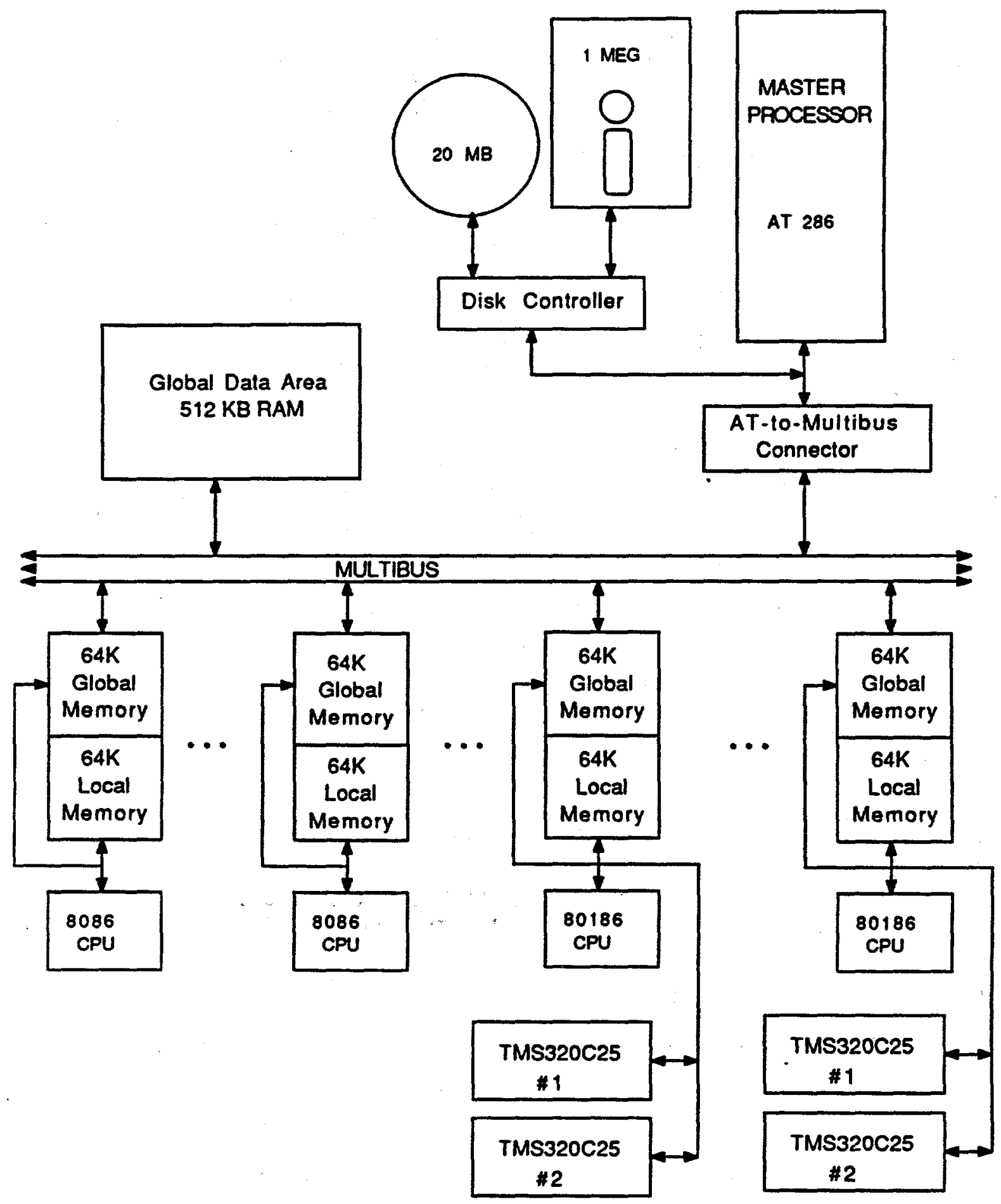

Figure 3: The $\mathrm{H}^{2}$ ART Multiprocessor Architecture 


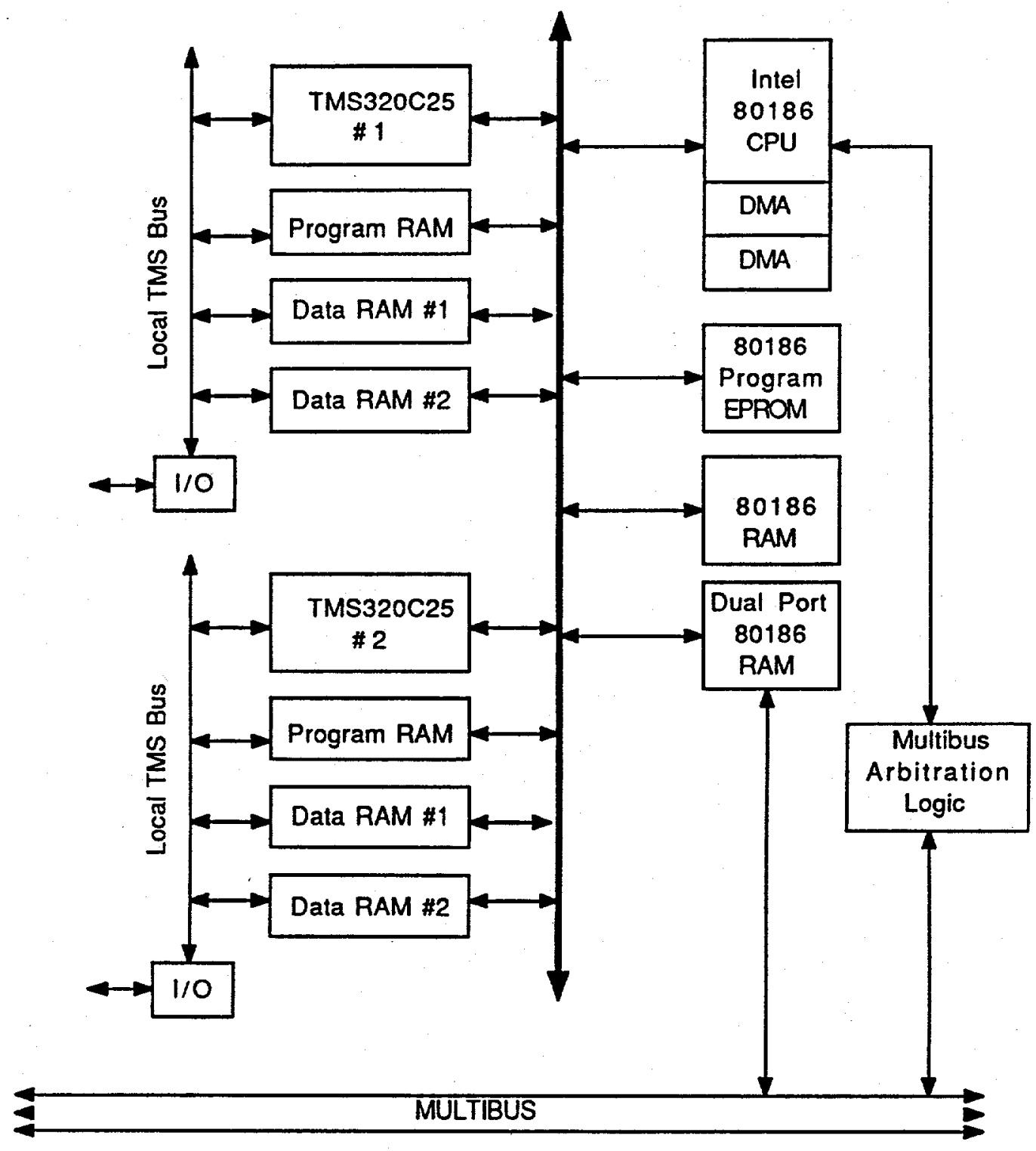

Figure 4: $\mathrm{A} \mathrm{H}^{2} \mathrm{ART}$ Multiprocessor Processing Node 
sor environment from the AT. This allows the user to manipulate the multiprocessor by providing basic execution functions, system monitoring functions and simple debugging tools. MPC provides utilities for system initialization and configuration. Processors are installed and removed through the functions instal and remove respectively. The function config allows the user to indicate the type of processor. The system configuration is stored by the system and only requires change in the event of a hardware change. The hardware connection between the AT and the multiprocessor must be explicitly initialized by the software before communication across the connection may begin. By default, this takes place when MPC is invoked but it can also be done by the user through the function imbus. The multibus itself can be reset through a hardware switch or through the MPC function reset. Finally, MPC allows the user to initialize semaphores in global memory by invoking the function isem with the name of the semaphore and the initial value.

Once an algorithm has been decomposed, coded and compiled, it must be downloaded to the individual processors. MPC contains a load function for this purpose. Due to the hierarchical nature of $\mathrm{H}^{2} \mathrm{ART}$, downloading is a two step process. Because the DSP program memory is not visible to the AT, the 80186 control processor and DSP code must first be downloaded from the AT to the control processor on each processing node. Is the responsibility of the control processor to place the DSP code in the appropriate program memory. Each control processor starts a DSP by giving it control of its program memory.

Execution in the multiprocessor is initiated through the start function which generates a start-up interrupt seen by all the control processors on the multibus. It is the responsibility of the control processor on each processing node to initiate execution of the DSPs on that node.

System monitoring is also available through MPC. A processor can be running, terminated or suspended awaiting data or synchronization information. The function psw allows the user to examine the state of the processor and if the processors execution is suspended, it also indicates why the processor is waiting. multibus memory can be dumped and modified through the dump and modify utilities. This provides a limited amount of debugging from the AT.

Some miscellaneous functions available through MPC are exec, wait and help. Exec allows the user to execution a program on the AT from within the MPC environment. Programs often executed include "command.com" for DOS commands, a compiler or an editor. The programs executed, however, are not intended to interact with the multiprocessor environment since the transfer of data across the hardware connection is relatively slow. The function wait, however, does allow the AT to receive messages from the multiprocessor environment for program development purposes. Finally there is a help facility. This provides online help for MPC functions. It presents a brief description of each command and its associated syntax. 


\subsection{APPLICATION SOFTWARE}

Pre-processors and run-time extensions have been developed to aid in application program development along with predefined routines for processor synchronization and data transfer.

The $\mathrm{H}^{2}$ ART system requires process code for individual processes to be written by the user. Presently, the code to be executed on the 80186 control processor is written in Turbo ' $C$ ' and the code to be executed by the DSP is written in TMS320C25 Assembly Language. Recently, a ' $C$ ' cross compiler for the TMS320C25 has become available. This would allow the DSP code to be written in ' $C$ ' relieving the user from the programming details of Assembly Language. The code produced by this compiler is being evaluated to determine its performance (See Section 11 of this report).

Predefined routines for processor synchronization and data transfer are also available to the user. Standard semaphore and message passing capabilities are included in system libraries for use by the programmer. The routines available under MPC are based on those used in the HART system [9], and have been modified to incorporate the low level details of $\mathrm{H}^{2} \mathrm{ART}$.

The process of coding an application for a $\mathrm{H}^{2} \mathrm{ART}$ processing node currently requires several steps. The pseudo code for this application can be found in Figure 5. First the DSP modules are coded in TMS320C25 Assembly Language as shown in Figure 6. This code is then assembled into a hexadecimal format. This hexadecimal file is input to a preprocessor called "tms2 $c$ " which converts the code into ' $C$ ' structures which can be included, via a ' $C$ ' include file, into the ' $C$ ' program written for the 80186 control node. The structure produced for the example file can be found in Figure 7.

To hide low level system details, there are several predefined functions available for use by the programmer. These include functions to load, start and terminate a DSP from the 80186 control processor. For example, to load a DSP, the user calls a function load_dsp with parameters indicating the DSP and the program to be loaded. Sample code written for the 80186 control node is presented in Figure 8 . Line \#1 includes a system file which contains all the definitions for the DSPs. Throughout the code, all variables beginning with '-' are predefined in this file. The include file specified on line \#3, is the file generated by the preprocessor, the same as shown in Figure 7. Sze_resource and rel_resource are predefined functions called by the control processor to obtain and give up control of the DSP resources (i.e., memories). This is necessary because, as mentioned previously, the 80186 is responsible for controlling possession of the DSP memories. Load_tpgm and start_tms are also predefined functions to load a DSP's program memory with the appropriate program and start the DSP, respectively. Finally, the code on lines \#16 through \#17 serve to establish when the DSPs have completed execution. This is currently done through examining a status port by way of the function check_status. This busy waiting loop will no longer be necessary when interrupts are added to the DSP/80186 communica- 


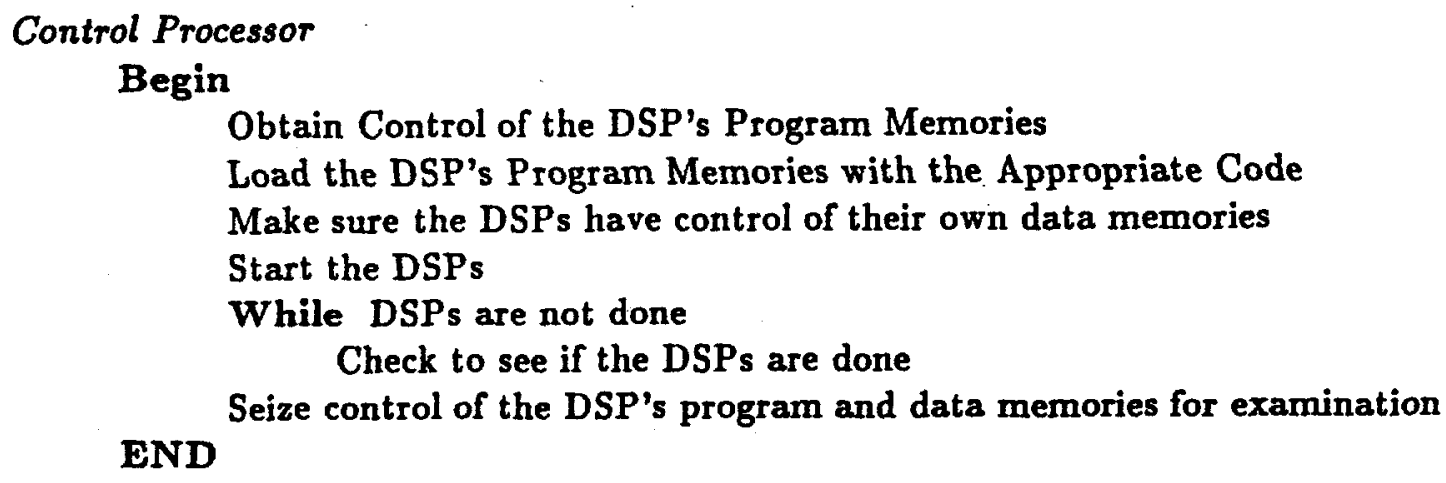

DSP Code

Begin

Indicate Start to the 80186 Control Processor

Fill Data Memory One with Ones

Fill Data Memory Two with Twos

Indicate FInish to the 80186 Control Processor

END

Figure 5: Pseudo Code for the Example Application 
This program runs on a TMS320C25 processor.

It loads the first data memory with one's and the second data memory with two's

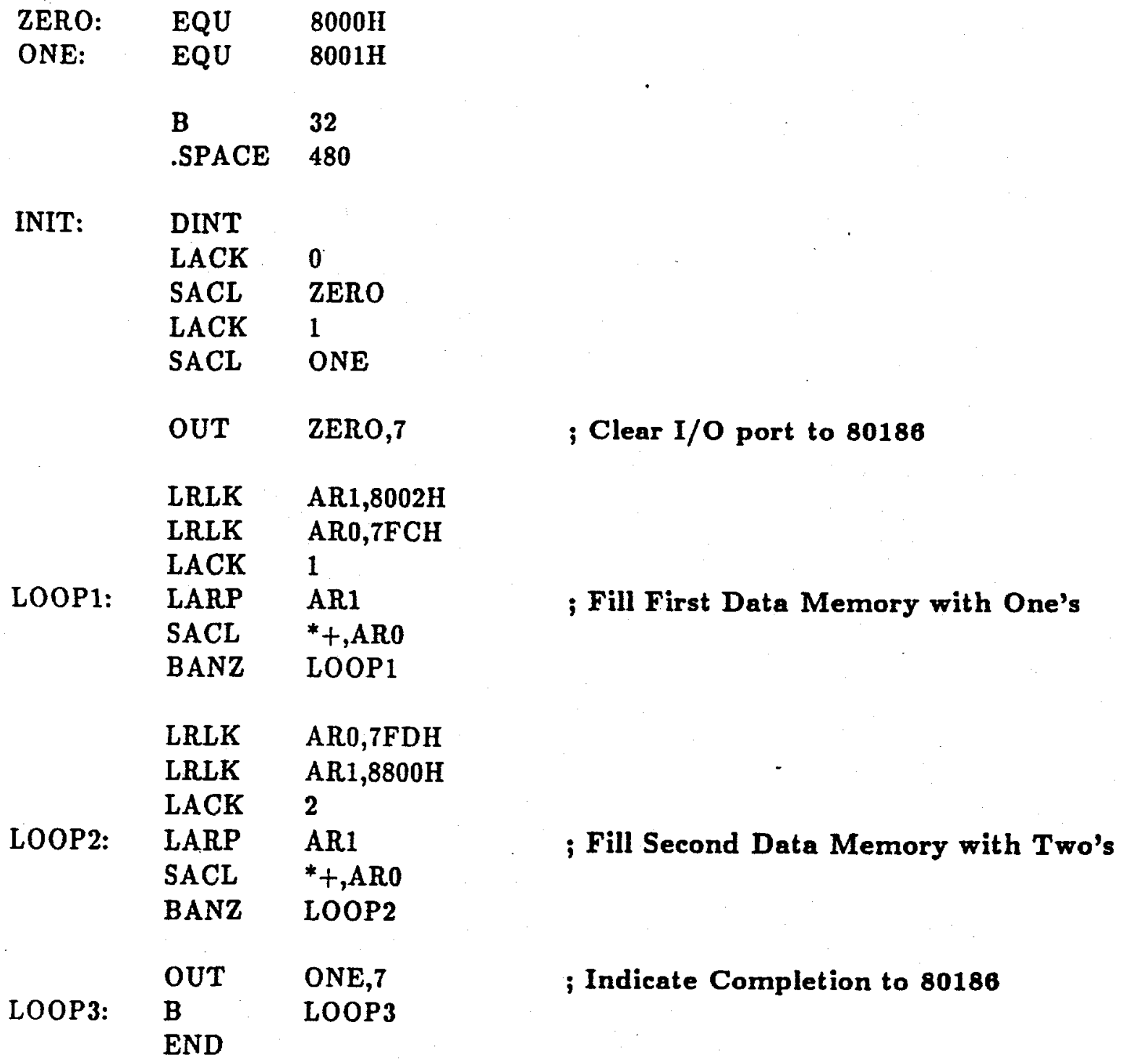

Figure 6: TMS320C25 Assembly Language Example Code 
$1^{*}$

TMS\#0's Program

const int $\_$topgm[ $]=\{$

0xFF80, 0x0020, 0x0000, 0x0000, 0x0000, 0x0000, 0x0000, 0x0000, 0x0000, 0x0000, $0 \times 0000,0 \times 0000,0 \times 0000,0 \times 0000,0 \times 0000,0 \times 0000,0 \times 0000,0 \times 0000,0 \times 0000,0 \times 0000$, $0 \times 0000,0 \times 0000,0 \times 0000,0 \times 0000,0 \times 0000,0 \times 0000,0 \times 0000,0 \times 0000,0 \times 0000,0 \times 0000$, $0 \times 0000,0 \times 0000,0 \times C E 01,0 \times C A 00,0 \times 6000,0 \times C A 01,0 \times 6001,0 \times E 700,0 \times D 100,0 \times 8002$, 0xD000, 0x07FC, 0xCA01, 0x5589, 0x60A8, 0xFB90, 0x002B, 0xD000, 0x07FD, 0xD100, $0 \times 8800,0 \times C A 02,0 \times 5589,0 \times 60$ A8, 0xFB90, 0x0034, 0xE701, 0xFF80, 0x0039, 0x0000\};

$1^{*}$

Size of TMS\#0's program in words

const int _t0pgmsize $=60$;

/*

const int _tlpgm[] =

TMS\#1's Program

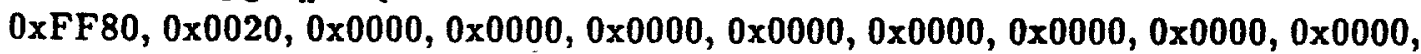
$0 \times 0000,0 \times 0000,0 \times 0000,0 \times 0000,0 \times 0000,0 \times 0000,0 \times 0000,0 \times 0000,0 \times 0000,0 \times 0000$, $0 \times 0000,0 \times 0000,0 \times 0000,0 \times 0000,0 \times 0000,0 \times 0000,0 \times 0000,0 \times 0000,0 \times 0000,0 \times 0000$, $0 \times 0000,0 \times 0000,0 \times C E 01,0 \times C A 00,0 \times 6000,0 \times C A 01,0 \times 6001,0 \times E 700,0 \times D 100,0 \times 8002$, 0xD000, 0x07FC, 0xCA01, 0x5589, 0x60A8, 0xFB90, 0x002B, 0xD000, 0x07FD, 0xD100, $0 \times 8800,0 \times C A 02,0 \times 5589,0 \times 60 A 8,0 \times F B 90,0 \times 0034,0 \times E 701,0 \times F F 80,0 \times 0039,0 \times 0000\}$;

$l^{*}$

const int $-t 1$ pgmsize $=60$;

\section{Size of TMS\#1's Program}

Figure 7: Output of the 'TMS2C' Preprocessor : A 'C' Include File 
tion path. The DSP will simply interrupt the 80186 to indicate completion, allowing the 80186 to do useful work while the DSPs are executing.

Work is being done to simplify the program development procedure. The idea is to totally automate this procedure so that the programmer only indicate the DSP code an the 80186 source code and the 80186 start-up code will take care of the downloading, making it transparent to the user.

\subsection{FUTURE WORK}

The prototype hardware and software described here are operational. Replication of the prototypes in printed circuit form will provide eight to sixteen nodes (sixteen to thirty two dsp's) for further experimentation. Circuit capture and layout are underway. Working boards should be available within several months.

The primary focus of this work in the next year will be the integration of the $H^{2}$ ART multiprocessor into Precision Engineering applications. The first of these, an improved controller for the diamond turning machine, is discussed in Section 9 of this report.

Digital control of the scanning-tunneling microscope with real-time graphics is another candidate application for the $\mathrm{H}^{2} \mathrm{ART}$ multiprocessor (See Section 11 of this report).

Also, algorithms such as fft's, matrix calculations, etc., will be re-examined. These and other related computations will be implemented on the $\mathrm{H}^{2} \mathrm{ART}$ multiprocessor. Performance statistics will be collected and evaluated. 


\section{/*}

TMS definitions

1: \#include <tmsdef.h>

2: \#include <stdio.h> $1 *$

3: \#include "TMS.h"

TMS 320 programs ready to be downloaded

4: main ( ) \{

5: $\quad$ int done0, done1;
/* Seize TMS\#0's andTMS\#1's program memory
6: $\quad$ sze_resource ( _TMS0, PGM_RES );
7: $\quad$ sze_resource (-TMS1, PGM_RES );

$1^{*} \quad$ Load TMS\#0's program memory and TMS\#1's program memory

8: $\quad$ load_tpgm ( -TMSO);

9: $\quad$ load_tpgm ( -TMS1);

\section{$1 *$}

Release data memory \#1 and \#2 for TMS\#0 and TMS\#1 rel_resource ( -TMSO, DTAO_RES ); rel_resource (-TMS1, DTA0_RES); rel_resource (-TMSO, DTAI_RES); rel_resource ( -TMS1, DTA1_RES );

\section{$1^{*}$}

start_tms ( -TMSO);

Start TMS\#0 and TMS\#1 start_tms ( -TMS1);

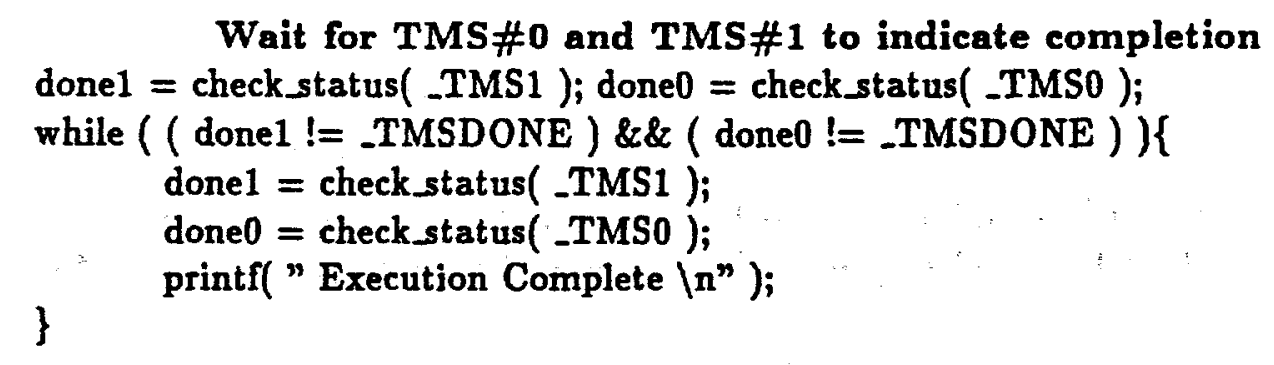

Wait for TMS\#0 and TMS\#1 to indicate completion

Seize the program and data memories for examination sze_resource ( TMSO, PGM_RES ); sze_resource (-TMS1, PGM_RES); sze_resource ( -TMSO, DTA0_RES ); sze_resource (-TMS1, DTA0_RES ); sze_resource (-TMSO, DTA1_RES ); sze_resource ( -TMS1, DTA1_RES );

Figure 8: Sample Code for a H $^{2}$ ART Control Node 


\section{References}

[1] Stankovic, John A., “Real-Time Computing Systems: The Next Generation”, 8806, COINS TECHNICAL REPORT, Department of Computer Science, University of Massachusetts, Amherst,Ma., 1988.

[2] Grossman, David D., "Opportunities for Research on Numerical Control Machining", Comm. of the ACM, Vol. 29, No. 6, June 1986, 515-522.

[3] Wirth, Niklaus, "Towards a Discipline of Real-Time Programming", Communications of the ACM, Aug 1977, Vol.20. No.8, reprinted in Real-Time Software by Robert L. Glass, 1983, p. 129 - 143.

[4] Taylor, Lauren W., Fornaro, R.J., and Garrard, K.P., "The Architecture of an 8086 Based Multiprocessor", TR-86-22, Department of Computer Science Technical Report, North Carolina State University, Raleigh, NC, December 1986.

[5] Fornaro, R.J., Garrard, K., and Taylor, L., "A Structured Operating Systems Approach to High Speed Real-Time Control", Precision Engineering Annual Report, North Carolina State University, Raleigh, NC, Vol. IV, p.48, December 1986.

[6] Luttrell, Dan. E., "Development of a High Speed System to Control Dynamic Behavior of Mechanical Structures", M. S. Thesis, Department of Mechanical and Aerospace Engineering, North Carolina State University, Raleigh, NC, 1987.

[7] Skroch, D.A., Garrard, K.P., Taylor, L.W., and Fornaro, R.J., "Algorithm Decomposition and Performance Evaluation on a Real-Time Multiprocessor", Precision Engineering Annual Report, North Carolina State University, Raleigh, NC, Vol. V, p. 146, December 1987.

[8] Garrard, K.P., Fornaro, R.J., and Taylor, L.W., "System Software for an 8086 Based Multiprocessor", TR-86-21, Computer Science Technical Report, North Carolina State University, Raleigh, NC, December 1986.//

[9] Garrard, K.P., Fornaro, R.J., Taylor, L.w., and Skroch, D.A., “ Programmer's Manual for A Real-Time Heterogenous Multiprocessor", TR-87-05, Computer Science Department Technical Report, North Carolina State University, Raleigh, NC, August 1987. 



\title{
11 MULTIPROCESSOR PROGRAMMING ENVIRONMENT
}

\author{
Mary Beth Smith \\ Graduate Student \\ Robert Fornaro \\ Associate Professor, CSE
}

Programming tools and techniques have been well developed for traditional uniprocessor computer systems. The focus of this research project is on the development of a programming environment for a high speed real time heterogeneous multiprocessor system, with special emphasis on languages and compilers. The new tools and techniques will allow a smooth transition for programmers with experience only on single processor systems.

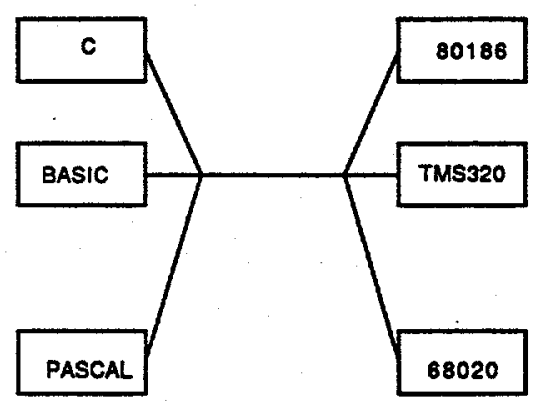

UNCOLMOOEL N+M

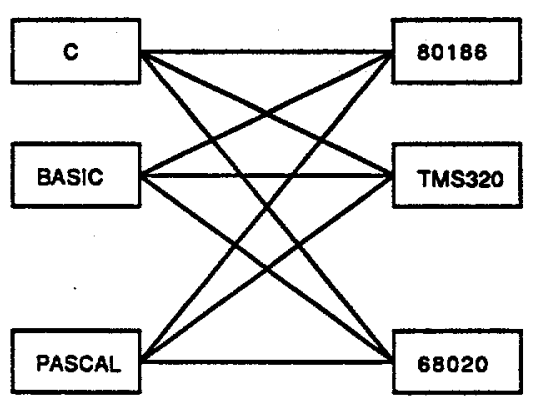

STANDARD MODEL N*M 


\subsection{INTRODUCTION}

The $\mathrm{H}^{2}$ ART computer is a powerful multiprocessor system designed for general purpose high speed real time control applications. To make the system usable by everyone, a user-friendly programming environment needs to be developed. Progress has been made in terms of a user interface for downloading programs and for communication among the various processors as discussed in Section 10. The objective of this section is to report on the status of languages and compilers for the $\mathrm{H}^{2} \mathrm{ART}$ system.

This section begins with a discussion and evaluation of the Texas Instruments $\mathrm{C}$ compiler for the TMS320C25 digital signal processor. Code segments from several common programming routines (loops, matrix multiplication, floating point arithmetic) are analyzed to determine how well the $C$ compiler can translate high level code into assembly code. Next is an outline of some fundamental issues to consider when programming a multiprocessor system. These issues of languages, communication, and architecture are drawn from the experience of working with the application of digital control for a scanning tunneling microscope (STM). Finally, the programming language and compiler currently under development are discussed.

\subsection{TI COMPILER}

The $\mathrm{H}^{2} \mathrm{ART}$ architecture currently consists of a host 80286 processor, an 80186 processor and two TMS320C25 digital signal processors. Compilers are readily available for the Intel $80 \times 86$ series in many of the common programming languages such as C, Basic or Pascal. Traditionally, programs for DSP chips have been written in assembly language. However, Texas Instruments has made available a TMS320C25 C compiler. Such a compiler is attractive because assembly language programming is tedious and often error-prone. It is also more difficult for others to read and modify, and requires a knowledge of the underlying hardware. Higher level programming languages, like $\mathrm{C}$, are closer to algorithmic style which makes the code easy to write, portable, and also easy to read and modify. Portability implies that a change in hardware does not necessitate a complete change in software. In general, programming in a higher level language is much less time consuming than programming in assembly language.

There is also a potential disadvantage in using the $\mathrm{C}$ compiler. The TMS320C25 is a high-performance microprocessor with an instruction set optimized for time critical digital signal processing applications [1]. An examination of the TI C compiler shows that the translated code does not always utilize the special purpose instructions that would normally be used if the programmer had coded in assembly language. Some specific examples are demonstrated next. 


\subsubsection{Compiler Evaluation}

The TI compiler actually takes two stages to complete. When invoked, the compiler first translates the $\mathrm{C}$ code into assembly language instructions. In the second stage, the assembly code is translated into machine code to be run on the TMS320C25. The efficiency of the compiler was evaluated by examining the output of the first stage. The translated code was compared to hand-coded assembly code for several sample programs. Shown below are code segments demonstrating some common programming routines for loops, matrix multiplication and floating point arithmetic. For each routine, the $\mathrm{C}$ code and corresponding assembly instructions are compared to a "hand-coded" routine.

Loops Looping constructs are common to most any program. It involves executing a segment of code repeatedly for a specified number of times.

\section{Code}

The following segment of code declares two integers, $x$ and $j$. The function of the code is to loop 10 times using $x$ as a loop control variable and increment $j$ each iteration.

$$
\begin{array}{ll}
\begin{array}{l}
\text { main }() \\
\text { int } x ;
\end{array} & ; \text { declare } x \text { as integer } \\
\text { int } j=0 ; & ; \text { declare } j \text { as integer and initialize to } 0 \\
\text { for }(x=0 ; x<10 ; x++)\{ & ; \text { loop } 10 \text { times } \\
j++; & ; \text { increment } j \text { each iteration }
\end{array}
$$

\section{Hand-Coded Assembly}

One way of writing this same segment of code in TMS320C25 assembly code is:

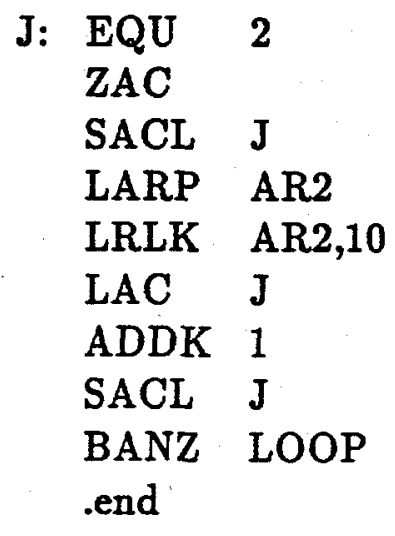

; $\mathrm{J}$ is data memory location 2

; zero the accumulator

; initialize $\mathrm{J}$ to 0

; set current auxiliary register to AR2

; set AR2 as loop control counter

; load $\mathrm{J}$ into accumulator

; add 1 to the accumulator

; store accumulator in data location $\mathrm{J}$

; branch to loop if AR2 is not zero - decrement . 
Translated Assembly

L3:

$\begin{array}{ll}\text { LARK } & \text { AR2,2 } \\ \text { MAR } & * 0+ \\ \text { LAC } & * \\ \text { ADDK } & 1 \\ \text { SACL } & *- \\ \text { LAC } & * \\ \text { ADDK } & 1 \\ \text { SACL } & * \\ \text { LAC } & * \\ \text { SUBK } & 10 \\ \text { BLZ } & \text { L3 }\end{array}$

; load register 2 with address of $x$ ; increment register 2 to address of $j$

; load accumulator with value of $j$

; increment $j$ by 1

; store new value of $j$

; load accumulator with value of $x$

; increment $x$ by 1

; store new value of $x$

; load accumulator with value of $x$

; subtract from upper limit of loop

; repeat if limit has not been reached

The translated code followed the hand-coded assembly code very closely. The accumulator is used in combination with the subtract and branch instructions to loop until the count is equal to 10. In the hand-coded version, an auxiliary register is used in conjunction with a BANZ (Branch on Auxiliary Register Not Zero) instruction which combines the branch and subtraction into a single step.

Matrix Multiply Matrix multiplication and summation of products are useful in many DSP applications. The TMS320C25 has special instructions for their efficient computation. $C$ Code

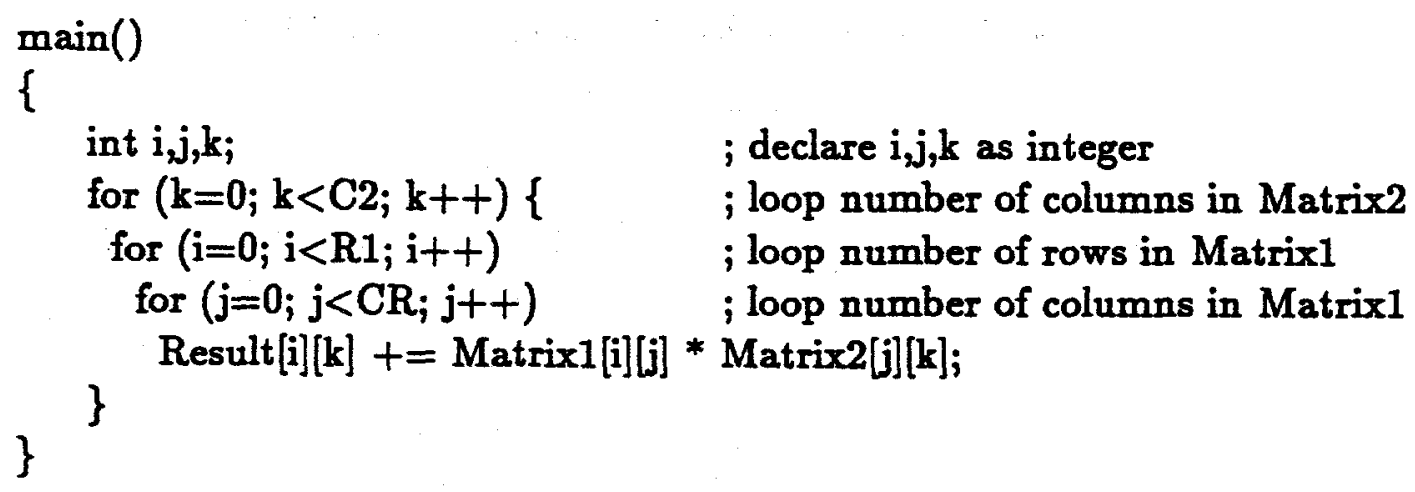




\section{Hand-Coded Assembly}

Matrix multiplication is repeated execution of row by column multiplication. The following code segment multiplies one row by one column.

$\begin{array}{ll}\text { MPYK } & 0 \\ \text { ZAC } & \\ & \\ \text { RPT } & \mathrm{N} \\ \text { MAC } & \text { FF00, }{ }^{*}+ \\ \text { APAC } & \end{array}$

; clear accumulator and $\mathrm{P}$ register

; repeat instruction $\mathrm{N}+1$ times

; multiply and accumulate

; add $\mathrm{P}$ register to accumulator

\section{Translated Assembly}

The actual code produced is too long to include here. However, the key issue in this example is that the TI compiler does not always utilize the RPT instruction which will repeat an instruction a specified number of times. Instead, the translated code uses a less efficient subtract and branch combination. Also, the compiler does not take advantage of the multiply/accumulate, MAC, command. This specialized command adds the contents of the product register to the accumulator and simultaneously reads two more numbers to be multiplied. Without the use of the RPT/MAC combination, several nested loops must be utilized to access the elements of each row and column.

Floating Point The TMS320C25 operates only on fixed point numbers. To implement floating point, operands must be converted to fixed point for arithmetic operations, and then converted back to floating point[1]. The floating point numbers are represented in two words, a mantissa and an exponent. Depending on the precision desired, different $\mathrm{Qx}$ formats can be used. The $\mathrm{x}$ is a number between 1 and 15. This is the number of bits to the right of an assumed binary point which represents the fractional portion of the number. The remaining bits in the word represent the integer portion.

The various conversions and formats can become confusing and even more complex for extended precision arithmetic operations. Use of the $\mathrm{C}$ compiler drastically reduces the amount of detail for the programmer. 


\section{Code}

This segment of code illustrates the ease of programming arithmetic operations, fixed or floating point, in a high level language.

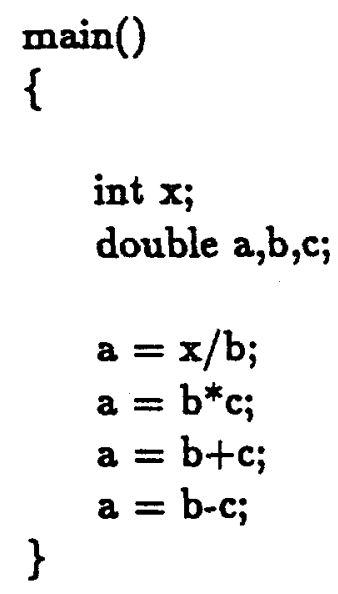

\section{Hand-Coded Assembly}

The hand-coded assembly involves shifting mantissas to obtain equal exponents, normalizing floating point numbers, and denormalizing fixed point numbers. Also, the division is actually implemented by repeated subtraction. The code is not shown here due to its length.

\section{Translated Assembly}

The translated code makes calls to the $\mathrm{C}$ floating point library routines. This library also contains trigonometric, exponential, and logarithmic functions. Since the TMS320C25 performs 32-bit arithmetic on 16-bit values, arithmetic overflow and underflow cannot be handled in a predictable way. Thus, there is no guarantee that code which is dependent on overflow/underflow handling will execute correctly[3].

Overall, the TI C compiler is an effective programming tool. As with any high speed, time intensive application, the most critical portions may still have to be written in assembly language for best results. This is a simple matter since the $\mathrm{C}$ compiler accepts in-line assembly code. This means that assembly language instructions preceded by an asm keyword can be inserted directly in with the rest of the $\mathrm{C}$ code. However, the compiler is able to produce reasonable code for most situations. The positive aspects of using a high level language far outweigh the few negative situations. 


\subsection{PROGRAMMING IN A MULTIPROCESSOR ENVIRON- MENT}

The complexity of programming increases when dealing with a multiple processor environment, especially a heterogeneous environment. Take, as an example application, the Scanning Tunneling Microscope (STM). A recent project, digital control of the STM, requires the use of two processors: an 80386 host and an Ariel board which contains a TI TMS320C25 digital signal processor (DSP). The Ariel board is an off-the-shelf data acquisition board which plugs into a PC bus. What follows is a discussion of some of the issues considered when programming for this application, as well as some limitations of this hardware configuration.

\subsubsection{Languages}

Figure 1 shows a simplified block diagram of the software functions and communication paths needed to control the STM. Running on the AT host are modules written in both $\mathrm{C}$ and Basic. The program running on the DSP is written in assembly code, assembled into a hex format and downloaded onto the Ariel board for execution. This particular application needs three languages, two compilers and one assembler. A user (programmer) of the system must learn three languages and the idiosyncracies of each compiler. For one application, the user must write and compile/assemble three separate programs.

\subsubsection{Communication}

Multiplicity of languages is not the only complexity that faces the user of a multiprocessor system. Other issues include synchronization and communication of tasks running on various processors. For STM control, a critical aspect is communication time, or time to transfer data, from the Ariel board to the host. One goal of the STM project is real time display, that is, graphically display the image as it is being scanned. As shown in Figure 1, one scan line (256 data points) is obtained in the DSP memory and then passed back to the host for display on the terminal.

The method of transfer on the Ariel Board which was found to be the most efficient means of moving that number of data points is not sufficient. The results of three possible communication methods are pictured in Figure 2. Using the DRDS method, it takes $4.2 \mathrm{~ms}$ to transfer 256 data points during which time there can be no axis control or error compensation. This time is barely tolerable. Figure 3 is an early version of the HART architecture consisting of an Intel 8086 and a TI TMS32010. Data transfer from the 32010 to the 8086 takes only $55 \mu \mathrm{s}$ as compared to $190 \mu \mathrm{s}$ to transfer a word from the Ariel board to the 80186. The bottleneck in this situation is data memory. If the host processor wants to access DSP data memory, the TMS320C25 must be put on hold. The following section 
Zenith 80386

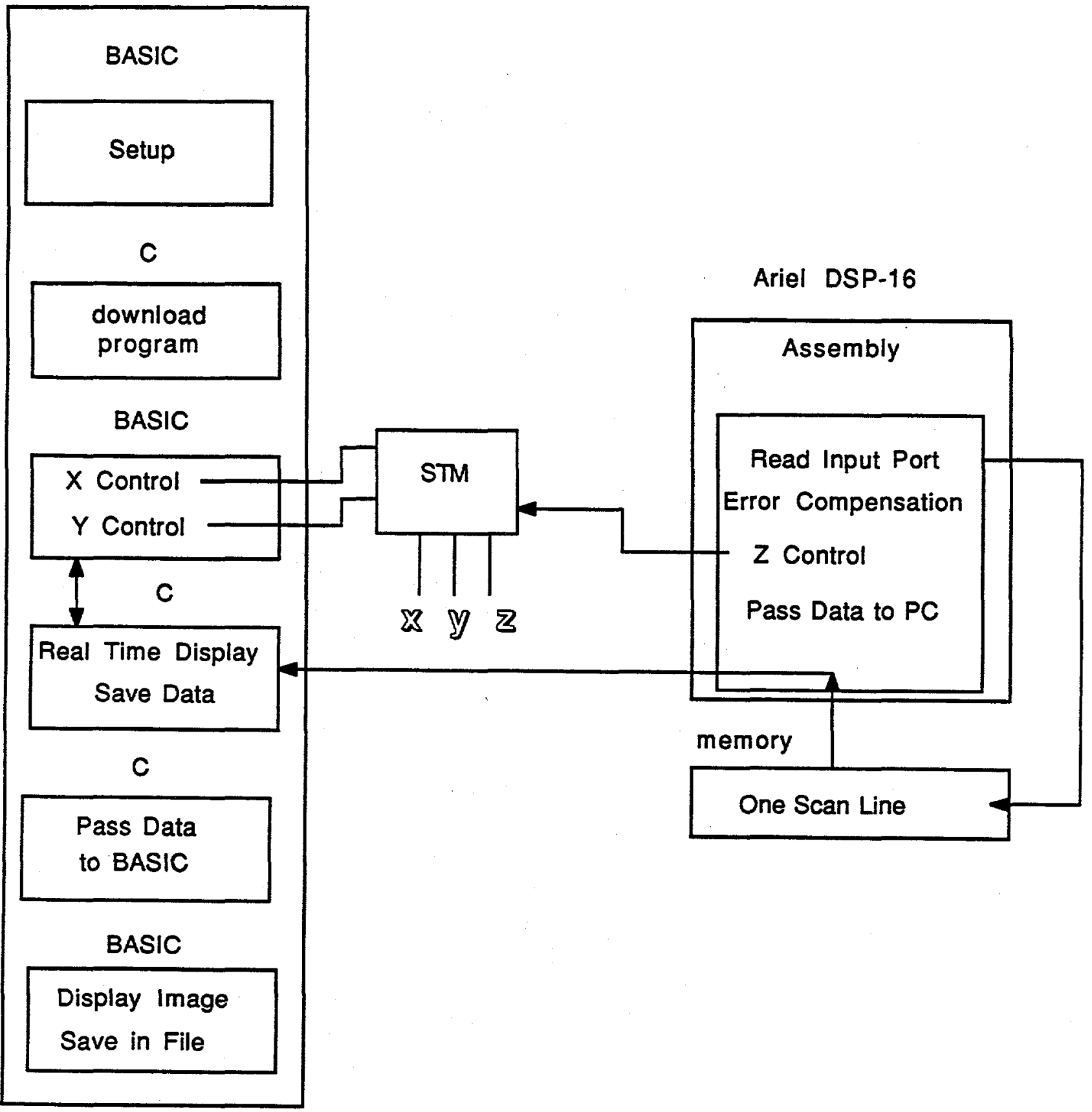

Figure 1: Software for STM Control 


\section{Transfer Rates}

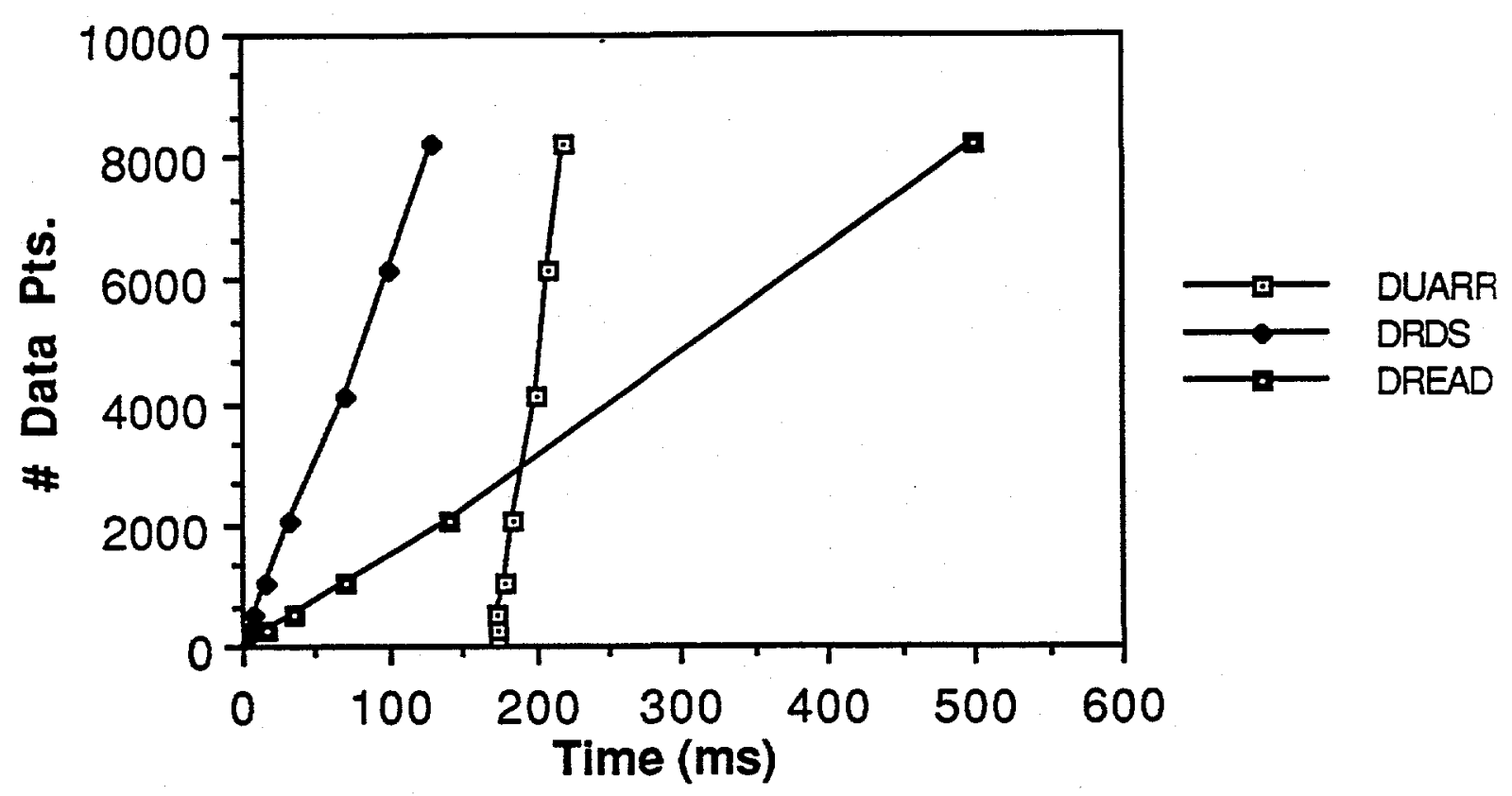

Figure 2: Data Transfer Rates for Ariel Board

discusses an architecture solution to this problem.

\subsubsection{Architecture}

A prominent feature of $\mathrm{H}^{2} \mathrm{ART}$ is the dual ported, double buffered memory associated with the TMS320C25 DSP chips. Double buffered implies that the data memory can be thought of as two separate memories. Dual ported implies that there are two paths to reach the memories. The significance of this is that the $\mathbf{8 0 1 8 6}$ may access one portion of DSP memory while the DSP is writing into the other memory area. Following through with the STM application example, this would completely resolve the aforementioned complications of moving data back to the host quickly enough for real time display. Communication in this type of architecture is much more efficient, especially for high-speed, time critical control applications.

\subsection{ACK COMPILER TOOL KIT}

To ease the task of programming for a heterogeneous multiprocessor system, an integrated compiler is needed. That is, a single compiler that can be used for several target processors. The target processors are commonly called back-ends. Current research ef- 


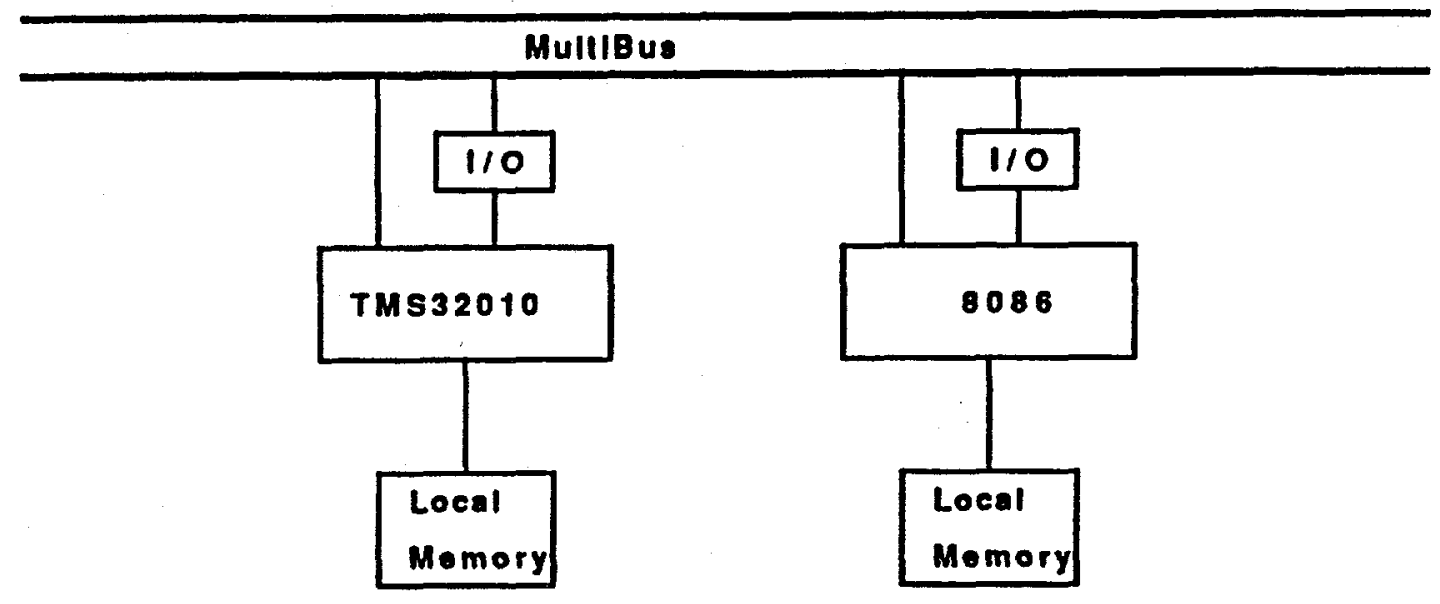

Figure 3: Previous Version of HART Architecture

forts are being focused on creating such a compiler based on the Amsterdam Compiler Kit (ACK) [2]. ACK is based on an idea known as UNCOL which stands for UNiversal Computer Oriented Language. It solves the problem of having to write $\mathrm{N}^{*} \mathrm{M}$ compilers for $\mathrm{N}$ front-ends (high level languages) and $\mathrm{M}$ back-ends (target processors). All source code is translated to a common intermediate form, EM. That intermediate form is then used by the back-ends to compile to machine code for a specific target. This means only $N+M$ rather than $N^{*} M$ compilers be written. Figure 4 illustrates these two compilation models. A heterogeneous multiprocessor system implies several different back-ends. Therefore, a compiler for a system such as this one is well suited to the UNCOL method.

The proposed $\mathrm{H}^{2} \mathrm{ART}$ programming language is based on the $\mathrm{C}$ language, provided by ACK, with extensions for process definition, communication, and timing. These extensions make $\mathrm{C}$ a real time concurrent programming language. It is "concurrent" because it allows the definition of parallel tasks and it is "real time" because it can have primitives for enforcing timing constraints of these concurrent tasks.

The process definitions are compiler directives that indicate which target processor that a particular module is to be run on. Figure 5 shows how the STM application software might look if it were written in the proposed real-time C. The module xyControl will run on the 80186. It contains subroutines to control the $x$ and $y$-axes and to plot the image. Everything between the square brackets [ is included in that module. The other module, $z$ Control, will be compiled for the TMS320C25. The programmer need only write 


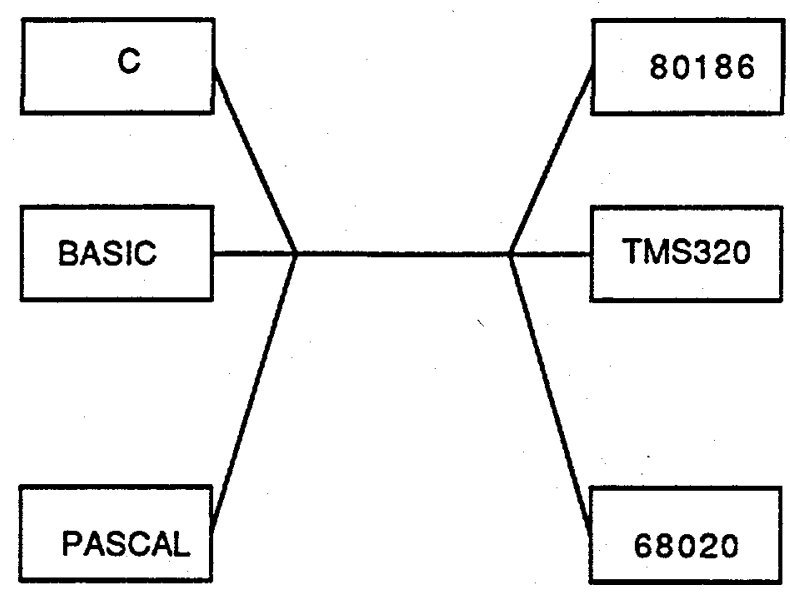

UNCOL MODEL N+M

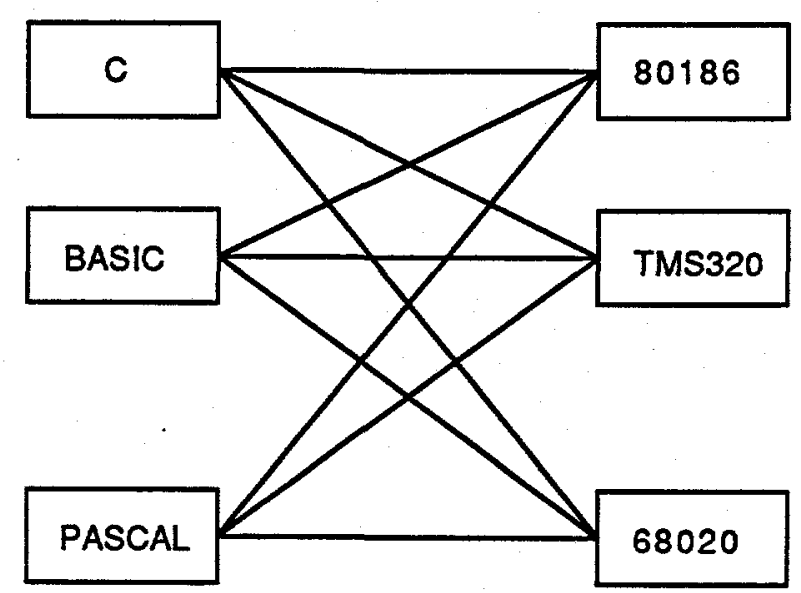

STANDARD MODEL $N^{*} M$

Figure 4: Compilation Models for Multiple Front-Ends and Back-Ends 
module xyControl : 80186

I

xyRoutine( )

\{

C code to control $x$ and $y$

\}

Display( )

\{

C code to plot image

]

\}

module $z$ Control : TMS320C25

[

zRoutine( )

\{

C code to control z

]

Figure 5: Sample Real Time C code for STM Control

in one language and invoke one compiler. The code is translated as depicted in Figure 6.

\subsection{FUTURE WORK}

Other extensions to the language will include primitives for communication and timing. Communication primitives allow the modules to pass data to each other and also use that as a means of synchronization. The timing primitives ensure a proper ordering of events and enforce timing constraints. 


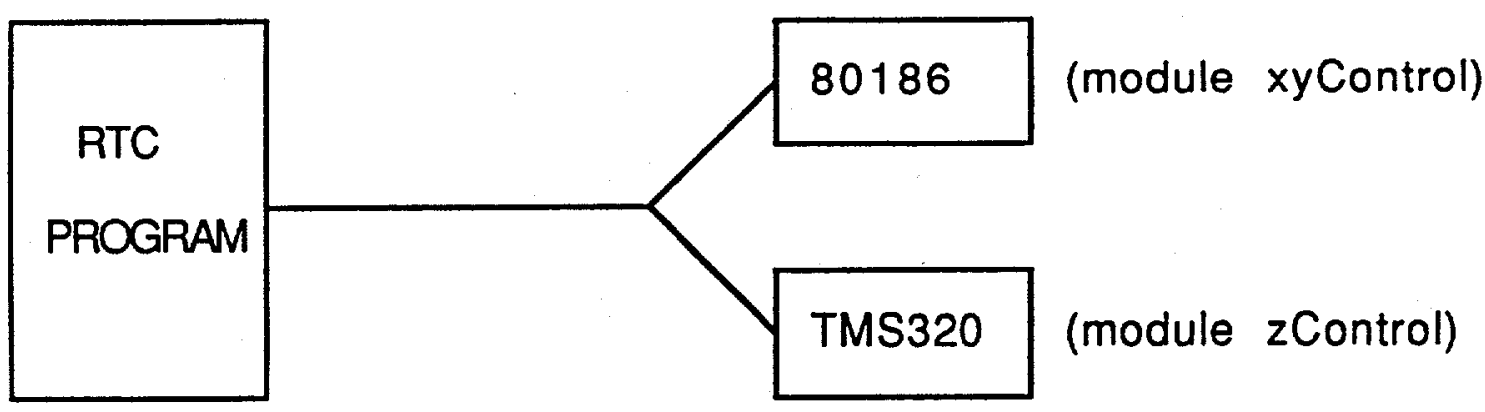

Figure 6: Translation of STM Control Code

\section{References}

[1] "Second-Generation TMS320 User's Guide", Texas Instruments, Inc., 1987.

[2] "Amsterdam Compiler Kit Reference Manual", Unipress Software, Inc., 1987, pp 1.1.

[3] "TMS320C25 C Compiler Reference Guide", Texas Instruments, Inc., 1988, pp 5-17. 



\title{
12 FABRICATION OF BRITTLE MATERIALS - CURRENT STATUS
}

\author{
Ronald O. Scattergood \\ Professor, MTE
}

\subsection{INTRODUCTION}

The research initiatives in the area of precision fabrication will be continued in the upcoming year. Three students, T. Bifano (PhD), P. Blake ( $\mathrm{PhD}$ ) and E. Smith (MS), finished their research programs in the last year. Sections 13 and 14 will summarize the essential results from the work of the Materials Engineering students Blake and Smith. Further details will be presented in forthcoming publications that are now in preparation [1-2]. The results from Bifano's thesis have been published in adequate detail and need not be summarized further [3-6].

Three new students, S. Blackley (MS), H. Paul ( $\mathrm{PhD}$ ), and S. Smith ( $\mathrm{PhD}$ ) have joined the program and will continue the research efforts in precision fabrication. The programs for these students will be outlined in Sections 15 and 16. Because of the success of the the earlier work in establishing new process models and experimental techniques for the study of diamond turning and diamond grinding, the new programs will, in part, build upon the earlier work. This is especially true for investigations concerned with brittle materials. The basic understanding of material response of nominally brittle materials during machining or grinding operations remains as a challenge. The precision fabrication of brittle materials will continue as an area of emphasis for the Precision Engineering Center.

\subsection{CURRENT STATUS - OVERVIEW}

A brief overview of the current status of the materials-oriented research on precision fabrication of brittle materials is appropriate, both as a summarization of the progress made to date and as the format for continuing research. Brittle materials such as glasses and ceramics are not machinable in the conventional sense. However, if the contact loads and depth of penetration of sharp tools are kept sufficiently small, then plastic flow can occur at the tool tip/workpiece interface without the formation of cracks. Material removal will then occur via plastic flow processes, similar to the case of ductile metals. However, the key issue with brittle materials is that if certain bounds on the cutting forces or depths are exceeded, then fracture occurs and, in general, the components produced are not acceptable. This need not always be the case, however, and "controlled damage" fabrication remains as an area for further research. In the current efforts, the primary aim is to establish the limits for the plastic flow vs fracture regimes. 
Prior to the research initiated at the Center, there was no basic rationale for describing what constitutes and limits ductile-regime machining or grinding of glasses and ceramics. The research efforts of Bifano [7] and Blake [8] have led to physically-based models of the process, along with the identification of certain critical machining parameters and material properties that limit the success of the process in any real application. Furthermore, their pioneering research has led to the development of experimental techniques that can aid in systematizing the study of process and material variables. Rather than applying a multiparameter test-matrix doctrine for investigations, the measurement of key, physically derived quantities, such as a critical depth of cut, allows a much more rational and efficient interpretation of experiments. Future research will build upon and expand these ideas.

The underlying theme of ductile-regime machining or grinding follows from a deceptively simple concept adapted from a large body of literature on indentation of brittle materials using sharp diamond (or other) indenters [9]. That these results, which were almost always obtained under static loading conditions, can be applied to precision machining or grinding phenomena is not apriori obvious in view of the very high deformation rates and different geometries applicable to the machining processes. The common underlying theme in the process models is that there exists a critical penetration depth $d_{\mathfrak{c}}$ for the diamond tool, such that when this depth is exceeded, fracture will ensue. The basic result, when properly formulated, is essentially independent of the particular model chosen for representation. If the key material factors are elastic response, plastic flow and fracture, then the pertinent material parameters must be, respectively, elastic modulus $E(\mathrm{MPa})$, hardness or plastic flow resistance $H(\mathrm{MPa})$, and fracture toughness $K_{c}\left(\mathrm{MPa}-\mathrm{m}^{\frac{1}{2}}\right)$. A critical depth $d_{c}(\mathrm{~m})$ for plasticity-fracture transition would then have the following form, this being the simplest form consistent with dimensional requirements

$$
d_{c}=\beta\left[\frac{K_{c}}{H}\right]^{2}\left[\frac{E}{H}\right]
$$

The constant $\beta$ depends upon the details of the plastic zone size and shape, ie., the actual machining-geometrical conditions. For establishing the role of material properties, only the parametric dependence on $E, H$ and $K_{c}$ is needed if standard machining conditions are employed, as in Bifano's work [7]. Among the different material properties, fracture toughness is the most important since. this is most sensitive to the microstructure in modern ceramics. Fracture toughness is also more difficult to measure than are the other properties. To understand the role of machining parameters such as rake angle, tool edge radius, cutting speed, etc., for a given material, a much more detailed and elaborate critical-depth model is needed since these effects will be manifest in the $\beta$ value in Equation (1). It is here that techniques such as finite-element analysis of the ductile-brittle machining process will play a central role.

A more specific, fracture mechanics-based argument involving stress intensity factors $K$ or energy balance requirements produces the same result as conjectured above for Equation (1). For example, the maximum stress intensity factor due to a localized plastic zone is 
finite because the maximum possible stress is bounded by the yield stress (hardness), ie., plastic flow removes any stress singularities associated with sharp tools. Commensurate with this, the stress intensity factor increases with plastic zone size (tool penetration depth d) simply because of the increased spatial extent of the stress field. The stress intensity factor is essentially the product of stress times the square root of a dimension. Accordingly, the maximum stress intensity factor is proportional to the maximum stress (yield stress) times the square root of a mean plastic zone size dimension. When the maximum possible stress intensity factor $K_{\max }$ reaches the fracture limit $K_{\max }=K_{c}$ (as plastic zone size or cutting depth $d$ increases), then the transition from plastic flow to fracture processes can occur. A careful consideration shows that this is a lower bound for $d_{c}$, i.e. the worst case $[8]$.

From an energy balance point of view, the elastic strain energy stored in the plastic zone must at least be equal to the energy required to produce or drive a crack, otherwise fracture processes cannot occur. The energy available relative to that required increases with plastic zone size and so fracture processes ultimately occur [7]. Both of these viewpoints lead to Equation (1). Often in the indentation-fracture literature, these concepts are expressed as a critical indentation load for fracture $P_{c}$, but this is exactly equivalent to a critical depth $d_{c}$ via the fact that load and indentation depth are uniquely related by the indenter geometry.

The application of the critical depth concept embodied in equation (1) has been successfully applied to precision machining and grinding. A key factor is the realization that tool geometry plays a critical role. For example, in single-point diamond turning the tool geometry is well defined, and careful consideration shows that the effective depth of cut for round-nosed tools varies along the tool cutting edge. The actual ductile-regime machining process is in fact a combined action of plastic flow and fracture. Success depends upon both the value of $d_{c}$ and the specific tool geometry involved, as has been clearly demonstrated for $\mathrm{Si}$ and $\mathrm{Ge}$ single crystals by Blake [8]. Recognition of these factors led to an experimental technique for the direct evaluation of $d_{c}$. Furthermore, this process model concept leads immediately to the identification of critical machining parameters such as tool infeed. Further work is needed to extend and verify these concepts for additional material systems.

In the work carried out by Bifano [7], the tool geometry for grinding was simplified by conducting studies using a plunge-mode grinding test. This leads to a different, but comparable experimental technique for the direct evaluation of $d_{c}$.

Precision diamond grinding is a more versatile technology than single-point diamond turning in that many more ceramics and glasses are amenable to grinding. The tool geometry is more difficult to characterize in grinding, however, and this remains as a critical area of research. In the studies carried out by Bifano, tool geometry was not a variable, and emphasis was placed on material property variations, as well as certain other machine or process variables. These initial material studies suggested that in the more complex, advanced ceramics, the fracture toughness $K_{c}$ for short cracks may be much less than the 
conventional toughness values measured for long cracks. Crack-resistance (R-curve) behavior of this kind has only recently been recognized in ceramic systems, but it may well be a limiting factor for applying the ductile-regime grinding technology to ceramic-based systems. The novel results related to $\mathrm{R}$-curve behavior obtained in the completed research at the Center require more detailed and quantitative study [6].

The results discussed above represent a significant advance in the conceptual understanding and process models underlying precision diamond turning and grinding. That a relatively simple physical picture can be successfully applied to complex machining processes, viz., a critical cutting depth for ductile-to-brittle transition, coupled with a proper recognition of tool geometry effects, opens the way for further research and more specific studies into various process parameters and material variables. The initial research is by no means complete, and the new initiatives described Sections 15 and 16 are intended as critical extensions of the existing knowledge base. Much of the materials-related research is inextricably linked to machine properties and machine parameters, for example, stiffness, grinding wheel properties, wheel dressing technique and tool characteristics. The material research efforts are continuing in close collaboration with the efforts in machine properties, sensors, control and process modelling. The precision fabrication of ductile materials is, by necessity, interdisciplinary in approach.

In the following sections, completed projects will be reviewed and new initiatives will be described. The latter are coupled to the earlier research studies.

\section{References}

[1] P. Blake and R. O. Scattergood, to be submitted, Jour. Amer. Cer. Soc. (1989).

[2] E. Smith and R. O. Scattergood, to be submitted, Prec. Engr. (1989).

[3] T. Bifano, T. Dow and R. O. Scattergood, Proc. Adv. in Opt. Fab., SPIE, 966, (1988).

[4] T. Bifano, T. Dow and R. O. Scattergood, Ultraprecision in Manuf. Engr., M. Weck and R. Hartel, eds., Springer-Verlag, 22 (1988).

[5] P. Blake, T. Bifano, T. Dow and R. O. Scattergood, Amer. Cer. Soc. Bull., 67, 1083 (1988).

[6] R. O. Scattergood, S. Srinivasan, T. Bifano and T. Dow., in press, Proc. 7th Int. Symp. on Ceramics (7th SIMCER), Bologna, Italy, Dec. 14-16 (1988).

[7] T. Bifano, PhD dissertation, North Carolina State University (1988).

[8] P. Blake, $\mathrm{PhD}$ dissertation, North Carolina State University (1988).

[9] B. R. Lawn and T. R. Wilshaw, Jour. Mat. Sci., 10, 1049 (1975). 


\title{
13 DIAMOND TURNING OF SI AND GE SINGLE CRYSTALS
}

\author{
Peter Blake \\ NASA/Goddard Space Center \\ Ronald O. Scattergood \\ Professor, MTE
}

Single-point diamond turning studies have been completed on Si and Ge crystals. A new process model was developed for diamond turning which is based on a critical depth of cut for plastic flow-to-brittle fracture transitions. This concept, when combined with the actual machining geometry for single-point turning, predicts that "ductile" machining is a combined action of plasticity and fracture. Interrupted cutting experiments also provide a means to directly measure the critical depth parameter for given machining conditions.

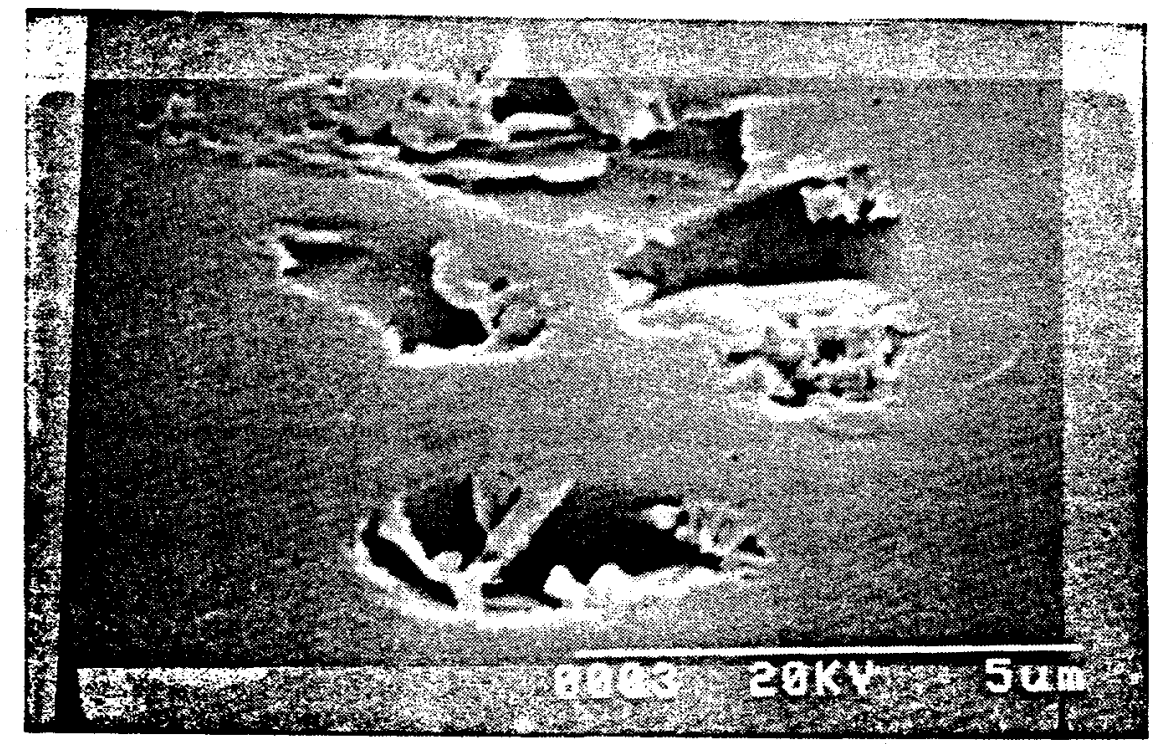




\subsection{INTRODUCTION}

The research on single-point diamond turning of $\mathrm{Si}$ and Ge single crystals has been completed. Various aspects of the work have been published [1-3] or are in preparation [4]. In this section, critical experimental results and interpretation of the research studies will be addressed. Single point diamond turning experiments were made on $\mathrm{Ge}$ and $\mathrm{Si}$ wafers of various orientation. The diamond turning was done using the PAUL lathe. The details of the experimental procedures and a description of the lathe and machining geometry are available in previous progress reports [5].

When the research was initiated, no process model for diamond turning of a brittle material was available. Of course, experienced diamond turners clearly recognized the important role played by certain machining parameters, such as tool rake angle, infeed, etc. One of the major aims of the work was to try an develop a suitable model in order to provide a basis for investigating the specific influence of machining or material variables. Compared to precision diamond grinding, relatively few brittle materials can be diamond-turned for actual production technologies. Thus, rather than attempt to introduce a systematic variation of material properties, the research focussed on diamond turning of two important, and prototypical brittle crystalline materials, viz., Ge and Si. Both of these were obtained in single-crystal form, eliminating any complications due to microstructural features such as grain size or grain orientation. These monolithic materials form ideal brittle model systems with which to study the machining process.

\subsection{EXPERIMENTAL RESULTS}

The key experimental results are linked to the machining geometry shown in Figure 1. As the round-nosed diamond tool cuts into the workpiece, the in-feed motion of the tool requires that the chip removed have the shape shown. This is of course a geometrical construction based on the undeformed chip shape. Two successive tool passes form the sector bounded by circular arcs, which is the projected area of the tool in the workpiece viewed along the direction of cut. The critical feature enforced by this tool geometry is that the effective depth of cut $d$ (or chip thickness) varies along the tool edge. The depth is zero on the cut surface and increases to a maximum value at the upper shoulder near the uncut surface. The effective depth $d$ is easily related to distance along the tool edge by the geometry.

Once the chip geometry is properly recognized, the proposed process model due to Blake follows from a critical depth of cut approach. If the effective cutting depth $d$ is larger than a critical value $d_{c}$, then fracture processes will be initiated. This is shown schematically in Figure 2, where a zone of microfracture damage extends into the shoulder. The microfracture damage initiates at $d=d_{c}$ and extends along the tool edge for $d>d_{c}$ as shown. The microfractures will extend down into the substrate, however, if $d_{\mathrm{c}}$ is located sufficiently 


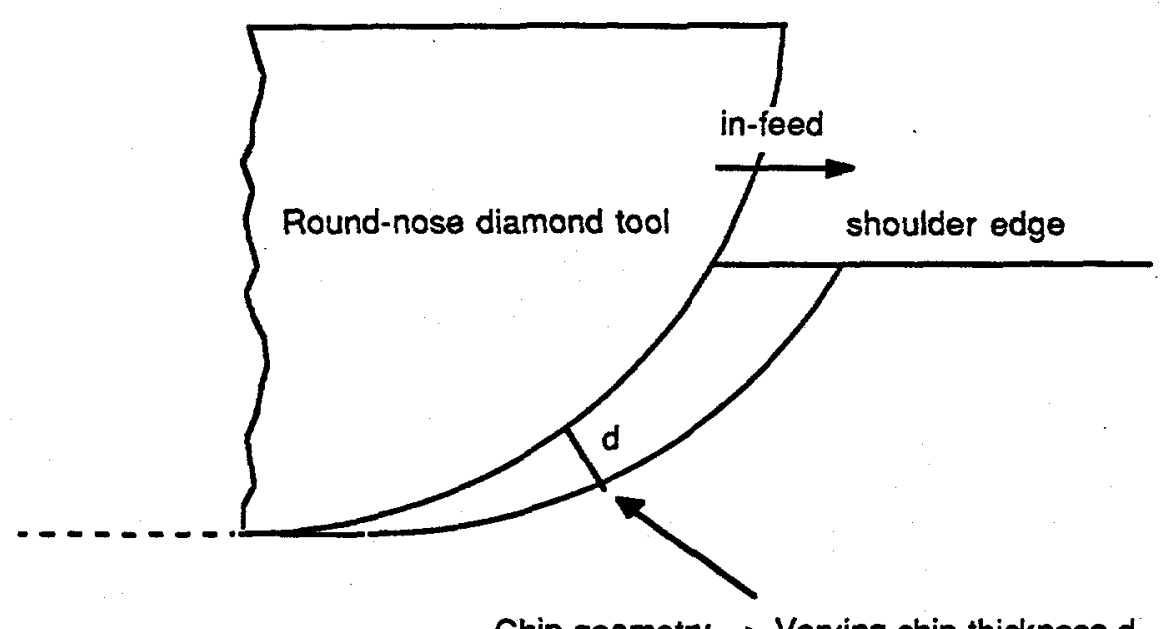

Chip geometry --> Varying chip thickness d

Figure 1: Schematic of geometry for single-point turning. View is along the cutting direction. The effective chip thickness varies along the tool edge as shown. 
far from the plane of the cut surface, then the fracture damage will not replicate into the finished surface and a fracture-free precision finish can be obtained. Fracture damage replicated into the shoulder, above the finish-cut plane, is irrelevant because it will be removed by later tool passes. Single-point turning on a brittle material therefore occurs as a combined action of plastic flow and brittle fracture. True ductile-regime cutting only need take place at the lower flank of the tool edge, where the effective cutting depth $d<d_{c}$ (Figure 2). The critical role of infeed is easily explained from Figure 2. As infeed increases, the value for $d=d_{c}$ moves lower along the tool edge, carrying the microfracture damage closer to the cut surface plane. At large infeed, depending on the actual value of $d_{c}$, a fracture-free surface will not be possible. This same process model should apply to precision grinding with crossfeed, however, the tool shape and chip geometries are considerably harder to characterize for grinding.

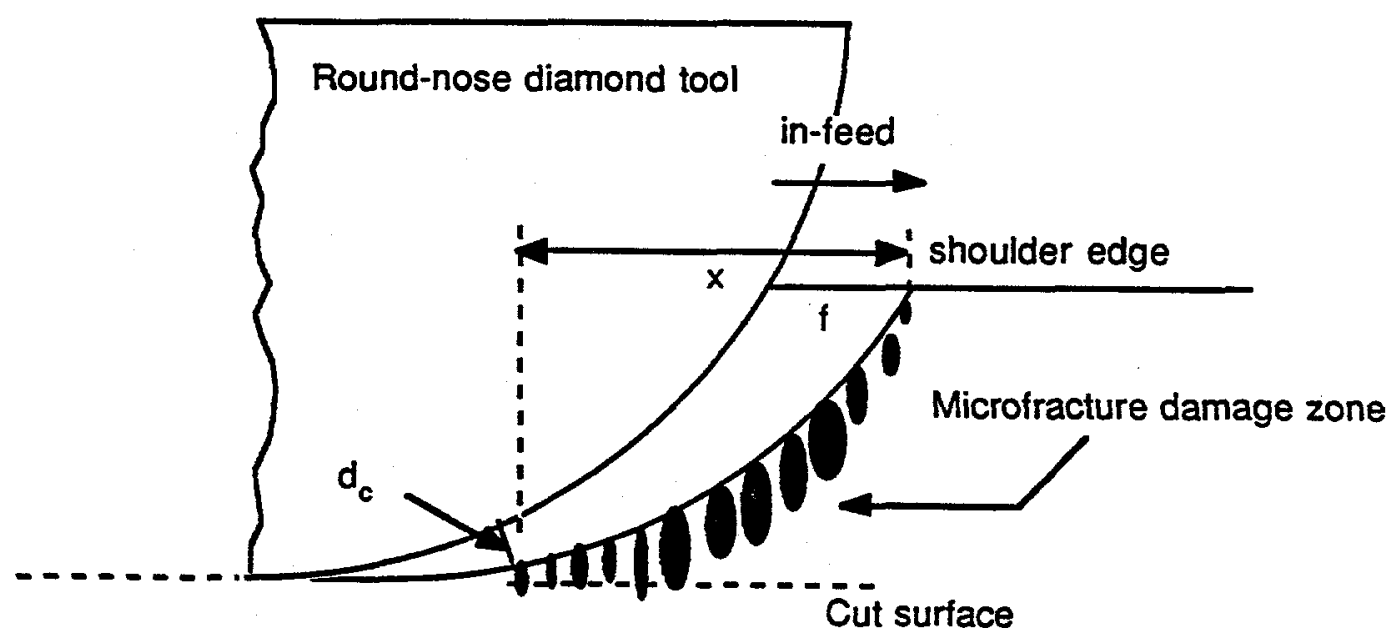

Figure 2: Schematic of critical depth model for machining of brittle materials. Below a critical depth $d_{c}$ (distance $x$ from shoulder edge) fracture does not occur. The microfracture damage for depths $d>d_{c}$ will be removed by successive tool passes if it does not replicate below the plane of the cut surface. From the geometry shown, $d_{c}=f x / R$ where $f$ is infeed and $R$ is tool radius.

The critical depth $d_{c}$ is related to material properties via a critical depth of cut model, which was discussed in Section 12. The pertinent relationship in terms of material properties is 


$$
d_{c}=\beta\left[\frac{K_{c}}{H}\right]^{2}\left[\frac{E}{H}\right]
$$

where $E, H$ and $K_{\mathrm{c}}$ are the elastic modulus, hardness and fracture toughness, respectively. $\beta$ is a constant that depends on cutting conditions, for example, rake angle, cutting speed, coolant chemistry, etc.

A series of experiments were carried out on $\mathrm{Ge}$ and, to a lesser extent, $\mathrm{Si}$ to test the process model embodied in Figure 2. These experiments were designed to both verify the underlying concepts and to provide an experimental means to evaluate $d_{c}$. The critical feature of the experiments was the use of interrupted cuts. A smooth reference surface was first machined on the sample, and then a test cut was made for given infeed, rake angle, etc. During the cut, the tool was rapidly withdrawn from the sample using a fast-tool servo, and the shoulder edge of the cut was examined using Nomarski or SEM techniques. Figure 3 shows a typical example of the experimental results. The cessation of the microfracture damage along the shoulder is clearly seen. The nominal depth of cut (distance between cut surface and shoulder edge - Figure 1) plays no role in the process.

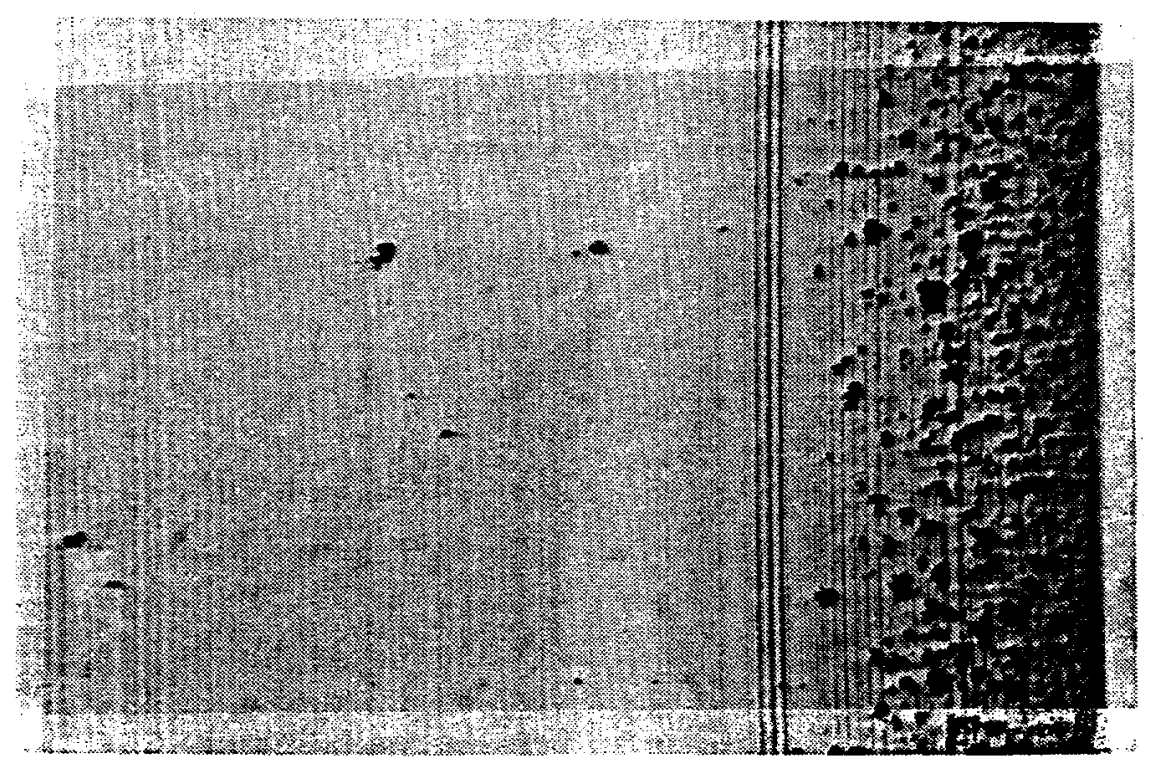

Figure 3: Nomarski micrograph of shoulder damage due to an interrupted cutting test on Ge. THe shoulder edge is on the right side. The termination line for the microfracture damage determines the critical depth of cut $d_{c}$ via the tool geometry.

The geometry in Figure 2 dictates a method whereby $d_{c}$ can be measured experimentally. Equation (1) cannot be used to determine a value of $d_{c}$, because of the unknown factor $\beta$. If 
the distance $x$ from the shoulder edge at which microfracture damage ceases is measured, then using the known tool radius, $d_{c}$ can be calculated (Figure 2). The magnitude of $d_{c}$ is central to the process because, coupled with the tool geometry, it is the essential (limiting) physical parameter that controls ductile-regime machining. Furthermore, if $d_{c}$ can be measured experimentally, it then provides a means to systematize the influence of machining parameters on the process itself. For example, the influence of rake angle on machining can be quantitatively described through its effect on $d_{c}$; large negative rake angles, which are often beneficial for machining brittle materials, would be expected to increase $d_{c}$.

Figure 4 shows a compilation of data for critical depth measurements on a (100) Ge crystal, plotted as $d_{c} v s$ infeed $f$. A large number of tests at different nominal cutting depths were made at each infeed, and several different samples with the same (100) face orientation were used. Other machining parameters were held constant in these tests. To eliminate a small effect of cutting direction in the (100) surface, all evaluations were made along $\langle 110\rangle$ directions. The error bars represent standard deviations for the data. An unexpected dependence of $d_{c}$ on $f$ is apparent in Figure 4. At first sight, the process model in Figure 2 suggests that the results will be independent of infeed, ie., $f$ merely determines the specific value of $\mathrm{x}$ for which $d=d_{c}$.

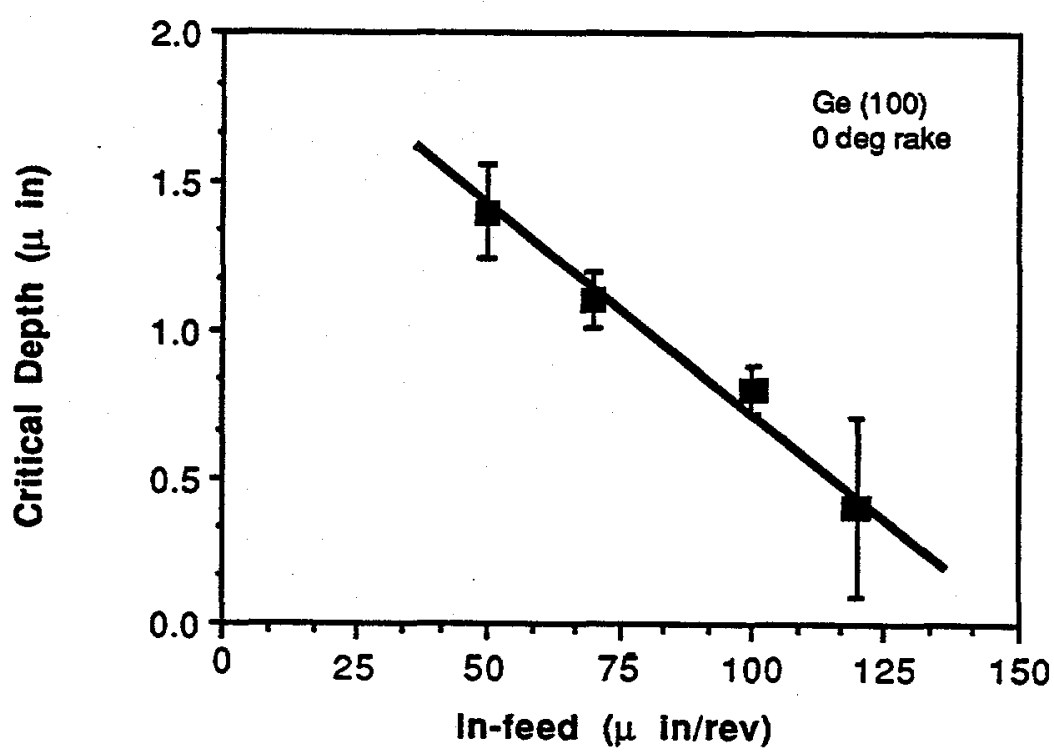

Figure 4: Plot of measured critical depths $d_{c}$ vs infeed $f$ for (100) Ge samples. The error bars represent the standard deviation. The true $d_{c}$ should be obtained by extrapolation to $f=0$.

The rationalization for the trend in Figure 4 is shown in Figure 5. Microfracture damage 
replicated into the shoulder for interrupted cuts will contain a history effect. That is, damage induced by prior cuts will overlap, and the final cut at the interruption (tool withdrawal) point will include the summation of the overlapped damage profiles on the shoulder. This clearly gives a smaller apparent value of $d_{c}$. For relatively small infeeds of the kind used for the turning experiments, the effect should be linear and thus $d_{c}$ should increase as $f$ decreases. A proper method to extract the true $d_{c}$ value then follows from data of the kind obtained in Figure 4. The correlation line is extrapolated to $f=0$, which gives the $d_{c}$ value free of any overlap or history effects. For the conditions shown in Figure 4 , the result is $d_{c}=2.2 \mu$ in $=55 \mathrm{~nm}$. A complete study was not made for $\mathrm{Si}$, however, the results obtained for $\mathrm{Si}$ indicate that $d_{c}$ will be slightly larger than for $\mathrm{Ge}$, with $d_{c} \approx$ $70 \mathrm{~nm}$ for conditions comparable to Figure 4. One of the main accomplishments of the research has been the development of an appropriate experimental method to measure $d_{c}$ along with the verification of the critical depth model approach using interrupted cutting tests.

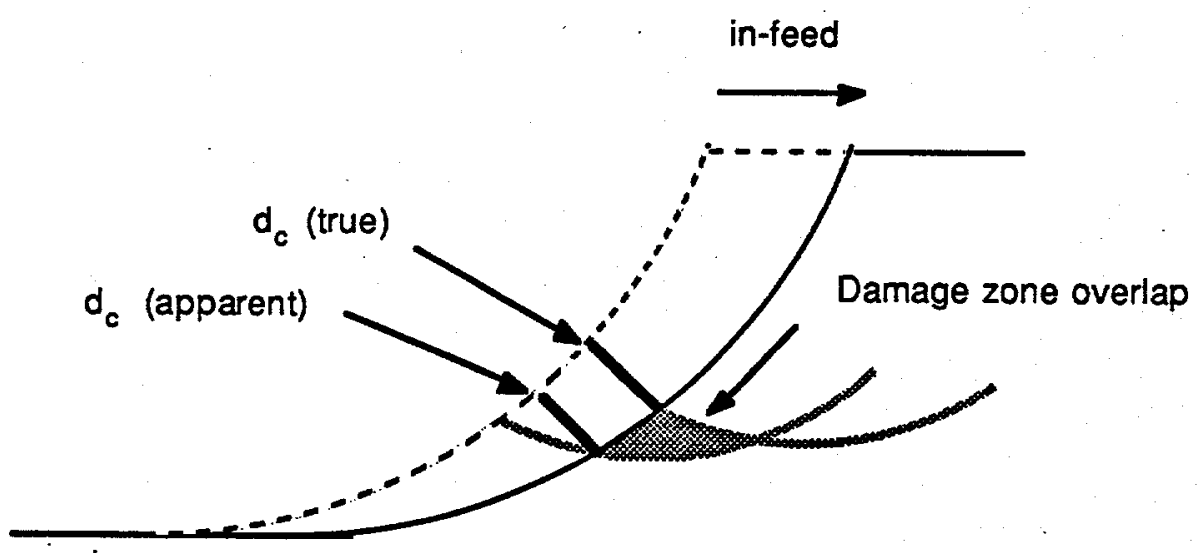

Figure 5: Schematic of overlap damage on successive infeeds. The overlaps replicate damage into the shoulder as shown. Because of this effect, the apparent $d_{c}$ is less than the true $d_{c}$. Larger infeed $f$ causes a proportional decrease in $d_{c}$ for sufficently small infeed values. 


\title{
Table 1
}

\author{
$d_{c}$ vs Rake Angle-(100)Ge \\ Rake (deg) \# tests $d_{c}(\mu$ in)

$\begin{array}{ccr}0 & 9 & .8 \pm .08 \\ -10 & 4 & .5 \pm .24 \\ -30 & 4 & 6.5 \pm 1.8\end{array}$ \\ $d_{c}$ vs Clearance Angle-(100)Ge \\ Clearance (deg) $\quad d_{c}(\mu \mathrm{in})$ \\ $6 \quad 0.6$ \\ $16 \quad 1.7$
}

The critical depth $d_{c}$ can also be used to systematize the effect of machining parameters. Table 1 shows results for $d_{c}$ measurements for varying rake angle and different tool clearance angles in $\mathrm{Ge}$ (other parameters held constant, as appropriate). For these results, $d_{c}$ was evaluated at a fixed infeed $f$ since the effects shown in Figures. 4 and 5 were not recognized in the early work. Nevertheless, the data shown in Table 1 are sufficient to show that $d_{c}$ can be used as a monitor of process parameters. For example, at sufficiently large negative rake angles, $d_{c}$ values increase substantially, consistent with the qualitative observation that Ge is easier to machine at large negative rake angles, ie; there is more ductility at larger negative rake. The implication here is that, rather than using a test-matrix approach, and often subjective assessments of performance improvements, measurement of a critical depth parameter $d_{c}$ can be used to investigate more systematically and efficiently changes in process parameters. Further application of this approach is clearly an avenue for future research.

\subsection{SUMMARY}

The completed research work has led to a successful process model for single-point diamond turning. The key feature is the combination of a critical depth $d_{c}$ for fracture initiation along with proper recognition of the tool geometry effects. Ductile-regime machining using single-point diamond turning is a viable fabrication process for brittle materials, but the term ductile regime is in a sense misleading. The actual process proceeds as combined plastic-flow fracture mechanisms. The role of material parameters have not been studied in detail for single-point turning, although equation (1) suggests the functional dependence for correlation studies, ie., evaluate $d_{c}$ for a range of different materials under standard 
machining conditions (fixed $\beta$ ), and then develop correlations using equation (1) along with appropriate measurements of $E, H$ and $K_{c}$. This approach was adopted in the grinding studies carried out by Bifano [6].

One feature of process model development for diamond turning requires comment. A predictive model for critical depth $d_{c}$ requires that the term $\beta$ in equation (1) be calculated. This can only be realized by using finite-element techniques for the plasticity problem and then combining the results with fracture mechanics. If the elastic stress fields can be obtained for given conditions (rake angle, cutting speed, etc.), then fracture mechanics should allow a lower bound on $d_{c}$ to be obtained $[7,8]$. Such modeling studies, combined with further experiments of the kind developed for this research, have the potential for providing a predictive approach for further developing a process model for single-point diamond turning, and diamond grinding fabrication processes. 


\section{References}

[1] P. Blake, T. Bifano, T. Dow and R. O. Scattergood, Amer. Cer. Soc. Bull., 67, 1083 (1988).

[2] P. Blake and R. O. Scattergood, in press, Proc. of Symp. on Machining of Ceramic Materials and Components, ACS-ASME, Dec. 1988.

[3] T. Bifano, P. Blake, T. Dow and R. O. Scattergood, Proc. Symp. on Machining of Adv. Ceramics and Components, R. E. Barks, et. al., eds., ACS-ASME (1987).

[4] P. Blake and R. O. Scattergood, to be submitted, Jour. Amer. Cer. Soc. (1989).

[5] Precision Engineering Center - NCSU, Annual Reports 1985-1987.

[6] T. Bifano, PhD Thesis, North Carolina State University (1988).

[7] S. S. Chiang, D. B. Marshall and A. G. Evans, Jour. Appl. Phys., 53, 298 (1982).

[8] P. Blake, PhD Thesis, North Carolina State University (1988). 


\title{
14 DIAMOND TURNING OF THERMOPLASTIC POLYMERS
}

\author{
Elizabeth Smith \\ Graduate Student \\ Ronald O. Scattergood \\ Professor, MTE
}

Single point diamond turning studies were made using a series of thermoplastic polymers with different glass transition temperatures. Variations in surface morphology and surface roughness were observed as a function of cutting speed. Lower glass transition temperatures facilitate smoother surface cuts and better surface finish. This can be attributed to the frictional heating that occurs during machining. Because of the very low glass transition temperatures in polymers, compared to inorganic glasses, the precision machining response can be very speed sensitive.

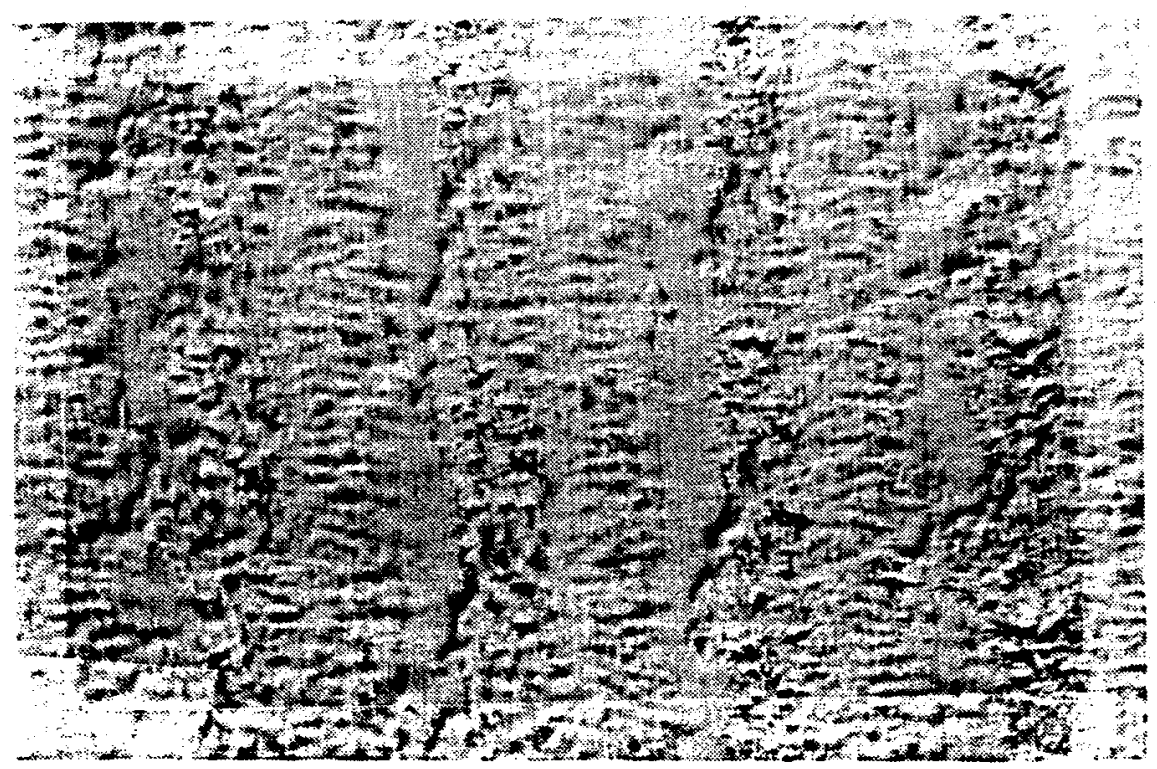




\subsection{INTRODUCTION}

The research on diamond turning of amorphous polymers has been completed. This report will address the most dramatic results of the work done on the laboratory scale diamond turning machine (PAUL). The polymers chosen to machine - polystyrene, Plexiglas, and polycarbonate - were profiled in an earlier report [1]. Cutting conditions were selected to determine the role of intrinsic heat, generated by the cutting action itself, in ductile machining. Surface characterization was by optical Nomarski microscopy and surface profilometry. The surface finish was measured using a Talysurf surface profilometer and was evaluated using the $R_{a}$ value, which is the center-line-average, or arithmetic average, roughness. Because of the importance of the polymers' thermal and mechanical properties, the surface generated from a set of conditions is totally dependent on the polymer type. The glass transition temperature $T_{g}$ is the most important property related to mechanical behavior of these polymers. Above $T_{g}$, the polymers flow in a viscous fashion whereas below $T_{g}$ they become brittle and show rather complex tearing and fracture phenomena. The approximate $\mathrm{T}_{g}$ values measured under slow strain-rate conditions for the polymers are $\mathrm{T}_{a}=155,110$, and $100^{\circ} \mathrm{C}$ for polycarbonate, Plexiglas and polystyrene, respectively. Below $\mathrm{T}_{g}$, the flow resistance (yield stress) of polycarbonate is appreciably less than that of the other two polymers. More details on the mechanical properties will be reported elsewhere [1]. The cutting speed is the machining parameter that most strongly controls the amount of intrinsic heat produced and associated softening.

\subsection{EXPERIMENTAL RESULTS}

\subsubsection{Polystrene}

Having the lowest glass transition temperature of the three polymers, polystyrene exhibits the most response to an increase in cutting speed from. 100 to 500 RPM. Using an infeed rate of $50.8 \mu \mathrm{m}$ per revolution $(2000 \mu \mathrm{in} / \mathrm{rev})$ at a rake angle of $0^{\circ}$, the $100 \mathrm{RPM}$ surface, shown in the top of Figure 1, has a rugged topography, with tearing at the feed marks. At a cutting speed of 500 RPM, with other parameters held constant, the surface topography is smoother, as seen in the bottom of Figure 1. There appears to be more ductile machining at the higher speed. The surface finish parameter, given in Table 1, confirms this decrease in roughness.

\begin{tabular}{|c|c|c|}
\hline $\begin{array}{c}\text { Tool Nose Radius, } \\
c m(i n)\end{array}$ & $\begin{array}{c}\text { Cutting Speed, } \\
\text { RPM }\end{array}$ & $\begin{array}{c}R_{a}, \\
\mu m(\mu i n)\end{array}$ \\
\hline 0.3175 & 500 & $0.163(6.4)$ \\
$(0.125)$ & 100 & $0.381(15)$ \\
\hline
\end{tabular}

Table 1: Surface Roughness Values for Polystyrene Cuts 


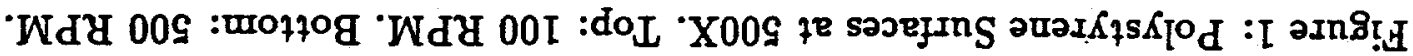
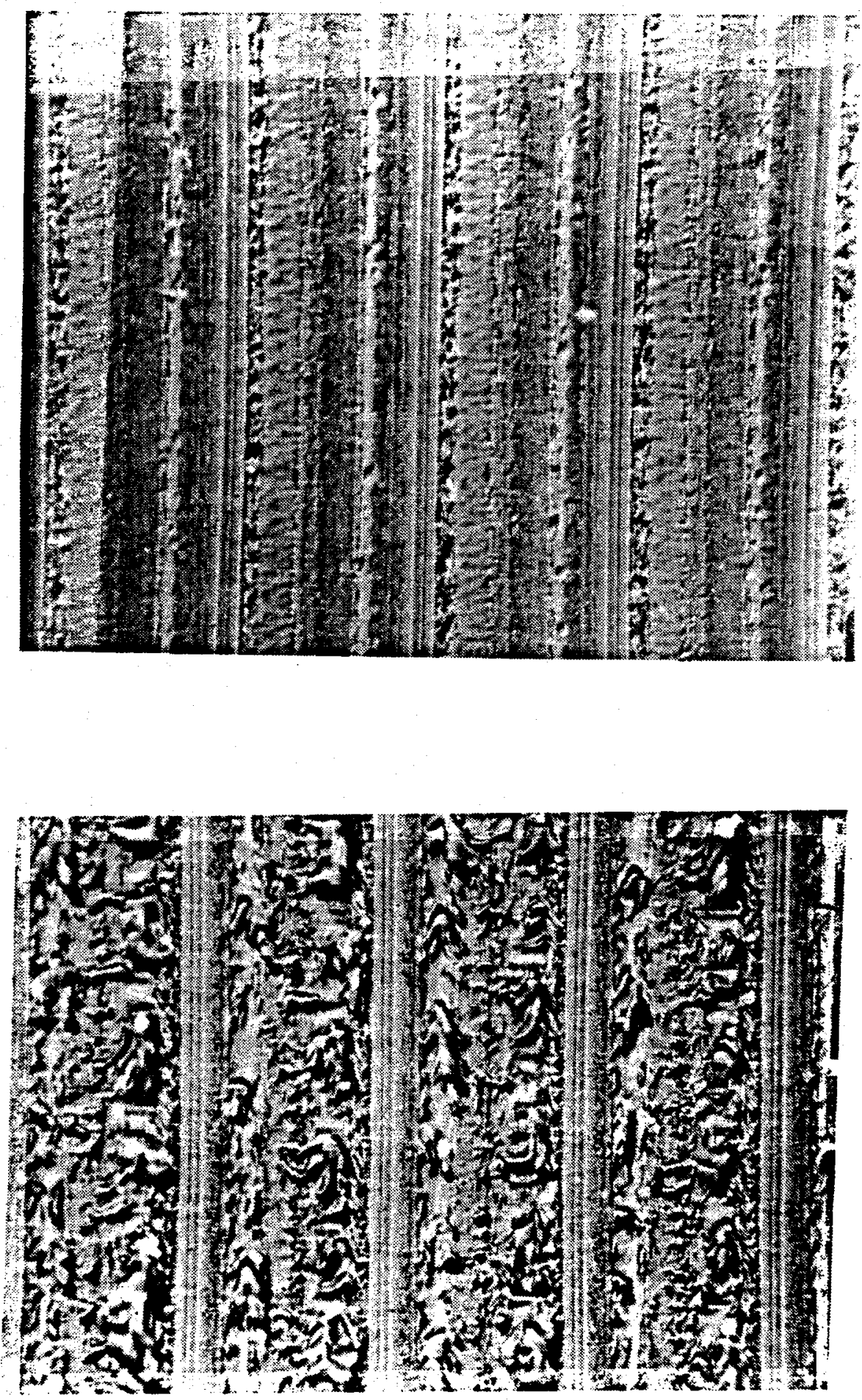


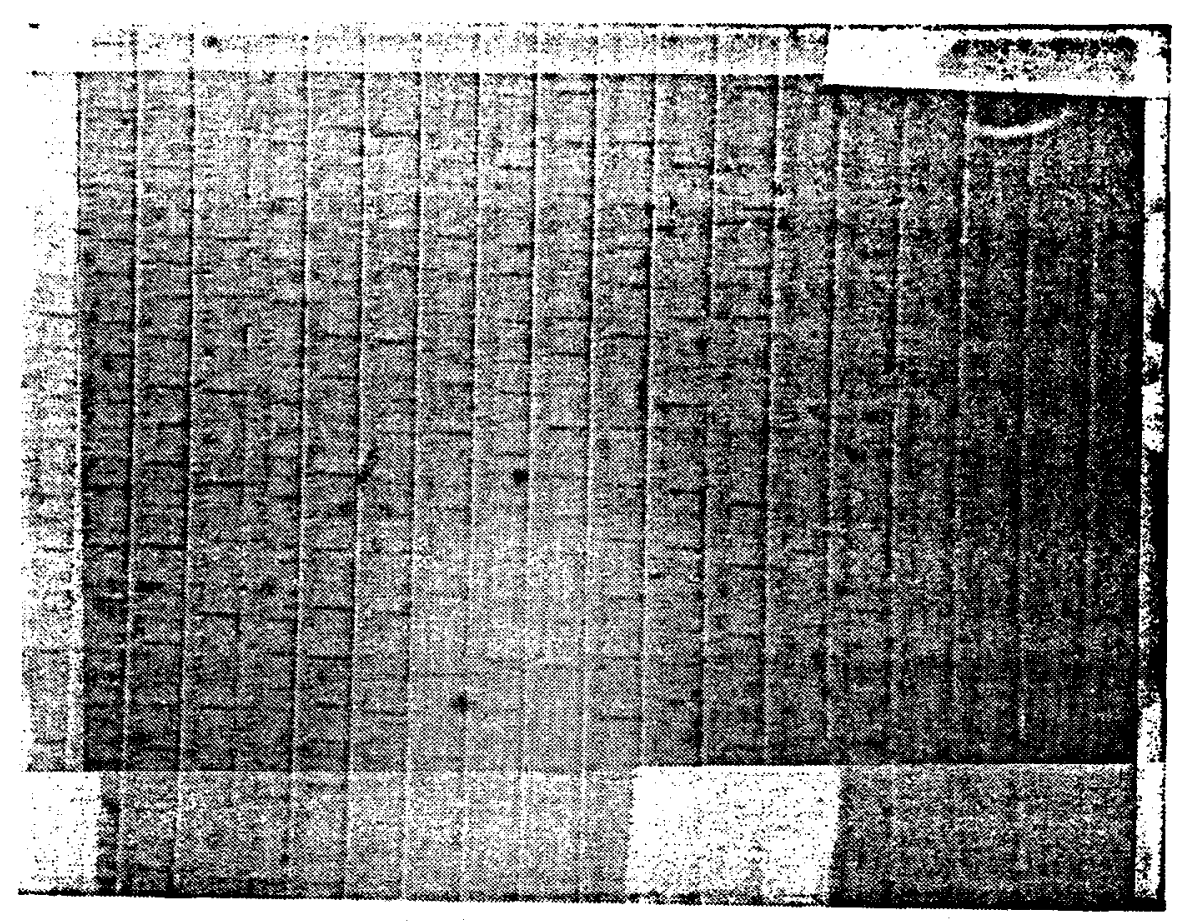

Figure 2: Plexiglas Surface Machined at 2500 RPM at 500X.

\subsection{PLEXIGLAS}

The best surface finish was obtained on Plexiglas (polymethyl methacrylate) at the highest cutting speed and the medium infeed rate. An $R_{a}$ value of $0.015 \mu \mathrm{m}(0.58 \mu \mathrm{in})$ was measured on the Plexiglas surface cut with the following conditions: speed $=2500$ RPM, infeed $=10.16 \mu \mathrm{m} / \mathrm{rev}(400 \mu \mathrm{in} / \mathrm{rev})$, tool nose radius $=0.0762 \mathrm{~cm}(0.030 \mathrm{in})$, and rake angle $=$ $0^{\circ}$. The surface topography, shown in Figure 2, exhibits the theoretical finish expected for turned surfaces. Plexiglas has the median transition temperature and the highest tensile strength and elastic modulus of the three polymers. Since these properties control the material response to the cutting action, Plexiglas must have the proper combination to machine a ductile surface.

\subsection{POLYCARBONATE}

With the highest transition temperature and ductility of the three polymers, polycarbonate responds differently than the others. Using the same parameters as mentioned above for Plexiglas, the machined surface on polycarbonate has an unusual feature, as seen in Figure 3. There is an apparent alignment of structural features lying nearly orthogonal to the cutting directions, as observed in STM studies on machined metals (Section 19). Another point about this surface, it has an $R_{\sigma}$ value of $0.042 \mu m(1.31 \mu i n)$. This small roughness value was certainly not expected from the appearance of the topography. 


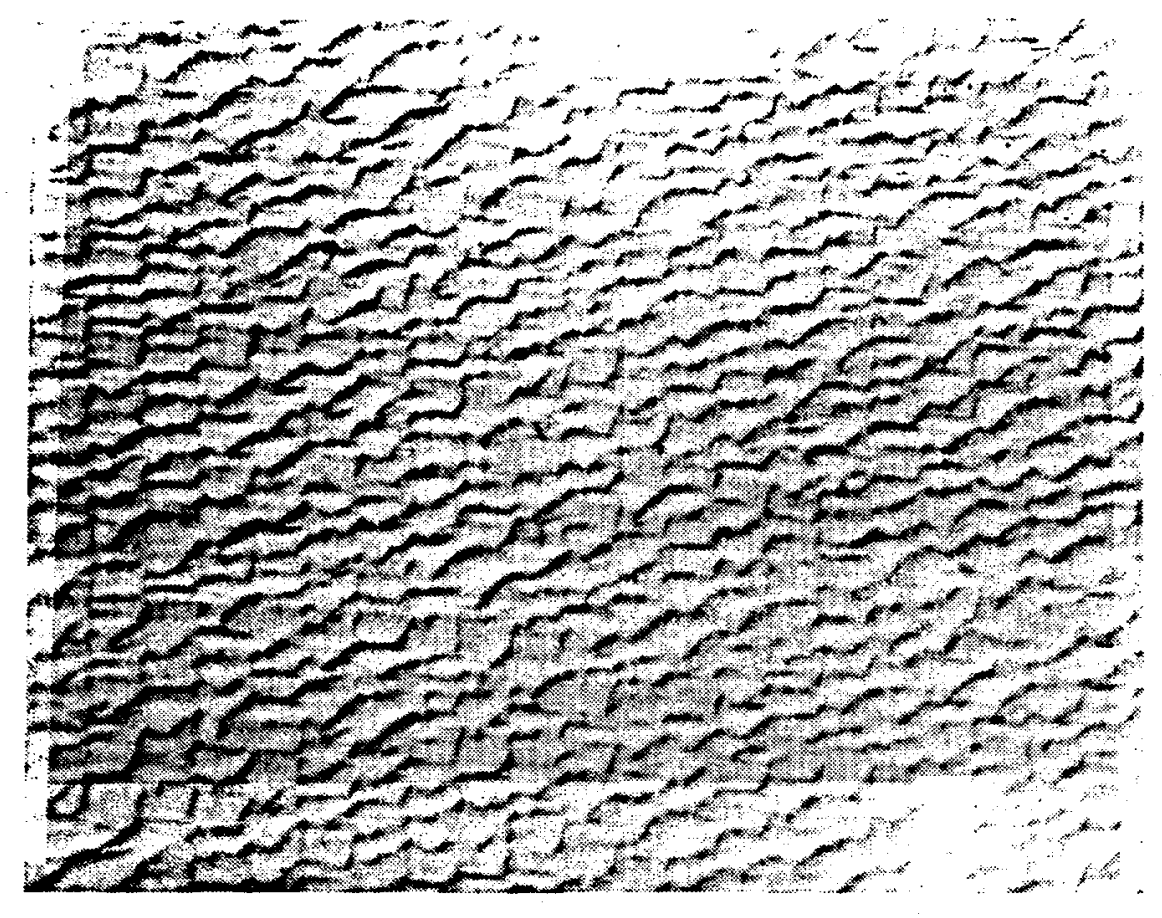

Figure 3: Polycarbonate Surface Machined at 2500 RPM at 500X.

\subsection{CONCLUSIONS}

The polymer type determines the finish and topography of the machined surface. The differences in the thermal properties of these polymers were proven by comparing their responses at identical cutting conditions. Polystyrene, having the lowest glass transition temperature, was most affected by the increase in intrinsic heat generated at 500 RPM, as compared to 100 RPM. Plexiglas and polycarbonate did not thermally soften under the higher speed, due to their higher transition temperatures. The mechanical properties of the polymers were also found to affect the machined surface, as in the case of the drastic change in topography from Plexiglas to polycarbonate, under the same conditions. The higher ductility of polycarbonate appears to be the reason for the unusual surface features shown in Figure 3. The combination of high tensile strength and elastic modulus found in Plexiglas must contribute to the smooth surface produced. These materials appear to have a unique response to the cutting process. Further details are presented in a forthcoming publication [2]. 


\section{References}

[1] Blake, P., Smith, E., Scattergood, R., "Single Point Turning of Brittle Materials", Precision Engineering Center Annual Report, Vol. 5, p. 245-247, Dec. 1987

[2] Smith, E. and Scattergood, R. "Single-Point Turning of Thermoplastic Polymers", to be submitted to Precision Engineering, 1989. 


\title{
15 DIAMOND TURNING OF GLASS
}

\author{
Winston Scott Blackley \\ Graduate Student \\ Ronald O. Scattergood \\ Professor, MTE
}

A new research initiative will be undertaken to investigate the critical cutting depth concepts for single point diamond turning of brittle, amorphous materials. Inorganic glasses and a brittle, thermoset polymer (organic glass) are the principal candidate materials. Interrupted cutting tests similar to those done in earlier research are Ge and Si crystals will be made to obtain critical depth values as a function of machining parameters. The results will provide systematic data with which to assess machining performance on glasses and amorphous materials.

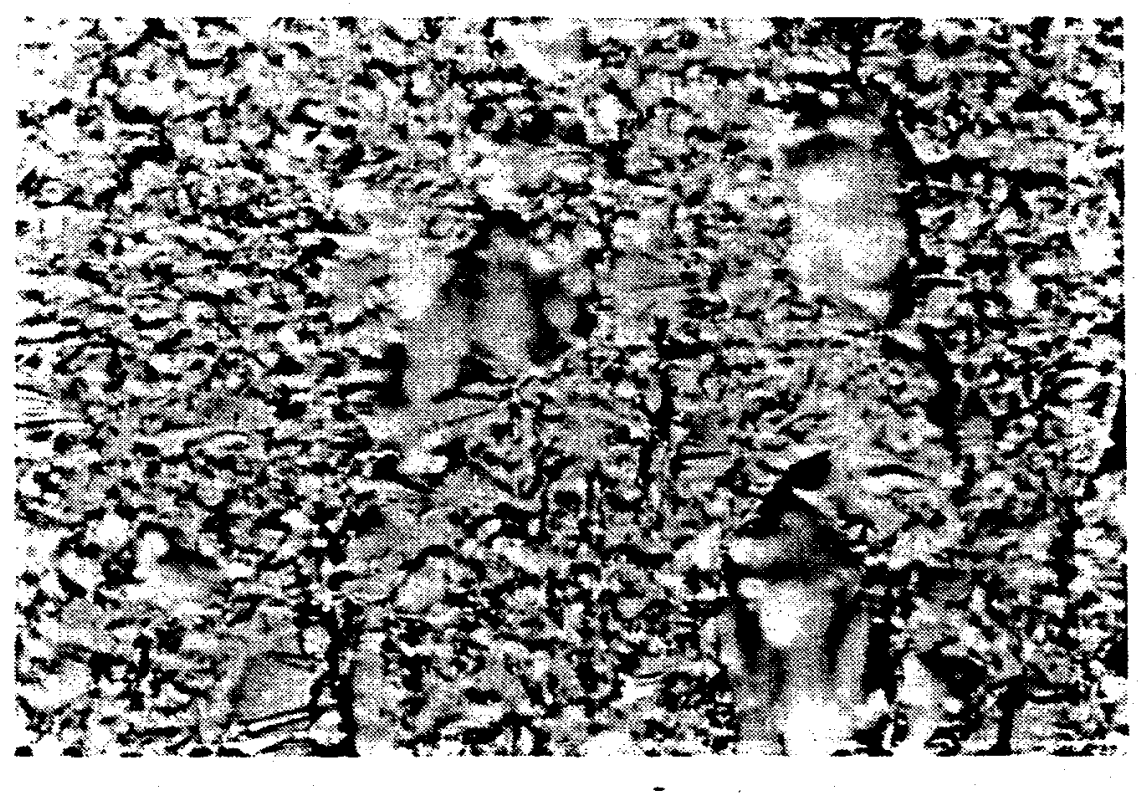




\subsection{INTRODUCTION}

Diamond turning of glass has been of interest to many researchers in the optics industry. The well-defined geometry associated with single-point diamond turning is an advantage for producing parts with a complex form. In order to produce an optically useful surface the glass must be removed in a ductile manner. However, brittle materials behave such that the fracture stress is less than the yield stress.

$$
\sigma_{F}<\sigma_{Y}
$$

To successfully turn glass the previous inequality must be reversed so that [1]

$$
\sigma_{Y}<\sigma_{F}
$$

This reversal takes place only at the tip of the tool resulting in small amounts of material and is affected by many different variables(glass composition, cutting rate, temperature, environment, etc.). The mechanism of ductile removal in glass and other amorphous materials is inhomogeneous shear [2] which tends to occur in the presence of network modifiers [3]. Modifiers tend to weaken the network structure of glass which promotes ductile removal by providing more paths on which inhomogeneous shear may occur. Of all the cutting parameters, the one that appears to have a very significant effect on the machining of glass is the rake angle of the tool. In brittle materials the rake angle is optimized between $-30^{\circ}$ to $-40^{\circ}$ [2]. Work done at Lawrence Livermore Laboratories indicates that glass with network modifiers lends itself to diamond turning more than does fused silica which has no network modifiers.

In itself, diamond turning of glass may not be economically desirable for two main reasons; 1) the amount of material removed is too small for economically producing parts and 2) tool wear is high leading to inconsistencies in the turning process. For these reasons the diamond turning experiments on glass will be performed with the aim of using the information gained to model the material removal that takes place when diamond grinding glass. These experiments will be evaluated using a method similar to the experiments performed on germanium and silicon [4] using the critical chip thickness as process model parameter.

\subsection{RESEARCH PLAN}

Initial experiments will be performed using SF-6 glass which is a silicate glass with a large amount of lead(modifier). This glass was selected because of previous success turning this glass at Lawrence Livermore Laboratories. These experiments will consist of first 
facing the glass samples ( 3 inches in diameter and $1 / 2$ inch thick) and then turning the glass in a final cut. After a smooth, fracture-free reference surface is obtained, a series of interrupted cutting tests will be made on the sample following the method developed by Blake [4], as described in Section 13. These tests allow the critical depth to be measured as a friction of machining parameters. One important aspect of the research will be to extend this testing methodology to non-crystalline systems and to critically assess the role of machining parameters.

In the event that unacceptable results are produced from the glass turning experiments, for example, if a smooth reference surface cannot be turned, work will be performed on other brittle amorphous materials. The first of the other materials to be examined will be CR39(diallyl diglycol carbonate). CR-39 is a thermoset polymer that is highly scratch resistant and when diamond turned shows fracture morphologies similar to germanium, silicon or glass. This material is chosen as an alternative for three reasons. First, like glass, CR-39 is an amorphous material and will perform similarly to glass under comparable cutting conditions. Second, CR-39 has a much lower $T_{g}, 85^{\circ} \mathrm{C}$, than does glass (typically $T_{g}>$ $500^{\circ} \mathrm{C}$ ) and will show thermal effects due to high-speed machining conditions. The last reason for machining CR-39 is that it also is used as an optical material and precision machining has practical applications. It is the aim of this project to use CR-39 as a model amorphous brittle material. An extended set of critical cutting experiments will be performed, similar to that of Blake. However, building upon the past results, it will now be possible to systematically investigate effects such as rake angle, tool radius, cutting speed and coolant using the critical depth model approach.

Another material of related interest is a two phase glass-ceramic Macor produced by Corning. This material consist of approximately $45 \%$ residual glass and $55 \%$ crystalline ceramic and is presently machinable using a carbide tool. Diamond turning experiments will be performed to see how a complex two-phase system adapts to precision machining process.

In all cases once a desirable surface finish has been achieyed to serve as a reference surface, a systematic study of the machining parameters will be made. The parameters of interest will be the rake angle, cutting speed, feed rate and the depth of cut. From the results of the analysis of the critical chip thickness for brittle materials(germanium and silicon)[4] two very important parameters should be the rake angle and the feed rate.

\subsection{DISCUSSION}

To date, turning experiments using the glass have been initiated and have produced the following results. The first experiment was performed in order to face the sample using a $0^{\circ}$ rake angle tool with a depth of cut equal to $5 \mu \mathrm{m}$ and a feed rate of $1 \mu \mathrm{m} / \mathrm{rev}$. This fracture surface is shown in Figure 1 and is an example of a classic fracture morphology. Ductile removal of the glass was not seen after inspection of the surface. Figure 2 shows the fracture for another cut made using a $0^{\circ}$ tool to face the sample and then a $-30^{\circ}$ rake 
angle, a $5 \mu \mathrm{m}$ depth of cut and a $1 \mu \mathrm{m} / \mathrm{rev}$ feed rate for the final cut. Larger scale fracture is evident in this picture and may be the result of the damage incurred when the facing cut was made. Future experiments will be performed using only the $-30^{\circ}$ tool for the cuts in hope of eliminating the subsurface damage which hampers ductile material removal. Preliminary work will begin on CR-39 material, which has just been procured.

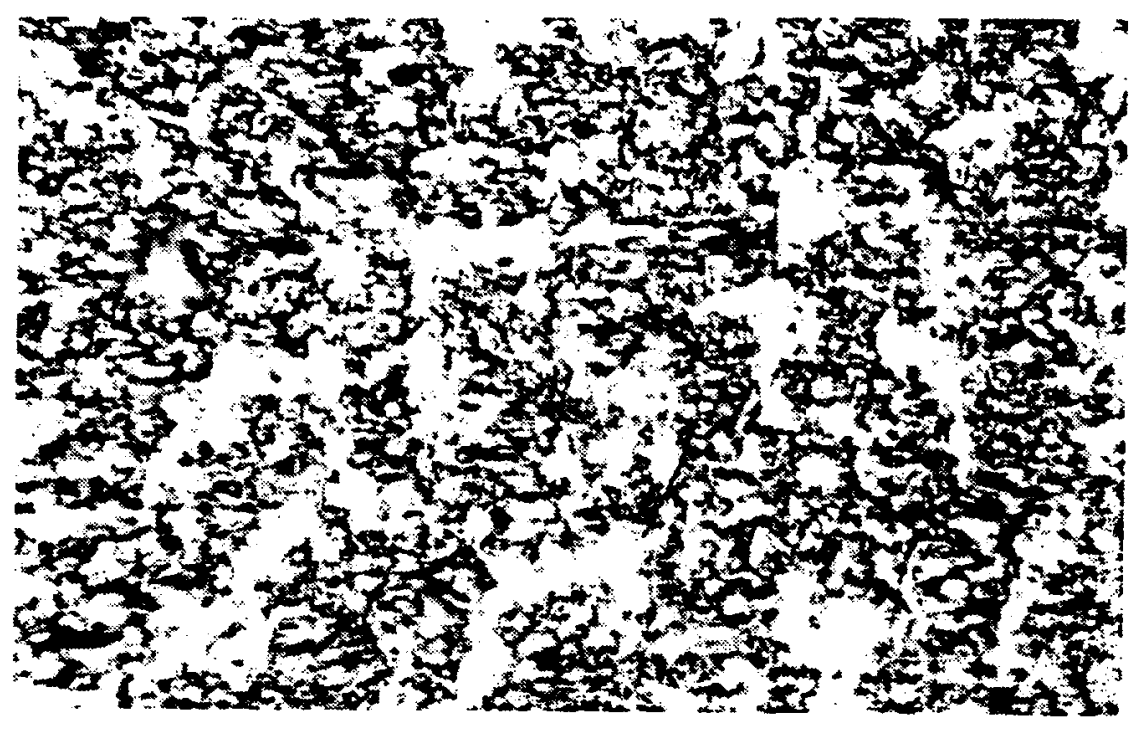

Figure 1: Example of Classic Fracture Morphology in Machined Glass (500x)

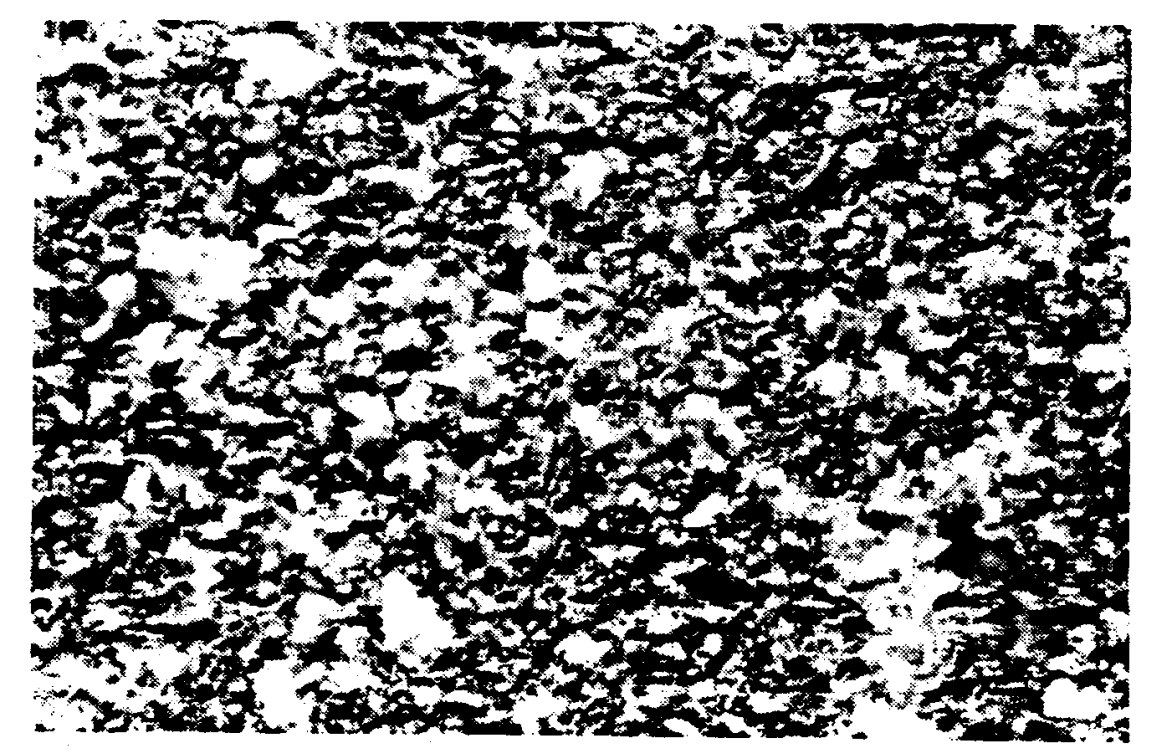

Figure 2: Fractured Glass Surface Due to Facing Operation (500x) 


\section{References}

[1] Giovanola, J.H. and Finnie, I., "On the Machining of Glass," Journal of Materials Science, Vol.15, 1980, p. 2508-2514.

[2] Sanger, G.M. and Baker, P.C.,Diamond Turning of Glass, Final Report, Lawrence Livermore National Laboratory, University of California, 1980.

[3] Peter, K., "Densification and Flow Phenomena of Glass in Indentation Experiments," Journal of Non-Crystalline Solids, Vol. 5 1970, p. 103-115.

[4] Blake, P.N. "Ductile-Regime Turning of Germanium and Silicon", PhD Thesis, North Carolina State University, 1988. 



\title{
16 PRECISION DIAMOND GRINDING OF CERAMICS AND GLASS
}

\author{
Stan Smith \\ Graduate Student \\ Harlow Paul \\ Graduate Student \\ Ronald O. Scattergood \\ Professor, MTE
}

A new research initiative will be undertaken to investigate the effect of machine parameters and material properties on precision diamond grinding of ceramics and glass. The critical grinding depth to initiate the plastic flow-to-brittle fracture regime will be directly measured using plunge-grind tests. This information will be correlated with machine parameters such as wheel bonding and diamond grain size. Multiaxis grinding tests will then be made to provide data more closely coupled with production technology. One important aspect of the material property studies involves measuring fracture toughness at the very short crack sizes commensurate with grinding damage. Short crack toughness values can be much less than the long-crack toughness values measured in conventional fracture tests.

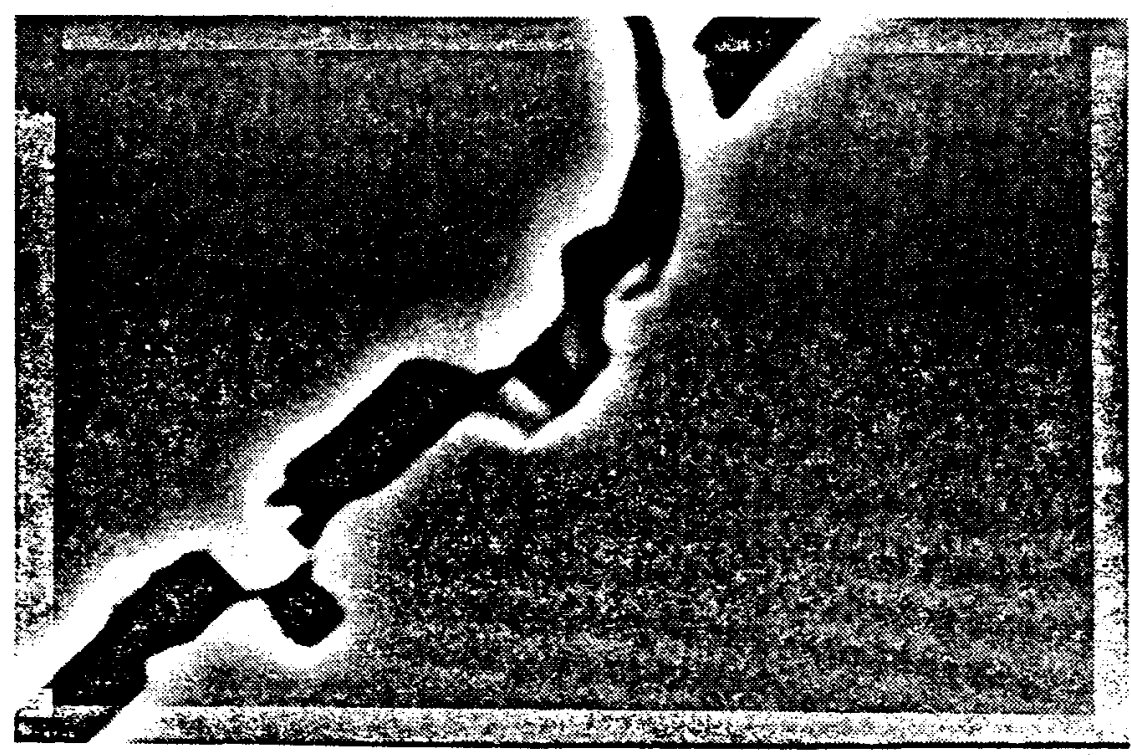




\subsection{INTRODUCTION}

Precision grinding of brittle ceramics and glasses in which the major mode of material removal is ductile is possible. Precision ductile regime grinding of brittle materials is important in the production of optical components characterized by high surface contour accuracy and smooth surface finish. Evidence for the ductile removal of material from normally brittle materials has been observed by diamond lapping of ceramics which results in an extremely smooth finish. Bifano has shown that for a wide range of ceramics and glasses, ductile regime grinding can be attained when the grinding machine possesses high stiffness, low wheel runout, vibration isolation and precisely controlled material feed-rate [1]. Materials properties such as fracture toughness, elastic modulus and hardness were shown in Bifano's work to be important in the determination of the critical depth of cut at which a transition from brittle to ductile grinding occurred. However, experimental values of critical depth of cut obtained did not always correlate well with the proposed model, especially for materials that are likely to exhibit decreasing toughness with decreasing scale of material removal. The work to be carried out will look specifically at the variability of precision ductile regime grinding with mechanical properties of a wide range of glasses and ceramic materials.

\subsection{RESEARCH PLAN}

The initial phase of research will be to obtain selected ceramics and glasses with varying values of fracture toughness, hardness and elastic modulus. With materials characterized by a wide range of properties, it will be possible to correlate these properties to the performance of a precision ductile grinding regime apparatus. Initially, the plunge-grinder (PEGASUS) designed by Bifano will be used by $\mathrm{H}$. Paul to carry out grinding experiments. Later, a precision grinding machine capable of grinding flat surfaces will be fitted to the diamond turning machine and will be used to carry out experiments where the chip geometry will be altered due to cross-feed of the workpiece.

Based on analyses of the indentation of brittle materials, the critical depth to which an indenter can penetrate without producing irreversible fracture is $[2,3]$

$$
d_{c}=\beta\left[\frac{K_{c}}{H}\right]^{2}\left[\frac{E}{H}\right]
$$

where $\beta$ is a constant, $E$ is elastic modulus, $H$ is hardness, and $K_{c}$ is fracture toughness. Fracture toughness will be measured by an indentation/four-point bend method designed to give toughness values which approach the short-crack limit which is the relevant value for the material removal rates encountered in ductile grinding.

Development of the test procedure to measure fracture toughness as a function of crack 
size is not a standard nor simple test procedure. Methods will be developed to polish the four-point bend bars used for testing purposes. Each test sequence requires $60-80$ bend bars. In addition, data analysis and deconvolution methods must be developed to analyze the test results and to extract the crack-resistance curve (R-curve) [4].

The data reduction method for analyzing the indentation four-point bend tests will be developed using fracture mechanics. The total stress intensity factor for the indentationbend test is

$$
K=\alpha \sigma c^{\frac{1}{2}}+\beta P c^{\frac{-3}{2}}
$$

where $\alpha$ and $\beta$ are constants, $\sigma$ is the bend stress, $P$ is indentation load and $c$ is crack length [4]. The first term corresponds to the bending stress field while the latter is due to residual stress in the indentation zone. $\alpha$ can be obtained in handbooks, but $\beta$ must be calibrated via experiment. Let $T=T(c)$ be the crack-size dependent fracture toughness. The idea of the indentation-bend test is that the load $P$ generates an initial crack, which can be changed by varying $P$, and the failure stress $\sigma_{f}$ is related to the toughness at that crack size. The conditions that determine failure at critical crack size $c=c *$ and stress $\sigma=\sigma_{f}$ follow from the conditions

$$
\begin{array}{r}
K=T \\
d K / d c=d T / d c
\end{array}
$$

The solution to equations (2) and (3) requires that a shape function $T=T(c)$ be input. This can be arbitrary or an assumed form based on physical models. In any case, the solution is facilitated by solving the following mathematically equivalent problem. From Equation (2) at fixed $P$,

$$
d \sigma / d c=\frac{1}{2}(K / \alpha) c^{\bar{\zeta}} 2+(c / \alpha)(d T / d c)+2(\beta / \alpha) P c^{3}=0
$$

The root $c=c *$ from Equation (4) corresponds to a maximum in $\sigma$. Substitution of $c^{*}$ into equation (2) for $K=T$ then gives $\sigma_{f}$ for any $P$ and any choice of $T=T(c)$. The data reduction proceeds by calculating $\sigma_{f}$ for all loads $P$ used in the test and then minimizing the residuals $R=\sum_{\text {over } P}\left\{\left(\sigma_{f}^{\text {meas }}-\sigma_{f}^{\text {calc }}\right)\right\}^{2}$ with respect to the $T(c)$ shape function parameters. Considerable care must be exercised in obtaining confidence limits for the fitted $T(c)$ curve if it is to be used for predictive analysis.

A four-point-bend fixture has already been designed and fitted to an ATS mechanical testing machine. The fixture and testing machine are shown in Figures 1 and 2 respectively. 
Vickers indentations of various loads will be introduced into polished surfaces of test bars by a new Zwick macro/microhardness testing machine which allows loads from $10 \mathrm{~g}$ to $30 \mathrm{~kg}$. Hardness of the materials will also be determined by use of the hardness tester. The elastic modulus of the materials will be determined using acoustic wave analysis.

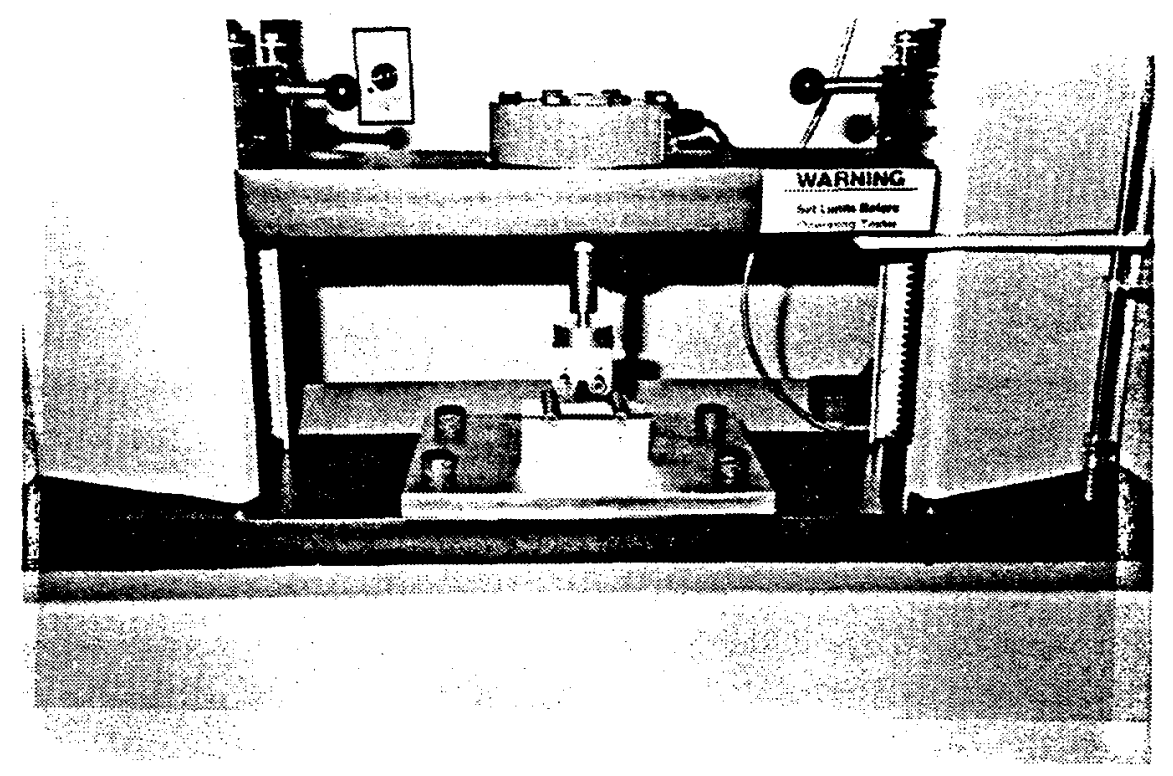

Figure 1: Four-point-bend fixture.

The ductile to brittle grinding transition will be characterized initially by analysis of the ground surfaces. Fracture sites as observed by SEM or Nomarski will be converted to a percent of fracture on the ground surface. Later, image analysis methods will be employed to expect the amount of fracture surface analysis.

\subsection{DISCUSSION}

Correlation of short-crack toughness and appropriate scale values of hardness and elastic modulus should provide insight into the modeling of ductile material removal processes during precision grinding. The determination of the short-crack fracture toughness of materials will provide direct information on the properties relevant to precision grinding. In many cases the high toughness of advanced ceramics pertains only to large cracks, and is not relevant for precision grinding. Often $R$-curve effects [4] result in short-crack toughness values being significantly less than the value measured for long cracks. The data will also help select optimal materials for improved grinding performance. 


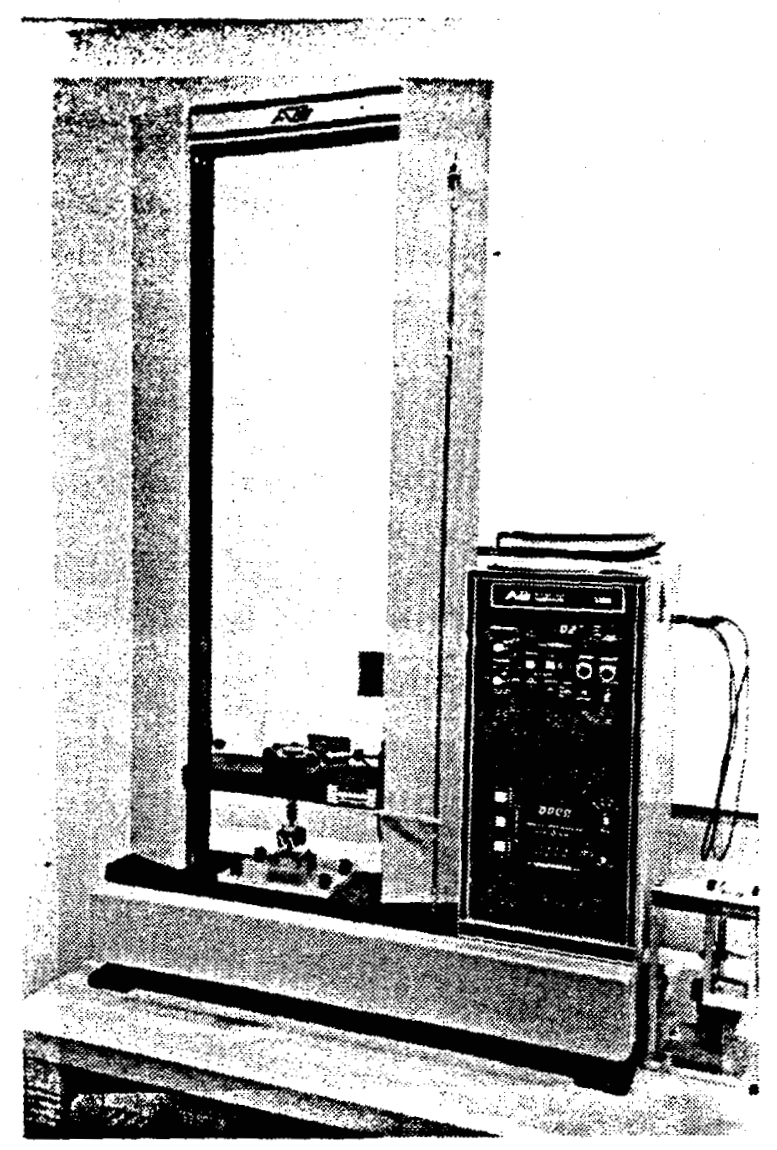

Figure 2: ATS testing machine with four-point-bend fixture.

Design of a multi-axis precision grinder will facilitate the grinding of flats on brittle materials. The complex chip geometry generated by the grinding wheel cross-feed will require modification of the model of critical grinding depth formulated by indentation studies. In addition, the precision surfaces produced by grinding of flats on four-point bend test bars can be used to assess the grinding damage using methods based on Weibull statistics. 


\section{References}

[1] Bifano, Thomas Gary, "Ductile-Regime Grinding of Brittle Materials", PhD Thesis, (1988).

[2] Lawn, B.R., Jensen,T, Arora, A., J. Matl. Sci. Lett., [11], p. 575, (1976).

[3] Marshall, D.B., Lawn, B.R., Microindentation Techniques in Materials Science and Engineering, p. 26, (1985).

[4] Broek, David, Elementary Engineering Fracture Mechanics, Fourth Edition, Martinus Nijhoff Publishers, Dordrecht, The Netherlands, p.22 (1986). 


\title{
17 THE EFFECTS OF MACHINE PARAMETERS ON RESIDUAL STRESS DETERMINED USING MICRO-RAMAN SPECTROSCOPY
}

\author{
R. G. Sparks and W. S. Enloe \\ Graduate Students \\ Michael A. Paesler \\ Associate Professor, Physics
}

The effects of machine parameters on residual stresses in single point diamond turned silicon and germanium have been investigated using micro-Raman spectroscopy. Residual stresses were sampled across ductile feed cuts in $<100>$ silicon and germanium which were single point diamond turned using a variety of feed rates, rake angles and clearance angles. High spatial resolution micro-Raman spectra ( $1 \mu \mathrm{m}$ spot) were obtained in regions of ductile cutting where no visible surface damage was present. The use of both $514.5 \mathrm{~nm}$ and 488.0nm excitation wavelengths, by virtue of their differing characteristic penetration depths in the materials, allowed determinations of stress profiles as a function of depth into the sample. Previous discussions have demonstrated that such Raman spectra will exhibit asymmetrically broadened peaks which are characteristic of the superposition of a continuum of Raman scatterers from the various depths probed. Depth profiles of residual stress were obtained using computer deconvolution of the resulting asymmetrically broadened raman spectra.

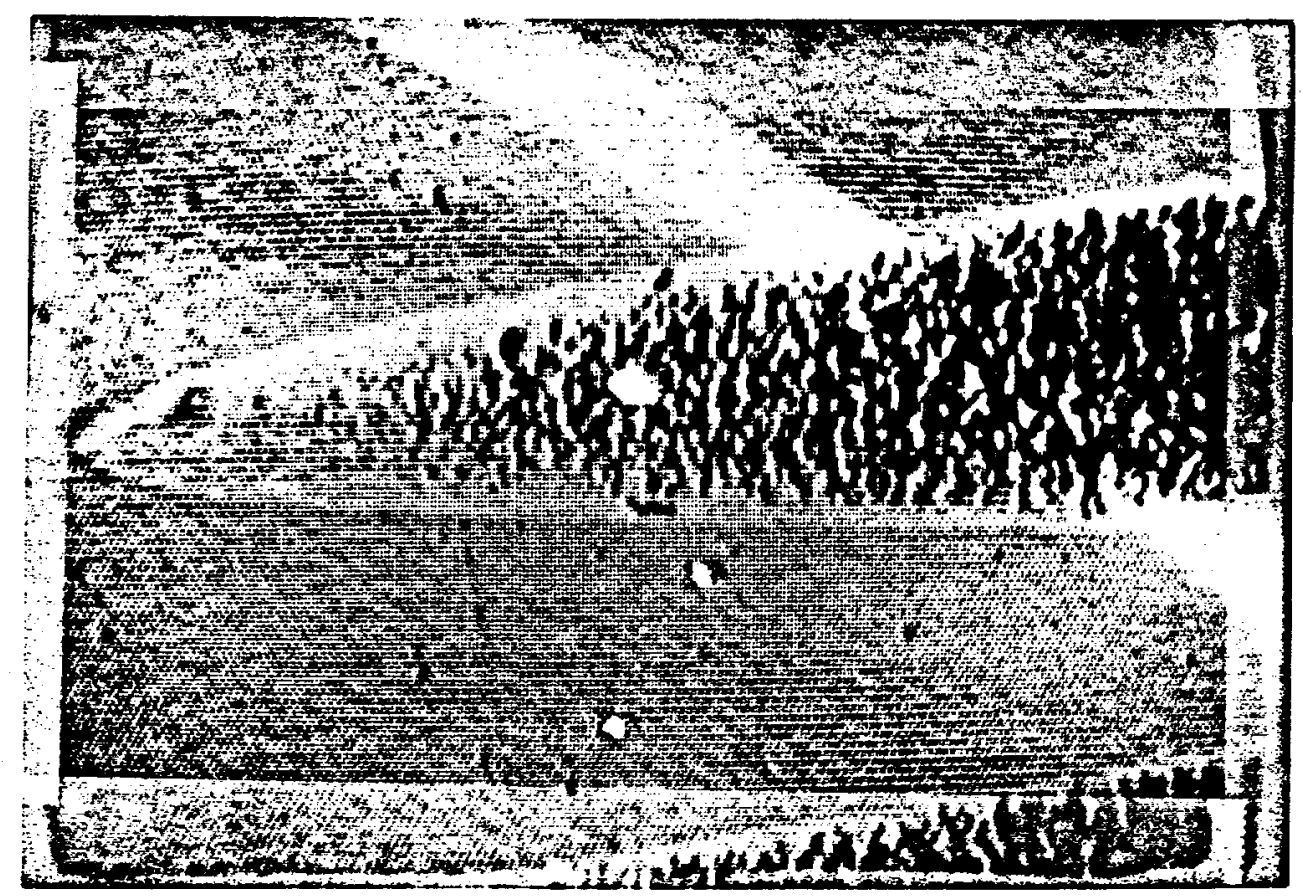




\subsection{INTRODUCTION}

Raman spectroscopy has been proven to be a widely useful technique for measuring a broad range of material properties such as phase and composition identification, the determination of long and short range order, impurity configurations, and superlattice properties [1]. It has been demonstrated that the Raman spectra of crystals is sensitive to the strain in the lattice and can be used to measure stress in such samples. The Raman microprobe technique has been used to map local stresses in patterned islands of laser recrystallized silicon and bulk glass [2], in epitaxial silicon on sapphire substrates [3], and in silicon interfaces on quartz [4]. Initial measurement determinations of residual stresses at the PEC for single point diamond turned silicon and germanium were reported in [5]. To more fully understand the role of machine parameters and their effects on the physics of the material removal process, a variety of machined samples have been studied. These samples were machined using various feed rates, rake and clearance angles, and spindle speeds. Raman spectra were obtained as outlined in earlier reports $[5,6]$, and the resulting shifts and broadened peaks were analyzed using the computer deconvolution techniques discussed in Section 4.

\subsection{EXPERIMENTAL RESULTS}

Machine parameters such as feed rate (1 to $2.5 \mu \mathrm{m} / \mathrm{rev}$ ) and spindle speed (1000 and 2500 RPM) showed little differences in their spectra and the resulting surface stresses were always compressive $\left(-10^{\circ}\right.$ rake angle, $6^{\circ}$ clearance angle). The depth for the transition from compressive to tensile stress was approximately constant for all feed rates and spindle speeds and occurred at a depth of $0.65 \pm 0.01 \mu \mathrm{m}$. The results indicate that the effects of these particular feed rates and spindle speeds on the residual stress field are not significant. Further investigation using a wider range of feed rates and spindle speeds is therefore indicated to more fully understand the effects of these parameters. Quite marked differences are evident, however, in the case of rake and clearance angles. Germanium samples turned using $0^{\circ},-10^{\circ}$, and $-30^{\circ}$ rake angles $(2.5 \mu \mathrm{m} / \mathrm{rev}$ feed rate, $1000 \mathrm{RPM}$ spindle speed, $6 \mu \mathrm{m}$ depth of cut, $6^{\circ}$ clearance angle) show clear evidence of superposition broadening with significant variations in the spectra obtained using $514.5 \mathrm{~nm}$ and $488.0 \mathrm{~nm}$ probes (Figures 1 and 2). These differences indicated changes in both the magnitude of the residual surface stresses and the depth at which the stress changed from compressive to tensile. Models of stress obtained from hardness indents associate such rapid sign changes with a transition from a plastic to elastic deformation zone [7]. Differences in the maximum magnitudes of the compressive and tensile stresses were also noted in the samples under consideration.

Depth profiles of residual stress calculated from the Raman data for $0^{\circ}$ and $-30^{\circ}$ rake angles are shown in Figure 3. The residual compressive surface stress was measured to be 1.1 and $1.3 \pm 0.1 \mathrm{kbar}$ respectively for the $0^{\circ}$ and $-30^{\circ}$ cases. The compressive stress increased to a maximum of $3.5 \pm 0.1 \mathrm{kbar}$ at a depth of $0.06 \pm 0.01 \mu \mathrm{m}$ for the $0^{\circ}$ rake angle while the 


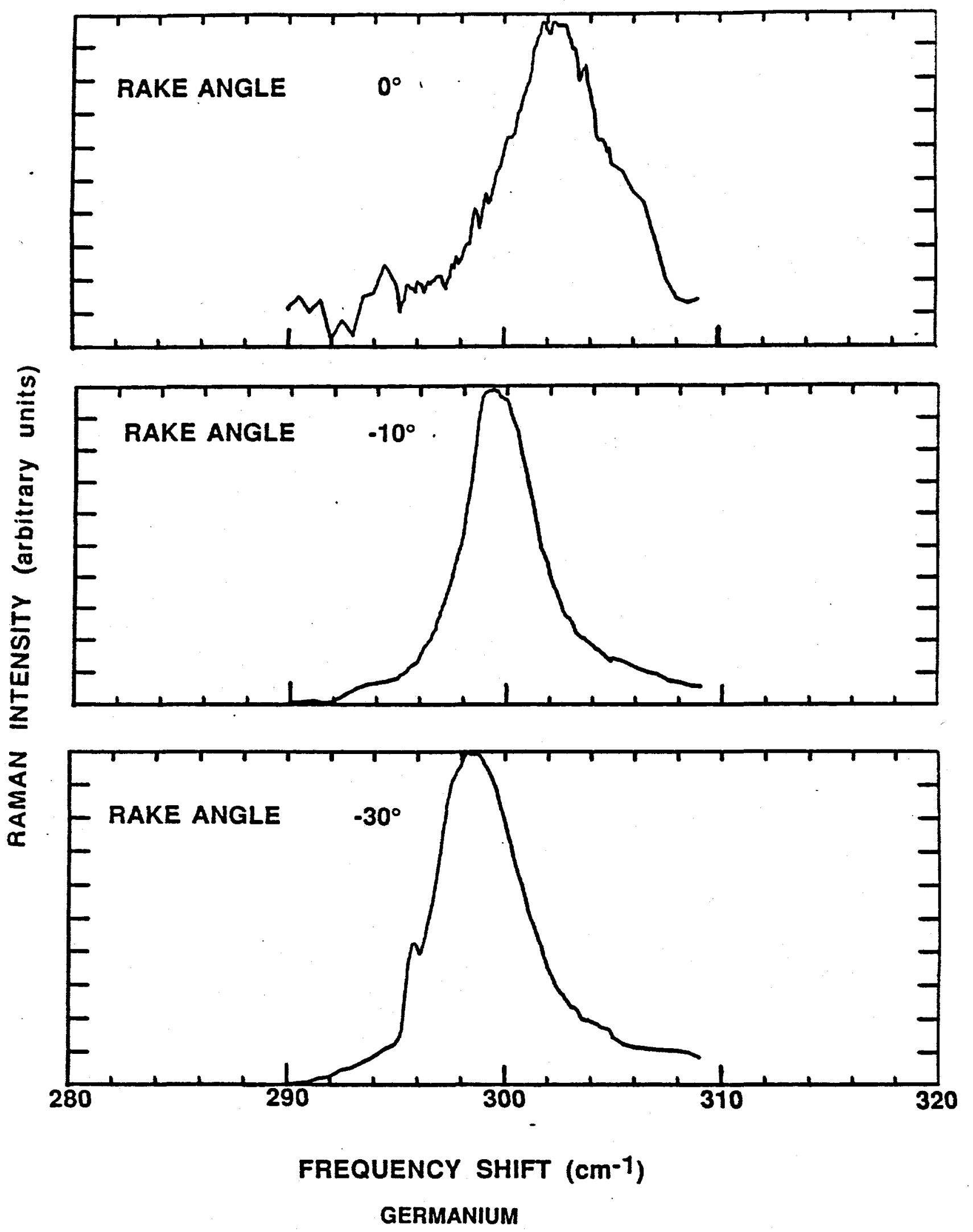

$514.5 \mathrm{~nm}$

Tigure 1: Raman spectra for various rake angles. Raman spectra were taken using a 514.5 nm probe wavelength. Note the asymmetrical broadening and shifts of the resulting peaks. 


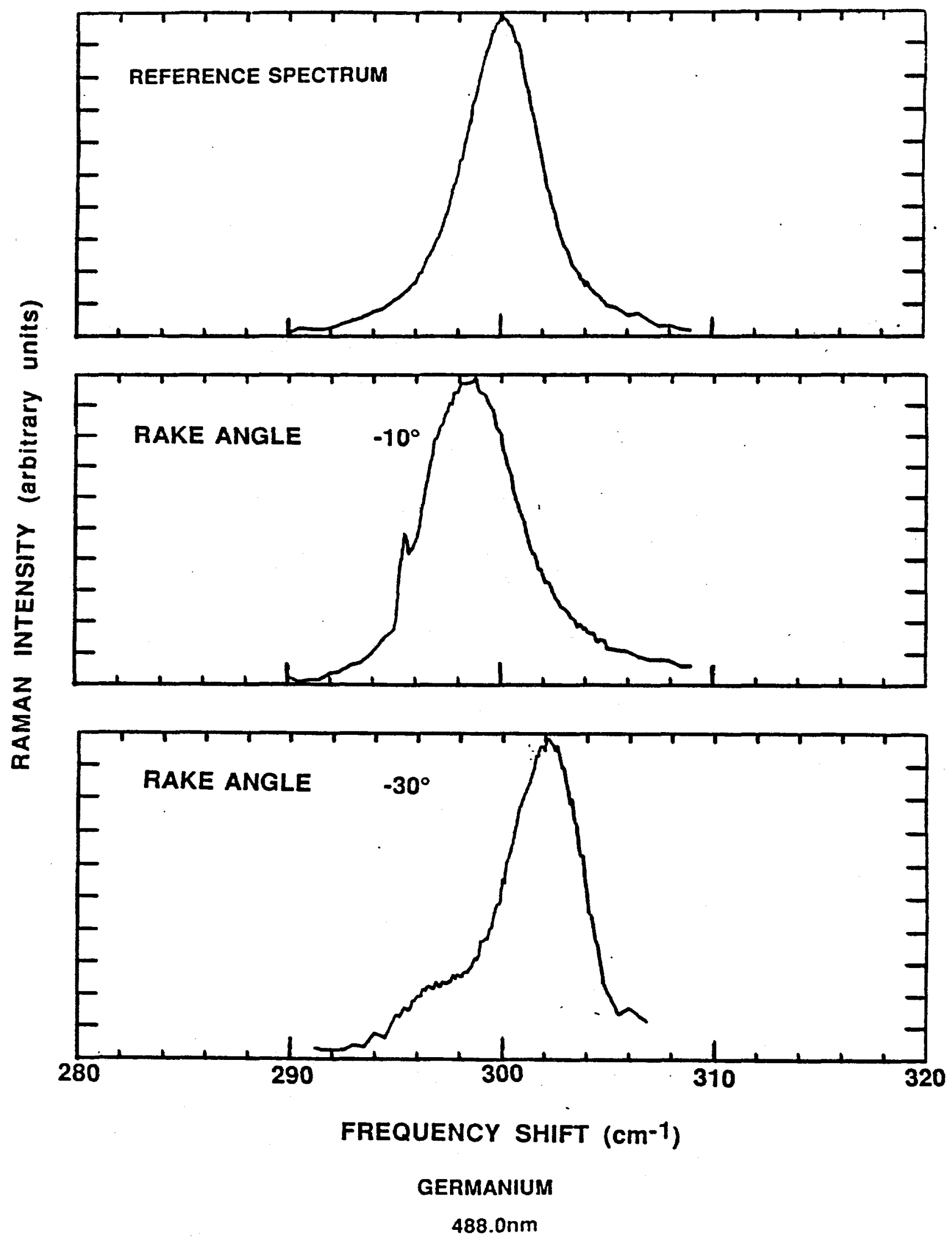

Figure 2: Raman spectra for various rake angles. Raman spectra were taken using a $488.0 \mathrm{~nm}$ probe wavelength. Again asymmetrical broadening and shifts in the peak locations are evident though quite different from Fig. 1. 
$-30^{\circ}$ rake angle exhibited a maximum compressive stress of $4.8 \pm 0.1 \mathrm{kbar}$ at a depth of $0.04 \pm 0.01 \mu \mathrm{m}$. In both cases the magnitude of the stress rapidly changed sign resulting in a discontinuity in the measured stress profile. The depth at which this discontinuity occurred was determined to be 0.07 and $0.06 \pm 0.01 \mu \mathrm{m}$ for the $0^{\circ}$ and $-30^{\circ}$ rake angles. The maximum magnitudes of the tensile stresses were $4.8 \pm 0.1$ and $7.3 \pm 0.1 \mathrm{kbar}$ for the $0^{\circ}$ and $-30^{\circ}$ rake angles with characteristic depths of 0.99 and $0.07 \pm 0.01 \mu \mathrm{m}$ respectively. In both cases this tensile stress was observed to relax to zero smoothly with the stress vanishing at a depth of $0.35 \pm 0.01 \mu m$.

Raman spectra obtained using $514.5 \mathrm{~nm}$ and $488.0 \mathrm{~nm}$ wavelengths on germanium samples turned with a $-10^{\circ}$ rake angle showed dramatic differences in their shifts and asymmetries. The resulting stress profile of Figure 4 indicated that a compressive stress exists near the surface of magnitude $1.8 \pm 0.1 \mathrm{kbar}$ and gradually decreased to a value of $0.78 \pm 0.1 \mathrm{kbar}$ at a depth of $0.05 \pm 0.01 \mu \mathrm{m}$. The compressive stress was observed to then rapidly increase to a maximum value of $7.3 \pm 0.1 \mathrm{kbar}$ at a depth of $0.06 \pm 0.01 \mu \mathrm{m}$. The stress then changed sign reaching a maximum tensile stress of $6.1 \pm 0.1 \mathrm{kbar}$ at a depth of $0.08 \pm$ $0.01 \mu \mathrm{m}$ with the transition from compressive to tensile stress occurring at a depth of 0.07 $\pm 0.01 \mu \mathrm{m}$. A smooth relaxation to zero stress was not observed in this case; the resulting stress profile showed evidence of secondary tensile maxima at depths of 0.11 and $0.13 \pm$ $0.01 \mu \mathrm{m}$ with a broad region of slowly varying tensile stress between depths of 0.14 and $0.22 \pm 0.01 \mu \mathrm{m}$. The tensile stress then rapidly relaxes to zero stress at a depth of $0.26 \pm$ $0.01 \mu \mathrm{m}$.

The effects of standard and high clearance angles $\left(6^{\circ}\right.$ and $16^{\circ}$ respectively) were also investigated $\left(-10^{\circ}\right.$ rake angle, $6 \mu \mathrm{m}$ in depth of cut, $2.5 \mu \mathrm{m} / \mathrm{rev}$ feed rate, $1000 \mathrm{RPM}$ spindle speed). The effects of $-10^{\circ}$ rake angle with a $6^{\circ}$ clearance angle were discussed in reference to Figure 4. The use of the same machine parameters with a clearance angle of $16^{\circ}$ resulted in a depth profile shown in Figure 5 . In this case the specimen had a tensile surface stress of magnitude $0.5 \pm 0.1 \mathrm{kbar}$. This small tensile surface stress changed sign and increased to a maximum compressive stress of $2.8 \pm 0.1 \mathrm{kbar}$ at a depth of $0.05 \pm$ $0.01 \mu \mathrm{m}$. The magnitude of the residual stress again changed sign with the next maximum $(4.7 \pm 0.1 \mathrm{kbar})$ of tensile stress occurring at a depth of $0.10 \pm 0.01 \mu \mathrm{m}$. Secondary peaks in the tensile stress were observed as in the case of $-10^{\circ}$ rake angle and $6^{\circ}$ clearance angle. These secondary peaks occurred at depths of 0.14 and $0.18 \pm 0.01 \mu \mathrm{m}$ with magnitudes of $2.9 \pm 0.1$ and $2.5 \pm 0.1 \mathrm{kbar}$ respectively. A broad region of slowly varying tensile stress was again observed, although in this case the relaxation of the residual tensile stress to zero did not occur until a depth of $0.54 \pm 0.01 \mu \mathrm{m}$ was reached.

\subsection{CONCLUSIONS}

Micro-Raman spectroscopy coupled with the use of computer deconvolution of the resulting spectra has yielded a wealth of information concerning the effects of machine parameters 

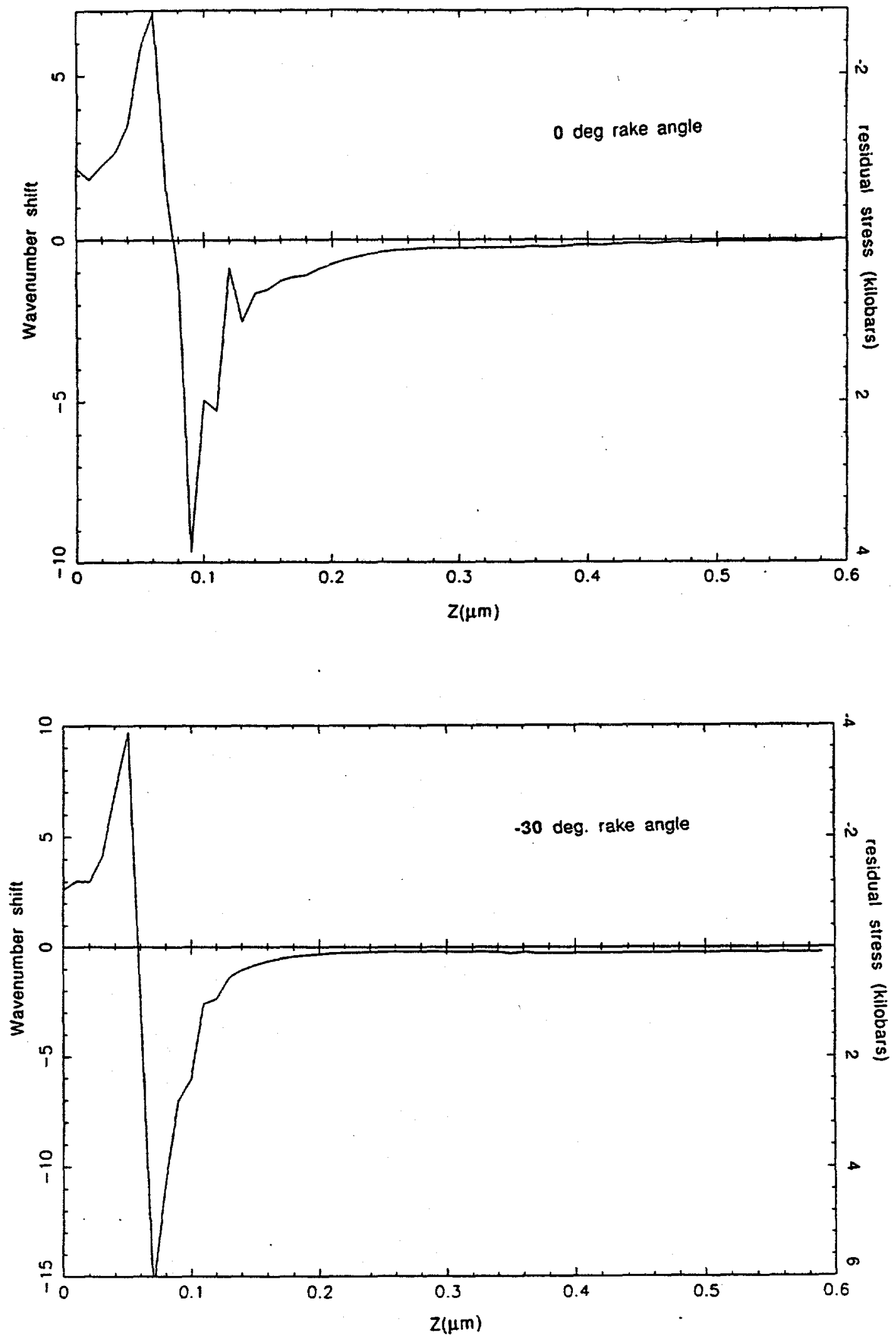

Figure 3: Depth profiles for $0^{\circ}$ and $-30^{\circ}$ rake angles. Wavenumber shift/stress is plotted as a function of depth into tile sample. Both profiles exhibit compressive surface stresses, the plastic/elastic transition is clearly evident. 


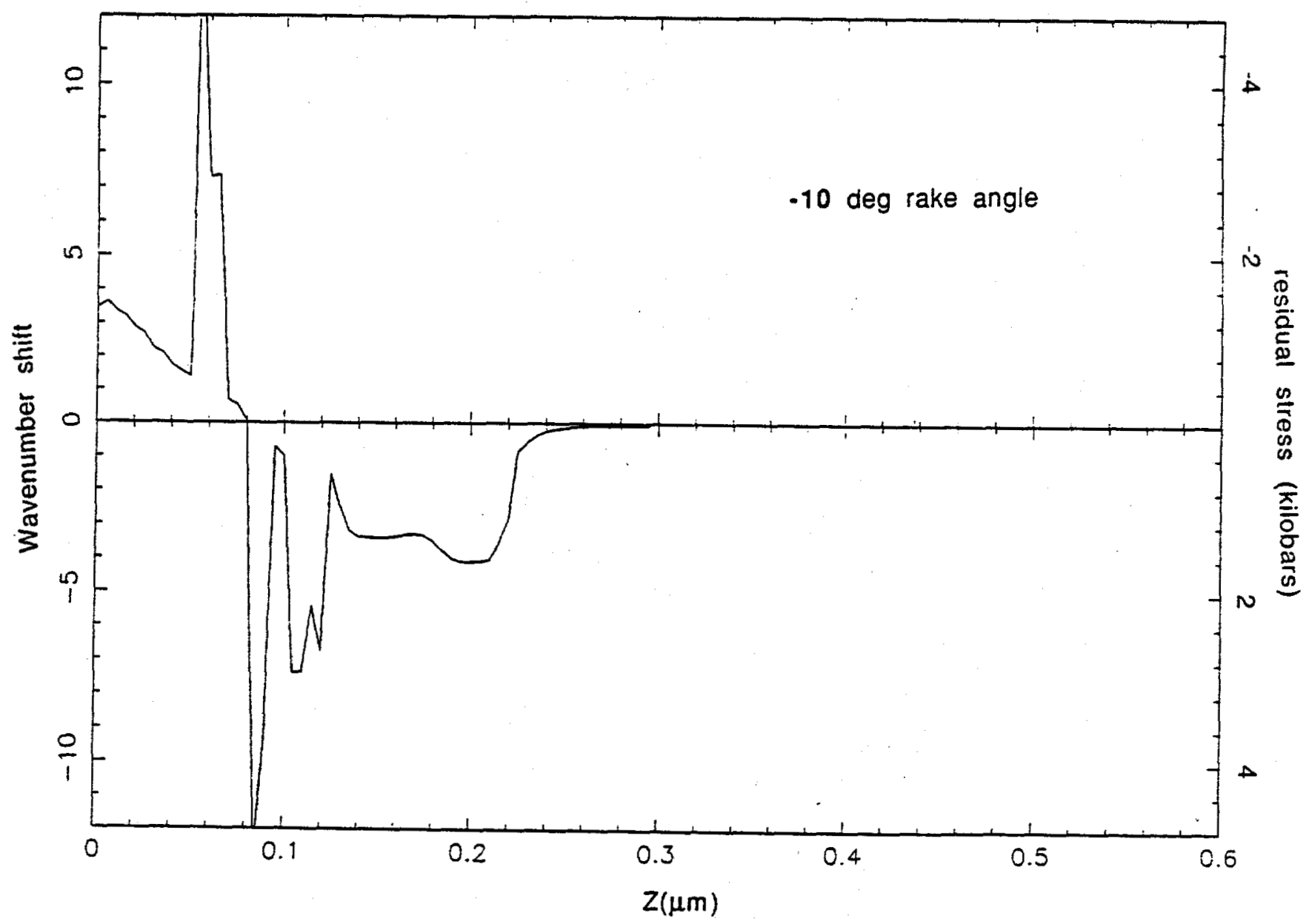

Figure 4: Depth profile for $-10^{\circ}$ rake angle. The secondary tensile stress peaks as well as the plastic/elastic transition region are shown. 


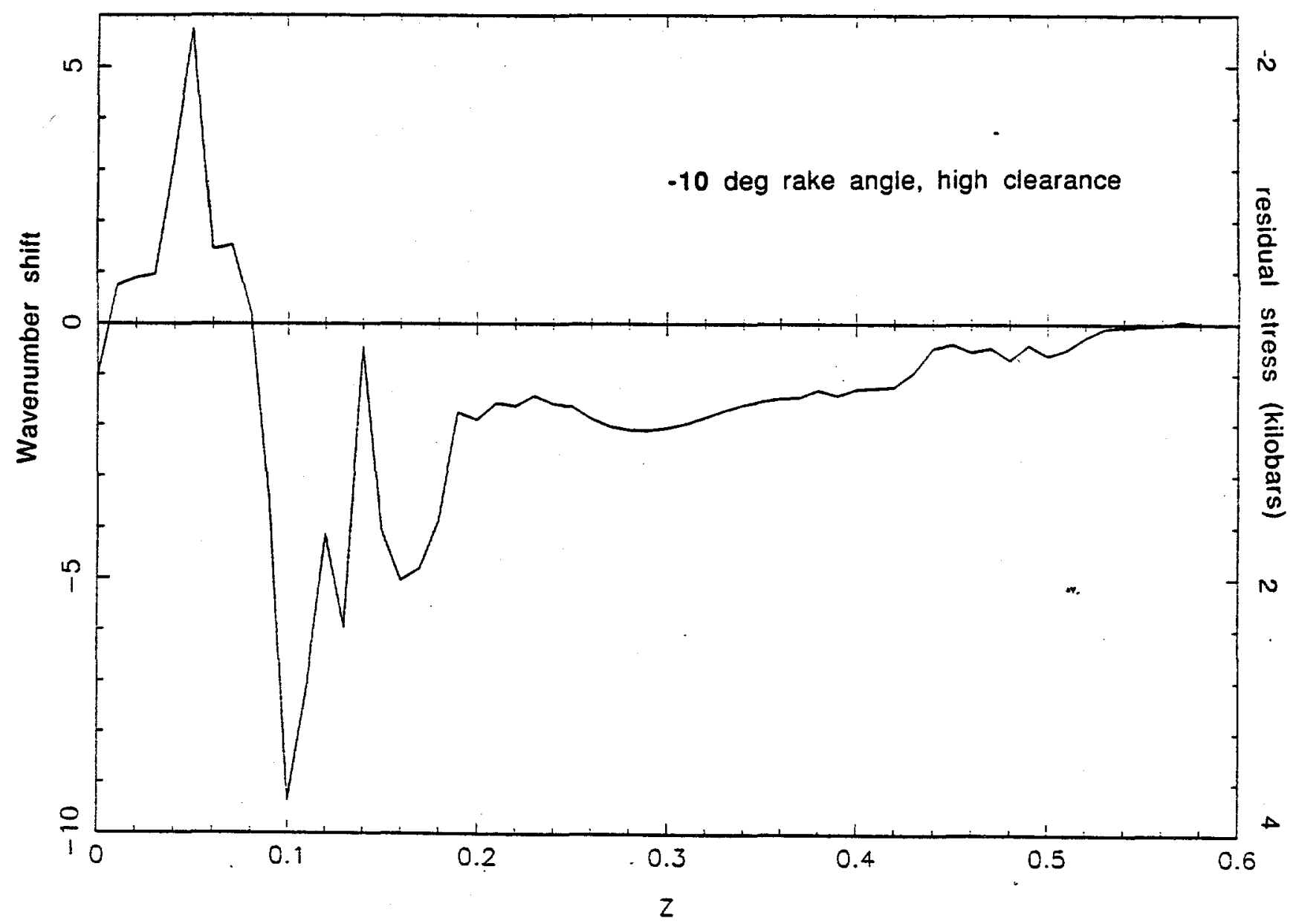

Figure 5: Depth profile for $-10^{\circ}$ rake and $16^{\circ}$ clearance angles. The profile exhibits the same general features, however the plastic/elastic transition has shifted. The tensile stress also penetrates deeper into the sample. 
on the residual stress field in single point diamond turned silicon and germanium. It has been found from these investigations that for the range of feed rates and spindle speeds studied, no significant variations in the residual stress field are noted. All feed rates and spindle speeds resulted in a compressive surface stress which showed an rapid increase to a maximum value of $3 \mathrm{kbar}$ at depth of $0.05 \mu \mathrm{m}$. The magnitude of the stress then changes sign and reaches a maximum tensile stress of approximately the same magnitude at a depth of $0.07 \mu \mathrm{m}$. Significant variations in the stress field were noted, however, for the various rake and clearance angles studied. These variations seem to indicate changes in the both the magnitude of the surface stress as well as significant variations in the region of plastic/elastic transition in such samples. 


\section{References}

[1] Nemanich, R. J. Mat Res Soc Symp. Proc. Vol. 69, 1986 p. 23.

[2] Lyon, S. A., Nemanich, R. J., Johnson, N. M. and Biegelsen, D. K. Appl Phys Lett Vol. 40, 1982 p. 316.

[3] Englert, Th., Abstreiter, G. and Pontcharra, J. Solid State Elect Vol. 23, 1980 p. 31.

[4] Cheong, Y. M., Marcus, H. L. and Adar, F. J Mater Res 2, 1987

[5] Sparks, R. G. and Paesler, M. A. Prec Eng Vol. 10, 1988 p. 4.

[6] Enloe, W. S., Sparks, R. G. and Paesler, M. A. NCSU Precision Engineering Center Mid-Year Report 1988, VI.

[7] Johnson, K. L. J Mech Phys Solids Vol. 18, 1970. 


\title{
18 ATOMIC STRUCTURE OF MACHINED SEMICONDUCTING CHIPS: AN X-RAY ABSORPTION SPECTROSCOPY STUDY
}

\author{
Michael Paesler \\ Associates Professor, Physics \\ Dale Sayers \\ Professor, Physics
}

$X$-ray absorption spectroscopy (XAS) has been used to examine the atomic structure of chips of germanium that were produced by single point diamond machining. It is demonstrated that although the local (nearest neighbor) atomic structure is experimentally quite similar to that of single crystal specimens, information from more distant atoms indicates the presence of considerable stress. An outline of the technique is given and the strength of $X A S$ in studying the machining process is demonstrated.

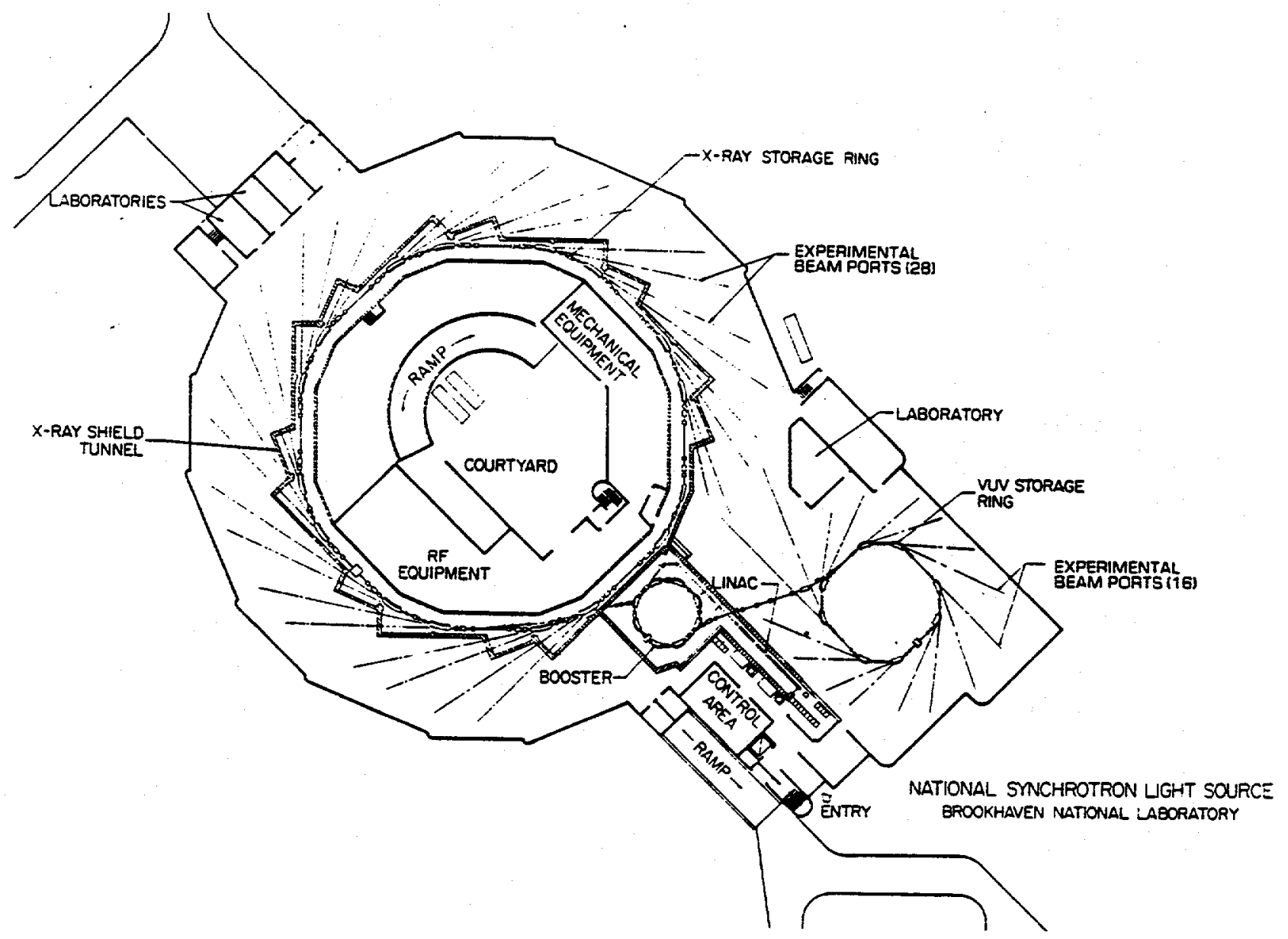




\subsection{INTRODUCTION}

The technique of $x$-ray absorption spectroscopy (XAS) involves analysis of the fine structure observed on the high energy side of an $x$-ray absorption edge [1]. By Fourier analyzing this structure, one can study atomic environments in the neighborhood of the particular species of atom that is absorbing the $x$-ray. This technique is applied to samples of germanium that were obtained as chips in a machining situation to draw conclusions about the stress in the chips and the temperature that they experienced during the machining process.

\subsection{EXPERIMENTAL PROCEDURE}

Samples of Ge chips were gathered from a machining operation using a diamond tool. Since the machining was done under a flow of distilled water, the chips were dried before evaluation. A collection of chips were pressed into a pellet approximately $1 \mu \mathrm{m}$ thick and $1 \mathrm{~cm}^{2}$ cross section. The pertinent machining parameters were a $2.5 \mu \mathrm{m}$ inch depth of cut, $80 \mu \mathrm{m}$ per revolution feed rate and $\mathrm{a}-10^{\circ}$ tool rake angle.

XAS data were taken at $77 \mathrm{~K}$ on beam line X-11A at the National Synchrotron Light Source at Brookhaven National Laboratory. In addition to the $\mathrm{Ge}$ chips, samples of crystalline $\mathrm{Ge}(\mathrm{c}-\mathrm{Ge})$ and $\mathrm{GeO}_{2}$ were also measured for comparison spectra. After appropriate background subtraction and Fourier transformation [1], the data resulted in the transforms shown in Figures 1 and 2.

\subsection{RESULTS AND DISCUSSION}

For elemental samples such as germanium, the pair distribution (which results from Fourier transforming a corrected absorption spectrum) is interpreted in a straightforward manner. As shown in Figure 1, the ordinate is related to the probability of finding a neighboring $\mathrm{Ge}$ atom at the distance from the absorbing atom given on the abscissa. Thus for the sample of c-Ge shown in Figure 1, the large peak at $2.1 \AA$ represents the first nearest neighbor Ge atom. Second and third nearest neighbor peaks near 3.8 and $4.2 \AA$ are likewise clearly interpretable. Identification of higher order peaks is somewhat problematic due to the transformation process. The general overall decrease in the magnitude of subsequently higher peaks is a general feature of XAS techniques and is a result of the fact that scattering amplitudes (not intensities) determine the XAS signal. This is to be distinguished from $x$-ray diffraction studies where the resultant radial distribution functions (RDF's) exhibit peaks at higher shells with generally greater amplitudes at larger distances. 


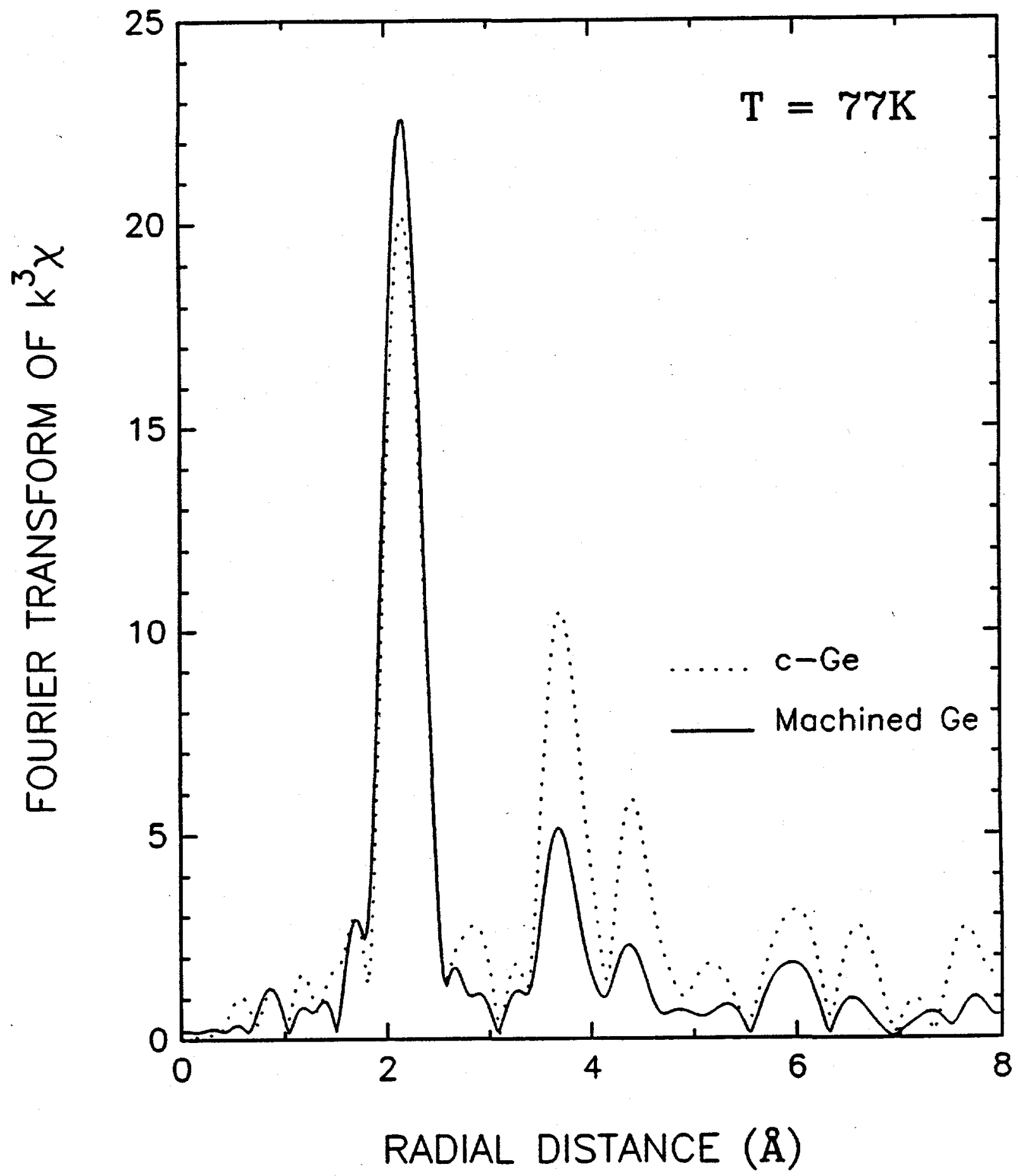

Figure 1: The weighted Fourier transform of the corrected extended $x$-ray absorption fine structure taken on the K-edge of germanium for samples of crystalline germanium (dotted) and chips of machined germanium (solid line). The features at approximately 2.1, 3.8 and 4.2 Arrepresent the 1st $2 \mathrm{nd}$ and 3rd nearest neighbors of the germanium atoms in the samples. The decrease in the magnitude in the 2nd and 3rd shell features of the machined specimens relative to the crystal indicates the presence of stress in the machined chips. 


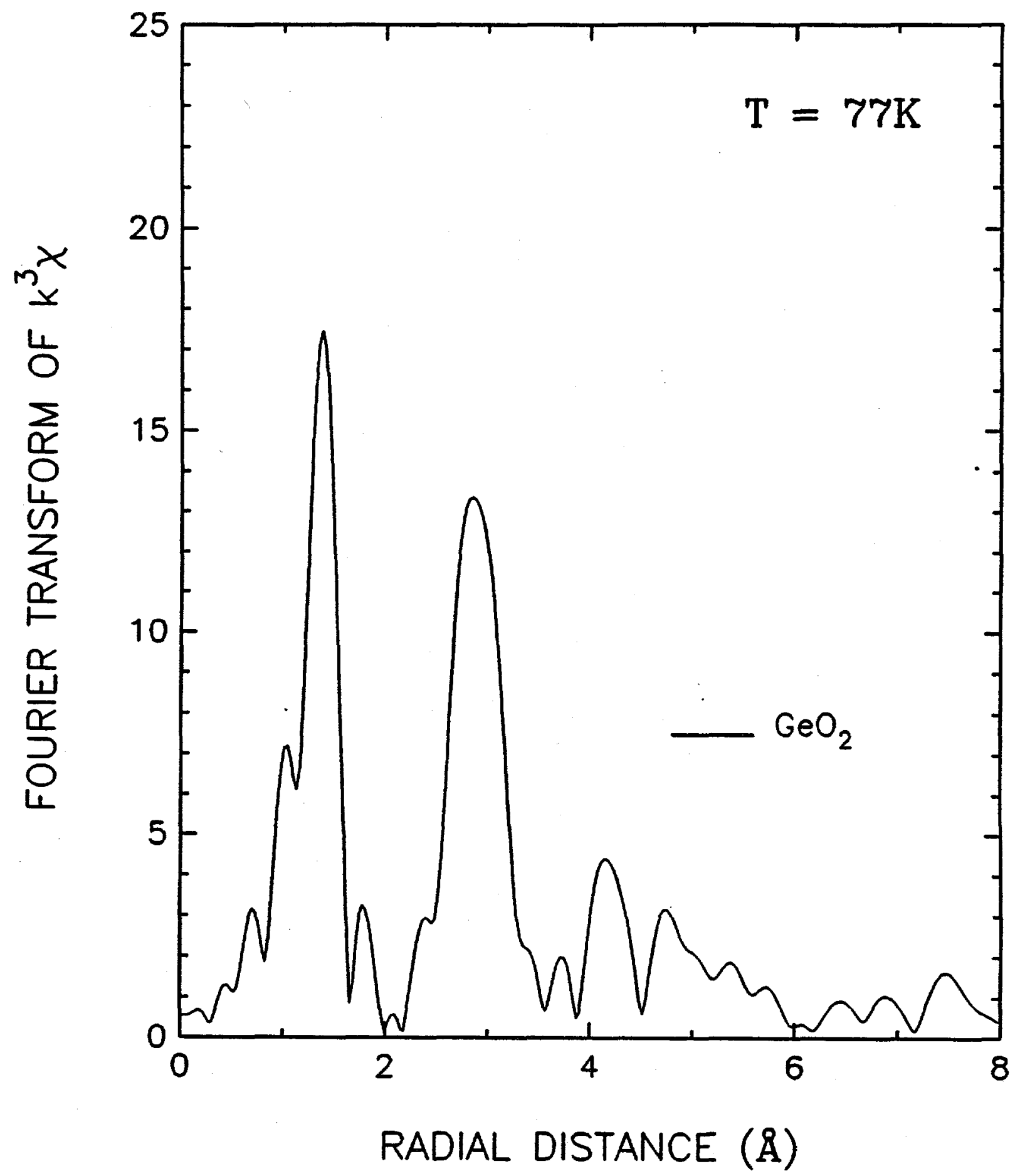

Figure 2: The same plot as for Figure 1 for a sample of $\mathrm{GeO}_{2}$. The large feature at approximately 1.4 Arepresents the oxygen neighbor of the germanium atoms. The absence of any feature at this location in Figure 1 suggests that the chips are not oxidized and therefore did not reach a high temperature during the machining process. 
The XAS data from the machined chips show peaks at the position of the first three neighbors of $\mathrm{c}-\mathrm{Ge}$, but the relative intensities are quite different from the $\mathrm{c}-\mathrm{Ge}$ data. The slightly lower peak from the nearest neighbor atoms (near $1.2 \AA$ ) is due to a thickness effect [1] and should not be interpreted as significant. The smaller magnitude of the second and third peaks in the machined specimen coupled with the relatively small change in the position of these peaks as compared to the $\mathrm{c}-\mathrm{Ge}$ data indicates the presence of considerable stress of both signs - compressive and tensile - in the machined chips. A spread in the position of atoms in these higher shells without a general change of the average neighbor separation, suggests that some bonds are stretched while others are compressed. It is not possible using XAS to determine the size of the regions of compressive and tensile stress. There may be microscopic regions of stress of opposite sign distributed throughout the chips, or large macroscopic portions of each chip may contain stress of a fixed sign.

In these initial studies, neither a survey of different machine parameters nor extensive analysis of the data has been undertaken. However, further analysis will make it possible to more accurately determine the position of the higher shells of atoms and thus to more precisely - and quantitatively - determine the actual stress values in the chips.

In a measurement of the XAS of $\mathrm{GeO}_{2}$, the transform shown in Figure 2 was obtained. The Ge-O distance is seen to be near $1.4 \AA$. The absence of any feature at this distance in the data of Figure 1 indicate that the chips were not oxidized during the machining process. This precludes the possibility of the chips having been raised to a high temperature during the process of material removal. Although a quantitative upper limit on the chip temperature cannot be predicted at the present time, controlled studies to determine the effect of high temperatures on the existing chips are underway.

\subsection{ACKNOWLEDGEMENTS}

The help of Quazi Islam in taking and processing the data is gratefully acknowledged. The experiments were performed with the assistance of the staff of the National Synchrotron Light Source at Brookhaven National Laboratory. 


\section{References}

[1] "X-ray Absorption" edited by D.C. Koningsberger and R. Prins, John Wiley and Sons, New York, 1988. 


\title{
19 OBSERVATION OF DIAMOND TURNED OFHC COPPER USING SCANNING TUNNELING MICROSCOPY
}

\author{
David A. Grigg \\ Graduate Student \\ Phillip E. Russell \\ Associate Professor, MTE \\ Thomas A. Dow \\ Professor, MAE
}

Diamond turned OFHC copper samples have been observed within the past few months using the Scanning Tunneling Microscope. Initial results have shown evidence of artifacts which may be used to better understand the diamond turning process. The STM's high resolution capability and three dimensional data representation allows observation and study of surface features unobtainable with conventional profilometry systems. Also, the STM offers a better quantitative means by which to analyse surface structures than the SEM. This paper discusses findings on several diamond turned $O F H C$ copper samples having different cutting conditions. Each sample has been cross referenced using STM and SEM.

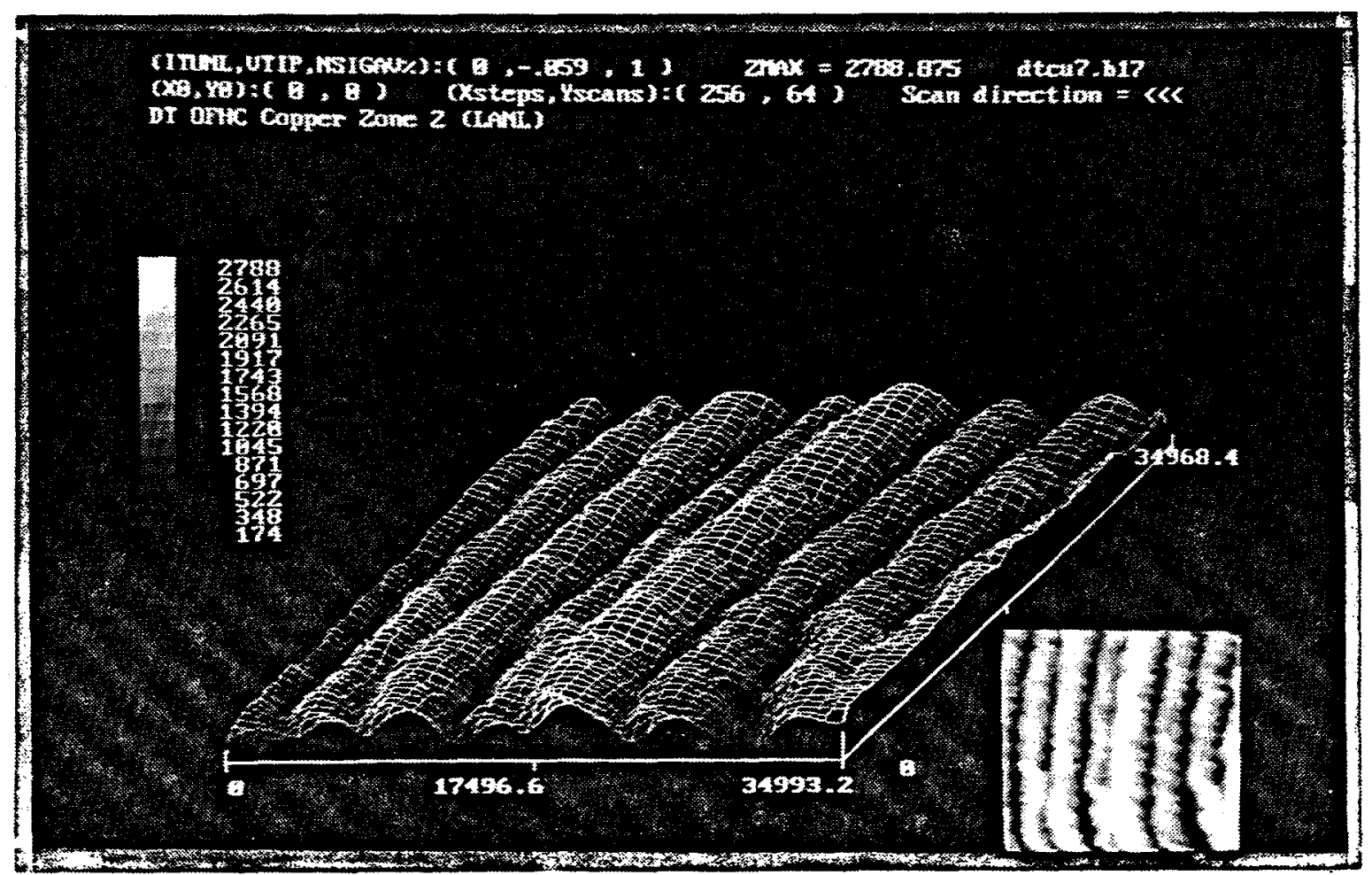




\subsection{INTRODUCTION}

Few papers have been published showing the effectiveness of the STM to image machined surfaces. In a paper by $R$. Dragoset [1], results are shown of a diamond turned gold sample observed with an STM. A second paper, by M. Gehrtz, H. Strecker, and H. Grimm [2], shows the result of an STM used to observe an individual diamond turned mark on an aluminium sample. The paper also makes a comparison to Scanning Electron Microscopy (SEM) images of the same sample showing that STM can reveal a much more quantitative result from surfaces than SEM.

Several samples have been prepared by diamond turning and imaged using the STM and SEM at the Precision Engineering Center. The first sample was cut in the Center and second sample was cut at Los Alamos National Laboratory with slightly different cutting conditions to emphasize the feed marks. Features observed using the STM have given new insights into the dynamics of the turning process. Similar features have been observed on polymers and plastics using SEM or optical microscopes (See Section 14), but, they have not been demonstrated on metals.

\subsection{SAMPLE \#1 (P.E.C.)}

The first sample studied was an OFHC copper sample diamond turned in the Center on a Rank Pneumo ASG 2500 diamond turning machine. All samples in this study were turned from 3 inches diameter blanks. Observation of these samples at $200 \mathrm{X}$ on a Zeiss ICM 405 optical microscope reveals a grain size of approximately $50 \mu \mathrm{m}$.

\subsubsection{Sample Preparation}

Several face cuts were made to prepare the sample surface for the final cut. Mineral oil was used as a lubricant during all cuts because previous experience has shown better surfaces finish results when cuts were made using mineral oil with OFHC copper. Table 1 lists the cutting conditions used for this experiment.

\begin{tabular}{||l|l||}
\hline Cutting Parameters, Sample \#1 \\
\hline Tool Radius & $0.76 \mathrm{~mm}$ \\
Feedrate & $1 \mathrm{~mm} / \mathrm{min}$ \\
Spindle Speed & $1900 \mathrm{rev} / \mathrm{min}$ \\
Feed & $0.52 \mu \mathrm{m} / \mathrm{rev}$ \\
Depth of Cut & $7.5 \mu \mathrm{m}$ \\
\hline
\end{tabular}

Table 1: Cutting Parameters for Sample \#1 
Surface preparation, after cutting, to remove the residual Diala oil becomes important in possible interpretations of the surfaces features. After the samples were turned, the residual mineral oil was removed in two stages. First, methanol was applied across the entire sample and allowed to sit for approximately half a minute. A single sheet of Kimwipes tissue was lowered onto the sample and allowed to rest until saturated with the methanol. The Kimwipe was then rolled off of the surface to minimize any possible scratching. Acetone was applied to the surface and a second Kimwipe was used in a similar manner. The Kimwipe was rolled off of the surface slowly and steadily to keep pace with the evaporation rate of the acetone. This is a widely used technique to produce optically clean diamond turned surfaces after cutting.

\subsubsection{SEM of Surface}

Figure 1 shows an SEM micrograph of sample \#1. This micrograph is characterized by striations in the cutting direction with feature spacings $0.25 \mu \mathrm{m}$ and larger. However, from this micrograph it is difficult to pick out repeated features at the $0.52 \mu \mathrm{m}$ feed rate set in the controller. There may be several reasons for this difficulty. First, the $1 \mathrm{~mm} / \mathrm{min}$ feed rate was at the least significant but on the digital output to the slide drive motor. Therefore, variations in the feed rate of the slide as a fraction of time are possible. Second, the theoretical finish for this tool/feedrate condition is less the $1 \AA$. There are potentially many features on the tool edge which are larger than this theoretical finish and will be replicated on the surface masking the theoretical finish. Third, the motion of the tool normal to the workplace caused by vibration can produce apparent changes in infeed due to changes in the depth of cut.

\subsubsection{STM of Surface}

The STM images from Sample \#1 give a different picture of the surface features than did the SEM images. A $3.2 \mu \mathrm{m}$ square section of the surface is shown in Figure 2. The sample was oriented in the STM such that the tool motion is from bottom right to upper left. The feed rate is $0.52 \mu \mathrm{m} / \mathrm{rev}$ and therefore nine(9) feeds should be visible from the upper right to the lower left. Surprisingly the picture shows none of the features expected. In fact the surface is characterized by marks which lie about 45 degrees to the tool path and a vertical vibration at an extremely high frequency of $10 \mathrm{MHz}$. This frequency is well above any machine resonances but may be due to oscillations of the diamond tool itself.

Figure 3 is an image from a different region of the same specimen at twice the magnification. The tool motion is still from lower right to upper left and again features are observed at an angle to this direction. At this magnification, feeds should be visible from lower left to upper right. The top view in Figure 3 does indicate about four rows of features but they are not in the expected direction. From observations of several different STM images of 


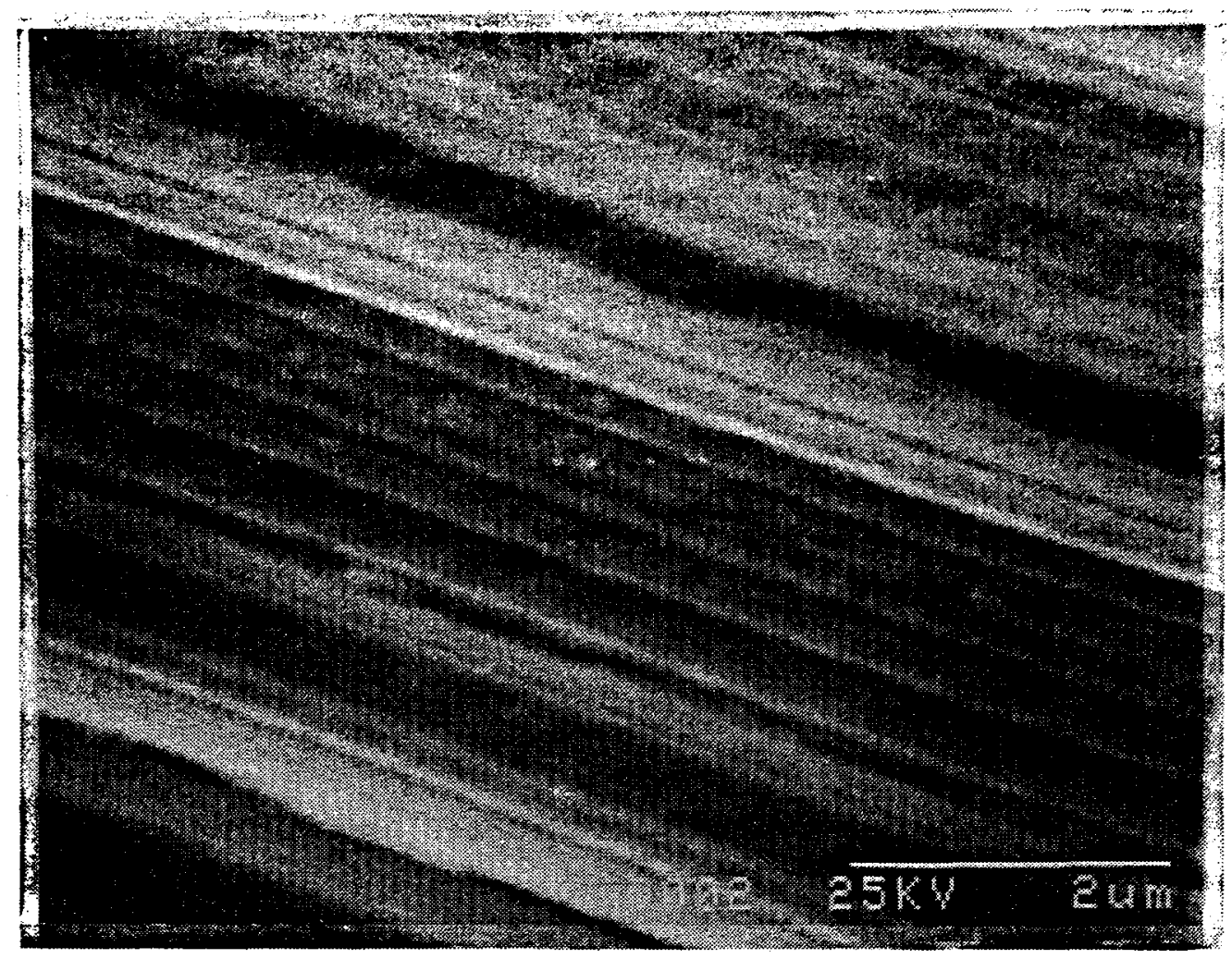

Figure 1: Scanning Electron Microscope micrograph of Sample \#1.

the specimen, a directionality was present in each image, but the direction was random from image to image. This may be due to the random grain directions of the different grains imaged.

The picture of the turned copper surface from Figures 2 and 3 is quite similar to that observed for the polycarbonate specimen described in Section 14 but at a smaller scale. Further work will be necessary to explain the features observed.

One of the difficulties in analyzing the features in Figures 2 and 3 is the uncertainty of the tool geometry and contact region. To simplify the analysis, another specimen was machined with a sharp tool and the resulting images are discussed in the next section.

\subsection{SAMPLE \#2 (LOS ALAMOS NATIONAL LAB)}

The second sample was prepared at Los Alamos National Lab (LANL) on a Moore \#5 diamond turning machine. This sample was slightly different from the previous sample, in that, a zero radius, $90^{\circ}$ nose angle tool was used such that a diamond turned grating was produced. The grating, was turned such that a triangular waveform would be produced with a peak to peak separation distance of 0.5 and $1.0 \mu \mathrm{m}$. This sample was used to demonstrate the effectiveness of the STM to image a precision turned surface of OFHC $\mathrm{Cu}$ with known spacings and high corrugation. 


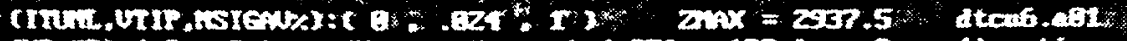

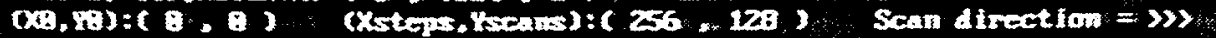

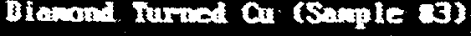

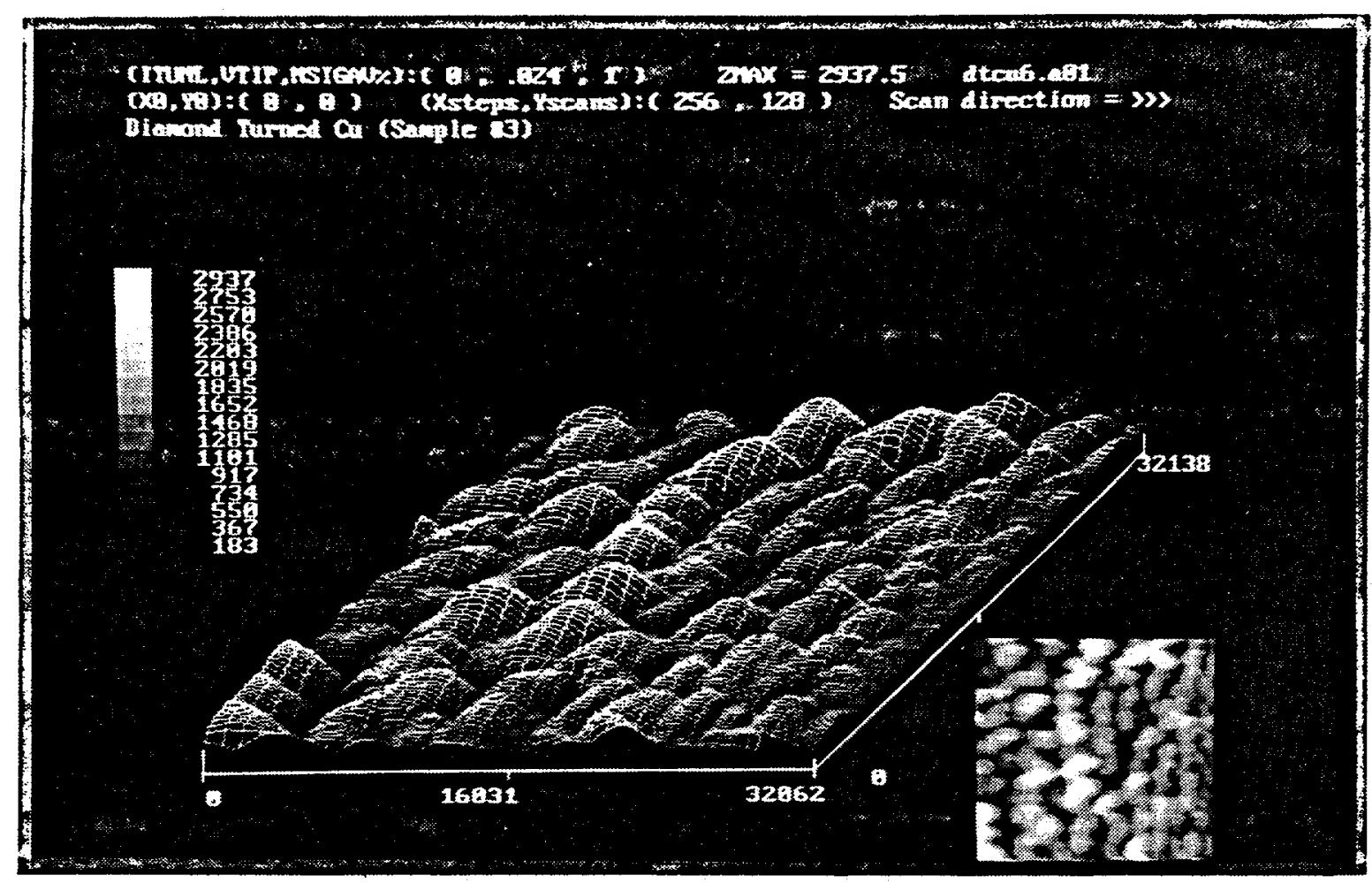

Figure 2: STM image of Sample \#1.

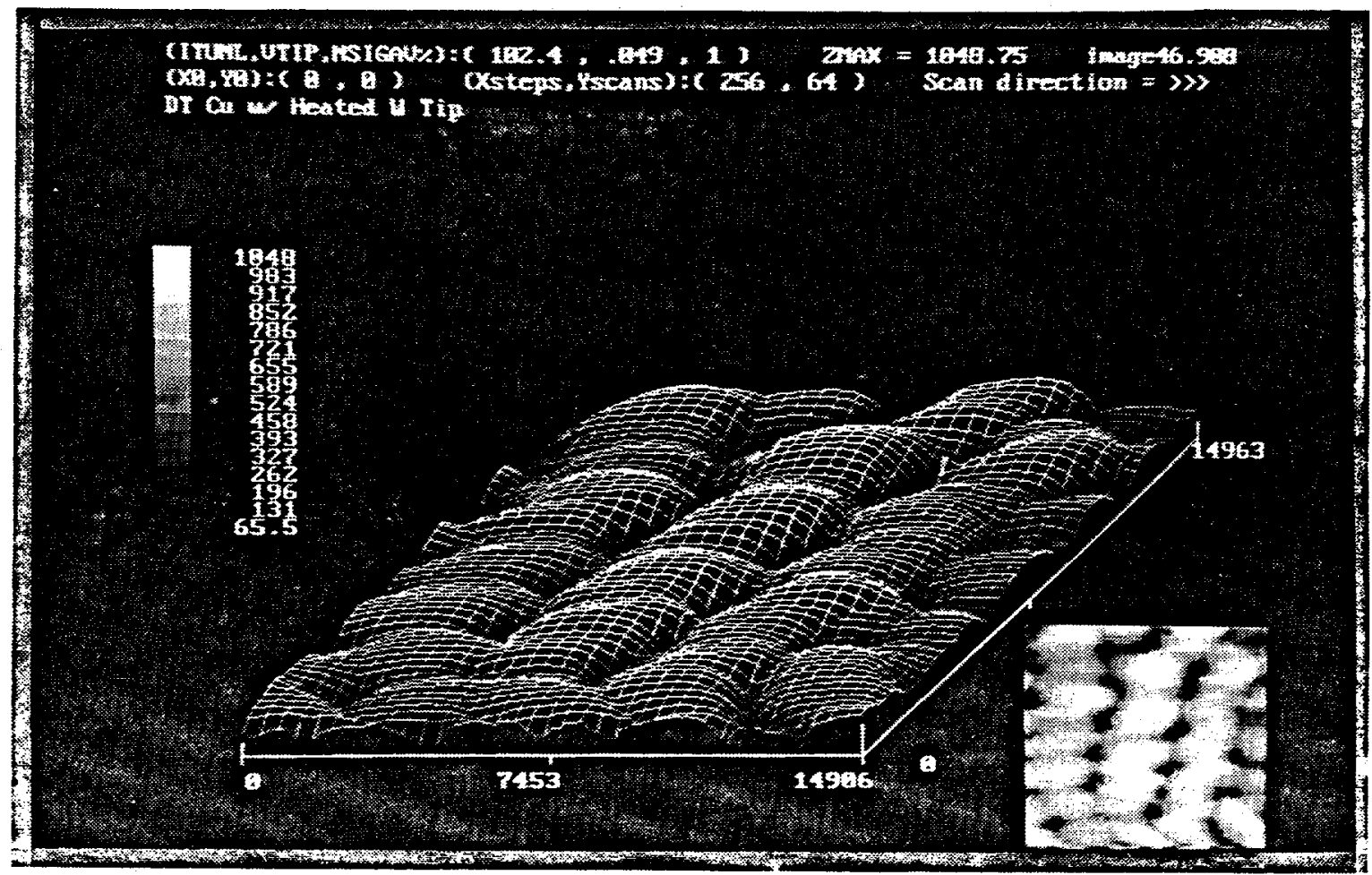

Figure 3: STM image of Sample \#1 


\begin{tabular}{||l|l||}
\hline Cutting Parameters, Sample \#2 \\
\hline Tool Radius & 0 in \\
Feedrate & $0.25 \& 0.5 \mu \mathrm{m} / \mathrm{min}$ \\
Spindle Speed & $250 \mathrm{rev} / \mathrm{min}$ \\
Feed & $0.50 \& 1.0 \mu \mathrm{m} /$ rev \\
Depth of Cut & $0.1 \mu \mathrm{m}$ \\
\hline
\end{tabular}

Table 2: Cutting Parameters for Sample \#2

\subsubsection{Sample Preparation}

Table 2 lists the cutting conditions used in this experiment. Two gratings were turned; one having $0.5 \mu \mathrm{m}$ spacings and the other having $1.0 \mu \mathrm{m}$ spacings. Instead of turning the sample in a spiral pattern, each groove was produced by plunge cutting. After machining a groove, the tool was raised approximately $5 \mu \mathrm{m}$ above the surface and incremented radially for the next cut. Then, the tool was fed at a rate of $25 \mu \mathrm{m} / \mathrm{min}$ into the sample for half a minute. Therefore, cutting at a speed of 250 revolutions/minute, the effective depth of cut per revolution was approximately $0.1 \mu \mathrm{m}$. The tool was then retracted and incremented to the next position. This was repeated over each grating which contains 762 grooves.

A light mineral oil was used during cutting. The process of removing the oil after cutting was slightly different than for Sample \#1. The sample was sprayed with a chloroethane solution to remove the oil. After application of the chloroethane, dry air was blown over the surface to remove the excess fluid. Ethanol was then applied to the surface and removed using dry air. This eliminated the possibility of any scratching due to Kimwipes or optical paper brought into contact with the surface.

To ship the sample from LANL, a container having a conical indentation with a diameter approximately equal to that of the part was used. By placing the part face down into the container, the bands of gratings were suspended such as not to contact any of the surrounding container. In this manner the part could be shipped without contact to the face.

\subsubsection{Talystep Trace of Surface}

Figure 4 shows a Talystep trace of the $1.0 \mu \mathrm{m}$ and $0.5 \mu \mathrm{m}$ sections turned at LANL. The radial direction of the part is traced from right to left in each trace. These traces shows the overall width of the section and the dimensional stability of the machine during cutting. The slope seen in both sections is approximately $30 \AA / \mu \mathrm{m}$. This is most likely due to thermal drifts. The time involved with cutting each section was 5.5 hours which would allow thermal drift to become a factor in the overall shape of the part.

Figure 5 shows Talystep traces of each section at higher magnification. In the case of 


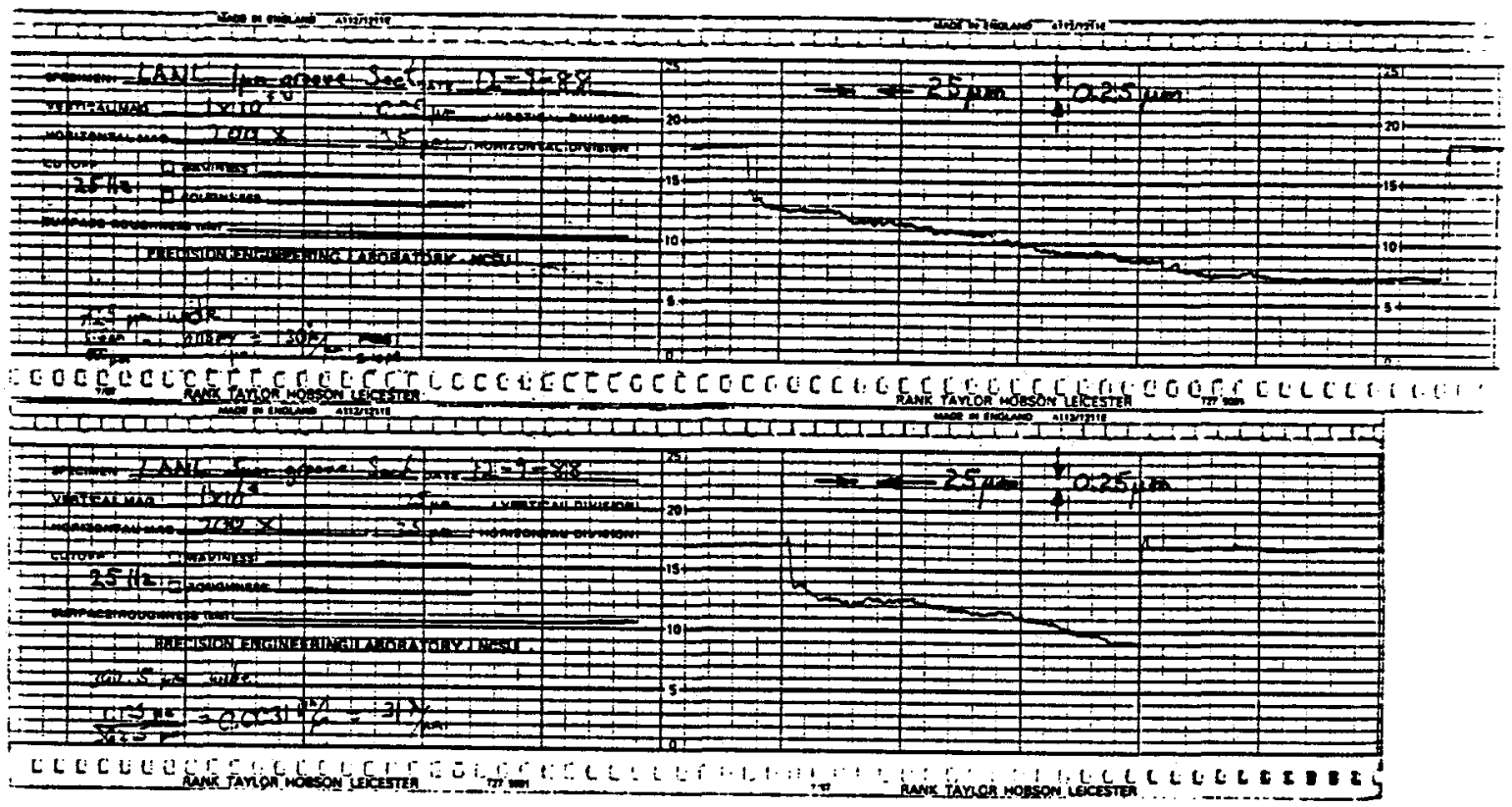

Figure 4: Talystep traces of the 1 (top) and 0.5 (bottom) $\mu \mathrm{m}$ gratings.

the $1.0 \mu \mathrm{m}$ section, the surface had enough dimensional instability from groove to groove to hinder the Talystep from tracing individual feedmarks. The Talystep has a $10.5 \mu \mathrm{m}$ diamond stylus. The large stylus tip has the effect of averaging the higher frequency components of the surface. This is believed to be the reason for the inability of visualizing the gratings using the Talystep. Only the longer wavelength errors in the surface can be seen. Also, from Figure 5 it can be seen that there are several spots were the surface features appear to be very sharp. Knowing that the radius of the stylus is $10.5 \mu \mathrm{m}$, these features cannot be real. Difficulties at higher magnifications with the Talystep are generally due to dirt [3]. However, the sample was cleaned thoroughly before the traces were taken.

\subsubsection{SEM of Surface}

Figure 6 is an SEM micrograph of the $1.0 \mu \mathrm{m}$ grating section of Sample \#2. From this micrograph it can be seen that the dimensional stability of the machine was adequate for $1 \mu \mathrm{m}$ infeed. The laser used for positioning of the infeed has a resolution of $25 \mathrm{~nm}$ and the variation in spacing is about $100 \mathrm{~nm}$. Inside of each of the grooves there exists some surface features. This may be due to stress relaxation, flow of the material or dirt. Also, the grooves are not triangular shaped as expected. The tops of each groove are rounded rather than sharp. This may be due to stress relaxation or flow of the OFHC copper during cutting as well as the action of chip removal, or tearing, at the edges of each groove. 


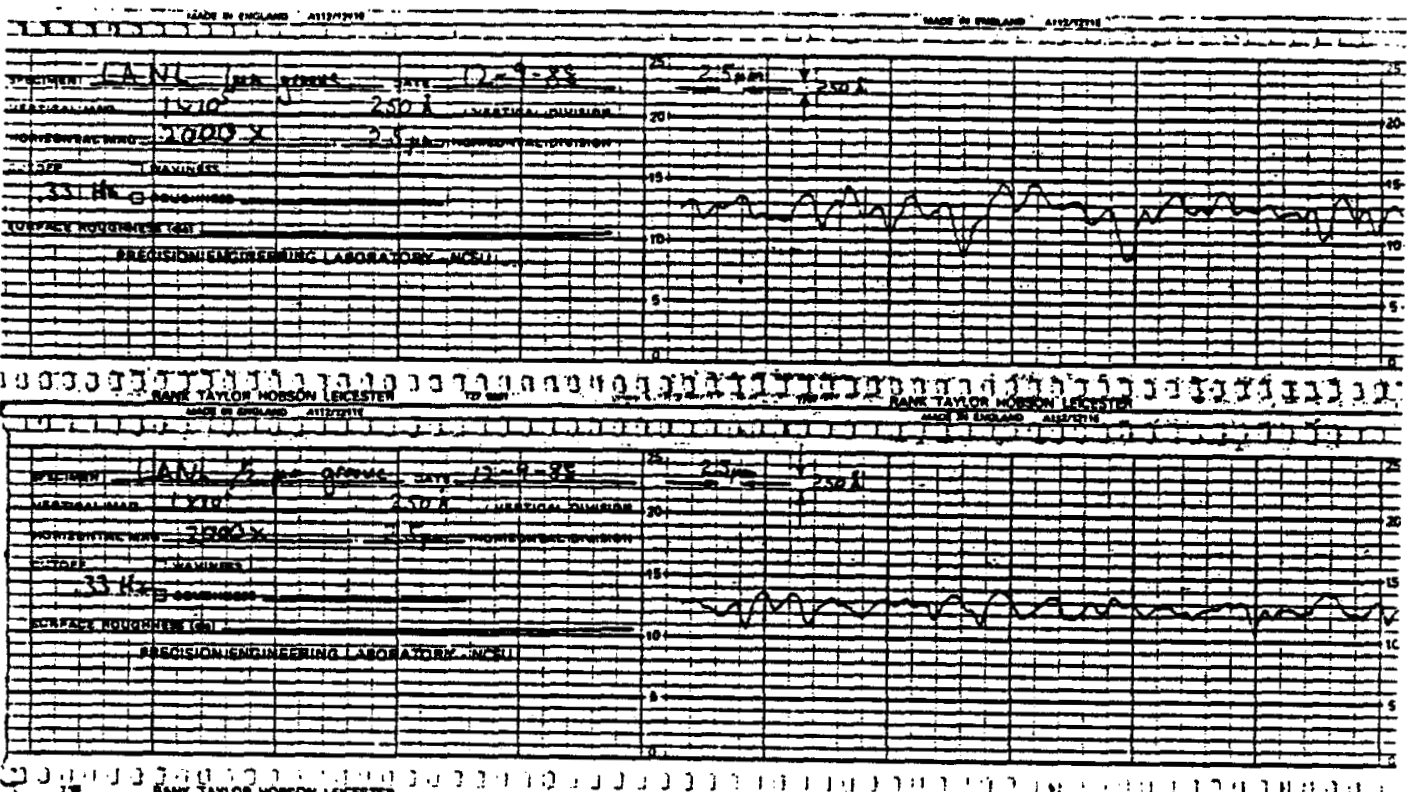

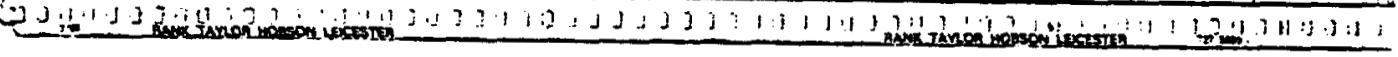

Figure 5: Talystep traces of the 1 (top) and 0.5 (bottom) $\mu \mathrm{m}$ gratings.

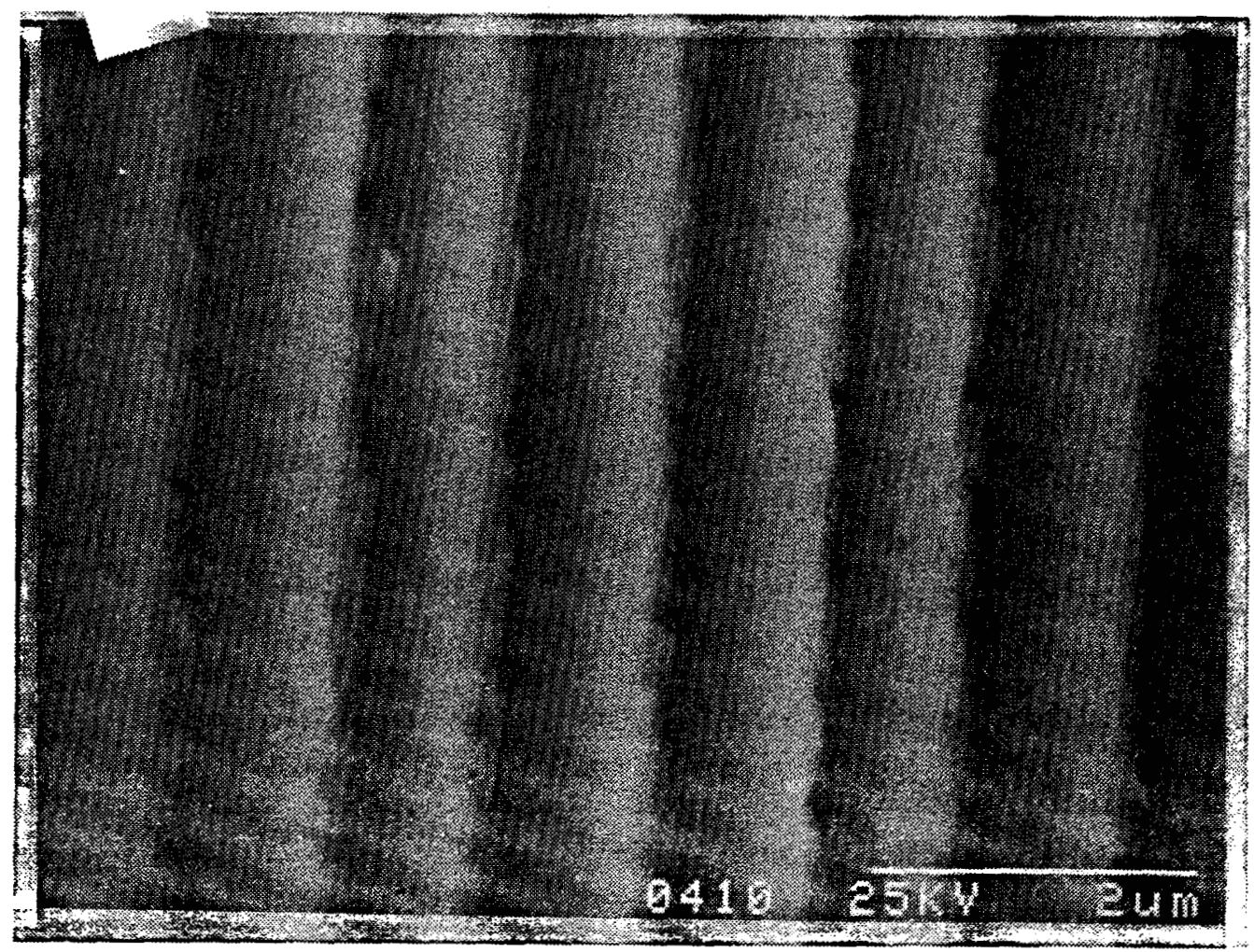

Figure 6: Scanning Electron Microscope micrograph of $1.0 \mu \mathrm{m}$ grating from LANL. 


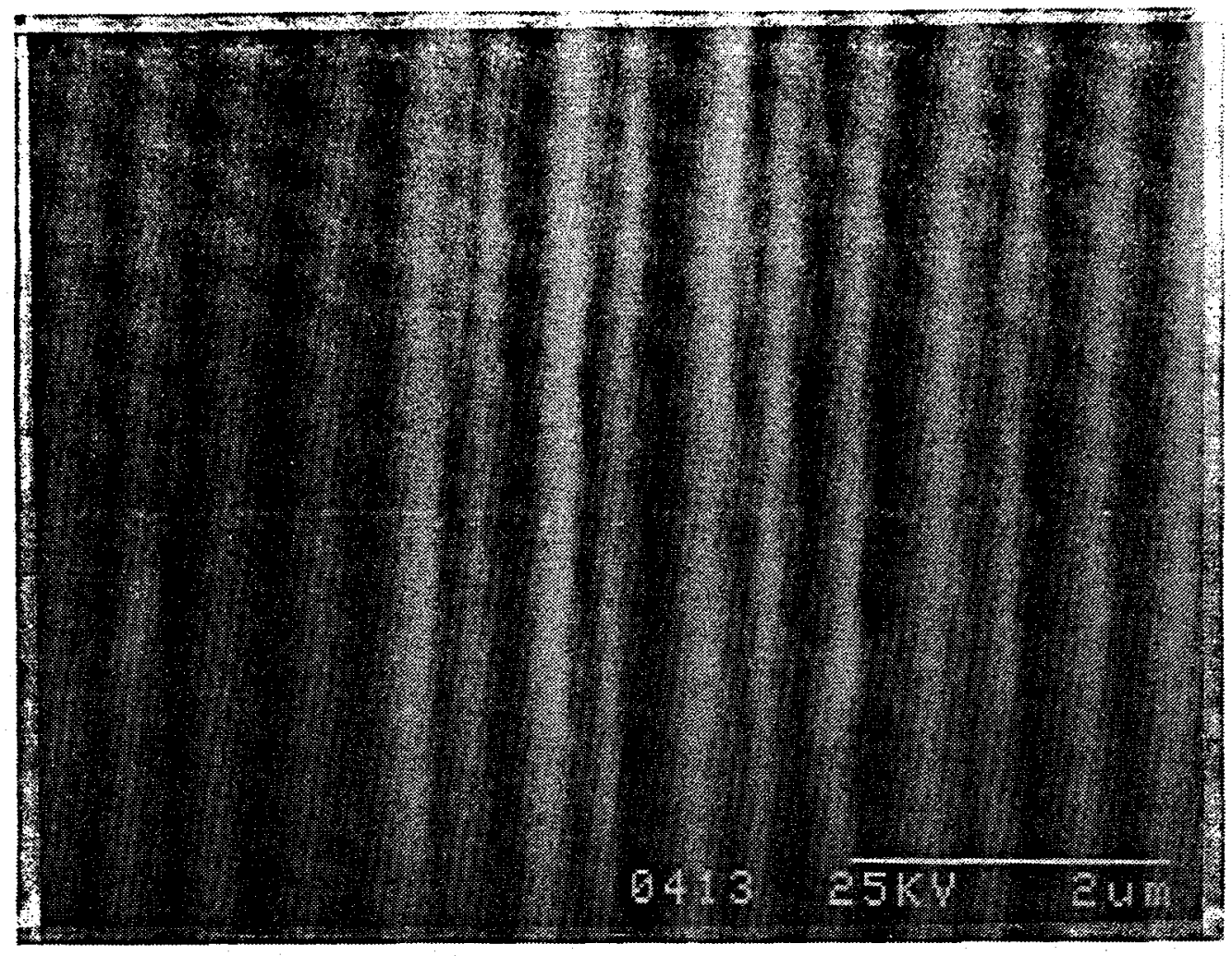

Figure 7: Scanning Electron Microscope micrograph of $0.5 \mu \mathrm{m}$ grating from LANL.

Figure 7 is an SEM micrograph of the $0.5 \mu \mathrm{m}$ grating section turned at LANL. The dimensional stability of these gratings are more visible than that for the $1 \mu \mathrm{m}$ gratings. The variation in separation of each groove is the same as in the $1.0 \mu \mathrm{m}$ section or about $100 \mathrm{~nm}$. Features within the grooves appear to cause the lines, in sections, to actually run together.

\subsubsection{STM of Surface}

Figure 8 shows an STM image taken of the $1.0 \mu \mathrm{m}$ grating on Specimen \#2. The STM scan directions were reoriented with respect to the sample such that the infeed direction is along the $\mathrm{X}$ axis and the direction of the tool pass is in the positive $\mathrm{Y}$ axis direction. The same inner groove structure seen by the SEM can be seen in the STM image. Also, the rounded tops between each groove is quantified by the STM image.

Figure 9 shows an STM image taken of the $0.5 \mu \mathrm{m}$ section. Similarities can be seen between the SEM micrographs and the STM image. Evidence of machine instability and sample tool interactions can be seen. Vibration effects can also be observed in Figure 9. The frequency seen within the groove along the $\mathrm{Y}$ axis direction corresponds to approximately $2.2 \mathrm{MHz}$. This is slightly different from that seen in sample \# 1 , however, both the machine and the diamond tool were different for these two samples. 


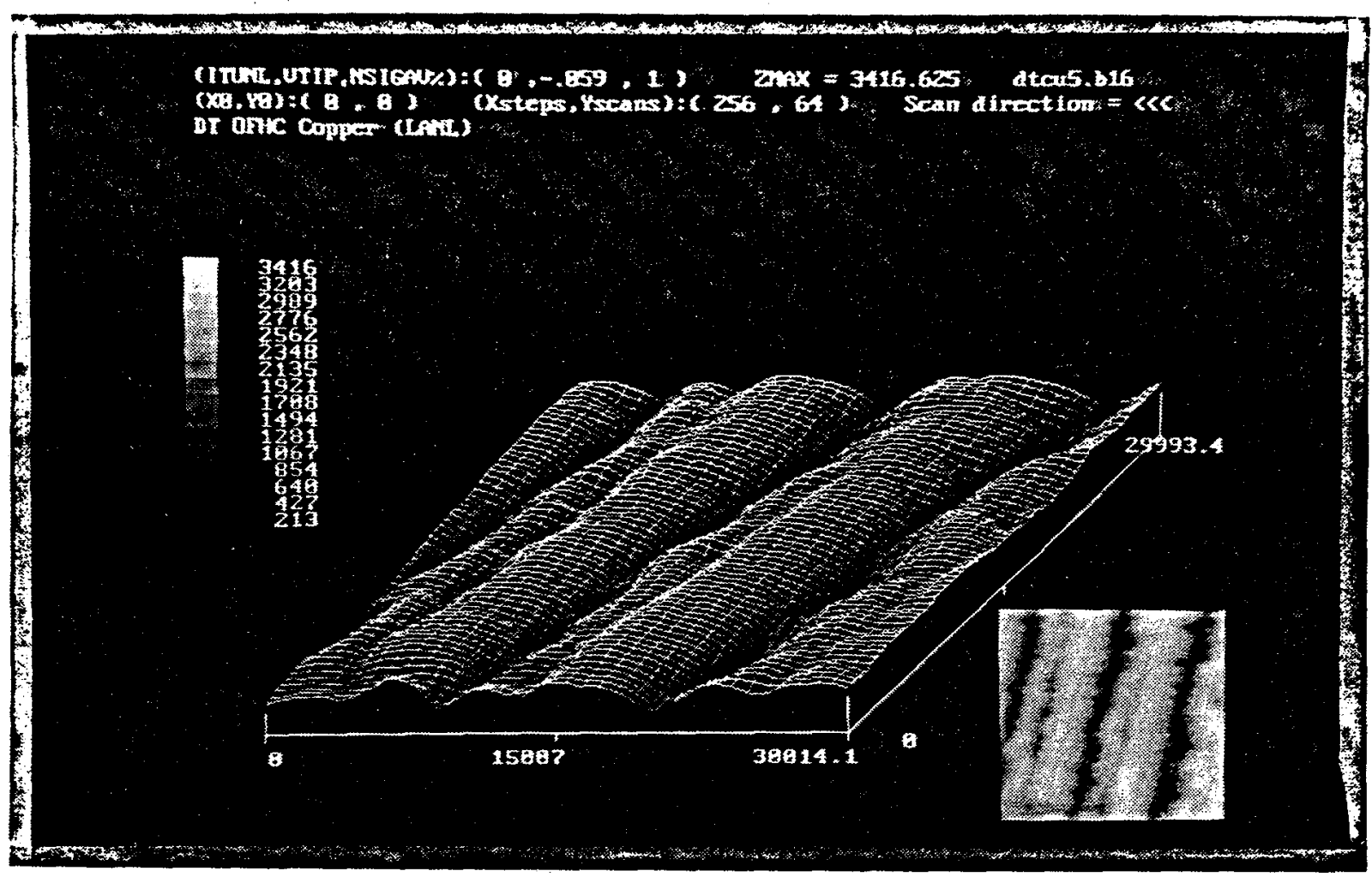

Figure 8: STM image of $1: 0 \mu \mathrm{m}$ gratings

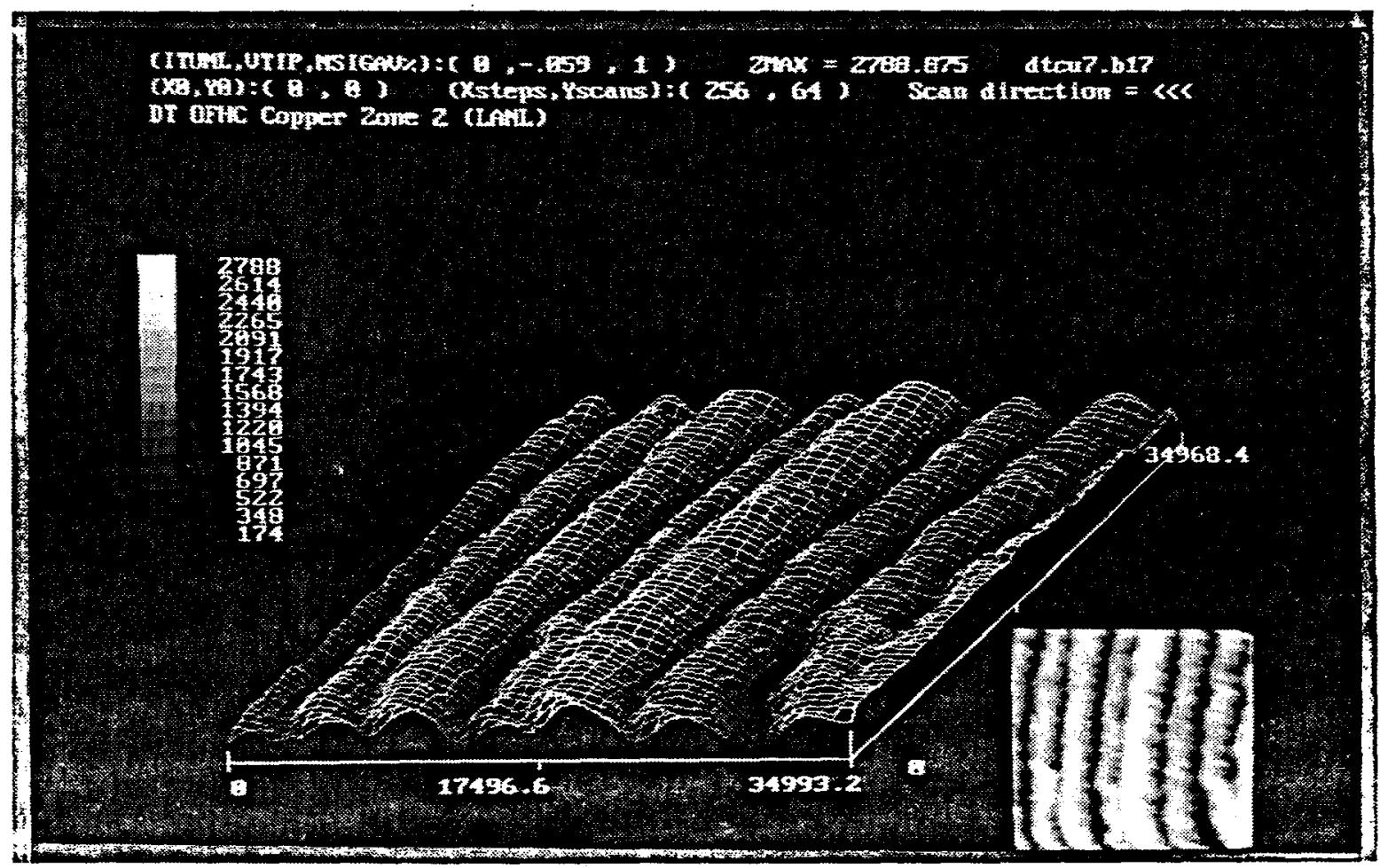

Figure 9: STM image of $0.5 \mu \mathrm{m}$ gratings 


\subsection{COMPARISON OF SAMPLES}

The surface profile of the sample machined with a large nose radius tool showed unexpected feature orientation. How the SEM and STM images compare continues to be a subject of debate in these samples. Several interpretations have risen as to the explanation of the features seen using the STM on sample \#1. The obvious tool marks in the SEM images were not visible using the STM. Further experiments will be needed to understand the differences between these different imaging techniques. Hypotheses for the striated lines in the STM images include; slip planes developed during the cutting process and tool tip vibrations.

The features measured on Sample \#2, turned at Los Alamos National Laboratory, fit predictions of spacing and height. SEM micrographs of this sample demonstrated how closely the SEM and STM matched. Surface structure which demonstrate vibration effects of the tool tip during cutting, not seen in the SEM, are useful in understanding features in the first sample. The rounded tops between each groove may be due to the chip removal processes, ie, tearing of the chip at the edge of the tool.

\subsection{CONCLUSIONS AND FUTURE WORK}

The full implications of the surface features measured by the STM on Sample \#1 has not yet been determined. Several theories are under consideration. Tool wear, vibration, or the cutting process itself have been suggested as possible causes. An experiment is in progress which will use UBAC copper [4] samples. This material has a much finer grain size and is harder than OFHC copper. Similar cuts will be made on these samples for comparison. Strict attention will be placed upon post-sample preparation, ie, no contact will be made to the surface other than the use of solvents or dry air. This will eliminate several unknowns due to sample preparation and will also prove or disprove theories thus far made upon the initial results.

Sample \#2 revealed results that are closer to that expected. SEM and STM correlated closely with this sample. The reason for the rounded tops between each of the grooves is not clear at this time, however, by diamond turning the less ductile UBAC copper, the effects of material flow may be deferred. If the assumption is correct that induced flow was the cause for a decrease in edge definition on the peaks of the grooves, then the UBAC copper should increase the edge sharpness. This will be studied by cutting new gratings into the UBAC copper sample with the same tool used at LANL.

The usefulness of STM imaging of diamond turned samples has been clearly demonstrated. The program is now to a turning point, from STM instrument development and verification to the application of STM techniques. The next section presents a preliminary analysis of the influence of dynamics in Diamond Turning. The STM will play a key role in defining the effects of dynamics on surface features. 


\section{References}

[1] R. Dragoset, R. Young, H. Layer, S. Mielczarek, E. Teague, and R. Celotta, "Scanning Tunneling Microscopy Applied to Optical Surfaces".

[2] M. Gehrtz, H. Strecker, and H. Grimm, "Scanning Tunneling Microscopy of Machined Surfaces", Journal of Vacuum Science and Technology, Vol. 2, March/April 1988, pp. 432-435.

[3] "Talystep Operating Instructions", Taylor-Hobson, Leicester, England, p. 19.

[4] UBAC Samples Provided by Lawrence Livermore National Laboratory, Lawrence Livermore, California. 


\title{
20 ANALYSIS OF THE INFLUENCE OF TOOL DYNAMICS IN DIAMOND TURNING
}

\author{
Steven C. Fawcett and Dan E. Luttrell \\ Graduate Students \\ Richard F. Keltie \\ Associate Professor, MAE
}

This report describes the progress in defining the role of machine and interface dynamics on the surface finish in diamond turning. It contains a review of literature from conventional and diamond machining processes relating tool dynamics, material interactions, and tool wear to surface finish. Data from experimental measurements of tool/workpiece interface dynamics are presented as well as machine dynamics for the DTM at the Center.

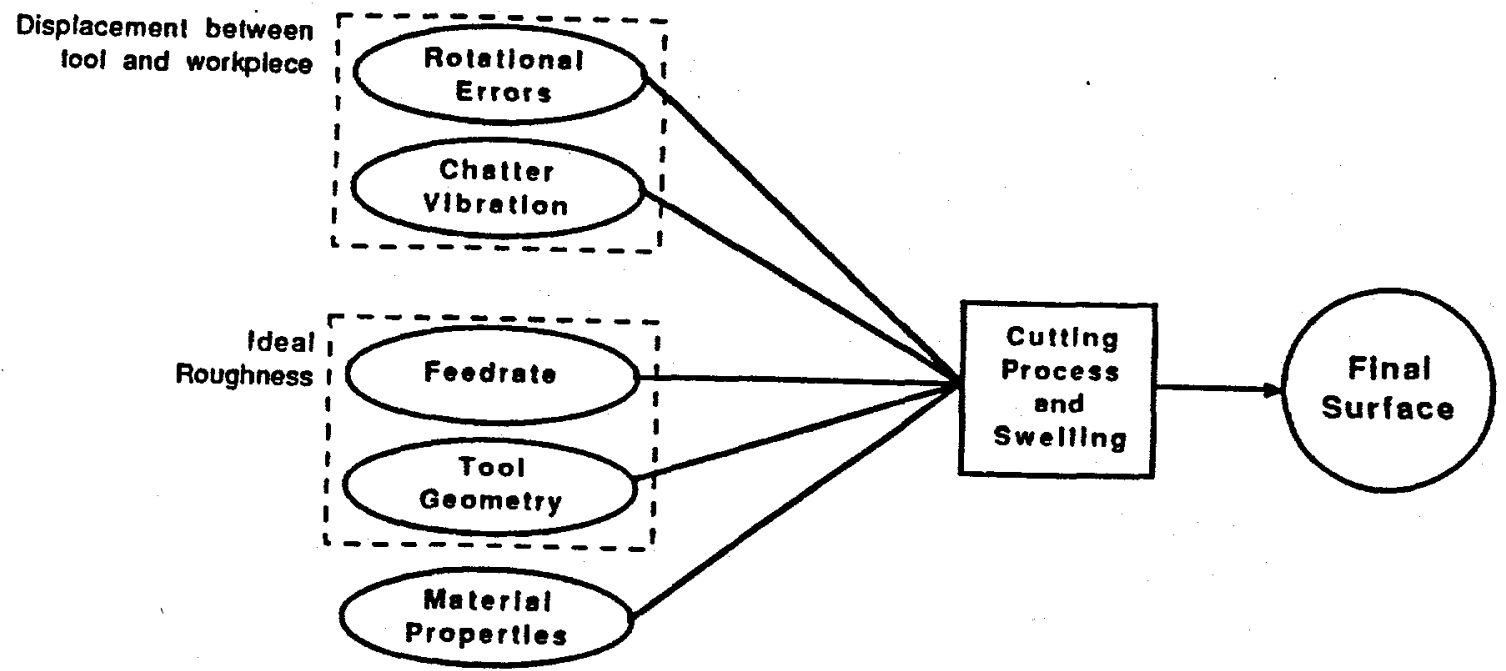




\subsection{INTRODUCTION}

The quality of the surface obtained by diamond turning is influenced by a number of factors including the material, the tool, and the machine. Material properties such as alloy phases, grain boundaries, and fracture toughness leave their signature on the finished surface roughness. In addition, features on the tool edge will be patterned in the finished surface. Finally, relative motion between the tool and workpiece due to dynamic effects will appear in the finished surface.

The preceeding section (Section 19) described measurements of a diamond turned surface using the STM. The results indicate a wealth of information is available from the surface texture about the dynamics of the cutting process. The relative motions of the tool with respect to the workpiece result in surfaces with a roughness larger than the theoretical roughness calculated from the tool radius and infeed parameters. To improve these surfaces one needs to understand the specific dynamics involved. For this reason, an investigation is underway to identify the cutting dynamics as well as potential frequencies and amplitudes of vibrations that can occur during the machining of metals on a diamond turning machine.

\subsection{BACKGROUND}

Several papers have been reviewed that investigated the dynamics of the turning operation $[1,2,3]$. These articles provide an overview of the standard turning operation. Tlusty [1] showed that there is a closed loop relationship between the machine structure and the cutting process. He states that the primary sources of dynamic excitation are the machine structure and the "feedback between subsequent cuts" and are not attributed to the chip formation mechanics. This may not be entirely true when dealing with precision diamond turning where the chip thickness is several orders of magnitude smaller than in the standard turning operation. Tlusty also defines two important parameters that are used extensively in research involving the tool/workpiece interactions. When referring to the removal of waviness already present in a surface, he calls the forces produced on the tool the outer modulation of the cutting force. In the case of cutting a periodic motion into a surface by the vibrations of a tool normal to the workpiece, the forcing function is referred to as the inner modulation.

The article by $\mathrm{Wu}[2]$ provides a mathematical basis for defining the governing equations of cutting dynamics. He includes shear flow stress effects as well as thermal effects in the model. The shear flow stress in the cutting of metals is a function of strain, strain rate and temperature. But, the variations in these parameters appear to cancel each other and the shear flow stress remains fairly constant for various cutting conditions on the same metal. One major conclusion is that variations in shear flow stress for different materials alter only the amplitude of vibrations and have little effect on the phase. Therefore the phase of the vibrations can be considered independent of the cutting substrate. Srinivasan 
[3] utilizes measurements of the input to the tool and the subsequent tool response to identify the parameters in the cutting force models. The motivation in most of this work has been to better understand regenerative chatter or to improve a machine's productivity by maximizing material removal rate. Only a limited effort has been made in the area of diamond cutting [4] where the objective is to improve the quality of the workpiece form and finish. The purpose of this research was to determine the dynamic characteristics of the tool/workpiece interaction in single point diamond cutting and, in an attempt to better understand the process, speculate on the origins of cutting forces.

\subsubsection{Micromachining}

The term micromachining is used when the final cut is made at depths of $5 \mu m$ or less. The process is used to produce surface finishes of extremely high quality. The accuracy of the machined surface is determined by the stiffness and repeatability of the machine tool. The surfaces produced are specifically related to both the static and dynamic characteristics of the device. To achieve surface roughness and figure in the microinch range, stiff, well controlled apparatus such as a diamond turning machine is used. The analysis of these machines introduces a microscopic element into the cutting dynamics $[4,5,6,7,8]$. An investigation of certain characteristics of the machine structure was done by Myler [4]. He concluded that surface finish is dependent on the finite compliance of the machine structure resulting in small scale vibrations... and that the stiffness of the workpiece is a primary consideration. The fixturing for both the tool and the workpiece can result in unrestrained directions that are prone to vibration. By using a vacuum chuck instead of the usual clamping method, one can effectively increase the damping and stiffness offered by the workpiece and improve surface quality. Myler states that the proper selection of rotational speeds and cutting depths can eliminate some of the vibration induced errors.

Another article that specifically investigates the dynamics involved in micromachining was written by S. Takasu [5]. He analyzed the surfaces obtained by cutting an aluminum alloy with a single point diamond tool experiencing simple harmonic motion of low amplitude $(0.1 \mu \mathrm{m})$ and low frequency $(0.1$ to $10 \mathrm{~Hz})$. The experiment used two different tool geometries, a straight tool (Radius $=\infty$ ) and a round tool (Radius $=R$ ). The theoretical surface finish was estimated analytically and experimentally verified. The investigation concluded that surface finish can be improved by using the straight edge tools and optimizing the phase shift between the disturbance input and the revolution of the workpiece. A surface finish with a roughness of $0.01 \mu \mathrm{m}$ maximum was obtained even when the disturbance input was $0.03 \mu \mathrm{m}$ in amplitude by the proper optimization of the cutting parameters.

The features cut into a surface can be attributed to tool geometry,specific motions of the tool, material effects and machine motion errors. T. Sata [6] reports that the material effects experienced after turning aluminum can be larger than the geometric surface features due to tool and infeed parameters. Therefore, the total error in the surface features is due to many error sources as shown in Figure 1. When a Fourier transform of the surface 


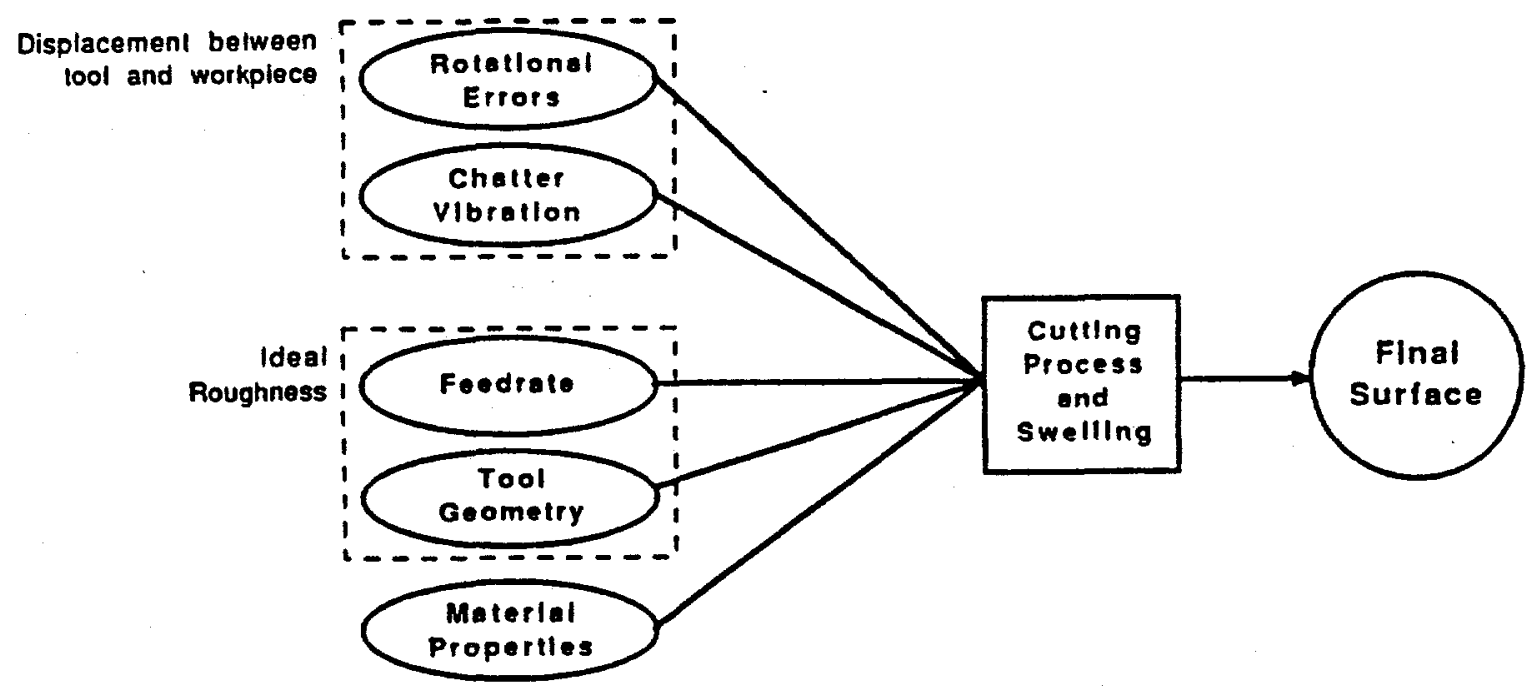

Figure 1: Total surface error sources, Sata (1985).

roughness data from an ideal surface (e.g. free cutting brass)is compared to an aluminum surface the peaks in the spectrum increase in height and width. This result gives a method of identification of what the author called post-machining swelling.

A study by Furukawa [7] analyzed the effects of the material properties of the workpiece on the force measurements from the diamond tool in a precision planing machine. The force measurements were made in the axial direction $\left(F_{z}\right)$ and the cutting directions $\left(F_{y}\right)$ at the base of the tool with a Kistler 9251A force transducer. This same transducer was used in the dynamic force measurements described in [4] and in Section 21 of this report and in the system identification measurements discussed later in this section. It was found that the cutting force per area of cut (denoted Specific Cutting Force) increased exponentially at the smaller cutting depths (less than $3 \mu \mathrm{m}$ ). The actual cutting data is shown in Figure 2 for the various materials. This data exhibits similar features to that observed in Section 21 of this report. The increase in specific cutting force, or flow stress, for the copper at small depths of cut has been attributed to a thin work hardened layer produced by previous machining.

The microstructure effects on the cutting force were also investigated by Furukawa. When the aluminum samples were heat treated to produce different grain sizes, the mean force was almost identical but that the dynamic responses differed greatly. The maximum frequency range of the axial force component was much less in the samples with the larger grains than it was in the samples with smaller grains. When examined more closely, it was found that the mean value of the force component increased by a factor of two at the grain boundaries. The cutting force did not exhibit the same trend and remained fairly constant. Furukawa theorizes that since the material is in the ductile regime, the chip produced is continuous and therefore the cutting force would show very little frequency content. When cutting brittle germanium, the chip is not continuous and the cutting force did not remain constant. In the amorphous resin (PMMA) both the forces remained 


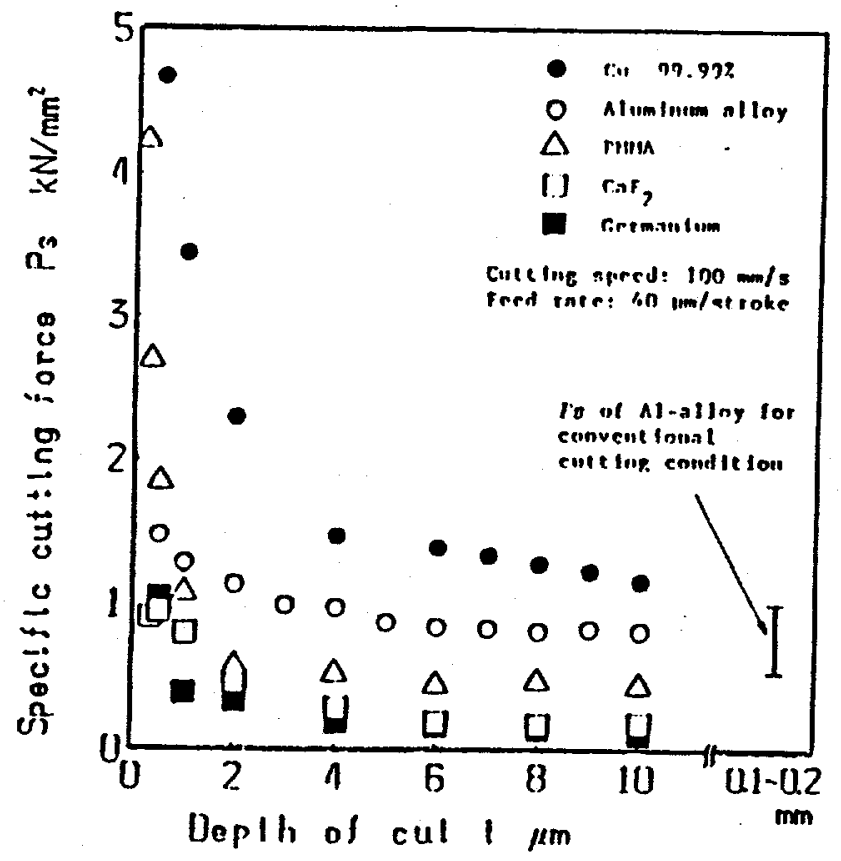

Figure 2: Cutting force data for Copper,Aluminum,Acrylic Resin (PMMA) Fluorite (CaF2) and Germanium, Furukawa (1988).

constant. Section 14 of this report shows the excellent surfaces possible with this material and the correspondence with the theoretical finish. This was also the case when single crystal materials were cut.

\subsubsection{Diamond Tools}

The diamond tool may experience wear after prolonged use. The determination of the extent of this wear is the subject of several papers $[8,9,10,11]$. Sugano [8] measured the forces on the tool and related them to the tool's total cutting distance. Both forces (normal and tangential) increased as the cutting distance increased but showed different trends. Figure 3 shows that the increase in the cutting (main) force was almost linear but the axial (thrust) force initially showed a quadratic increase and then remained fairly constant after about $700 \mathrm{~km}$ total cutting distance. Jiang [9] used accelerometers mounted on a standard carbide tool to measure the accelerations in the cutting and infeed direction. The time-domain signals were converted to the frequency-domain for analysis. It was found that the magnitude and frequency content of the acceleration signals varied greatly as tool wear progressed. He also found that there was a transition zone of micro-breakage before complete failure of the tool that could be detected. A method of returning the signal analysis problem back to the time domain was also presented to increase the speed with which the wear detection can be completed. Giusti [10] used a television camera routed 


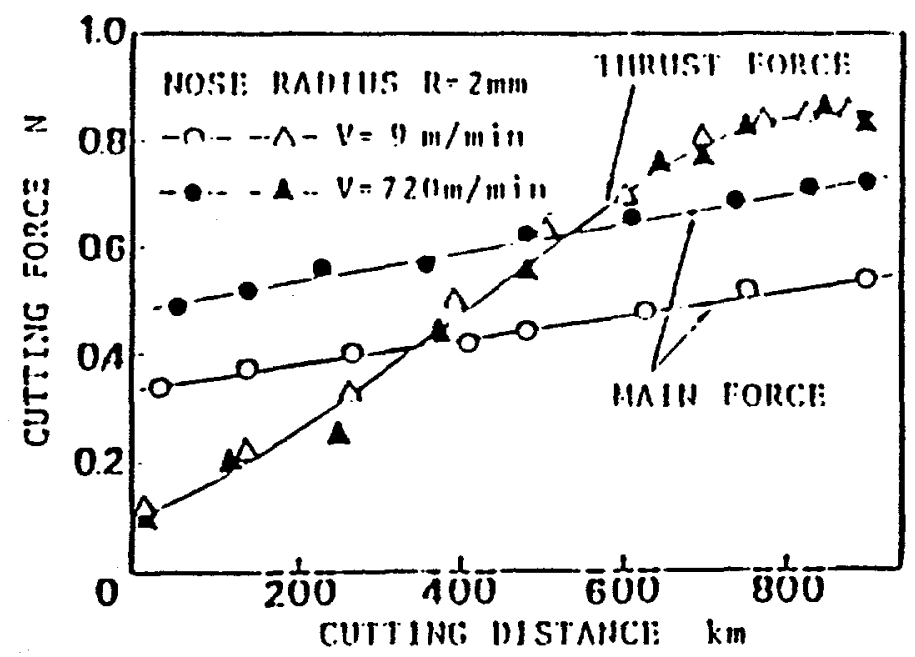

Figure 3: Cutting force versus total cutting distance for aluminum, Sugano (1987).

through optical fibers to determine the extent of tool wear during the cutting process. The system gave information on the total wear area and location for carbide cutting tools and could possibly be adapted for use with diamond cutting tools.

To completely understand the wear experienced by a diamond tool, there must be a way to categorize the strength of the specific tool. Ilawa [11] developed two non-destructive strength evaluations to estimate the fracture strength of the diamond. One method, Infrared Absorbtion (IRA), analyzes the absorbtion coefficient at a specific wavelength (7.3 $\mu \mathrm{m})$ and is correlated with the fracture strength. The correlation showed a linear trend decreasing strength with increasing absorbtion. The other method, Electron Spin Resonance (ESR), which has two different types of spectra that depend on the phase difference between the magnetic-field of the modulation signal and the intensity. The first type measures the relative intensity of the spectrum and corresponds to the nitrogen impurity defects. This relative intensity again shows a decreasing linear trend in fracture strength as the intensity increases. The other method, ,correlates the line width of the spectrum to the number of unpaired electrons due to vacancies or unbound electrons. Therefore, the line width is proportional to the fracture strength of the diamond sample. An increase in the density of the unpaired electrons decreases fracture strength and is not necessarily related to the nitrogen content.

\subsubsection{Tool Fixturing}

The stiffness of the tool is not only related to its inherent physical properties but also to the stiffness of the tool fixturing. A study into the contact rigidity of the tool/fixture interface was reported by Marui [12]. The analysis modeled the contact area of the tool shank with the fixture as an elastic interface similar to a beam on an elastic foundation. The paper presents theoretical equations as well as experimental results. Since current efforts in the 
Center revolve around the Rank Pneumo ASG-2500 diamond turning machine (DTM), the specific tool holder is being modeled as a finite element project to determine relative stiffness and natural frequencies. These results will be reported at a later date.

\subsection{PRELIMINARY RESULTS}

\subsubsection{Machine Dynamics}

To better understand the effects of the machine tool/workpiece vibrations on tool life and surface quality, one must first determine the natural modes and frequencies of the critical components of the machine. The Pneumo DTM discussed in Section 9, has two slides that move in orthogonal $x$ and $z$ directions. The layout is shown in Figure 5. Normal operation has the part rotating clockwise with the chuck mounted on the z-axis. The depth of cut is therefore controlled by the motion of the z-axis. The $x$-axis controls the infeed of the cut (right to left).

The inherent dynamics of the slides affect the amplitudes and frequencies observed at the tool tip. The natural frequencies for the translation slide system were determined by the following method. A piezoelectric accelerometer was attached to the slide oriented in a specified direction. The slide was then excited in the same direction with a small rubber hammer. The output from the charge amplifier attached to the accelerometer was input into a spectrum analyzer and six averages of the transient were made for the desired frequency range. Figure 6 shows the response spectrum when the $x$-slide is excited in the $x$-direction. The response shows a sharp peak at the natural frequency of $89 \mathrm{~Hz}$. The $y$ and $z$ directions are shown in Figures 7 and 8 respectively. The slide is much stiffer in the $y$-direction since this direction is restrained by the fluid bearing and it had a fundamental natural frequency of about $320 \mathrm{~Hz}$. The response of the $x$-slide in the z-direction is not a true representation of the actual response of the slide because the frequency detected is due to the motion of the z-axis which is transmitted through the machine structure. This was proven through subsequent investigation of the relative amplitudes of the motions of the two slides in the z-direction. Figure 9 verifies that that the fundamental frequency of the z-slide is at $67.5 \mathrm{~Hz}$ as previously detected. Fundamental modes for the z-slide in the $x$ and $y$ directions were not detectable due to the increased stiffness in the fluid film bearing. The natural frequencies determined in these experiments will be valuable in isolating their contributions the overall tool motion spectrum.

\subsubsection{Indentiflcation of Cutting Dynamics}

Measured tool forces and depths of cut were analyzed using system identification techniques from the field of automatic control [14] to develop a model of the tool/workpiece interaction. Figure 9 illustrates the experimental configuration and resulting model of the measured 


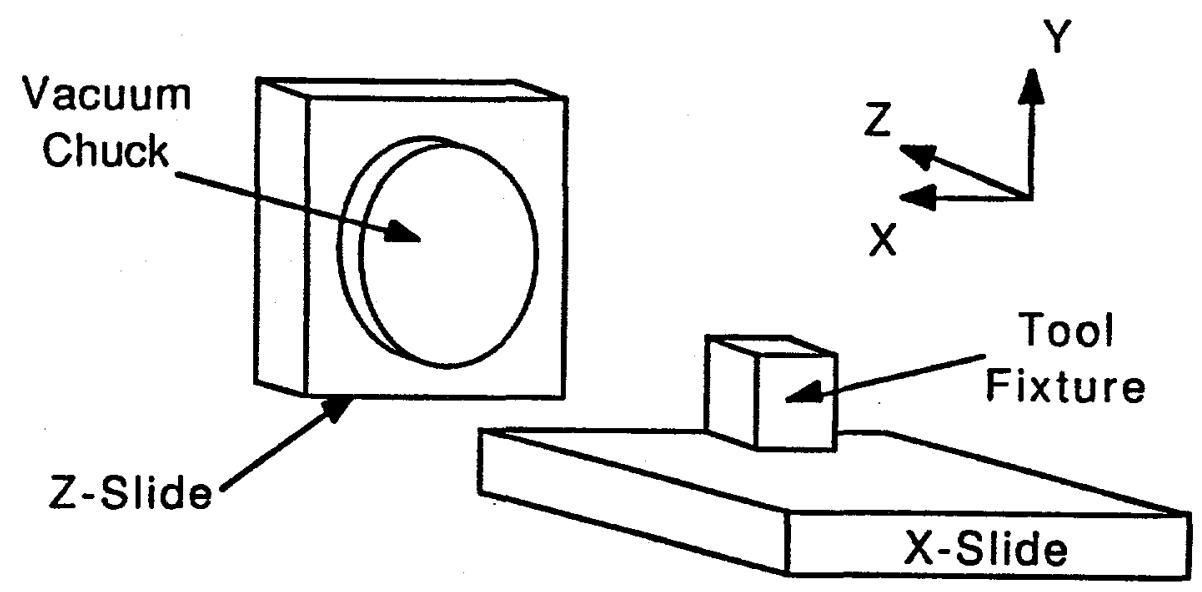

Figure 4: Layout of the diamond turning machine.

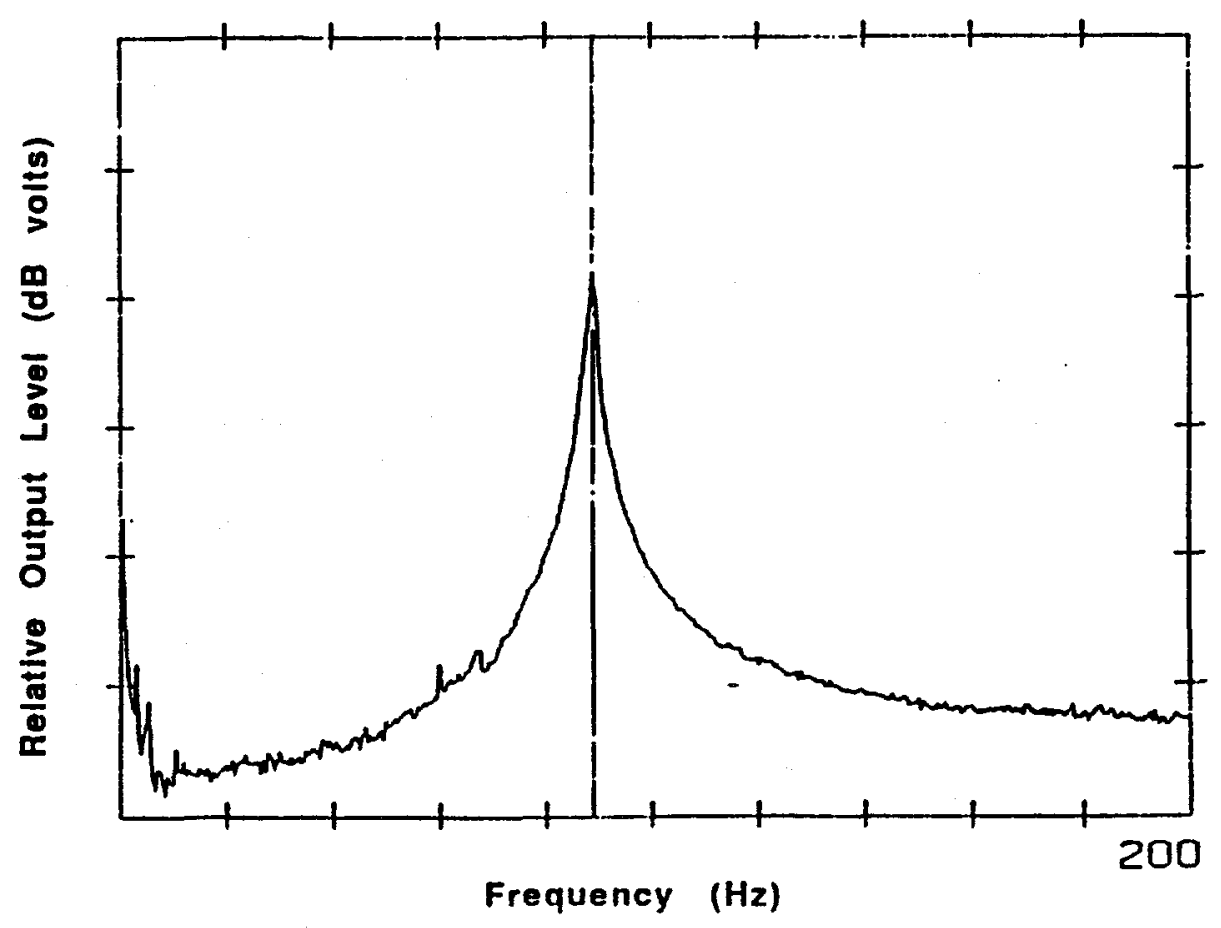

Figure 5: Response spectrum for the $\mathrm{x}$-slide in the $\mathrm{x}$-direction. 


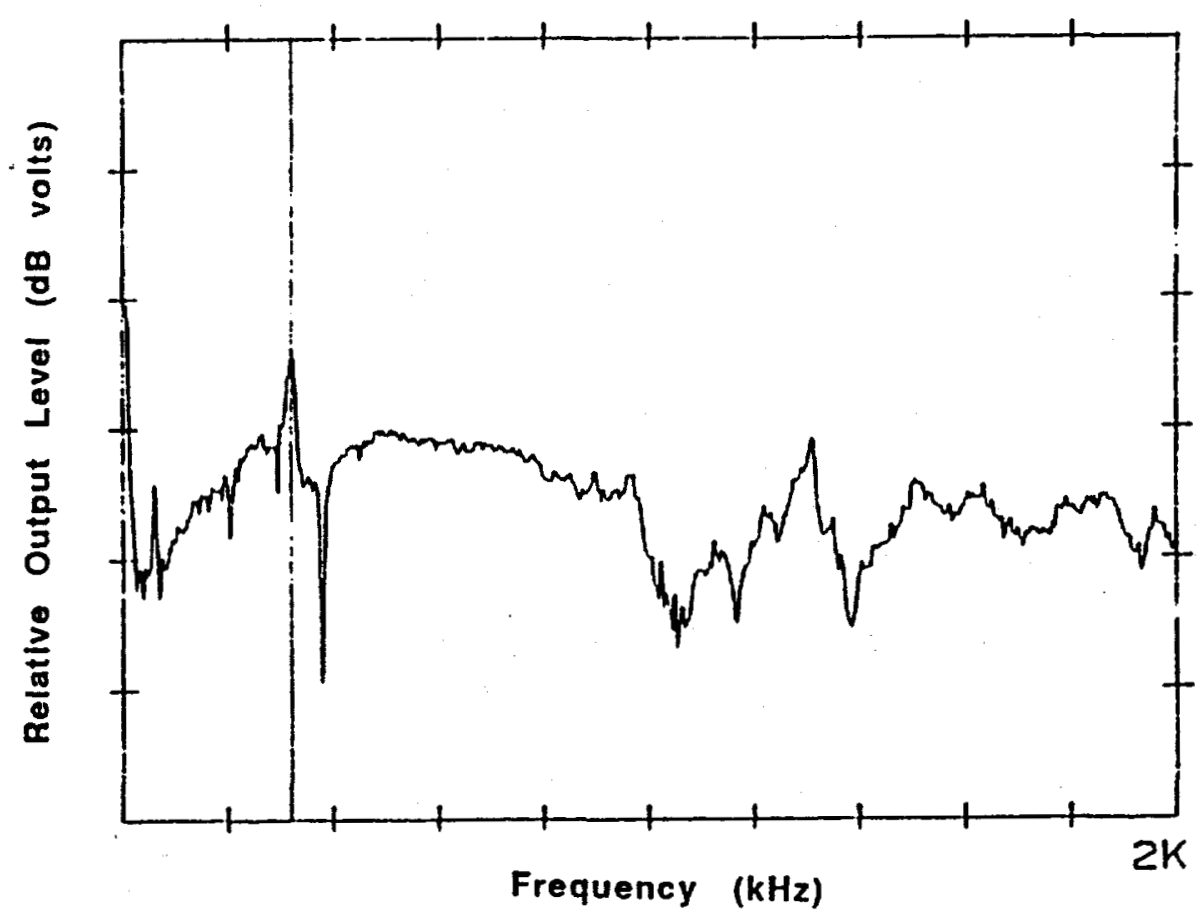

Figure 6: Response spectrum for the $x$-slide in the $y$-direction.

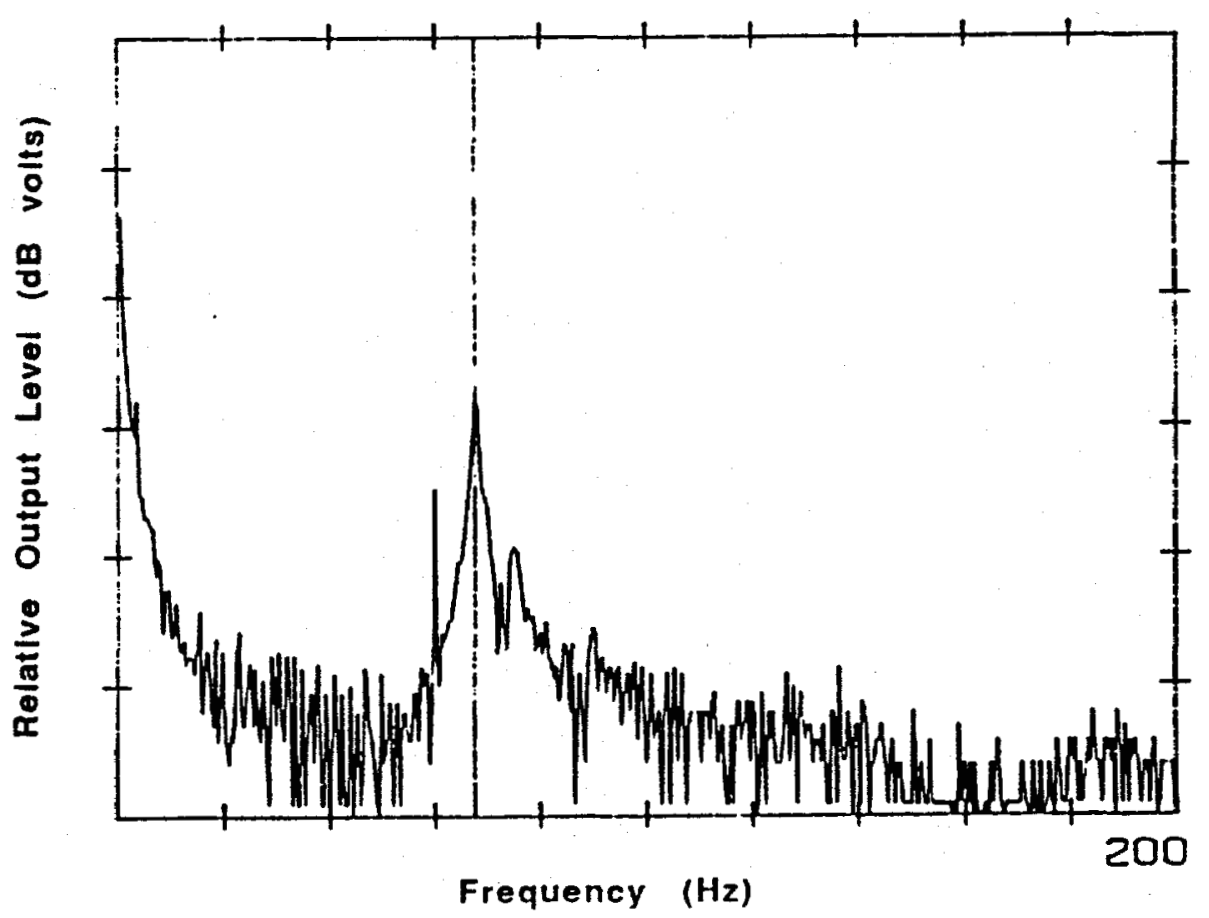

Figure 7: Response spectrum for the $x$-slide in the z-direction. 


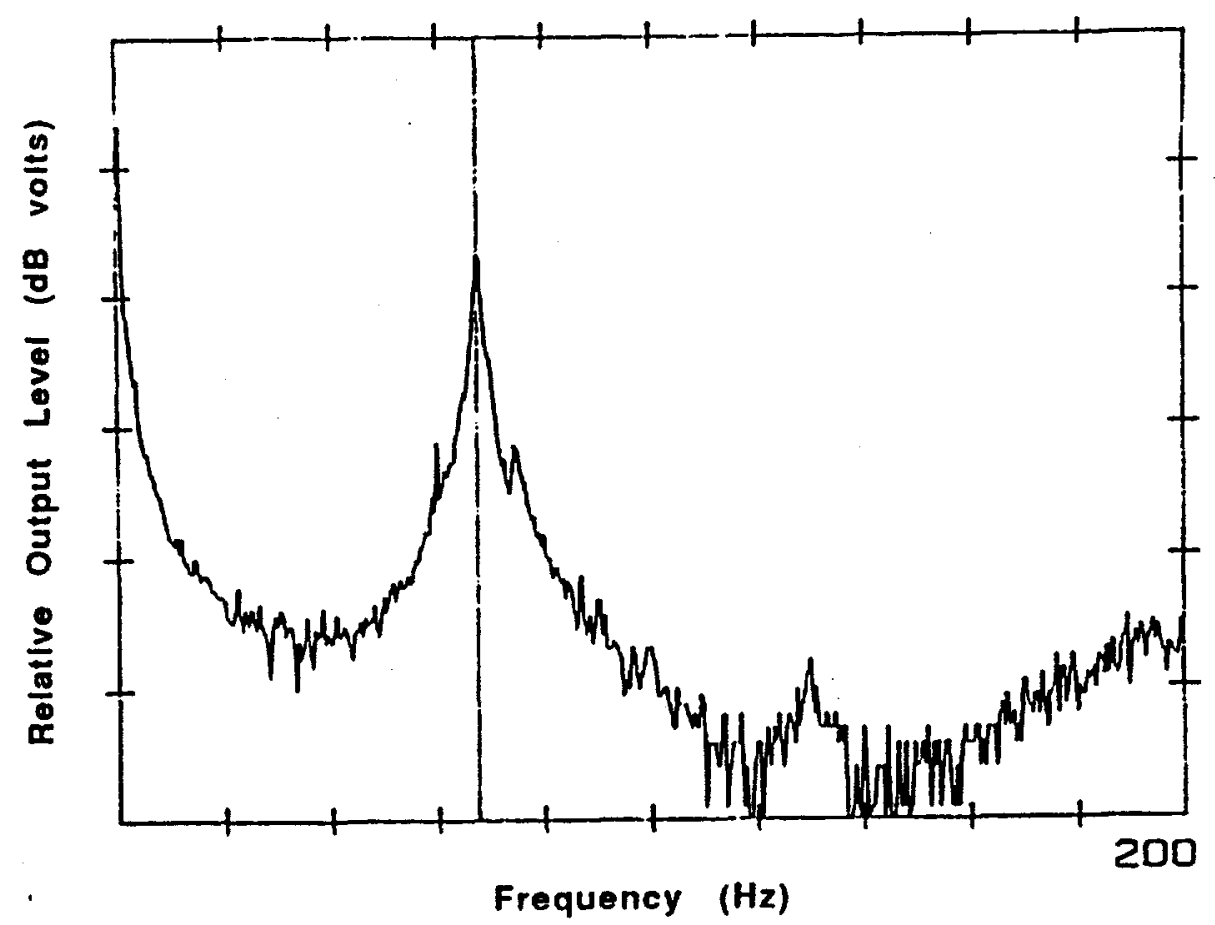

Figure 8: Response spectrum for the z-slide (chuck) in the z-direction.

data. The tool was driven with small random displacements around some nominal cutting depth and the resulting forces normal to the workpiece surface were measured. Analysis of the data used the Weighted Recursive Least Squares algorithm, and yielded parameters that describe the cutting process dynamics.

Experiments were conducted over a wide range of cutting conditions. These tests were facilitated by the use of a Fast Tool Servo [15] modified to accommodate a piezoelectric force transducer. With this device the cutting depth could be varied by as much as 25 $\mu m$ and directly measured with an integral capacitance gage. Sensitivity of the force transducer was less than $0.01 \mathrm{~N}$ and proved to be sufficient for this study. The Fast Tool Servo was mounted on a commercial diamond turning machine. Displacement and force data were recorded by a high-performance data acquisition system and transferred to a minicomputer for analysis.

Tests were conducted in workpieces of OFHC copper, 60-40 brass and 6061-T6 aluminum using three different tools over a wide range of cutting conditions. Tools used for cutting had circular nose radii of $0.76,1.52$ and $2.54 \mathrm{~mm}$ with $0^{\circ}$ rake angle. Initial rests were designed so that grooves produced by the cutting tool did not overlap in successive revolutions of the workpiece. This was done to prevent the small depth variations of one cutting groove from affecting the cutting force of subsequent passes. Displacements of the tool around the nominal cutting depth were random in nature but limited to below $500 \mathrm{~Hz}$ and amplified so that depth variations were approximately $.75 \mu \mathrm{m}$ peak-to-peak. 


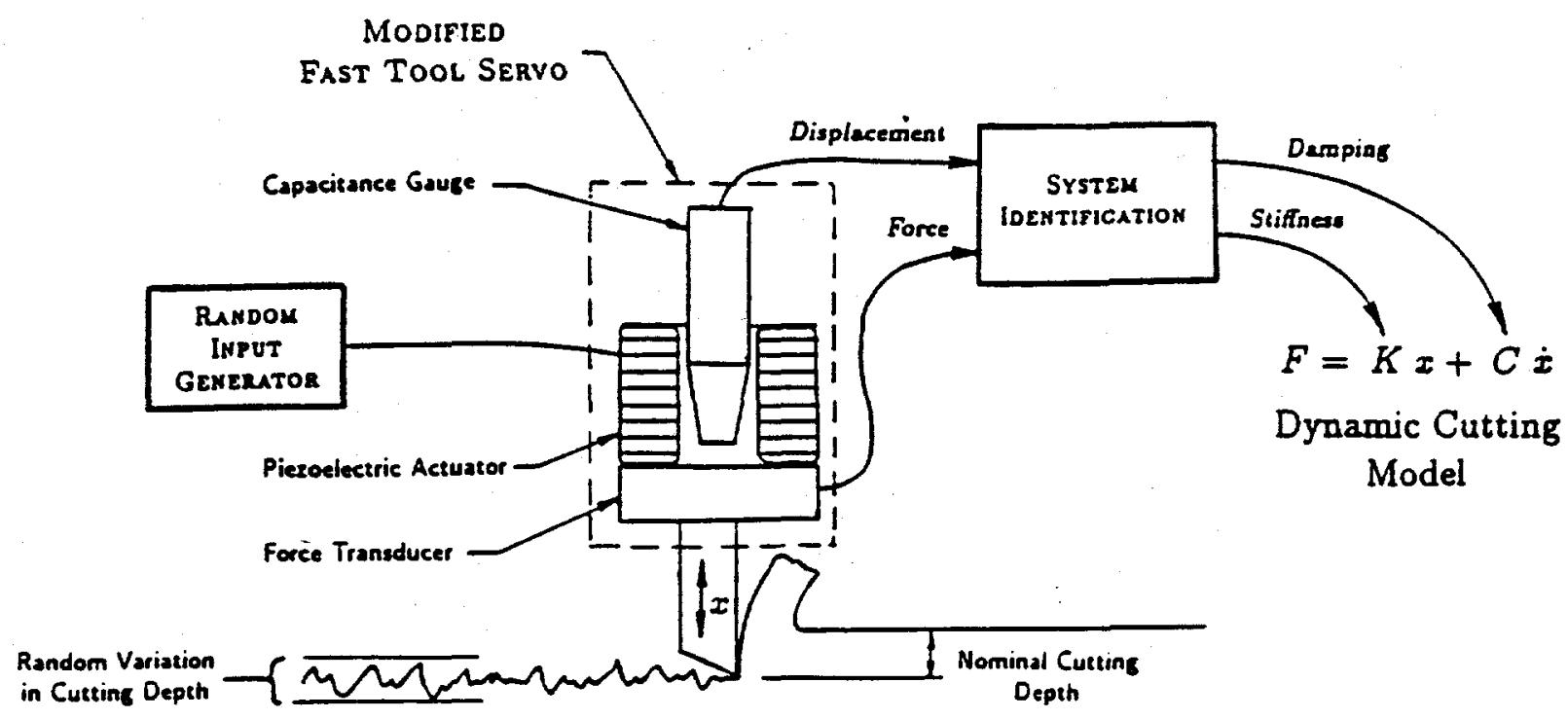

Figure 9: Diagram illustrating the experimental configuration and system identification method used to characterize the dynamics of single point diamond cutting.

Analysis of the measured data showed that a model of the form

$$
F_{x}=K x+C \dot{x}
$$

could be used to describe the tool/workpiece interface. In this model the $K x$ term is that part of the normal cutting force proportional to cutting depth and the $C \dot{x}$ term that part dependent on the rate of change of cutting depth. Together these two terms (the static component, $K x$, and the damping component, $C \dot{x}$ ) may be considered to make up the effective dynamic stiffness.

Results of the system identification process have led to curves for the coefficients $K$ and $C$ such as shown in Figure 10. In this figure the static coefficient, $K$, is plotted as a function of nominal cutting depth for the three test materials. Error bars accompanying each point show variance in the data at each depth as a result of four different cutting speeds. Plots were made in this way because no correlation was found between the identified coefficients and cutting speed. For this particular tool, the static coefficient was essentially independent of depth of cut and was largest for the aluminum workpiece and smallest for the copper. The damping coefficient, $C$, is also plotted in Figure 10. Once again the coefficient is independent of the depth of cut and cutting speed but the ranking is reversed with the largest value measured for the copper and the smallest for the aluminum.

Dynamic stiffness of the tool/workpiece interface measured in this study is small when compared to the stiffness of the axes of a diamond turning machine which are on the order of $25 \mathrm{~N} / \mu \mathrm{m}$. For example, the maximum stiffness encountered using the Fast Tool 


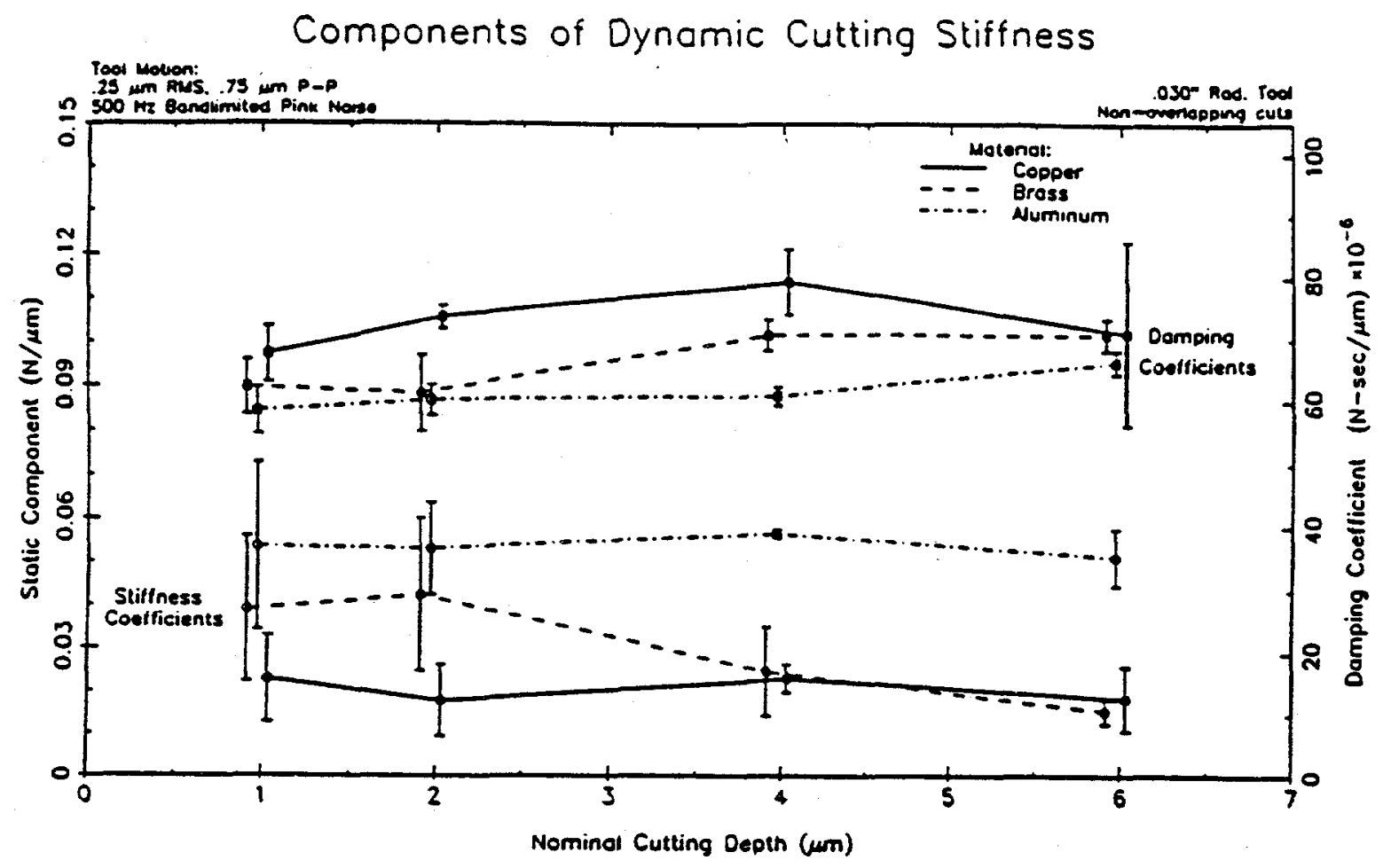

Figure 10: Dynamic cutting stiffness components identified from tests in copper, brass and aluminum with a .030 in. radius tool.

Servo at its maximum bandwidth can be calculated by considering a sinusoidal input at a frequency of $1000 \mathrm{~Hz}$. If the input is $x=x_{\max } \sin (2 \pi f)$ then $\dot{x}=x_{\max } 2 \pi \cos (2 \pi f)$ which implies $\dot{x}_{\max }=x_{\max } 2 \pi f$. Rewriting Equation (1) in terms of maximum dynamic stiffness gives

$$
\frac{F_{x}}{x}=K+\frac{C \dot{x}_{\max }}{x}
$$

Substituting the values of $f=1000 \mathrm{~Hz}, K=.04 \mathrm{~N} / \mu \mathrm{m}$ and $C=70 \times 10^{-6} \mathrm{~N}-\mathrm{s} / \mu \mathrm{m}$ results in a maximum dynamic stiffness of $\frac{\underline{F}_{x}}{x}=.48 \mathrm{~N} / \mu \mathrm{m}$. This stiffness is $2 \%$ of the machine stiffness and, while it will impose some phase delay in the tool motion due to the damping, the net effect should be extremely small.

This result influences the control algorithm for tool position when using a Fast Tool Servo. If the tool/workpiece interface adds only a small component to the forces and is virtually the same for different material properties, it can be ignored in the control algorithm. Thus the controller algorithm discussed in Section 9 can be independent of the material being machined. 


\subsection{FUTURE INVESTIGATIONS}

Several areas of investigation are currently being pursued. The first area of interest is in the detectability of tool wear thru remote dynamic measurements. These measurements will use accelerometers on or near the tool and the force transducer capable of measuring the forces in the three orthogonal directions. The tool to be used is a new diamond tool from Norton Tools with a $1.5 \mathrm{~mm}$ by 30 degree nose radius with a 10 degree clearance angle and 0 degree rake. The diamond tool is mounted in a $6 \mathrm{~mm}$ square by $20 \mathrm{~mm}$ length steel shank. The total cutting distance will be monitored with cut samples and force/acceleration data stored for discrete stages of wear. The samples will be examined in conventional as well as scanning tunnelling microscopes. An STM technique is bearing developed to study the change in edge radius of the tool. The data from the sensors will then be correlated to the specific surface features on the samples. The replication of the tool dynamics into the cut surface is the second area of interest. The data collected can be used for both the tool wear and the replication studies. It is hoped that the features can be identified with the modes of vibration of the tool and that the data will also give an indication of the point where the surface irregularities due to severe vibrations or tool wear occurred. The cuts will be made on 2024 aluminum and free machining yellow brass samples. The cuts will be made at several speeds,depths and infeeds to provide a broad range of data.

The motion of the tool with respect to the workpiece will also be measured by attaching an optical fiber sensor to the tool. The sensor will be parallel to the tool shank and aimed at the cut surface. The output from the sensor will provide information on the actual surface roughness [13] as well as the relative motions of the tool and workpiece. A frequency domain analysis of the signal from the optical gage and the other transducers will provide some insight into the dynamic effects on the cutting process. 


\section{References}

[1] J. Tlusty, "Analysis of the State of Research in Cutting Dynamics", Annals of the CIRP, Vol. 27/2/1978, p. 583.

[2] D.W. Wu, "Comprehensive Dynamic Cutting Force Model and Its Application to Wave-Removing Processes", Journal of Engineering for Industry, Vol. 110, May 1988, p. 153.

[3] K. Srinivasan and C.L. Nachtigal, "Investigation of the Cutting Process Dynamics in Turning Operations", Journal of Engineering for Industry, Vol. 100, August 1978, p. 323.

[4] J. Franse, and R.J.P. Schrama, "The Precision Cutting Process as a Non-Linear Closed Loop System", Precision Engineering, Vol. 10 No. 4, 199, 1988.

[5] J.K. Myler, "The Influence of Machine Tool Vibration on the Surface Texture of Diamond Turned Components", British Aerospace, 1987.

[6] S. Takasu, M. Masuda and T. Nishiguchi, "Influence of Steady Vibration with Small Amplitude Upon Surface Roughness in Diamond Turning", Annals of the CIRP, Vol. 34/1/1985.

[7] T. Sata et al., "Analysis of Surface Roughness Generation in Turning Operation and its Applications", Annals of the CIRP, Vol. 34/1/1985, p. 473.

[8] Y. Furukawa and N. Moronuki, "Effect of Material Properties on Ultra Precise Cutting Processes", Annals of the CIRP, Vol. 37/1/1988, p. 117.

[9] T. Sugano and K. Takeuchi, "Diamond Turning of an Aluminum Alloy for Mirror", Annals of the CIRP, Vol. 36/1/1987, p. 17.

[10] C.Y. Jiang, Y.Z. Zhang and H.J. Xu, "In-Process Monitoring of Tool Wear Stage by the Frequency Band-Energy Method", Annals of the CIRP, Vol. 36/1/1987, p. 45.

[11] F. Giusti, M. Santochi and G. Tantussi, "On-Line Sensing of Flank and Crater Wear of Cutting Tools", Annals of the CIRP, Vol. 36/1/1987, p. 41.

[12] N. Ikawa, S. Shimada and H. Tsuwa, "Non-Destructive Strength Evaluation of Diamond for Ultra-Precision Cutting Tool", Annals of the CIRP, Vol. 34/1/1985, p. 117.

[13] E. Marui, S. Ema and S. Kato, "Contact Rigidity at Tool Shank of Turning Tools", Journal of Engineering for Industry, Vol. 109, May 1987, p. 169.

[14] S.C. Fawcett and R.F. Keltie, "Use of a Fiber Optic Displacement Probe as a Surface Finish Sensor", submitted for publication, Sensors and Actuators, 1988. 
[15] Goodwin, and Sin, Adaptive Filtering Prediction and Control, Prentice-Hall, 1984.

[16] Falter, P.J., and T.A. Dow, "A Diamond Turing Apparatus for Fabrication of NonRotationally Symmetric Surfaces", Proceedings of the International Congress for Ul. traprecision Technology, Aachen FRG, Weck \& Hartel Editors, Springer-Verlag, May 1988. 



\title{
21 MEASUREMENT OF TOOL FORCES IN DIAMOND TURNING
}

\author{
Joe Drescher \\ Graduate Student \\ T. A. Dow \\ Professor, MAE
}

A dynamometer has been designed and built to measure forces in diamond turning. The design includes a 9-component, piezo-electric transducer. Initial experiments with this dynamometer system included verification of its predicted dynamic characteristics as well as a detailed study of cutting parameters. Many cutting experiments have been conducted on OFHC Copper and 6061-T6 Aluminum. Tests have involved investigation of velocity effects, and the effects of depth and feedrate on tool forces. Velocity has been determined to have negligible effects between \& and $21 \mathrm{~m} / \mathrm{s}$. Forces generally increase with increasing depth of cut. Increasing feedrate does not necessarily lead to higher forces. Results suggest that a simple model may not be sufficient to describe the forces produced in the diamond turning process.

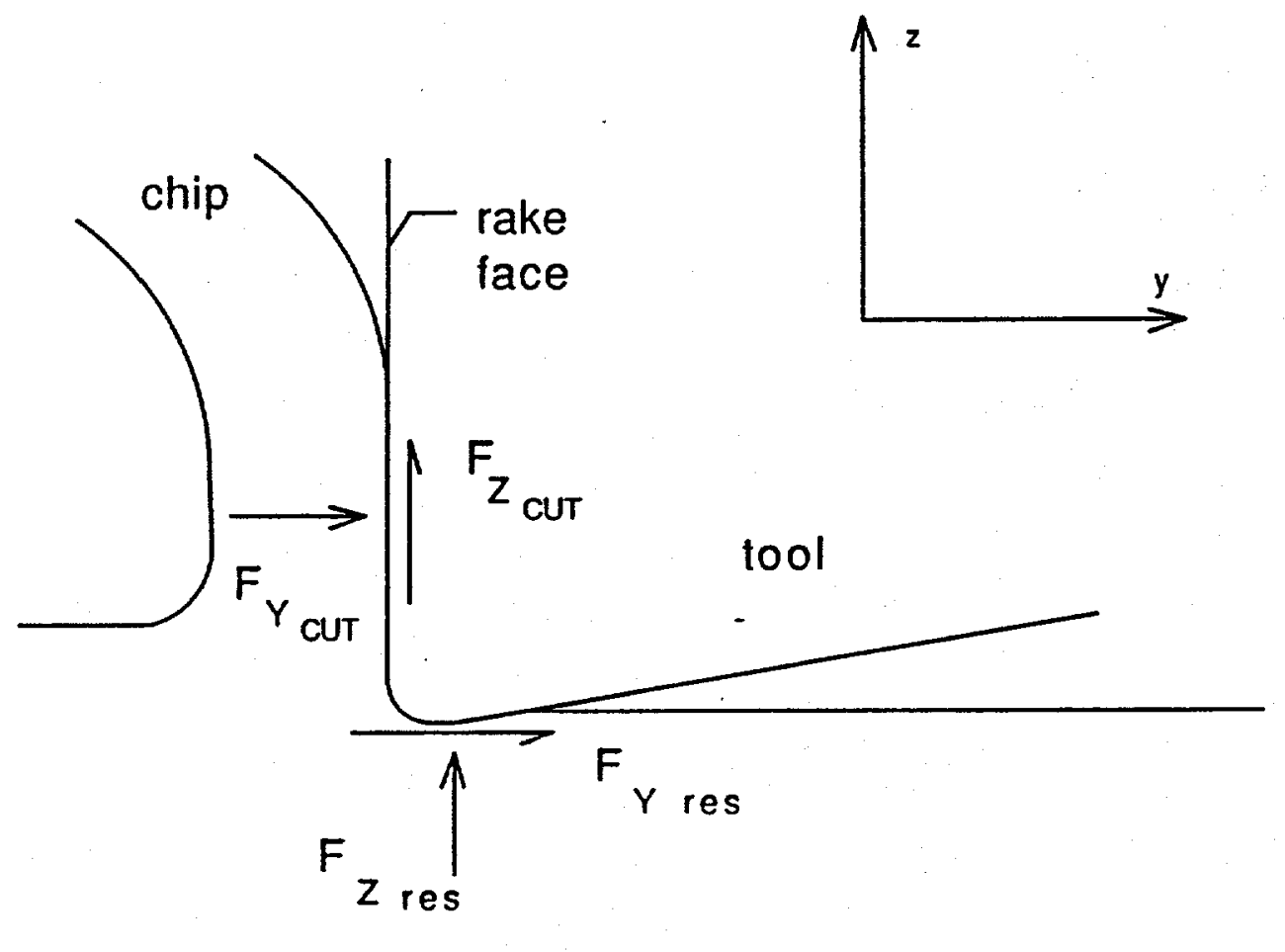




\subsection{INTRODUCTION}

A model which can quantitatively describe tool forces in diamond turning is essential for understanding and controlling this process. Starting with a simple model, we can predict forces and compare them to experimental data. The model can then be refined. Development of the model is by this iterative procedure.

A preliminary model was based on a flow stress acting over the chip's cross-sectional area to predict force in the cutting direction. The flow stress was taken as three times the material yield strength for both copper and aluminum. The force perpendicular to the cutting direction was estimated using a friction coefficient of 0.5 multiplied by the cutting force. This perpendicular force was split into two components, one in the feed direction, and one along the axis of the tool. To test and develop this model, the ability to accurately measure tool forces is very important.

A dynamometer has been designed to measure tool forces in diamond turning. The measurement system, including piezo-electric transducer, charge amplifiers, filters, and output device, yields average, steady-state forces in three orthogonal directions. It has been designed to aid in the development of a diamond turning model and is also seen as a possible tool for process control feedback.

\subsection{DYNAMOMETER}

\subsubsection{Dynamometer Design}

A basic goal in the dynamometer design was very high stiffness. The sensitivity of the dynamometer was also an important factor. Figure 1 illustrates the importance of this factor by showing the magnitudes of the predicted force components. The $y$-direction is the main cutting direction, parallel to the face of the workpiece. The force in the $y$-direction is expected to be the greatest for a sharp tool. The z-direction force is perpendicular to the workpiece and along the tool axis. The force in the $x$ or feed direction is expected to be the smallest of the three forces due to the low feedrates used in typical DT applications. For the two requirements of high stiffness and high sensitivity, piezo-electric crystals are ideal. The Kistler 9251A, a 3-component transducer used in similar applications [1], [2], [3] has a z-axis stiffness of $1000 \mathrm{~N} / \mu \mathrm{m}$ and a lower force threshold of about $1 \mathrm{gm}$.

There were three possible orientations for the 3-axis transducer as shown in Figure 2. Figure 2(a) shows the transducer mounted with its $z$-axis perpendicular to the workpiece. Figure 2(b) shows the $z$-axis parallel with the face of the workpiece and in the direction of the cut. Figure 2(c) has the $z$-axis parallel to the workpiece face and in the feed direction.

The stiffness of the transducer is highest in the $z$-direction when oriented as shown in 


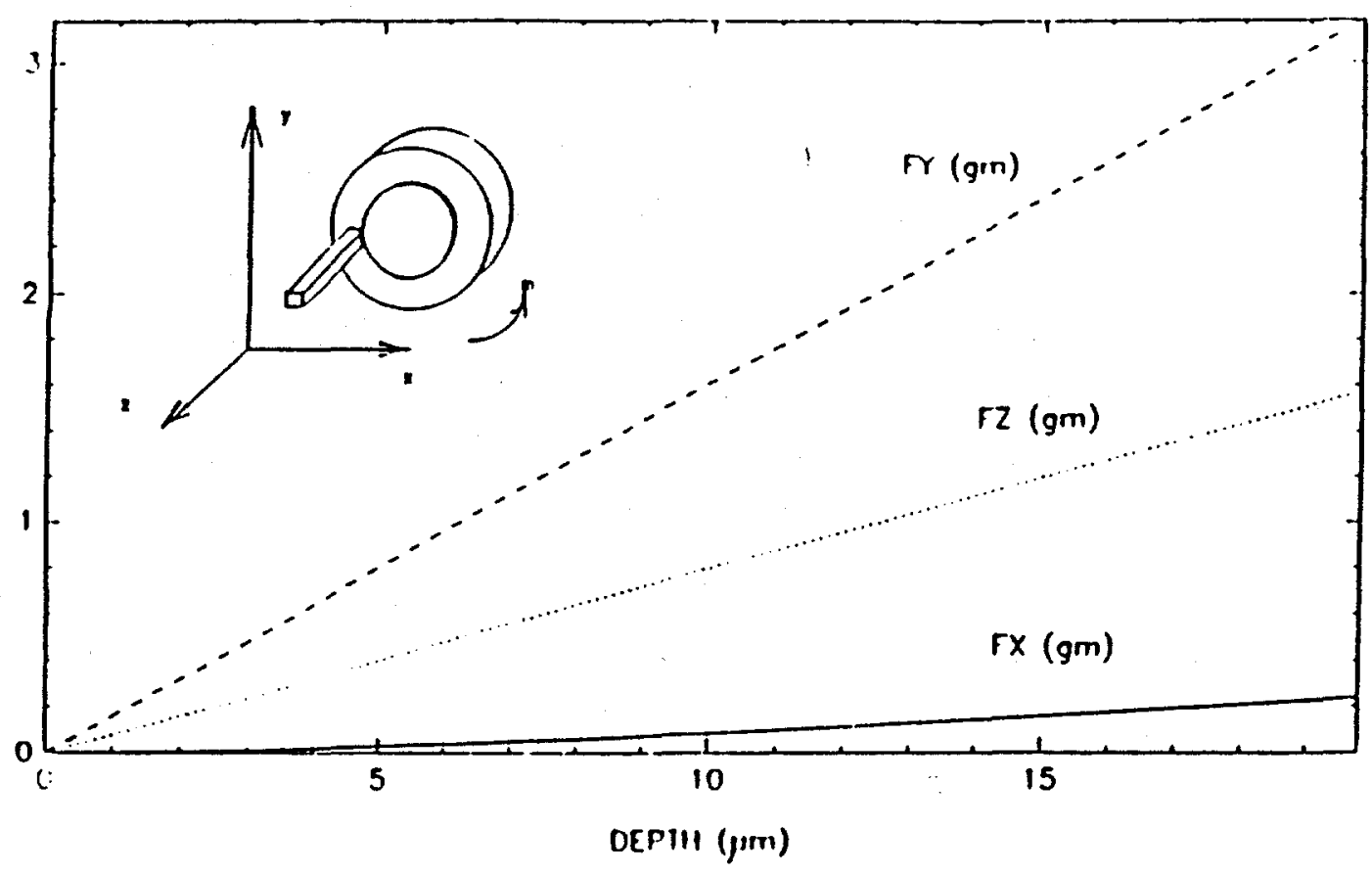

Figure 1: Analytical prediction of 3-dimensional tool forces

Figure 2(a). Also, the sensitivity was best in this direction. This axis should therefore be aligned with the greatest expected force component. Although the analytical model predicts the highest force parallel to the workpiece in the direction of the cut, indications [1] are that the force perpendicular to the workpiece becomes highest as the diamond tool wears. For these reasons it was decided that the configuration in Figure 2(a) would be the most stiff and most sensitive orientation.

In designing the structure of the dynamometer, stiffness was the main concern. With the dynamometer mounted on the DTM slide, the combined stiffness could be no higher than the slide stiffness. The slide stiffness is $27 \mathrm{~N} / \mu \mathrm{m}$ and $101 \mathrm{~N} / \mu \mathrm{m}$ in the horizontal and vertical directions respectively. The stiffness of the dynamometer was to be as high as possible to maximize overall stiffness. There were also constraints dictated by the use of the transducer. One was the necessity of a preloading mechanism to allow transverse forces to be transmitted by friction. Normally a bolt is placed through the center of the transducer for this preload. The stiffness requirement, accessibility of the bolt head, and mounting dimensions on the DTM were important factors in determining the structure shown in Figure 3. The base shown in Figure 5(a) attaches to the DTM. A preloading bolt will be inserted through the horizontal hole in the base, pass through a piezo-electric transducer, and then be threaded into the toolholding assembly shown in Figure 3(b).

The stiffness of this structure was estimated in each of the three orthogonal directions before fabrication. The combined stiffness of the slide and dynamometer base was estimated using the formula:

$$
K_{x, y, z}=\left[\frac{1}{K_{0}}+\frac{1}{K_{d}}\right]_{x, y, z}^{-1}
$$

where 

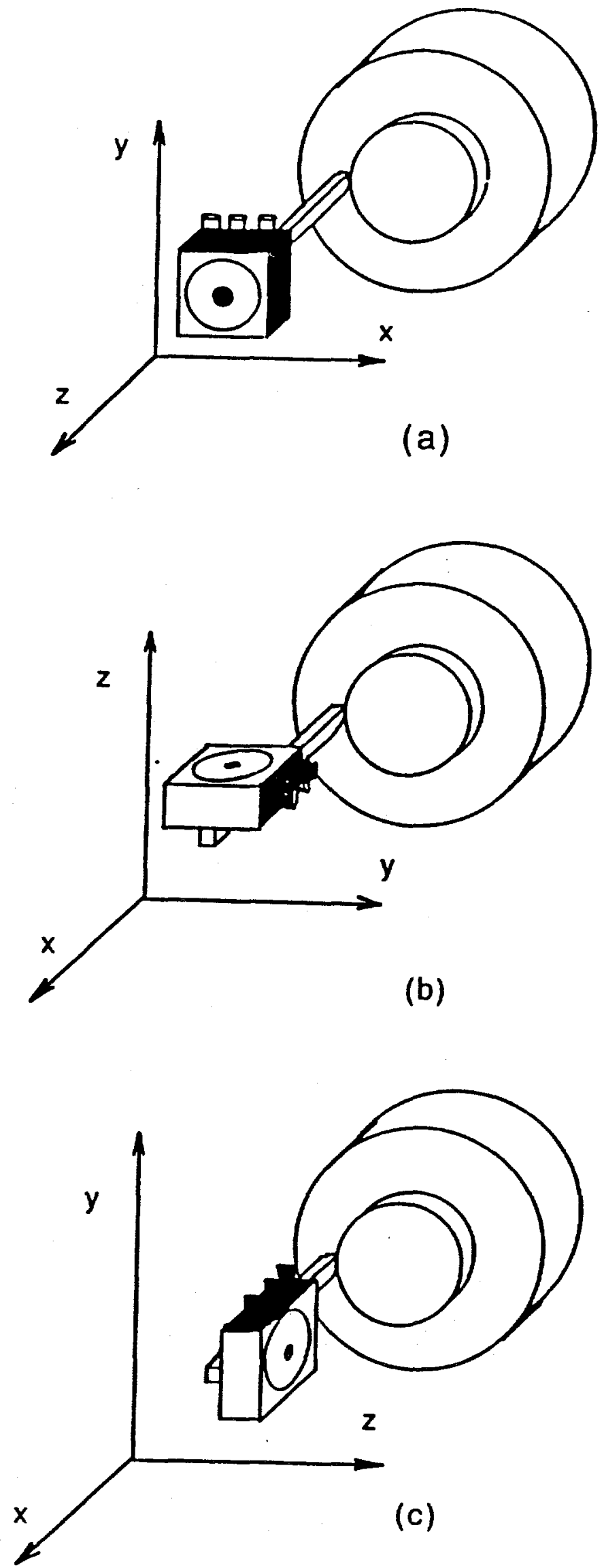

Figure 2: Possible orientations for the transducer 


$$
\begin{aligned}
& \mathrm{K}=\text { combined stiffness } \\
& K_{d}=\text { dynamometer stiffness } \\
& K_{d}=\text { slide stiffness }
\end{aligned}
$$

The combined stiffness estimated in this way in all three directions was very close to the machine stiffness without the tool force dynamometer. This was the maximum achievable combined stiffness.

\subsubsection{Dynamic Characterization}

In designing a measuring instrument to study a moving system, it is important to understand how the dynamic behavior of the measuring instrument will affect the measured quantity. With this in mind, the candidate structure of Figure 3 was modeled as a two degree of freedom system. The model consisted of two masses, one being the DTM slide together with the dynamometer base, and the other the tool and toolholding assembly. This model is shown in Figure 4. The diagram shows only one dimension of the actual three dimensional system. By summation of forces the equations of motion are written:

$$
\begin{gathered}
m_{1} \ddot{x_{1}}=k_{2}\left(x_{2}-x_{1}\right)+c_{2}\left(\dot{x_{2}}-\dot{x_{1}}\right)-k_{1} x_{1}-c_{1} \dot{x_{1}} \\
m_{2} \ddot{x_{2}}=f_{2}(t)-k_{2}\left(x_{2}-x_{1}\right)-c_{2}\left(\dot{x_{2}}-\dot{x_{1}}\right)
\end{gathered}
$$

where,

$$
\begin{aligned}
& x_{1}=\text { displacement of the slide } \\
& x_{2}=\text { displacement of the tool } \\
& k_{1}=\text { stiffness of the slide } \\
& k_{2}=\text { stiffness of the transducer } \\
& c_{1}=\text { viscous damping coefficient of slide } \\
& c_{2}=\text { viscous damping coefficient of transducer } \\
& m_{1}=\text { mass of slide and dynamometer base } \\
& m_{2}=\text { mass of tool, toolholder, and transducer } \\
& f_{2}(t)=\text { tool force }
\end{aligned}
$$

Solution of these equations yielded relationships between displacements of the two masses and the forcing function. Figure 5(a) is a plot showing these displacements obtained by using $y$-direction parameters. The difference between these displacements, that is the relative motion of dynamometer base and tool, when multiplied by the stiffness of the transducer $\left(k_{2}\right.$ in Figure 4$)$ gives the relationship between force input and transducer output. This is seen in Figure $5(\mathrm{~b})$. The system should accurately read tool forces in the $y$-direction at frequencies up to about $153 \mathrm{~Hz}$. At higher frequencies resonance is 


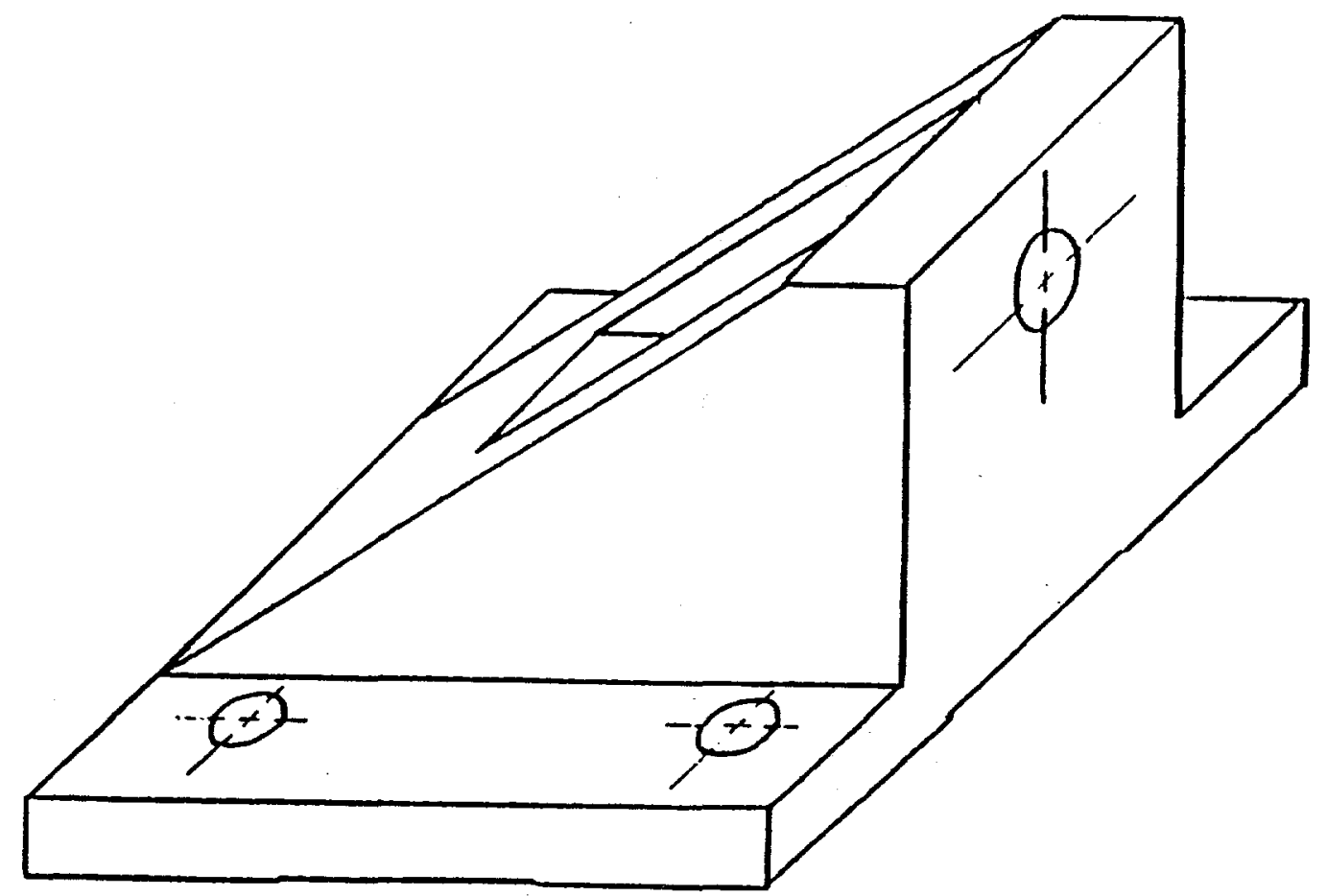

(a) Dynamometer base

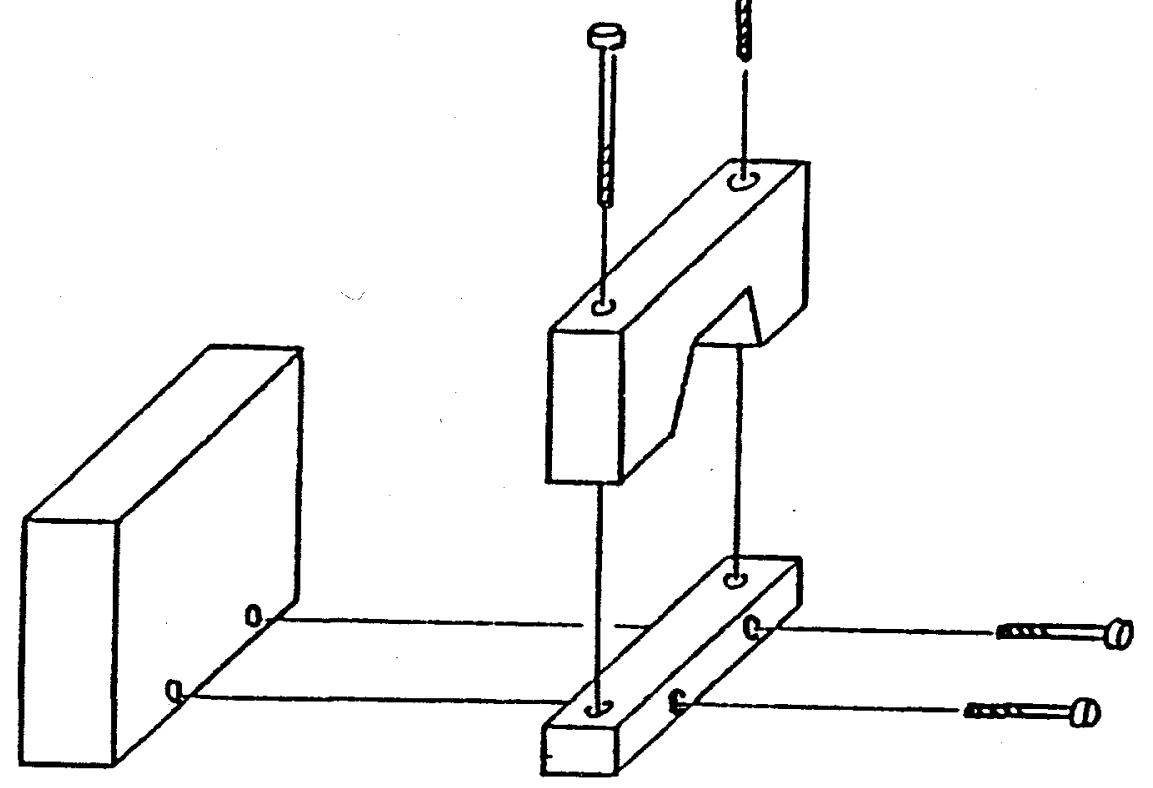

(b) Toolholding assembly

Figure 3: Dynamometer structure 


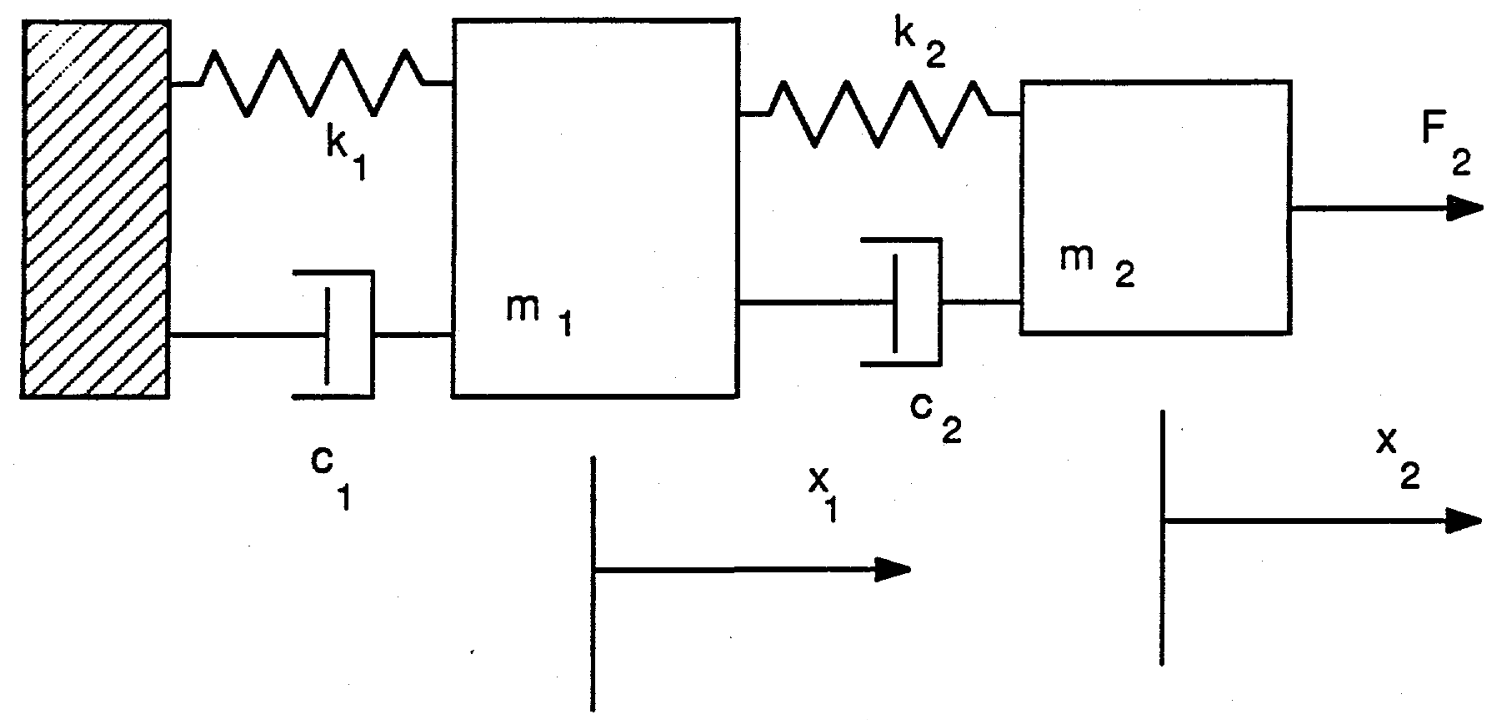

Figure 4: Model for dynamic analysis NOTE: $x_{1}$ and $x_{2}$ in this figure represent degrees of freedom and not directions.

encountered and the masses begin to move out of phase. With $x$ and $z$ direction parameters for stiffness, damping, etc., the results were different. Rather than the $150 \mathrm{~Hz}$ limit for the $y$-direction, illustrated in Figure 5 , the $x$ and $z$ axes are limited to 78 and $67 \mathrm{~Hz}$ respectively.

After fabrication the structure's resonant frequencies were verified using an accelerometer and frequency analyzer. The dynamometer was attached to the DTM as in normal cutting. The structure was then excited by tapping with a hammer and frequency spectra were obtained. The frequency response was as expected for the $x$ and $z$-directions. However, the spectrum obtained experimentally for the $y$-direction indicated that no resonance occurred below $500 \mathrm{~Hz}$ in the structure's main cutting direction. The fact that the peak in frequency response did not occur at $150 \mathrm{~Hz}$ as predicted might be attributed to high damping in the hydrostatic slide bearing.

\subsection{FORCE MEASUREMENT}

One basic goal of this project is to develop a measurement system applicable to closed loop DTM control. Therefore, cutting experiments are needed to quantify cutting parameterturning process relationships. Although research in this area has been going on for several years, diamond turning is still considered an art by many people. Trial and error procedures are common when new materials and tolerances are encountered. 


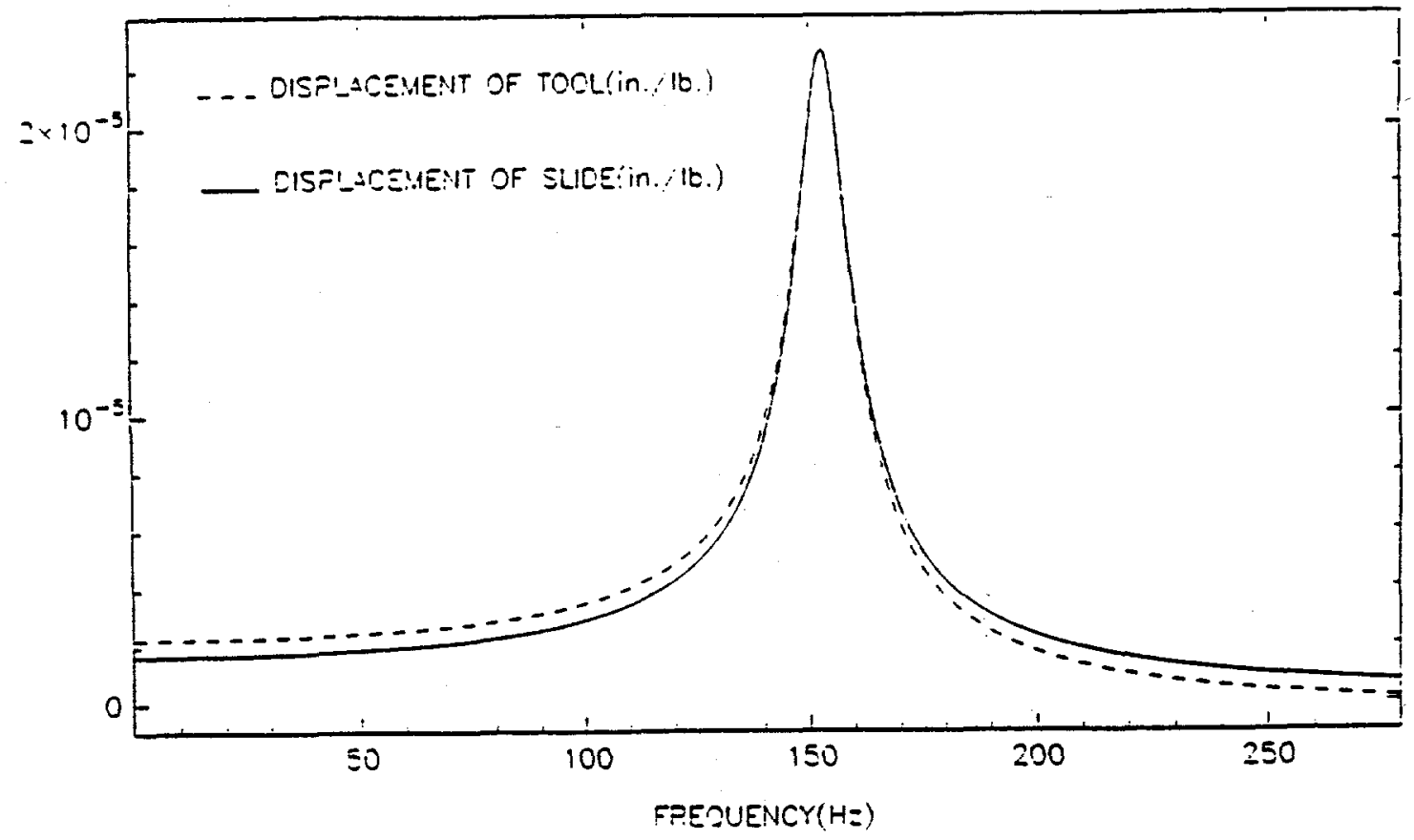

(a) Relative displacements of tool and slide as function of forcing frequency

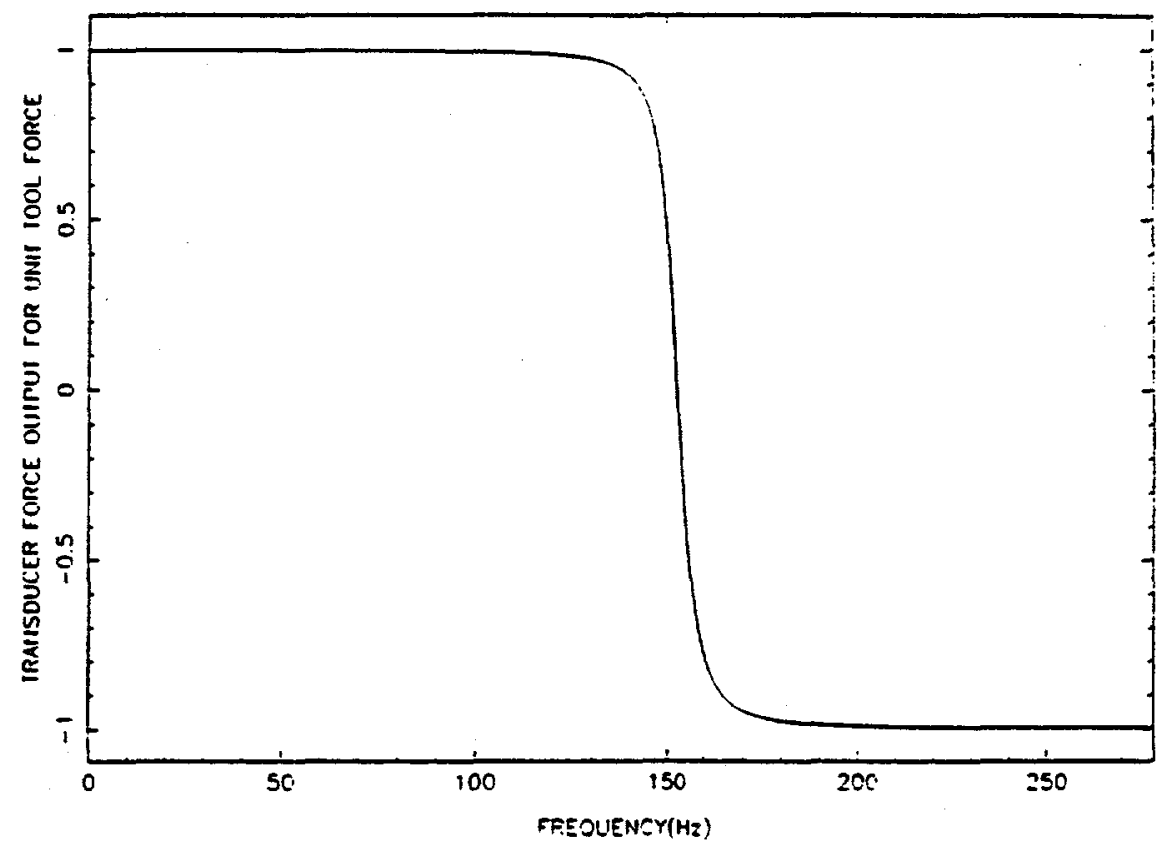

(b) Predicted relationship between force input and transducer output

Figure 5: Calculated dynamometer frequency response in $y$-direction 


\begin{tabular}{|l|c|c|c|c|c|}
\hline \multicolumn{5}{|c|}{$3.0 \mathrm{~mm}$ Tool Radius, COPPER, $5 \mu \mathrm{m}$ DEPTH, 6/8/88 } \\
\hline CUTTING & FORCE & \multicolumn{4}{|c|}{ FORCE (Newtons) } \\
\cline { 3 - 6 } SPEED & COMP. & $5 \mu \mathrm{m} / \mathrm{rev}$ & $10 \mu \mathrm{m} / \mathrm{rev}$ & $20 \mu \mathrm{m} / \mathrm{rev}$ & $40 \mu \mathrm{m} / \mathrm{rev}$ \\
\hline $20.8 \mathrm{~m} / \mathrm{s}$ & $\mathrm{Y}$ & 0.270 & 0.280 & 0.350 & 0.430 \\
& $\mathrm{Z}$ & 1.70 & 1.50 & 1.36 & 1.13 \\
\hline $16.7 \mathrm{~m} / \mathrm{s}$ & $\mathrm{Y}$ & 0.250 & 0.270 & 0.320 & 0.420 \\
& $\mathrm{Z}$ & 1.59 & 1.44 & 1.32 & 1.19 \\
\hline $12.5 \mathrm{~m} / \mathrm{s}$ & $\mathrm{Y}$ & 0.230 & 0.250 & 0.310 & 0.380 \\
& $\mathrm{Z}$ & 1.54 & 1.36 & N $/ \mathrm{A}$ & 1.22 \\
\hline $8.3 \mathrm{~m} / \mathrm{s}$ & $\mathrm{Y}$ & 0.230 & 0.250 & 0.310 & 0.380 \\
& $\mathrm{Z}$ & N $/ \mathrm{A}$ & N $/ \mathrm{A}$ & N $/ \mathrm{A}$ & N $/ \mathrm{A}$ \\
\hline $4.2 \mathrm{~m} / \mathrm{s}$ & $\mathrm{Y}$ & 0.220 & 0.260 & 0.324 & 0.408 \\
& $\mathrm{Z}$ & 1.47 & N/A & 0.91 & N/A \\
\hline AVERAGE & $\mathrm{Y}$ & 0.240 & 0.262 & 0.324 & 0.408 \\
& $\mathrm{Z}$ & 1.58 & 1.43 & 1.20 & 1.18 \\
\hline
\end{tabular}

Table 1: Data showing effect of velocity on tool forces for OFHC Copper

\subsubsection{Study of Parameters}

Cutting Speed To maximize the number of different cutting conditions on a single test piece, $200 \mathrm{~mm}$ disks are used. If the spindle speed is not changed, cutting velocity varies over this $100 \mathrm{~mm}$ radius. Therefore, one of the first concerns was to determine the effect of cutting speed on tool forces. The materials investigated in these experiments were OFHC Copper and Aluminum alloy, 6061-T6. At a constant depth of $5 \mu \mathrm{m}$ and constant feedrate of $5 \mu \mathrm{m} / \mathrm{rev}$, five cutting velocities ranging from 4 to $21 \mathrm{~m} / \mathrm{s}$ were used. The same series of cuts was repeated for feedrates of 10,20 , and $40 \mu \mathrm{m} / \mathrm{rev}$ The three orthogonal forces were monitored during all cuts. The results for copper are given in Table 1 . They show no consistent variation in forces with respect to velocity in either material. The $x$-direction forces are omitted since the magnitude was insignificant in comparison to $y$ and $z$ forces. This is as expected since the cutting is largely planar when cutting at typical rates of infeed.

These results agree with those of Iwata, Moriwaki, and Okuda of Kobe University in Japan. [4] They report that cutting speed has no influence on cutting forces in copper in the range from 9.4 to $22 \mathrm{~m} / \mathrm{s}$. It was therefore concluded that cutting velocity would not be a concern in subsequent tests as long as velocities were kept in this range.

Cutting Depth A second test was devised in which feedrate was again held constant and depth varied in a single cut. The feedrate was $5 \mu \mathrm{m} / \mathrm{rev}$ and the depths were $1,2,5,8$, and 
$10 \mu \mathrm{m}$. Three tools were used with different nose radii. Figure 6 shows measured cutting and axial forces as functions of depth of cut for the three tools as well as the corresponding theoretical curve. The theoretical curves (Figure 6(a)) are based on a flow stress acting over the chip cross-sectional area to calculate $y$-force and this value multiplied by a friction factor of 0.5 for the $z$-force. The flow stress is estimated as three times the material yield stress. The yield stress is assumed constant at $0.22 \mathrm{MPa}$ for copper. Although tool nose radius is not considered a factor in the model, Figure 6 indicates that there is dependance on the particular tool.

Effect of Feedrate The effect of feedrate on tool forces was also investigated. Separate cuts were made at feedrates from 5 to $40 \mu \mathrm{m} / \mathrm{rev}$ with a programmed depth of $5 \mu \mathrm{m}$. Copper and aluminum were again the test materials. Disks of $200 \mathrm{~mm}$ diameter were prepared by cutting $6.5 \mathrm{~mm}$ concentric grooves on a turned surface. The grooves were 6.5 $m m$ apart and were $25 \mu m$ deep. There were seven separate test surfaces. Starting at the outer ring on the part a cut was made at $5 \mu \mathrm{m} / \mathrm{rev}$. Subsequent cuts were made at 10 , 20 , and $40 \mu \mathrm{m} / \mathrm{rev}$.

Forces vs. feedrate are plotted in Figure 7. Also the corresponding theoretical curves are given. As with the depth experiments, the predicted forces are lower than the measured ones. While $y$-direction forces increase almost linearly with feedrate, $z$-directional forces tend to decrease in both copper and aluminum as feedrate is increased.

\subsection{MODEL DEVELOPMENT}

The procedure of the feedrate tests made it possible to distinguish between the forces during chip formation and the forces due to the tool/part contact alone. Figure 8 is a typical plot of force vs. time. The initial step in force was caused as the tool came into contact with the test part and began steady state cutting. Before the tool traversed the entire $6.5 \mathrm{~mm}$ step, the feed was stopped abruptly but the tool was kept in contact with the work as the spindle continued to turn. At this point the force levels dropped by a discrete amount where they again remained relatively constant. Therefore, there is a residual force which seems to be independent of the force associated with material removal.

In Table 2, for each feedrate, the force in the $y$ and $z$-directions are listed. The total force measured during cutting is listed as well as the residual force measured when the feed was stopped. The differences between these two components are also listed. These three columns are plotted as functions of feedrate in Figure 9. The $y$-direction force is almost linearly proportional to increase in feedrate. The total force increases with feedrate although the residual component becomes less of the total. A similar situation is evident in the $z$-forces in that the residual component of force decreases with increasing feedrate. However, the magnitude of the residual forces are much different. At low feedrates the 


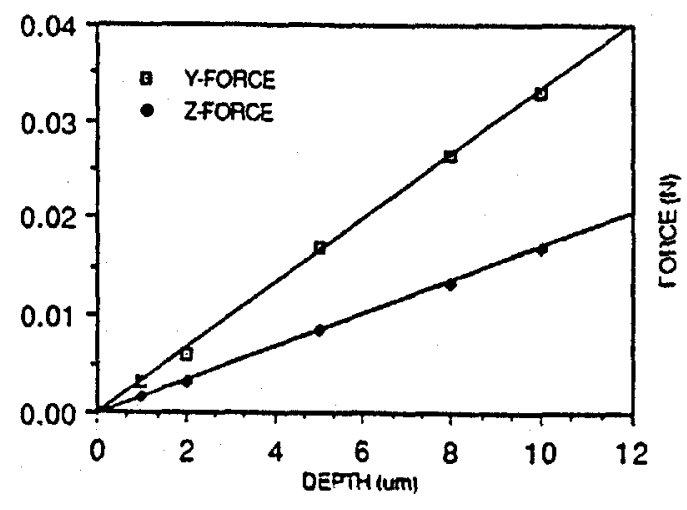

(a) Theoretical cutting forces for OFHC copper

岛

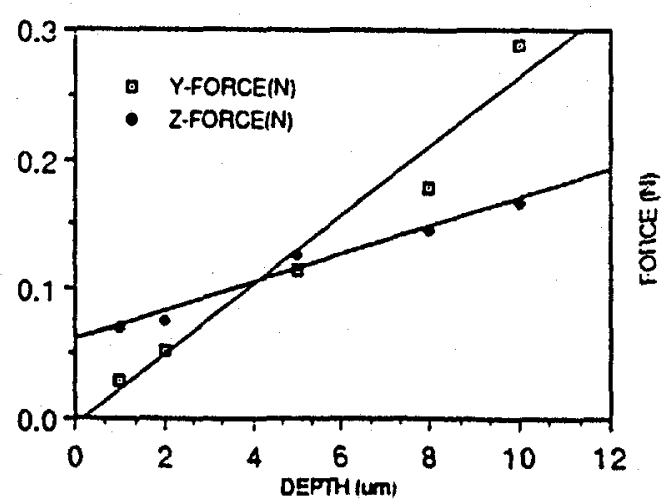

(c) $1.5 \mathrm{~mm}$ tool radius

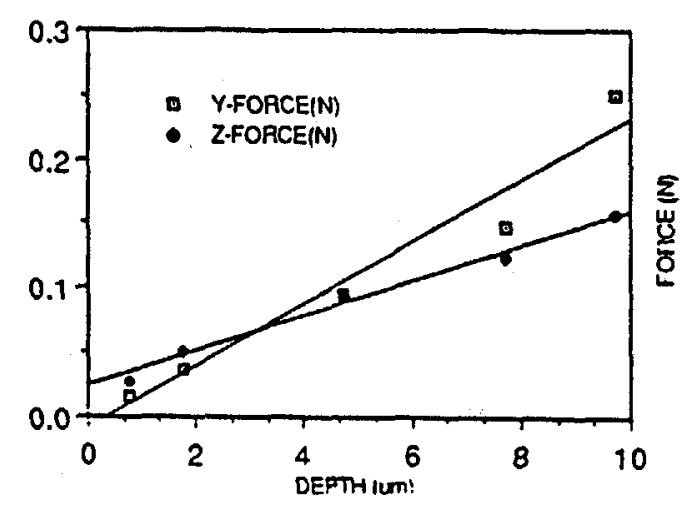

(b) $0.5 \mathrm{~mm}$ tool radius

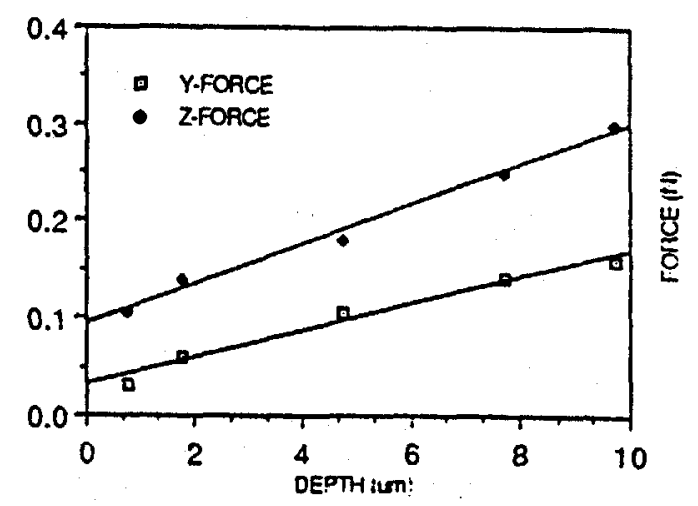

(d) $3.0 \mathrm{~mm}$ tool radius

Figure 6: Theoretical and experimental force curves for various tools showing effect of cutting depth (Feedrate is $5 \mu \mathrm{m} / \mathrm{rev}$; Material is OFHC copper) 


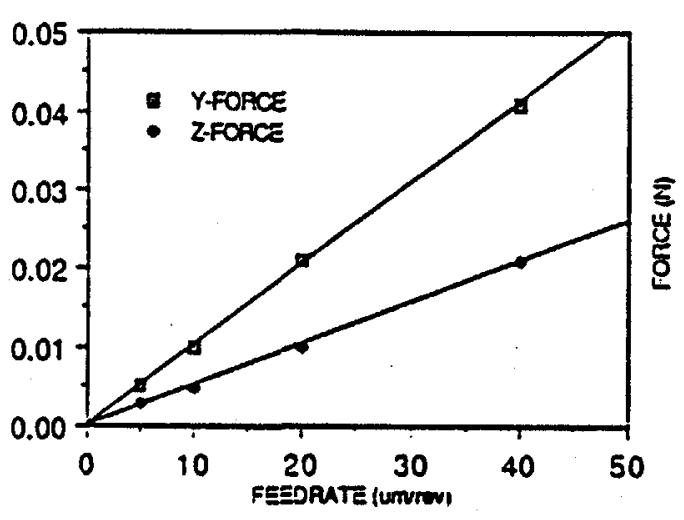

(a) Theoretical forces for OFHC copper

岕

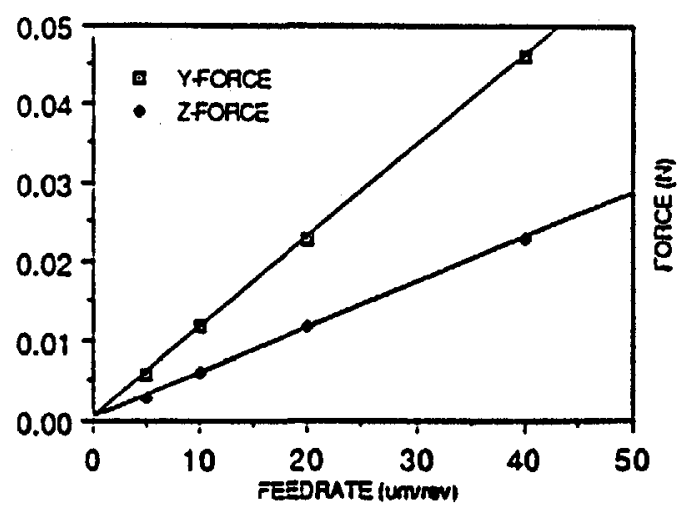

(c) Theoretical forces for 6061-T6 aluminum

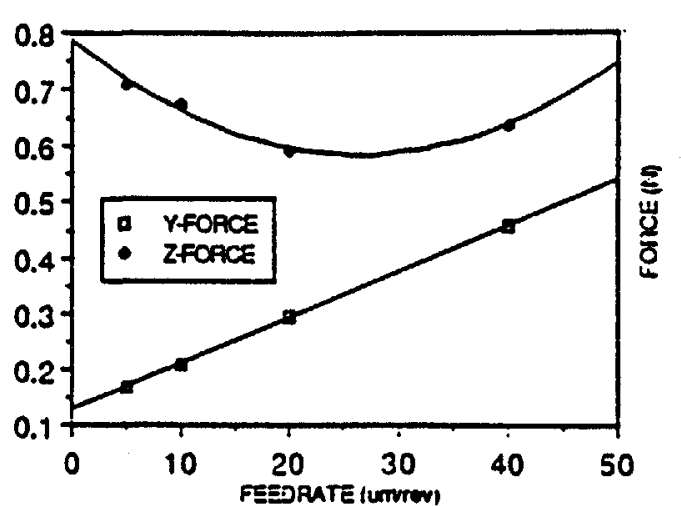

(b) Measured forces for OFHC copper

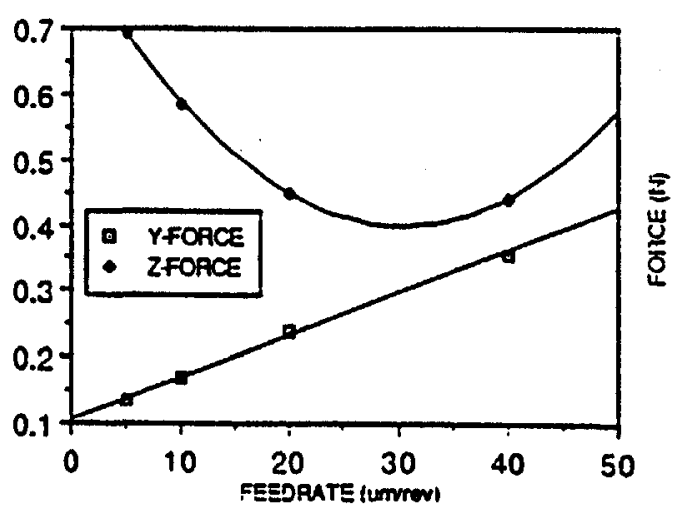

(c) Measured forces for 6061-T6 aluminum

Figure 7: Theoretical and experimental data of feedrate cutting test 


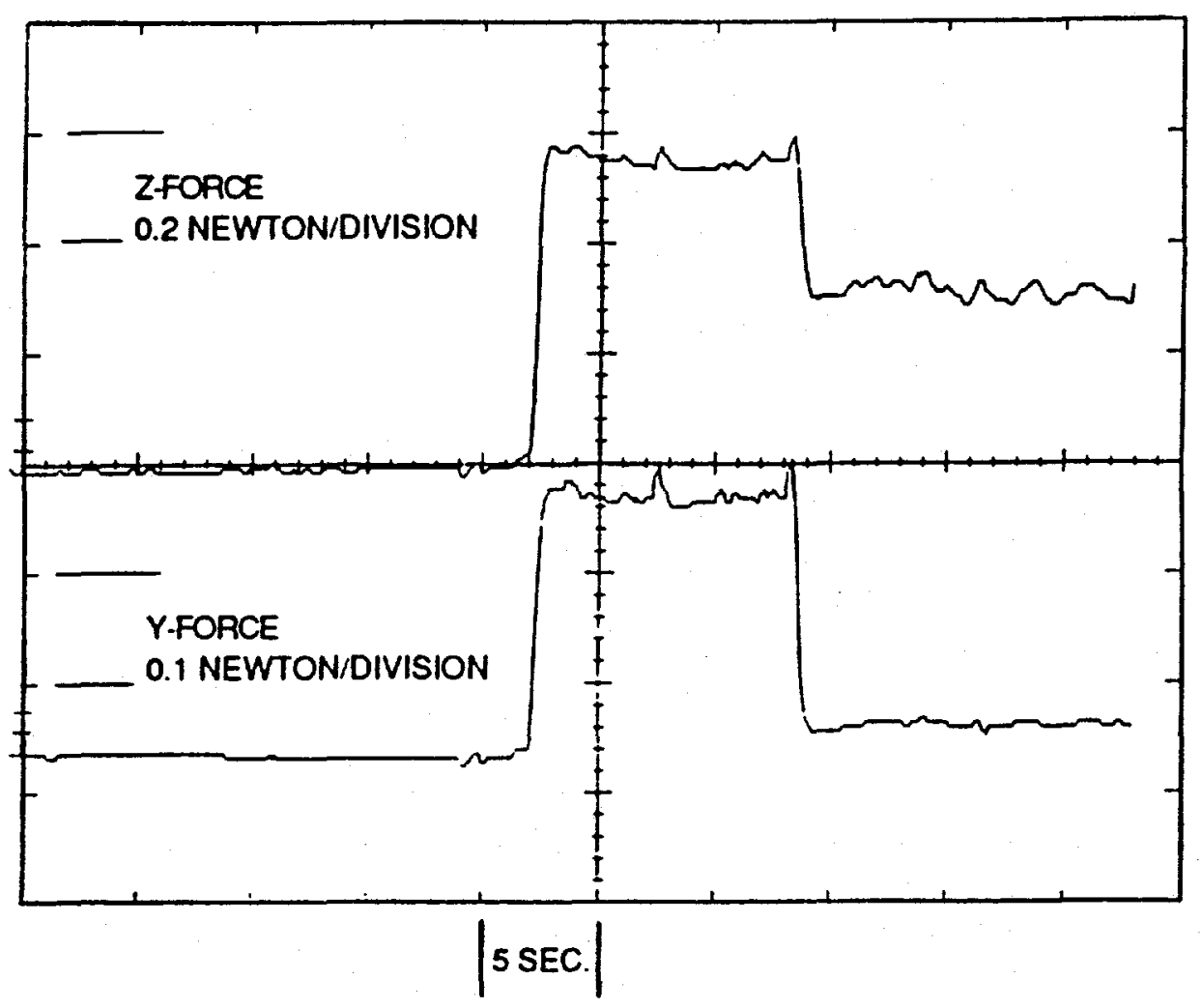

Figure 8: Typical oscilloscope trace of forces from feedrate experiment

\begin{tabular}{|c|c|c|c|c|c|c|}
\hline 0.06 in. Tool Radius, ALUMINUM, $5 \mu$ m DEPTH, 6/29/88 \\
\hline \multirow{2}{*}{$\begin{array}{c}\text { FEEDRATE } \\
(\mu \mathrm{m} / \mathrm{rev})\end{array}$} & Y-FORCE (Newtons) & \multicolumn{2}{|c|}{ Z-FORCE (Newtons) } \\
\cline { 2 - 7 } & Tot. & Res. & Diff. & Tot. & Res. & Diff \\
\hline 5 & .126 & .074 & .052 & .718 & .670 & .048 \\
\hline 10 & .171 & .052 & .119 & .582 & .499 & .083 \\
\hline 20 & .229 & .030 & .199 & .456 & .262 & .194 \\
\hline 40 & .356 & .025 & .331 & .439 & .166 & .273 \\
\hline
\end{tabular}

Table 2: Components of cutting forces 
residual force in the $z$-direction is a major component of the total force. Therefore, as this component decreases the total force also decreases.

These results lead to a more detailed picture of the cutting model. There appear to be four separate force components in the $y-z$ plane. Two of these forces exist only when a chip is forming. These forces apparently act on the rake face of the tool. The other two remain on the tool as long as the tool is in contact with the workpiece even if a chip is not forming. Figure 10 shows forces on the tool including:

$$
\begin{aligned}
& F_{z_{\text {zee }}}=\text { residual } z \text { force } \\
& F_{z_{\text {est }}}=z \text { force on the rake face } \\
& F_{y_{\text {yes }}}=\text { residual } y \text { force } \\
& F_{\text {yeat }}=y \text { force on the rake face }
\end{aligned}
$$

The forces on the rake face are the differences between measured total and residual forces. Although not yet verified experimentally, there is a hypothesis which explains the differences between the measured forces in Figure 9 and the theoretical forces shown in Figure 1. This modification accounts for the higher measured forces and also for the non-linear change in the axial component of tool force with increasing feedrate.

It has been shown [5] that diamond turned, copper surfaces exhibit a material hardness which is inversely proportional to the depth below the finished surface. The depth and magnitude of this hardness variation are dependant upon the sharpness of the tool edge. The hardness at the surface is as much as 10 times the bulk material hardness. and decreases exponentially to the bulk hardness at some depth below the surface. This depth is equal to the extent of the plastic work zone and is on the order of $1.0 \mu m$.

In diamond turning applications a large fraction of the tool-workpiece interaction is in this region where the hardness is much greater than the bulk material hardness. Higher cutting forces are predicted with this model than with the previously described model in which the hardness was assumed constant and equal to three times the yield strength. The cutting component of force along the axis of the tool, $F_{z_{\text {eut }}}$ in Figure 10, is proportional to the cutting force in the cutfing direction, $F_{y e s t}$, and both increase with increasing chip area. The residual component of the axial force, $F_{2 \text { ree }}$, decreases with increasing feedrate when hardness is not constant. This force is proportional to the length of the tool's contact edge. At low feedrates this perimeter area cuts through the harder material near the surface. As feedrate increases a greater and greater portion of this edge length goes through the bulk, non-worked material. Figure 11 is a view of the workpiece showing the tool/workpiece interaction. For simplification, the variation in hardness is illustrated by two distinct zones having high hardness near the surface and low hardness in the bulk material. Therefore the magnitude of $F_{\text {ree }}$ decreases and this decrease is exponential if 


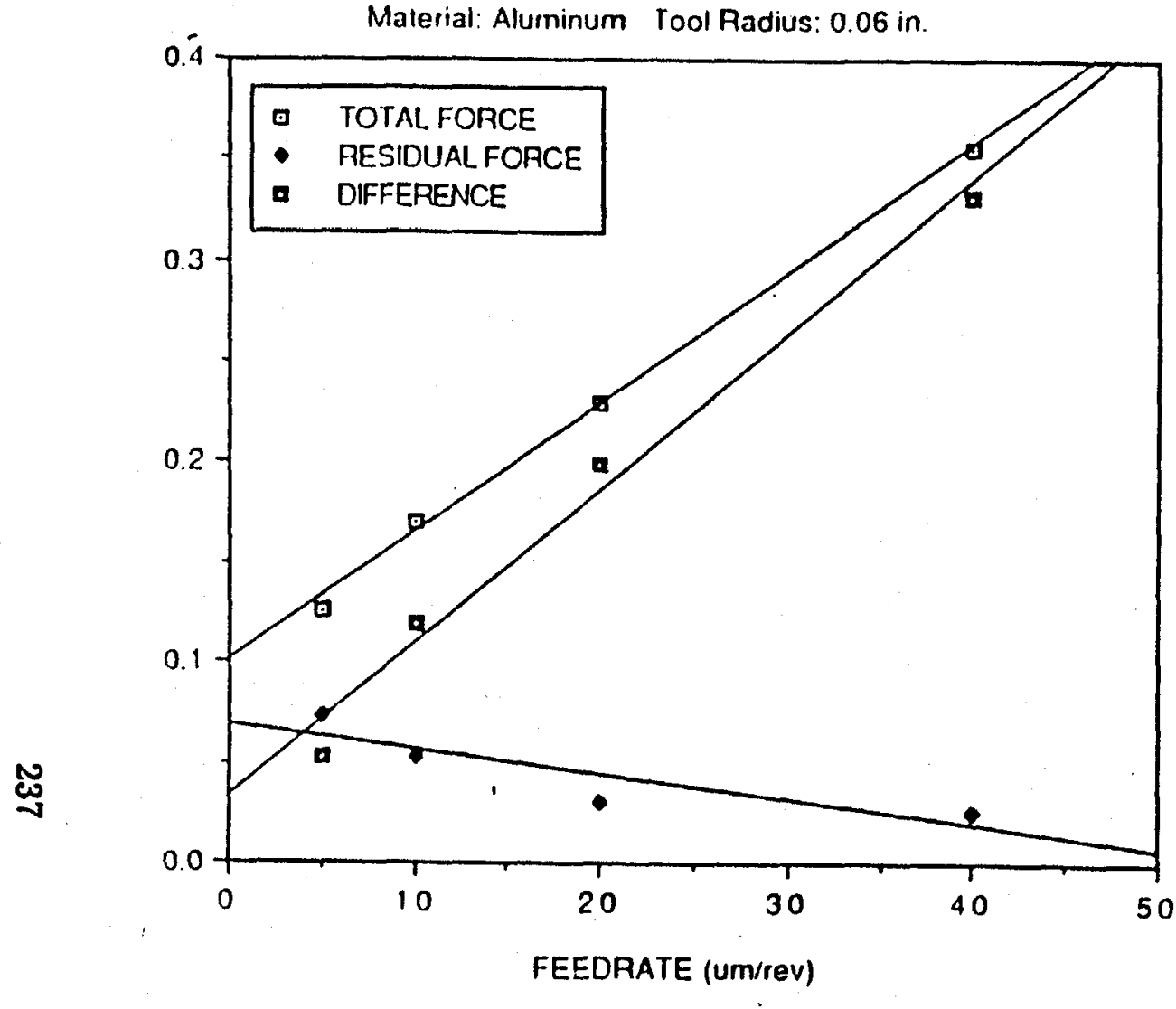

(a) Components of "cutting" force

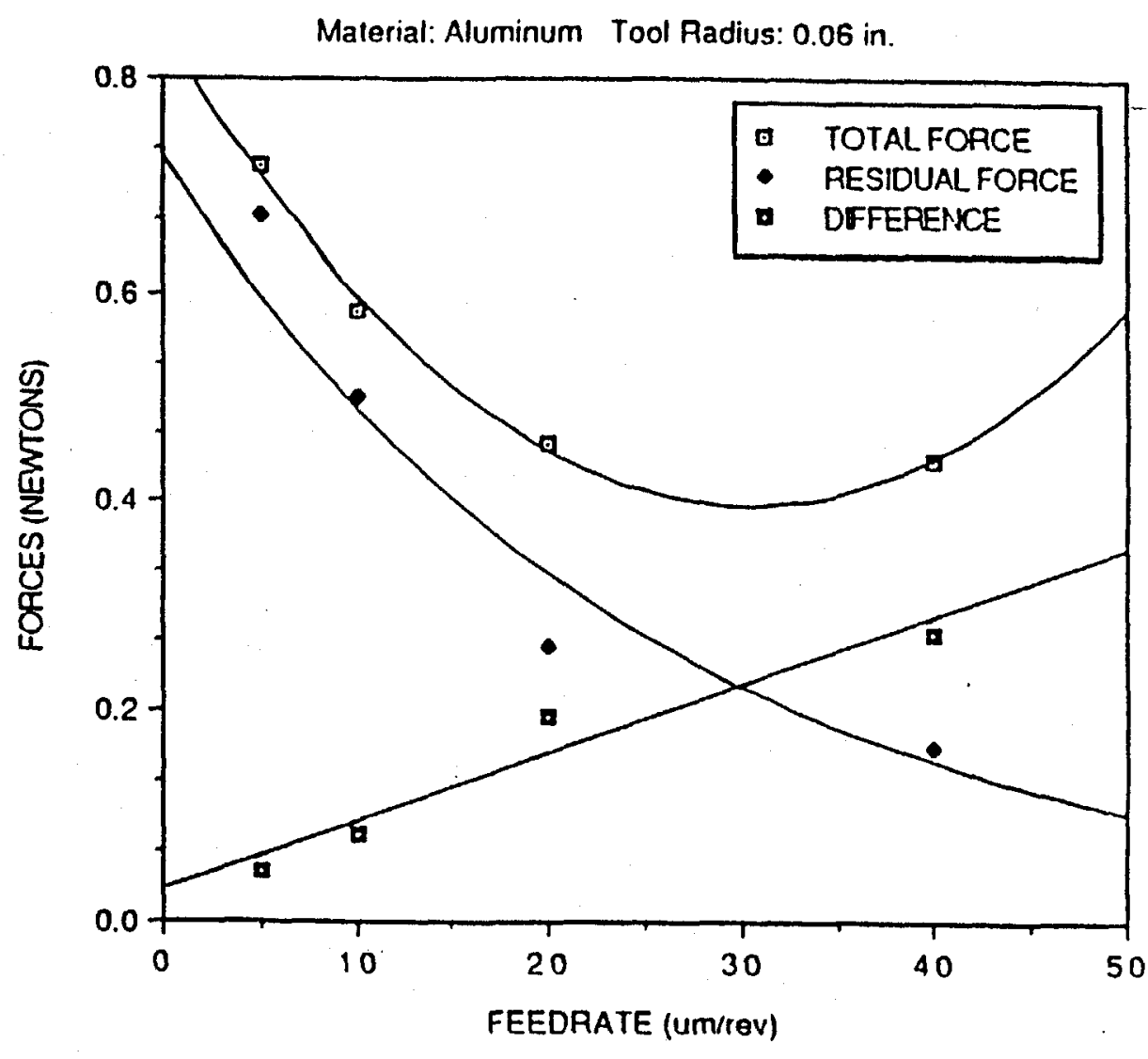

(b) Components of axial tool force

Figure 9: Plots showing effect of feedrate parameter on cutting force components for 6061-T6 aluminum Tool radius is $1.5 \mu \mathrm{m}$ 


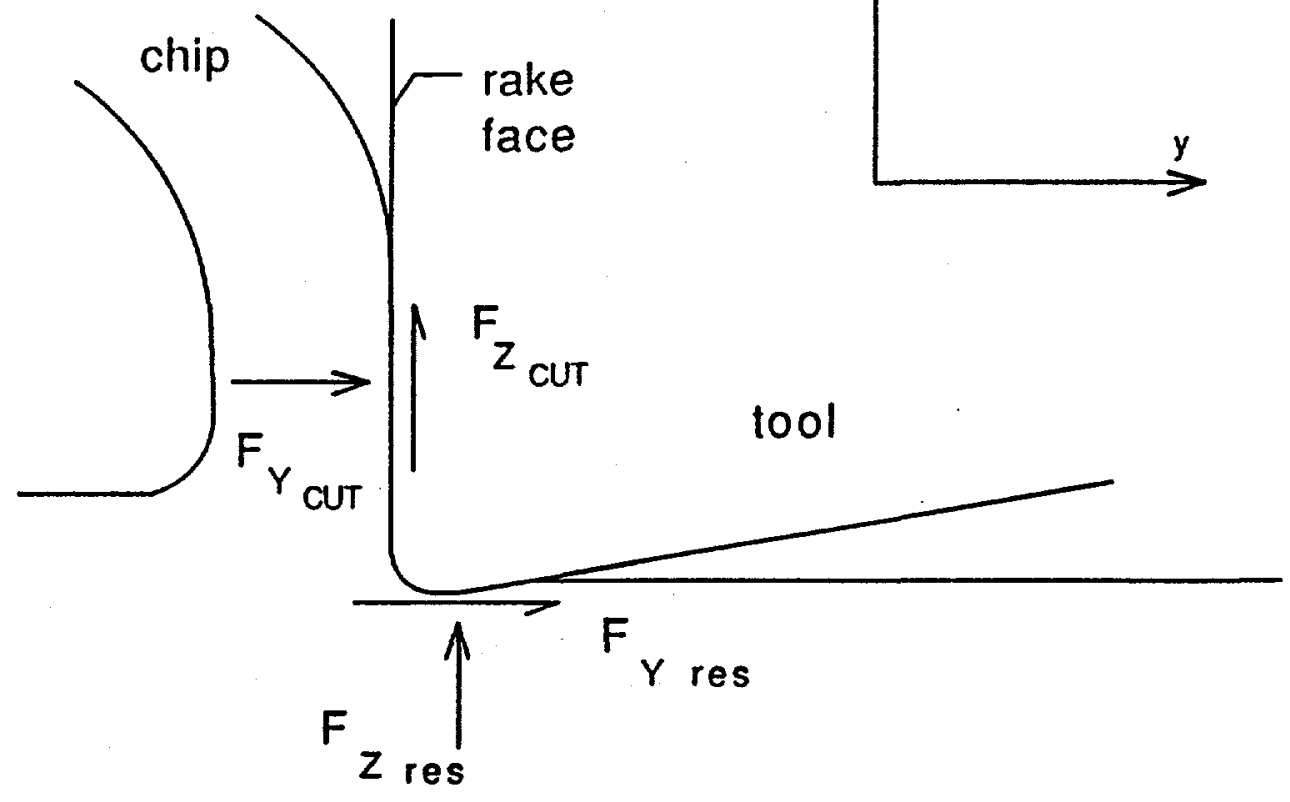

Figure 10: Force component diagram in the $y-z$ plane

an exponential hardness profile is assumed. The residual force in the cutting direction, $F_{\text {yest }}$, also decreases since it is related to the residual axial force by a coefficient of friction. The results of this hypothesis correspond, at least qualitatively, to the measured forces as represented in Figure 9. However, additional experiments with other materials exhibiting different work hardening behaviour are necessary to develop more quantitative correlation between the measured forces and this theoretical model.

\subsection{CONCLUSIONS}

It has been determined that cutting velocity has little or no effect on tool forces for OFHC copper and 6061-T6 aluminum in the range from 4 to $21 \mathrm{~m} / \mathrm{s}$. This result is corroborated by other researchers. [4]

Measured forces are almost an order of magnitude higher on average than predicted by the simple model. As overall depth of cut is increased, all else remaining the same, both $y$ and $z$-direction forces increase linearly. In 11 of the 12 series of cuts made on copper the $z$ force is higher than the $y$-force at very low depths. The two curves cross and $y$-force is higher at the higher depths. This same trend is apparent in 9 of the 12 tests made on aluminum. 


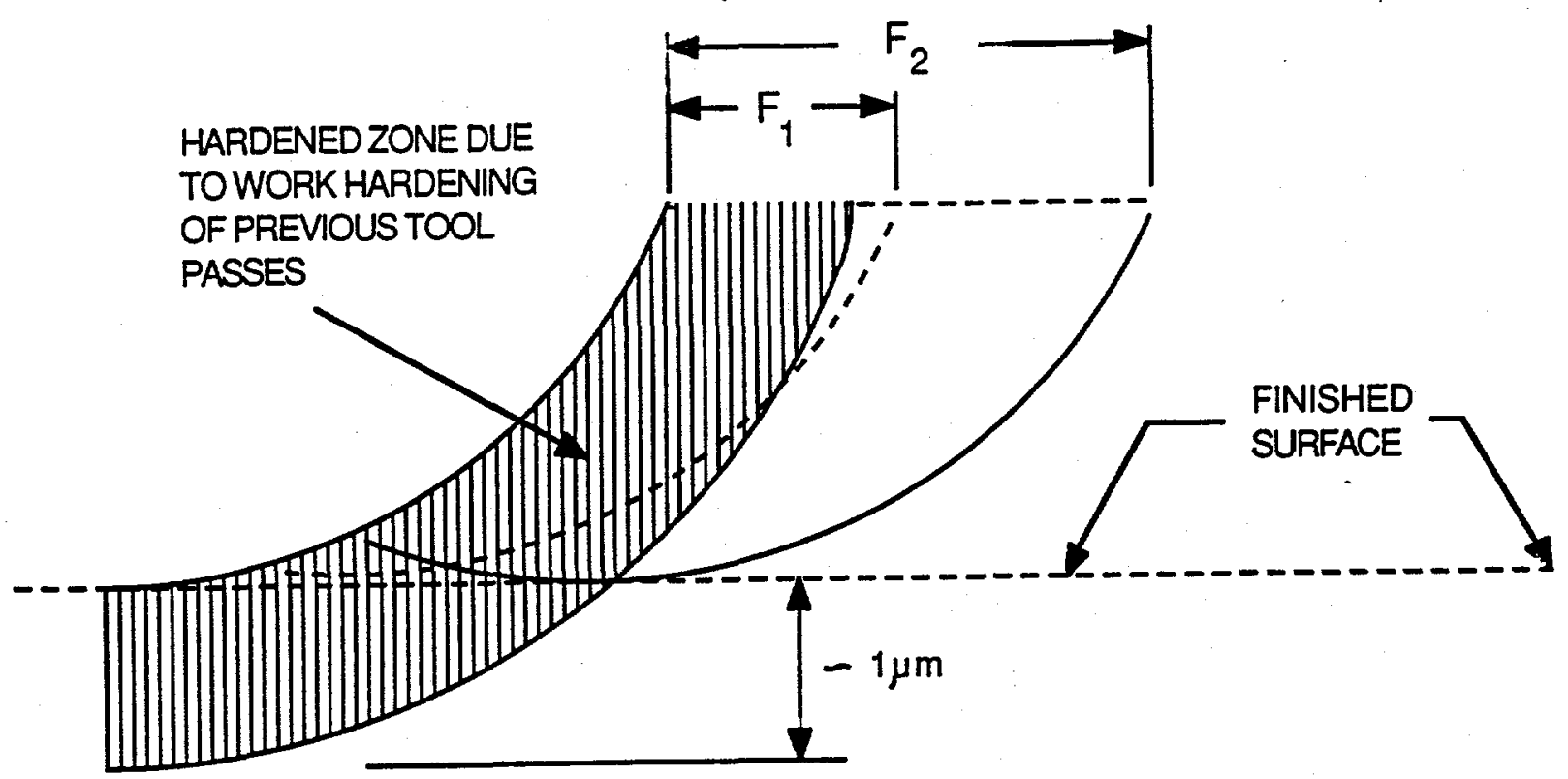

Figure 11: Simplified model showing relationship between hardness variation and feedrate in axial tool force components

With increasing feedrate the cutting force component is similar to the curve for increasing depth. It is approximately an order of magnitude greater than predicted but increases linearly as predicted. The axial force component decreases with increasing feedrate at low feedrates for the depths used in the experiments. As feedrate is increased further the axial force begins to take an upward trend. These results can possibly be explained if a hardness is included in the model which varies as an exponential function of depth below the surface.

The unexpected values and trends of measured force data show that the simple model involving flow stress and friction on the tool rake face will be inadequate in predicting forces. An ongoing part of the force measurement project is in modifying and refining the model.

\subsection{FUTURE WORK}

Work in the immediate future will be to:

- incorporate into the model a variation in material hardness due to work hardening during machining. This work hardening is described by Evans et. al. [5] It is a function of the tool sharpness or edge "radius".

- use the refined model to predict forces and attempt to correlate these with new cutting data using copper. 


\section{References}

[1] R.J.P. Schrama, J. Franse; "The precision cutting process as a non-linear closed loop system", PE, Vol 10, pp. 199-207, Oct.,1988

[2] Kazuaki, Toshimichi, Koichi; Society of Manfg. Engineers, May, 1987

[3] Furukawa, Moronuki; "Effect of Material Properties on Ultra Precise Cutting Processes", CIRP Vol 37, p. 113, Jan, 1988

[4] Iwata, Moriwaki, Okuda; "A Study of Cutting Temperature in Ultra-High Precision Diamond Cutting of Copper",SME North American Manufacturing Research Conference Proceedings , May 1987

[5] C.Evans, R. Polvani, M. Postek, R Rhorer; "Some observations on tool sharpness and sub-surface damage in single point diamond turning", SPIE Vol 802, pp.52-66, 1987 


\title{
22 SIMULATIONS OF DUCTILE FLOW IN BRITTLE MATERIAL PROCESSING
}

\author{
Michael H. Luh \\ Graduate Student
}

Dr. John S. Strenkowski

Associate Professor, MAE

Research is continuing on the effects of thermal properties of the cutting tool and workpiece on the overall temperature distribution. Using an Eulerian finite element model, diamond and steel tools cutting aluminum have been simulated at various speeds and depths of cut. The relative magnitude of the thermal conductivity of the tool and the workpiece is believed to be a primary factor in the resulting temperature distribution in the workpiece. This effect is demonstrated in the change of maximum surface temperatures for diamond on aluminum vs. steel on aluminum. As a preliminary step toward the study of ductile flow in brittle materials, the relative thermal conductivities of diamond on polycarbonate is simulated. In this case, the maximum temperature shifts from the rake face of the tool to the surface of the machined workpiece, thus promoting ductile flow in the workpiece surface.

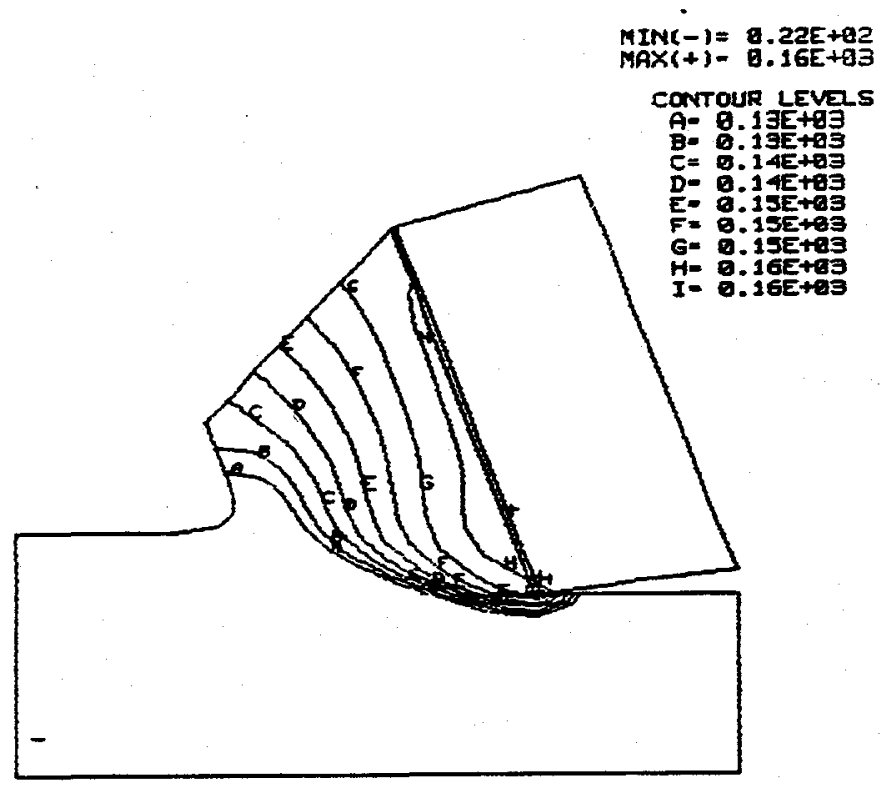




\subsection{INTRODUCTION}

The occurrence of ductile flow in cutting is influenced by the thermal response of the workpiece and tool. The objective of this study is to investigate the effect of the thermal properties of the workpiece and tool on the overall temperature distribution. Using an Eulerian cutting model, various workpieces and tools can be simulated for various cutting conditions, such as depth of cut, rake angle, and cutting speed. With this approach, the cutting conditions necessary to achieve ductile flow in a brittle material can be determined. Ultimately, the model will lead to an improved understanding of the material removal mechanisms in brittle materials.

\subsection{WORKPIECE AND TOOL MATERIAL SELECTION}

The relative magnitude of the thermal conductivities of the workpiece and tool play a dominant role in the overall temperature distribution resulting from cutting. The importance of the thermal conductivity takes on added significance because it can vary over a wide range for commonly-used tool and workpiece materials. Table 1 shows the thermal conductivities for steel and diamond tools, as well as several workpiece materials. The ratio of the thermal conductivities of various workpiece materials and steel and diamond tools is also shown. For example, cutting of aluminum with a steel tool gives a ratio of 0.28 versus 15.0 for a diamond tool. Not only is the magnitude of the thermal conductivity significantly different, but cutting of aluminum with diamond also results in an extremely large heat sink for the tool. The transfer of this large amount of heat from the chip into the tool results in a significant change in the temperature distribution in the workpiece. Ultimately, this will change the cutting conditions required for ductile flow to occur in a brittle material.

The first phase of this study explored the cutting of aluminum and copper with both steel and diamond tools. Although aluminum and copper are not brittle materials, their temperature-dependent thermal and material properties are more readily available then the other workpiece materials. Thus, a more realistic simulation can be performed with these materials to assess the utility of the model for investigating the effects of thermal conductivity.

The second stage of this study involved studying polymers which show characteristics similar to those of brittle materials [2]. These materials exhibit thermally softening regions which are much larger than either silicon or germanium and which occur at lower temperatures. Finally, extension of this study to silicon and germanium is discussed [1]. 


\begin{tabular}{||c|c|c|c||}
\hline \hline Material & $\begin{array}{c}\text { Thermal Conductivity }\left(\mathrm{k}_{\boldsymbol{w}}\right) \\
\left(\mathrm{W} / \mathrm{m}^{\circ} \mathrm{C}\right)\end{array}$ & $\mathrm{k}_{\text {steel }} / \mathrm{k}_{\boldsymbol{w}}$ & $\mathrm{k}_{\text {diamond }} / \mathrm{k}_{\boldsymbol{w}}$ \\
\hline \hline Steel & 57 & & \\
\hline Diamond & 3000 & & \\
\hline Aluminum 6061-T6 & 204 & 0.28 & 15.0 \\
\hline Copper & 400 & 0.14 & 7.5 \\
\hline Polycarbonate & 0.26 & 220.0 & $11,500.0$ \\
\hline Polystyrene & 0.13 & 440 & $23,000.0$ \\
\hline Silicon & 84 & 0.68 & 36.0 \\
\hline Germanium & 59 & 0.97 & 51.0 \\
\hline \hline
\end{tabular}

Table 1: Thermal conductivities for selected materials.

\begin{tabular}{||c|c||}
\hline \hline Rake Angles & $\begin{array}{c}\text { Depth of Cuts } \\
\text { ( } \mu \text { inches) }\end{array}$ \\
\hline \hline $0^{\circ}$ & $200,300,350,400,450,500$ \\
\hline$-10^{\circ}$ & $200,300,350,400,450,500$ \\
\hline$-20^{\circ}$ & $200,300,350,400,450,500$ \\
\hline \hline
\end{tabular}

Table 2: Simulation cases run of diamond and steel tools on aluminum at a constant velocity of $0.78 \mathrm{~m} / \mathrm{sec}$.

\subsection{PRELIMINARY MODELING RESULTS FOR 6061-T6 ALUMINUM ALLOY}

Because of the large amount of thermal and material data available, aluminum was first chosen. The cases studied for aluminum consisted of rake angles of $0,-10$, and $-20^{\circ}$, cutting speeds varying from $0.53 \mathrm{~m} / \mathrm{sec}$ to $2.6 \mathrm{~m} / \mathrm{sec}$ and depths of cuts ranging from 200 to $500 \mu$ inches. Identical cases were run for both the steel and diamond tools. The models simulated the cutting of aluminum with a sharp tool.

The mesh used was the 460 node, 406 element, two dimensional Eulerian model shown in Figure $1[3,7]$. The conditions which were initially modeled for the orthogonal cutting of aluminum with both a steel and a diamond tool at a constant cutting speed are listed in Table 2. The material properties were modeled such that the yield strength for the workpiece was a linear function of the temperature and the thermal conductivity was a second order function of the temperature. Each case was run on a DEC VAXstation II/RC with an average CPU time of approximately four hours.

Typical temperature contour plots for aluminum cut with steel and diamond tools are shown in Figures 2 and 3, respectively. Notice that the maximum temperature for the same cutting conditions moved down on the rake face for the diamond tool. This movement 


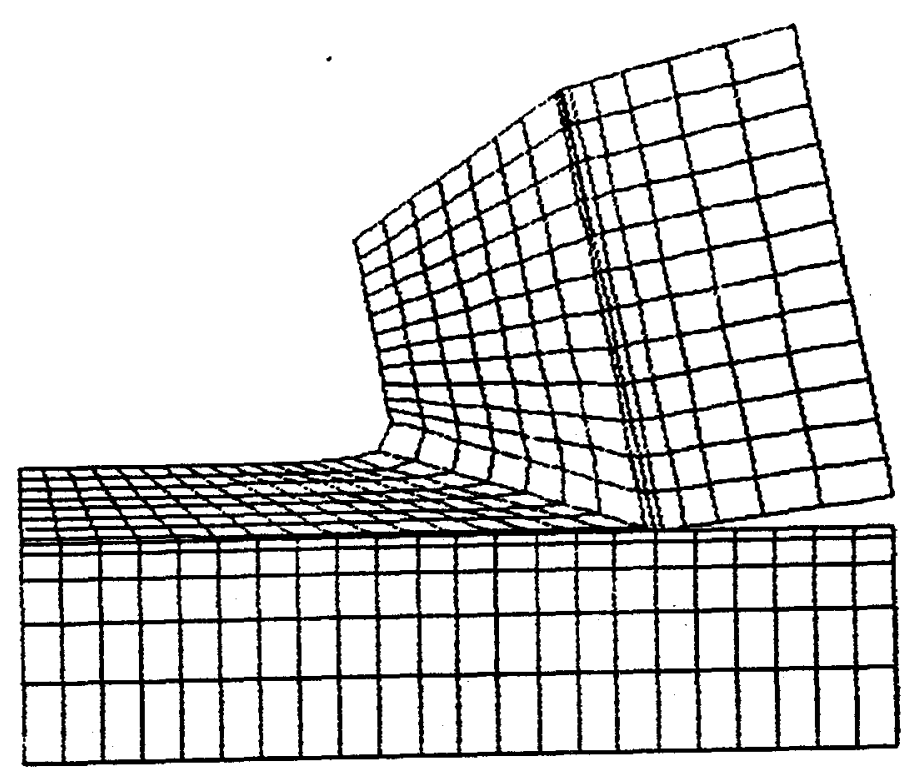

Figure 1: Typical mesh used for a $-10^{\circ}$ rake angle

down the rake face is believed to be related to the thermal conductivity ratios previously mentioned. This will become more evident when polycarbonate is discussed.

Figures 4 and 5 show the cutting and thrust force components versus depth of cut, respectively. In both of these figures, the simulation results for three rake angles $0^{\circ},-10^{\circ}$, and $-20^{\circ}$ at a constant speed of $0.78 \mathrm{~m} / \mathrm{sec}$ are displayed for cutting of aluminum with both diamond and steel cutting tools. Different cutting force levels resulted for the diamond and steel tools only for the largest negative rake angle of $-20^{\circ}$. For the thrust component shown in Figure 5, a higher force occurred for the steel tool as compared to the diamond, which became more pronounce as the depth of cut and negative rake angle was increased. It is believed that the primary factor for this difference is the coefficient of friction. The coefficients of friction for the diamond and steel on aluminum were 0.12 and 0.2 , respectively.

As a measure of the effect of the different thermal conductivities of the two cutting tools on the overall cutting force, the total force is shown in Figure 6 as a function of maximum temperature. The total force was calculated as the sum of the squares of the cutting and the thrust forces.

For all depths of cut, the temperature range was larger for the steel tool as compared to the diamond. This is a direct result of the higher thermal conductivity of diamond, which acts as a heat sink to reduce the overall cutting temperatures. In spite of this difference, however, the force versus temperature curves are similar for the steel and diamond tools. 


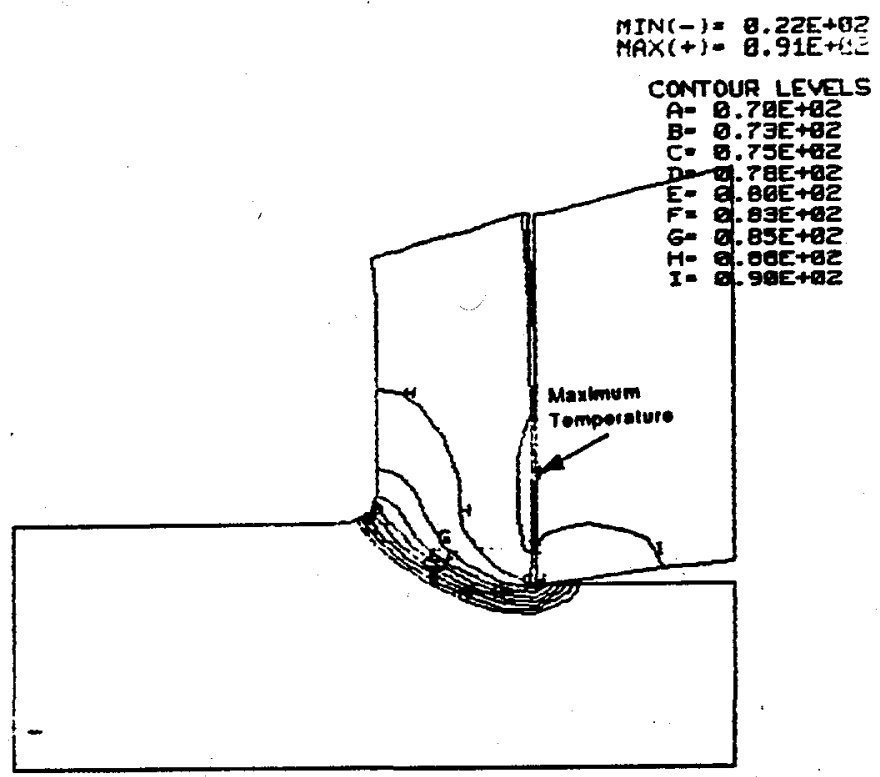

Figure 2: Typical temperature contour for aluminum cut with a steel tool for a $0^{\circ}$ rake angle, $200 \mu$ inch depth of cut, and a cutting speed of $0.78 \mathrm{~m} / \mathrm{sec}$.

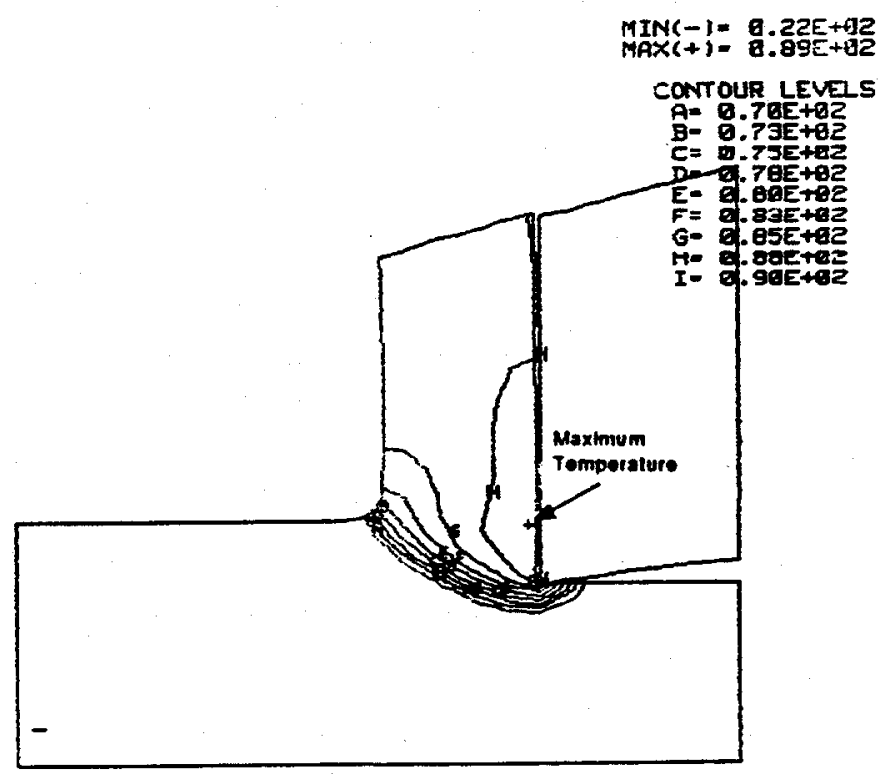

Figure 3: Typical temperature contour for aluminum cut with a diamond tool for a $0^{\circ}$ rake angle, $200 \mu$ inch depth of cut, and a cutting speed of $0.78 \mathrm{~m} / \mathrm{sec}$. 


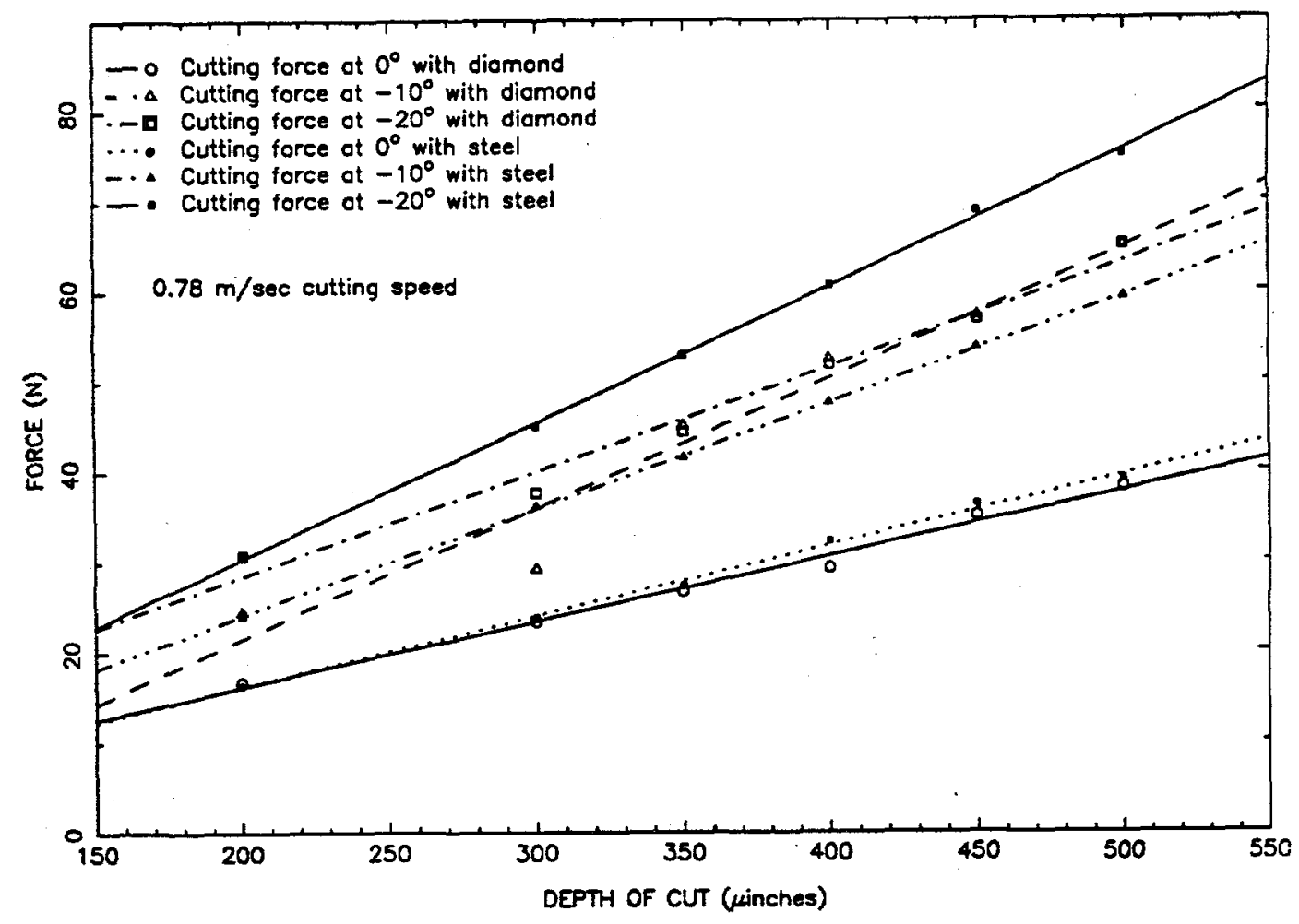

Figure 4: Cutting force vs. depth of cut for aluminum at various rake angles.

The resulting forces calculated from the models appeared to be reasonable when scaled for the amount of material actually removed. This removal area when linearly scaled to experimental values (Section 20) seemed to underestimate the values only slightly. As an example, for a $160 \mu \mathrm{m}$ infeed and a $4.27 \mu \mathrm{m}$ depth of cut the area of material removed would be $672 \mu \mathrm{m}^{2}$ while for the simulation the removal rate would be $22,606 \mu \mathrm{m}^{2}$ for a unit width of cut. Using a ratio of the two areas of 0.0297 , the force found from the simulation was on the order of $1.0 \mathrm{~N}$, compared with an experimentally measured force on the order of $1.0 \mathrm{~N}$.

Several cases were studied for the cutting of aluminum at a constant depth of cut (Table 3). A depth of cut of $500 \mu$ inches was chosen because several ongoing experiments at the PEC were performed at that depth with various materials. Figure 7 shows the maximum temperature as a function of the square root of the cutting speed. Figures 8 and 9 show the cutting and thrust force components, respectively, as a function of cutting speed. In contrast to the depth of cut, the cutting speed does not influence the tool forces.

\subsection{PRELIMINARY MODELING RESULTS FOR POLYCAR- BONATE}

One of the primary reasons for studying polymers is that these materials exhibit brittle characteristics similar to some ceramics but at more moderate temperatures and with a 


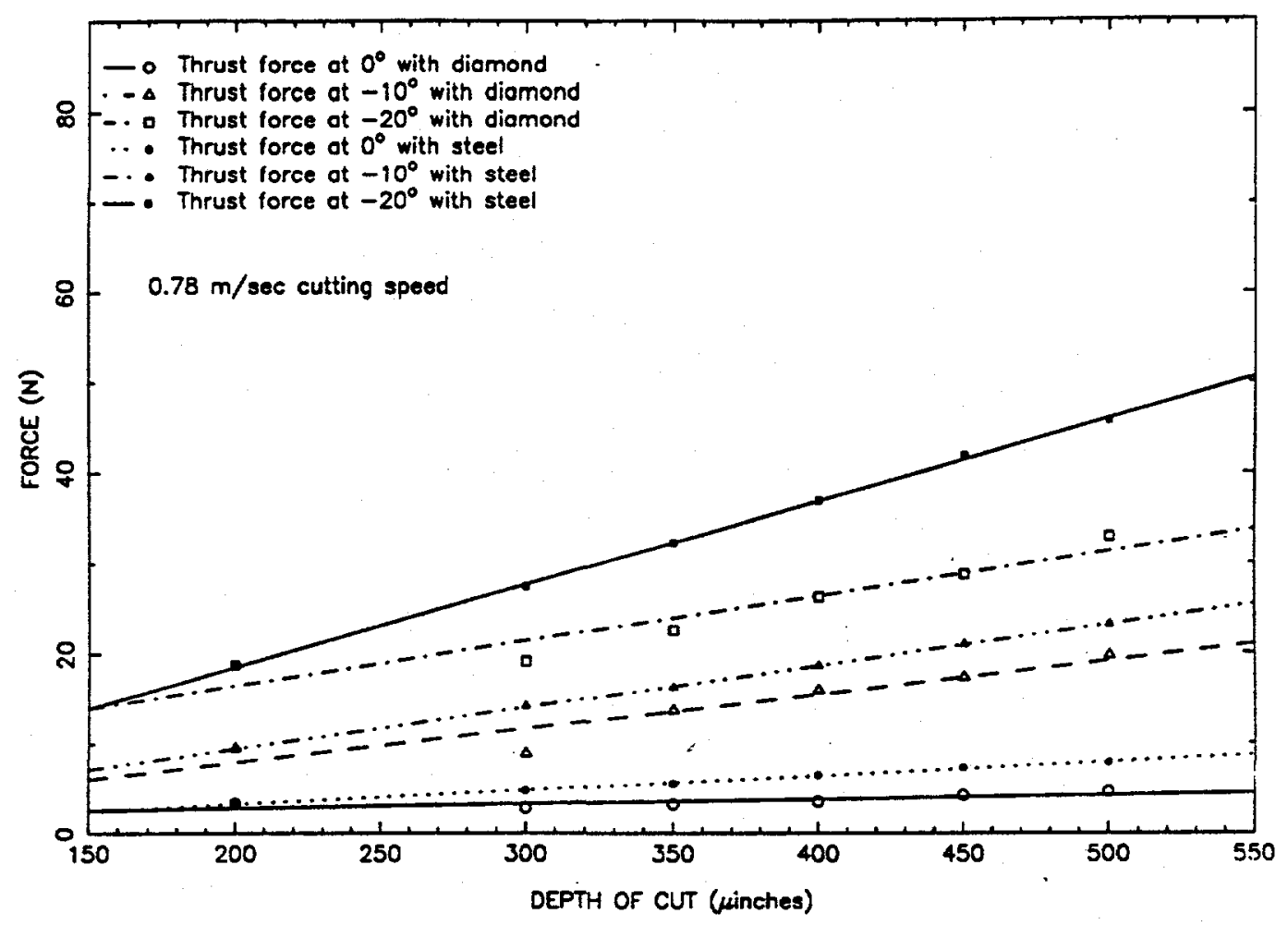

Figure 5: Tinrust force vs. depth of cut for aluminum at various rake angles.

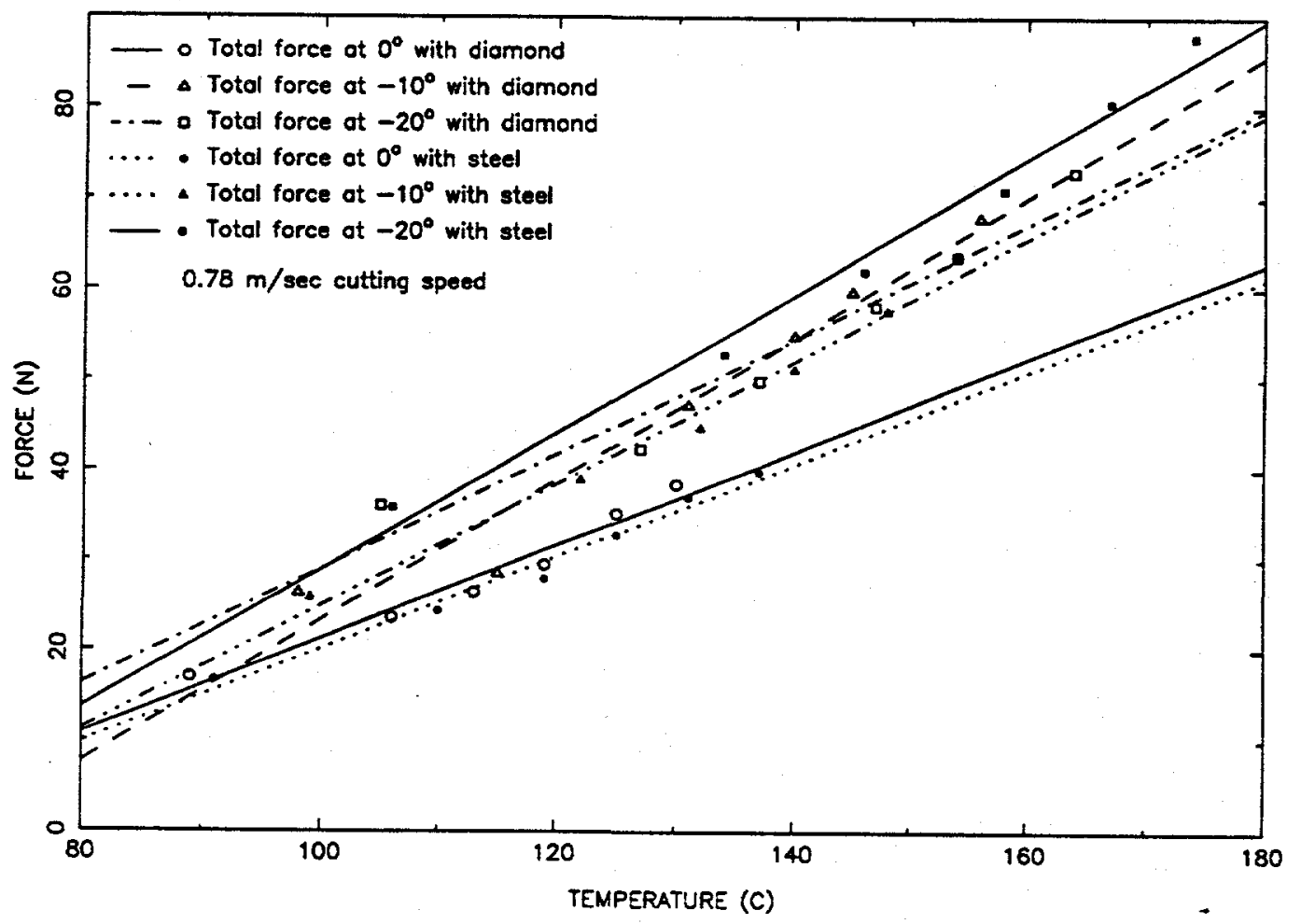

Figure 6: Total force vs. maximum temperature for aluminum at various rake angles. 


\begin{tabular}{||c|c||}
\hline \hline Rake Angles & $\begin{array}{c}\text { Cutting Speeds } \\
(\mathrm{m} / \mathrm{sec})\end{array}$ \\
\hline \hline $0^{\circ}$ & $0.27,0.53,0.78,1.33,2.66$ \\
\hline$-10^{\circ}$ & $0.27,0.53,0.78,1.33,2.66$ \\
\hline$-20^{\circ}$ & $0.27,0.53,0.78,1.33,2.66$ \\
\hline
\end{tabular}

Table 3: Simulation cases run of diamond and steel tools on aluminum at a constant depth of cut of $500 \mu$ inches.

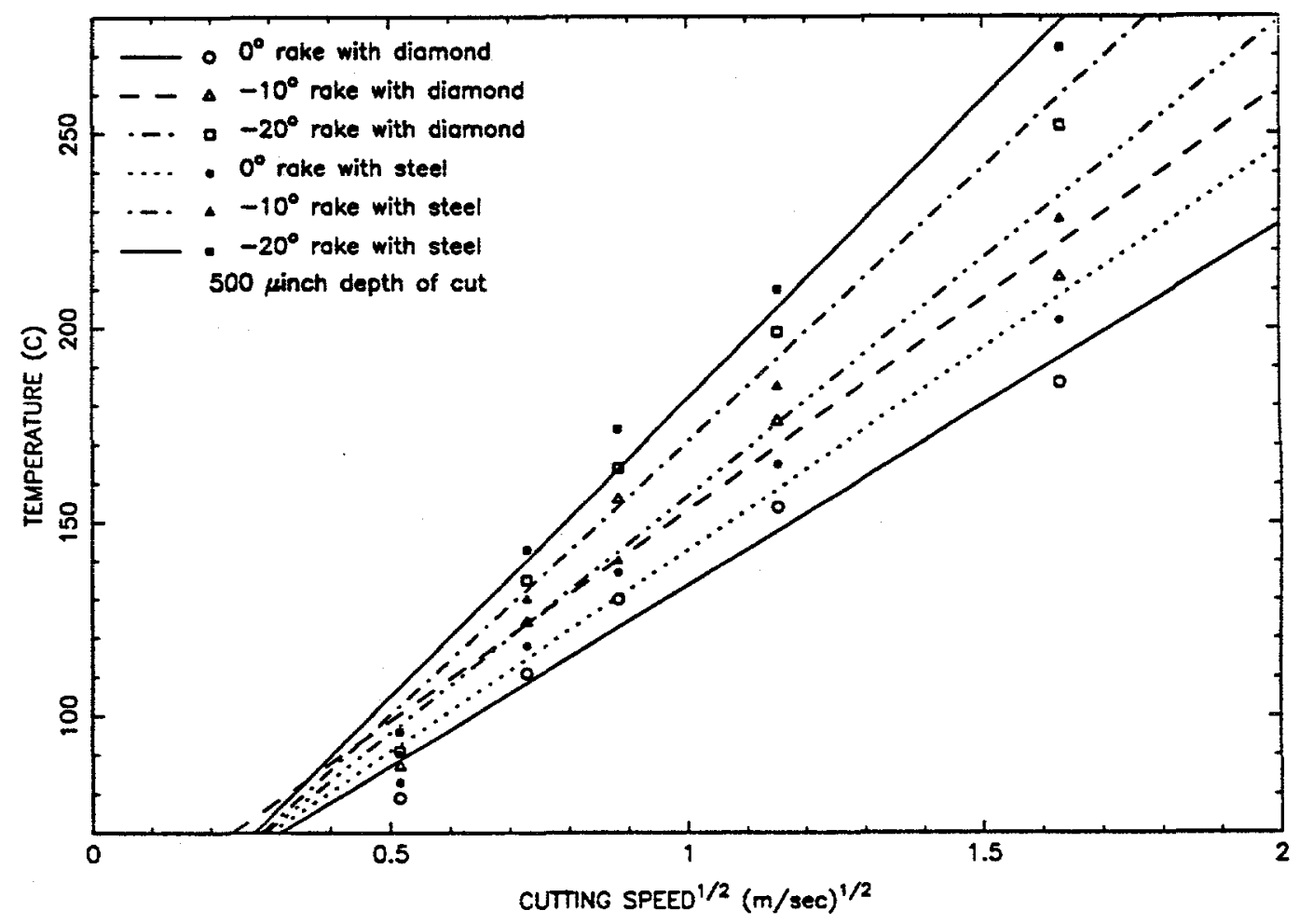

Figure 7: Temperature vs. cutting speed ${ }^{1 / 2}$ for aluminum at various rake ang: 


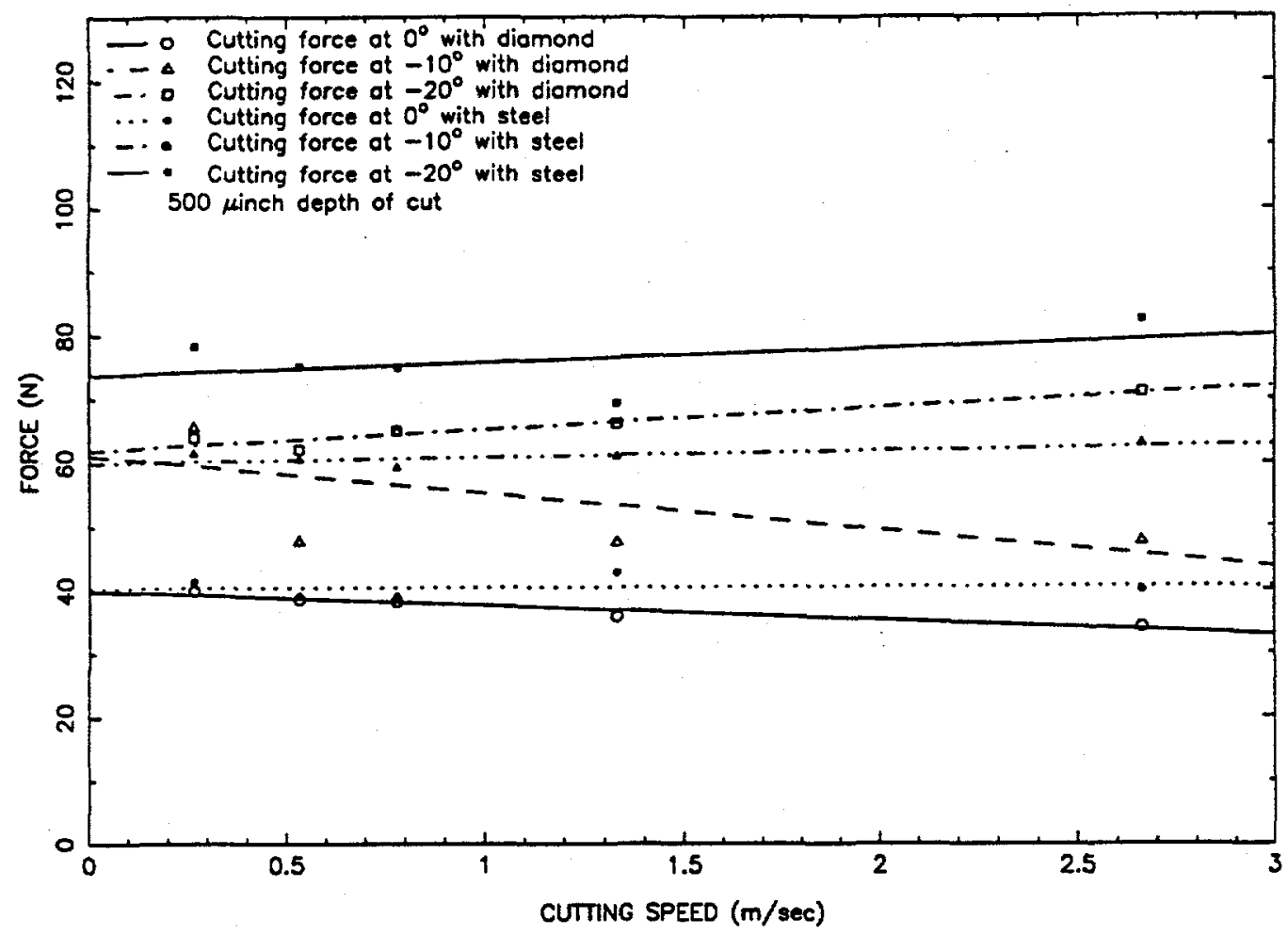

F: jure 8: Cutting force vs. cutting speed for aluminum at $v$ :ious rake angles.

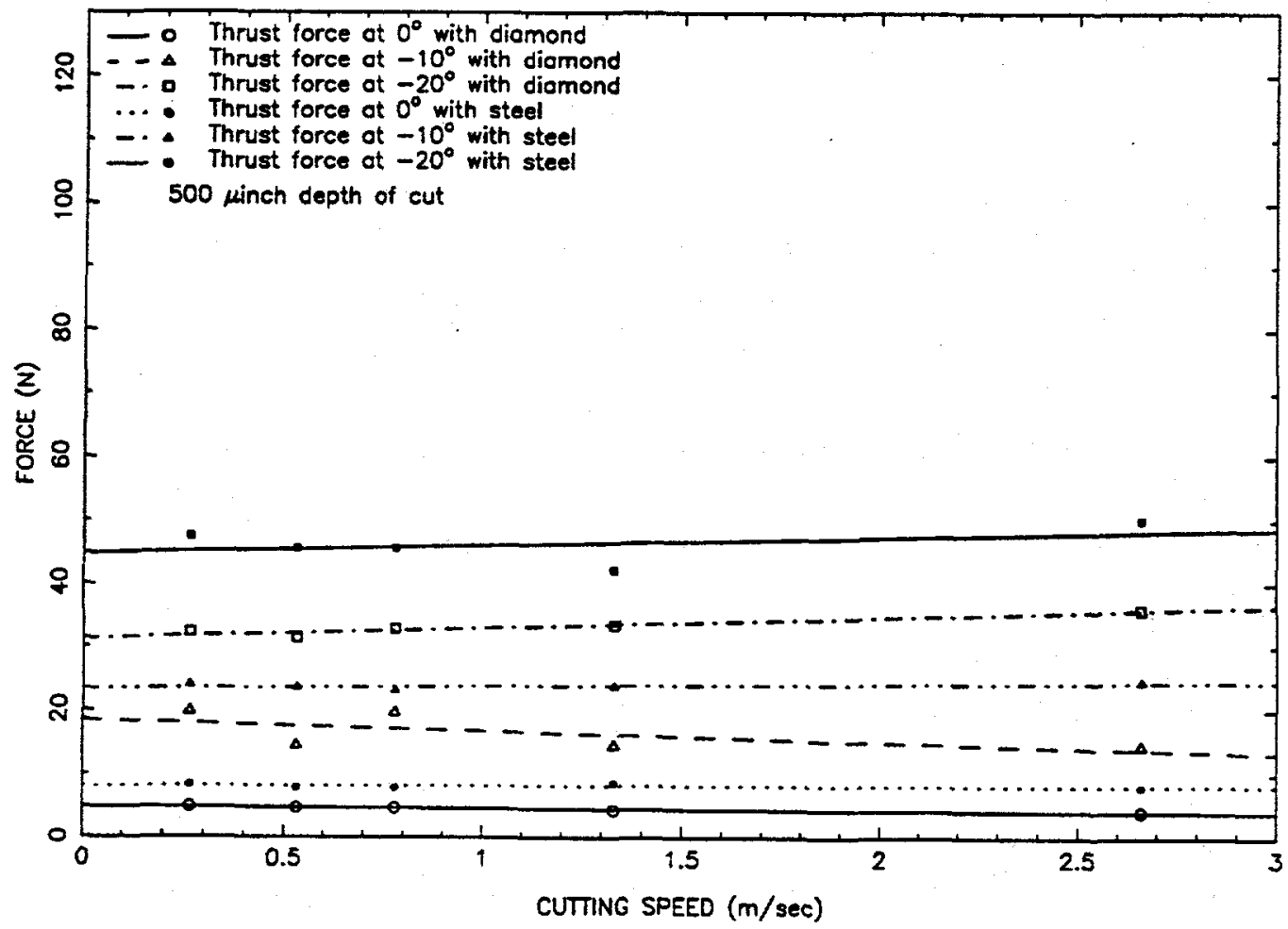

Figure 9: Thrust force vs. cutting speed for aluminum at various rake angles. 


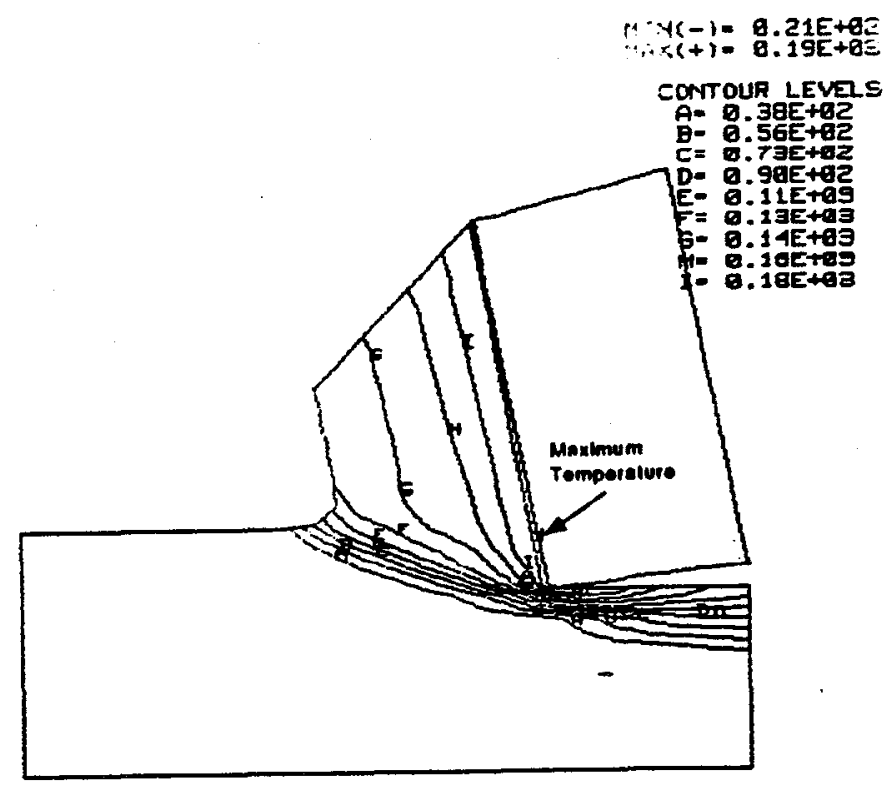

Figure 10: Typical temperature contour of polycarbonate cut with a diamond tool.

larger softening regime. From some of the Center's previous publications [2] [6] [12] it was generally believed that a study of these polymers would help improve the understanding of the cutting process of other brittle materials such as silicon and germanium.

Figure 10 shows the temperature contours for cutting of polycarbonate with diamond. Although the maximum temperature is still along the rake face, the temperature of the surface behind the tool edge is nearly equal to the maxima. This can be explained by the extremely large conductivity ratio for diamond on polycarbonate, 23,000. Thus the diamond acts as an enormous heat sink, drawing away most of the heat generated. The significance of this result is that the maximum temperature is now in the workpiece surface rather than in the chip. The elevated temperature in the surface would cause the thermal softening to occur, thus increasing the likelihood of ductility in the finished workpiece surface.

\subsection{SUMMARY OF RESULTS}

Initial simulations have been conducted to elucidate the role of thermal conductivity between the workpiece and tool in influencing ductile flow. From this study, it has not been possible to completely separate the effects of the relative conductivities from the friction effects because the two tool materials have different friction coefficients. In addition, the yield strength for aluminum decreases slowly with respect to temperature, so that thermal 
effects would not be as significant as for the polymers which show a sharp drop in yield strength at the softening temperature. Also, preliminary models of polycarbonate indicate that the thermal effect can be recognized more readily, because the coefficients of friction between the steel and diamond tools with polycarbonate are similar. Finally, the brittle materials have the most pronounced drop in yield strength over the softening temperature and hence, the thermal effects would be even more pronounced.

\subsection{PLANS FOR FUTURE WORK}

Several areas of study remain in which further efforts should be placed. Foremost is the need to further the material characterization of copper, polystyrene, silicon, and germanium. The work on polycarbonate will be completed with emphasis placed not only on the thermal effects but also on material properties which are strain dependent. It is believed that a family of curves can be obtained in which given the material surface temperature an approximation can be made on the material's surface quality. Figure 11 characterizes this hypothesis which can be accomplished by basing the models on experimental results as discussed in Section 20. In these experiments, the conditions were chosen to produce a spiral cut on polycarbonate. Figures 12,13 , and 14 show the surface quality for three cutting speeds. Within one spiral, various surface topography can be seen. Referring to Figure 12, one can see that at and near the edges of one turn, ductile flow occurred resulting in a smooth surface. However as one approaches the center of a groove, a sharp change in the surface arises which is believed to be brittle fracture at larger depths. Since the tool actually has a varied depth of cut from 0 to $500 \mu$ inches, a critical depth in which brittle fracture will occur can be determined (Figure 15). Hence a family of curves can be found similar to those seen in Figure 11.

Another area in which further research will be conducted is a study on the improvement of friction simulations. Currently the friction is given as a simple numerical value as shown in Figure 16. It is believed in order to obtain a more realistic model, the friction must be characterized as a viscoplastic boundary layer, as shown in Figure 17. Other friction models similar to this will be studied in the coming year. 


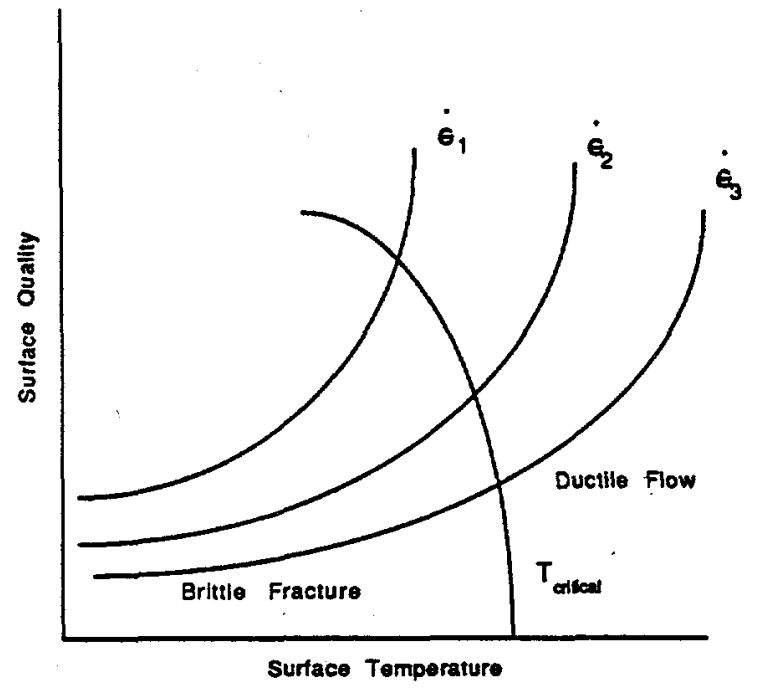

Figure 11: Proposed surface quality vs. maximum surface temperature for a family of strain rate curves.
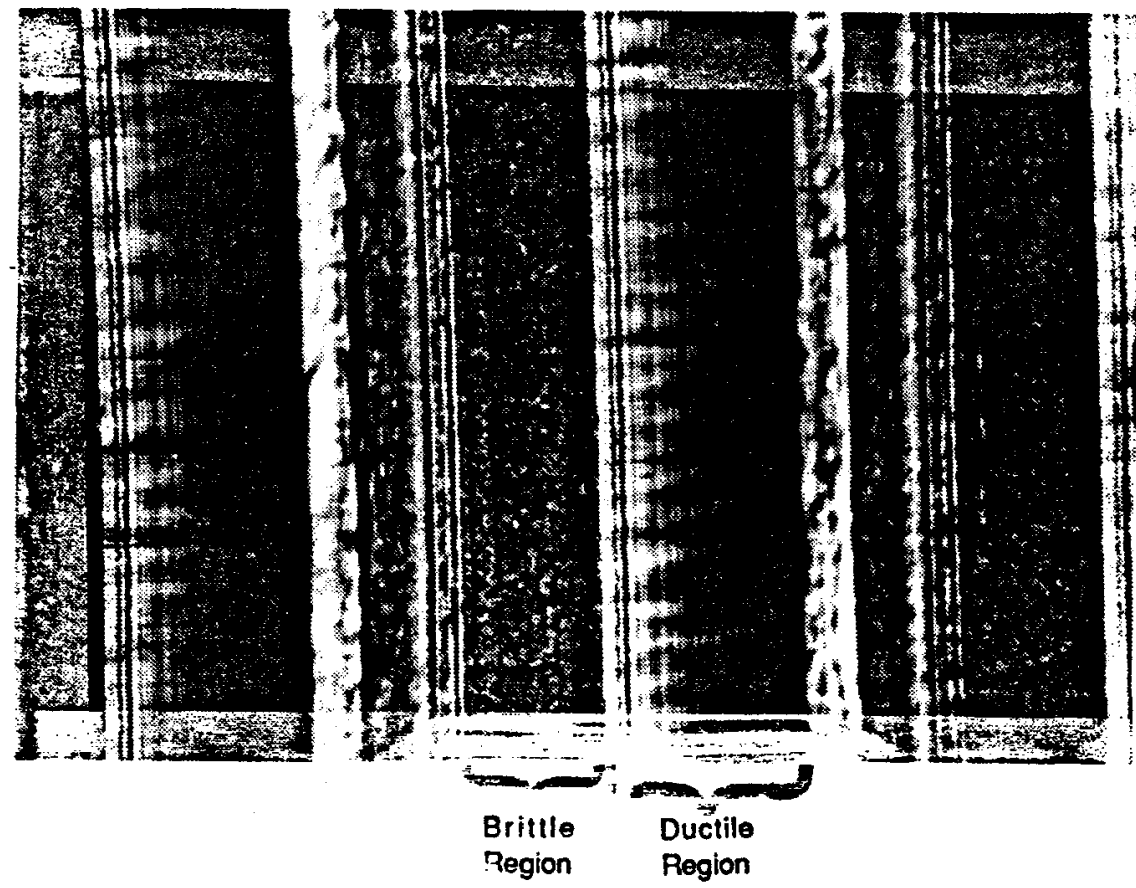

Figure 12: Spiral cuts of polycarbonate with a depth of cut of $500 \mu$ inches, $288.0 \mathrm{in} / \mathrm{sec}$, $0^{\circ}$ rake, and $200 \mathrm{X}$. 


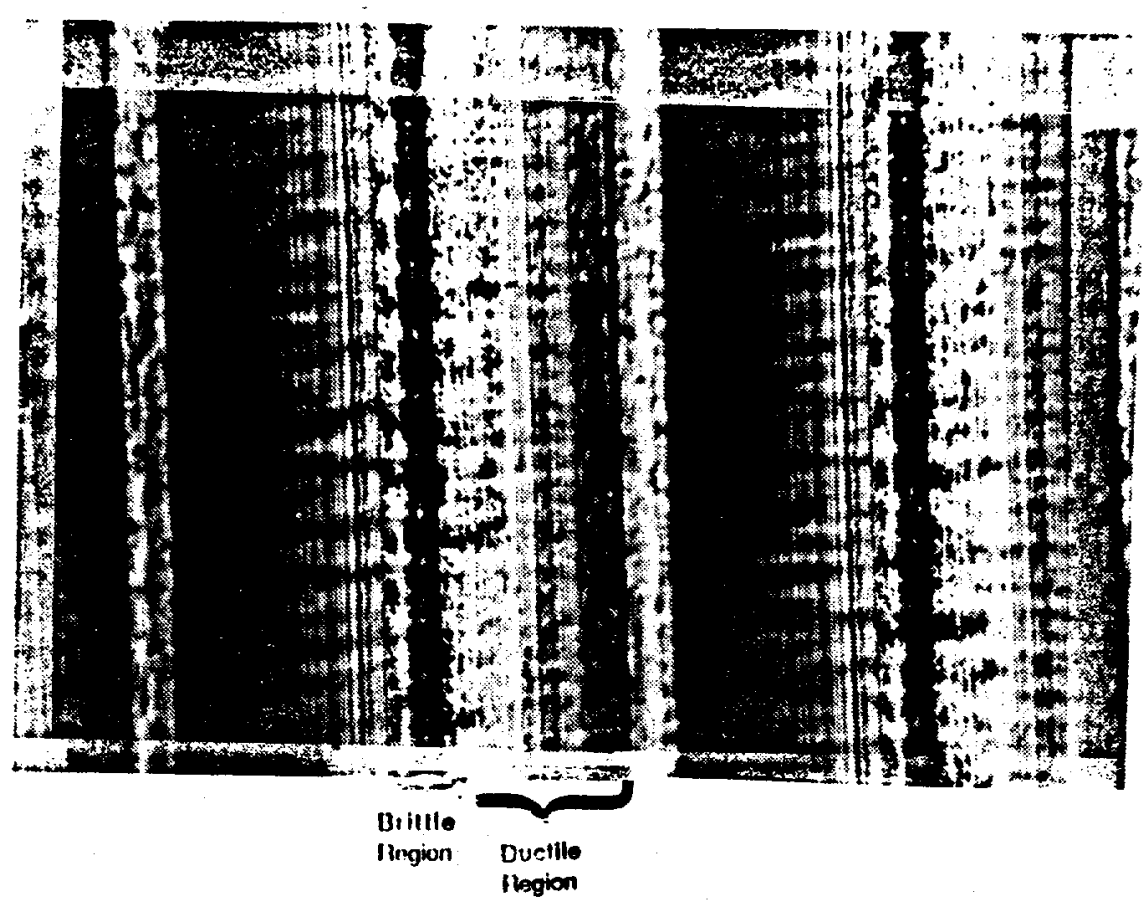

Figure 13: Spiral cuts of polycarbonate with a depth of cut of $500 \mu$ inches, $55.0 \mathrm{in} / \mathrm{sec}, 0^{\circ}$ rake, and $200 \mathrm{X}$.

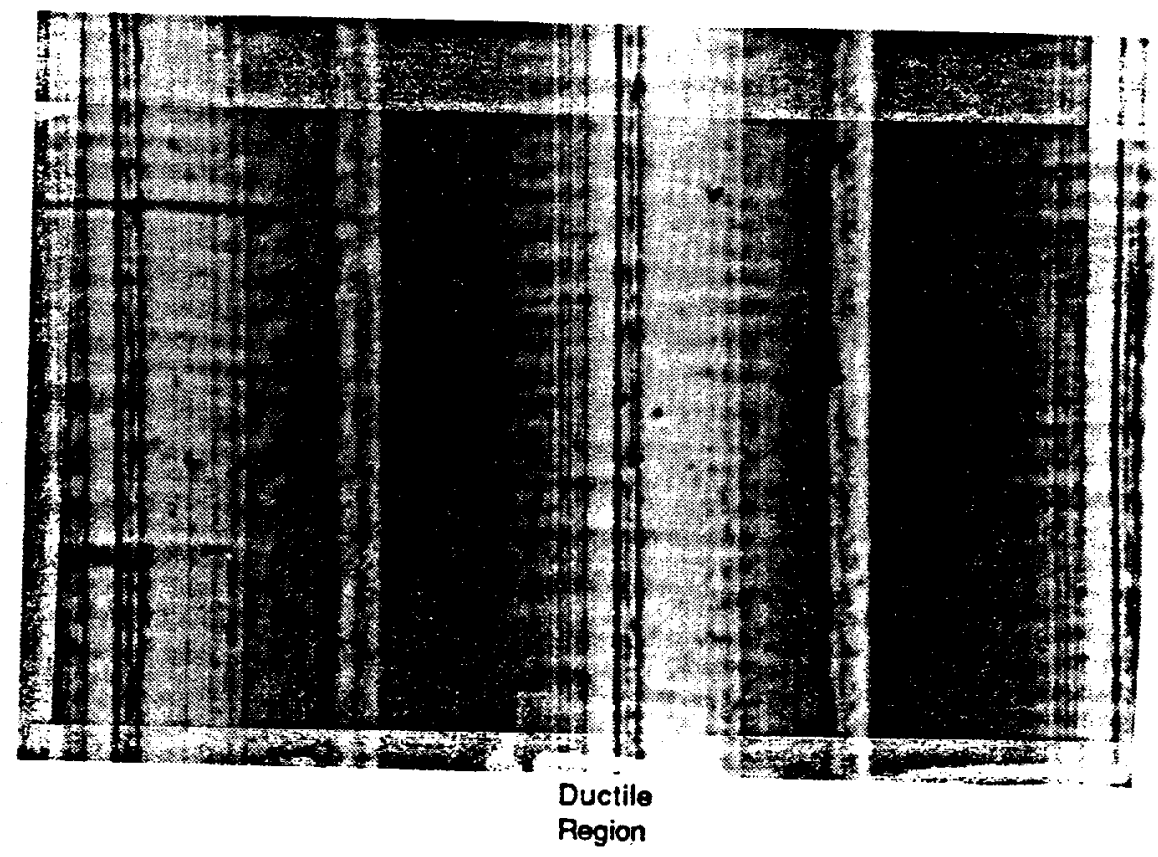

Figure 14: Spiral cuts of polycarbonate with a depth of cut of $500 \mu$ inches, $7.8 \mathrm{in} / \mathrm{sec}, 0^{\circ}$ rake, and $200 \mathrm{X}$. 


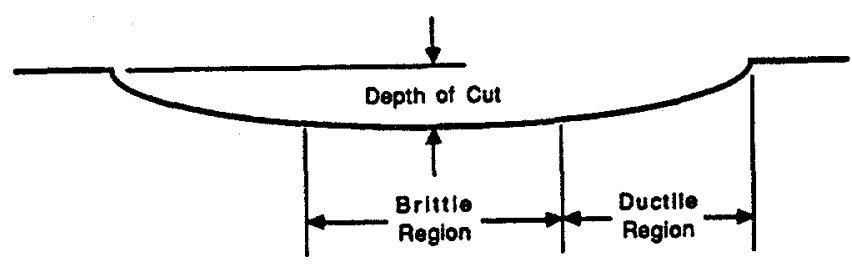

Figure 15: Profile of a spiral cut.

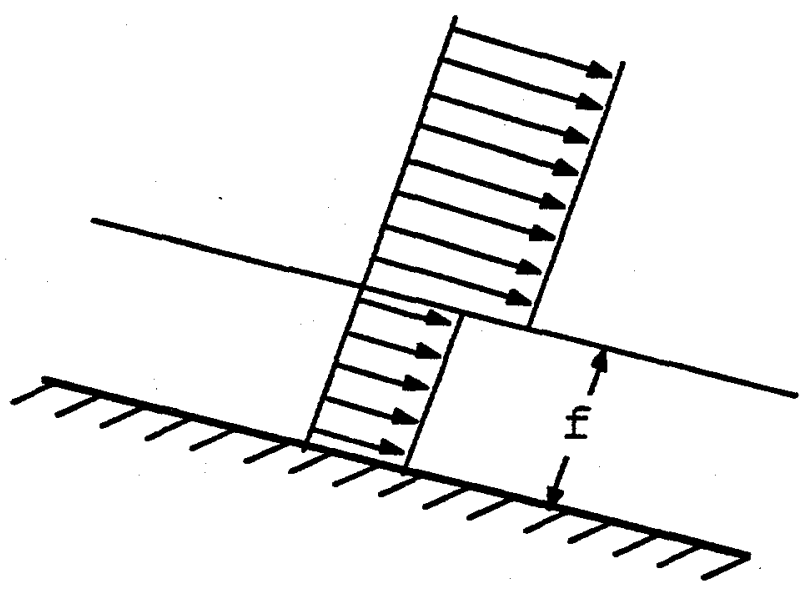

Figure 16: Current friction simulation. 


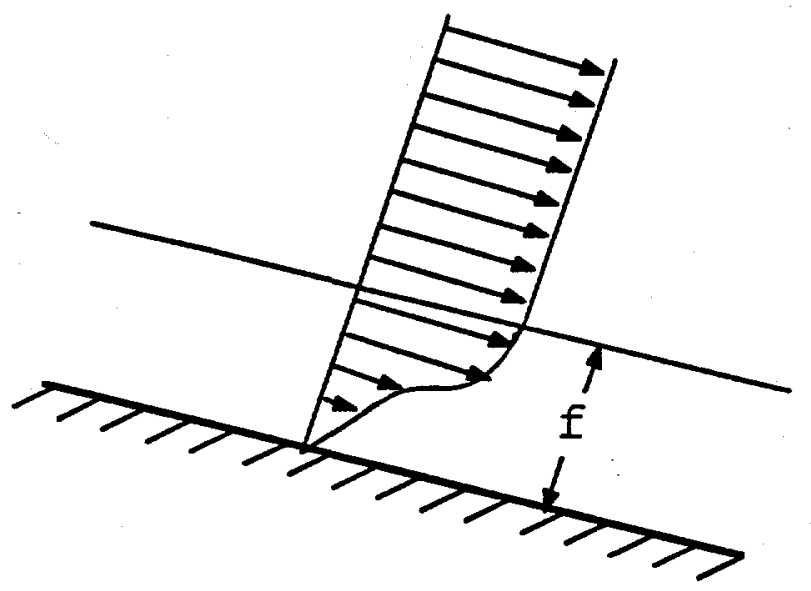

Figure 17: Proposed future improvement of friction simulation as a boundary value problem.

\section{References}

[1] Blake, Peter N., and Scattergood, Ronald O., "Diamond Turning of Germanium and Silicon," Precision Engineering Center Annual Report, 1986, Vol. iv, pp. 180-201.

[2] Blake, Peter N., Smith, Elizabeth F. and Scattergood, Ronald O., "Single Point Diamond Turning of Brittle Materials," Precision Engineering Center Annual Report, 1987, Vol. v, pp. 224-248.

[3] Carroll, John Thomas III, "A Numerical and Experimental Study of Single Point Diamond Machining," PhD. Dissertation, North Carolina State University, Raleigh, NC, 1986.

[4] Hallquist, J.O., "NIKE2D-A Vectorized, Finite Deformation, Finite Element Code For Analyzing the Static and Dynamic Response of 2-D Solids," Lawrence Livermore National Laboratory, Report Number UCID-19677.

[5] Luh, Michael H. and Strenkowski, John S., "Simulations of Ductile Flow in Brittle Material Processing," Precision Engineering Center Annual Report, 1987, Vol. v, pp. 284-293.

[6] Luh, Michael H. and Strenkowski, John S., "Simulations of Ductile Flow in Brittle Material Processing,"Interim Report Precision Engineering Center, 
July 1988 , pp. 6-7.

[7] Moon, K.J., "Transient Cutting Model Based on Eulerian Finite Element Approach," $\mathrm{PhD}$. Dissertation, North Carolina State University, Raleigh, NC, 1988.

[8] Schinker, Martin G. and Döll, Walter, "Basic Investigations into the High Speed Processing of Optical Glasses with Diamond Tools," SPIE Proc., Vol. 381. pp. 32-38.

[9] Schinker, Martin G. and Döll, Walter, "Plasticity and Fracture Induced by Scratching Optical Glasses at High Speed," Strength of Inorganic Glass, 1985, pp. 115-133.

[10] Schinker, Martin G. and Döll, Walter, "Turning of Optical Glasses at Room Temperature," SPIE Proc., Vol. 802.

[11] Smith, Elizabeth F. and Scattergood, Ronald O., "Diamond Turning of Optical Glass," Precision Engineering Center Annual Report, 1986, Vol. iv, pp. 202-217.

[12] Smith, Elizabeth F. and Scattergood, Ronald O., "Diamond Turning of Amorphous Polymers," Interim Report Precision Engineering Center, 1988, pp. 4-5.

[13] Strenkowski, J.S. and Carroll, J.T. III, "A Finite Element Model of Orthogonal Metal Cutting," Journal of Engineering for Industry, Vol. 107, November 1985, pp. 349-354. 


\title{
23 FRACTURE MECHANICS APPLIED TO THE MACHINING OF BRITTLE MATERIALS
}

\author{
Gary D. Hiatt \\ Graduate Student \\ John S. Strenkowski \\ Associate Professor, MAE
}

Research has begun on incorporating fracture mechanics into a model of the orthogonal cutting of brittle materials. Residual stresses are calculated for the machined material by a combination of Eulerian and Lagrangian finite element models and then used in the calculation of stress intensity factors by the Green's Function Method.

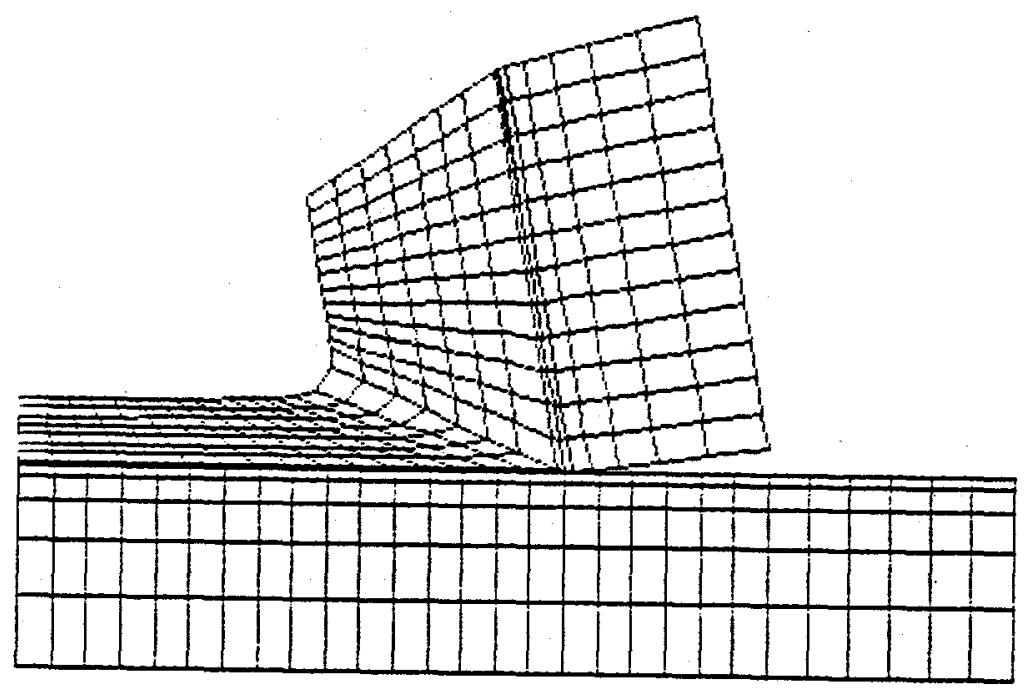




\subsection{INTRODUCTION}

Two important engineering ceramics, silicon and germanium, have been turned with diamond tools without extensive fracture or surface damage [1]. To obtain quality surfaces consistantly, it is necessary to possess a fundamental understanding of how different parameters affect the machining of ceramics. Factors which affect the process include the depth of cut, rake angle, and cutting speed. The finite element method is a powerful tool which can provide insight as to how different parameters affect the role of fracture in the machining of ceramics. Finite element analysis has been applied to the machining of brittle materials [2]. In that study a crack was placed at the tip of the tool and mode I fracture was assumed. This section describes a more realistic model including mixed mode fracture.

\subsection{ANALYSIS OF THE MACHINING PROCESS}

Assuming that the material to be used possesses flaws, Figure 1 illustrates what happens (assuming plane strain conditions) when the tool approaches the crack. As the tool approaches the crack the stresses acting on the crack are compressive and shearing. The shear stresses drive the crack and are opposed by sliding friction between the crack faces. The effective shear stress on the crack is given by Equation (1),

$$
\tau_{e f f}=\tau-\mu \sigma
$$

where $\tau$ is the shear stress, $\sigma$ is the compressive stress, and $\mu$ is the coefficient of friction for the material sliding on itself. The crack can grow only when the right hand side of Equation (1) is positive. As the tool advances towards the crack, the shear stress decreases with respect to the compressive stress, and can no longer overcome the crack-face friction. After the tool passes the stress changes to tension and shear resulting in crack opening and growth.

Two theories are available to predict the direction of crack growth: the maximum hoop stress theory and the minimum strain energy density theory [3]. The maximum hoop stress theory simply predicts that the crack extends in the direction perdendicular to the maximum principal stress. The minimum strain energy density theory predicts that the crack grows in the direction for which the strain energy density is a minimum. Both theories predict that when the tool is approaching the crack, the front end of the crack propagates deeper into the material and the back end propagates towards the surface. Figure 2 shows the directions for the predicted crack growth.

After the tool has passed by, the principal stress changes to tension, but the directions for crack growth predicted by the two theories remain the same as when the tool was approaching. These predictions seem to be contrary to what one would expect for the formation of brittle chips. When the crack is close to the surface, there may be a boundary 


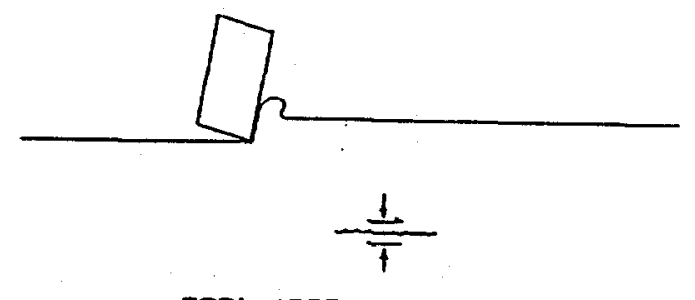

TOOL APPROACHINO CRACK

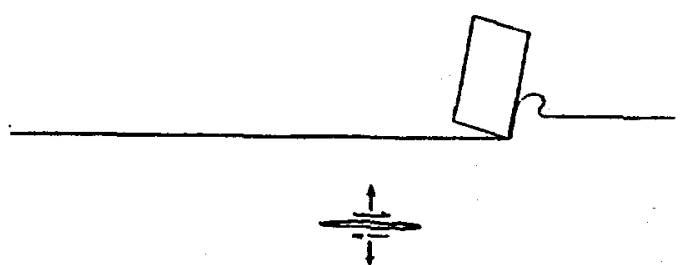

TOOL has passed CRACK

Figure 1: Stresses Acting on Crack During Wlachining

effect present which would invalidate the two theories.

Two competing effects can lead to the creation of the residual stresses; plastic deformation and thermal effects.

- The material behind the tool point will be in a state of tension [4]: If the yield stress is exceeded, the top layers of the workpiece will be plastically deformed. Beneath the plastically deformed layers will be layers which are elastically extended. When the tool has been removed or passed far enough away, the elastically extended layers will contract, compressing the plastically extended layers which are unable to return to their former state as illustrated in Figure 3.

- Because of the heat produced during machining, a temperature gradient will appear and produce thermal stresses [5]. The largest temperature will appear at the surface, with temperatures decreasing below the surface. The upper layers will expand more than the lower layers with the result that the top layers are in state of compression while the deeper layers are in tension. The higher temperatures lower the yield point, and if the compressive stresses exceed the yield point, then permanent deformation will appear in the top layers. Upon cooling the top layers will contract leading to a state of tension as illustrated in Figure 4. 

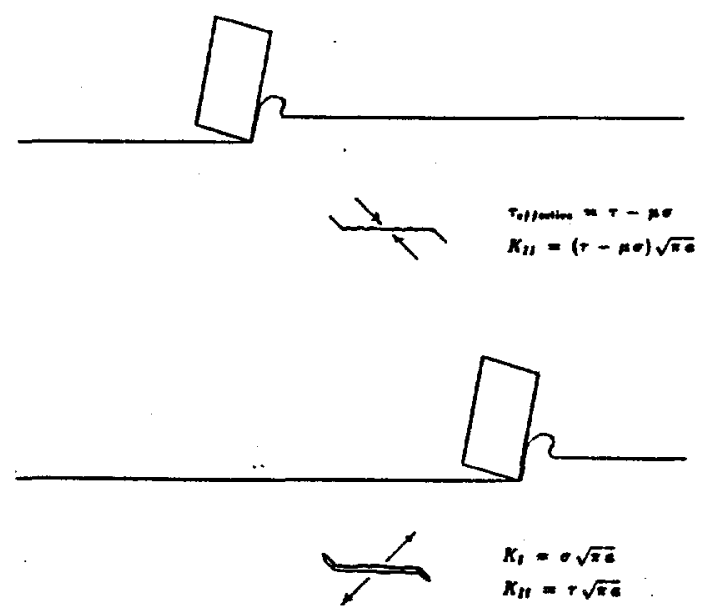

CRACK GROWTH DIRECTION PREDICTED EY

1. MaxTMUM HOOP STRESS THEORY

z. MINIMUM STRAIN ENERGY THEORY

Figure 2: Growth of Crack During Machining

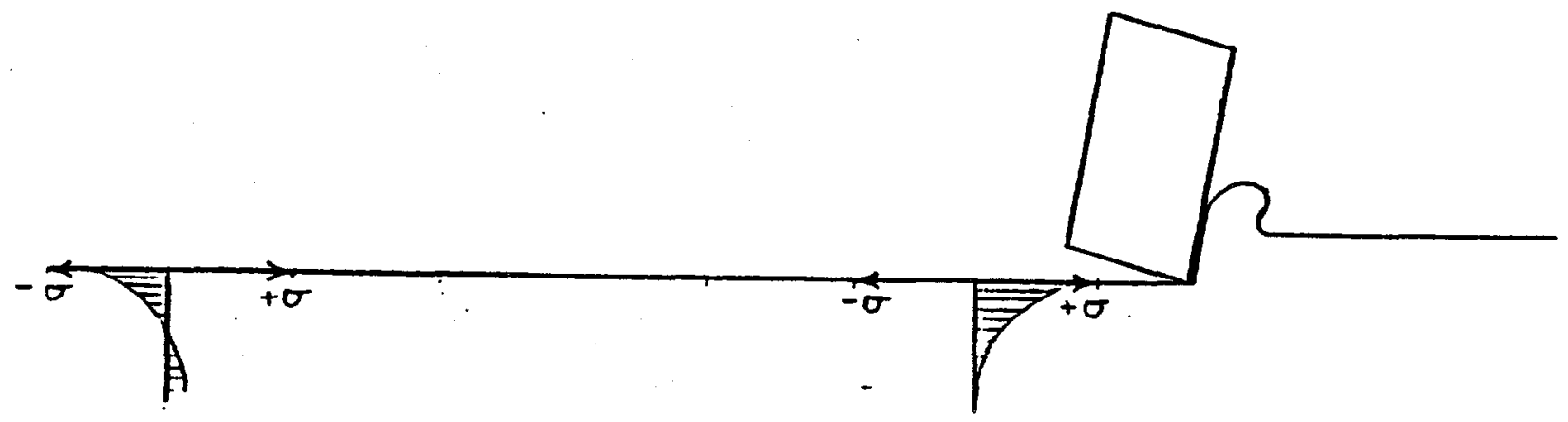

Figure 3: Stress Fields in the Surface Layers due to the Tool 


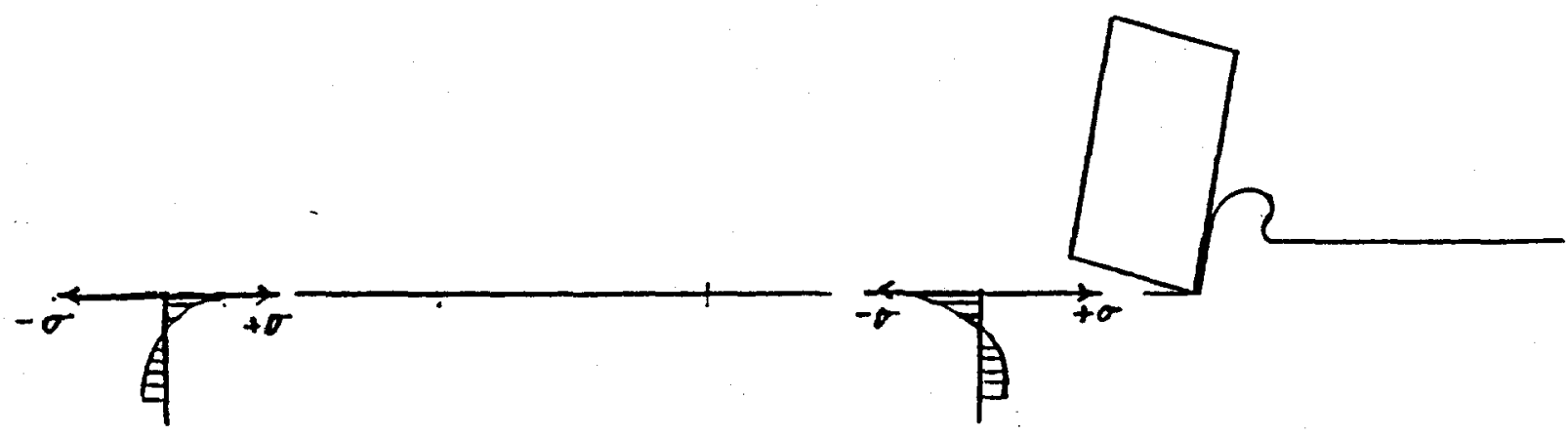

Figure 4: Stress Fields in Surface Layers due to Temperature Effects from Heat Generated during Machining

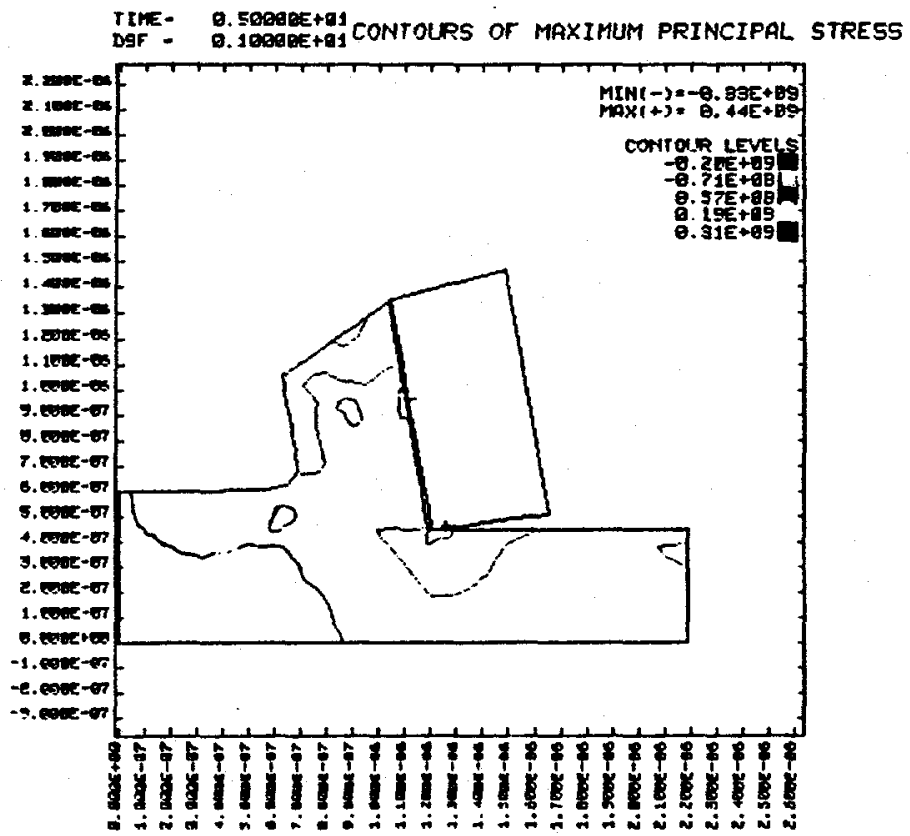

Figure 5: Eulerian Model of the Machining of Siiicon 


\subsection{PRELIMINARY RESULTS}

Several problems exist in the application of fracture to the cutting process. The distribution and size of pre-existing cracks is a statistical phenomenon. The crack size and depth of cut approaches the order of microstructural features which limits the continuum foundation on which fracture is derived. The effort described here is then a start towards applying fracture mechanics to the micro-machining of silicon.

An Eulerian model [6] of the orthogonal cutting process has been used to model the ductile flow of silicon. This model is based upon a perfectly-rigid/perfectly-plastic stress-strain relationship, and so does not include an elastic response. When the tool is removed from the workpiece there should be an elastic springback. Zienkiewicz [7] shows that the elastic rebound can be obtained by inputting the model geometry into an elastic finite element program, removing the tool, and applying the inverse of the tool forces upon the workpiece. Thus, in order to obtain the residual stress in the machined silicon, the Eulerian model was used to determine the stress field as well as the forces acting upon the workpiece from the tool. Then the deformed workpiece geometry was used in the NIKE2D program [8] along with the inverse of the tool forces to calculate another stress field. The two stress fields were then combined to give the residual stress by this procedure. Figures 6,7 and 8 show the residual stresses calculated at depths of cuts of $0.075 \mu \mathrm{m}, 0.15 \mu \mathrm{m}$, and $0.30 \mu \mathrm{m}$ respectively. These stresses were calculated for a rake angle of $-10^{\circ}$ and a speed of $1 \mathrm{~m} / \mathrm{sec}$.

The top portion of Figures 6,7 and 8 shows the stress distribution in the cutting direction in the surface layer and in a lower layer parallel to the surface. These stresses would cause median cracks (cracks perpendicular to the surface). The bottom portion of Figures 6,7 and 8 shows the stress distribution in the thrust direction just behind the tool edge and a distance further behind the tool. These stresses would cause lateral cracking. One point integration was used in the model in order to reduce numerical inaccuracies. Thus the stresses are computed at the center of each element. The jagged appearance of the graphs is due to the variation of stress from element to element.

Common trends in the graphs show that the stresses in the top layers are the largest, and that the stresses in the lower layers level off to a fairly constant value. What was not expected was the alternating nature of the cutting stresses in the top layer of the material. These stresses were expected to be eithor totally compressive or tensile, but not a combination of the two.

Sparks, Enloe, and Paesler in Section 17 of this report show the average in-plane residual stress profile obtained from Raman spectroscopy for a $-10^{\circ}$ rake angle for Germanium. The stress profiles for the thrust direction of Figures 6, 7, and 8 directly under the tool are somewhat similar to the one obtained using Raman spectroscopy. The largest stress obtained in the computer model was $0.801 \times 10^{9} \mathrm{~Pa}$ at a depth of $0.074 \mu \mathrm{m}$ for the $0.30 \mu \mathrm{m}$ depth of cut. The largest stress obtained in the Raman spectroscopy study was $0.61 \times 10^{9}$ $\mathrm{Pa}$ at a depth of $0.08 \mu m$. The largest difference is that the Raman spectroscopy shows 
a compressive residual stress in the lower layers while the finite element model shows a tensile stress in those layers. These differences may be due to the fact that the Raman stresses are averaged in-plane stresses while the finite element stresses are in. the thrust direction. Also the finite element model used in the study had a relatively coarse grid. More accurate stresses would be given by a finer grid.

The Eulerian model uses many different material properties such as thermal conductivity, density, specific heat, shear modulus and yield strength (see Figures 9 and 10). Figure 9 shows the thermal conductivity of diamond. Diamond was modeled as the material for the cutting tool. The very high thermal conductivity of diamond along with the high thermal conductivity of silicon plus the low coefficient of friction for diamond suggests that the temperature rise during the machining of silicon would be lower than what would be expected.

Obviously accurate material properties are required for the model to give useful results. Obtaining valid data for the flow stress of silicon at different strain rates and temperatures has been a problem. Figure 11 shows how the stress strain curve for silicon varies with temperature. At low temperatures silicon will fracture before it plastically deforms. At higher temperatures plastic flow occurs and there is a decrease in the yield stress as seen in Figure 11. The upper yield stress is the stress at which yielding first begins. The flat part of the curve is referred to as the lower yield stress. The lower yield stress at different temperatures was used to compose Figure 10. However, there is a difference in the lower yield stress for different strain rates as shown by Figure 12. The Figure 10 data was obtained for a strain rate of $2 \times 10^{-5} / \mathrm{sec}$. Data is needed for much higher strain rates.

\subsection{FRACTURE FROM RESIDUAL STRESSES}

Indentation studies of ceramics have shown that residual stresses are very important in the development of lateral cracks [13]. To see whether the residual stresses in machined silicon are great enough to cause crack propagation, a fracture mechanics model is being developed.

Most equations used to calculate stress intensity factors are based on uniform stress fields. This is certainly not the case in machining. One method that can overcome this problem and that has been used on residual stress fields is the Green's Function Method [14]. To use this method the stress field in the absence of a crack must be found first. The crack is then inserted at the desired location and tractions are applied to the crack faces to satisfy boundary conditions. These tractions are used along with the appropiate Green's Weighting Function to calculate the stress intensity factor. For a crack in an infinite plate the stress intensity factor is

$$
K=\frac{1}{\sqrt{\pi a}} \int_{-a}^{a} \sigma(x)\left[\frac{a+x}{a-x}\right]^{1 / 2} d x
$$


Rosenfield [15] shows that an approximate solution is

$$
K=\left(\frac{\sigma_{T}+2 \sigma_{o}}{3}\right)(\pi a)^{1 / 2}
$$

$\sigma_{T}$ is the stress applied at $x=a$ and $\sigma_{o}$ is the stress applied at the crack midpoint.

Keefe-Fraundorf [16] gives the predominant defects in p-type silicon as being $0.05 \mu m$ to $0.3 \mu \mathrm{m}$ irregularly shaped inclusions. The largest inclusion was treated as an omnidirectional crack to be placed at critical sites.

In order for linear elastic fracture mechanics to apply, the stress field must be less than the yield stress. Puttick [17] gives the yield stress of silicon at room temperature as $3 \mathrm{x}$ $10^{9} \mathrm{~Pa}$. The residual stresses calculated for use in this study were all below Puttick's value for yielding.

Blake [1] was able to obtain ductile machining of silicon at depths of cuts of $0.15 \mu \mathrm{m}$ and below. Three depths of cuts were used in this study including $0.075 \mu \mathrm{m}, 0.15 \mu \mathrm{m}$, and $0.30 \mu \mathrm{m}$. Using the fracture toughness of $0.6 \mathrm{MNm}^{-3 / 2}[18]$ crack propagation was observed in the $0.30 \mu \mathrm{m}$ depth of cut model but not in the others.

\subsection{SUMMARY}

Fracture mechanics has been applied to the residual stresss field created by the machining of silicon and preliminary results indicate that the model agrees with experimental data. Future work is planned for obtaining better material data from published sources for use in the model, specifically the variation of flow stress with strain rate. A finer grid will be used to obtain greater accuracy and ease of comparison with experimental data. Future plans also include the development of a model for crack initiation during the cutting process. 

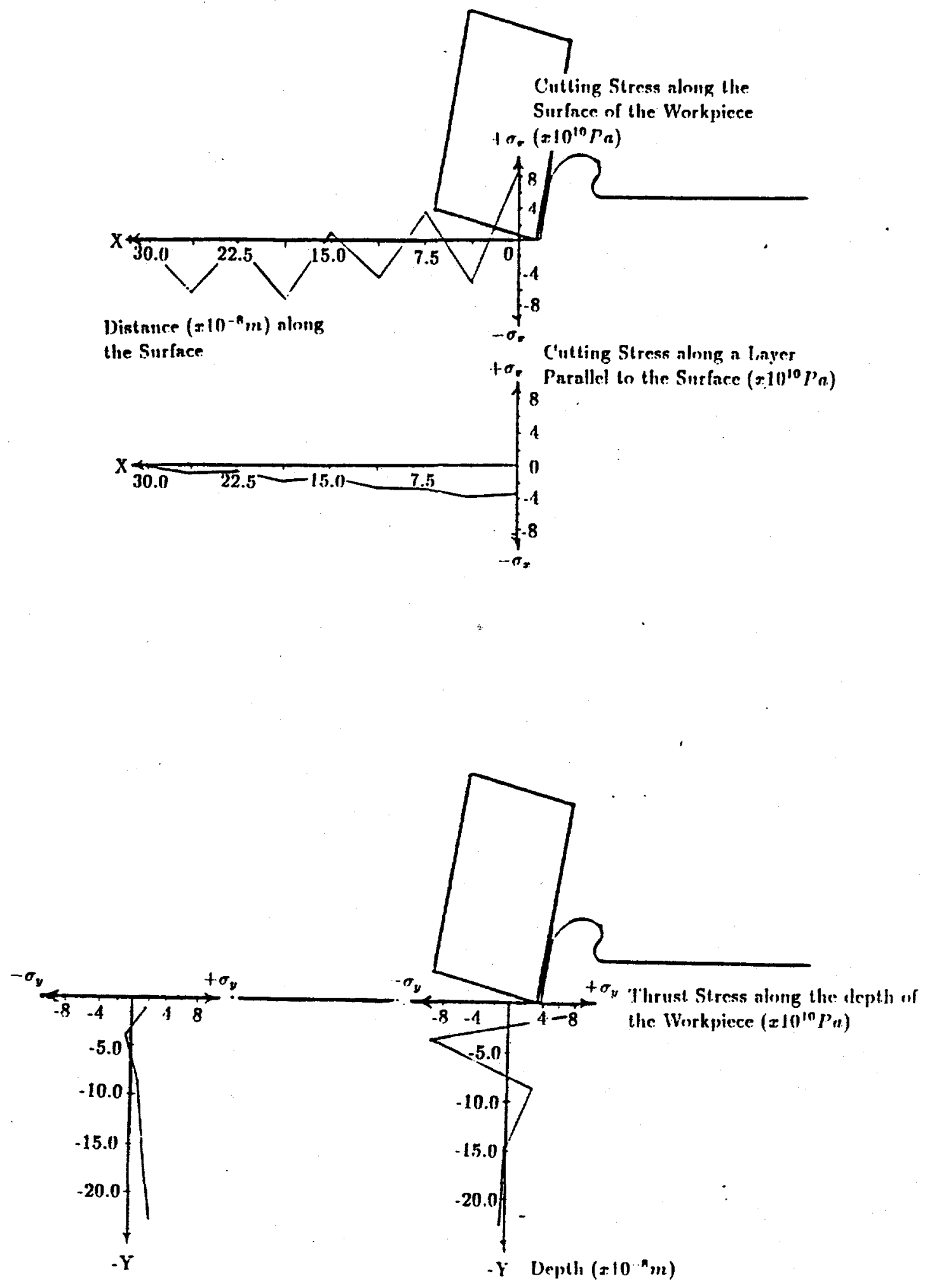

Figure 6: The variation of $\sigma_{x}$ along the machined surface and at a depth of $0.189 \times 10^{-6} \mathrm{~m}$ below the surface and variation of $\sigma_{y}$ below the edge of the tool and $0.915 \times 10^{-6} \mathrm{~m}$ behind the tool for a depth of cut of $0.075 \mu m$. 

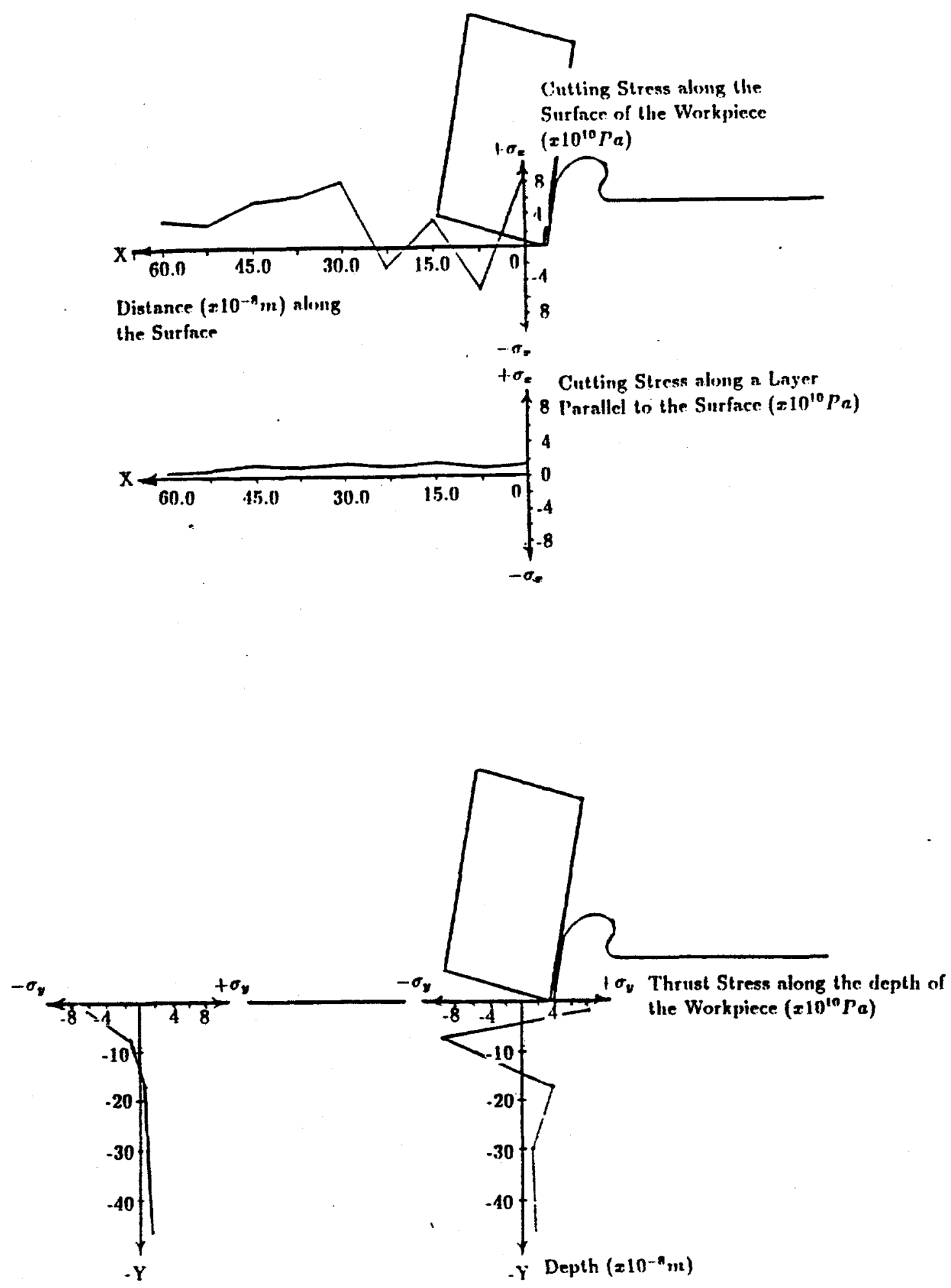

Figure 7: The variation of $\sigma_{z}$ along the machined surface and at a depth of $0.378 \times 10^{-6} \mathrm{~m}$ below the surface and variation of $\sigma_{y}$ below the edge of the tool and $0.366 \times 10^{-5} \mathrm{~m}$ behind the tool for a depth of cut of $0.15 \mu m$. 

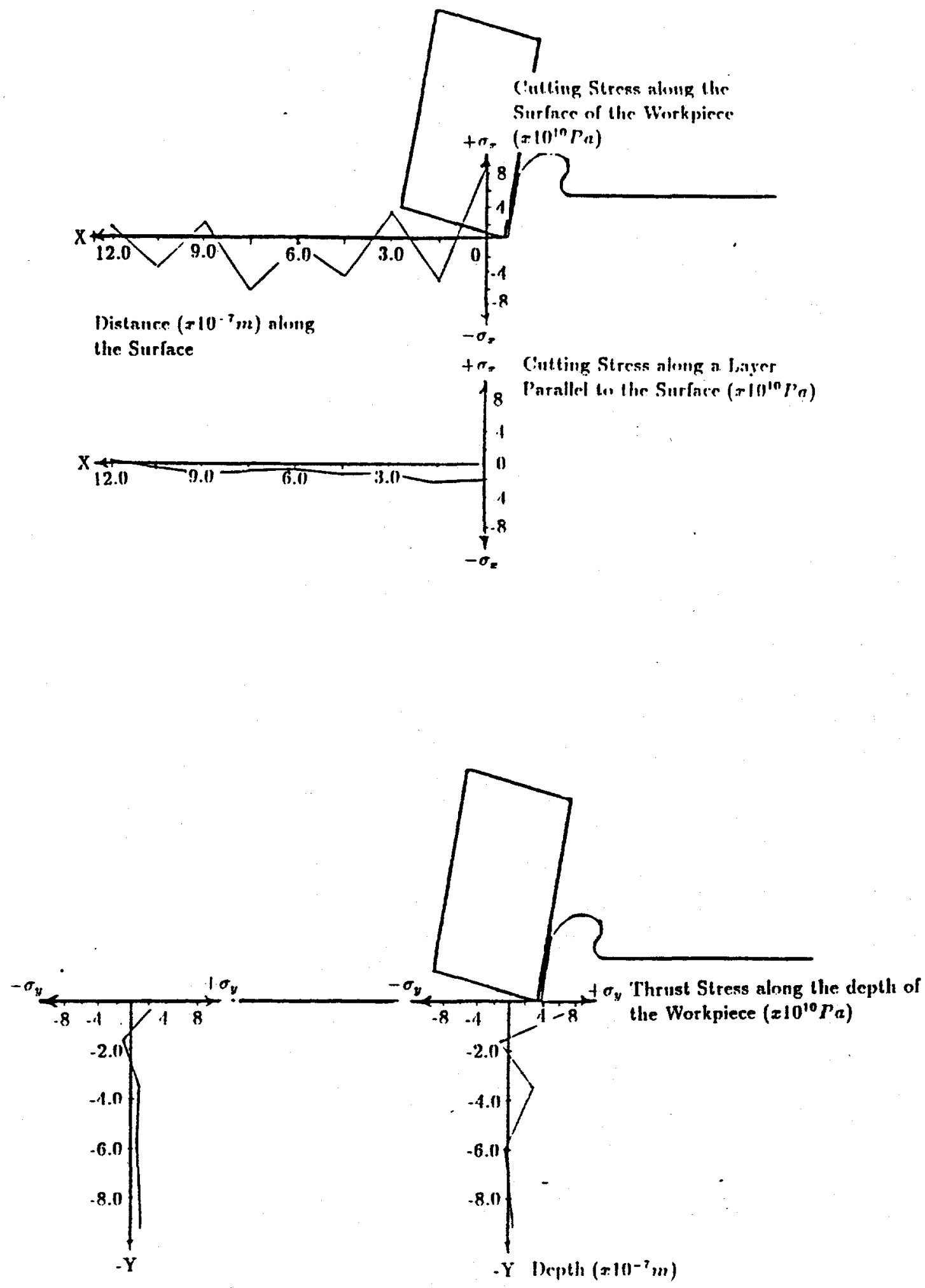

Figure 8: The variation of $\sigma_{x}$ along the machined surface and at a depth of $0.378 \times 10^{-6} \mathrm{~m}$ below the surface and variation of $\sigma_{y}$ below the edge of the tool and $0.915 \times 10^{-6} \mathrm{~m}$ behind the tool for a depth of cut of $0.30 \mu m$. 


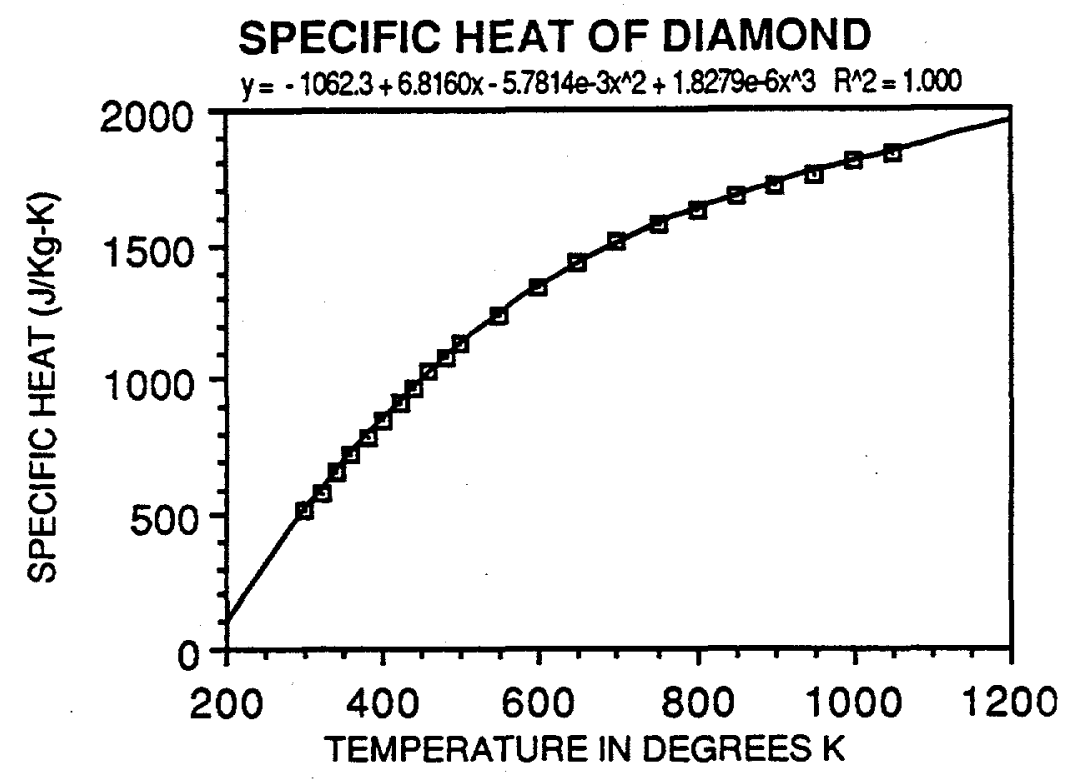

Figure 9: The Variation of Specific Heat of Diamond with Temperature [9]

FLOW STRESS OF SILICON IN Pa

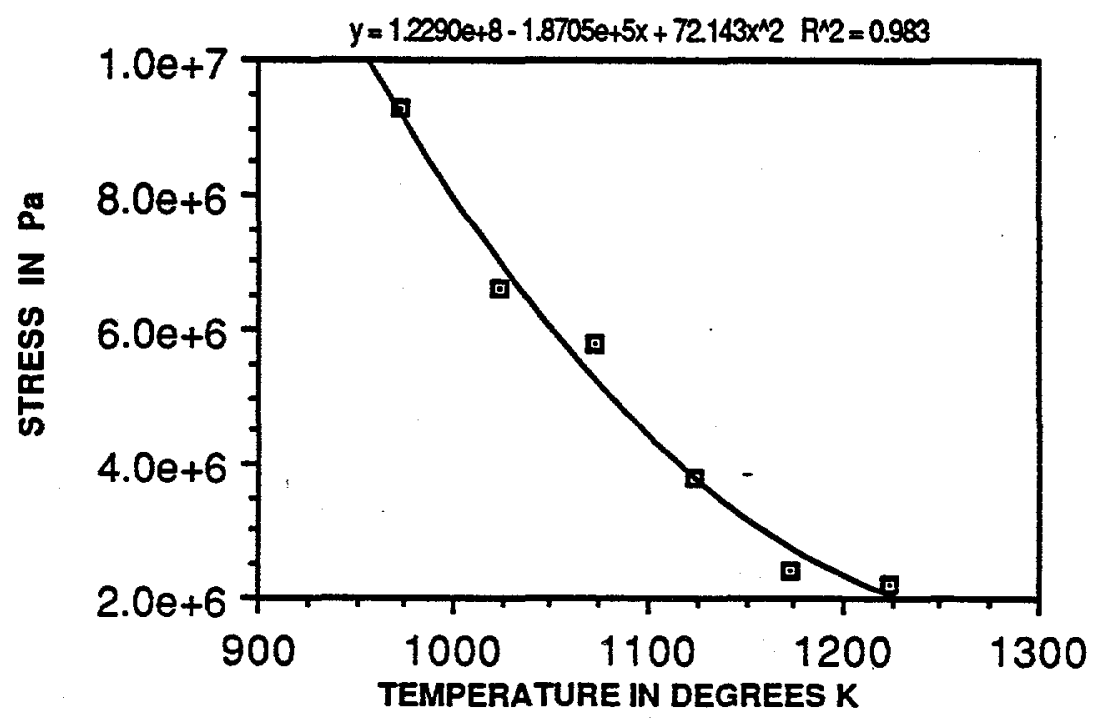

Figure 10: Lower Yield Stress of Silicon as a Function of Temperature [10? 


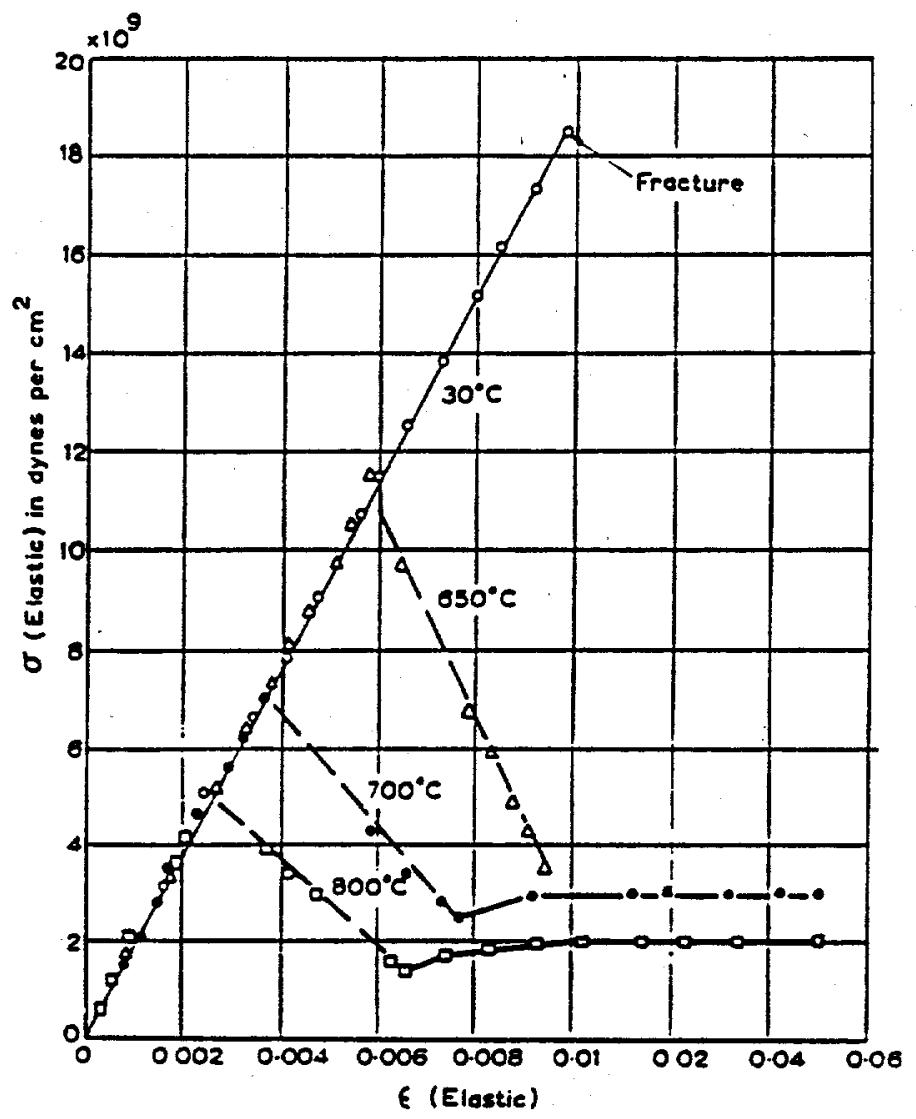

Figure 11: Stress strain curves for silicon at different temperatures [11]

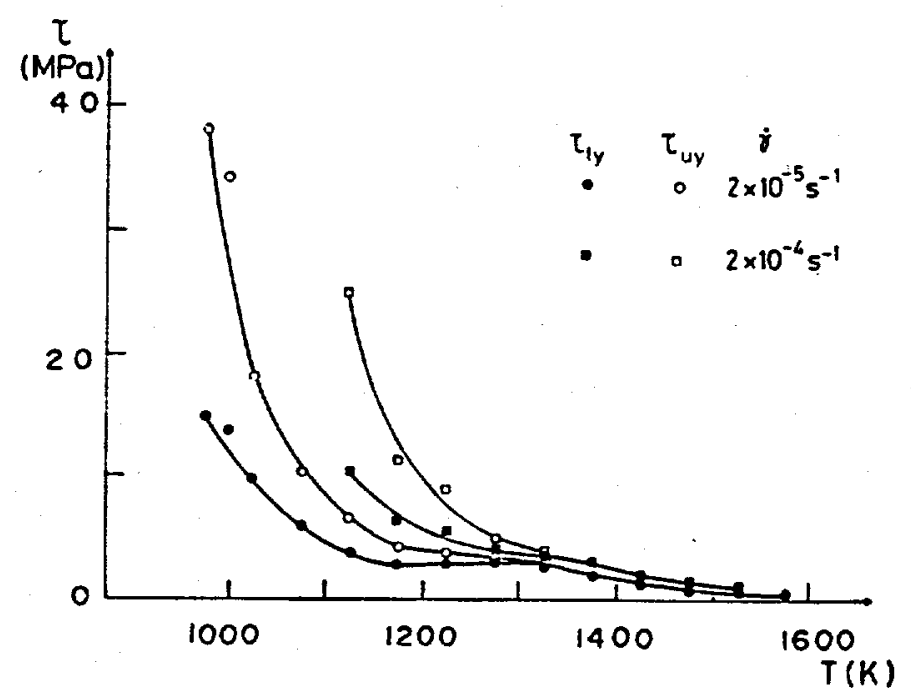

Figure 12: The temperature dependence of the upper and lower yield stresses at two strain rates [12] 


\section{References}

[1] Blake, Peter, "Ductile-Regime Diamond Turning of Germanium and Silicon," PhD. Dissertation, North Carolina State University, Raleigh, NC 1988

[2] Ueda, K., and Tsuwa, H., "Application of Fracture Mechanics in Micro-Cutting of Engineering Ceramics," Annals of the CIRP, Vol. 32, 1983, pp.83-86.

[3] Swedlow, J.L., "Criteria for Growth of the Angled Crack," Cracks and Fracture, ASTM STP 601, 1976, pp.506-521.

[4] Ramalingam,S., and Lehn, L.L., "A Photoelastic Study of Stress Distribution During Orthogonal Cutting, "Journal of Engineering for Industry, May 1971, pp.527-537.

[5] Kraczmarek,J., Principles of Machining by Cutting, Abrasion and Erosion, Peter Pergrinus Ltd., Stevenage, Poland, 1976, pp.152-154.

[6] Moon,K.J., "Transient Cutting Model Based on Eulerian Finite Element Approach", PhD.Dissertation, North Carolina State University, Raleigh, NC, 1988.

[7] Zienkiewicz,0.C., "Flow Formulation for Numerical Solution of Forming Processes," Numerical Analysis of Forming Processes, John Wiley \& Sons Ltd., 1984, pp.1-43.

[8] Hallquist,J.O., "NIKE2D-A Vectorized, Implicit Finite Deformation Finite Element Code for Analyzing the Static and Dynamic Response of 2-D Solids", Lawrence Livermore National Laboratory Report No UCID-19677.

[9] Touloukian,Y.S., Thermophysical Properties of Matter, Vol. 2, Plenum Data Corp., New York, 1970, p.12.

[10] Castaing,J., "The Plastic Deformation of Silicon between $300 \mathrm{C}$ and $600 \mathrm{C}$," Philosophical Magazine A, Vol.44, No.6, 1981, pp.1407-1413.

[11] Rhodes,R.G., Imperfections and Active Centres in Semiconductors, MacMillan, New York, 1964, p.99.

[12] Omri,M., Tete,C., Michel,J., and George,A., "On the Yield Point of Floating-Zone Silicon Single Crystals", Philosophical Magazine A, Vol. 55, No.5, p.606.

[13] Evans,A.G., and Marshall,D.B., "Wear Mechanisms in Ceramics," Fundamentals of Friction and Wear of Materials, American Society for Metals, 1980, p.441.

[14] Rooke,D.P., Baratta,F.I., and Cartwright,D.J, "Simple Methods of Determining Stress Intensity Factors, "Engineering Fracture Mechanics, Vol.14, 1981, pp.397-426.

[15] Rosenfield,A.R., "A Fracture Mechanics Approach to Wear", Wear, 61, 1980, pp.125132. 
[16] Keefe-Fraundorf,G., and Craven,R.A., "Microdefects Near the Surface of Oxidixed Silicon Wafers", Defects in Silicon, The Electrochemical Society, 1983, p.406.

[17] Puttick,K.E., and Hosseini,M.M., "Fracture by a Pointed Indenter on near (111) Silicon," J.Phys.D:Appl.Phsy. 13, 1980, p.877.

[18] Bhaduri,S.B., and Wang,F.F.Y., "Fracture Surface Energy Determination in 110 Planes in Silicon by the Double Torsion Method," Journal of Materials Science, Vol.21, 1986, pp.2489-2492. 



\title{
24 ELASTIC EMISSION POLISHING
}

\author{
Michael Loewenthal, Kyle Loseke \\ Graduate Students
}

Thomas A Dow

Professor, MAE

Ronald O. Scattergood

Professor, MTE

Elastic emission polishing, also called elastic emission machining (EEM), is a process where a stream of abrasive slurry is used to remove material from a substrate and produce damage free surfaces with controlled surface form. It is a noncontacting method utilizing a thick elasto-hydrodynamic film formed between a soft rotating ball and the workpiece to control the flow of the abrasive. An apparatus was built in the Center, which consists of a stationary spindle, a two-axis table for the workpiece, and a pump to circulate the working fluid. The process is controlled by a programmable computer numerical controller $(C N C)$, which presently can operate the spindle speed and movement of the workpiece in one axis only. This apparatus has been used to determine material removal rates on different material samples as a function of time, utilizing zirconium oxide $\left(\mathrm{ZrO}_{2}\right)$ particles suspended in distilled water as the working fluid. By continuing a study of removal rates, the process should become predictable, and thus create a new, effective, yet simple tool for ultra-precision mechanical machining of surfaces.

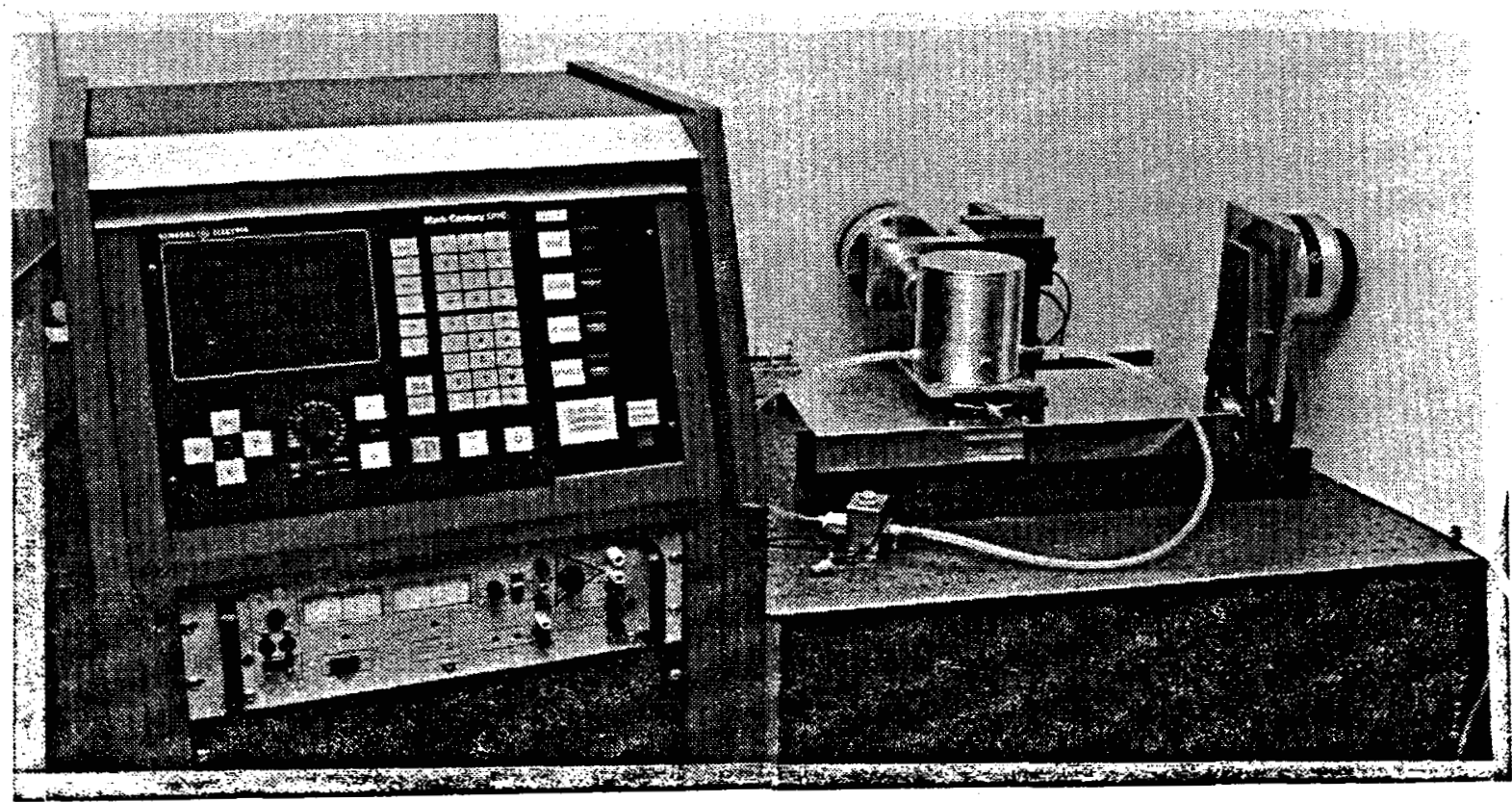




\subsection{INTRODUCTION AND BACKGROUND}

Ultraprecision manufacturing of components requires the use of creative nontraditional manufacturing techniques. Currently, ultraprecision machining means the achievement of dimensional tolerances in the order of $10 \mathrm{~nm}$ and surface roughness of $1 \mathrm{~nm}$ [1]. Traditional manufacturing has relied on grinding and lapping processes to produce products with superior surface finish and figure. As the world's competitive environment stimulates the creation of better products, the equipment to produce these products must also improve. Today, the tolerances on assemblies or individual components may be as small as $1 \mu \mathrm{m}$ and resolution and repeatability of the machines used must be on the order of $10 \mathrm{~nm}$.

Thus machining technology must progress from microtechnology $(1 \mu \mathrm{m}$ accuracy capability) to nanotechnology ( $1 \mathrm{~nm}$ accuracy capability). Nanotechnology, the most advanced manufacturing technology, is the theoretical limit of accuracy of machining of substances since the size of an atom or molecule which makes up each substance is on the order of 0.2 to $0.4 \mathrm{~nm}$. The state-of-the-art in grinding and lapping techniques is limited to $0.1 \mu \mathrm{m}$ figure accuracy. To produce higher levels of accuracies, nontraditional techniques such as mechanochemical lapping, noncontacting lapping, ion beam machining, elastic emission machining, and others must be used.

Noncontacting lapping is a polishing method where no contact is made between the lapping plate and the workpiece. The lapping plate contains specially designed grooves which develop a hydrodynamic fluid film between the plate and the workpiece much like a hydrodynamic thrust bearing. The fluid contains abrasive which interacts with the workpiece as the lap rotates to remove material. Polished silicon wafers with $10 \dot{A}$ surface roughness have been achieved with this process [2]. Elastic emission machining (EEM) is another example of this machining method but differs in that the contact patch of a soft ball is used to localize the polishing action. This technique holds the promise of a polishing method that can improve both the figure and the surface finish of optical surfaces.

The previous Annual Report (Volume V, December 1987, page 263) contains the details this polishing process and the apparatus design. This section will add to the information on: 1) apparatus development; 2) ball fabrication, and 3) experimental results.

\subsection{APPARATUS DEVELOPMENT}

The surface finish of the polyurethane sphere is a key factor in the ability to produce polished surfaces. To prevent any contact between the rotating sphere and the surface to be polished, the surface roughness peak must be much less than the elasto-hydrodynamic film thickness which was estimated to be $1 \mu \mathrm{m}$. 


\subsubsection{Ball Fabrication Technique}

The test sphere was fabricated from a bar of polyurethane manufactured by Mearthane Products which was mounted on a mandrel and machined in a lathe using a variety of methods. The problem is that the polyurethane tends to tear in the cutting process leaving a jagged surface. This tearing seemed to be independent of the cutting tool as both diamond and conventional tools were used. Both conventional and diamond turning have been used to produce an acceptable spherical surface.

Best results were obtained with soapy water as a lubricant, $1200 \mathrm{rpm}$ spindle speed, and a 1/50 rpm feed rate of the tool on the Shore 85 polyurethane ball. A high speed steel (HSS) tool with a $0.38 \mathrm{~mm}$ tool nose radius, $15^{\circ}$ positive rake angle, and $40^{\circ}$ clearance angle was used. The cutting edge was hand sharpened with a stone hone. Using this tool, a $0.8 \mu m$ Ra surface finish with $2.5 \mu m$ peak-to-valley was achieved.

Since this finish was on the order of the film thickness, additional finishing was required. Hand sanding using 1200, 2400, and 4000 grit silicon carbide sandpaper aided in lowering the roughness to $0.23 \mu \mathrm{m}$ Ra. 750 to $1200 \mathrm{rpm}$ spindle speeds seem to work the best. Higher spindle speeds were also tried (up to $2000 \mathrm{rpm}$ ) but the heat generated caused the abrasive to debond from the paper.

A method was then necessary to remove the abrasive particles trapped in the polyurethane ball from the sanding process. The method developed was to polish the surface with fine $\mathrm{Al}_{2} \mathrm{O}_{3}$ abrasives manufactured by Buehler. The freehand polishing procedure began with $3.0 \mu m \mathrm{Al}_{2} \mathrm{O}_{3}$ and finished with $0.05 \mu m \mathrm{Al}_{2} \mathrm{O}_{3}$ at a spindle speed between 750 to $1200 \mathrm{rpm}$. This polishing process, while slightly improving the surface finish, greatly reduced the amount of abrasive embedded in the ball .The final ball surface finish was $0.20 \mu \mathrm{m} \mathrm{Ra}$ with $0.3 \mu \mathrm{m}$ peak-to-valley. The final size was $47.2 \mathrm{~mm}$ with a $15.2 \mu \mathrm{m}$ TIR.

While the single point turning method has the advantage of controlling shape and producing a contaminate free surface, the low modulus of the polyurethane limited the minimum surface finish obtainable. Cutting the polyurethane at a lower temperature may be a way to improve the surface finish. The sanding method produced the best surface finish but contaminated the ball with abrasive. Sanding also appears to have a limit to the minimum surface finish that can be obtained.

\subsubsection{Influence of Ball Runout}

There was some concern that runout of the ball would adversely affect the EEM process. To determine the effect of this runout on the film thickness, a comparison of the ball stiffness and fluid film stiffness was made. From this information the magnitude of the ball runout can be used to determine the change in fluid thickness.

To calculate the ball stiffness, the change in displacement of the polyurethane for a change 
in load is needed. The following elasticity equation is used to determine $y$, the approach of the center of a sphere to a flat [3].

$$
y=1.040 \sqrt[3]{\frac{F^{2} C_{E}^{2}}{D}}
$$

where,

$a=$ sphere property

$b=$ work material property

$C_{E}=$ effective modulus, $\frac{1-\nu_{a}^{2}}{E_{a}}+\frac{1-\nu_{b}^{2}}{E_{b}}$

$D=$ diameter of sphere

$E=$ modulus of elasticity

$F=$ normal applied load

$\nu=$ Poisson's ratio

The change in $y$ is nonlinear as the load is increased. Therefore, a small increment in the working range was chosen. A change in load from 4.17 to $4.91 \mathrm{~N}$ (425 to $500 \mathrm{gm}$ ) results in a change of $y$ of $13.0 \mu \mathrm{m}$ for Shore 85 polyurethane and a $47.2 \mathrm{~mm}$ ball diameter. This yields a stiffness of $56.6 \mathrm{kN} / \mathrm{m}$.

The fluid film stiffness can be determined from Eq 1. A change in load from 4.17 to 4.91 $N$ results in a change in film thickness of $.028 \mu \mathrm{m}$. This shows that the fluid film is very stiff $(26285.7 \mathrm{kN} / \mathrm{m})$; in fact 464 times stiffer than the ball. Because the stiffness of the fluid and the ball act as springs in series, the following relationship can be developed for the change in fluid film thickness, $\Delta H$.

$$
\Delta H=\frac{K_{1}(T I R)}{K_{1}+K_{2}}
$$

where,

$K_{1}=$ stiffness of ball

$K_{2}=$ stiffness of fluid film

TIR = total indicator runout of the ball

Using the values obtained previously for the finished ball TIR (15.2 $\mu \mathrm{m})$, a $\Delta H$ of 0.032 $\mu m$. This is $1 / 25$ the fluid film thickness. Therefore, the ball runout for these conditions has no appreciable effect on the fluid film thickness and the material removal process. 


\subsubsection{Direction of Ball Rotation}

The first experiments were performed with the spindle axis perpendicular to the motion of the slideway. With this geometry, the ball rotation will be in the direction of feed. This turned out to be a major problem as the surface roughness of the ball was replicated in the substrate. Since the ball had a peak-to-valley surface roughness of $0.3 \mu \mathrm{m}$, the finish of the resulting surface had a similar roughness.

The solution was to turn the spindle 90 degrees so that the ball rotation was perpendicular to the feed direction. This had the effect of averaging the ball roughness and the resulting surfaces are reported in the experimental results section.

\subsubsection{Abrasive Circulation System}

In the initial experiments, material removal rates appeared to decrease with increased test time. One explanation was that the abrasive tended to settle out of the slurry changing the abrasive concentration and affecting the material removal rate. To keep the slurry mixed and circulated, an immersible centrifugal vane type pump with a flow rate of $19 \mathrm{l} / \mathrm{min}$ was used. A valve on the pump outlet controlled the flow rate. Various flow arrangements were tried with best results achieved with the nozzle in the bottom of the container creating a swirling motion. Another nozzle at the top of the container was used to direct a stream of slurry directly into the inlet side of the polishing ball. This stream also washed any settled abrasive off of the surface of the wafer.

However it was found that the centrifugal pump produced enough heat that the solution temperature rose 14 to $16^{\circ} \mathrm{C}$ in an hour. Mori's work showed that temperature increase would decrease material removal. For example, a $30 \%$ decrease in removal rate occurred for a fluid temperature change from 15 to $25^{\circ} \mathrm{C}$. This was based upon removal rates on glass with $0.6 \mu m$ zirconia at $4.0 \mathrm{wt}$. percent [4]. As a simple temperature control method the pump was immersed in an ice bath. This greatly reduced the heat transfer from the motor to the solution. The slurry temperature was maintained at $19^{\circ} \mathrm{C} \pm 1{ }^{\circ} \mathrm{C}$ with this method.

\subsection{EXPERIMENTAL RESULTS}

\subsubsection{Experimental Procedure}

Before conducting an experiment, the entire container/pump system was flushed at least three times with distilled water. The silicon used was polished wafers manufactured by Monsanto, and were $<100\rangle$ p-type $76.2 \mathrm{~mm}$ in diameter.The germanium was obtained from Eagle-Picher in the form of sawed $<111>$ wafers and was subsequently diamond 


\begin{tabular}{||l|l||}
\hline \multicolumn{2}{|c||}{ Test Parameters } \\
\hline Spindle speed & $1200 \mathrm{rpm}(178 \mathrm{~m} / \mathrm{min}$ surface speed $)$ \\
Load & $425 \mathrm{gm}(4.169 \mathrm{~N})$ \\
Z-Axis feedrate & $2 \mathrm{~mm} / \mathrm{min}$ \\
Ball diameter & $47.2 \mathrm{~mm}$ \\
Ball runout & $15.2 \mu \mathrm{m}$ TIR \\
Slurry & 8 wt. \% of $0.1-0.2 \mu \mathrm{m} \mathrm{ZrO}_{2}$ and $\mathrm{H}_{2} \mathrm{O}$ \\
Slurry temperature & constant \\
Pump system & centrifugal pump \\
\hline
\end{tabular}

Table 1: Test parameters

turned to a flat surface. The Best mounting results for silicon, germanium, and float glass were similarly obtained by bonding the specimen to an aluminum sample holder using low melting point $\left(65^{\circ} \mathrm{C}\right)$ glue. The abrasive supplied by Z-Tech Corporation was weighed and mixed with distilled water to give the proper weight percent. Spindle speeds, feedrates, and traverse direction were operated by a controller program. At the end of the experiment, the abrasive solution was collected for possible reuse in later experiments.

Data Collection Numerical values of material removal were determined by the use of Talysurf and Talystep profilometry instruments. The Talysurf has a $120 \mathrm{~mm}$ traverse range with a maximum magnification of $2 \times 10^{5}$. The Talystep has a $2 \mathrm{~mm}$ traverse range with a $2 \times 10^{6}$ magnification. While the Talystep provides higher magnification, it does not have the traverse length to measure the profile across an EEM contact.

In some tests the wafers were traced before and after the polishing process. A scribed line on the surface was used to reference the traces. This method would account for a crown or depression in the original surface. If this method was not used, it was assumed the surface was originally flat over the polished area.

\subsubsection{Silicon Material Removal}

To determine silicon material removal, a multipass test was conducted with the test parameters shown in Table 1 . Using a CNC controller, the following machining procedure was performed during the test to give 1, 2, and 4 pass material removal areas. Starting at the $30 \mathrm{~mm}$ position, the z-axis was traversed to the zero position. The direction reverses and the axis returns to the $20 \mathrm{~mm}$ position. Direction changes again and returns to the $10 \mathrm{~mm}$ position. Finally the axis travels to the $20 \mathrm{~mm}$ position and stops. This pattern of movement creates three areas $10 \mathrm{~mm}$ in length, each with a different amount of integrated polishing time.

Results of polishing silicon with these test parameters showed a smooth surface with very 


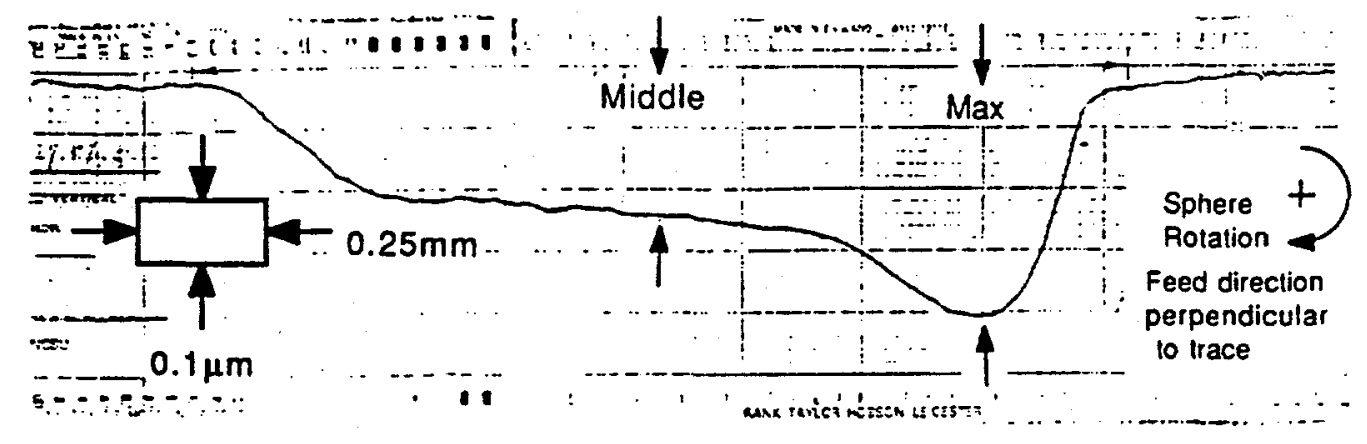

Figure 1: Typical profile of silicon material removal

few scratches. When the workpiece moves parallel to the spindle axis, there is a very significant averaging action of the polishing process. The pronounced grooving obtained in the tests with workpiece motion perpendicular to the spindle axis is absent, i.e., it has been averaged out. Higher magnification micrographs show very faint lines (grooves) on the surface perpendicular to the the direction of motion $4.0 \mu \mathrm{m}$ apart, almost like tool feedmarks. If one spot on the ball was making these marks, a single point cut would have feedmarks $1.7 \mu \mathrm{m}$ apart for these machining parameters.

The Talysurf trace, of Figure 1, shows the surface profile to have more material removal at the inlet side. This rapidly decreases and then levels off toward the outlet side. All three pass areas show material removal and in a similar fashion to the profile in Figure 1. The width of removal is approximately 1.78 to $2.03 \mathrm{~mm}$ and does not noticeably increase as the number of passes is increased. A Talystep of the polished area shows the roughness to be better than $25 \stackrel{A}{\text { peak }}$ to valley in the direction of travel. The roughness across the profile is also better than $25 \AA$.

Results from five tests of maximum material removal rate verses number of passes for silicon are show in Figure 2. The maximum material was measured from the Talysurf traces at the inlet side. Two traces were taken in each of 1,2 , and 4 pass areas for a total of six traces per test. The line is the best fit of the data, which correlates very well to a straight line. The slope of the line shows the material removal rate to be $0.122 \mu \mathrm{m}$ per pass. The shift of $0.031 \mu m$ from passing through the origin is small. The plot shows error bars for two standard deviations $( \pm \sigma)$. The maximum variability of $0.076 \mu \mathrm{m}$ approaches the order of the resolution of the Talysurf.

Silicon Treated with Sodium Pyrophosphate A correlation has been shown between the zeta potential and the measured hardness for silicon [5]. The zeta potential is a measure of the electrostatic potential between the Stern layer of the double layer at a liquid-solid interface and the bulk solution. With the addition of sodium pyrophosphate 


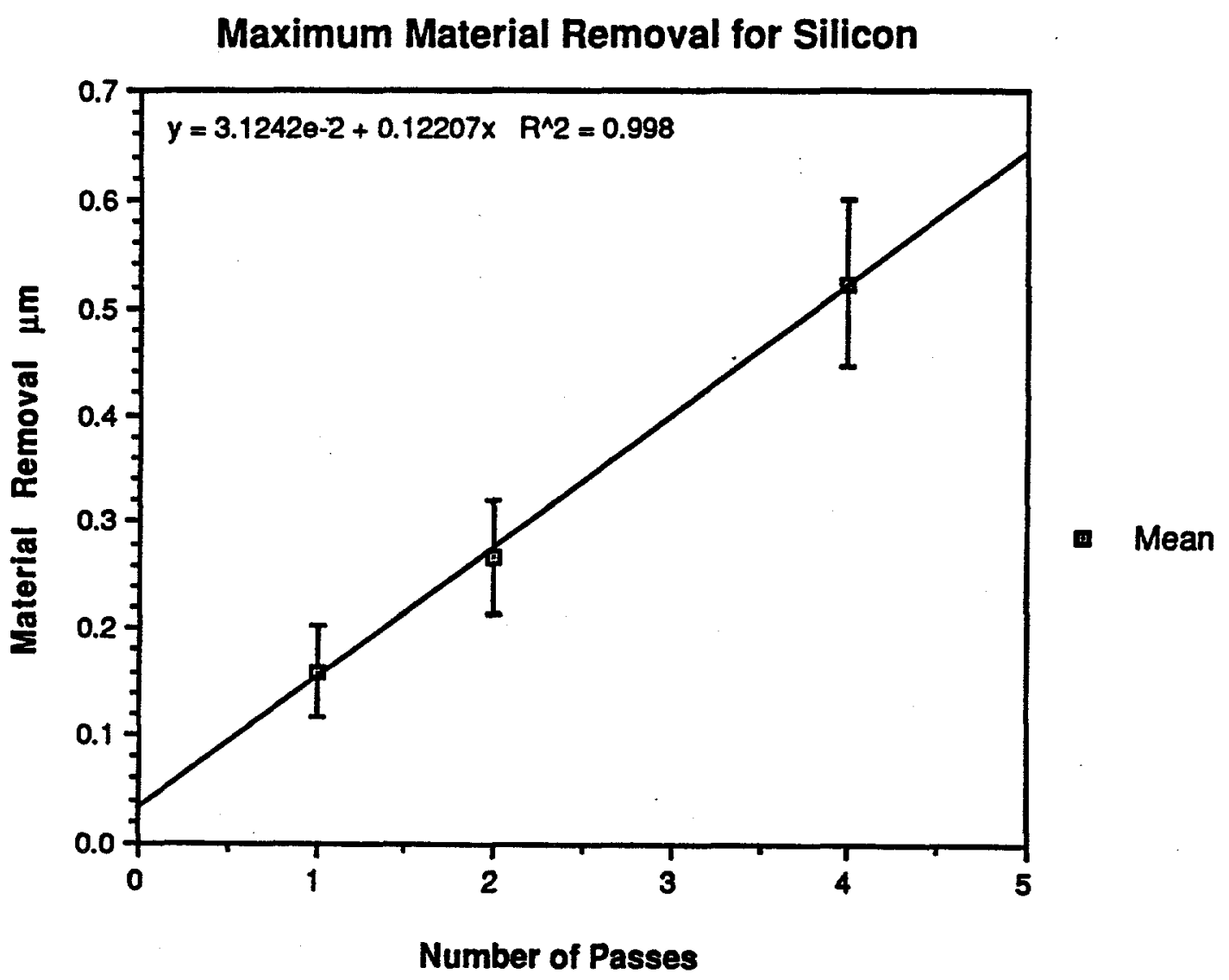

Figure 2: Maximum material removal for silicon 


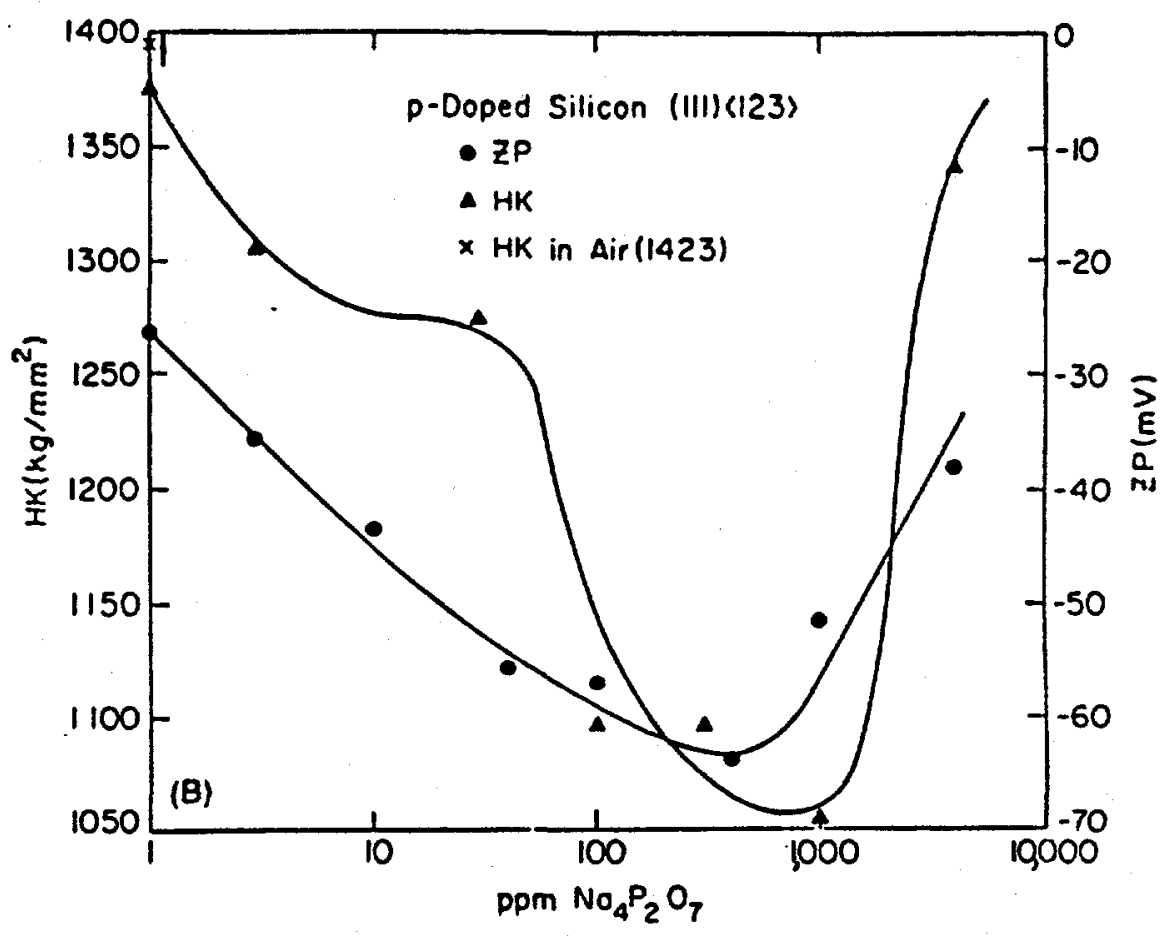

Figure 3: Relationship between zeta potential and Knoop hardness as a function of concentration of $\mathrm{Na}_{4} \mathrm{P}_{2} \mathrm{O}_{7}$ for p-type $<111>$ silicon wafer [5]

$\left(\mathrm{Na}_{4} \mathrm{P}_{2} \mathrm{O}_{7}\right)$ the zeta potential is lowered and hence the surface hardness. Figure 3 shows the relationship between zeta potential and Knoop hardness as a function of concentration of $\mathrm{Na}_{4} \mathrm{P}_{2} \mathrm{O}_{7}$ for p-type $<111>$ silicon wafer. It can be seen that at $700 \mathrm{ppm}$ (by weight) solution of $\mathrm{Na}_{4} \mathrm{P}_{2} \mathrm{O}_{7}$ and deionized water results in a $330 \mathrm{Kg} / \mathrm{mm}^{2}$ lowering of the surface hardness, or, a reduction of about $23 \%$.

Based upon this, an experiment was performed repeating the test parameters shown in Table 1 to see the effects of hardness (or strictly zeta potential) on material removal. Results showed the same visual material removal as for untreated silicon. Talysurf traces of the 1, 2, and 4 pass areas showed profiles typical of the previous silicon surfaces.

The combined results of the maximum material removed shown in Figure 4 plot as a linear removal rate. The slope, $28 \mathrm{~nm}$ per pass is $23 \%$ of the maximum removal rate for untreated silicon. Thus, the reduced surface hardness has also reduced the material removal rate by approximately the same percentage.

Material Removal for Silicon with Water Only To determine a base line for material removal on silicon, the test parameters were repeated, but without the abrasive present in the distilled water. The base line test showed significantly less material removal than with the $\mathrm{ZrO}_{2}$ present. However, some material was removed from the silicon surface. This 


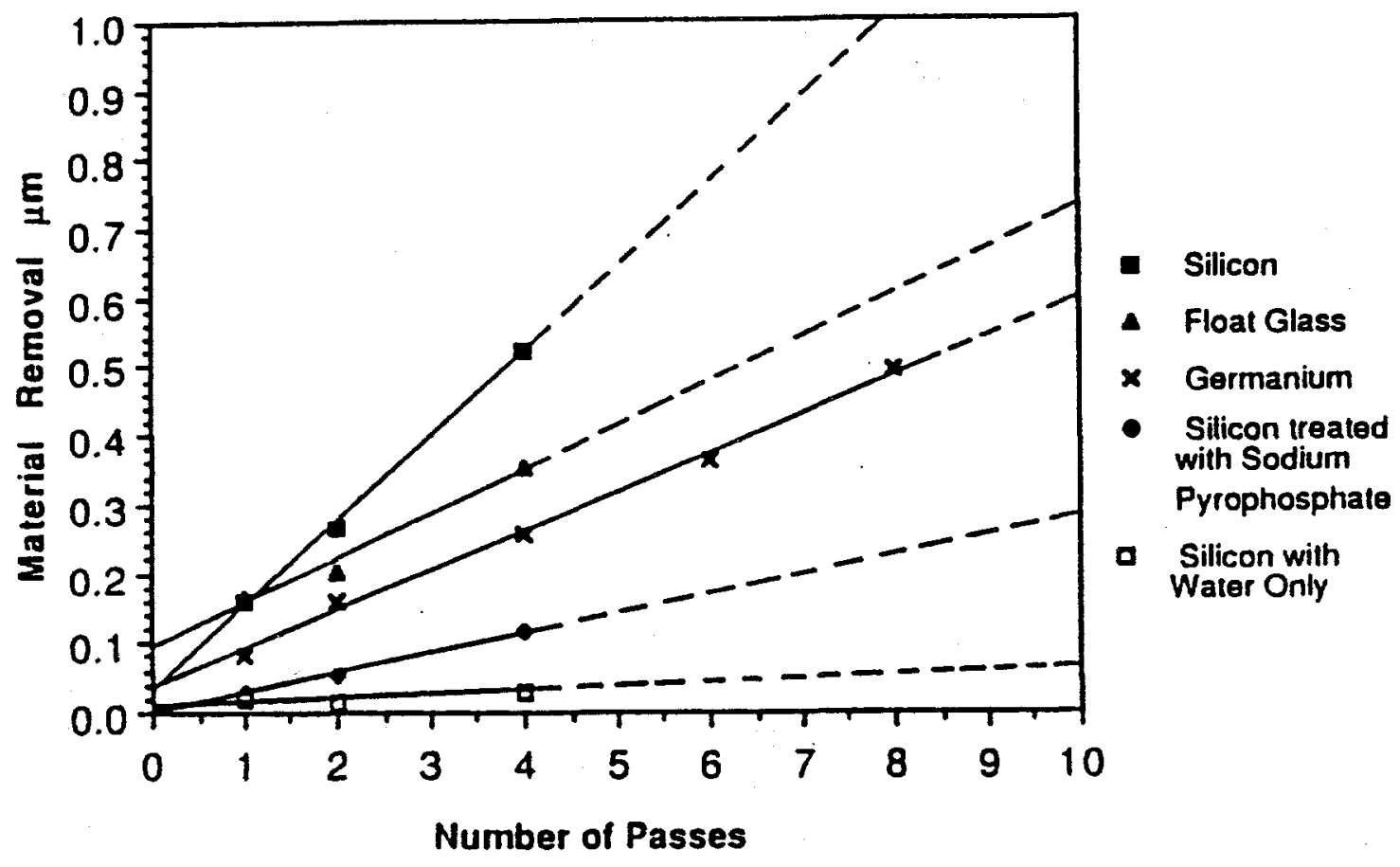

Figure 4: Maximum depth of the polished region for multiple passes 
could be due to residual $\mathrm{ZrO}_{2}$ on the ball or contaminants in the system that were not removed in the cleaning precess. The material removal was on the inlet side and was only in the first half of the conjunction zone.

Determining the exact material removal rate from the profile traces is difficult since the depth was extremely small and no traces were taken before the test to measure the wafer curvature. The plot of silicon with water alone is included in Figure 4. The removal rate without abrasive is only $5 \%$ of that with the abrasive and thus can be considered insignificant.

\subsubsection{Float Glass Material Removal}

The material removal rates on float glass were determined using the test parameters shown in Table 1. Due to the difficulty in focusing on the top surface of the glass in the microscope, a reference line was scribed on the surface. The ball was located adjacent to the scribed line at the start of the test.

A polishing test was performed using float glass and an abrasive solution that had been previously used for two hours. For this test, the center of the polished region had the maximum material removal and not the inlet side as seen in the silicon tests. The original surface was not flat but somewhat convex, which makes some error in measuring material depth. Repeating the test twice with a new solution of $8 \mathrm{wt \%} \mathrm{ZrO}_{2}$ resulted in three and a half times more material being removed. Using the data generated with the fresh abrasive, the removal rate was $64 \mathrm{~nm}$ per pass with a $38 \mathrm{~nm}$ maximum standard deviation. The removal rate is $53 \%$ of the maximum for silicon as shown in the plot of Figure 4, which contains the material removal rate for all of the materials studied.

The surface finish of float glass before the experiment is on the order of $25 \dot{A}$ peak-to-valley. A roughness trace in the direction of travel after EEM appears much smoother because the high frequency component over the length of trace has been removed. However, the peak-to-valley surface roughness was of the same order as the original surface.

\subsubsection{Germanium Material Removal}

Experiments were conducted to determine the material removal rates on a diamond turned germanium (111) surface. Using the test parameters shown in Table 1, one and two passes did not remove all of the diamond turned tool marks. The test was repeated, but increasing the number of passes to 4, 6, and 8. This completely removed the tool feed marks. The surface finish was not as smooth as the silicon or float glass surfaces due to the appearance of grooves about $3.5 \mu \mathrm{m}$ apart. It is interesting to note that what appears to be subsurface damage from the turning operation is visible after the EEM polishing.

Figure 4 includes the results of the maximum material removal for the germanium experi- 
ments. The removal rate is $56 \mathrm{~nm}$ per pass with a maximum standard deviation of $74 \mathrm{~nm}$. A Talystep trace in the direction of travel shows the peak-to-valley roughness to be on

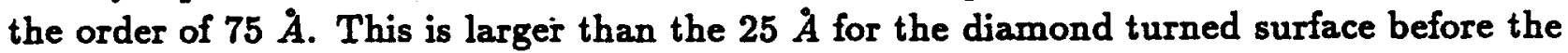
experiment.

\subsection{Residual Stress Measurement}

Raman spectroscopy is a method of studying vibrational and electronic properties of semiconductors and insulators which has been discussed in several sections of this report. In the Raman effect, a photon is scattered inelastically by the crystal, with the creation or annihilation of a phonon. It is possible to determine existing stresses in silicon and germanium by measuring the location of the Raman peak and comparing it to the value obtained for the unstressed material.

Raman spectroscopy using green light with a penetration depth of $1 \mu \mathrm{m}$ was used to study the stresses in EEM regions. In a region with four passes of the polishing ball, a shift of less than $5.0 \times 10^{7} \mathrm{~Pa}$ was measured compared to the as received wafer surface. This small shift indicated that the EEM polished surface had virtually no change in stress.

To see if this polishing process could actually remove stress, a piece of silicon wafer was sanded with 4000 grit silicon carbide sandpaper to create a $5 \mathrm{~mm}$ wide patch. Using EEM, a pass was polished through the sanded area using Table 1 test parameters without constant temperature. This was repeated 15 times to remove enough material to polish through the sanded surface.

Raman spectroscopy was measured in the as received wafer, the sanded area, the EEM polished sanded area, and the EEM polished as received wafer. Choosing the as received surface for the reference Raman peak, Figure 5 shows the normalized data. This normalization allows easier comparison of the shifts and broadening. One wave number is 2.5 $x 10^{8} \mathrm{~Pa}$ of stress. Higher wave numbers indicate compressive stress while lower wave numbers indicate tensile stress. For a material like silicon, a compressive stress indicates a ductile material removal while a tensile stress tends to indicate a brittle removal.

The Raman spectrum in Figure 5 shows broadening in the sanded zone as an indication of the large induced damage. The rougher surface gives a lower count value (less Raman signal) and a noisier plot as more light is scattered. The shift indicates the average stress to be $1.25 \times 10^{8} \mathrm{~Pa}$ compressive. The sanded area which was polished by EEM showed that most of the damage had been removed. The peak is approximately the same width and just slightly shifted, showing $3.0 \times 10^{7} \mathrm{~Pa}$ of compressive stress. This shows that the EEM process is capable of relieving stresses in the $1 \mu m$ surface depth region. 
Normalized Raman Spectroscopy of EEM Silicon

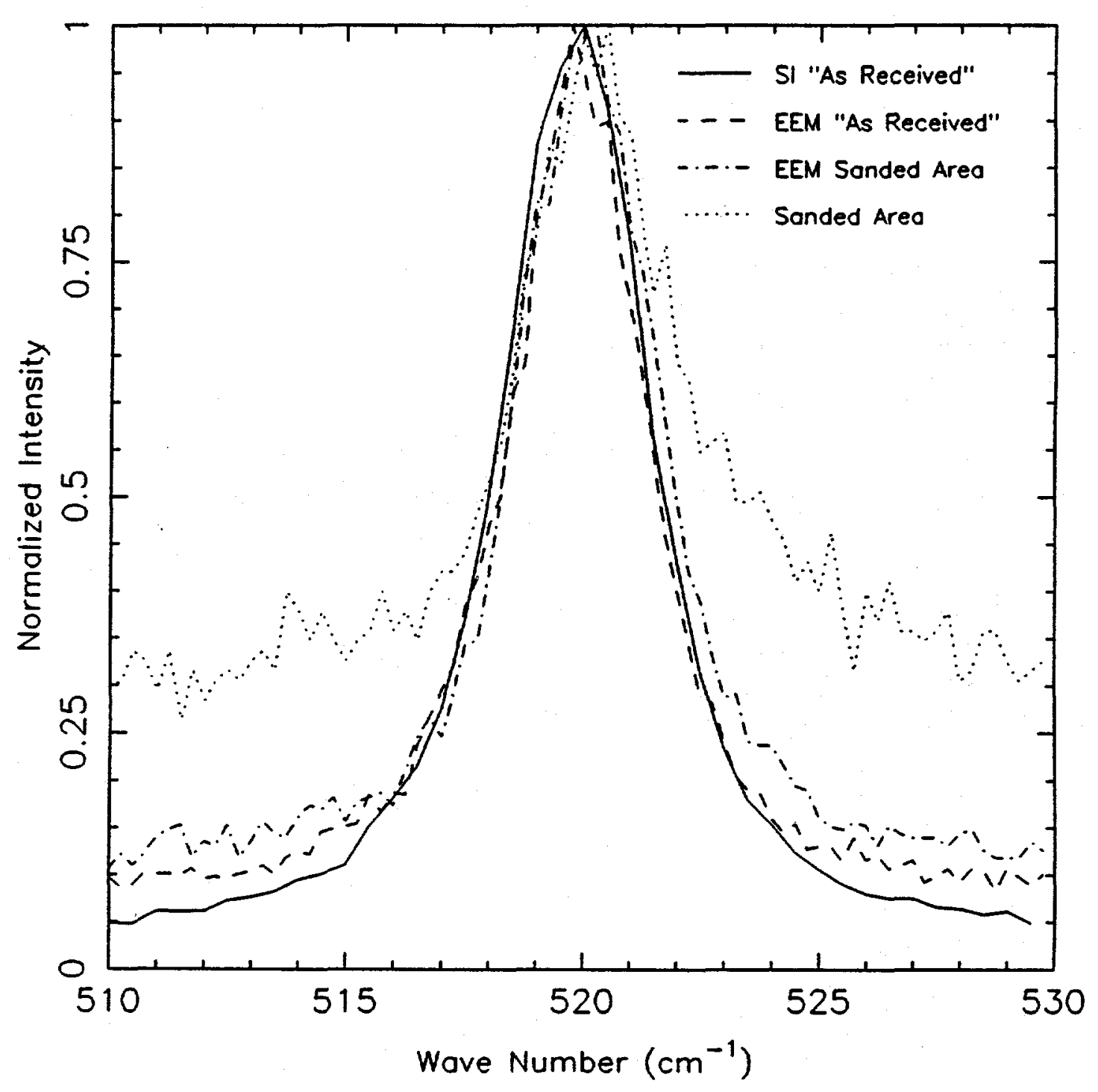

Figure 5: Normalized Raman spectroscopy of EEM silicon 


\begin{tabular}{|c|c|c|c|}
\hline \multicolumn{4}{|c|}{ Material Removal Determined from Profile Traces } \\
\hline Test Material & Area per Pass & Volume per Particle & Cubic Depth \\
\hline Silicon & $1.29 \times 10^{-4} \frac{\mathrm{mm}}{\mathrm{pad}}$ & $212 \frac{n m^{3}}{\text { particle }}$ & $5.9 \mathrm{~nm}$ \\
\hline Float Glass & $9.87 \times 10^{-5} \frac{\mathrm{mm}}{\mathrm{pae}}$ & $162 \frac{n m^{3}}{\text { particle }}$ & $5.5 \mathrm{~nm}$ \\
\hline Germanium & $4.27 \times 10^{-5} \frac{\mathrm{mm}}{\mathrm{pas}}$ & $70 \frac{\mathrm{nm}^{3}}{\text { particle }}$ & $4.1 \mathrm{~nm}$ \\
\hline $\begin{array}{l}\text { Silicon treated } \\
\text { with } \mathrm{Na}_{4} \mathrm{P}_{2} \mathrm{O}_{7}\end{array}$ & $3.01 \times 10^{-5} \frac{\mathrm{mm}}{\mathrm{pas}}$ & $50 \frac{\mathrm{nm}^{3}}{\text { particle }}$ & $3.7 \mathrm{~nm}$ \\
\hline
\end{tabular}

Table 2: Material removal determined from profile traces

\subsection{MATERIAL REMOVED PER ABRASIVE PARTICLE}

The amount of material removed per abrasive particle provides a measure of the severity of the polishing process. Knowing the amount of material removed and estimating the number of particles that pass thru the conjunction zone, the material removed per abrasive particle can be determined.

The number of abrasive particles per unit volume of fluid can be determined from the volume fraction of abrasive present and the volume of an abrasive particle. Assuming the abrasive is spherical in shape and the mean particle size, the concentration of particles can be determined. The contact area of the ball and the minimum hydrodynamic film thickness is assumed to define the volume of the conjunction zone. The number of abrasive particles in the conjunction zone can be determined from the product of the concentration of abrasive particles, and the volume of the conjunction zone. Thus, $3.3 \times 10^{7}$ abrasive particles exist in the conjunction zone for the $8 \mathrm{wt} . \%$ solution of $\mathrm{ZrO}_{2}$.

The amount of material removed can be determined from the experimental profile traces. Using the 4 pass traces obtained from polishing, the area of material removed was measured from each of the traces and divided by four. The mean value of the area of material removed per pass for silicon, float glass, germanium, and silicon treated with sodium pyrophosphate is shown in Table 2.

Since a linear velocity flow profile exists in the hydrodynamic film, then the average velocity of the fluid is one half the surface speed of the ball. Assuming the average velocity of the fluid is the average velocity of the abrasive particles and every particle removes material, then the volume of the material removed per particle can be calculated.

Table 2 shows the volume of material removal per particle for the parameters shown in Table 1. If each particle were to impact the surface and remove a cubic volume of material, then the cube root of the volume per particle is the depth of material removed per particle. Table 2 also shows the cubic removal depth for the materials tested. Of the materials tested, silicon has the maximum removal depth per particle of $5.9 \mathrm{~nm}$. Interestingly, these results are less than the $20 \mathrm{~nm}$ critical depth for fracture shown to exist in diamond turning and grinding of silicon and germanium [6]. Therefore, on the average, the material removal 


\begin{tabular}{|c|c|c|c|}
\hline \multicolumn{4}{|c|}{ Material Removal Determined from Slope of Removal Rates } \\
\hline Test Material & Slope & Volume per Particle & Cubic Depth \\
\hline Silicon & $1.22 \times 10^{-1} \frac{\mu m}{p a r d}$ & $393 \frac{\mathrm{nm}^{3}}{\text { particle }}$ & $7.3 \mathrm{~nm}$ \\
\hline Float Glass & $6.40 \times 10^{-2} \frac{\mu m}{p a s s}$ & $206 \frac{\mathrm{nm}^{3}}{\text { particle }}$ & $5.9 \mathrm{~nm}$ \\
\hline Germanium & $5.64 \times 10^{-2} \frac{\mu m}{p a s .}$ & $182 \frac{n m^{3}}{\text { particle }}$ & $5.7 \mathrm{~nm}$ \\
\hline $\begin{array}{l}\text { Silicon treated } \\
\text { with } \mathrm{Na}_{4} \mathrm{P}_{2} \mathrm{O}_{7}\end{array}$ & $2.84 \times 10^{-2} \frac{\mu m}{p a s e}$ & $92 \frac{n m^{3}}{\text { partide }}$ & $4.5 \mathrm{~nm}$ \\
\hline
\end{tabular}

Table 3: Material removal determined from slope of removal rates

process is in the ductile material removal regime.

A different approach to estimating the volume of material removed per particle is from the removal rates plotted in Figure 4. The slope of the plot is the material removal per pass. It is assumed that the material removed is uniform across the conjunction zone, that is, it is the maximum removal rate times the contact diameter. The slope needs to be converted to a material removal per unit time. The time for the ball to totally pass over a given point on the surface being polished is determined by the conjunction diameter divided by the $\mathrm{z}$-axis feedrate. Therefore, the material removal rate, $d h / d t$, can be determined. The number of abrasive particles passing thru the conjunction zone per unit time, $d n / d t$, is determined by assuming the average velocity of the abrasive particles is the average velocity of the fluid and using the concentration and volume of the conjunction zone. The volume of material removal per abrasive particle can be determined by dividing the material removal rate by the number of abrasive particles passing thru the conjunction zone per unit time.

Table 3 shows for each of the materials tested the volume of material removed per particle for the maximum material removed slopes. If each abrasive particle removes a cubic volume of material, then the depth of material removed per particle, cubic depth, is shown in Table 3. These results are comparable to those obtained from the profile traces shown in Table 2. Silicon has the maximum removal depth per abrasive particle of $7.3 \mathrm{~nm}$. This is still less than the $20 \mathrm{~nm}$ critical depth for fracture. This method again shows that on the average the material removal process is in the ductile material removal regime.

\subsection{MATERIAL REMOVAL PROCESS}

The material removal mechanism in EEM results from a complicated interaction among the ball, workpiece, fluid flow, particle trajectory, and possibly chemistry. The work reported here has begun to define these relationships but much work remains to be done.

Comparing the stock removal rate from Mori's work with that in Figure 4 shows a significant difference. To compare the graphs, the data in Figure 4 must be put in terms of machining time. The feedrate is $2 \mathrm{~mm} / \mathrm{min}$ and the width of the contact patch is approx- 
imately $2 \mathrm{~mm}$, so the machining time per pass is $1 \mathrm{~min}$. Thus, the five minute machining time of Mori's experiments would correspond with 5 passes. Comparing these results:

\begin{tabular}{rcc} 
& Loewenthal & Mori \\
\cline { 2 - 3 } silicon & .65 & 6.25 \\
float glass & .4 & 4 \\
germanium & .3 & 2
\end{tabular}

A specific comparison is difficult to perform because the details of Mori's experiments are not known. However, the major components of the two systems are similar so, while the trends are the same, the large difference between the material removal rates in the two experiments is puzzling. One possibility is that the material removal regimes of the two are different. Could chemical effects have been enhanced in Mori's experiments to produce the higher wear rate?

The material removal shown in Figure 4 has been assumed to be a mechanical erosion process. This conclusion is based on the results of the material removal experiments where the surface hardness was reduced. Based on an erosion hypothesis, the particles in the slurry would be accelerated toward the workpiece surface and impact at a small incident angle. Reducing the hardness should reduce the erosion rate for brittle materials as was measured in the experiments and this has lead to the erosion hypothesis. An erosion model would also account for the higher removal rate in the inlet side of the contact.

\subsection{FUTURE WORK}

In view of the complexity of the EEM process, further research is necessary before a viable fabrication method can be implemented. The effects of the many process parameters are as yet unknown. Therefore, further experimentation must be performed to determine the results on the process by varying:

- Rotating Ball

1. Surface Finish

2. Geometry - Alternatives to Spherical

3. Material

- Workpiece Materials

- Abrasive Slurry

1. Abrasive Material

2. Particle Size and Concentration 


\section{Working Fluid}

Future work requires some prioritization of the experiments. Si will be used as a standard material. The influence of the ball geometry and especially ball finish will be investigated further. Direct molding of a polyurethane ball in a diamond-turned mold will be attempted. Machining of a polyurethane ball at lower temperature is also a possibility. Other materials and geometries will be considered. For example, elastomer O-rings on a spindle could also be used for EEM machining. The primary intent of this work will be to produce a smooth, well-defined polishing spot geometry that is amenable to $x-y$ motion and eventual implementation for control of the form of a polished part.

A second priority item is the polishing slurry. Particle size distribution will be measured and experiments will be conducted to more systematically study the effect and reproducibility of different slurry mixtures. It is essential to understand these properties to control the process. Finally, further evaluations will be made on material property effects and material removal mechanisms.

\section{References}

[1] Taniguchi, Norio, "Current Status in, and Future Trends of, Ultraprecision Machining and Ultrafine Materials Processing", Annals of the CIRP, Vol 32,No 2, 1983, pp 573582.

[2] Watanabe, J. and J. Suzuki, "High Precision Polishing of Semiconductor Materials Using Hydrodynamic Principle", Annals of the CIRP, Vol. 30, No. 1, 1981, pp. 91-95.

[3] Roark, Raymond J. and Warren C. Young, Formulas for Stress and Strain, McGrawHill Book Company, 1982.

[4] Mori, Yuzo, Toru Okuda, Kazuhisa Sugiyama, and Kozuto Yamauchi, ${ }^{-}$Numerically Controlled Elastic Emission Machining- Consideration of Machining Process by Motion Analysis of Powder Particles in Fluid", Journal of Japan Society of Precision Engineering, Vol.51, No. 5, 1985, pp. 1033-1039.

[5] Yost, Gerald H., Wendell S. Williams, "Chemomechanical Effect in Doped and Intrinsic Silicon",Journal of The American Ceramic Society, Vol.61, No.3-4, Mar.-Apr. 1978, pp. 139-142.

[6] Blake, Peter, N. and Ronald O. Scattergood, "Ductile-Regime Turning of Germanium and Silicon", Second Intersociety Symposium of Ceramic Materials and Components, ASME Winter Annual Meeting, Chicago, Illinois, Nov. 30 to Dec. 2, 1988. 



\title{
25 DEVELOPMENT OF A FOCUSED ION BEAM MICROMACHINING SYSTEM
}

\author{
John G. Pellerin \\ Graduate Student \\ Dieter Griffis \\ Research Associate \\ Analytical Instrumentation Facility \\ Phillip E. Russell \\ Associate Professor, MTE
}

Focused ion beams are currently being investigated for many submicron fabrication and analytical purposes. An FIB micromachining system consisting of a UHV vacuum system, a liquid metal ion gun, and a control and data acquisition computer has been constructed. This system is being used to develop nanofabrication and nanomachining techniques involving focused ion beams and scanning tunneling microscopes.

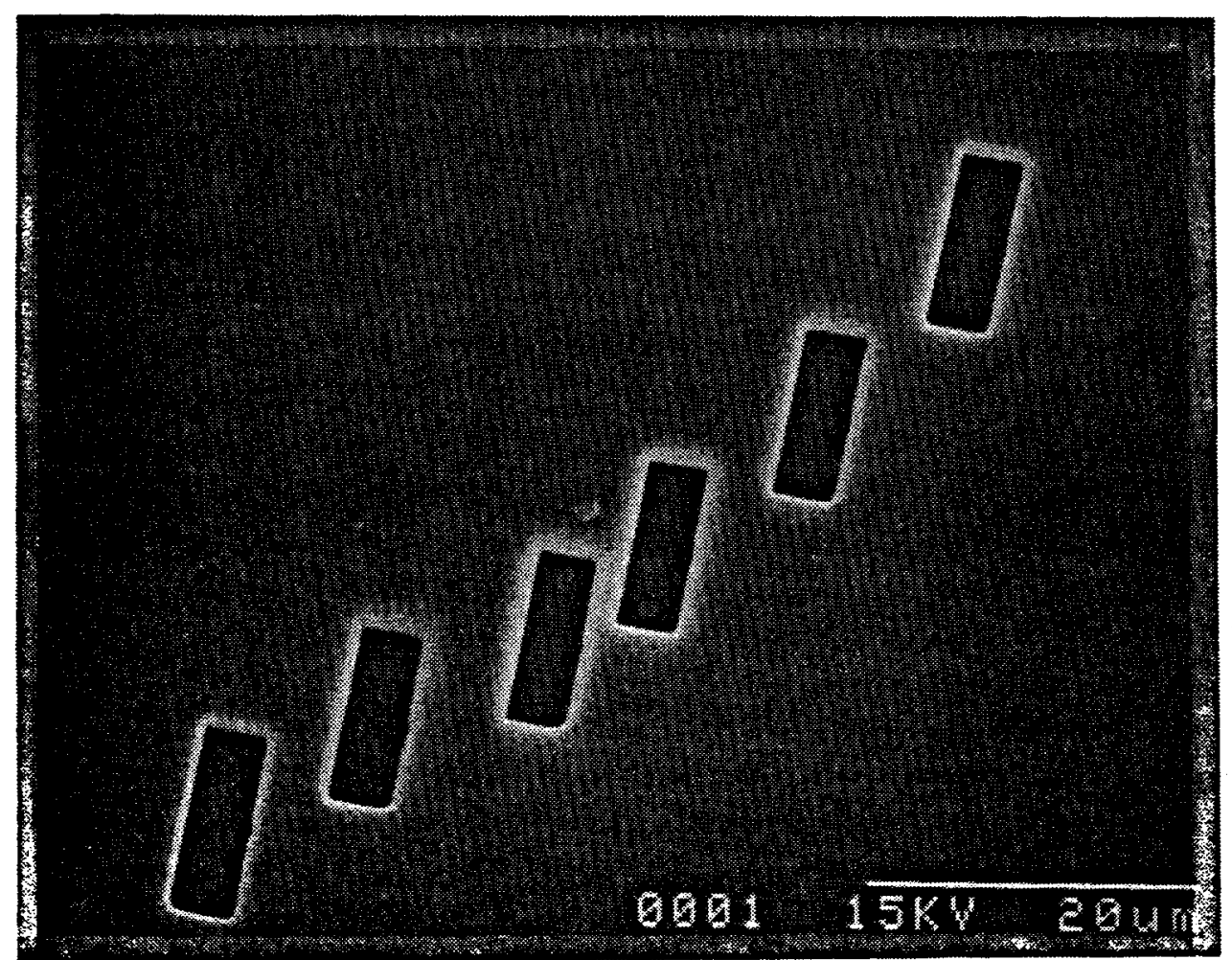




\subsection{INTRODUCTION}

Many of the precision fabrication processes used today are capable of producing high quality surfaces over relatively large areas. However, the need to produce similar surfaces over localized areas of tens of square microns or less excludes most machining techniques. Focused ion beam (FIB) micromachining is a technique under investigation at the Precision Engineering Center that can machine features of micron lateral dimensions. In addition, the ion beam can be scanned across the sample to generate a microscopic image for selecting a specific area to be machined as well as providing in situ feedback after a feature has been made.

Machining is accomplished by generating a narrow beam of high energy ions which can be moved in a desired pattern. When the ions impact the surface of the sample,' material is sputtered away. Sputtering is a purely physical process where momentum is transferred from the impinging ion to atoms in the proximity of the impact resulting in removal rates of 1 to 10 sputtered atoms per incident ion. To be considered a practical precision machining tool, the ion beam current density should be high to maximize the material removal rate. Also, the beam diameter must be significantly less than the dimensions of the feature to be machined to maintain feature definition. These requirements have been met with the development of the liquid metal ion source (LMIS).

Many industries using micro- and nano-fabrication techniques have found potential applications for FIB micromachining. Semiconductor micro- and opto-electronic device developers have made features such as 3-d interconnections, waveguides, laser facets, and mirrors [1]. Post-processing applications include integrated circuit testing and modification, such as cutting via to underlying layers for test probing [2] or microsectioning devices for voltage contrast measurements [3]. Focused ion beam techniques are currently applied to the repair of lithography masks [4]. Opaque defects are removed by sputtering away the excess material while clear defects can be repaired by depositing material through the exposure of the ion beam with an organometallic gas on the mask surface. Other applications of focused ion beams include direct-write lithography and implantation, surface chemical analysis techniques, and material deposition. All of these uses take advantage of the high spatial resolution and current density of the focused ion beam.

To conduct FIB machining experiments, a laboratory scale micromachining system has been constructed and tested over the past year. The tasks involved in developing this system were the design and construction of an ultrahigh vacuum system and the implementation of computer control to the machining process. 


\subsection{MACHINING SYSTEM HARDWARE}

\subsubsection{Focused Ion Beam Column}

The focused ion beam column incorporates a liquid metal ion source and an electrostatic optical column as shown in Figure 1. The source consists of a small reservoir of gallium metal suspended on a tungsten wire hairpin. The metal is melted by passing current through the wire fixture allowing the metal to flow and wet the emitter needle. By applying a high negative potential between the source and the extractor, a Taylor cone is formed at the tip of the needle from which ions are emitted [5]. The emitted ions are accelerated down the column by a 0 to $25 \mathrm{keV}$ potential between the source and the third electrode.

After the ion beam has been generated, it is initially collimated by a beam defining aperture. The ion beam then passes through an electrostatic lens that focuses the ion beam at the sample surface. The remainder of the column allows the beam to be moved and corrects the beam for astigmatism introduced by the lens elements. Since exposure to the ion beam results in sputtering of the sample, beam blanking is needed to sweep the ion beam off the sample. Voltages applied to parallel blanking plates deflect the beam onto a blanking aperture located below the plates. Movement of the beam in the scan field is done by applying voltages to an eight-plate assembly known as an octupole. Stigmation plates are included to make the beam cross-section circular by applying a correcting field to the beam. A noncircular beam is a result of geometric or electrical asymmetries in the electro-optic column.

With this single lens arrangement, a minimum spot size of $0.2 \mu \mathrm{m}$ is attainable at $25 \mathrm{keV}$ having a current density of $0.7 \mathrm{~A} / \mathrm{cm}^{2}$.

\subsubsection{Ultrahigh Vacuum System}

The intent of designing and building the UHV system at the Precision Engineering Center was to have a dedicated FIB micromachining station that would also have the versatility for future experiments involving the scanning tunneling microscope discussed in Section 25.

The vacuum system, shown in Figure 2 is made up of three main chambers; the sample introduction chamber, the FIB chamber, and the STM chamber. For high resolution FIB machining and microscopy the entire vacuum system is rigidly mounted to a vibration isolation table. The table, manufactured by Kinetic Systems Inc., has a $2800 \mathrm{lb}$ load capacity, $1.3 \mathrm{~Hz}$ natural frequency, and a $25 \%$ critical damping rate [6]. The sample introduction chamber allows quick and efficient changing of samples or apparatus without having to vent 


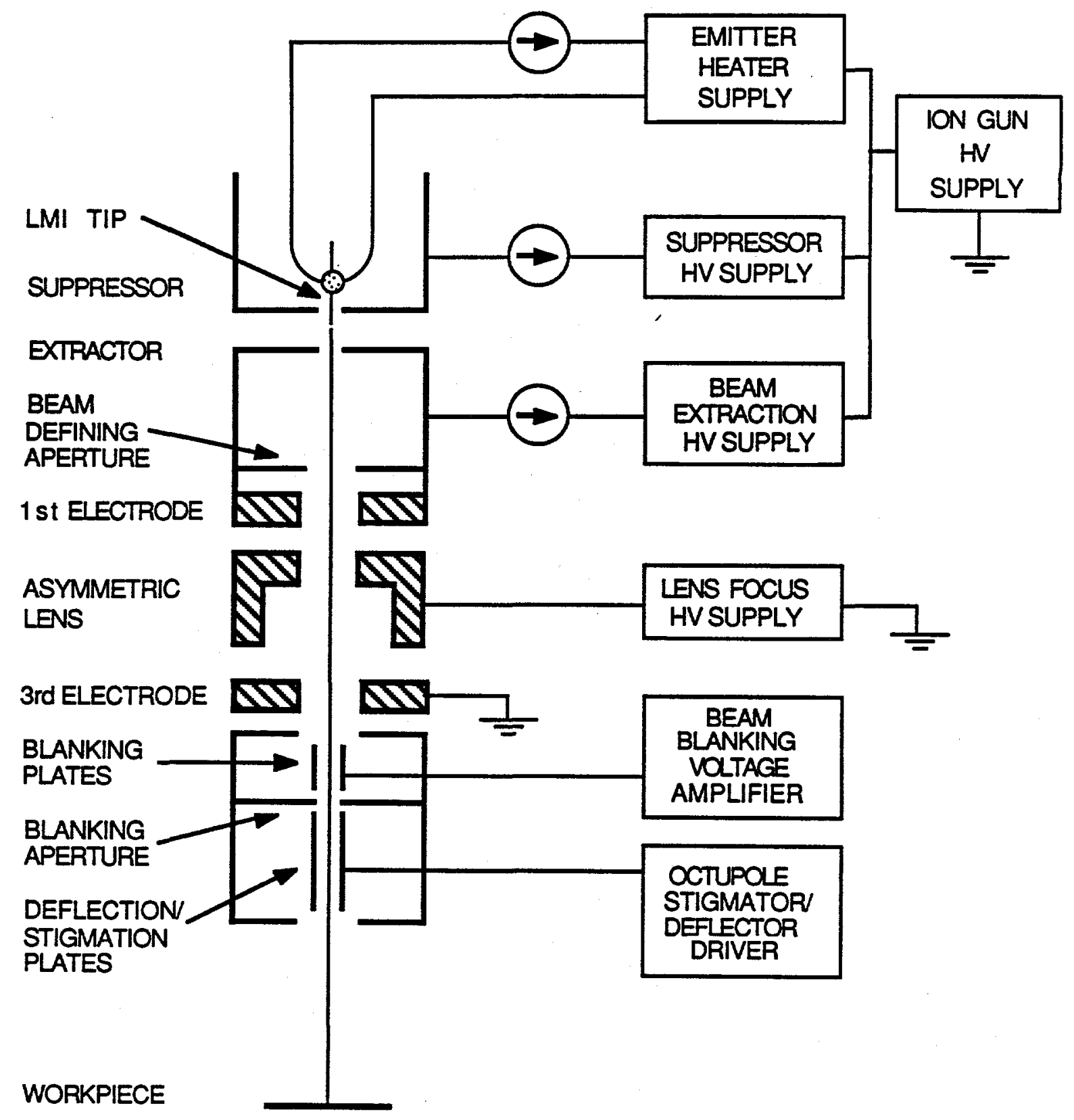

Figure 1: Focused ion beam column 
щว7s

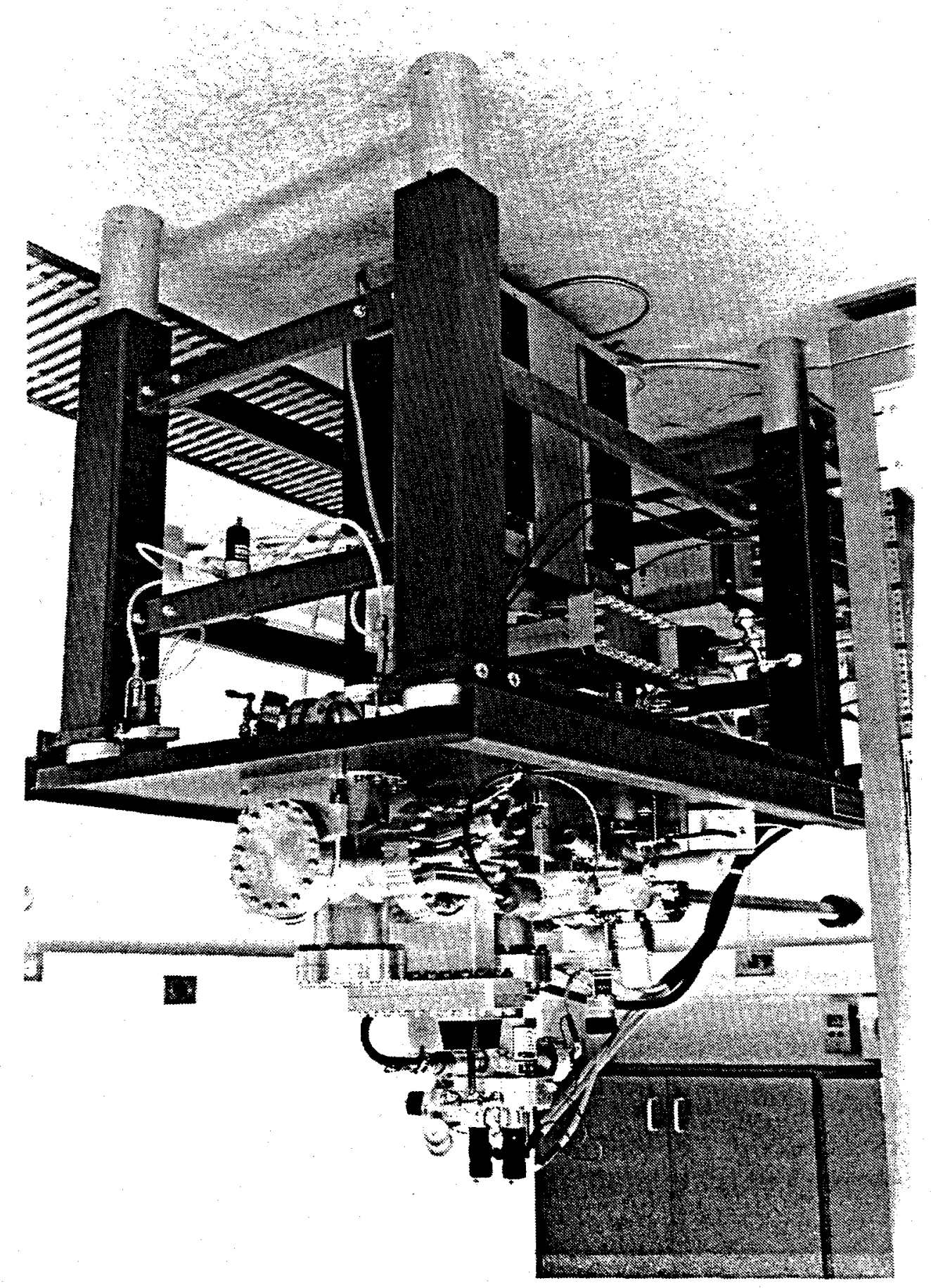


the main experiment chambers. This feature greatly reduces the time between performing experiments and prevents rapid contamination of the other chambers by reducing their exposure to atmosphere. Specimens are put into the chamber which is then evacuated by a turbomolecular pump to a pressure in the range of $10^{-6}$ torr. The turbomolecular pump also serves as the roughing pump to the entire vacuum system. A gate valve is then opened and the sample is moved into the high vacuum environment with a magnetically-coupled linear translator.

The FIB chamber houses the ion gun, an X-Y-Z specimen stage, an ionization pressure gauge, and a residual gas analyzer, shown in Figure 3. At present, a microchannel plate secondary electron detector is being built to be incorporated into this chamber. The chamber is based on a simple six way cross which was chosen to keep the distance from the ion gun to the sample stage as short as possible. This is necessary to maintain the submicron ion beam spot size at the sample. To increase the versatility of the six way cross chamber, a multi-port fitting was designed and built to be attached to one of the main flanges. This allows the addition of viewports and feedthroughs without adding complexity to the overall chamber design. The manipulation stage allows sample movement of $1 \mathrm{~cm}$ with $20 \mu m$ resolution in the $x, y$, and $z$ directions. Electrical feedthroughs are included in the stage for beam current and diameter measurements, and for providing electrical connections for future STM experiments. Contacts between the stage and sample holder allow absorbed current from the specimen to be amplified outside the vacuum and used as an imaging signal. To measure UHV pressures, a Bayard-Alpert type ionization gauge is used. A residual gas analyzer (RGA) has been included to detect leaks and monitor the partial pressures of various gaseous species in the system. The RGA is necessary to detect contamination that may affect the performance of the vacuum system or be harmful to sensitive experiments. The pressure gauge, RGA, and turbomolecular pump connections are mounted to a feedthrough collar attached to the bottom of the FIB chamber. The FIB chamber is connected to a high volume ion pump through an isolation gate valve. The ion pump was chosen for long service life, mechanical simplicity, and vibration-free UHV pumping.

The STM chamber is also based on a six way cross and is equipped with an ionization gauge and various electrical feedthroughs for the development and implementation of a UHV STM. Additions to this chamber will include sample introduction and manipulators for STM placement in the system, scanning tip replacement, etc.

\subsubsection{Computer Scanning System}

The Macintosh II is being used to control scanning and blanking of the ion beam and to acquire beam current and secondary electron signals as illustrated in Figure 4. The input 


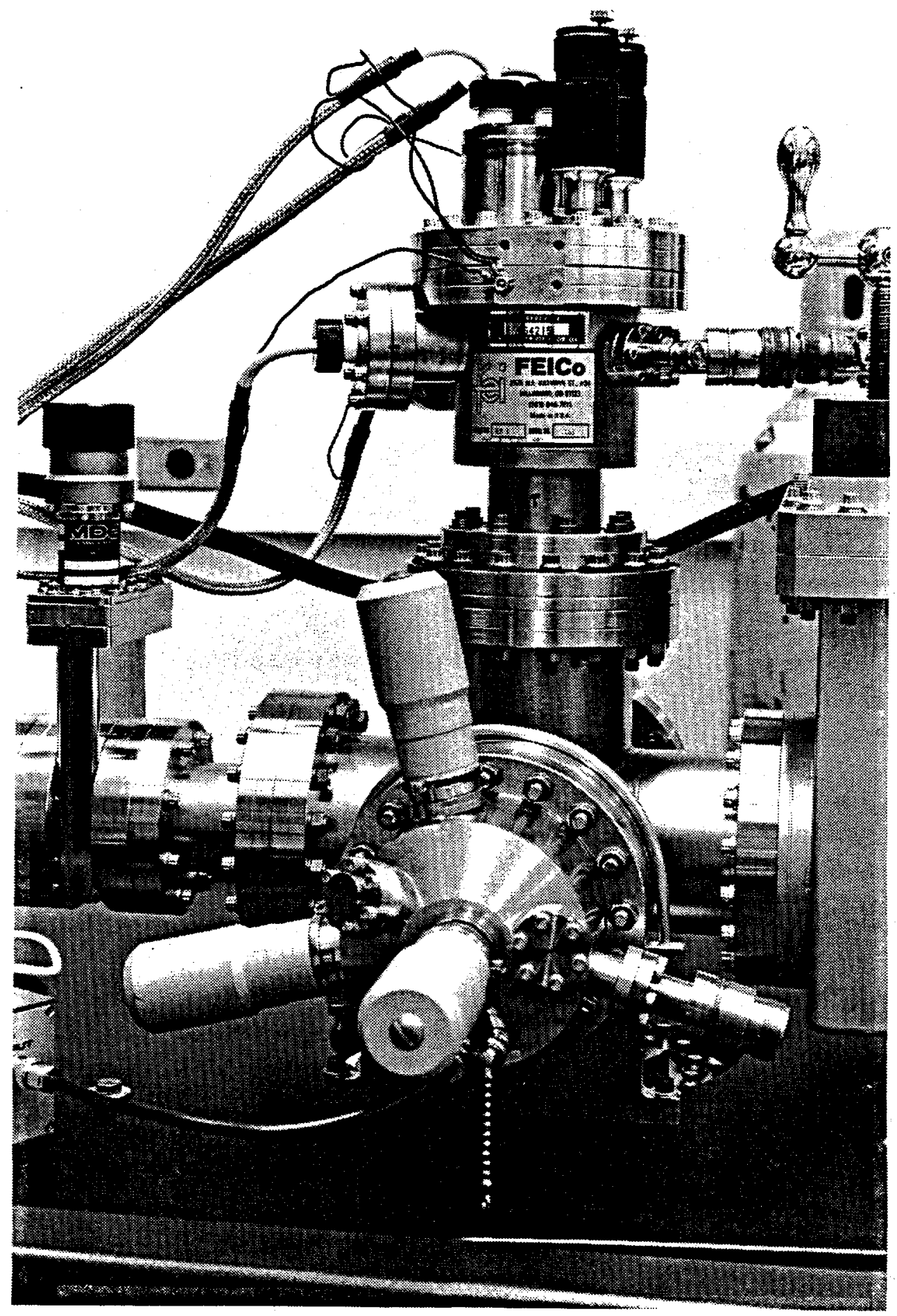

Figure 3: FIB chamber with ion gun and specimen manipulator. 


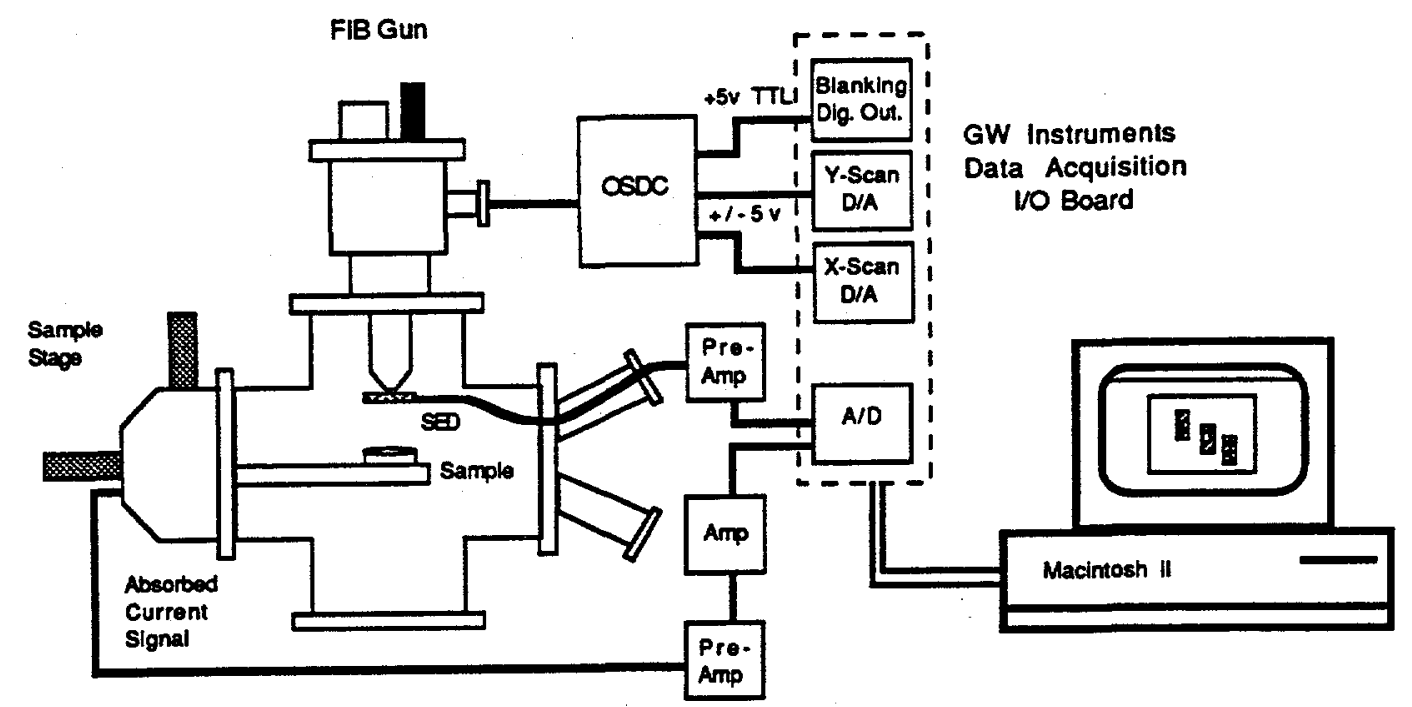

FIB Chamber

Figure 4: Schematic of the Macintosh II ion beam control and image acquisition system

and output hardware to accomplish these tasks is provided by a data acquisition board from GW Instruments [7].

To position the ion beam, 2 12-bit D/A converters output voltages in the range of $+/-5$ volts at a maximum rate of $111 \mathrm{kHz}$. The scanning, or positioning, signals are input to the octupole/stigmator deffector controller (OSDC) which then generates the 8 octupole signals necessary to move the beam in the scan field.

Blanking is done by the computer through 1 of 8 available lines of digital output. The form of this output is a +5 volt TTL signal that drives a blanking circuit in the OSDC. This in turn applies a 150 volt potential across two parallel blanking plates in the ion gun column.

Beam current, absorbed current, and secondary electron signals are acquired by a 12-bit A/D converter having a $200 \mathrm{kHz}$ sampling rate and an input range of 0 to 10 volts. This signal is used to generate images and can be plotted versus scan distance across an aperture to measure beam diameters. 


\subsection{SYSTEM SOFTWARE DEVELOPMENT}

The main objectives of developing software for the Macintosh II-based micromachining system are twofold; first, to control the ion beam for machining features under various parameters and geometries and second, to display and post-process acquired images from the beam induced signals.

A machining program has been written to cut rectangular well features. User inputs to the program are working distance, magnification, spot size, feature dimensions, and machining times. From these parameters, the program calculates the corresponding voltages to be input to the OSDC to scan the beam in a raster pattern. The increment between successive scan lines is also calculated so that the beam profiles overlap by half of the beam diameter. Since the beam profile can be considered having a Gaussian distribution, overlapping scans in this manner will closely approximate a uniform ion dose. Scan rates are determined by the dwell time chosen and the total machining time is determined by the number of frame scans selected by the user. This sequence is seen in the flowchart of Figure 5. To avoid the retrace artifacts seen in previously machined features, the beam is blanked at the end of each scan and unblanked at the beginning of the next line scan. Figure 6 illustrates a beam position ramp with the beam blanking signal applied at the end of a scan and turned off at the beginning of the next scan. After beam rastering is completed, the beam is automatically blanked to eliminate any unintentional exposure to the sample.

Future steps in developing FIB micromachining software are to investigate schemes to machine more complex structures. Two such schemes are: (1) to specify dwell times for each individual pixel and therefore have different amounts of material removal at each point and (2) to control which pixels are to be on (beam unblanked) or off (beam blanked) for each successive frame scan [8].

Imaging software development has resulted in the ability to acquire an image from a single frame scan of the ion beam. An acquired image of micromachined features is shown in Figure 7. A problem encountered with this imaging method has been the time required to obtain an image and display it on the Macintosh II monitor. Possible solutions are the adaptation of public domain programs developed primarily for real-time electron and optical microscope image acquisition and processing. Progress is continuing in the area of real-time image acquisition and display. 


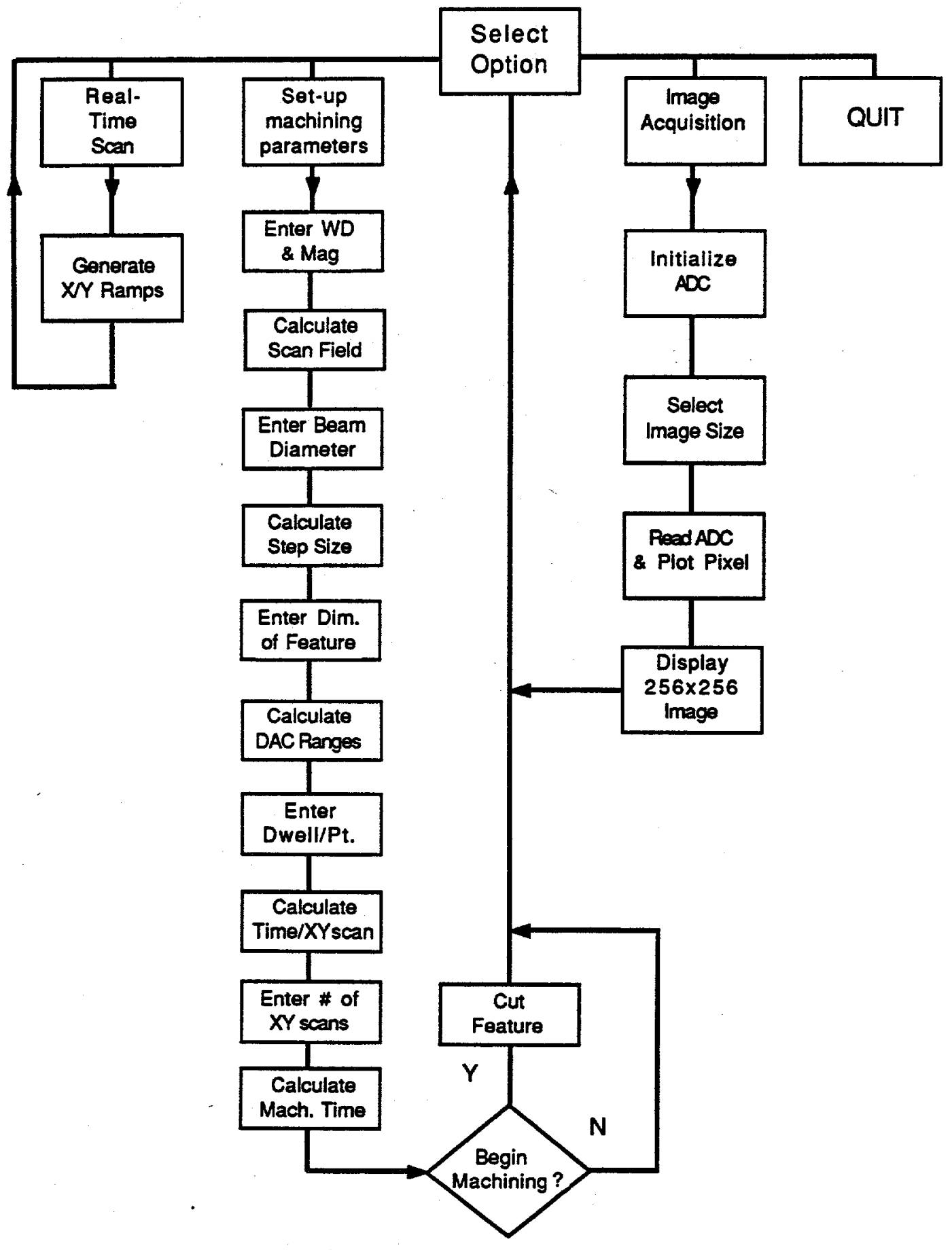

Figure 5: Flowchart of the machining/imaging program. 


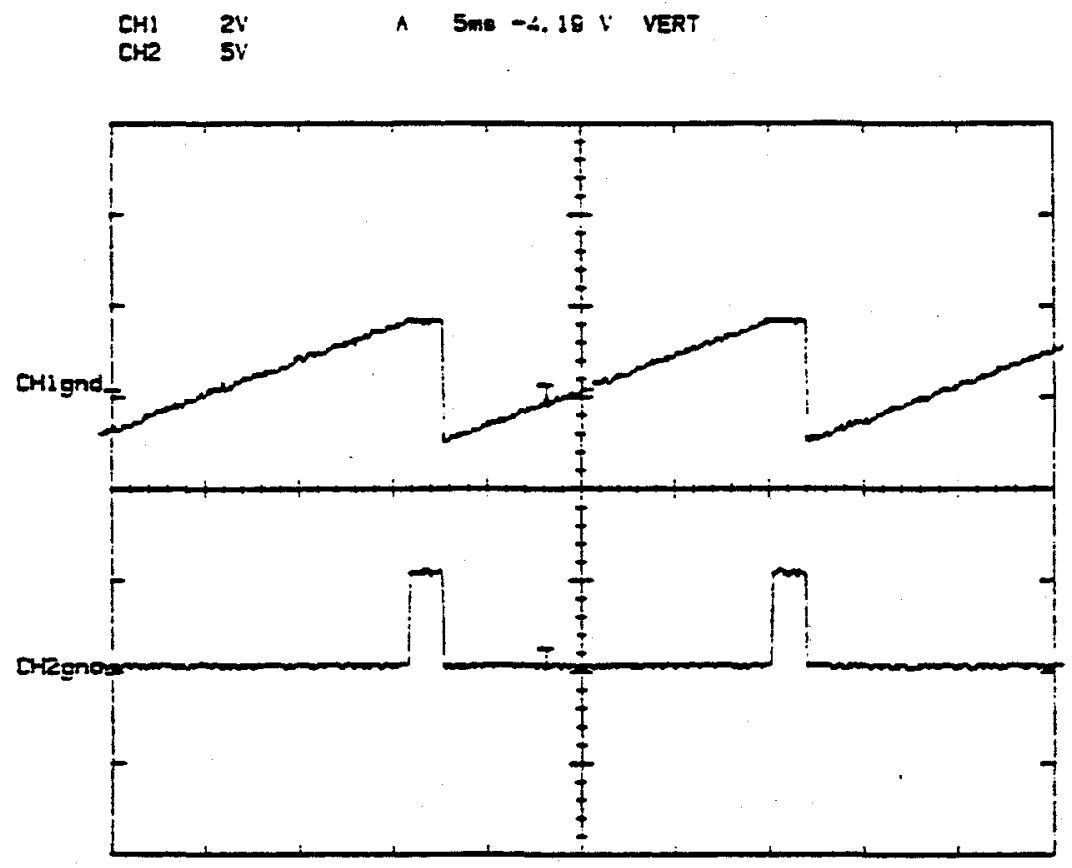

Figure 6: Full range $(+/-5$ volt $)$ beam scan with blanking signal

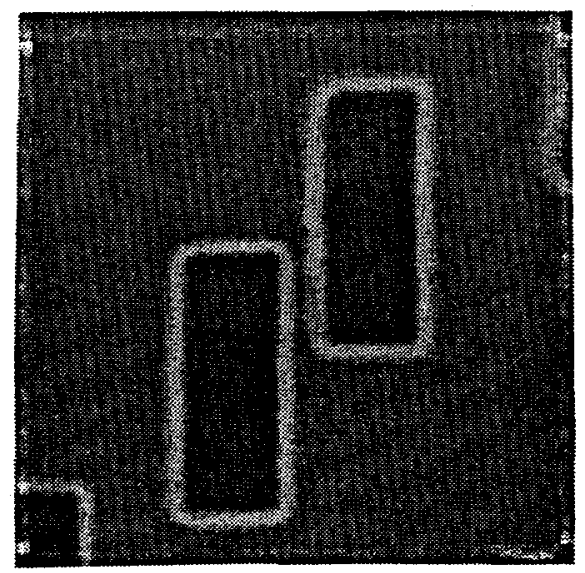

Figure 7: Acquired image of micromachined features using the absorbed current mode. 


\subsection{ION BEAM MACHINING EXPERIMENTS}

\subsubsection{The Material Removal Process}

As stated previously, the mechanism of material removal in FIB micromachining is sputtering. The sputter yield of a material is a function of both beam and substrate parameters as seen in the expression[9]:

$$
Y=\frac{\rho \times d}{\Delta}
$$

where:

$$
\begin{aligned}
& Y=\text { sputter yield (atoms/ion) } \\
& \rho \doteq \text { sample density (atoms } / \mathrm{cm}^{3} \text { ) } \\
& d=\text { depth of feature }(\mathrm{cm}) \\
& \Delta=\text { beam current density (ions } / \mathrm{cm}^{2} \text { ) }
\end{aligned}
$$

When considering the case of perfect material removal, i.e. all sputtered material leaves the area of the machined feature as seen in Figure 8, the material removal can be calculated for a 3-dimensional machined feature given by the equation [10]:

$$
V=\frac{1.04 x 10^{-5} I t Y A}{\rho}
$$

where:

$$
\begin{aligned}
& Y=\text { sputter yield (atoms/ion) } \\
& \left.\rho=\text { sample density (grams } / \mathrm{cm}^{3}\right) \\
& V=\text { volume of material removed }\left(\mathrm{cm}^{3}\right) \\
& t=\text { total sputtering time }(\mathrm{s}) \\
& I=\text { beam current (amps) } \\
& A=\text { at. wt. of sample (grams) }
\end{aligned}
$$

For example, to machine features in silicon having a $4 \mu m$ width, $10 \mu m$ length, and $4 \mu m$ depth using a sputter yield of 2.6 atoms/ion [9], and a beam current of $480 p A$ requires 17 minutes total machining time. If a beam current of $4 \mathrm{nA}$ is used the total machining time is reduced to 2 minutes. 


\section{Focused \\ Ion Beam}

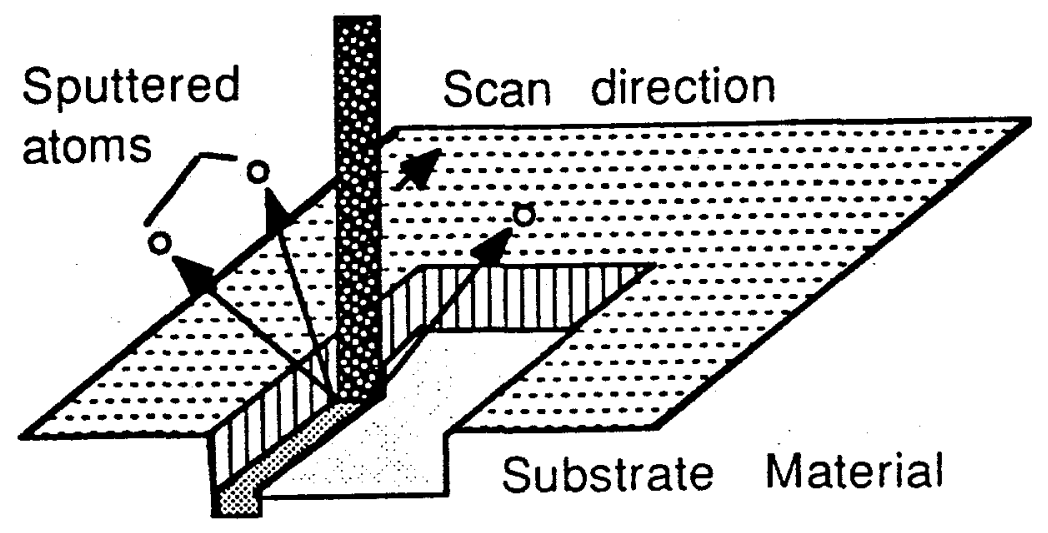

Figure 8: Machining by a scanning focused ion beam.

However, in actuality, material removal is accompanied by redeposition of sputtered atoms. Redeposition is dependent on such parameters as beam dwell time [11] and the aspect ratio of the machined features [10]. For a given total machining time, the dwell time will be determined by the number of times that the beam is scanned over the feature. When considering a single raster to machine a feature, the beam will leave behind a large number of redeposited atoms. For many scans, the number of redeposited atoms will be less for each scan and these can be removed with subsequent scans. The maximum depth that a feature may be machined can be described by a simple cosine distribution of the sputtered atoms. When the depth of the feature becomes sufficiently deep that sputtered atoms can not escape without striking the sidewalls, appreciable redeposition will occur [12]. For example, a $4 \mu \mathrm{m}$ deep feature may be degraded by sidewall redeposition on the order of $0.5 \mu \mathrm{m}$ of accumulated material on the sidewalls perpendicular to the beam scanning direction. This phenomena is depicted in Figure 9.

\subsubsection{Experimental Procedure}

To understand the fundamentals of focused ion beam micromachining, it will be necessary to conduct a multiparametric machining study involving both beam and substrate parameters. Beam parameters being examined are beam energy, current, and.dwell time. Substrate parameters are type of material ( $\mathrm{Si}, \mathrm{GaAs}$, and $\mathrm{Au})$ and crystallographic orientation in the case of $\mathrm{Si}((100)$ and (111)). 


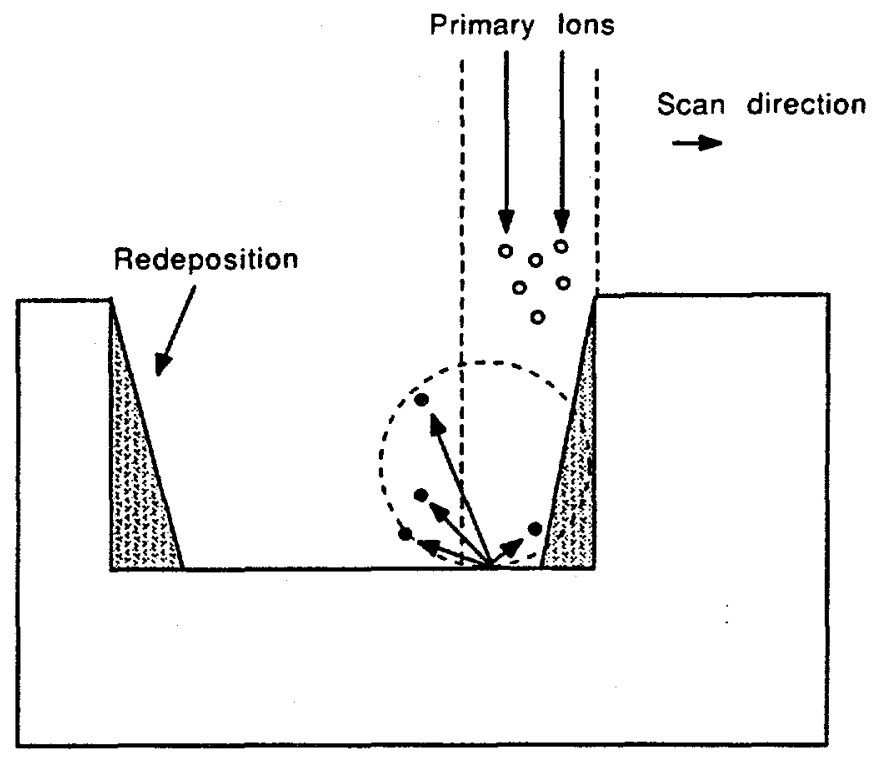

Figure 9: Redeposition due to feature aspect ratio

Experiments are being conducted to determine sputter yields for conditions where redeposition may be considered negligible. This information will be used as a basis on which redeposition rates will be quantified in future experiments. Figure 10 illustrates a $4 \mu \mathrm{m}$ $\times 10 \mu m \times 4 \mu m$ feature machined into (100) silicon with a $480 p A$ ion beam, $0.025 \mathrm{~ms}$ dwell time, and 20 min total machining time ( 825 frame scans). In contrast, Figure 11 shows a feature of the same size and beam current, but a $2.5 \mathrm{~ms}$ dwell time. The $20 \mathrm{~min}$ total machining time in this case results in 8 frame scans. The features cut with a $2.5 \mathrm{~ms} \mathrm{dwell}$ time exhibit significant redeposition compared to the $0.025 \mathrm{~ms}$ dwell time features. Material removal will be measured by cross-sectional SEM of the rectangular features. The depth of the features will be determined by cleaving the sample along a scribed line that passes through one of the rectangular wells. In addition, the effect of aspect ratio on the redeposition process may be readily observable. From the initial values of sputter yields, redeposition rates will be examined for varying ion accelerating voltages, beam currents and dwell times.

In addition to planview and cross-sectional SEM, other means of examining machined features will include interferometry TEM (STM), micro-Raman spectroscopy. Information about the interaction of high energy ions and a crystalline lattice can be gained from changes in the Raman signal. TEM will also give information regarding lattice damage from the ion beam. 


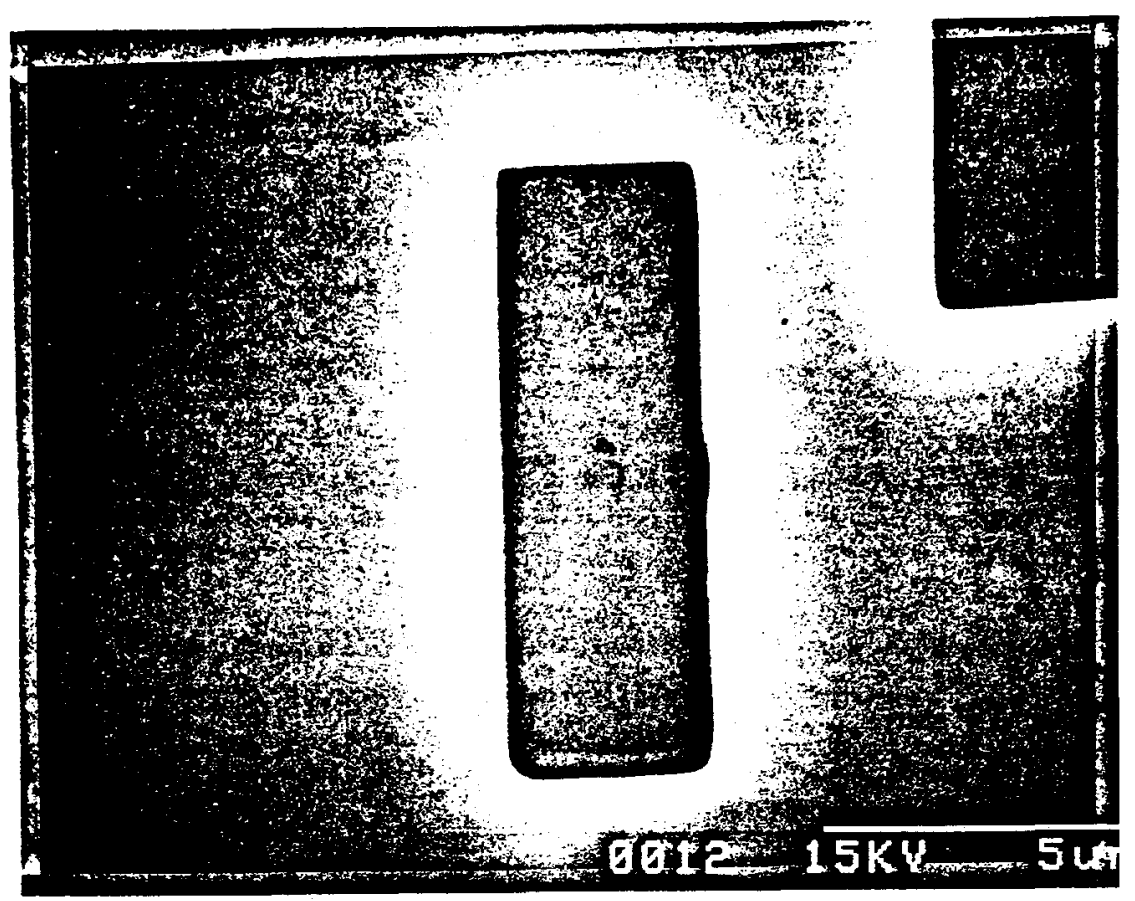

Figure 10: SEM micrograph of feature cut with $0.025 \mathrm{~ms}$ dwell time and $480 \mathrm{pA}$ beam.

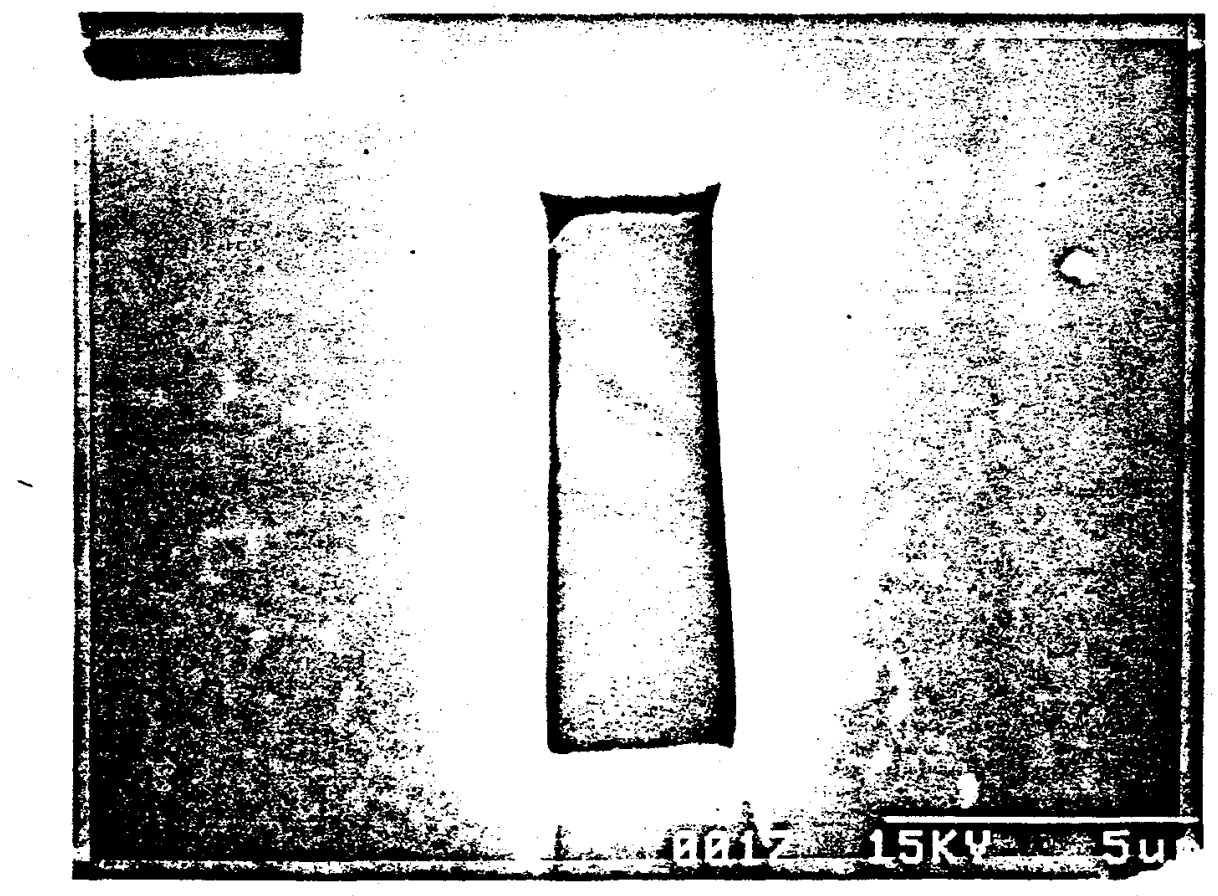

Figure 11: SEM micrograph of feature cut with $2.5 \mathrm{~ms}$ dwell time and $480 \mathrm{pA}$ beam. 


\subsection{CONCLUSION}

With the completion of the FIB micromachining vacuum system, plans are to conduct the multiparametric machining study. Work is continuing to expand the capabilities of the computer system in the areas of imaging and complex feature machining.

The results of this study are providing a fundamental understanding of material removal and redeposition from focused ion beam micromachining. In the course of these experiments, the feasibility and applicability of this new machining tool is being examined. The merging of this technique with other nanofabrication tools, such as STM, may further expand the capabilities of industry to manufacture complex devices of decreasing dimension. 


\section{References}

[1] Puretz, J., DeFreez, R.K., Elliott, R.A., Orloff, J., "Focused Ion Beam Micromachined AlGaAs Semiconductor Laser Mirrors", Electronics Letters, Vol. 22, No. 13, June 19, 1986.

[2] Shaver, D.C., Ward, B.W., "Integrated Circuit Diagnosis Using Focused Ion Beams", Journal of Vacuum Science and Technology, B, 4(1), Jan/Feb 1986.

[3] Cleaver, J.R.A., Kirk, E.C.G., Young, R.J., Ahmed, H., "Scanning Ion Beam Techniques for the Examination of Microelectronic Devices", Journal of Vacuum Science and Technology, B, 6(3), May/June 1988.

[4] Ward, B.W., Shaver, D.C., Ward, M.L., "Repair of Photomasks with Focused Ion Beams", SPIE Vol. 597 Electron Beam, X-Ray, and Ion Beam Techniques for Submicron Lithographies IV, 1985.

[5] Kingham, D.R., Bell, A.E., "In Defence of the Taylor Cone Model: Application to Liquid Metal Ion Sources", Journal de Physique, C9, No. 12, Dec 1984.

[6] Kinematic Systems, Inc., 20 Arboretum Rd. P.O. Box K, Roslindale, MA 02131.

[7] GW Instruments, 35 Medford St., Somerville, MA 02143.

[8] Crow, G., Puretz, J., Orloff, J., DeFreez, R.K., Elliott, R.A., "The Use of Vector Scanning for Producing Arbitrary Surface Contours with a Focused Ion Beam", Journal of Vacuum Science and Technology, B, 6(5), Sept/Oct 1988.

[9] Melngailis, J., "Critical Review: Focused Ion Beam Technology and Applications", Journal of Vacuum Science and Technology, B, 5(2), Mar/Apr 1987.

[10] Swanson, L.W., "Method of Evaluating Milling Rates Using Focused Ion Beams", May 12, 1987.

[11] Yamaguchi, H., Shimase, A., Haraichi, S., Miyauchi, T., "Characteristics of Silicon Removal by a Fine Focused Gallium Ion Beam", Journal of Vacuum Science and Technology, B, 3(1), Jan/Feb 1985.

[12] Betz, H., Heuberger, A., Economou, N.P., Shaver, D.C., "Influence of Sputter Effects on the Resolution in X-Ray Mask Repair", SPIE Vol. 692 Electron Beam, X-Ray, and Ion Beam Techniques for Submicrometer Lithographies V, 1986. 



\title{
26 NANOFABRICATION WITH THE SCANNING TUNNELING MICROSCOPE
}

\author{
Gordon M. Shedd \\ Graduate Student \\ Phillip E. Russell \\ Associate Professor, MTE
}

The Precision Engineering Center has recently begun a research program into applications of STM to Nanotechnology. Few tools permit humans to control events and processes at the nanometer level, and of those, the STM is the most well-suited to the task. A versatile new ultra-high-vacuum (UHV) STM is being built to study the use of STM for the manipulation of nanometer-scale particles. Part of the STM's usefulness will be due to its being positioned directly beneath the focused ion beam (FIB). The interface of the STM with the FIB will allow the STM to take advantage of the FIB for long-range imaging and as a particle source; the FIB can in turn use the STM for in situ, high-resolution imaging of micromachined features.

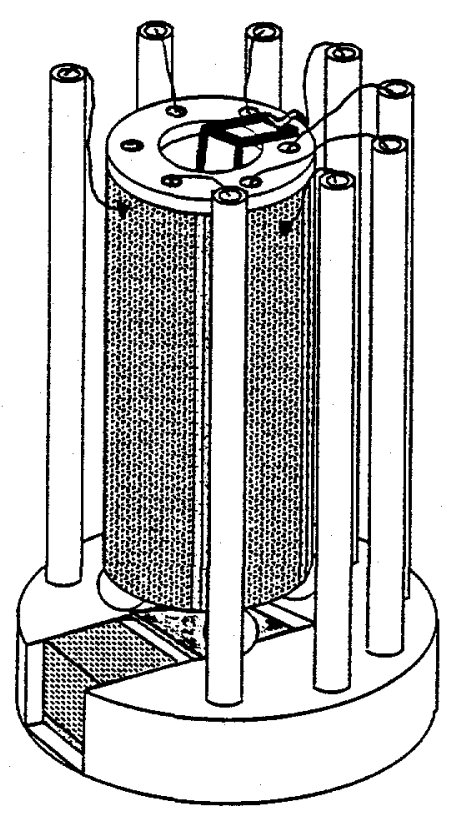




\subsection{INTRODUCTION}

The scanning tunneling microscope (STM) has found increasing use as a means to produce nanometer-scale modifications to surfaces [1-7]. The same characteristics that allow the STM to image with atomic resolution also permit it to function as a high-spatial-resolution probe, capable of influencing a very localized region on a surface. Used as a tool, the STM can exert human influence upon individual atoms [8], provoking thoughts of genetic surgery, quantum-electronic devices built from individual atoms, chemistry induced at the molecular level, and data storage measured in Terrabytes per square centimeter.

If these ideas (and others) are to be realized, however, the control and use of STM as a tool must be more clearly understood than is presently the case. To participate in and contribute to progress in this area, a program of research into the use of STM for nanofabrication has been initiated at the Precision Engineering Center. This report includes a perspective on the role of STM in nanotechnology, a summary of work done in the field to date, a description of the UHV STM that has been designed for this project, and an explanation of how this STM will interface with the vacuum system and focused ion beam (FIB) (see Section 25), and a discussion of the initial focus of research.

\subsection{STM AND NANOTECHNOLOGY}

\subsubsection{Tools for Nanotechnology}

As the prefix nano implies, nanofabrication refers to techniques that create nanometer-scale structures or motions (nanometer-scale encompasses dimensions from atomic diameters up to $100 \mathrm{~nm}$ ) [9]. The end products of nanotechnology are so small that the choice of tools that can be used to produce them is limited. Few tools can address a region of only nanometers in extent, let alone precipitate a controlled response within that region; examples of such tools are described in this section.

Lens Focused Beams Almost all of the tools that have been used or suggested for nanofabrication can be categorized as focused beams. Focusing is normally done by the use of lenses, but can also be achieved by proximity focusing (which will be described below). A lens-focused beam is, in optical terms, an image (often de-magnified) of a source of particles or photons. If the source is a good approximation of a point source, then, in the vicinity of the image plane, a very fine beam occurs.

Short wavelength photons (e.g. $x$-rays) can theoretically be focused into a beam that might be useful for nanofabrication, but the current state of the art in x-ray optics can do no better than about a $50 \mathrm{~nm}$ beam diameter [9]. Primary among the lens-focused beams useful for nanotechnology are electron beams, which are routinely formed into sub-nanometer probes for scanning transmission electron microscopy (STEM) and high-resolution SEM. 
Contact-Induced Modification The site of an inadvertent tip touch, or crash is doubtless the most commonly created nanostructure, but not all tip touches are accidental. The effects of controlled tip touches have been explored in detail, with some perhaps unexpected results. Normally, it would be assumed that contact between a sharp probe and a flat surface would leave an indentation in the surface. This is not always the case for very slight contact.

Abraham [2] performed a series of experiments in which he touched a gold surface with a tungsten tip after first determining that the surface was flat. The severity of the touch was controlled by the choice of tunneling resistance for a fixed tip bias (the lower the resistance, the closer the tip moves toward the surface). When the resistance was set to $200 \Omega$ for several minutes, subsequent images of the region showed a hole approximately $3 \mathrm{~nm}$ deep and $15 \mathrm{~nm}$ in diameter. In addition, the surface exhibited deformations over an area 70 $\mathrm{nm}$ in diameter. However, when the resistance was increased to $5 \mathrm{k} \Omega$ in other experiments, the post-contact images revealed protrusions on the surface, rather than depressions. The authors conjectured that the $20-25 \mathrm{~nm}$ diameter hillocks were re- depositions of gold that had been picked up by the tip during previous crashes.
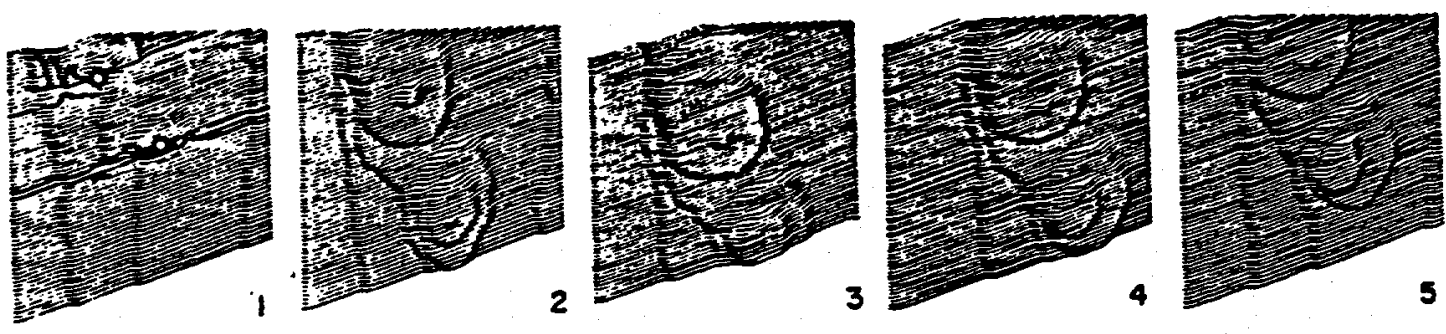

Figure 1: Time-lapse STM images of two craters created by touching a Au (111)surface with a tungsten tip. Image field is $40 \mathrm{~nm} \times 40 \mathrm{~nm}$, and the time between images is 8 minutes [15].

Jaklevic and Elie [15] recorded time-lapse STM images of indentations that they had intentionally created on a single crystal gold surface during a study of surface diffusion. The holes created are approximately $15 \mathrm{~nm}$ in diameter and 2-3 atomic layers deep, although, as Figure 1 indicates, the diffusion of gold atoms fills in the craters rather quickly.

Work by Gimzewski and Möller [4] explored the effects of more delicate tip-sample contact, and they too observed both indentations and protrusions, but their explanation of the results was different. In these experiments, instead of varying the tunneling resistance (i.e. changing the feedback control of the z-position), the STM was first brought into tunneling range, then the feedback signal was fixed, and the tip was moved towards the surface by directly applying additional voltage to the $z$ piezo. This method allows the z-motion control to be much more linear than the method utilized by Abraham.

The authors were able to correlate their results with the state of contamination of the tip. 
Tunneling current vs. tip bias measurements for clean and dirty (i.e. after several hours of use) tips showed identifiable characteristics, and the results of touches by the two tip types were also easily distinguished-dirty tips left depressions; clean tips left protrusions. It was concluded that the clean tips adhered to the surface when contact was made, a hypothesis that was supported by hysteresis in the current vs. z-displacement data which showed that, to return the current to its pre-touch level, the tip had to be retracted further than it had been driven in. Tip contamination prevents adhesion to the surface, so that retracting the dirty tips after contact did not pull the surface back up, but left the it indented. The features created for both tip conditions were $10 \mathrm{~nm}$ in diameter and $\pm 2 \mathrm{~nm}$ in the $\mathrm{z}$ direction when the tip is driven in $2.5 \mathrm{~nm}$.

Scanning the tip transversely while in contact with the surface causes linear scratches or grooves, which, as reported by Ringger [1] can as narrow as several tens of nm. Packard [7] have extended the process to two dimensions to perform what they term sanding. A related, and slightly larger-scale example of this method of surface modification is a series of 360-nm-wide grooves (Figure 2) that were created by scratching a 20-nm-thick film of calcium fluoride [3]. The film is an insulator on a conducting substrate, so the tip had to penetrate deep into it to achieve tunneling. When the tip was scanned along the surface, the film peeled off.
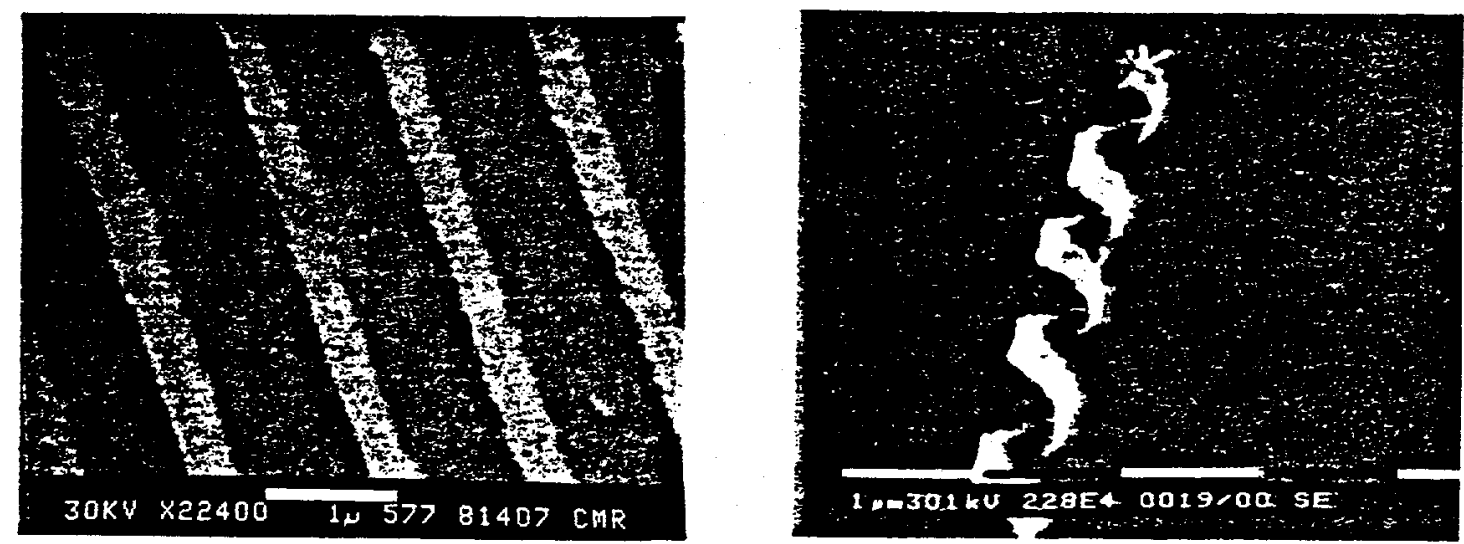

Figure 2: $360 \mathrm{~nm}$ grooves micromachined in a calcium fluoride film with the tip of an STM. At right is the shaving that results when aluminum fluoride is machined [3].

Beam and/or Field-Induced Modification Some of the beam-induced phenomena used for STM nanomodification have been exploited previously using conventional lensfocused beams. Beam-induced exposure of resist for nanolithography has been performed with focused beams of electrons, ions, and x-rays. Given the hundreds of millions, if not billions; of dollars that have been spent on improving the resolution of lithographic techniques, it is understandable that there should be interest in exploring the STM's ca- 
pabilities in this area. McCord and Pease [16] first demonstrated STM-based lithography in 1985 when they formed $100 \mathrm{~nm}$ lines by contamination writing (polymerization of hydrocarbon surface contaminants), and by exposing a thin layer of resist . Resist exposure requires that the incident electrons have energies exceeding $10 \mathrm{eV}$, so the STM is operated in the field emission mode (farther from the surface, and with a higher tip bias), much as was done in Young's forerunner of the STM, the Topografiner [17]. Although operating in the field emission mode causes the beam diameter to increase, McCord and Pease have been able to expose 10-20 nm lines in PMMA and CaF2 resists, and 20-nm-wide metal lines have been created using these patterns [18].

Another nanomodification process that has been developed with conventional focused beams is beam-induced deposition. A surface, upon which it is desired to cause deposition, is exposed to the vapor of a compound that contains the substance to be deposited (e.g. a metal). The beam is used to control the chemical decomposition reaction, which occurs only where the beam supplies energy or creates reaction sites.

Following the beam-induced deposition procedure, Silver [19] has used the STM to deposit 20-30 nm cadmium dots and lines on silicon substrates. For deposition to occur, however, the STM must be operated at a tip bias high enough to cause field emission. Whether this is necessitated by a need for high field strength, or for energetic electrons, is unclear. The authors have hypothesized that a plasma may be created between the tip and the sample.

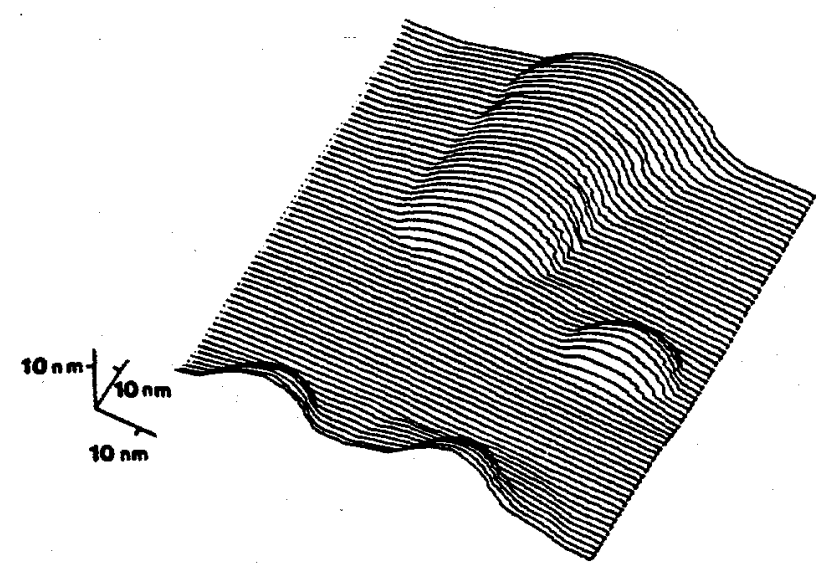

Figure 3: An STM image of several hillocks produced by increasing the tip bias and tunneling current above points on a glassy metal substrate. The hillocks are $<20 \mathrm{~nm}$ in diameter [20].

One of the more interesting modes of STM nanomodification is a combination of beaminduced and field-induced effects that Staufer [20] refers to as hillock formation. While the STM tip is held above a spot on the surface, the tunneling current is raised to several hundred nanoamperes (normal imaging currents are $; 10 \mathrm{nA}$ ). The high current density causes the substrate to melt, at which point the liquid is pulled up into a Taylor cone by 
the negatively biased tip. Reducing the current to $1 \mathrm{nA}$, and keeping the tip stationary, allows the cone to cool while the field is maintained. The result is a hillock, as shown in Figure 3, that can be as small as 3-5 $\mathrm{nm}$ in diameter. Densities of 1 hillock per $100 \mathrm{~nm}$ 2 have been claimed, which, if each hillock were to represent a bit, converts to over 100 Gigabytes of data per $\mathrm{cm}^{2}$. However, small structures are more prone to become unstable and perhaps disappear after relatively short periods of time-an unacceptable characteristic for any long term data storage medium.

Hillocks cannot be formed on all surfaces, however. Obviously, the melting temperature of the substrate determines how much heating is required, but how much heating actually occurs is related not only to the current density, but also to the electron mean free path and thermal conductivity of the substrate. For these reasons, disordered solids with moderate melting temperatures are used (e.g. splat-cooled glasses), and additional disordering, induced by ion etching the surface, improves the heating efficiency.

\subsubsection{STM Nanomanipulation}

In addition to being useful for modifying surfaces, the STM can also be used to manipulate particles that are not actually part of the surface, but sit upon it (e.g. clusters, protruding atoms, or adsorbates). Manipulation might mean moving a particle from one place to another, or it could mean causing the particle to undergo a chemical change or interaction. These capabilities would be useful for building devices out of small particles, perhaps from particles of different materials. Nanomanipulation may also be useful for studying interactions between particles, and between particles and substrates. Eventually it may become possible to perform molecular surgery, building or dismantling a molecule atomby-atom.

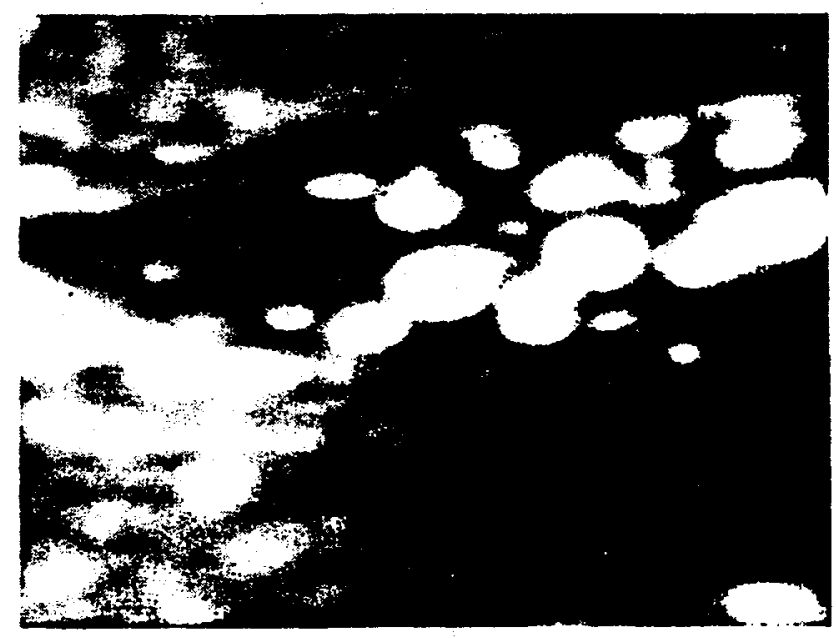

Figure 4: An atomic resolution STM image of a 10-atom cluster of the semiconductor bismuth iodide on a graphite substrate. The image field is $2.5 \mathrm{~nm} \times 2 \mathrm{~nm}$ [24]. 
Particles On Substrates The ability of STM to detect particles on substrates has been demonstrated convincingly; examples of particles that have been imaged include metal clusters, $[21,22]$ semiconductor clusters (Figure 4),[24] benzene molecules, [25] bacterial cell sheaths, [26] amino acids, [27] and individual adsorbed atoms [28]. The greatest advantage of using the STM for manipulation is that before and after imaging can be done in situ, without using a highly energetic beam that might disturb the subject being observed.

Manipulating Particles On Substrates A theoretical analysis by Gomer [29] discussed several physical processes by which atoms might be transferred from a sample to a tip, and the reverse. As is the case for electrons, when two surfaces are brought close together it is possible for an atom to tunnel from one surface to the other; although, for a given separation distance, the tunneling probability is much lower for an atom than it is for an electron. Gomer concluded that atom tunneling is a likely avenue for transfer only when the atom is essentially in contact with both surfaces, as might occur if the tip were intentionally moved toward the sample.

Field-assisted, thermally-activated desorption is another possible transfer mechanism. The field between the tip and the sample can be made relatively high with a modest tip bias due to the close proximity of the two electrodes. In the presence of a high field, the amount of energy required to ionize an atom can become less than the amount required to cause desorption of the neutral atom. If ionization occurs the ion will desorb, following the electric field lines away from the surface where it was adsorbed. The energy barrier to desorption is effectively replaced by the lowered barrier to ionization as the new thermal activation energy.

The controlled manipulation of individual atoms was first reported by Becker [8] in early 1987. In a process referred to as bit writing, the authors first prepared the tungsten tip by touching it to the surface of a germanium sample, then the tip was used to image the sample. After determining that a region of the sample was sufficiently flat, the tip was brought to a stop above the point where it was desired to deposit a bit. The tip bias was changed from the value used for imaging $(-1.0$ volt $)$ to -4.0 volts, while the tunneling current was left unchanged ( $20 \mathrm{pA})$. If the writing was successful, the tip would be retracted suddenly by the control circuit, indicating that the gap between the tip and sample had changed abruptly. After resetting the tip bias to -1.0 volt, subsequent images of the region revealed new atomic-dimension features,as shown in Figure 5. [4] 


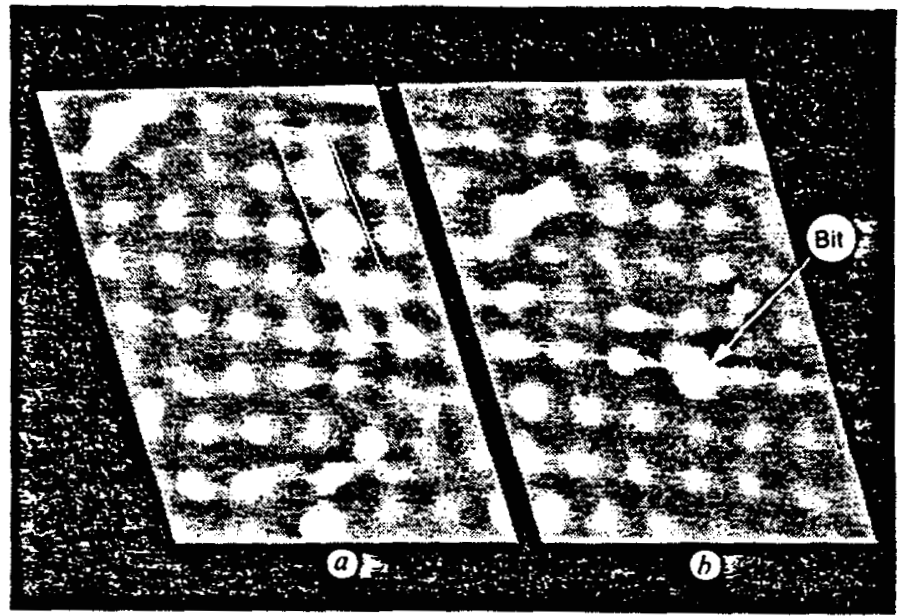

Figure 5: Before and after images of the writing of an atomic bit on a germanium substrate. The recurring defect, which appears in the upper left-hand corner of $a$, serves to relate the topography of the $5 \mathrm{~nm} \times 6.5 \mathrm{~nm}$ images [8].

The authors presented arguments that the new features had come from the tip, and that the success of attempts to transfer atoms from tip to sample depends on the strength of the bonding between the species to be transferred and species of the tip. For example: Si and $W$ form an alloy, but $G e$ and $W$ do not; therefore, it is expected that Ge atoms should be more loosely bound to the $W$ tip, and should be more easily transferred from the tip than $\mathrm{Si}$ atoms. Indeed, the authors were never successful at depositing bits after touching the $\mathrm{W}$ tip to Si samples, but could achieve success rates of up to $90 \%$ after touching $\mathrm{Ge}$ samples.

Perhaps surprisingly, only a few indirect mentions of material transfer between tip and sample have been published in the past two years-the buildup of carbon whiskers on a tip during graphite imaging has been observed [30], as well as the reproducible transfer of aluminum clusters to a tip during the imaging of $\mathrm{Al}$ (111) [31]. 
A nanomanipulation experiment that did not have atom transfer as its goal was the attempt to modify individual strands of a polymer that had been deposited on a graphite substrate [32]. By applying short voltage pulses to the tip, the authors were able to disrupt $5 \mathrm{~nm}$ diameter regions along the linearly oriented chains. Presuming that this effect is similar to that which occurs during the exposure of electron-beam resists, the technique could be used to improve the spatial resolution of lithography.

\subsection{RESEARCH PROGRAM}

With the background provided by the previous sections, specific research plans for the STM Nanotechnology program will be presented. The following sections describe the equipment, both existing and under construction, and outline the emphasis of the experiments to be performed.

\subsubsection{Nanomanipulation of Particles}

Several factors have lead to the decision to concentrate on nanomanipulation as the subject for this research program. One is that, compared to the effort that has been devoted to nanomodification, little work has been done to explore the mechanistic aspects of particle manipulation. The immediate payoffs that may be derived from nanomodification-based improvements in lithography and data storage density have no doubt driven the disparity in research emphasis thus far. However, nanomanipulation will eventually provide even greater control over nanofabrication; only by nanomanipulation could structures be built from nanometer-sized bricks of different elements, making possible such developments as new electronic devices in which electron transport is dominated by quantum-mechanical size effects [8].

To reach the stage at which nanomanipulation will be technologically useful, the practical problems involved in moving particles from point $A$ to point $B$, and in transporting particles of different elements, must first be addressed. Most of the published work that relates to STM nanomanipulation has not been performed with manipulation in mind. STM studies of particles on substrates concern the topography, growth, electronic properties, or bonding behavior of the particles [21-28]. The few indications that can be gleaned from the literature regarding the possibilities of nanomanipulation are promising. 
Another important factor is the convenience of a focused ion beam (FIB) in the same vacuum system with the STM. A FIB can be used as a source of multi-atom clusters, so nanometer-scale particles can be procured. A third factor that favors the chosen subject is the motivation to design a UHV-compatible STM capable of being interfaced with a FIB or SEM.

\subsubsection{Source of Nanometer-Scale Particles}

The single most important component in a FIB is the source of the ions, a liquid metal ion source (LMIS). A tungsten needle, wetted with liquid metal (e.g. Ga, In, Au) from a resistively-heated reservoir, is pointed down along the axis of the cylindrically symmetric, beam-focusing column. Less than a $\mathrm{mm}$ below the point of the needle is the extraction aperture, a flat metal plate with a circular hole centered about the beam axis. When the tip is held at a high positive potential relative to the extraction aperture $(10 \mathrm{kV})$, the electric field pulls the liquid into a sharp cone, the tip of which serves as a bright, point-like ion emitter. It is the emitter's brightness and small size that make the high current density and small beam diameter of the FIB possible.

The FIB can be used as a scanning ion microscope, capable of image resolution on the micron scale, and as a tool for micromachining [33]. As was alluded to above, the LMIS can also be used as a source of atom clusters, which are microscopic collections of from two to $>10^{6}$ atoms [34]. As the extraction field is increased beyond the level required for normal ion emission, a larger and larger proportion of the beam current will be due to clusters of atoms, until the field finally induces the Taylor cone to become unstable, and emission takes the form of charged droplets of the liquid metal [35]. The electrostatic lenses and scanners of the FIB column can be used to focus and deflect the charged clusters and droplets, permitting accurate control of their placement on a substrate [36].

In order to be able to manipulate a particle, it is first necessary to find one. STM certainly possesses adequate resolution to see nanometer-scale particles, but the difficulty associated with locating one relates to the particle size, and the density with which particles are distributed on the surface. To maintain adequate resolution to detect a single atom sitting on a surface, the field of view probably needs to be restricted to $25 \times 25 \mathrm{~nm}$ or smaller during the search ( $1 \AA^{2}$ per pixel for a $256 \times 256$ pixel image). Although the full range of the STM might be microns, it would be almost like searching for a needle in a haystack to find a single atom in a $1 \mu \mathrm{m}^{2}$ area $(1600 \times 25 \times 25 \mathrm{~nm})$. 
The probability that a search will be successful increases as the particle size, or the areal density of particles increases. It is expected that a FIB, when used as a particle source, can control the density and (to some extent) the size of the deposited particles, as well as position the deposition region on the sample [36]. Added to the FIB's ability to change the particle species by changing sources, this provides a great deal of experimental flexibility.

\subsubsection{UHV STM/FIB Combination}

To take the best advantage of the FIB as more than just a versatile particle source, the UHV STM has been designed around the concept of the down-the-tubes STM/FIB interface shown schematically in Figure 6. The most apparent feature of this interface is the high degree of collinearity of the ion beam and tip axes, achieved by shooting the FIB down the center of the STM tube scanner. This configuration is optimal for STM imaging of FIBproduced particles and micromachined features, and for combined FIB / STM imaging.

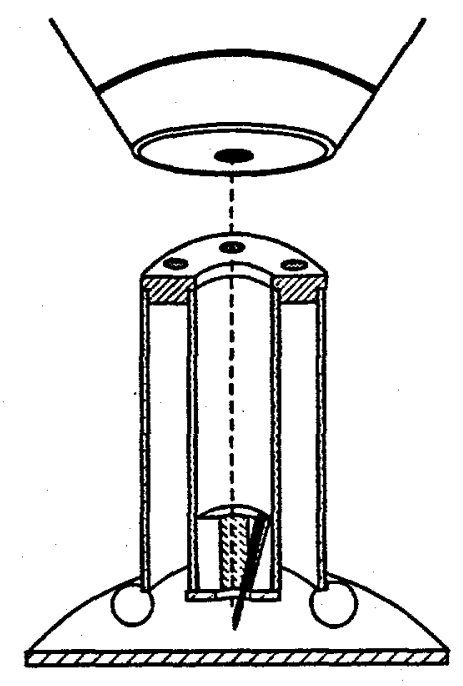

Figure 6: A schematic view of the UHV STM situated under the nose of the FIB gun. The ion beam can pass down through the center of the STM tube scanner, to image the tip, or to create features to be imaged by the STM. 
Several previous designs have combined STM with SEM to improve the ability to locate features of interest for examination $[37,38]$. Like the SEM, the FIB can be used to image regions of from $1 \mu \mathrm{m}^{2}$ up to $\mathrm{cm}^{2}$; whereas, most STM's have a maximum field size of tens of $\mu \mathrm{m}^{2}$. Larger-scale images are useful not only for locating features, but also for placing STM images within a larger context. Unlike the SEM, the FIB can easily create subjects to be examined by the STM (e.g.atom clusters or micromachined features). It should be pointed out that consideration must be given to the alteration of the sample that occurs due to ion impacts during FIB imaging.

Tube-In Tube STM The UHV STM itself is a variation on two tube-in-tube designs that have appeared in the literature $[39,40]$. Two piezoelectric tubes of different diameters are attached concentrically to a rigid base plate. The inner tube, to which the tip is mounted, operates as a conventional STM tube scanner. The sample is in contact with the outer tube, which is used for $x, y$, and $z$ offsets.

A major advantage of the tube-in-tube design is the rigidity of the tip-to-sample structural loop; the vibrational eigen frequencies reported in references 39 and 40 are $20-100$ $\mathrm{kHz}$. The increased stiffness reduces the vibration isolation requirements by improving the STM's common mode rejection (it is relative motion between the tip and sample that affects images). Another beneficial side-effect of the design is the inherently reduced thermal drift: If the inner tube expands, sending the tip toward the sample, then the outer tube expands also, pushing the sample away by an almost identical distance. The primary difference between the UHV STM and the other tube-in-tube designs is the orientation and loading of the outer tube. As shown in Figure 7, the entire double-tube assembly of the UHV STM is supported on three spherical feet mounted to the end of the outer tube; whereas, the published designs use the outer tube to support the sample (and holder, if any), rather than to support the STM. Using the outer tube to support the STM's mass may reduce the tube's resonant frequency, but the design keeps the supported mass to a minimum; furthermore, the advantages of convenience, compactness, and versatility that accrue to this configuration should be worth the price of a possible slight degradation in ultimate performance.

Z Approach and Coarse Transverse Motion Both of the previously published designs use inertial stick / slip motion induced by the outer tube to accomplish coarse sample positioning; for coarse $\mathrm{z}$ approach in one design, for coarse $\mathrm{x}-\mathrm{y}$ position in the other. To allow inertial stick / slip motion, the sample (or sample holder) must not be mechanically constrained by more than the force of friction along the direction in which motion is to occur. For example, in the referred-to design that incorporates coarse $x-y$ motion, the tubes are oriented vertically and the tip points upward, while the sample rests on three contact points about the perimeter of the outer tube. Gravity and the three contacts exert forces in the $\mathrm{z}$ direction, but the sample is unconstrained in the transverse directions. 
If the outer tube is bent slowly to one side, then straightened almost instantaneously, the sample will slip relative to its three contact points. This sequence can produce a sub-micron step, or be repeated at $\mathrm{kHz}$ rates to produce macroscopic motions [41].

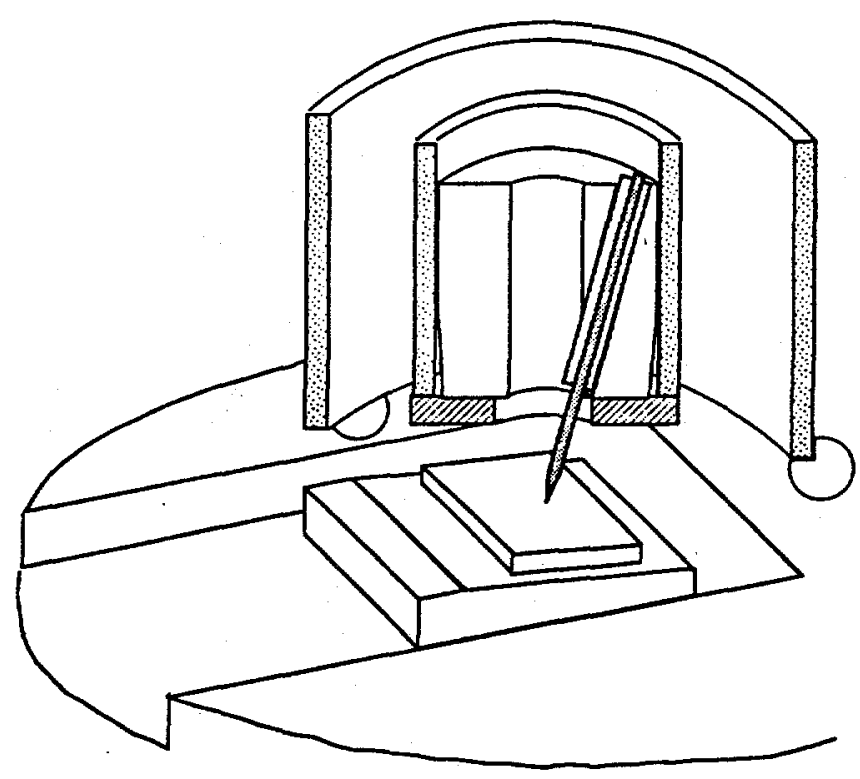

Figure 7: A closer view of the tip sample interface and the supporting feet attached to the outer STM tube. The STM may walk toward the inclined sample by stick-slip motion, bringing the tip within the $\mathrm{z}$ range of the piezoelectric scanner.

Two modes of inertially-stepped, coarse, tip-to-sample positioning are under consideration for the UHV STM. The first mode uses a variation on the slow bend, fast straighten, $x-y$ motion just described to walk the STM relative to a fixed sample. On a large, flat sample this mode would permit long-range $x-y$ motion, but would not provide motion in the $z$ direction. If a small sample were placed on a wedge-shaped sample holder beneath the tip (see Figure 7), then transverse STM motion toward the uphill part of the wedge would result in a reduced tip-to-sample separation distance. Wedge angle determines the ratio of the change in $z$ separation to y motion; a wedge with a 1:10 slope will give $100 \mu \mathrm{m}$ of $z$ motion for each $\mathrm{mm}$ of $\mathrm{y}$ travel. Motion across the wedge's slope (in the $\mathrm{x}$ direction) will not change the $z$ height macroscopically, so coarse $x$ motion should be possible without moving the tip out of the $z$ piezo offset range. Considering the case of an STM with a 1:10 wedge and a $z$ piezo capable of a $5 \mu \mathrm{m}$ offset, the area on the sample accessible for imaging would be $50 \mu \mathrm{m}$ wide (in the y direction) by several $\mathrm{mm}$ in the $\mathrm{x}$ direction-quite a large region by STM standards. 
If the STM walking principle proves impracticable, the second mode of coarse positioning will be used. It is still possible to achieve the same combination of $y$ and $z$ motions by placing the wedge on top of an inertial stepper; leaving the STM stationary while the sample is stepped in the y direction beneath it. In this case, a thin piezoelectric plate is placed under the wedge. The plate is poled so that a voltage applied across its thickness causes the top face to shear in the $y$ direction relative to its bottom face. Then, either the wedge can be made to slip relative to the piezo plate, or feet attached to the underside of the piezo could slide relative to the surface beneath them [41].

Vacuum Interface Designing an instrument to work in vacuum makes problematic issues out of such normally straightforward details such as wiring, tip and sample changing, and even moving the STM from one place to another. Doing all of these things inside a vacuum chamber increases their complexity considerably.

The UHV STM is designed to sit on top of a 1" diameter FIB sample holder, which fits down into a spring-loaded socket on the 3-axis, sample-positioning stage in the FIB compartment. To get the the sample holder and STM into the vacuum, the FIB's sample introduction port is used. The two tines of a fork attached to the sample transfer rod slide around the outside of the sample holder, supporting the holder by the protruding lip around its top edge. After the introduction chamber has been evacuated, the holder and STM are pushed into the FIB chamber until they are directly above the stage socket. The stage is raised until the socket surrounds the holder, then the fork is withdrawn. Electrical signals pass to the STM through contacts in the socket that mate to contacts on the sample holder.

The STM is to operate not only under the FIB, but also in the STM chamber of the UHV system. Transfer of the STM from the FIB compartment to the STM compartment will also be accomplished using the FIB sample-transfer rod. Once inside the STM chamber, a UHV 3-dimensional translator (wobble stick), and a separate STM sample introduction port will permit the tip and sample to be exchanged without breaking vacuum.

Tip Changing To avoid contaminating the UHV environment as much as possible, it is desirable to minimize the number of items that must be taken in and out of the vacuum chamber. By leaving the STM in vacuum at all times, and limiting introduced items to tips and samples, the contamination potential is greatly reduced. Therefore, the tip changing procedure for the UHV STM has been designed to be performed inside the STM vacuum chamber. 
The central element of the tip exchange design is the removable tip holder assembly. The tip holder is a plug-shaped piece of insulating boron nitride that fits inside the inner STM tube, where it rests on the washer-shaped silicon disc that covers the bottom of the tube. A hole drilled through the tip holder (at a $15^{\circ}$ angle to the central axis) contains a metal sleeve into which the shank of an STM tip can be placed. The STM tip protrudes beyond the end of the scanning tube, through the hole in the center of the silicon disc.

Another hole, bored down through the center of the tip holder, gives the FIB access to the sample and tip. If the FIB is aimed down the central axis of the STM tube, then the FIB should intersect the STM tip 0.1" below the end of the tip holder. The distance that the tip extends from the tip holder can be adjusted outside the vacuum, so that the asinstalled tip-to-sample distance is within the range of the coarse $z$ adjustment ( $200 \mu \mathrm{m}$ ). Electrical contact to the tip is made via the wire handle by which the tip holder assembly is carried inside the vacuum. The handle extends from the tip holder to above the top of the inner tube. Figure 8 illustrates how a tapered fitting attached to the end of a manipulator can be used to wedge the handle under a contact pad. This slight spring-loading of the tip holder will produce adequate frictional force at the interface with the surface of the silicon washer to prevent transverse slippage of the tip assembly during scanning. When the tapered fitting is wedged between the opposite side of the handle and the STM tube it can free the wire from beneath the contact.

Sample Changing Final design of the exchangeable sample holder must wait for the results of testing to determine the UHV STM's ability to walk. If the STM proves mobile, then the sample holder will be a small metal block with an electrically-isolated, inclined surface on its top. The block will slide into a slot in the FIB sample holder upon which the STM stands. If the STM is stationary, then the metal block will include a shear-mode piezoelectric plate to be used for sample motion. 
The STM sample exchange rod will likely be terminated with a threaded extension that will mate with a corresponding threaded hole in the STM sample holder, as is done for sample intro systems on UHV SEM's. Electrical connections will be made to the FIB sample holder by slide contacts.

Predicted Performance From previous experience with other tube-based STM's such as are described in Section 1, it is expected that the scanning tube of the UHV STM should be able to image an area more than $1 \mu \mathrm{m}^{2}$ in size ( $\mathrm{x}$ and $\mathrm{y}$ sensitivity of $>50 \AA$ per volt), and the outer tube should provide offsets of 2-6 $\mu \mathrm{m}$ (sensitivities of $100 \AA$ per volt). On the basis of the published double tube STM designs, it is thought that the resonance frequencies will be $<5 \mathrm{kHz}$, and perhaps higher than $10 \mathrm{kHz}$.

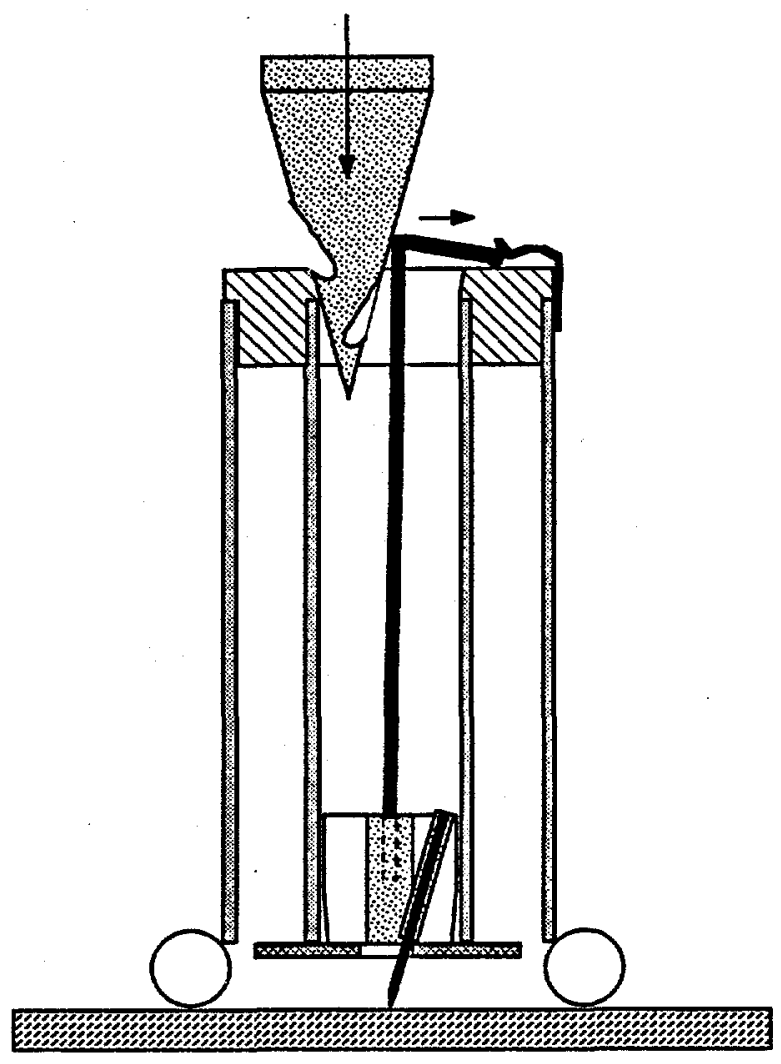

Figure 8: The UHV STM is designed to accommodate tip exchange inside the vacuum chamber. Two slots cut in opposite faces of the tapered fitting serve as hooks to grab the handle of the tip holder. The fitting also wedges the handle under the spring contact during tip installation, or frees the handle during tip removal. 
Immediate Plans Two initial steps must be completed before any nanomanipulation studies can be attempted:

1. The UHV STM must be brought to operational status

2. The techniques required to make deposition of clusters and droplets routine must be dereloped.

Step 1 will be aided by the fact that most of the electronic hardware and computer software needed already exists. The STM will be operated in air during initial evaluation of the coarse positioning technique and the imaging capability. Once the STM is operational it will be placed in the vacuum chamber and the STM / vacuum chamber interface will be debugged.

Development of the FIB particle deposition technique will be accomplished using visual feedback provided by SEM and STM images. The parameters of initial concern will be the particle size and density as functions of extracted ion current and beam energy. When the UHV STM becomes operable in vacuum, the first experiment will be an attempt to image FIB-created particles in situ.

\subsection{CONCLUSION}

This report has provided a brief introduction to nanotechnology, and particularly to areas in which STM can be applied. It is clear that the field is in its infancy, and that much remains to be learned; however, it is also clear that the STM's combination of abilities makes it uniquely suited as a tool for nanofabrication.

- a nanometer-sized, low-energy, high-current-density, reversible electron beam

- nanometer-resolution tip-positioning and imaging for placing and verifying modifications

- the ability to initiate highly localized, field-induced, or field-assisted phenomena.

The initial emphasis of research will be to study the use of STM for manipulating nanometerscale particles (or nanomanipulation). To do this, a new UHV-compatible STM is being built to fit under the FIB. The FIB will serve as the particle source for the nanomanipulation studies, but, in addition, the interface of the STM and the FIB should open up a wide range of possible experiments for both instruments. 


\section{References}

[1] M. Ringger, H.-R. Hidber, R. Schlögl, P. Oelhafen, and H.-J. Güntherodt, "Nanometer Lithography with the STM," Appl. Phys. Lett. 46, 832 (1985).

[2] D. W. Abraham, H. J. Mamin, E. Ganz, and J. Clarke, "Surface Modification With the Scanning Tunneling Microscope," IBM J. Res. Develop. 30, 492 (1986).

[3] M. A. McCord and R. F. W. Pease, "Scanning Tunneling Microscope as a Micromechanical Tool," Appl. Phys. Lett. 50, 569 (1987).

[4] J. K Gimzewski and R. Moller, "Transition from the Tunneling Regime to Point Contact Studied Using Scanning Tunneling Microscopy," Phys. Rev. B 36, 1284 (1987).

[5] T. R. Albrecht, M. M. Dovek, C. A. Lang, P. Grütter, C. F. Quate, S. W. J. Kuan, C. W. Frank, and R. F. W. Pease, "Imaging and Modification of Polymers by Scanning Tunneling and Atomic Force Microscopy," J. Appl. Phys. 64, 1178 (1988).

[6] R. Emch, J. Nogami, M. M. Dovek, C. A. Lang, and C. F. Quate, "Characterization and Local Modification of Atomically Flat Gold Surfaces by STM," Paper presented at STM '88 Conference (Oxford, England), July, 1988.

[7] W. E. Packard, Y. Liang, N. Dai, H. A. Blackstead, J. D. Dow, R. C. Jaklevic, and W. J. Kaiser, "Nanomachining of Gold Surfaces," Poster presented at STM '88 Conference (Oxford, England), July, 1988.

[8] R. S. Becker, J. A. Golovchenko, and B. S. Swartzentruber, "Atomic-Scale Surface Modification Using a Tunnelling Microscope," Nature 325, 419 (1987).

[9] T. H. P. Chang, D. P. Kern, E. Kratschmer, K. Y. Lee, H. E. Luhn, M. A. McCord, S. A. Rishton, and Y. Vladimirsky, "Nanostructure Technology," IBM J. Res. Develop. 32, 462 (1988).

[10] N. D. Lang, "Theory of Single-Atom Imaging in the Scanning Tunneling Microscope," Phys. Rev. Lett. 56, 1164 (1986).

[11] A. E. Bell, K. Rao, and L. W. Swanson, "Scanning Tunneling Microscope LiquidMetal Ion Source for Microfabrication," J. Vac. Sci. Technol. B 6, 306 (1988).

[12] A. Zangwill, Physics at Surfaces, Cambridge University Press, Cambridge, England, 1988 , ch. 14.

[13] T. R. Albrecht and C. F. Quate, "Atomic Resolution with the Atomic Force Microscope on Conductors and Nonconductors," J. Vac. Sci. Technol. A 6, 271 (1988).

[14] U. Dürig, J. K. Gimzewski, and D. W. Pohl, "Experimental Observation of Forces Acting During Scanning Tunneling Microscopy," Phys. Rev. Lett. 57, 2403 (1986). 
[15] R. C. Jaklevic and L. Elie, "Scanning-Tunneling-Microscope Observation of Surface Diffusion on an Atomic Scale: Au on Au(111)," Phys. Rev. Lett. 60, 120 (1988).

[16] M. A. McCord and R. F. W. Pease, "Lithography with the Scanning Tunneling Microscope," J. Vac. Sci. Technol. B 4, 86 (1986).

[17] R. Young, J. Ward, and F. Scire, "The Topografiner: An Instrument for Measuring Surface Microtopography," Rev. Sci. Instrum. 43, 999 (1972).

[18] M. A. McCord and R. F. W. Pease, "Lift-Off Metallization Using Poly (Methyl Methacrylate) Exposed with a Scanning Tunneling Microscope," J. Vac. Sci. Technol. B 6, 293 (1988).

[19] R. M. Silver, E. E. Ehrichs, and A. L. de Lozanne, "Direct Writing of Submicron Metallic Features with a Scanning Tunneling Microscope," Appl. Phys. Lett. 51, 247 (1987).

[20] U. Staufer, R. Wiesendanger, L. Eng, L. Rosenthaler, H.-R. Hidber, H.-J. Gütherodt, and N. Garcia, "Surface Modification in the Nanometer Range by the Scanning Tunneling Microscope," J. Vac. Sci. Technol. A 6, 537 (1988).

[21] D. W. Abraham, K. Sattler, E. Ganz, H. J. Mamin, R. E. Thomson, and J. Clarke, "Direct Imaging of $\mathrm{Au}$ and $\mathrm{Ag}$ Clusters by Scanning Tunneling Microscopy," Appl. Phys. Lett. 49, 853 (1986).

[22] A. M. Baró, A. Bartolome, L. Vasquez, N. García, R. Reifenberger, E. Choi, and R. P. Andres, "Direct Imaging of 13- $\AA$-Diameter Au Clusters using Scanning Tunneling Microscopy," Appl. Phys. Lett. 51, 1594 (1987).

[23] J. A. Stroscio, R. A. Dragoset, and R. M. Feenstra, "Structure and Electronic Properties of One-Dimensional Atomic Cs Chains on GaAs (110) by Scanning Tunneling Microscopy," Poster presented at STM '88 Conference (Oxford, England), July, 1988.

[24] D. Sarid, T. Henson, L. S. Bell, and C.J. Sandroff, "Scanning Tunneling Microscopy of Semiconductor Clusters," J. Vac. Sci. Technol. A 6, 424 (1988).

[25] H. Ohtani, R. J. Wilson, S. Chiang, and C. M. Mate, "Scanning Tunneling Microscopy Observations of Benzene Molecules on the Rh (111) - (3×3) (C6H6 + 2CO) Surface," Phys. Rev. Lett. 60, 2398 (1988).

[26] B. Blackford, M. Watanabe, D. Dahn, and M. H. Jericho, "STM Imaging of the Complete Bacterial Cell Sheath of Methanospirillium Hungatei," Poster presented at STM '88 Conference (Oxford, England), July, 1988.

[27] L. Feng, C. Z. Hu, and J. D. Andrade, "Scanning Tunneling Microscopic Images of Amino Acids," Poster presented at STM '88 Conference (Oxford, England), July, 1988. 
[28] E. Ganz, K. Sattler, and J. Clarke, "Scanning Tunneling Microscopy of Silver, Gold, and Aluminum Monomers and Small Clusters on Graphite," J. Vac. Sci. Technol. A 6,419 (1988).

[29] R. Gomer, "Possible Mechanisms of Atom Transfer in Scanning Tunneling Microscopy," IBM J. Res. Develop. 30, 428 (1986).

[30] R. J. Colton, S. M. Baker, J. D. Baldeschwieler, and W. J. Kaiser, "'Oxide-Free' Tip for Scanning Tunneling Microscopy," Appl. Phys. Lett. 51, 305 (1987).

[31] J. Wintterlin, J. Wiechers, Th. Gritsch, H. Höfer, and R. J. Behm, "Images of Individual Atoms on an Aluminum (111) Surface by STM," Paper presented at STM '88 Conference (Oxford, England), July, 1988.

[32] T. R. Albrecht, M. M. Dovek, C. A. Lang, P. Grutter, C. F. Quate, S. W. J. Kuan, C. W. Frank, and R. F. W. Pease, "Imaging and Modification of Polymers by Scanning Tunneling and Atomic Force Microscopy," J. Appl. Phys: 64, 1178 (1988).

[33] J. G. Pellerin, P. E. Russell, and R. O. Scattergood, "Focused Ion Beam Micromachining," Precision Engineering Center Annual Report Volume V (1987), pp. 294-306

[34] M. Francois, K. Pourrezaei, A. Bahasadri, and D. Nayak, "Investigation of the Liquid Metal Ion Source Cluster Beam Constituents and Their Role in Properties of the Deposited Film," J. Vac. Sci. Technol. B 5, 178 (1987).

[35] A. Wagner, T. Venkatesan, P. M. Petroff, and D. Barr, "Droplet Emission in Liquid Metal Ion Sources," J. Vac. Sci. Technol. 19, 1186 (1981).

[36] P. Sudraud, G. Ben Assayag, and M. Bon, "Focused-Ion-Beam Milling, ScanningElectron Microscopy, and Focused-Droplet Deposition in a Single Microcircuit Surgery Tool," J. Vac. Sci. Technol. B 6, 234 (1988).

[37] Ch. Gerber, G. Binnig, H. Fuchs, O. Marti, and H. Rohrer, "STM Combined with a Scanning Electron Microscope," Rev. Sci. Instrum. 57, 221 (1986).

[38] L. Vázquez, A. Bartolomé, R. Garcia, and A. M. Baró, "The Combination of a Scanning Tunneling Microscope with a Scanning Electron Microscope," Poster presented at STM '88 Conference (Oxford, England), July, 1988.

[39] F. Besenbacher, E. Laegsgaard, K. Mortensen, U. Nielsen, and I. Stensgaard, "Compact, High-Stability, 'Thimble-Size' Scanning Tunneling Microscope," Rev. Sci. Instrum. 59, 1035 (1988).

[40] J. W. Lydings, S. Skala, J. S. Hubacek, R. Brockenbrough, and G. Gammie, "Variable-Temperature Scanning Tunneling Microscope," Rev. Sci. Instrum. 59, 1897 (1988). 
[41] Ph. Niedermann, R. Emch, and P. Descouts, "Simple Piezoelectric Translation Device," Rev. Sci. Instrum. 59, 368 (1988). 



\section{ACADEMIC PROGRAM}

Problems and limitations associated with precision manufacturing can originate in the machine, the process, or the material. In fact, most problems will probably be caused by a combination of these factors. Therefore, improvement of current processes and development of new manufacturing methods will require knowledge of a multi-disciplinary array of subjects. The educational goal of the Precision Engineering Center is to develop an academic program which will educate scientists and engineers in metrology, control, materials, and the manufacturing methods of precision engineering.

The graduate students involved in the Precision Engineering Center have an annual stipend as research assistants. They can take up to 3 classes each semester while spending about 20 hours per week on their research projects. These students will also work in the Center full-time during the summer months.

The Precision Engineering Center began in 1982 with an emphasis on the mechanical engineering problems associated with precision engineering. As a result, the original academic program proposed was biased toward courses related to mechanical design and analysis. However, as the research program has developed, the need for complementary research in sensors, materials, and computers has become obvious. A graduate student capable of making valuable contributions in the computer area, for example, will require a significantly different academic program than in mechanical engineering. For this reason, the Center faculty have set a core curriculum and each student in the program is required to take at least 3 of these core courses. The remainder of the courses for the MS or the $\mathrm{PhD}$ degree are determined by the university or department requirements and the faculty committee of the student.

The required courses are:

- MAE 589 Metrology and Precision Engineering

- PY 516 Physical Optics

- MAT 500 Modern Concepts in Materials Science

- CSE 574 Real Time Systems

\section{PhD DEGREE PROGRAM}

The PhD program in Precision Engineering has been set up as a multi-disciplinary program, drawing upon courses throughout the University to provide background and expertise for the students. It should contain required courses to insure solid grounding in the fundamentals plus electives to prepare the student in his area of specialization. 
Because Precision Engineering is concerned with an integrated manufacturing process, students interested in computer control, materials, machine structure, and measurement and actuation systems are involved in the program. Student research projects include the wide variety of topics addressed in this report Each student's thesis should have an experimental component because Precision Engineering is basically a hands-on technology.

\section{MS DEGREE PROGRAM}

The Master of Science degree will have a higher percentage of application courses than the $\mathrm{PhD}$ degree. The emphasis will be to develop the foundation for involvement in precision engineering research and development. A total of 30 credits including 6 credits for the MS thesis is required. The thesis, while less comprehensive than the $\mathrm{PhD}$ dissertation, will be directed at important problems in Precision Engineering. Typically the MS program will take four semesters plus one summer.

\section{UNDERGRADUATE PROGRAM}

The undergraduate degree broadly prepares an engineering student for industrial activities ranging from product design and engineering sales to production implementation. Because a large share of engineers only have the BS degree, these will be the people who must implement the new technology developed in research programs like the Precision Engineering Center. Therefore, a way must be found to acquaint engineers at the BS level with the techniques, problems, and potential of precision manufacturing.

In most undergraduate degree programs only limited time is available for technical electives. However, these electives offer the student the opportunity to expand his knowledge in many different directions. Specific lecture and laboratory courses are being planned for the University undergraduate curriculum. These will be offered by Physics, Computer Science, Mechanical, and Materials Engineering Departments and will include:

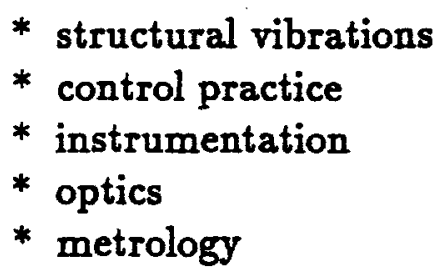

Undergraduate projects and summer employment have also been utilized to include undergraduate students into the research program of the Center. Two Material Science students were involved in the Center during the summer of 1988 under NSF funding directed by 
Prof. Russell. In addition, two undergraduate students in Mechanical Engineering built a prototype laser interferometer as a senior project.

\section{STUDY PLANS}

Study plans for several example students are given below both for the MS and the $\mathrm{PhD}$ degree. Because of the breadth of the field and the wide range of thesis topics, few if any study plans will be exactly the same. The plan will depend upon the student's background, his interests, his thesis topic, the department, and the chairman and members of his committee.

\section{Ph.D. PROGRAM IN MECHANICAL ENGINEERING}

\section{Major Courses:}

- MAE 541 Advanced Machine Design I

- MAE 640 Advanced Machine Design II

- MAE 505 Heat Transfer Theory \& Applications

- MAE 513 Vibrations of Mechanisms \& Structural Components

- MAE 560 Computing Fluid Dynamics

- MAE 589 Metrology in Precision Engineering

- MAE 615 Nonlinear Vibrations

- MAE 619 Random Vibrations

- MAE 614 Mechanical Transfer \& Machine Vibrations

- MAE 642 Machine Design Analysis

- MAE 699 Research

\section{Minor Courses:}

- MA 511 Advanced Calculus I

- MA 514 Methods of Applied Mathematics

- MA 530 Numerical Analysis II

- PY 516 Physical Optics

- ECE 516 System Control Engineering

- MAT 500 Modern Concepts in Materials Science

- ECE 613 Advanced Feedback Control

- ECE 555 Digital Image Processing 


\section{Ph.D. PROGRAM IN MATERIALS ENGINEERING}

\section{Major Courses:}

- MAT 610 X-ray Diffraction

- MAT 699 Research

- MAT 500 Modern Concepts in Materials Science

- MAT 556 Composites

- MAT 615 Transmission Electronic Microscopy

- MAT 595b Defect Analysis

- MAT 633 Advanced Mechanical Props

- MAT 589 Scanning Electron Microscopy

\section{Minor Courses:}

- PY 414 Electricity \& Magnetism

- STAT 515 Probability \& Statistics

- MAE 541 Advanced Machine Design

- MAE 640 Advanced Machine Design II

- MAE 589 Metrology in Precision Engineering

- PY 516 Optics

- MA 401 Advanced Differential Equations

\section{Ph.D. PROGRAM IN ME (FOR STUDENT WITH MS DEGREE)}

- ECE 516 System Control Engineering

- ECE 591 Gate Array Design

- MAT 500 Modern Concepts in Materials Science

- PY 516 Physical Optics

- MA 502 Advanced Mathematics for Engineers

- MA 524 Math Methods in the Physical Sciences

- MA 530 Numerical Analysis II

- MAE 532 Fundamentals of Metal Machining Theory

- MAE 541 Advanced Machine Design I

- MAE 589 Metrology in Precision Engineering

- MAE 619 Random Vibrations

- MAE 640 Advanced Machine Design II 


\section{MS PROGRAM FOR ME STUDENT}

- MAE 513 Vibrations of Mechanical \& Structural Components

- MAE 541 Advanced Machine Design I

- MAE 589 Metrology in Precision Engineering

- MAT 500 Modern Concepts in Material Science

- PY 516 Physical Optics • MA 501 Advanced Math for Engineers and Scientists I

- MA 502 Advanced Math for Engineers and Scientists II

- MAE 699 Mechanical Engineering Research

\section{MS PROGRAM FOR COMPUTER SCIENCE STUDENT}

- CSE 501 Operating Systems

- CSE 506 Computer Architecture

- CSE 512 Compiler Construction

- ECE 520 Fundamentals of Logic Systems

- CSE 606 Concurrent Software Systems

- MAE 589S Metrology

- MAE 589W Digital Control Systems

- ECE 558 Digital Image Processing

\section{MS PROGRAM FOR MATERIAL SCIENCE STUDENT}

- MAT 500 Modern Concepts in Material Science

- MAT 510 Crystallography

- MAT 515 Transmission Electron Microscopy

- MAT 595 Scanning Electron Microscopy

- MAT 612 Advanced SEM

- MAE 589S Metrology

- PY 516 Physical Optics

- ECE 539 IC Technology and Fabrication

- MAT 699 Research

\section{MS PROGRAM FOR PHYSICS STUDENT}

- PY 516 Physical Optics

- PY 552 Introduction to Structure of Solids I 
- PY 553 Introduction to Structure of Solids II

- PY 581 Quantum Mechanics I

- PY 582 Quantum Mechanics II

- PY 583 Advanced Classical Mechanics

- PY 585 Advanced Electricity and Magnetism I

- PY 586 Advanced Electricity and Magnetism II

- MAT 500 Modern Concepts in Material Science

- MAE 589S Metrology

- PY 699 Research

\section{SHORT COURSES AND TV COURSES}

Six graduate level courses, Scanning Electron Microscopy (MAT 512), Advanced SEM Surface Analysis (MAT 612), Modern Concepts in Materical Science (MAT 500), Mechanical Properties of Materials (MAT 505), and Advanced Mechanics of Materials (MAE 541) have been developed and taught as video courses offered nationwide via National Technological University. This past year approximately 120 students from industry and national laboratories participated in these courses. Future plans call for a MS program in Precision Engineering to be offered via the television network. 


\section{PUBLICATIONS}

\section{Papers}

1. Bifano, T.F., Dow, T.A., and Scattergood, R.O., Ductile-Regime Grinding of Brittle Materials: Experimental Results and the Development of a Model, Proceedings of Advances in Optical Fabrication and Metrology Including Large Optics, SPIE Vol. 966, August 1988.

2. Bifano, T.G., Dow, T.A., and Scattergood, R.O., Ductile-Regime Grinding: A New Technology for Machining Brittle Materials, to be published ASME TransactionsJournal of Engineering in Industry, 1988. 8-18-88.

3. Bifano, T.G., Dow, T.A., and Scattergood, R.O., Ductile-Regime Grinding of Brittle Materials, Ultraprecision in Manufacturing Engineering, edited by Manfred Weck, and Robert Hartel, published by Springer-Verlag, p. 22, May 1988.

4. Blake, P., Bifano, T., Dow, T.A., and Scattergood, R.O., Precision Machining of Ceramic Materials, American Ceramic Society Bulletin, Vol. 67, No. 6, p. 1038, June 1988.

5. Blake, P.B. and Scattergood, R.O., Ductile-Regime Turning of Germanium and Silicon, to be published, Proc. of Symposium on Machining of Ceramic Materials and Components, ACS-ASME, December 1988.

6. Carroll, J.T., III and Strenkowski, J.S., Finite Element Models of Orthogonal Cutting with Application to Single Point Diamond Turning, to be published, International Journal of Mechanical Sciences, 1988.

7. Falter, P.J. and Dow, T.A., A Diamond Turning Apparatus for Fabrication on NonRotationally Symmetric Surfaces, Ultraprecision in Manufacturing Engineering, edited by Manfred Weck, and Robert Hartel, published by Springer-Verlag, p. 187, May 1988.

8. Fawcett, S.C. and Keltie, R.F., Use Of A Fiber Optic Displacement Probe As $A$ Surface Finish Sensor, to be published Sensors and Actuators, 1989.

9. Fornaro, R.J., and Dow, T.A., A High-Performance Machine Tool Controller, to be presented 23rd IEEE Conference, Pittsburg, PA, October 1988.

10. Grigg, D.A. and Russell, P.E., Vibration Isolation and Mechanical Designs for Several $S T M ' s$ in $A$ ir and $U H V$, Proc. STM Conference, Oxford, England, July 1988.

11. Hren, J.J., and Shedd, G., Field Electron Emission, The Atom Probe and Scanning Tunneling Spectroscopy, Ultramicroscopy 24, p. 169, 1988. 
12. Keltie, R.F. and Fawcett, S.C., A Dual Channel Fiber Optic Displacement Probe for Structural Power Flow Measurements, to be published Sensors and Actuators, 1989. 9-21-88.

13. Keltie, R.F. and Allred, C.K., Measurement of Structural Power Flow in Vibrating Systems, Proc. of the 6th International Model AnalysisConference, Orlando, Florida, pp. $1675-1681,1988$.

14. Keltie, R.F., and Fawcett, S.C., Characterization of Fiber Optic Displacement Sensors for Precision Measurements, Proc. of the 6th International Model Analysis Conference, Orlando, Florida, 1988.

15. Luttrell, D.E. and Dow, T.A., Control of Precise Positioning System with Cascaded Colinear Actuators, Proc. of the 1988 American Control Conference, Atlanta, GA, June 15-17, 1988.

16. Russell, P.E., and Grigg, D.A., Mechanical Design Considerations for the Scanning Tunneling Microscope, presented ACS Conference, Toronto, Canada, June 1988.

17. Russell, P.E., Griffis, D.P., Shedd, G.M., and Woodward, W.S., A New Design for STM Control and Spectroscopic Data Acquisition, ACS Conference, Toronto, Canada, June 1988.

18. Scattergood, R.O., Srinivasan, S., Bifano, T., and Dow, T.A., R-Curve Effects for Machining and Wear of Ceramics, presented at 7th International Symposium on Ceramics, Bologna, Italy, December 1988.

19. Sparks, R.G. and Paesler, M.A., Micro-Raman Analysis of Stress in Machined Silicon and Germanium, to be published, J. Precision Engineering, 1988.

\section{Thesis/Dissertations}

20. Bifano, T.G., Ductile-Regime Grinding of Brittle Materials, $\mathrm{PhD}$ Dissertation, North Carolina State University, 1988.

21. Blake, P.N., Ductile-Regime Diamond Turning of Germanium and Silicon, PhD Dissertation, North Carolina State University, 1988.

22. Fawcett, S.C., Analysis and Development of a Multi-Channel Fiber Optic Probe for Structural Vibration Measurement, M.S. Thesis, North Carolina State University, 1988.

23. Loewenthal, M.D., Elastic Emission Polishing, M.S. Thesis, North Carolina State University, 1988. 


\section{Technical Reports}

Annual Report 1987, December 1987, 336 pages.

Semi Annual Progress Report, July 1988, 24 pages.

\section{AMERICAN SOCIETY FOR PRECISION ENGINEERING}

The objective of the American Society for Precision Engineering ASPE is to advance the arts, sciences, and technology of precision engineering, and to promote its dissemination through education and training, and to promote its use by science and industry. Founded in 1986, $A S P E$ provides a new focus for a diverse but important community. Other professional organizations have covered aspects of precision engineering, always as a side-line to their principle goals. $A S P E$ is based on the core of generic concepts necessary to achieve precision in any application; independent of discipline, $A S P E$ intends to be the focus for precision technology - and to represent all facets, from research to application.

The third annual meeting of the ASPE was held in Atlanta, Georgia, from October 24-27, 1988. This meeting was attended by over 225 engineers, researchers, and entrepreneurs from all over the world. 36 papers were given in technical sessions covering the following areas: Dimensional Metrology, Survey of Precision Technology Programs World-Wide, Material Effects, Controls and Controllers, Structural Dynamics in Precision Engineering, Applications of Precision Engineering, Machine Tool Design, Metrology, Fabrication Processes-Cutting and Grinding and Polishing. In addition, two Open Forums were held for brief presentations of recent technological advances and new results.

$A S P E$ approaches its fourth year of operation with optimism - an active community structure is in place with over 400 members, an Editorial Board is publishing a quarterly newsletter, and a publication agreement with the journal Precision Engineering has been officially arranged. In addition, plans are underway to publish a Monograph Series of Papers and to hold a Spring meeting on Sub-Surface Damage in Glass. The fourth annual meeting in 1989 is to be held jointly with the 5th International Precision Engineering Seminar in Monterey, California.

The Precision Engineering Center at NCSU has been an important influence in the growth of the $A S P E$. In addition, the $A S P E$ is a positive force for emphasizing the field of precision engineering, one that benefits the Precision Engineering Center by providing a dynamic exchange of information and ideas with other university and national laboratory researchers, 
development engineers, and industrial entrepreneurs in their quest for improved repeatability and accuracy in their manufacturing processes. 


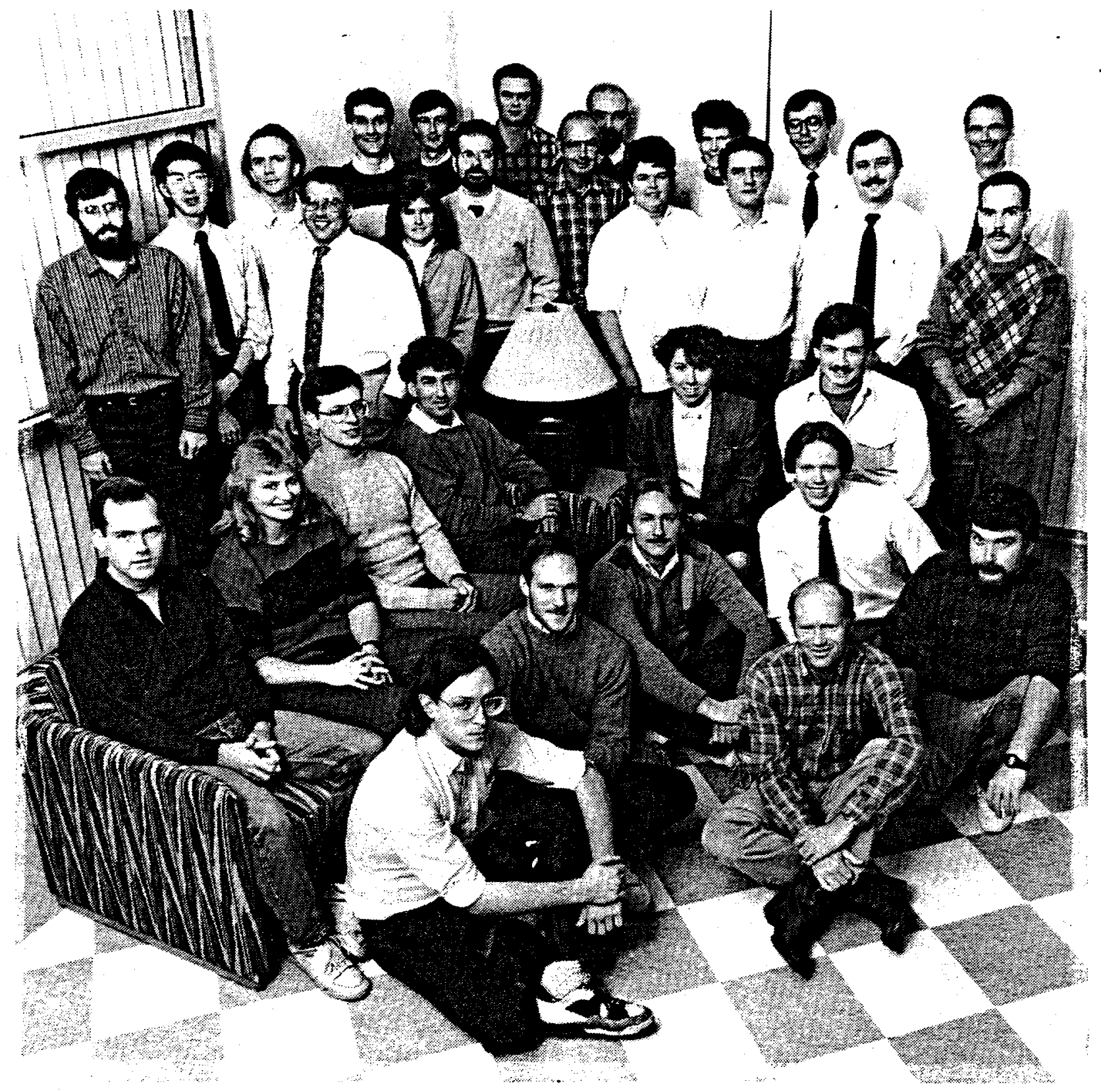

PRECISION ENGINEERING CENTER: FACULTY, STAFF, AND STUDENTS 


\title{
FACULTY
}

\author{
THOMAS A. DOW
}

Director, Precision Engineering Center

Professor, Department of Mechanical and Aerospace Engineering

BS, Mechanical Engineering, Virginia Polytechnical Institute, 1966

MS, Engineering Design, Case Institute of Technology, 1968

$\mathrm{PhD}$, Mechanical Engineering, Northwestern University, 1972

After receiving his $\mathrm{PhD}$ degree from Northwestern University in 1972, Dr. Dow joined the Tribology Section of Battelle Columbus Laboratories. For the next ten years, he performed and managed sponsored research programs ranging from the study of the wet-effectiveness of bicycle brakes to oil-in-water emulsions for large steel rolling mills to film thickness generated in a concentrated contact bearings. He developed test apparatuses, established analytical models, and corroborated those analysis with experimental measurements. Since joining the faculty at North Carolina State University in 1982, Dr. Dow has become involved in metrology, precision fabrication and control. He is one of the founders of the American Society for Precision Engineering.

During the coming year, Dr. Dow will continue to pursue research in the areas of metrology, apparatus design, and control. The long term goal is to develop schemes for in-process control of a precision fabrication process. To this end, research is continuing on studies of precision fabrication processes (diamond turning, grinding, and polishing) as well as high-speed control systems to control these processes. 


\section{ROBERT J. FORNARO}

Associate Professor

Computer Science Department

BA, Mathematics, St. Vincent College, 1963

MA, Mathematics, The Pennsylvania State University, 1965

$\mathrm{PhD}$, Computer Science, The Pennsylvania State University, 1969

Dr. Fornaro joined the Computer Science faculty at N.C. State in 1969. For the next several years he continued research in numerical analysis and contributed to the development of the Computer Science curriculum at NCSU. Since then he has been working in the computer systems area. He is especially interested in the relationship between operating systems software and multiprocessor architecture in real-time applications. He has developed an operating systems laboratory and an experimental concurrent programming environment in which high-level programming language constructs for concurrency and operating systems implementation can be compared and evaluated.

In the coming year, Dr. Fornaro will continue the development and evaluation of hardware and software for a high performance computer system to be used in control and signal processing applications. He will also investigate techniques for multiprocessor decomposition of high-speed real-time algorithms. 
RICHARD F. KELTIE

Associate Professor

Mechanical and Aerospace Engineering Department

BS, Mechanical Engineering, North Carolina State University, 1973

MS, Mechanical Engineering, North Carolina State University, 1975

$\mathrm{PhD}$, Mechanical Engineering, North Carolina State University, 1978

Dr. Keltie has a strong background in the study of the dynamics of elastic structures and their interaction either with other structures or with their surroundings. His research topics in this area have ranged from the study of the aerodynamic excitation of saw blades, to analytical modelling of truck tire carcass vibration and sound radiation, to the coupled vibro-acoustic response of elastic structures underwater. From 1978 to 1981, he was a member of the Senior Professional staff at the Applied Physics Laboratory of the Johns Hopkins University. Among his duties there, he initiated several studies regarding signal detection and tracking algorithms for use in sonar data processing. In 1981 he joined the faculty at North Carolina State University where he is currently Director of the Center for Sound and Vibration. Dr. Keltie's sponsored research activities have been in the areas of investigating the dynamic response of shells underwater and energy propogation and dissipation in large structures.

During 1989, Dr. Keltie will head the research efforts in machine tool structural dynamics. Development of precision machining processes has placed new demands on the ability to characterize and control the relative motion between the tool and workpiece. Two important aspects of this topic, that are presently under study, are the development of precise linear positioning techniques, and the investigation of the correlation between diamond machine tool dynamics and the resulting workpiece surface finish. The positioning project involves open loop control strategies implemented via ultra-high speed D/A converters to control tool position in diamond turning. The other project involves the measurement of dynamic tool force components during diamond turning, and the subsequent correlation between the dynamic characteristics of the tool, workpiece and machine structure and the resulting surface finish and accuracy of the workpiece. 
MICHAEL A. PAESLER

Associate Professor

Department of Physics

BS, Physics and Math, Beloit College, Beloit Wisconsin, 1968

MS, Physics, University of Chicago, 1971

$\mathrm{PhD}$, Physics, University of Chicago, 1975

After receiving his graduate degrees at the University of Chicago, Dr. Paesler spent one year as a Visiting Scientist at the Max-Planck-Institut fur Festkorperforschung in Stuttgart, FRG, working on the photo-Hall effect in gallium arsenide. From 1977-80 he was a Research Fellow at Harvard University in the Division of Applied Sciences. His work there involved investigations of hydrogenated amorphous silicon. Since joining the North Carolina State University Physics Department faculty in 1980, Dr. Paesler has been involved in a number of investigations in both fundamental and applied solid state physics.

At North Carolina State University, Dr. Paesler pursues research on two distinct fronts. In one program, his work is focused on understanding the structure and photo-induced structural changes observed in chalcogenide glasses. For this work optical probes as well as $x$-ray absorption spectroscopy are employed. In a separate program, research in Dr. Paesler's laboratory involves using micro-Raman spectroscopy to measure residual stresses in machined surfaces. These studies enable one to obtain stress profiles with microscopic resolution in three dimensions, and are crucial to understanding the physics of material removal.

During the coming year, Dr. Paesler will continue using micro-Raman spectroscopy in a comprehensive study of semiconductor machining using single point diamond turning. Other efforts will center on increasing lateral resolution using near-field optical techniques in micro-Raman applications. 
PHILLIP E. RUSSELL

Associate Professor

Department of Materials Science and Engineering

BS, Physics, Appalachian State University, 1975

MS, Physics, West Virginia University, 1977

$\mathrm{PhD}$, Materials Science and Engineering, University of Florida, 1982

After graduate work at the University of Florida, Dr. Russell joined the Solar Energy Research Institute (a DOE lab) in Golden Co. in 1980. There he developed a photovoltic materials and device characterization laboratory with emphasis on electron and ion beam analytical instrumentation. After three years at SERI, Dr. Russell joined JEOL, Inc. in Boston, Massachusetts, an electron optical instrumentation company where he lead the technical and application groups. One of his major projects was the development of an electron beam based integrated circuit metrology system. He was also involved in the development and application of focused ion beam systems and electron beam lithography systems, as well as numerous analytical instrumentation projects.

On joining North Carolina State University, Dr. Russell took on the role of Director of the Analytical Instrumentation Facility and has established graduate level courses in electron optics and electron optical instrumentation techniques. He was awarded the NSF Presidential Young Investigator Award in 1987. His research at NCSU in addition to Precision Engineering, are in the areas of Scanning Electron Microscopy, Auger Electron Spectroscopy and beam testing of integrated circuits.

Dr. Russell will act as principal investigator in the Scanning Tunneling Microscopy project and Focused Ion Beam Machining project during the coming year. This research group has 3 graduate students and very close interaction with the control fabrication and metrology groups within the Precision Engineering Center. Specifically, this group will complete the development of a scanning tunneling microscope this year with the goal of applying the technique to precision surface metrology. The Focused Ion Beam system will be automated and fundamental machining parameters investigated. 
RONALD O. SCATTERGOOD

Professor

Materials Science and Engineering Department

BS, Metallurgical Engineering, Lehigh University, 1961

MS, Metallurgy, Massachusetts Institute of Technology, 1963

$\mathrm{PhD}$, Metallurgy, Massachusetts Institute of Technology, 1968

R. O. Scattergood is a Professor in the Department of Materials Science and Engineering. He received BS degrees in Mining Engineering and Metallurgical Engineering from Lehigh University. His $\mathrm{MS}$ and $\mathrm{PhD}$ degrees were obtained in Metallurgy from M.I.T. In 1968 he became a member of the basic research staff in the Materials Science Division at the Argonne National Laboratory,. In 1981 he joined the faculty as a Professor of Materials Engineering at North Carolina State University.

Professor Scattergood's major research interests have been focused on the mechanical behavior of solids. He has worked in the areas of strengthening mechanisms in solids, dislocations in crystals, continuum theory of defects, radiation and diffusion effects and, most recently, in wear and fracture processes in ceramics and precision engineering, with emphasis on fabrication processes. He has expertise in both analytical methods and computer modeling as well as in mechanical testing methods and microscopy. He has published over 80 technical papers and reports.

During the coming year, he will direct several projects related to material removal characterization in precision fabrication processes including machining, grinding and EEM. 


\section{JOHN S. STRENKOWSKI}

Associate Professor

Mechanical and Aerospace Engineering Department

BS, Aerospace Engineering, University of Virginia, 1972

MS, Astronautics and Aeronautics, Massachusetts Institute of Technology, 1973

PhD, Applied Mechanics, University of Virginia, 1976

After completing his Ph.D., Dr. Strenkowski joined the Advanced Solid Mechanics Section of Battelle's Columbus Labs. While at Battelle, he participated in a variety of projects, which ranged from analyzing the vibrational and stress levels in offshore drilling platforms to predicting the residual stresses in weldments. This experience gave Dr. Strenkowski an awareness of the problems that face industry and the potential for their resolution through the application of advanced computational techniques.

Since joining the faculty of the Mechanical and Aerospace Engineering Department at North Carolina State University in 1978, Dr. Strenkowski has focused on computer-aided design, and finite element methods as applied to nonlinear problems involving large deformation and viscoplastic material behavior at elevated temperatures and high strain-rates. For the past five years he has been developing new computational models of the cutting process. This work has brought new understanding to the mechanics of material removal processes, which will signifiacntly change the way that cutting tools are designed to improve the productivity of cutting operations. Ultimately, this research is leading to new fundamental understanding that will result in significant advancements, such as the design of cutting tools for extended life and single-point diamond turning of ceramic materials.

For the coming year, Dr. Strenkowski will direct the development of advanced cutting models for precision fabrication processes. With these models, functional relationships can be found between cutting conditions and tool forces, chip and tool temperatures, tool wear, and subsurface residual stresses. The main emphasis in 1989 will be continuation of the study of the ductile-to-brittle transition in the cutting of brittle materials, and the development of a fracture mechanics model as applied to the residual stress field arising from cutting brittle materials. 


\section{STAFF}

KENNETH P. GARRARD

Research Assistant, Computer Science Department

BS, Computer Science, North Carolina State University, 1979

MS, Computer Studies, North Carolina State University, 1983

As a full-time research assistant, Mr. Garrard is studying the design of systems software that supports the development of high-speed real-time applications for special purpose multiprocessor computer systems. He has several years experience in academia and industry designing and implementing real-time systems. While in graduate school, Mr. Garrard's thesis work on concurrent programming environments was instrumental in the successful development of the Computer Science Department's Operating Systems Laboratory. He has also taught undergraduate courses in data structures, sorting, and systems programming. He has been employed as a scientific programmer for a pharmaceutical company, and as a consulting software engineer on a variety of projects, including real-time process control applications.

MARTHA U. NEELEY

Secretary, Precision Engineering Center

Ms. Neeley was a secretary for the Computer Survey Department, Research Triangle Institute and office manager for Executive Dimensions, Ltd. prior to joining the Precision Engineering Staff. This experience enabled Ms. Neeley to become proficient in word processing, especially using the VAX system. Ms. Neeley's varied background provides experience in secretarial, conference planning, public relations and administrative duties. 
Administrative Assistant, Precision Engineering Center

BS, Industrial Relations, University of North Carolina at Chapel Hill, 1983

Most recently, Ms. Smith served as Secretary to the American Society for Precision Engineering, located on North Carolina State University's Centennial Campus. Prior to joining the University system, she was employed as Human Resources Coordinator with the Dyestuffs \& Chemicals Division of CIBA-GEIGY Corporation, Greensboro, NC. With experience in both the areas of Human Resources and administration, she provides the Center with skills in supervision of personnel, budgetary management and public relations.

\section{LAUREN WILLIAM TAYLOR}

Research Assistant, Computer Science Department

AS, Electrical Engineering Technology, Behrend College, 1973 BS, Computer Science, North Carolina State University, 1977

Mr. Taylor, a full-time research assistant, is involved in designing both hardware and software to support fast, real time multiprocessor computer systems. He has worked mainly with microprocessor based systems and was involved in setting up the Computer Science Department's Microprocessor Laboratory. He has taught undergraduate courses in programming concepts, computer architecture and microprocessor interfacing. He has also been employed as a systems analyst with an energy management company. 


\section{GRADUATE STUDENTS}

Joseph D. Drescher is an MS student in Mechanical Engineering studying the precision diamond point turning process. He is concentrating on developing a model for diamond turning which can predict tool forces with the goal of correlating the model with experimentally measured forces. BSME (North Carolina State University), 1985

WILLIAM S. ENLOE is an MS student in Physics currently studying depth profiling of residual stresses using micro-Raman spectroscopy. He received a BS from the University of North Carolina at Ashville in 1986.

KARL J. FALTER is a MS candidate in Mechanical Engineering currently studying the measurement of structural power flow. The focus of his study is the identification and correction of error sources in the instrumentation and experimental apparatus used in previous power flow studies. He received a BS degree in 1987 from North Carolina State University.

PETER FALTER is a $\mathrm{PhD}$ candidate in Mechanical Engineering working on the real-time control of tool position in diamond turning. This work examines the feasibility of producing nonrotationally symmetric parts with a single point machining process. Mr. Falter joined the Lab in 1983 after receiving his BS and has been involved with several Center projects, most notably the construction of the PAUL diamond turning machine. Mr. Falter has accepted a position with Westinghouse, Baltimore.

STEVEN C. FAWCETT is a $\mathrm{PhD}$ student in Mechanical Engineering. Mr. Fawcett received his MS in Mechanical Engineering from NCSU in 1988. The title of his master's thesis is "Analysis and Development of a Multi-Channel Fiber Optic Probe for Structural Vibration Measurements". His current research activities include analysis of the dynamics of single point diamond turning and high speed data acquisition.

DAVID A. GRIGG is finishing his MS in Mechanical Engineering this spring (1989). His research has involved the design and building of Scanning Tunneling Microscopes and their use in the observation of machined surfaces. His studies have included vibrational analysis, piezoelectric technology, metrology, microscopy, and precision fabrication. He received a BS degree in Mechanical Engineering from North Carolina State University in 1986. His interests include metrology and precision actuation and measurement systems. 
GARY D. HIATT is a $\mathrm{PhD}$ student in Mechanical Engineering. Mr. Hiatt received his BS in Engineering Science \& Mechanics from North Carolina State University and a MS in Engineering Mechanics from Virginia Polytechnic Institute \& State University in 1981. After graduating from VPI, he worked as a mechanical engineer with the Naval Air Rework Facility at Cherry Point, North Carolina. Mr. Hiatt will work on developing a finite element model of the cutting of ceramics from a fracture mechanics viewpoint.

MICHAEL H. LUH is an MS student working on finite element modeling of brittle materials during orthogonal cutting. He received his BS in Mechanical Engineering from North Carolina State University in 1985. He joined the Precision Engineering Center in August 1987 to begin new research on the Modeling of Brittle Material Processing.

DAN E. LUTTRELL is a PhD student in Mechanical Engineering in the areas of Dynamic Systems and Controls. Mr. Luttrell received his BS degree in Mechanical Engineering from the University of Tennessee in 1977 and MS degree in Mechanical Engineering from North Carolina State University in 1987. After undergraduate school he served for six years in the U.S. Navy and then worked at DOE's Y-12 plant in Oak Ridge, Tennessee before joining the Precision Engineering Center. His research involved the application of system identification techniques to determine machine and cutting dynamics during the single point diamond turning process. Mr. Luttrell has accepted a position at Rank Pneumo in Keene, NH.

HAKAN OZISIK is a $\mathrm{PhD}$ student studying the development of an open loop control technique to minimize residual vibrations in positioning and cutting processes. Mr. Ozisik received his BSME (1982) and MSME(1986) from North Carolina State University. His MS thesis involved the numerical and analytical study of one-dimensional thermal phase change problems.

JOHN G. PELLERIN is an MS student in Materials Engineering currently studying focused ion beam micromachining. Mr. Pellerin received his BS in Materials Science and Engineering from the University of Florida in the Spring of 1987. His interests include semiconductor device processing, electron microscopy, and vacuum technology. 
GORDON SHEDD entered the Materials Science and Engineering PhD program, and the Precision Engineering Center, in Fall 1986 after moving to North Carolina from Boston. In Boston, he attended M.I.T. for two years, during which time he used a focused ion beam (FIB) to induce the selective deposition of sub-micron-wide strips of gold on silicon, work that lead to the M.S. degree in Nuclear Engineering (Sept. 1986). He received his undergraduate degree in Engineering Science from Penn State (May 1984). He has spent the past two years in STM development, and is currently interfacing a vacuum-compatible STM with the FIB. His next project is to use this STM to manipulate nanometer-scale particles that have been created with the FIB.

DENISE A. SKROCH received a BS in Physics and Computer Science from Appalachian State in 1986. Ms. Skroch is currently an MS student in Electrical and Computer Engineering. Her research work is in the area of Real Time Multiprocessor Architectures and associated systems software.

ELIZABETH F. SMITH has completed her thesis research on diamond turning of amorphous polymers. She is currently writing her Master's thesis, and expects to complete her MS in Materials Sciene and Engineering in early 1989.

MARY BETH SMITH is an MS student in Computer Studies. Ms. Smith received her BS degree in Computer Science from North Carolina State University (December 1987). Her research work is in the area of programming environments for multiprocessor systems, with emphasis on languages and compilers.

RONALD SPARKS is a $\mathrm{PhD}$ candidate currently engaged in research involving the use of Raman microprobe spectroscopy in measuring the residual stress in single point diamond machined crystalline silicon and germanium. He received his BS degree in Physics from the University of North Carolina at Charlotte in 1982. Mr. Sparks worked for one year testing digital electronics before enrolling in the graduate program. His interests include optical processes and measurements in semiconductors and ceramics. 


\section{NEW GRADUATE STUDENTS}

W. SCOTT BLACKLEY is an MS student in Materials Science and Engineering and is currently studying diamond turning of brittle materials with a specific interest in glass. He received his BS from North Crolina State University in Materials Science and Engineering in 1988.

ROBERT D. DAY is a PhD student in Materials Science as part of the Advanced Study Program at Los Alamos National Laboratory. He is investigating the application of the Atomic Force Microscope to Surface Metrology on Non-Conductors. He received a BSME and MSME from New Mexico State University in 1972 and 1974 respectively. He is employed by the Los Alamos National Laboratory where he has been working for the past 14 years.

KYLE W. LOSEKE is an MS student in Mechanical Engineering engaged in the study of nanofabrication technology. Specifically, he is researching the Elastic Emission Machining (EEM) process. Mr. Loseke received his BSME from North Carolina State University in 1988.

BYRON F. KNIGHT is an MS student in Mechanical Engineering currently studying Precision Linear Drives to develop systems capable of sub- $\mu$ in accuracies, including the Luttrell Constant Velocity Micro-Stepper. He received a BS (1982) from the University of Michigan. Mr. Knight was employed by Harris in Melbourne, FL and Unisys in Reston, VA prior to enrollment in the graduate program.

MICHELE MILLER is an MS student in Mechanical Engineering whose focus is developing a controller for the Diamond Turning Machine. Ms. Miller received her BSME from Duke University in 1986. Before joinging the Center in January 1989, she worked as a Manufacturing Engineer for General Motors in Detroit, Michigan.

HARLOW L. PAUL received his BS (1980) and MS (1982) degrees from the University of Texas at El Paso and is currently a PhD student in Mechanical Engineering. Mr. Paul's area of research is ductile grinding of brittle materials with an emphasis on machine design, dynamics and control.

STANLEY M. SMITH received his BS degree in Chemical Engineering from North Carolina State University in 1986. He was a student member of AICHE. During the summer of 1985, he worked for Glaxo, Inc., a pharmaceutical manufacturing company, as a Product and Process Investigation and kDevelopment technician. Mr. Smith will receive his MS in Materials Science and Engineering in December 1988 from NCSU. Under the auspices of the Thesis Parts Program Mr. Smith carried out his research at Argonne National Laboratory in the Materials and Components Technology Division. The title of his thesis is "Fabrication and Characterization of $\mathrm{Al}_{2} \mathrm{O}_{3} / \mathrm{SiC}$-whisker Composites". 
GRADUATES OF THE PRECISION ENGINEERING CENTER

$\underline{\text { Student }}$

Degree Date $\quad$ Company/Location

Kelly Allred

MS

June 88

Tom Bifano

PhD

June 88

Boston University

Boston, MA

Peter Blake

$\mathrm{PhD}$

Dec 88

NASA Goddard

Greenbelt, MD

Mark Cagle

MS

June 86

NASA-Langley

Norfolk, Virginia

John Carroll

$\mathrm{PhD}$

Jan 87

Cummins Engine Co.

Columbus, Indiana

Damon Christenbury

MS

June 85

Michelin Tire Co.

Spartanburg, SC

Jim Gleeson

MS

June 85

Battelle Columbus Labs

Columbus, Ohio

Jerry Kannel

PhD

June 86

Battelle Columbus Labs Columbus, Ohio

Mark Landy

MS

June 86

Battelle Columbus Labs Columbus, Ohio

Mike Loewenthal

MS

Dec 88

Cummins Engine Co.

Columbus, IN

Gary Mitchum

MS

June 87

Harris Corp.

Melbourne, Fla.

Larry Mosley

$\mathrm{PhD}$

June 87

Intel Corp.

Chandler, AZ 


\section{RESEARCH PLANS FOR 1989}

The research agenda of the Precision Enginieering Center since it began in 1983 has been to study and improve the capability of Precision Fabrication Processes such as diamond turning, grinding, polishing, focused ion beam machining, and nanofabrication. This emphasis will continue in 1989. In an effort to bring the multi-disciplinary group into each aspect of the problem, five (5) projects have been selected for 1989. Each of these projects involves faculty and students from several different disciplines.

\section{HIGH-PERFORMANCE COMPUTER CONTROL FOR PRECI- SION MACHINES AND INSTRUMENTS}

Objective: Definition and prototype implementation of high-speed real-time multiprocessor systems for use in precision engineering applications.

Background: A variety of precision engineering projects are computationally intensive and require fast real-time response to extend I/O activity. Commercially available computer systems are unsuitable for these and other related signal processing applications. Custom architectures (both hardware and software) are necessary to provide the computer power, speed and flexibility required. Also, new programming tools must be developed to describe and enforce timing information in these systems.

Scope of Work: This project will include hardware and software developments for computer systems and application to DTM and STM.

- Controller for Diamond Turning Machine including Real-Time control of Fast Tool Servo

- Digital Control and Real-Time Graphics for the STM

- Development of a Prototype Multiprocessor Architecture for Real-Time Applications

- Real-time Multiprocessor program development tools

Anticipated Results:Hardware and Software for a high performance computer system in useable prototype form. 


\section{PRECISION FABRICATION OF GLASS AND CERAMICS}

Objective: Establish the role of structural design and dynamics material properties and operating parameters for grinding and turning of brittle materials.

Background: Earlier work by Bifano and Blake demonstrated that ductileregime response can be achieved for sufficiently small cutting depths. The Eulerian model developed by Carroll provides useful information about the cutting process, however, it must be developed further to include residual stresses and fracture mechanics. The research needs to be extended to more fully characterize material response.

Scope of Work: This project will involve experimental grinding studies, material and surface characterization, model development, and process control.

- Modify PEGASUS and DTM for multiaxis grinding

- Perform systematic studies to determine ductile-regime transitions

- Study single-point turning of optical glass

- Measure properties of brittle materials using indentation-bend technique

- Characterize the surface damage using RAMAN, TEM and other appropriate methods

- Extend micro-Raman stress measurement analysis to include consideration of all stress components in the complete stress tensor

- Begin feasibility studies for micro-Raman control of single-point diamond machining

- Expand cutting models to include elastic stresses and fracture criteria.

- Develop correlations between machine, model and damage parameters

- Optimize the fabrication processes and develop real-time control

Anticipated Results: Determine the critical parameters that limit ductileregime response when fabricating brittle materials and control surface damage.

\section{DIAMOND TOOL FORCES AND TOOL WEAR}

Objective: To determine the influence of machine dynamics, tool edge condition, thermal effects, and materials on the static and dynamic tool forces encountered in diamond turning and the resulting surface finish.

Background: The work of students Drescher and Luttrell have indicated that the tool forces as well as the dynamic cutting stiffness are strongly related to the sharpness of the tool. Strenkowski's finite element model can be used to show the influence of the radius of the tool cutting edge on the stress state and tool forces. The model can also predict the temperature distribution in the workpiece, chip, and tool. 
Scope of Work: This project will require considerable instrumentation development as well as experimental cutting tests and analytical investigation. It will involve the following tasks:

- Develop a technique for tool edge sharpness measurement

- Measure the static and dynamic tool forces

- Relate the forces measured to the machine dynamics, tool edge condition and the resulting surface finish.

- Study minimum chip thickness as a parameter to evaluate the edge sharpness.

- Extend the finite element model to include cooling of the machined surface and the influence on the residual stress in the finished surface.

- Develop a wear model of the diamond tool based on the experiments and the analytical model.

Anticipated Results: An understanding of the relationship between the machine dynamics and the tool edge radius on the dynamic tool force components and surface finish. This understanding will be useful to monitor tool wear and will also result in an analytical cutting model which will predict the tool wear and residual stress.

\section{FUNDAMENTALS OF MICROMACHINING AND NANOMA- CHINING}

Objective: To examine potential machining processes with the fundamental capability of material manipulation on a sub-micrometer or nanometer scale. The processes to be studied include Elastic Emission Machining (EEM), Focused Ion Beam machining (FIB), and Scanning Tunnelling Machining (SEM). The objective will be to define the capability and limitations of these processes as machining techniques of the future.

Background: Loewenthal has demonstrated a first-generation EEM apparatus that can machine brittle materials and produce extremely smooth surfaces. A preliminary hypothesis for the material removal mechanism has been proposed but many issues remain unresolved. FIB has shown potential for machining feature sizes as small as $0.1 \mu \mathrm{m}$. Pellerin has developed a FIB system over the past year including a UHV chamber and a computer controlled scan system. He has demonstrated fabrication of line and square well features using this system on silicon wafers. An STM has been built by Shedd and others and demonstrated atomic resolution when imaging graphite specimens.

Scope of Work: The three proposed material removal/manipulation techniques will be studied to determine the parameters that influence the surfaces or features generated. 
- Systematic studies of EEM will be made to understand the material removal mechanisms, influence of chemistry, profile of material removed as a function of time, machine parameters (including ball properties, runout, etc.), and slurry effects. Experiments will also be undertaken to create a polished surface by scanning the ball over the surface.

- For FIB micromachining, a multiparametric study will be conducted to observe the effects of beam energy, current density, dwell time, and material parameters on the resulting machined feature. Profilometry and electron microscopy will be used to analyze the machined features and quantify removal and redeposition rates.

- Nanomanipulation will involve creating metal clusters using the FIB with sizes ranging from 2 to 100 atoms. These clusters will be characterized and manipulated with a in-situ, vacuum-compatible STM to be developed. The ability to move and group clusters and ultimately create nanometer scale structures will be investigated.

Anticipated Results: Analysis of the micromachined features will provide an understanding of the machining processes and allow selection of machining conditions to produce features of desired shape and surface finish. Capabilities and limitations of each micromachining technique will be defined as well as the potential for nanometer machining using the STM as a tool.

\section{TESTBED FOR PRECISION LINEAR MOTION}

Objectives: To develop expertise in the application of hydrostatic bearings, precision gaging systems, linear motion drive, and control systems to precision linear motion. The goal will be to improve the resolution and response of a . typical slide for a precision machine tool by open and closed loop techniques.

Background: The problems of controlling linear motion at the nanometer level have become clear during the implementation of the DTM controller. Precision machine tool slide are typically driven by dc motors through a lead/ball screw or a traction drive and supported by a hydrostatic fluid bearings or roller bearings. Laser interferometer feedback is used to produce the required resolution but the small motion of the motor dictated by this design is difficult to control at the limit of resolution of the sensor. In addition the stick/slip behavior of the slideway motion complicates the small resolution motion. High speed operation of a slide to dynamically correct for errors will need different systems for rapid response while retaining high resolution. Recent experimental results have demonstrated the ability to improve step response of a mechanical system by open loop signal tailoring techniques.

Scope of Work: This project will involve the design and construction of a test bed for the study of linear drives. It will include research into measurement systems, control systems, error compensation schemes, and electromechanical designs. The project will involve the following tasks: 
- Develop or purchase hydrostatic bearing

- Design and construct motor/slide coupling

- Integrate computer controller

- Design and fabricate traction drive

- Develop software correction for motion errors

- Characterize open loop and closed loop error correction schemes

- Build the constant-velocity PZT actuator

Anticipated Results: Current designs for linear motion are intended to be used in a quasi-static mode and not driven at high-speed. The results of this project are intended for drives for high-precision machine tools, measuring machines, and translation stages where high- resolution and high-speed disturbance rejection are required.

\section{DISCLAIMER}

This report was prepared as an account of work sponsored by an agency of the United States Government. Neither the United States Government nor any agency thereof, nor any of their employees, makes any warranty, express or implied, or assumes any legal liability or responsibility for the accuracy, completeness, or usefulness of any information, apparatus, product, or process disclosed, or represents that its use would not infringe privately owned rights. Reference herein to any specific commercial product, process, or service by trade name, trademark, manufacturer, or otherwise does not necessarily constitute or imply its endorsement, recommendation, or favoring by the United States Government or any agency thereof. The views and opinions of authors expressed herein do not necessarily state or reflect those of the United States Government or any agency thereof. 